Copyright

by

Žarko Pavlović

2008 


\section{The Dissertation Committee for Žarko Pavlović}

certifies that this is the approved version of the following dissertation:

\section{Observation of Disappearance of Muon Neutrinos in the NuMI Beam}

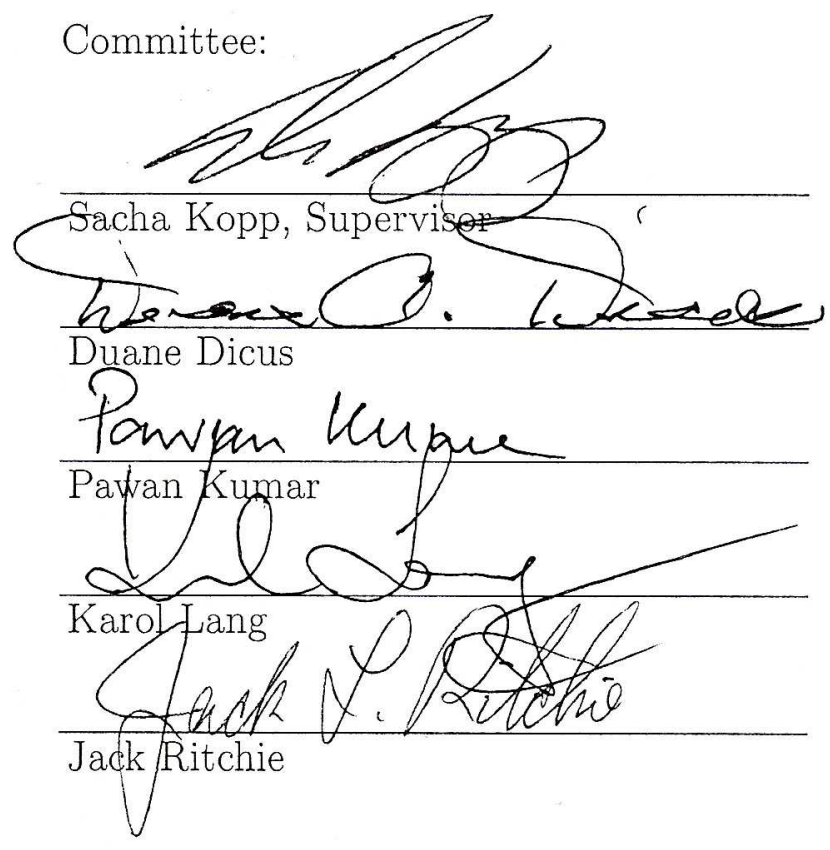




\title{
Observation of Disappearance of Muon Neutrinos in the NuMI Beam
}

\author{
by \\ Žarko Pavlović, BS
}

\author{
Dissertation \\ Presented to the Faculty of the Graduate School of \\ The University of Texas at Austin \\ in Partial Fulfillment \\ of the Requirements \\ for the Degree of \\ Doctor of Philosophy
}

\section{The University of Texas at Austin}

May 2008 
To my parents. 


\section{Acknowledgments}

This thesis would not have been possible if it wasn't for the help, knowledge, friendship and support from so many people. Many of them made the days I have spent at the University of Texas memorable.

I have had the great opportunity to have Prof. Sacha Kopp as my advisor. His passion, dedication and commitment to physics were a huge motivation, and I am greatly thankful for all his advice and guidance, as well as his genuine care about my progress.

I would like to thank Prof. Karol Lang for the valuable advices he gave me during the course of my study, and for his helpful comments on this thesis.

It would be hard for me to imagine the work in lab without the help of Marek Proga.

Many of my dear MINOS colleagues have often helped me with their knowledge, skills and friendship. Dharma Indurthy, Masaki Ishitsuka, Mike Kordosky, Laura Loiacono, Jasmine Ma, Jon Paley, Tom Osiecki, Rustem Ospanov, Tricia Vahle, Bob Zwaska, to only name a few. I would like you to know how grateful I am to you all. Thanks guys.

Mom, Dad, Maša, Radovan and Marija, you have given me so much love and support. Your enthusiasm for physics has always inspired me. 
My dear nephew Andrew, you brought me so much joy and happiness.

Finally, Ana - thank you for being there for me every step of the way.

I am so happy to have you with me on this journey.

\section{ŽARKo PAVLOVIĆ}

The University of Texas at Austin

May 2008 


\title{
Observation of Disappearance of Muon Neutrinos in the NuMI Beam
}

\author{
Publication No. \\ Žarko Pavlović, Ph.D. \\ The University of Texas at Austin, 2008
}

Supervisor: Sacha Kopp

The Main Injector Neutrino Oscillation Search (MINOS) is a two detector long-baseline neutrino experiment designed to study the disappearance of muon neutrinos. MINOS will test the $\nu_{\mu} \rightarrow \nu_{\tau}$ oscillation hypothesis and measure precisely $\Delta m_{23}^{2}$ and $\sin ^{2} 2 \theta_{23}$ oscillation parameters. The source of neutrinos for MINOS experiment is Fermilab's Neutrinos at the Main Injector (NuMI) beamline. The energy spectrum and the composition of the beam is measured at two locations, one close to the source and the other $735 \mathrm{~km}$ downstream in the Soudan Mine Underground Laboratory in northern Minnesota. The precision measurement of the oscillation parameters requires an accurate 
prediction of the neutrino flux at the Far Detector. This thesis discusses the calculation of the neutrino flux at the Far Detector and its uncertainties. A technique that uses the Near Detector data to constrain the uncertainties in the calculation of the flux is described. The data corresponding to an exposure of $2.5 \times 10^{20}$ protons on the NuMI target is presented and an energy dependent disappearance pattern predicted by neutrino oscillation hypotheses is observed in the Far Detector data. The fit to MINOS data, for given exposure, yields the best fit values for $\Delta m_{23}^{2}$ and $\sin ^{2} 2 \theta_{23}$ to be $\left(2.38_{-0.16}^{+0.20}\right) \times 10^{-3} \mathrm{eV}^{2} / c^{4}$ and $1.00_{-0.08}$, respectively. 


\section{Contents}

Acknowledgments $\quad$ v

Abstract vii

1 Neutrinos 1

1.1 Brief History of Neutrinos . . . . . . . . . . . . . . . . 1

1.2 Neutrino Oscillations ................ 5

1.3 Observation of Neutrino Oscillations . . . . . . . . . . . 10

1.4 Goal of this Thesis . . . . . . . . . . . . . 18

2 The MINOS Experiment $\quad 19$

2.1 NuMI Beamline . . . . . . . . . . . . . . . . . 21

2.2 MINOS Detectors . . . . . . . . . . . . . 34

3 Calculation of Neutrino Flux $\quad 57$

3.1 Meson Decay to Neutrinos . . . . . . . . . . . . . . 58

3.2 Meson Production in Nuclear Targets . . . . . . . . . . . . 62

3.3 The NuMI Target . . . . . . . . . . . . . . . . . 75

3.4 Particle Focusing . . . . . . . . . . . . . . 78

3.5 Target Hall . . . . . . . . . . . . . . . . . . 87

ix 
3.6 Decay Pipe and Hadron Absorber . . . . . . . . . . . . . . . . 90

3.7 Monte Carlo Biasing Techniques . . . . . . . . . . . . . . . . 92

3.8 Predicted Neutrino Flux at the ND . . . . . . . . . . . . 96

4 Predicted Neutrino Flux at the Far Detector 106

4.1 Neutrino Flux at ND and FD . . . . . . . . . . . . 107

4.2 Prediction of the FD Flux . . . . . . . . . . . . . . . . . 112

4.3 Error on the Predicted Flux at FD . . . . . . . . . . . . . . 117

5 Uncertainties in the Neutrino Flux Calculation 120

5.1 Methodology .................. 121

5.2 Alignment and Focusing Uncertainties . . . . . . . . . . . . 125

5.3 Hadron Production Uncertainty . . . . . . . . . . . . . . 158

5.4 Summary . . . . . . . . . . . . . . . . 166

6 Constraint on Flux uncertainties Using Near Detector Data 172

6.1 Introduction . . . . . . . . . . . . . . . . . 172

6.2 Parameterizing Hadron Production . . . . . . . . . . . . . 179

6.3 Fit Technique . . . . . . . . . . . . . . . . . . 183

6.4 Results of the Fit . . . . . . . . . . . . . . . . . . . . . . . 188

6.5 Neutrino Flux Uncertainty After Tuning . . . . . . . . . . . . 208

7 Changes in Running Conditions 210

7.1 Possible Explanations of Differences Between Two LE010/185kA

Data Sets . . . . . . . . . . . . . . . 211

7.2 Determination of Target Position . . . . . . . . . . . . 216

7.3 Impact on Oscillation Analysis . . . . . . . . . . . . . . . 220 
8 Observation of Neutrino Disappearance 223

8.1 Far Detector Data . . . . . . . . . . . . . . . 223

8.2 Background ..................... 230

8.3 Observed Energy Spectrum _. . . . . . . . . . . 235

9 Summary and Outlook $\quad 241$

9.1 Fit to Oscillation Hypothesis . . . . . . . . . . . . . . . . . . 242

9.2 Systematic Errors . . . . . . . . . . . . . . . . . 243

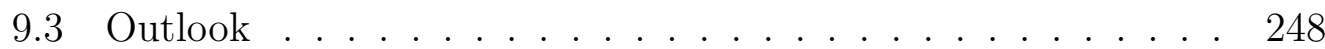

$\begin{array}{ll}\text { A Calculating The Beam Matrix } & 251\end{array}$

B Cross-check of NuMI Flux Using MiniBooNE Detector 253

B.1 Calculation of Offaxis Flux . . . . . . . . . . . . . . . 258

B.2 Hadron Production Uncertainties . . . . . . . . . . . . 265

B.3 Focusing Uncertainties . . . . . . . . . . . . . . 266

B.4 Angular Error . . . . . . . . . . . . . . . . . . 270

B.5 Summary of Off-axis $\nu$ Beam Uncertainties . . . . . . . . . . . 275

B.6 MiniBoone Data Analysis . . . . . . . . . . . . . . 275

B.7 Summary . . . . . . . . . . . . . . . 282

C Cross-check of NuMI Flux Using the Accompanying Muon $\begin{array}{ll}\text { Beam } & 283\end{array}$

C.1 Muon Alcove Acceptances . . . . . . . . . . . . . . . . . . . 285

C.2 Horn Current Scans . . . . . . . . . . . . . . . . . . . . . 295

C.3 Comparison to Muon Monitor Data . . . . . . . . . . . . . . . 300

C.4 Fit to Muon Monitor data . . . . . . . . . . . . . . . . 300

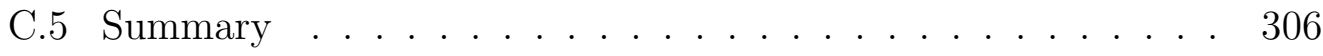


D Using GNuMI Ntuples for Muon Monitor Rates 312

D.1 Definition of Reference Frames . . . . . . . . . . . . . . 313

D.2 Angular Distribution of Daughters . . . . . . . . . . . 315

D.3 Energy Distribution of Daughters . . . . . . . . . . 318

D.4 Implementation into beam Monte Carlo . . . . . . . . . . . . 319

E Neutrino Flux Uncertainty 324

$\begin{array}{ll}\text { Bibliography } & 340\end{array}$

$\begin{array}{ll}\text { Vita } & 353\end{array}$ 


\section{Chapter 1}

\section{Neutrinos}

\subsection{Brief History of Neutrinos}

The history of neutrinos begins with Becquerel's discovery of radioactivity in 1886. Today we know that neutrinos interact only through the weak force, so the discovery of radioactivity, which was the first manifestation of the weak force in an experiment, opened the door to the discovery of a neutrino.

At the beginning of the twentieth century the known elementary particles were proton, electron and a photon. It was assumed that the nucleus $(\mathrm{A}, \mathrm{Z})$ was composed of $\mathrm{A}$ protons and $(\mathrm{A}-\mathrm{Z})$ electrons. The beta decay was explained as an emission of an electron from the nucleus. It followed from the conservation of energy and momentum that the energy spectrum of emitted electrons in beta decay should be discrete. By 1920s, studies performed by Meitner and Chadwick revealed that the spectrum of electrons is in fact continuous, so it appeared as if this process was violating the conservation laws. 
In 1930, Pauli proposed that introducing a new elementary particle could solve the problem. This particle had to be neutral to explain why it escapes unobserved and from the analysis of the electron spectra it followed that it had to be very light. Pauli suggested that the mass of this particle should be less than $1 \%$ of the proton mass and thus put the first limit on neutrino mass. Fermi dubbed Pauli's particle the neutrino and in analogy to quantum electrodynamics built the theory of beta decay [1]. The theory in its lowest order predicts the following processes ${ }^{1}$ :

$$
\begin{aligned}
& p \rightarrow n+e^{+}+\nu \\
& n \rightarrow p+e^{-}+\bar{\nu}
\end{aligned}
$$

It was immediately realized that observing such a particle would be very difficult. Bethe and Peierls [2] calculated the cross section for neutrino interactions using the Fermi theory of beta decay and found that it is extremely small. In fact, their conclusion was "...there is no practically possible way of observing the neutrino.".

It is not surprising that it took more than 20 years since neutrino was introduced to finally observe it in an experiment performed by Reines and Cowan [3]. To compensate for the minuscule cross section they used a large flux of neutrinos coming from the nearby nuclear reactor. In order to detect the neutrinos they used inverse beta decay process $\bar{\nu}+p \rightarrow n+e^{+}$. As a detector they used a liquid scintillator to which they added cadmium. The signature of a neutrino was observing two gammas from positron annihilation

\footnotetext{
${ }^{1}$ The neutron was discovered by Chadwick in 1932. and Fermi published his theory in 1933.
} 


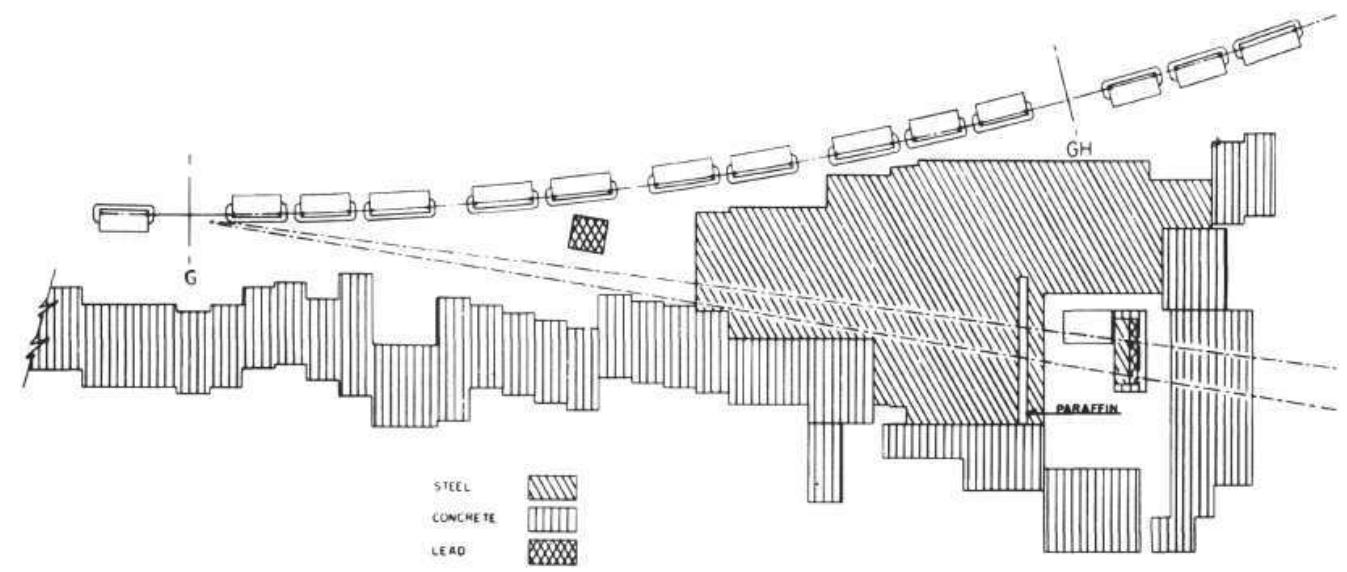

Figure 1.1: Plan view of the first accelerator neutrino experiment. The neutrino beam was produced by steering a proton beam on a nuclear target producing a beam of pions. The pions were allowed to decay in flight giving rise to neutrino beam. The shielding was used to remove all particles except for the neutrinos. (Figure taken from [4])

and a delayed gamma emitted after neutron capture on cadmium.

With the discovery of a pion in 1947 [5], a new source of neutrinos was discovered. It was observed that one of the products of the pion decay is a light neutral particle which was not a photon, so neutrino was a perfect candidate. Around 1960, Pontecorvo and Schwartz realized independently that one could use that to produce intense flux of neutrinos. The pions could be produced in great quantity by impinging high energy protons on a target. Schwartz, Lederman and Steinberger performed such an experiment in 1962 [4]. Figure 1.1 shows the plan view of the experiment. This was the first accelerator based neutrino experiment. Modern accelerator based neutrino experiments share similar features, using the proton beam to create a beam of mesons which decays into neutrinos. The detector is placed behind shielding which stops all particles but neutrinos. The outcome of the experiment was that they 
observed a different kind of neutrino than the neutrino coming from nuclear reactors. Since in pion decays a muon is created along with a neutrino, these neutrinos were dubbed muon neutrinos.

After the discovery of the $\tau$ lepton by Martin Perl in 1975 [6], it was expected that a third type of a neutrino will be found. Again it took more than 20 years before $\nu_{\tau}$ was finally observed at Fermilab in the DONUT experiment in 2000 [7].

Today neutrino interactions are described within the electroweak sector of the Standard Model (SM) which was developed in 1960s by Glashow, Salam and Weinberg. The model assumes the existence of three neutrinos (and three antineutrinos). The existence of exactly three weakly interacting light neutrinos was experimentally established [8]. The neutrinos in the model participate only in the weak force and thus couple only to $W^{ \pm}$and $Z^{0}$ bosons. Interactions mediated by $W^{ \pm}$are called charged current interactions and those mediated by $Z^{0}$ are called neutral current interactions. Figure 1.2 shows an example of two interaction types. The neutrinos played a crucial role in establishing the validity of the Standard Model. One of the predictions of the model was the existence of $Z^{0}$ boson. In 1973 , scientists at CERN used neutrinos to observe the neutral current events.

From the time Pauli introduced neutrino and placed the first limit on its mass, the limit was pushed by several orders of magnitude by various experiments. The current best limit on $\nu_{e}$ mass from direct measurement is $\mathrm{m}<$ $2.2 \mathrm{eV}$ (95\%CL) [10]. An even more stringent limit comes from cosmology, from which it follows that the sum of masses of the three neutrinos $\sum m_{\nu}<0.61 \mathrm{eV}$ (95\%CL) [11]. Since there was no direct evidence for neutrino masses, the neutrinos in the SM are assumed to be massless. This has several consequences, 


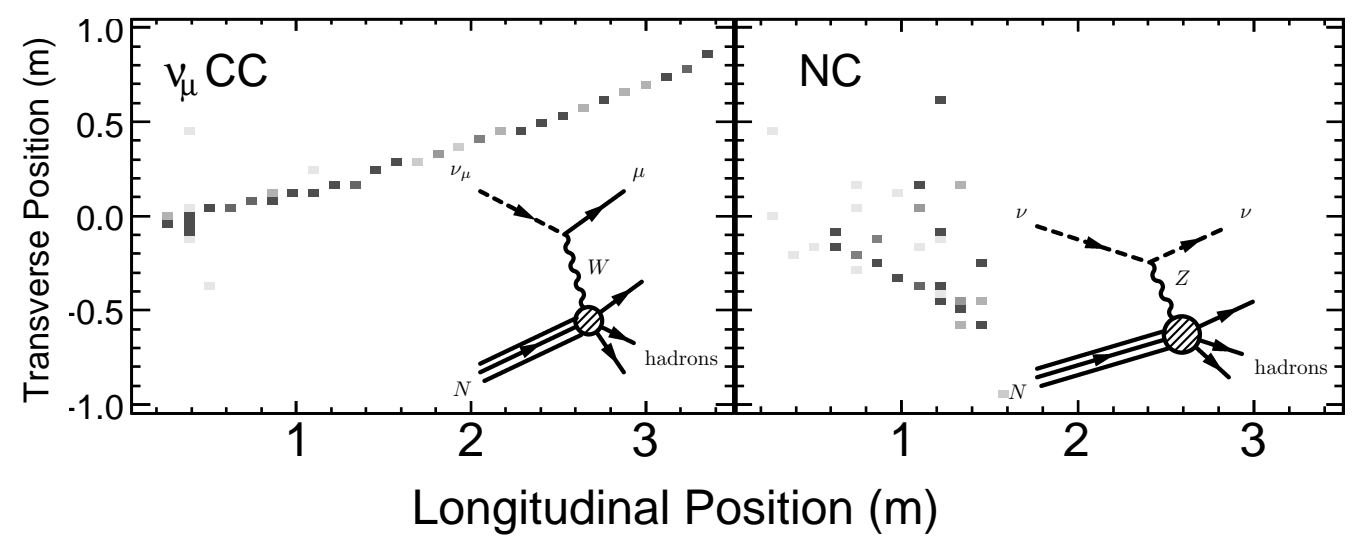

Figure 1.2: Muon-neutrino charged current (left) and neutral current event (right) simulated in the MINOS detector. Shaded rectangles indicate energy depositions (hits) in the detector. A muon created in CC interactions leaves a long track in the detector. (Figure taken from [9])

one of which is that mixing, observed in the quark sector, is not present in leptonic sector of SM.

\section{$1.2 \quad$ Neutrino Oscillations}

The idea of neutrino oscillations was first introduced by B. Pontecorvo in 1957 $[12,13]$. He proposed that in analogy to neutral kaon oscillations, neutrinos could change from one type into other. Since at that time only the electron type neutrino was observed, he considered the oscillations between neutrinos and antineutrinos. Maki, Nakagawa and Sakata [14] proposed in 1962 a new model involving two different flavors of neutrino, one associated with an electron and the other associated with a muon. This oscillation model resembles Cabbibo mixing seen in the quark sector. At that time the muon type neutrino was not yet observed, but there were some hints of its existence coming from the analysis of $\mu \rightarrow e^{-}+\gamma$ decays. In the model proposed by Maki, Nakagawa 
and Sakata, the neutrinos were assumed to be massive and the two neutrinos that interact with matter $\nu_{e}$ and $\nu_{\mu}$, the so called weak eigenstates, were in fact different mixtures of two mass eigenstates $\nu_{1}$ and $\nu_{2}$. In 1967, Pontecorvo developed the first phenomenological theory of two neutrino flavor oscillations $[15,16]$.

Assuming that there are only two neutrino flavors, the relation between the weak eigenstates $\nu_{\alpha}(\alpha=e, \mu)$ and mass eigenstates $\nu_{i}(\mathrm{i}=1,2)$ is given with $\left|\nu_{\alpha}\right\rangle=\sum_{j} U_{i j}\left|\nu_{j}\right\rangle$. The unitary matrix $U_{i j}$ can be parametrized using one mixing angle $\theta$.

$$
U=\left(\begin{array}{cc}
\cos \theta & \sin \theta \\
-\sin \theta & \cos \theta
\end{array}\right)
$$

The mass eigenstates are the eigenstates of the free particle Hamiltonian, so their time evolution is given with:

$$
\left|\nu_{i}(t)\right\rangle=e^{-i\left(E_{i} t-p_{i} L\right)}\left|\nu_{i}(0)\right\rangle
$$

where $E_{i}$ and $p_{i}$ are the energy and momentum of $\nu_{i}$. Here and in the following equations we use $c=\hbar=1$. Given that the neutrino mass is extremely small, in most of the cases the neutrinos can be considered as ultrarelativistic, so $E_{i}=\sqrt{p_{i}^{2}+m_{i}^{2}} \approx p_{i}+\frac{m_{i}^{2}}{2 p_{i}} \approx p_{i}+\frac{m_{i}^{2}}{2 E}$. Given that ultrarelativistic neutrinos move approximately at the speed of light distance $L \approx t$. Using the above equations, we can find the time evolution of the flavor eigenstate:

$$
\left|\nu_{e}(t)\right\rangle=\cos \theta e^{-i \frac{m_{1}^{2}}{2 E} t}\left|\nu_{1}(0)\right\rangle+\sin \theta e^{-i \frac{m_{2}^{2}}{2 E} t}\left|\nu_{2}(0)\right\rangle
$$

and similarly for $\left|\nu_{\mu}(t)\right\rangle$. The probability of finding a muon neutrino at some 
later time $t$ if at $t=0$ we start with pure $\left|\nu_{e}\right\rangle$ state is then given with:

$$
\begin{aligned}
P\left(\nu_{e} \rightarrow \nu_{\mu}\right) & =\left|\left\langle\nu_{\mu} \mid \nu_{e}(t)\right\rangle\right|^{2} \\
& =\sin ^{2} \theta \cos ^{2} \theta\left|e^{-i \frac{m_{1}^{2}}{2 E} t}+e^{-i \frac{m_{2}^{2}}{2 E} t}\right|^{2} \\
& =\sin ^{2}(2 \theta) \sin ^{2}\left(\frac{\Delta m_{12}^{2} L}{4 E}\right)
\end{aligned}
$$

where $\Delta m_{12}^{2} \equiv m_{1}^{2}-m_{2}^{2}$. Since initially we assumed that there are only two neutrino states, in this case the probability that we will observe a $\left|\nu_{e}\right\rangle$ at some later time $t$ is just 1 minus the right hand side of equation 1.2.

It is common to express $\Delta m^{2}$ in units of $\left[\mathrm{eV}^{2}\right]$ and also use units of $[\mathrm{km}]$ for distance $L$ and units of $[\mathrm{GeV}]$ for energy of neutrino $E$. When expressed in those units the following substitution can be made $\frac{\Delta m_{12}^{2} L}{4 E} \approx 1.267 \frac{\Delta m_{12}^{2} L}{E}$ and we find the widely used formula for two neutrino oscillation probability:

$$
P\left(\nu_{e} \rightarrow \nu_{\mu}\right) \approx \sin ^{2}(2 \theta) \sin ^{2}\left(1.267 \frac{\Delta m_{12}^{2} L}{E}\right)
$$

Since the oscillation probability depends on a difference in mass between the neutrino states, observing the neutrino oscillations implies that not all of the neutrinos are massless. It should also be noted that the oscillation probability is sensitive only to the difference in mass between the mass eigenstates and not to the absolute value of the mass.

The formalism complicates somewhat when third neutrino is introduced. The relation of three mass eigenstates to three weak eigenstates is again given through an unitary mixing matrix $U$, however, in this case more parameters are needed to parametrize the matrix. The matrix is usually parametrized [17] in terms of three mixing angles $\theta_{i j}$ and one complex phase 
$\delta$ :

$$
\left(\begin{array}{c}
\nu_{e} \\
\nu_{\mu} \\
\nu_{\tau}
\end{array}\right)=\left(\begin{array}{ccc}
c_{12} c_{13} & s_{12} c_{13} & s_{13} e^{-i \delta} \\
-s_{12} c_{23}-c_{12} s_{23} s_{13} e^{i \delta} & c_{12} c_{23}-s_{12} s_{23} s_{13} e^{i \delta} & s_{23} c_{13} \\
s_{12} s_{23}-c_{12} c_{23} s_{13} e^{i \delta} & -c_{12} s_{23}-s_{12} c_{23} s_{13} e^{i \delta} & c_{23} c_{13}
\end{array}\right) \cdot\left(\begin{array}{c}
\nu_{1} \\
\nu_{2} \\
\nu_{3}
\end{array}\right)
$$

where $c_{i j} \equiv \cos \theta_{i j}$ and $s_{i j} \equiv \sin \theta_{i j} .{ }^{2}$ The probability of finding $\nu_{\beta}$ some distance $\mathrm{L}$ away if initially it was $\nu_{\alpha}$ is given with:

$$
\begin{aligned}
P\left(\nu_{\alpha} \rightarrow \nu_{\beta}\right)=\delta_{\alpha \beta} & -4 \sum_{i>j} \Re\left(U_{\alpha i}^{*} U_{\beta i} U_{\alpha j} U_{\beta j}^{*}\right) \sin ^{2}\left(\frac{\Delta m_{i j}^{2} L}{4 E}\right) \\
& +2 \sum_{i>j} \Im\left(U_{\alpha i}^{*} U_{\beta i} U_{\alpha j} U_{\beta j}^{*}\right) \sin ^{2}\left(\frac{\Delta m_{i j}^{2} L}{2 E}\right)
\end{aligned}
$$

where $\Re$ and $\Im$ are real and imaginary part of the product.

Figure 1.3 shows the probability of Equation 1.4 as a function of $L / E$. The calculation assumes that the CP phase $\delta=0$. The non-zero CP phase would distort the probabilities somewhat and make the curves look different for neutrinos and anti-neutrinos. The values of mixing angles and mass splittings used in calculation come from current experimental data (Table 1.1), which will be discussed in the next section. The two different oscillation wavelengths are noticeable corresponding to two very different mass splittings $\Delta m_{12}$ and $\Delta m_{13} \approx \Delta m_{23}$. The amplitude of the oscillations or the strength of the mixing between the flavors is determined by mixing angles.

We have seen that neutrino oscillations imply that neutrinos have mass. Since neutrinos are massless in Standard Model the phenomenon of neutrino

\footnotetext{
${ }^{2}$ The most general form of mixing matrix would include two Majorana phases (if neutrinos are Majorana particles), however these were omitted here since oscillation experiments are not sensitive to them.
} 


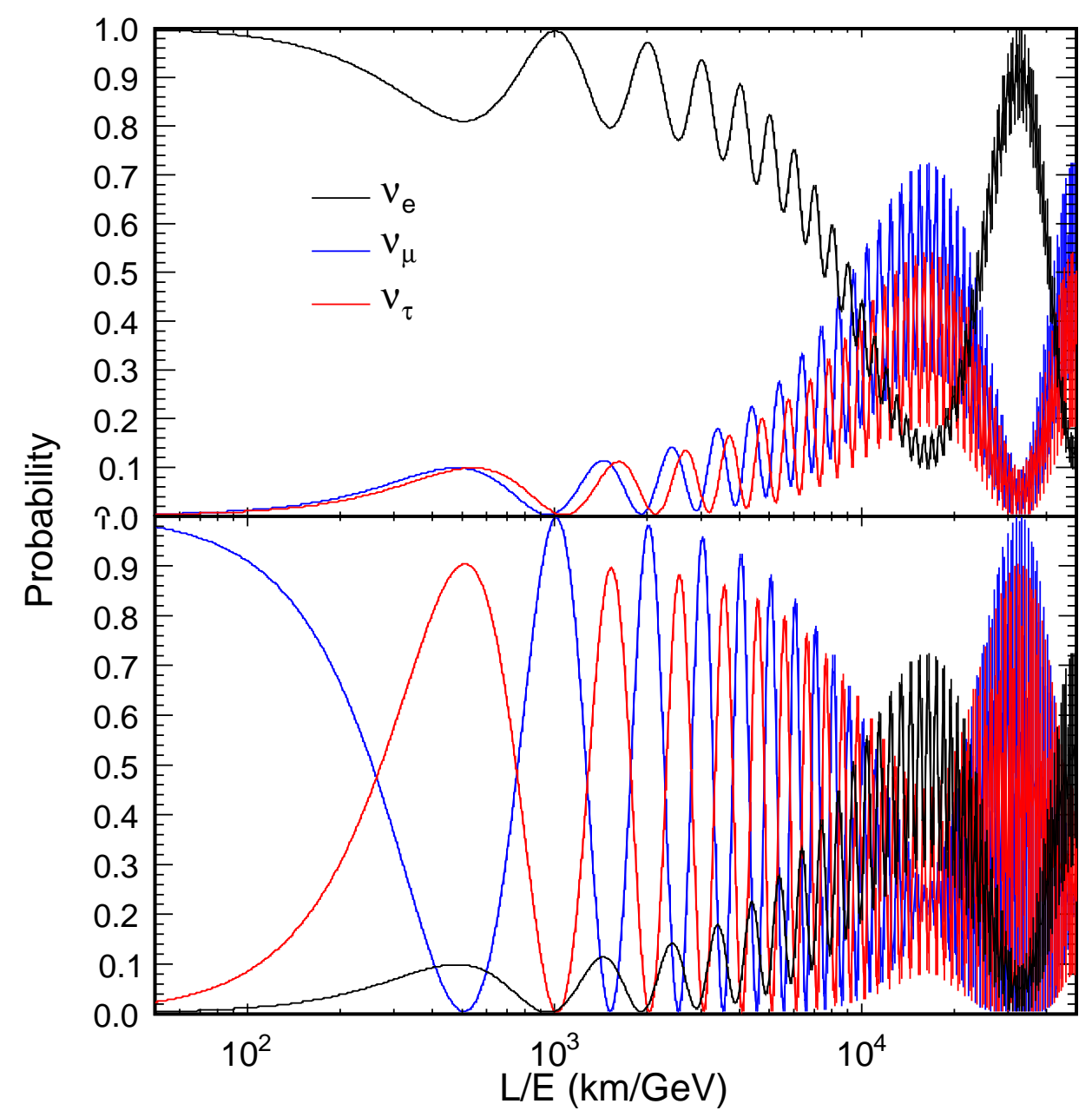

Figure 1.3: Probability of finding a neutrino of certain flavor as a function of $\mathrm{L} / \mathrm{E}$ if at $\mathrm{L}=0$ only electron neutrino (top) or muon neutrino (bottom) was present. It is assumed that $\Delta m_{21}^{2}=7.58 \times 10^{-5} \mathrm{eV}^{2}, \Delta m_{23}^{2}=2.4 \times 10^{-3} \mathrm{eV}^{2}$, $\theta_{23}=45^{\circ}, \theta_{12}=34^{\circ}, \theta_{13}=13^{\circ}$ and CP phase $\delta=0$. 
oscillations points toward the physics beyond the Standard Model. The model could in fact be extended to include neutrino masses. There is no fundamental reason why neutrino mass should be zero in Standard Model but it is not clear why would the neutrino mass be 6 orders of magnitude (or even more) smaller than it is for other fermions. A more plausible explanation is that the origin of neutrino mass comes from the physics beyond SM.

\subsection{Observation of Neutrino Oscillations}

There are 4 major categories of experiments studying neutrino oscillations divided depending on the source of neutrinos. These are solar, atmospheric, reactor and accelerator neutrino experiments. Among the 4 neutrino sources, the accelerator neutrino beams provide the greatest control over the source, while no control is possible in case of solar and atmospheric neutrinos. This makes the accelerator neutrinos essential tool for studying neutrino oscillations. The flexibility it offers makes it possible to test the oscillation hypotheses thoroughly and also to measure the oscillation parameters precisely. Several accelerator-based beams operate or are being built around the world [28, 30, $31,32,33]$.

Neutrino oscillation experiments typically are either appearance or disappearance experiments with detectors idealy located at L/E which corresponds respectively to either a peak or a valley of the oscillation probability shown in Figure 1.3. Appearance experiments, as the name suggests, measure the appearance of neutrinos of some flavor in the beam. To achieve that it is necessary to know the exact flavor composition of the beam coming from the source of neutrinos. The disappearance experiments usually measure flux of 
one particular flavor of neutrinos. The disappearance is established by knowing the flux of that particular flavor coming from the source.

A two detector technique, opposed to one detector, is often times used to make a precise measurements of oscillation parameters. The first detector is placed close to the source of neutrinos and measures the flux and neutrino flavor composition at $L / E \approx 0$ where neutrinos haven't yet oscillated. The other detector is placed further away at a convenient $\mathrm{L} / \mathrm{E}$, the peak or the valley of the oscillation probability depending on whether the experiment is appearance or disappearance. One detector experiments don't measure the flux at the source and therefore have larger uncertainties in the flux estimatees.

The neutrino oscillation experiments using accelerator neutrino beams can be divided into two categories depending on the length of the baseline, ie the distance of the detector from the source. The short-baseline experiments have a neutrino detector typically at the distance of $1 \mathrm{~km}$ or less from neutrino source. On the other hand long-baseline experiments have a detector several hundreds of kilometers away from the source.

The first hint of neutrino oscillations was seen in 1968 when Ray Davis performed a radiochemical experiment in which he was looking for the neutrinos coming from the Sun. The fusion processes in the Sun's core release a huge flux of electron neutrinos which pierce through the Earth. To detect neutrinos the experiment used a 100000 gallon tank filled with perchlorethylene, located 4800 feet underground in Homestake Mine. When a neutrino scatters of the chlorine atom it transforms it into a radioactive isotope of argon which can be extracted from the tank and counted. Comparing his observed neutrino rate with the expected rate calculated by John Bahcall, he saw a deficit. Observed rates were only $1 / 3$ of the expected rate. One of the possible explanations 


\begin{tabular}{ll} 
Experiment & Parameter \\
\hline \hline Chooz [19] & $\sin ^{2}\left(2 \theta_{13}\right)<0.19$ \\
SNO [20] & $\tan ^{2}\left(\theta_{12}\right)=0.45_{-0.10}^{+0.11}$ \\
KamLAND [21] & $\Delta m_{21}^{2}=7.58_{-0.20}^{+0.21} \times 10^{-5} \mathrm{eV}^{2}$ \\
Super Kamiokande [22] & $1.9 \times 10^{-3} \mathrm{eV}^{2}<\Delta m_{23}^{2}<3.0 \times 10^{-3} \mathrm{eV}^{2}$ \\
Super Kamiokande [23] & $\sin ^{2}\left(2 \theta_{23}\right)>0.92$
\end{tabular}

Table 1.1: The best measurements of neutrino oscillation parameters excluding the previously published MINOS [24] data. The parameters can be further constrained by combining data from various experiments.

was that some of the electron neutrinos on their long voyage oscillate into a different flavor which would pass through the detector unobserved.

The extremely long distance between the Sun and the Earth means that oscillation experiments involving solar neutrinos can probe very small mass splittings. Given the order of magnitude of the energy of solar neutrinos of $\sim \mathrm{MeV}$ and mean distance of $1.5 \times 10^{11} \mathrm{~m}$ the mass splittings down to the order of $\Delta m^{2} \sim E / L \sim 10^{-11} \mathrm{eV}^{2}$ can be probed. However, when data from solar experiments is combined with other neutrino oscillation experiments, the best agreement is achieved for values of $\Delta m^{2}$ of the order of $10^{-5} \mathrm{eV}^{2}$. This implies that what is observed on earth is in fact an averaged oscillated spectrum. To explain the solar neutrino data fully it is also necessary to include matter effects. The electron neutrinos as they move through dense matter are singled out since they can scatter of the electrons via both neutral and charged current interactions, while the muon and tau type neutrinos can only scatter via neutral currents. As a result, the oscillation probability is modified for neutrinos moving through matter. The effect is known as MSW effect named after S.P Mikheev, A. Yu. Smirnov and L. Wolfenstein who introduced it [18]. Since Davis's experiment neutrino oscillations have been observed in 
many other experiments $[25,26,23,27,20,21,28]$. The oscillations of neutrinos coming from the Sun were confirmed. In addition these experiments probed neutrinos coming from sources other than sun and helped built a consistent picture of neutrino oscillations. The four experiments providing the best measurements or limits on the oscillation parameters are listed in Table 1.1. What follows is a brief description of several experiments that tested the oscillation hypothesis.

\section{SNO}

The Sudbury Neutrino Observatory (SNO) is a 1 kton heavy water Cherenkov detector. The detector is capable of detecting neutrinos from ${ }^{8} B$ decays in the Sun which are on the higher energy end of the spectra. The neutrinos are detected through scattering on the deuterium either via CC or NC interactions. The detector is also capable of detecting the neutrino scattering on electrons (ES). While the NC reaction is sensitive to any neutrino flavor, the CC reaction is available only to $\nu_{e} \mathrm{~s}$. The ES is primarily sensitive to $\nu_{e}$, but it has some sensitivity to non-electron flavors as well. The fact that some reactions were available only for $\nu_{e}$ s while others were available to all flavors, enables SNO detector to measure both the total neutrino flux and separately the $\nu_{e}$ flux. SNO

measured flux from electron neutrinos to be $\phi_{C C}=1.68_{-0.05}^{+0.05}(\text { stat. })_{-0.09}^{+0.08}($ syst.) and flux of all neutrinos to be $\phi_{N C}=4.94_{-0.21}^{+0.21}(\text { stat. })_{-0.15}^{+0.15}$ (syst.) [20]. The total flux is in excellent agreement with the flux predicted from solar models, proving that electron neutrinos indeed change their flavor to other active neutrino types. 


\section{Super-Kamiokande}

Super-Kamiokande is a 50 kton water Cherenkov detector which is being used to study the neutrinos created by cosmic ray interactions in the atmosphere. The detector is capable of detecting and identifying interactions of both the electron and muon type neutrinos. The majority of atmospheric neutrinos are created in decays of charged pions $\pi^{ \pm} \rightarrow \mu+\nu_{\mu}\left(\bar{\nu}_{\mu}\right)$, and of daughter muons $\mu^{ \pm} \rightarrow e^{ \pm}+\nu_{e}\left(\bar{\nu}_{e}\right)+\bar{\nu}_{\mu}\left(\nu_{\mu}\right)$. Hence it is expected that the flux of muon type neutrinos is roughly double the flux of electron type neutrinos. The flux of atmospheric neutrinos is only known with $\sim 20 \%$, so to demonstrate the disappearance of neutrinos the Super-Kamiokande looked at the double ratio $R=\left(\nu_{\mu} / \nu_{e}\right)_{d a t a} /\left(\nu_{\mu} / \nu_{e}\right)_{M C}$. In the absence of oscillations the expectation would be that $R=1$, however the measured ratio was $R=0.61 \pm 0.03$ (stat.) \pm 0.05 (syst.) for events with energy below $\mathrm{GeV}$ and $R=0.66 \pm 0.06$ (stat.) \pm 0.08(syst.) for multi-GeV events. This was a clear evidence that atmospheric neutrinos disappear [29].

The Super-Kamiokande experiment also measured the oscillation parameters looking at the dependence of the $\nu_{\mu}$ flux on the zenith angle [23]. Furthermore, using a subset of data for which the distance from the creation of a neutrino L and the energy of neutrino E could be determined with high resolution, they found an expected sinusoidal dependence of Equation 1.3 [22]. Relatively big uncertainty in the flux, however results in the systematic error in the measured oscillation parameters. 


\section{KamLAND}

The Kamioka Liquid scintillator Anti Neutrino Detector (KamLAND) was designed to observe electron anti-neutrinos emitted from distant nuclear reactors. The heart of the detector is 1 kton of highly purified liquid scintillator. The detector is surrounded by 55 Japanese nuclear power reactors, each being an isotropic source of $\bar{\nu}_{e}$. The average distance between the reactor and the detector weighted by the flux is $L=180 \mathrm{~km}$ and the nuclear reactors emit neutrinos with energy in few $\mathrm{MeV}$ range. KamLAND observed 1609 events while the expected rate was $2179 \pm 89$ in absence of neutrino oscillations [21].

\section{CHOOZ}

The source of neutrinos for the $\mathrm{CHOOZ}$ experiment was a nuclear reactor as well. A liquid scintillator detector was used to detect the electron antineutrinos. The detector was located $1 \mathrm{~km}$ away from the reactor, while the energy of neutrinos was, similarly as for KamLAND $E \sim 3 \mathrm{MeV}$. The measured flux of $\bar{\nu}_{e} \mathrm{~s}$ agreed well with the calculated flux without any neutrino oscillations. Although the $\mathrm{CHOOZ}$ experiment did not see any oscillation signal, it provided the upper limit on the $\theta_{13}$ angle [19].

\section{$\mathrm{K} 2 \mathrm{~K}$}

Super Kamiokande detector which was used to observe the disappearance of muon neutrinos created by cosmic rays was also used in KEK to Kamiokande (K2K) experiment [28]. This was a first long-baseline neutrino oscillation experiment. The neutrino beam used in that experiment was produced using a $12 \mathrm{GeV}$ proton synchrotron accelerator at the High Energy Accelerator Re- 
search Organization (KEK) in Tsukuba city, Japan. The neutrino beam was steered toward the Super-Kamiokande detector which was $250 \mathrm{~km}$ away in Kamioka.

To check the direction and also measure the flux and energy spectrum of the neutrino beam precisely a near neutrino detector system was installed $300 \mathrm{~m}$ downstream from the proton target. The system was comprised of a 1 kton water Cherenkov detector and a fine grained detector system.

The K2K experiment started in 1999 and operated until 2004. Prior to the $\mathrm{K} 2 \mathrm{~K}$ experiment there were a few short-baseline accelerator based experiments that were sensitive to a higher $\Delta m^{2}$ region. Figure 1.4 shows the exclusion limits as well as the prefered parameter region by Super-Kamiokande experiment from the time before $\mathrm{K} 2 \mathrm{~K}$ result. It can be seen that these experiments were at such L/E that their sensitivity was at too high $\Delta m^{2}$.

During the period while $\mathrm{K} 2 \mathrm{~K}$ was running, $1.049 \times 10^{20}$ protons were delivered on production target. As a result, 112 beam-originated neutrino events were observed with an expectation of $158.1_{-8.6}^{+9.2}$ events without oscillation. For 58 events it was possible to reconstruct the neutrino energy and the spectrum distortion expected from oscillation was observed. The oscillation parameters measured in $\mathrm{K} 2 \mathrm{~K}$ experiment agreed well with those measured in Super Kamiokande detector using atmospheric neutrinos.

Even though $\mathrm{K} 2 \mathrm{~K}$ did not measure the oscillation parameters with more precision, it provided a complementary measurement and confirmed the disappearance of atmospheric neutrinos. The next generation long baseline experiments will use high intensity neutrino beams allowing for precise measurements of oscillation parameters and further testing of oscillation hypothesis. 


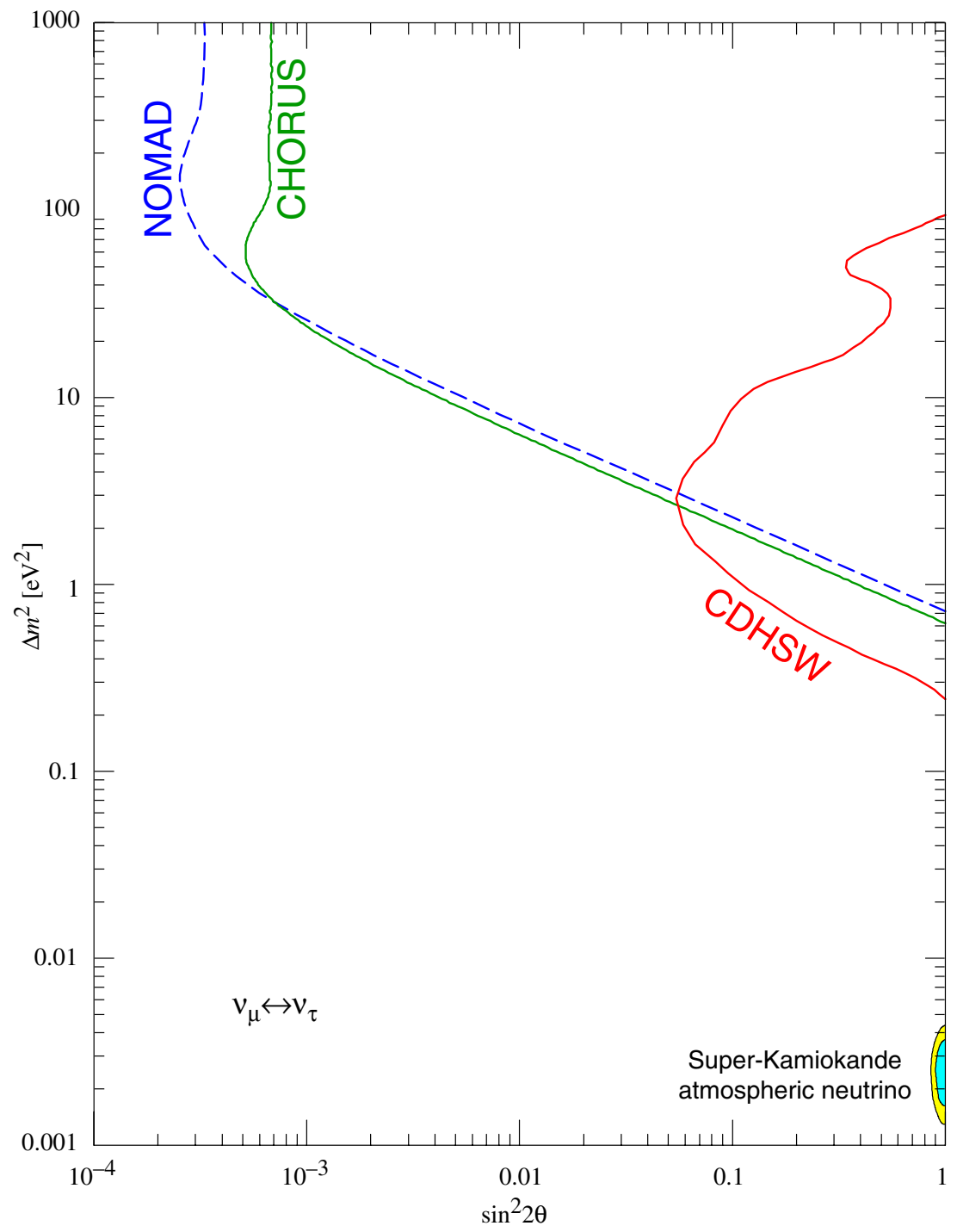

Figure 1.4: The exclusion limit and the prefered parameter region from $\nu_{\mu} \rightarrow$ $\nu_{\tau}$ oscillation experiments before K2K experiment (Taken from [34]). The experiments providing the exclusion limits at that time occured at too low $\mathrm{L} / \mathrm{E}$ and were senesitive only to higher $\Delta m^{2}$. 


\subsection{Goal of this Thesis}

To study the energy dependent modulation of neutrino flux predicted by neutrino oscillations it is necessary to know the energy spectrum of neutrinos coming from the source. The goal of this thesis is to accurately predict the neutrino flux for the Main Injector Neutrino Oscillation Search (MINOS) experiment whose primary physics goal is to test the $\nu_{\mu} \rightarrow \nu_{\tau}$ hypothesis and to measure precisely $\Delta m_{23}^{2}$ and $\sin ^{2} 2 \theta_{23}$. To achieve its goal MINOS uses an intense neutrino beam coming from Fermilab's NuMI (Neutrinos at the Main Injector) beamline. Like the K2K experiment, MINOS uses two neutrino detectors, the Near Detector which is close to the source of neutrinos and the Far Detector $735 \mathrm{~km}$ away. The thesis proceeds as follows:

In Chapter 2 the source of neutrinos for MINOS as well as the two MINOS neutrino detectors are described. The identification of neutrino interactions in MINOS detectors is discussed and the neutrino data from the Near Detector is presented.

Chapters 3-5 present the details of the calculation of neutrino flux coming from the NuMI beamline. Also discussed is how the measured neutrino energy spectrum in the Near Detector is used to predict the expected neutrino energy spectrum at the Far Detector and what are the systematic errors.

Chapters 6 and 7 show a technique that was developed for MINOS to further constrain the predicted flux using the measured neutrino energy spectrum in the Near Detector.

In Chapters 8 and 9 the comparison between the predicted neutrino spectrum and the spectrum measured at the Far Detector is shown and the implications are discussed. 


\section{Chapter 2}

\section{The MINOS Experiment}

The Main Injector Neutrino Oscillation Search (MINOS) is a two detector neutrino oscillation experiment. The source of the neutrino beam for MINOS is the Neutrinos at the Main Injector (NuMI) facility at the Fermi National Accelerator Laboratory (FNAL). MINOS uses two neutrino detectors to measure the neutrino beam composition and energy spectrum at two locations along the beam-line. The experiment was designed to measure the disappearance of muon type neutrinos from the beam.

Figure 2.1 shows the geographical layout of the two detectors. One of the detectors called Near Detector (ND) is located at Fermilab, close to the source of neutrinos and the other detector called the Far Detector (FD) is $735 \mathrm{~km}$ away in Soudan mine, Minnesota. The neutrino beam is produced at FNAL in a downward direction and goes through the Earth's crust before it reaches the FD. The ND samples the neutrino beam before neutrino oscillations occur, while the FD measures the oscillated neutrino energy spectrum. 

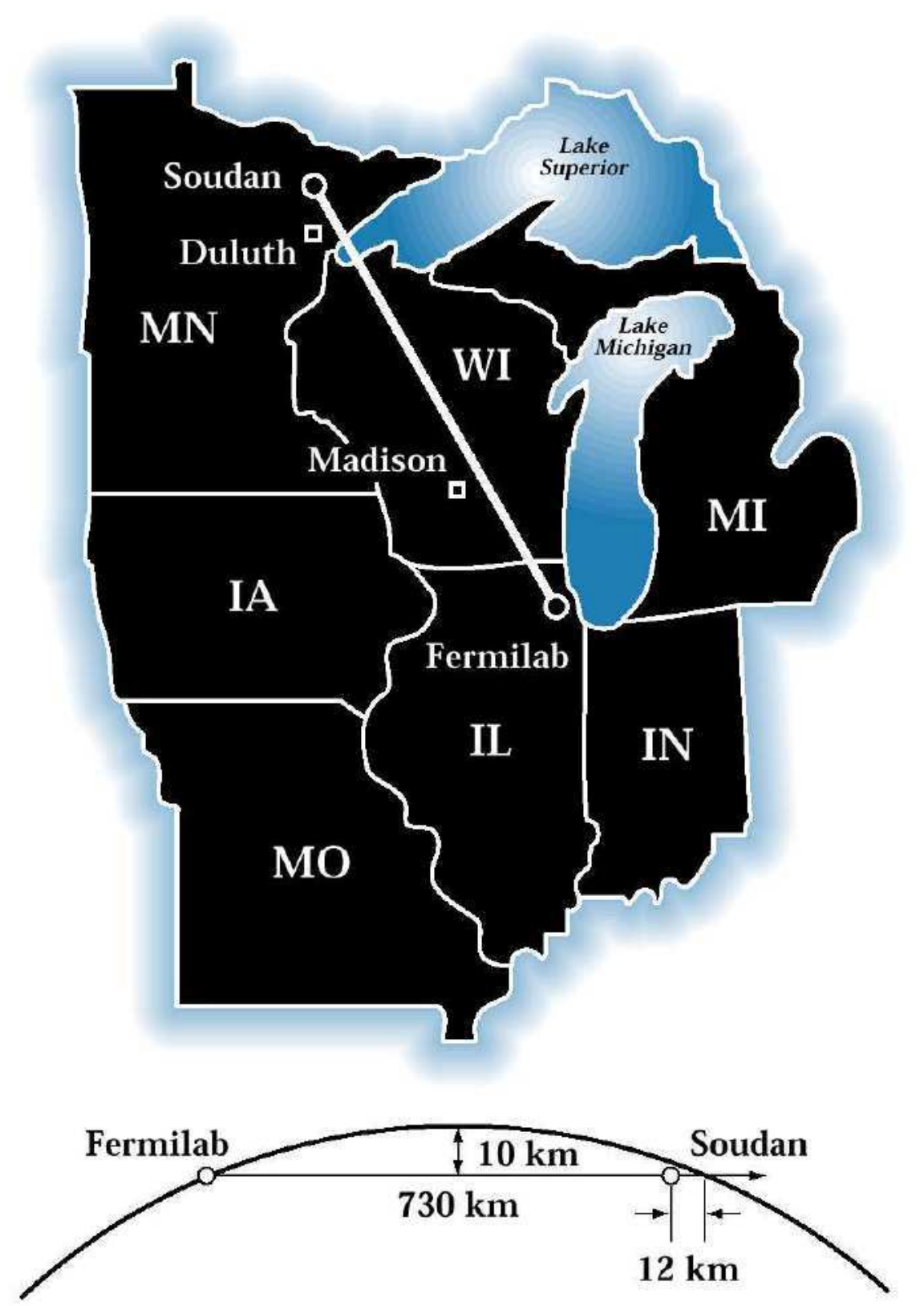

Figure 2.1: A geographical layout of MINOS detectors. The source of neutrinos for MINOS experiment is Fermilab's NuMI beam-line. The beam is steered toward the two MINOS detectors. The Near Detector (ND) is located close to the source of neutrinos on Fermilab site, while the Far Detector (FD) is $735 \mathrm{~km}$ away in the Soudan mine, Minnesota. Figure taken from [32]. 


\subsection{NuMI Beamline}

The advantage of accelerator beams over the other neutrino sources is that they provide much greater control of the source. The energy spectrum of neutrinos produced using accelerator beams can be adjusted. Also, neutrinos can be delivered in a controlled fashion allowing for better background rejection. The beam can be easily turned on and off and the duration of the beam pulse can be very short.

Neutrino beams are derived from meson decays. The branching ratios for those mesons that are most abundantly produced in beamlines make the production of muon type neutrino beams the easiest:

$$
\begin{aligned}
& \pi^{ \pm} \rightarrow \mu+\nu_{\mu} \quad \mathrm{BR} \approx 99.99 \%, \tau \approx 26 \mathrm{~ns} \\
& K^{ \pm} \rightarrow \mu+\nu_{\mu} \quad \mathrm{BR} \approx 63.4 \%, \tau \approx 12 \mathrm{~ns} \\
& K_{L}^{0} \rightarrow \pi+\mu+\nu_{\mu} \quad \mathrm{BR} \approx 27.2 \%, \tau \approx 52 \mathrm{~ns}
\end{aligned}
$$

The muons produced along with neutrinos can also give rise to neutrinos through $\mu \rightarrow e+\nu_{e}+\nu_{\mu}$ decay. Due to their relatively long, $2.2 \mu$ s lifetime in practice they are stopped before decaying and therefore they are not a significant source of muon neutrinos. For example, in the NuMI beam tunnel $(L=677 \mathrm{~m})$ approximately $70 \%$ of $10 \mathrm{GeV} \pi^{+}$will decay, while only $1 \%$ of $10 \mathrm{GeV} \mu^{+}$will decay. Muon decay is largely responsible for the $\nu_{e}$ component of the beam since the only other meson decays contributing to $\nu_{e}$ flux are $K_{L} \rightarrow \pi+e+\nu_{e}(\mathrm{BR} \approx 38.8 \%)$ and $K^{ \pm} \rightarrow \pi^{0}+e+\nu_{e}(\mathrm{BR} \approx 4.9 \%)$.

A beam of either neutrinos or anti-neutrinos can be selected by removing either negatively or positively charged mesons from the beamline. 


\subsubsection{Description of the NuMI Beamline}

Figure 2.2 shows a schematic view of the NuMI beamline. The NuMI beam uses $120 \mathrm{GeV}$ protons from the Main Injector that are extracted in a single turn. A $10 \mu$ s long proton spill is transferred over roughly $370 \mathrm{~m}$ to an underground target hall. In order to point the neutrino beam toward the MINOS detectors, the proton beam is steered downward.

The NuMI beam is operated in different running modes depending on the running mode of other experiments in the Fermilab accelerator complex. The Main Injector is filled with a number of batches from the $8 \mathrm{GeV}$ Booster Accelerator, whereupon it accelerates the protons to $120 \mathrm{GeV}$. Six booster batches can be consecutively fit in the Main Injector, but it is possible to put few more booster batches on top of the ones that are already inside. This batch structure remains in the spill that is delivered to NuMI. The beam spill is typically $10 \mu \mathrm{s}$ long when all of the protons from the Main Injector are delivered to NuMI, however occasionally either the first or the last batch is used for Fermilab's antiproton source in which case NuMI gets remaining 5 batches and the beam spill is $8.6 \mu$ s long.

Before the proton beam hits the target it passes through a collimating baffle as shown in Figure 2.3. The baffle provides protection to the target and focusing horns from stray protons. It is made of graphite and has a shape of a $1.5 \mathrm{~m}$ long cylinder with a $11 \mathrm{~mm}$ diameter inner bore.

The proton beam is steered onto a $\sim 1 \mathrm{~m}$ long graphite target which corresponds to almost 2 nuclear interaction lengths. The width of the target is only $6.4 \mathrm{~mm}$ in order to allow the secondary particles to easily exit. On the other hand it is relatively wide compared to the proton beams' transverse 

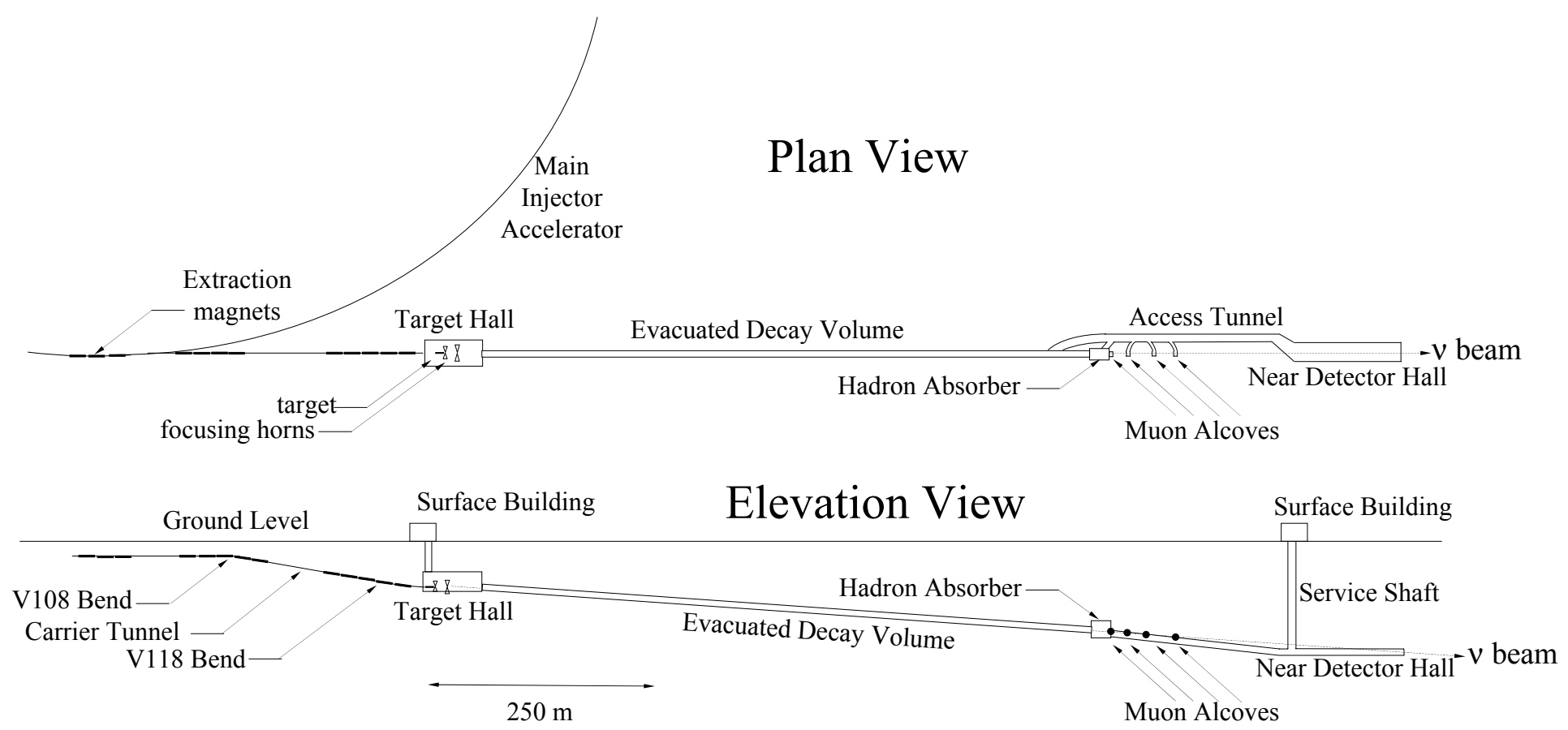

Figure 2.2: Plan and elevation views of the NuMI beam facility. A proton beam is directed onto a target producing a secondary pions and kaons. Positive mesons emanating from the target are focused into a decay volume using two magnetic horns. The mesons decay in flight giving rise to neutrinos. 
size. The size of the proton beam can not be arbitrarily small since that could damage the target [35].

Following the target are two magnetic horns [35] which are used to focus the positive particles down the decay volume (Figure 2.3). The horns bend $\pi^{+}$and $K^{+}$forward, in the direction of MINOS detectors, while bending away (defocusing) $\pi^{-}$and $K^{-}$. In addition the horns select a particular momentum of pion and kaon, which in turn establishes the mean neutrino energy, as discussed in Chapter 3.

The remnant hadrons that reach the end of the decay pipe are stopped by a hadron absorber [36, 37] comprised of aluminum, steel and concrete shielding, indicated in Figure 2.2. However, the absorber is not long enough to stop the muons which are produced along with neutrinos. Since the NuMI beam is steered through the Earth crust, the unexcavated dolomite rock is used to stop muons. The $240 \mathrm{~m}$ of rock separate the hadron absorber and the Near Detector, enough to stop even the most energetic muons coming from the decay pipe.

\subsubsection{Variable Energy Beam}

The NuMI beamline was designed to produce neutrinos that range from 1$16 \mathrm{GeV}$. However, the magnetic horns can typically focus only pions from a limited range of momenta and therefore produce a neutrino spectrum of limited range in energy. The NuMI beamline has the ability to adjust the energy spectrum of the neutrino beam [38]. This is achieved by changing the relative position of the target with respect to the focusing horns. Moving the target away from the horns has (Figure 2.3) the effect of focusing pions with 


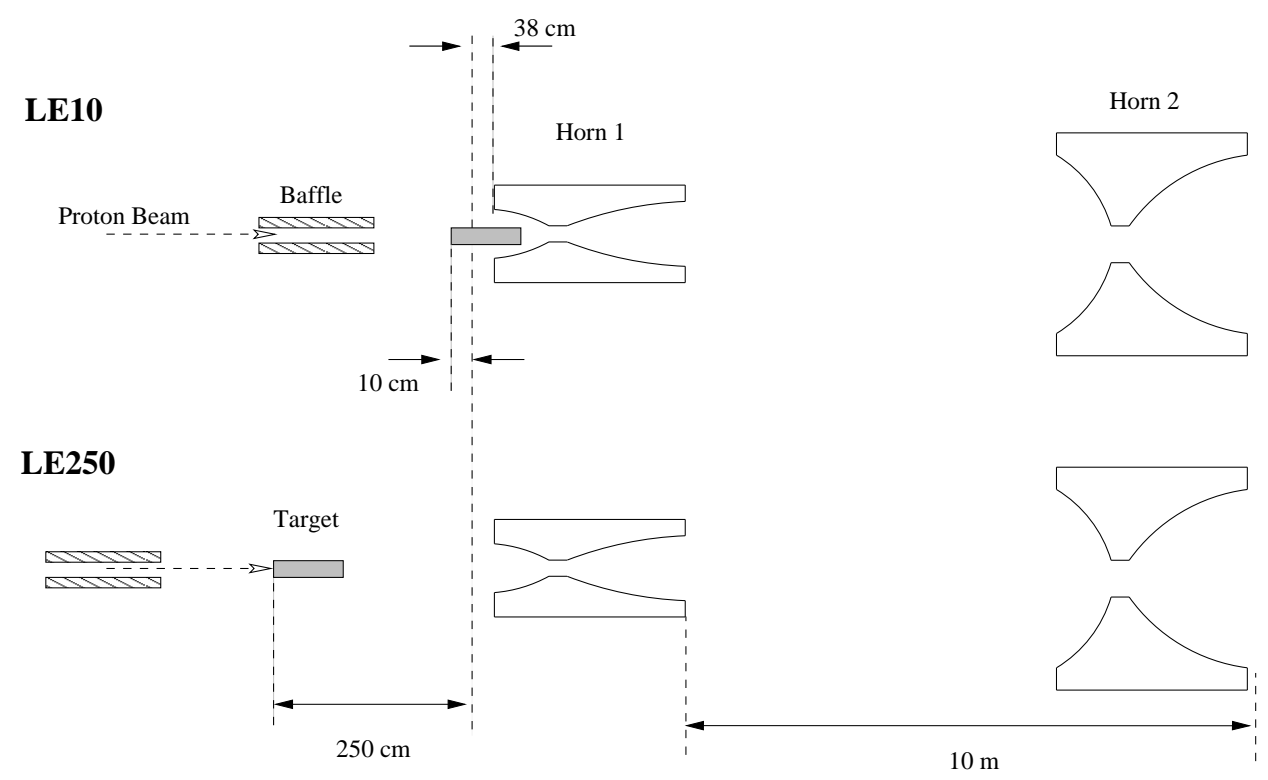

Figure 2.3: The NuMI target is fixed on a rail system which allows remote adjustment of target position relative to the horns. The NuMI beam has been operated in four different configurations with target at 10, 100, 150 and $250 \mathrm{~cm}$ away from first horn. Each configuration gives different neutrino energy spectrum. The beam configurations are named according to the distance between the target and the horns, ie LE10, LE100, LE150 and LE250. The point at $0 \mathrm{~cm}$ is the closest the target can be placed without touching the first horn.

higher momentum. Consequently this results in the neutrino spectrum shifted toward higher energies.

The target is mounted on a rail-drive system with $2.5 \mathrm{~m}$ of longitudinal travel. The system permits changing the target position remotely, without the need to directly access the horns and the target. Figure 2.3 shows the schematic view of the target and the focusing horns in two particular positions of the target. The NuMI beam has been operated in 4 different configurations, corresponding to 4 different target positions.

In addition to the target position it is also possible to adjust the strength of the horns' focusing magnetic field. This also has an impact on neutrino flux 
since it changes the amount of focusing received by the $\pi$ and $K$ mesons.

Figure 2.4 shows the calculation of the neutrino energy spectrum (described in Chapter 3) in three different beam configurations. The simulation indeed shows the shifting of the neutrino spectrum toward the higher energies as the target is pulled away from the horns. The same can be seen in Figure 2.5 which shows the position of the peak of neutrino energy distribution as a function of the relative distance between the target and the horns.

\subsubsection{Instrumentation}

The NuMI beam is monitored at each stage. Various devices monitor the properties of the primary proton beam from the point of extraction up to the target region. The secondary and tertiary beam comprised of the hadrons produced in the target and muons created along with neutrinos are being monitored by stations at the end of the NuMI decay pipe.

The proton beam intensity is measured using Toroid Intensity Monitor Integrators. One is located at Main Injector extraction point and the other is immediately upstream of the target station.

The position of the proton beam is measured at 24 capacitative "Beam Position Monitors" (BPMs). Each BPM consists of two cylindrical electrodes and measures the position in either horizontal or vertical plane by the image charge induced on the plates by the beam.

In addition to BPMs, Secondary Emission Monitors (SEMs) measure the beam position and profile [39]. These monitors consist of $5 \mu \mathrm{m}$ thick Titanium foil which is placed in the path of the proton beam. The secondary electrons ejected by the beam are used to make a measurement. Segmentation 


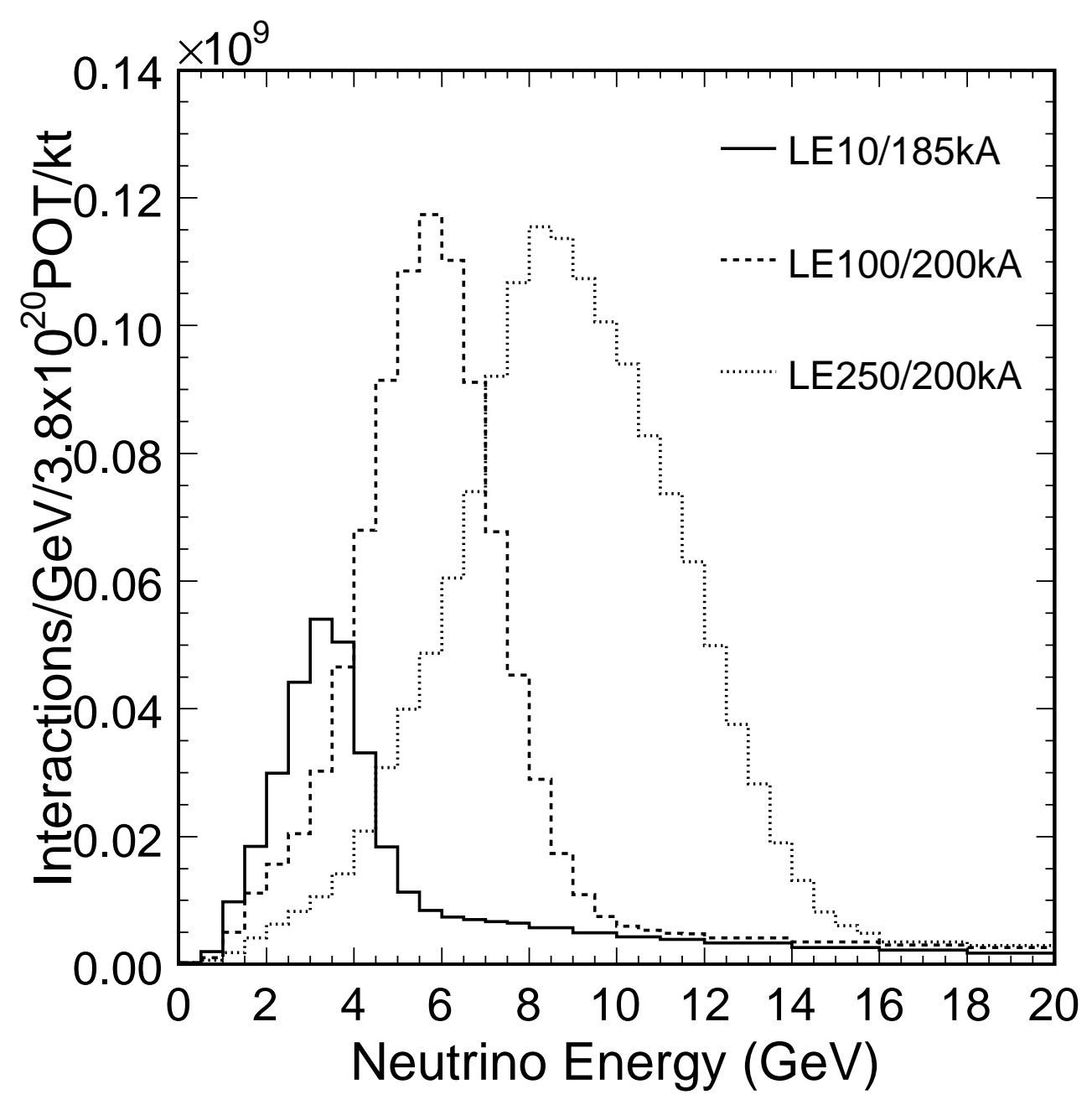

Figure 2.4: The calculation of the energy spectrum of neutrino Charged Current interactions for three different target positions (Figure 2.3). 


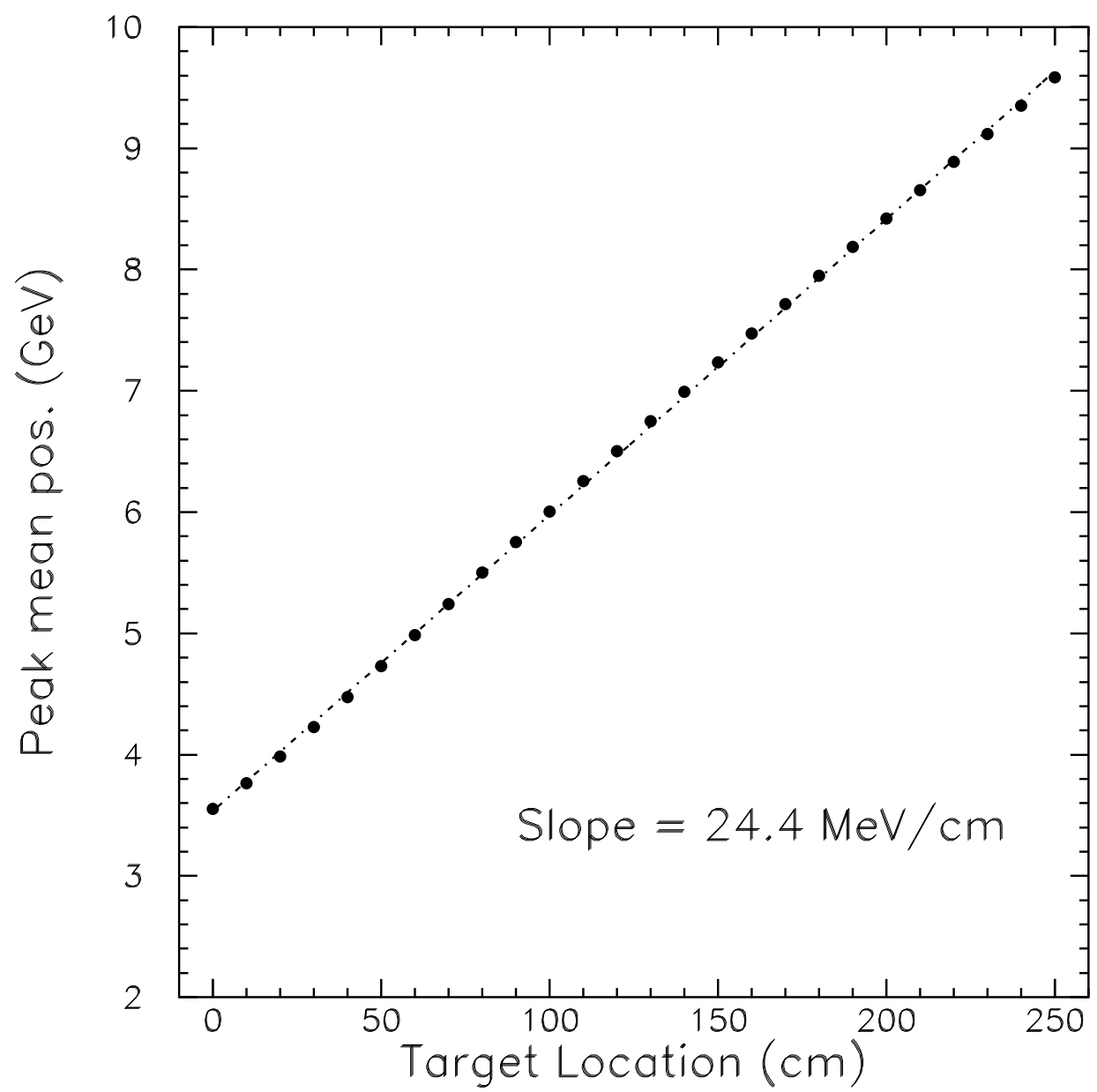

Figure 2.5: The peak position of the calculated neutrino energy spectrum (Chapter 3) as a function of target longitudinal position (Figure 2.3). The calculations for each target position were done with same horn current $I=$ $200 \mathrm{kA}$. 
of the SEMs permits measuring of the beam transverse dimensions.

As the proton beam passes through the collimating baffle on its way to target, some of the protons can end up hitting the baffle. The resulting temperature rise of the collimating baffle is monitored using two thermocouples. Based on this temperature it is possible to infer the number of protons scraping the baffle.

The secondary and tertiary beam are monitored using the arrays of ion chambers [40]. Figure 2.2 shows the placement of these monitors at the end of the decay pipe. The arrays are capable of measuring both the flux and spatial distribution. A $7 \times 7$ array of ion chambers spanning a $76 \times 76 \mathrm{~cm}^{2}$ area is in front of the hadron absorber measuring the flux of hadrons that reach the end of decay pipe. Three arrays of $9 \times 9$ ion chambers covering the $2 \times 2 \mathrm{~m}^{2}$ area are placed downstream of the hadron absorber. These so called muon monitors measure the muon beam flux. The two arrays further downstream are placed in the alcoves excavated from the dolomite rock. Each muon monitor is separated from the decay pipe by a layer of shielding and therefore measures the integrated flux of muons above some energy threshold. The thresholds are different for three alcoves due to different thickness of shielding. Use of these instruments as a means of measuring the neutrino flux is discussed in Appendix $\mathrm{C}$ and $\mathrm{D}$.

\subsubsection{Beam Performance}

The NuMI beam started its operation in December 2004. After a commissioning period, data taking commenced in April 2005. The data used in this thesis was accumulated up until April 2007, corresponding to a total of $2.5 \times 10^{20}$ 
protons on target (POT). Table 2.1 lists all the beam configurations in which data was taken. Each configuration is designated by a label LEXXX/YYYkA, where LE denotes the placement of the horns at $10 \mathrm{~m}$ separation, XXX is the location (in centimeters) of the target upstream of the first horn, and YYY is the current (in kiloamperes) in the horns. The LE10/185kA is utilized for the oscillation analysis. The other configurations are used for validation of the focusing properties of the beamline, as will be discussed in Chapter 6 .

\begin{tabular}{lccc} 
& Target Position & Horn Current & POT/1e18 \\
\hline \hline LE010/185kA (Run I) & $-10 \mathrm{~cm}$ & $185 \mathrm{kA}$ & 129.7 \\
LE010/185kA (Run II) & $-9 \mathrm{~cm}$ & $185 \mathrm{kA}$ & 122.9 \\
LE010/0kA (Run I) & $-10 \mathrm{~cm}$ & $0 \mathrm{kA}$ & 2.915 \\
LE010/170kA (Run I) & $-10 \mathrm{~cm}$ & $170 \mathrm{kA}$ & 1.466 \\
LE010/200kA (Run I) & $-10 \mathrm{~cm}$ & $200 \mathrm{kA}$ & 1.366 \\
LE100/200kA (Run I) & $-100 \mathrm{~cm}$ & $200 \mathrm{kA}$ & 1.168 \\
LE150/200kA (Run I) & $-150 \mathrm{~cm}$ & $200 \mathrm{kA}$ & 2.003 \\
LE250/200kA (Run I) & $-250 \mathrm{~cm}$ & $200 \mathrm{kA}$ & 1.596 \\
LE250/200kA (Run II) & $-250 \mathrm{~cm}$ & $200 \mathrm{kA}$ & 16.03
\end{tabular}

Table 2.1: Over the period between April 2005-April 2007, the NuMI beam was operated in several different beam configurations varying in target position and horn current. Each configuration yields a different neutrino energy spectrum (Figure 2.4). The data is split in two run periods, Run I which corresponds to data taken April 2005-February 2006 and Run II taken June 2006.-April 2007. Between the two runs the accelerator complex was shut down for maintenance. In September 2006, just prior to taking the Run II LE010/185kA data, the NuMI target was replaced.

The optimal yield of neutrinos is achieved only if the proton beam is centered on the target. Additionally, it is desirable that the collimating baffle is aligned on the same axis as target, ie on the beam axis. Such alignment reduces the number of protons hitting the baffle and maximizes the number of protons hitting the target. 
The alignment of the baffle and the target was checked using the proton beam [41]. Figure 2.6 shows the profile of the proton beam for one spill. The location of the target and baffle, derived from the beam based alignment, are indicated. As can be seen, the majority of the proton beam strikes the target, of importance for accurate counting of the number of proton on target. Further, very little beam strikes the baffle. Protons striking the baffle produce mesons further upstream in the beamline whose neutrino flux must be accounted for. Figure 2.7 shows the proton beam position and width over a longer period of running. It can be seen that majority of spills hit the center of the target. Also, the horizontal beam width was typically $\sim 1.1 \mathrm{~mm}$, small compared to the width of the target ensuring that majority of proton hit the target.

The alignment of baffle, target and horns can change any time when any of these components is moved. This can happen whenever some part needs to be replaced. The harsh radioactive environment in the target hall makes it impossible to design everything to last over the several years of running. The first NuMI target had to be replaced in September 2006. Before starting the new run, the alignment was checked again and the proton beam steered accordingly.

After a period of running with the replacement target, it was discovered that the longitudinal position of the target with respect to horns changed between two runs (see discussion Chapter 7). The target was $9 \mathrm{~cm}$ away from the first horn in second run, opposed to $10 \mathrm{~cm}$ during the first run. The change had a noticeable effect on the neutrino flux as is discussed in Chapter 7 .

The neutrino beam has to be steered precisely enough toward the FD which is $735 \mathrm{~km}$ away. The direction of the neutrino beam can be checked by 


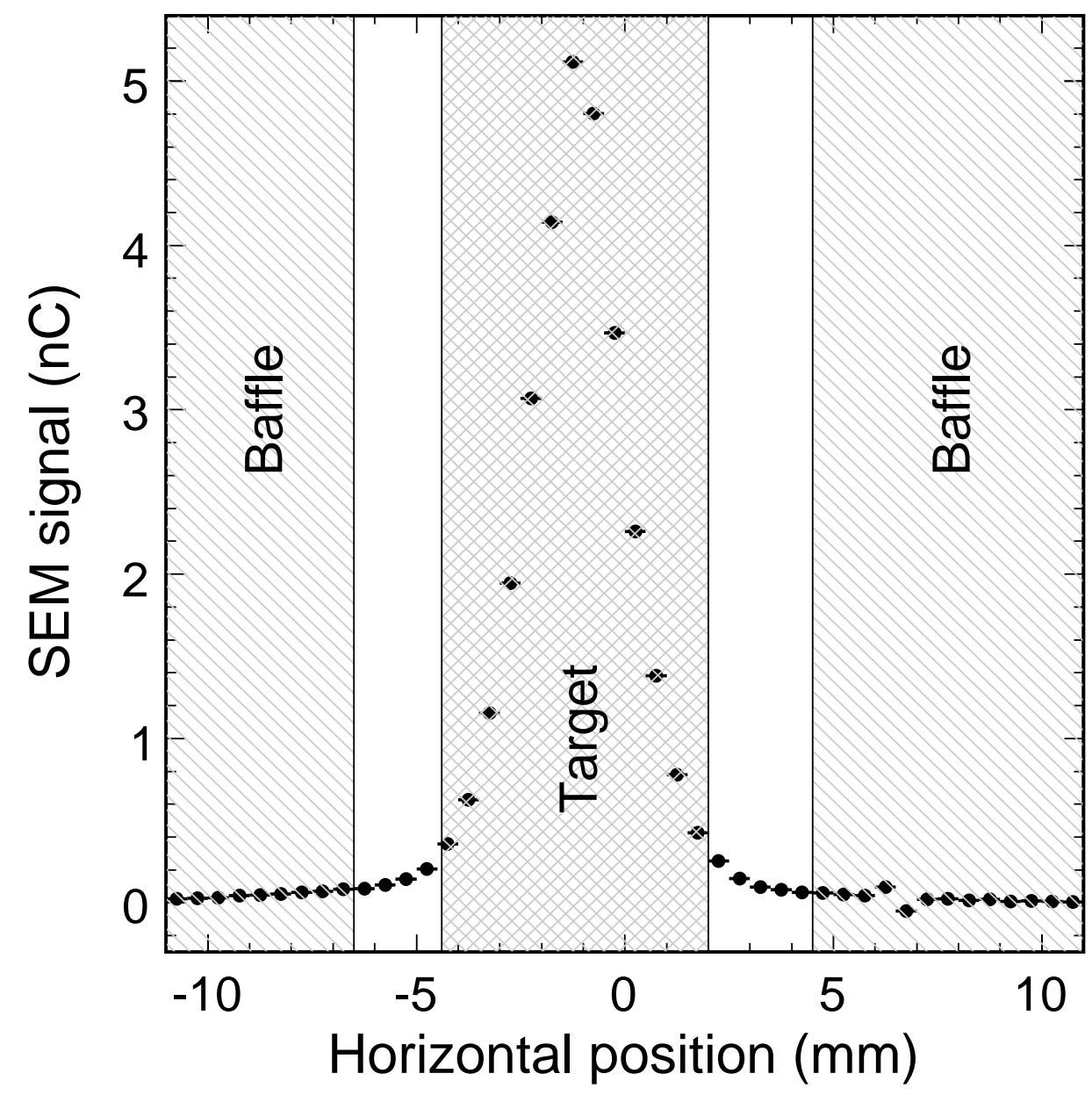

Figure 2.6: Distribution of protons in horizontal plane for a spill centered at $\mathrm{x}=1.1 \mathrm{~mm}$ with width $\sigma_{x}=1.2 \mathrm{~mm}$. Indicated is the location of the target and the baffle during the first run period as determined in [41]. 


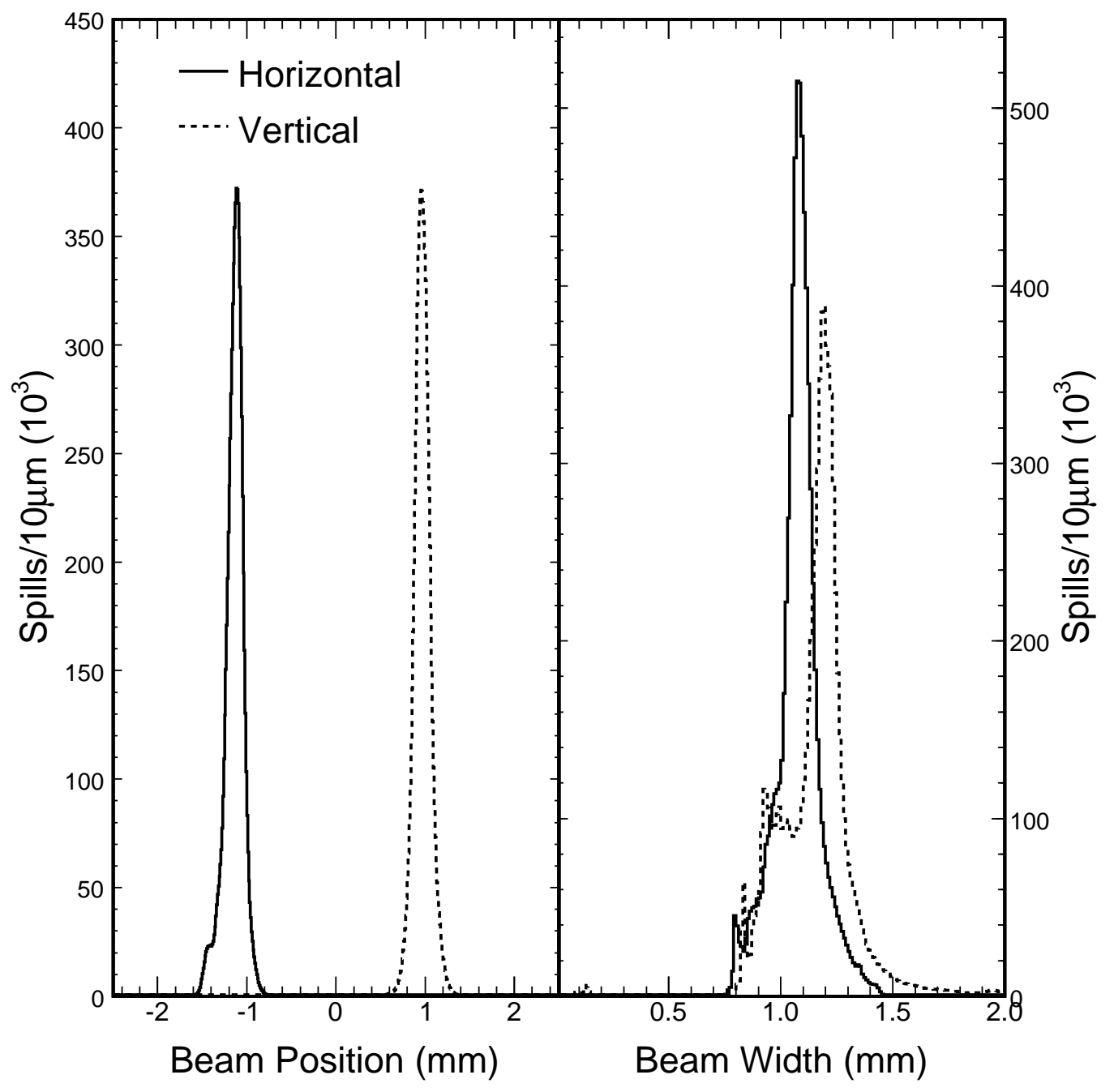

Figure 2.7: Primary proton beam position and width at the target. Data accumulated with first NuMI target corresponding to $1.57 \times 10^{20}$ POT. During the running the beam was centered on the target (Figure 2.6) and the beam width was relatively small compared to the $6.4 \times 18 \mathrm{~mm}^{2}$ profile of the target. 


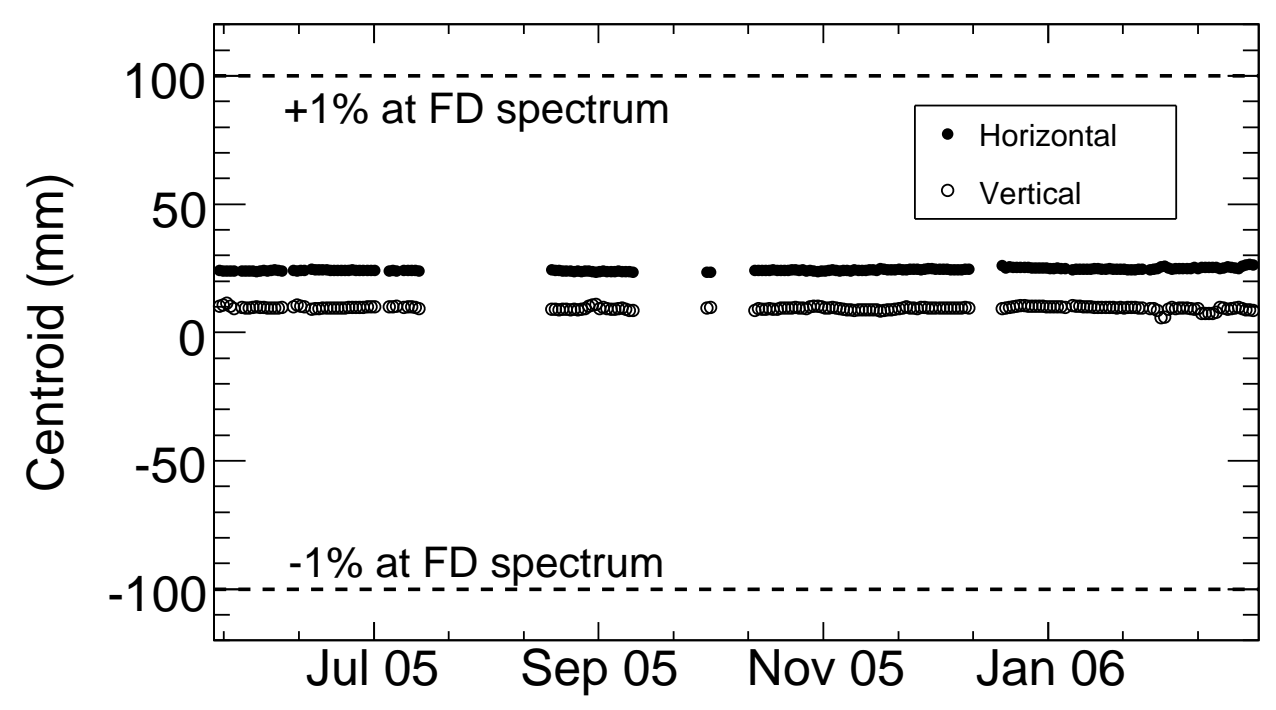

Figure 2.8: Horizontal and vertical muon beam centroid measured by first muon monitor station. The direction of the muon beam is the same as the direction of the neutrino beam. The observed variations in the centroid correspond to $20 \mu \mathrm{rad}$ variations in the beam angle. Such deviations are expected to have less than $0.2 \%$ effect on the $\nu_{\mu}$ energy spectrum at the Far Detector. Figure courtesy L. Loiacono.

measuring the direction of the muon beam. Figure 2.8 shows the center of the distribution of muons as measured with the muon monitor in the first alcove for a period of several months. Measurement of the centroid of the distribution provides a very precise measurement of the direction of the beam due to a long lever arm of $735 \mathrm{~m}$ that separates the target and the muon monitor.

\subsection{MINOS Detectors}

The two MINOS detectors (Figure 2.9) are functionally identical and were designed to detect muon neutrino charged current interactions with neutrino 
energies above $\sim 1 \mathrm{GeV}$. The almost identical design of the detectors allows for the cancellation of uncertainties related to neutrino interaction cross sections, or related to the detector response.

The difference in neutrino interaction rates, the neutrino beam size at the ND and FD and the desire to minimize the cost is why the two detectors are not completely identical. The ND which sees a greater flux of neutrinos is significantly smaller. On the other hand, the rate of events at the ND is much higher, requiring faster electronics used for read out.

The ND is on Fermilab site $104 \mathrm{~m}$ underground and $1040 \mathrm{~m}$ downstream from the target. The FD is located in the Soudan Underground Laboratory, $705 \mathrm{~m}$ underground and $735 \mathrm{~km}$ away from the NuMI target.

\subsubsection{Description of Detectors}

The detectors are steel-scintillator tracking calorimeters built out of planes consisting of $2.54 \mathrm{~cm}$ thick steel plane and a $1 \mathrm{~cm}$ thick scintillator. The steel planes act as a target for neutrino interactions and a passive absorber, while the scintillator is the active medium. The center-to-center distance between the planes in both detectors is $\sim 5.94 \mathrm{~cm}$.

Figure 1.2 shows an example of muon neutrino Charged-Current $\left(\nu_{\mu}+\right.$ $\left.F e \rightarrow \mu^{-}+X\right)$ and Neutral-Current $\left(\nu_{\mu}+F e \rightarrow \nu_{\mu}+X\right)$ interactions as seen in the detector. The relativistic muon created in the $\mathrm{CC}$ interaction looses its energy primarily through ionization. The energy deposited in the scintillator is observed and the segmentation of the detector allows the reconstruction of longitudinal and transverse position of the hit. The muons loose on the

average $\sim 2 \mathrm{MeV} /\left(\mathrm{g} / \mathrm{cm}^{2}\right)$, so given the density and the thickness of the steel, 

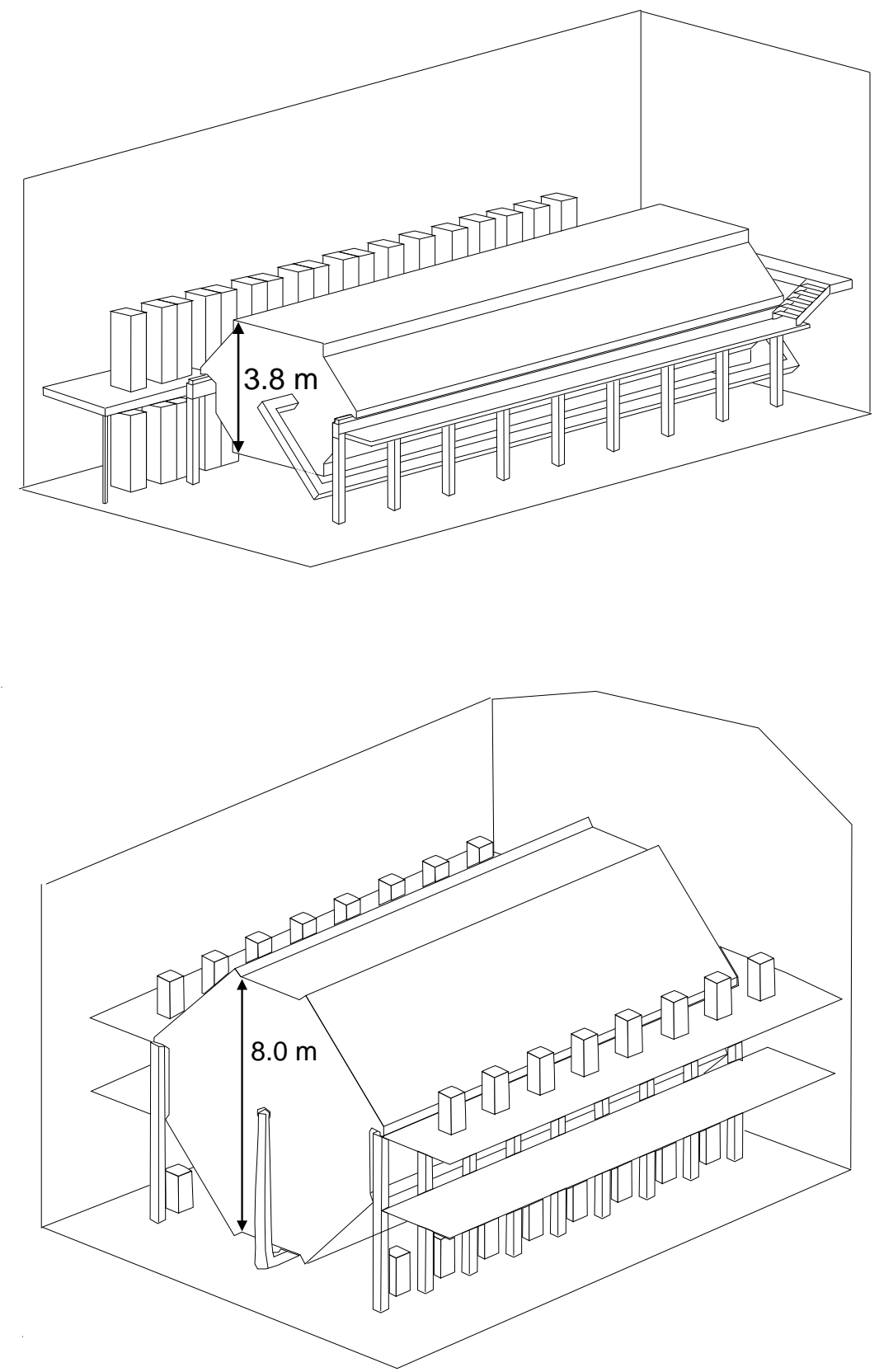

Figure 2.9: The MINOS Near (Top) and Far (Bottom) Detectors. The Near Detector is $16.6 \mathrm{~m}$ long, while the total length of the Far Detector is $30 \mathrm{~m}$. Only one of two functionally identical modules is shown for the Far Detector. (Figure taken from [9]) 
a muon looses $\sim 40 \mathrm{MeV}$ per MINOS detector plane. A $2 \mathrm{GeV}$ muon is thus expected to traverse $\sim 50$ planes before stopping. Identification of muon tracks is crucial for reconstructing $\nu_{\mu} \mathrm{CC}$ events. The hadronic shower $X$ is typically much shorter since hadrons loose energy faster.

The ND consists of 282 steel planes that have irregular $3.8 \times 4.8 \mathrm{~m}^{2}$ octagonal shape. The total mass of the detector is 0.98 kton. The ND is divided into 2 units. The first 121 planes form a calorimeter used to find the interaction vertex and the beginning of the muon track. The remaining 161 planes form a muon spectrometer which is used to measure the momentum of muons.

The FD has 486 steel, $8 \mathrm{~m}$ wide octagonal planes and the total mass of 5.4 kton. The FD consists of 2 modules, one comprised of 249 and the other of 237 planes with the air gap between the two modules of $1.1 \mathrm{~m}$.

In both detectors scintillator planes are comprised of $4.1 \mathrm{~cm}$ wide strips. The segmented design allows the identification of tracks left by energetic particles. Each strip is coated with $\mathrm{TiO}_{2}$ doped polystyrene reflective layer. The strips are oriented at $45^{\circ}$ with respect to the vertical axis. Consecutive planes have scintillator strips at $90^{\circ}$. The two orientations of scintillator strips define the so called $\mathrm{U}$ and $\mathrm{V}$ planes and allow the reconstruction of the track in three dimensions.

The light from the scintillator is transported via embedded wavelength shifting (WLS) fibers. The $1.2 \mathrm{~mm}$ diameter WLS fiber runs through a $2.3 \mathrm{~mm}$ deep grove in the center of the wide face of each scintillator strip, collecting the light from the entire strip.

The scintillator planes are somewhat shorter in the ND than in the FD, ranging from $2.5-6 \mathrm{~m}$. The $8 \mathrm{~m}$ long strips in the FD are long compared to the 
attenuation length of the light in the WLS fibers. Because of that the WLS fibers in the ND are read out only on one side, while the other side is covered with reflective material. In the FD both sides of a scintillator strip are being read out. The mentioned orientation of the planes at $45^{\circ}$ with respect to the vertical is in order to avoid any connections underneath the detector.

Light from the end of each WLS is carried by a clear fiber to a multianode photomultiplier tube (PMT) as shown in Figure 2.10. The two detectors use different types of PMTs. The ND uses 194 Hammamatsu sixty four pixel (M64) PMTs, while the FD uses 1452 Hammamatsu sixteen pixel (M16) PMTs. In the FD the fibers are "multiplexed", meaning that eight fibers from non-neighboring scintillator strips are mapped onto one pixel. On the opposite end of the scintillator plane, different mapping is used allowing for unambiguous demultiplexing.

Both detectors are magnetized with toroidal magnetic field. In the FD the current carrying coil runs through the middle of each plane along the length of the detector producing the average field of 1.4 T. The ND coil hole is offset $45.8 \mathrm{~cm}$ from the center of the plane. The neutrino beam is centered halfway between the coil hole and the left vertical edge. The average magnetic field in the region used as a target for neutrino interactions in the ND is $1.16 \mathrm{~T}$.

The momentum of the muon is primarily determined using the range of the muon in the detector. This however, is possible only for events which are contained in the detector. The magnetic field allows for the reconstruction of the momentum, for those more energetic muons that exit the detector, through the curvature of the muon track.

The magnetic field also allows for the separation between the negative and positive tracks. Since the charge of the outgoing muon in the muon neu- 


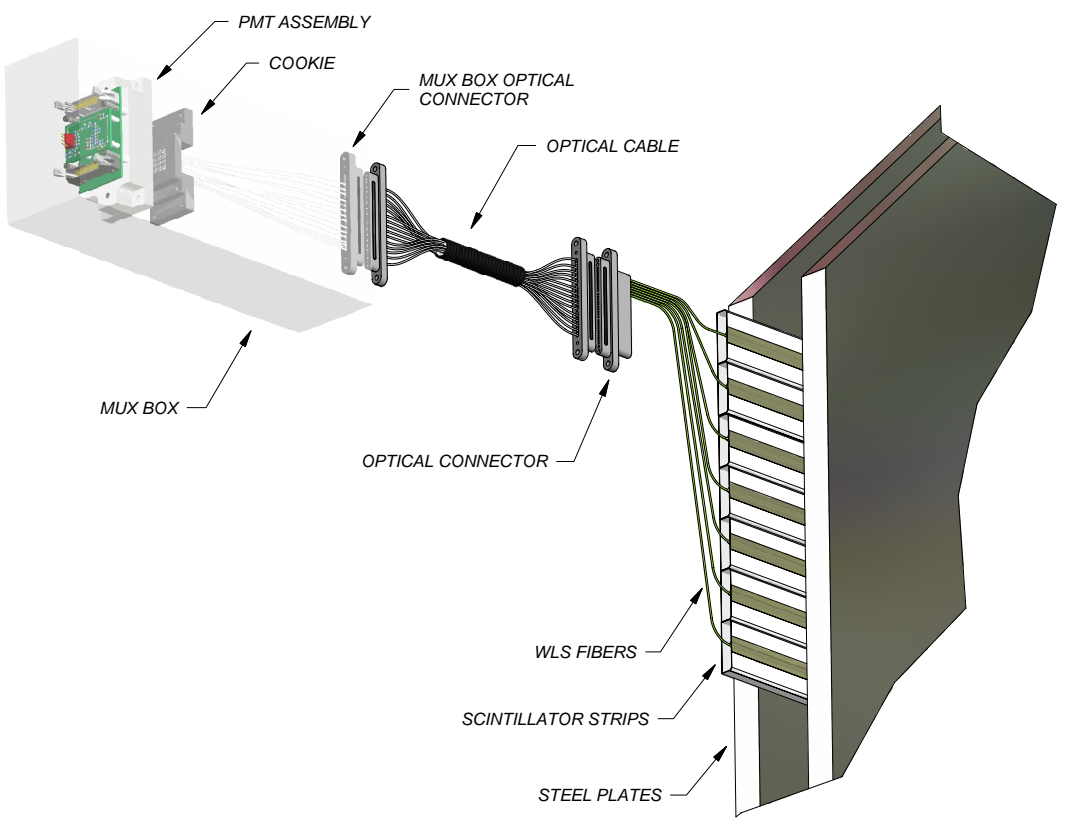

Figure 2.10: Optical readout of the MINOS detectors. Scintillation light is captured by wavelength-shifting fibers embedded in the scintillator and then transferred to multi-anode photomultipliers via clear optical fibers. (Figure taken from [9]) 
trino $\mathrm{CC}$ interactions depends on whether the incoming neutrino was a particle or an anti-particle.

It is possible to choose the polarity of the current flowing through the coil, however during the normal running, the current is flown in a way that it produces magnetic field lines that bend negative muons toward the center of the detector. This improves their containment making it easier to reconstruct such an event. The negative muons are created in CC interactions of muon neutrinos, while the positive ones are created by anti-neutrinos.

\subsubsection{Detector Calibration}

The MINOS detectors measure hadronic and electromagnetic shower energy by calorimetry. To measure the oscillation parameters with desired accuracy the detectors have to be calibrated relatively within $2 \%$ and have an absolute energy-scale uncertainty of $5 \%$.

The calibration of the MINOS detectors consists of several steps. The goal of the calibration is to correct the scintillator light output variations as well as non-uniformities of light transmission and collection in the fibers, PMTs and readout electronics. Finally the goal of calibration is to establish the absolute energy scale for the measured signal in the detectors.

Prior to the installation, the scintillator modules were exposed to radioactive sources. The response of each scintillator module, when irradiated at different position, was measured. The signal varies depending on the position at which the module is irradiated due to the attenuation in optical fibers. An empirical model of attenuation was developed that fits the data.

To relatively calibrate the signal from individual scintillator strips MI- 
NOS uses cosmic-ray muons. These muons constantly traverse the two MINOS detectors allowing the relative comparison of the signal between the strips. The rate at the FD is $\sim 0.5 \mathrm{~Hz}$, while at the ND it is $\sim 10 \mathrm{~Hz}$. Cosmic ray muons also allow the correction of the data for the temporal changes in the detector response.

Both MINOS detectors are equipped with LED based light-injection (LI) system. Over the course of running the wavelength shifting fibers in the scintillator modules are being illuminated with light from LEDs. The role of this system is twofold. First, it allows the data to be corrected for temporal variations in the signal. These variations occur mostly due to environmental conditions, but also due to aging of the scintillator. Second, the WLS are being illuminated with various intensity of light which allows the measurement of the nonlinearity of the PMT response.

The response of MINOS detectors to various particles was studied using a dedicated calibration detector. The calibration detector had the same steelscintillator design as the MINOS detectors and was exposed to the test beam at CERN. The response to hadrons, electrons and muons with momenta in the range $0.2-10 \mathrm{GeV} / \mathrm{c}$ was studied yielding the absolute energy scale $[42,43]$. The response of the detector was in good agreement with simulations done using GCALOR [44] shower code. Generally, the simulations reproduced the induced showers at the level of 1-5\% depending on the energy and particle type. The hadronic energy resolution was measured to be $56 \% / \sqrt{E[\mathrm{GeV}]} \oplus 2 \%$.

The muon energy resolution $\Delta E_{\mu} / E_{\mu}$ varies smoothly from $6 \%$ at low energies, where most tracks are contained and momentum is measured from range, to $13 \%$ at high energies, where curvature is used to measure the momentum. 
A relative calibration of the ND and FD is achieved by normalizing the energy scale of ND, FD and calibration detector to within the $2 \%$. This is achieved by using the muons that stop within the detector. As mentioned, the two MINOS detectors are constantly being bombarded by cosmic muons providing the measurement for free. In particular, the response of the three detectors to muons with momentum in $0.5-1.1 \mathrm{GeV}$ range is used since muon $\mathrm{dE} / \mathrm{dx}$ varies slowly in that region. The muons in that momentum window are found by tracing the muon track back from the point where it stopped. Using the known geometry of the detector and known muon $\mathrm{dE} / \mathrm{dx}$ the portion of the track where muon momentum was in 0.5-1.1 GeV range can be found.

More detailed discussion of the calibration of MINOS detectors can be found in $[9,45]$.

\subsubsection{Event Reconstruction}

The NuMI beam neutrinos have the same time structure as the proton beam and therefore come in short $\sim 10 \mu$ s spills. To reduce the background for the analysis of beam neutrinos, the detectors are read out in a time window spanning the beam duration. The Main Injector broadcasts a signal preceding the spill which triggers the ND and FD electronics.

The neutrinos can interact either via charged current (CC) $\nu_{\mu}+F e \rightarrow$ $\mu^{-}+X$ or neutral current (NC) $\nu_{\mu}+F e \rightarrow \nu_{\mu}+X$ as indicated in Figure 1.2. The two types of events look very different in the MINOS detectors. The typical CC $\nu_{\mu}$ event has a long muon track accompanied by a short hadronic shower $X$ from the breakup of the iron nucleus. The NC events are typically short with diffuse hadronic showers and look the same for all neutrino flavors. 
The NuMI beam also has a small $\nu_{e}$ component, so $\nu_{e} \mathrm{CC} \nu_{e}+F e \rightarrow e^{-}+F e$ events are present in the detectors. These events are characterized by short and dense electromagnetic showers. The goal of the reconstruction is to estimate the visible energy of neutrino interactions, as well as other quantities that are necessary to distinguish between $\mathrm{NC}, \nu_{\mu} \mathrm{CC}$ and $\nu_{e} \mathrm{CC}$ events.

Figure 2.11 displays the data from one beam spill in the ND. The typical spill produces a number of neutrino interactions throughout the ND, while for the FD it is very unlikely to have multiple interactions. The event reconstruction begins with identifying the strips with hits. In ND the timing as well as spatial information is used to separate individual neutrino events.

For each event, the reconstruction algorithm identifies the hadronic shower activity and finds and fits the tracks. Figure 2.12 shows the distribution of reconstructed neutrino vertices compared to the predicted distributions using the calculated neutrino flux and simulation of detector (§2.2.5). The distributions agree very well.

The tracks found by the reconstruction algorithm mostly belong to the muons created in the neutrino $\mathrm{CC}$ interactions. These tracks are expected to be preferentially in the direction of the beam. Again good agreement between the reconstructed data and prediction can be seen in Figure 2.13.

As mentioned, the event rate in the ND is high and multiple events are seen in each beam spill. The reconstruction algorithm does not show any biases due to higher rates. Figure 2.14 shows the number of selected events as a function of beam intensity. The linearity implies no dependence of the reconstruction on the instantaneous event rate.

For $\nu_{\mu} \mathrm{CC}$ events, the total reconstructed energy is obtained by summing the reconstructed muon energy and the visible energy, $E_{\text {shower }}$, of the 

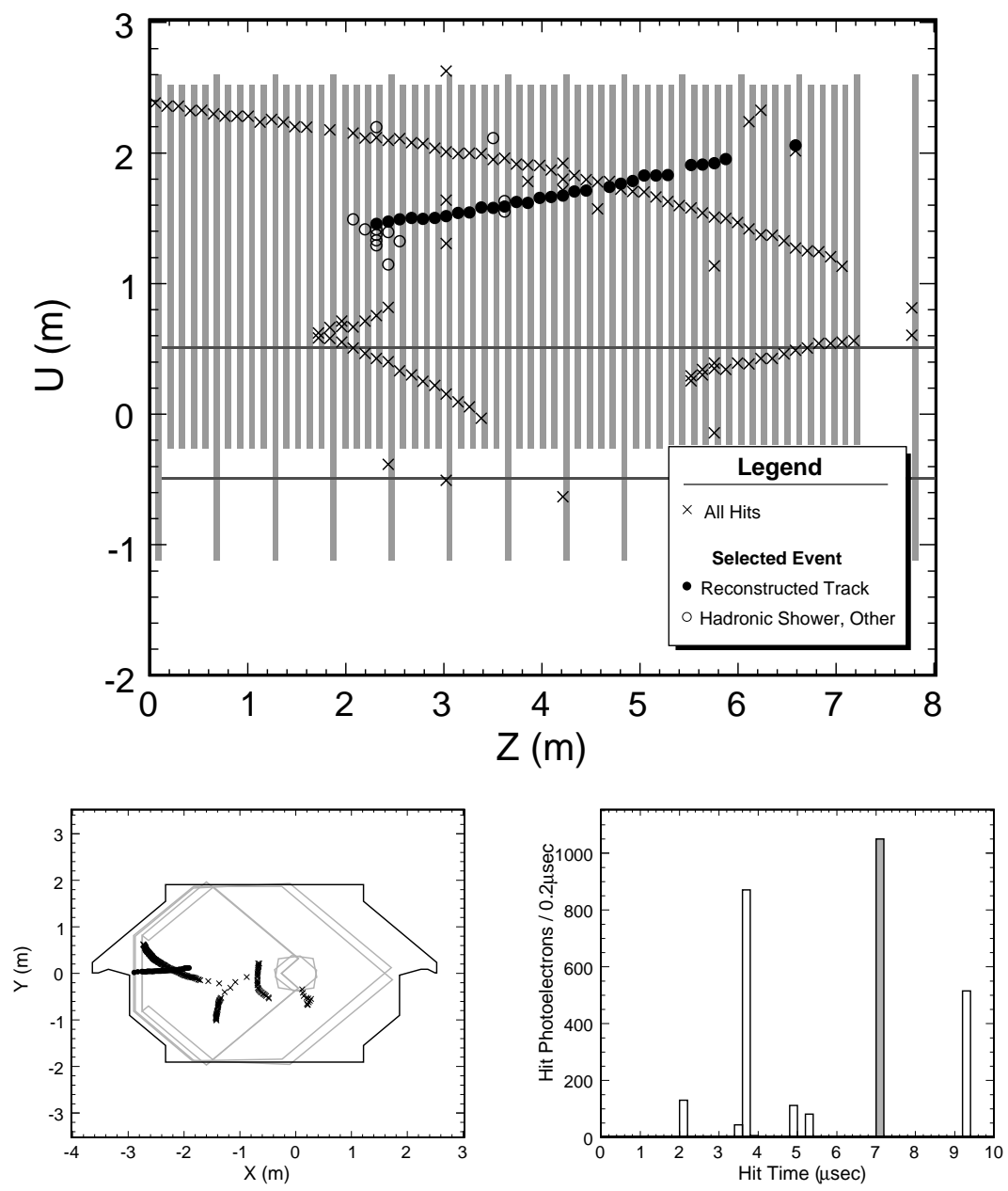

Figure 2.11: One beam spill observed in the Near Detector. This spill has four separate neutrino interactions visible. For clarity, a spill containing smaller than average number of neutrino events was chosen. The data in the detector $\mathrm{U}$ view (a), beam's eye view (b) as well as timing (c) is shown. Event topology and timing are used to select individual neutrino events. Of the four events shown only the event with a $5.6 \mathrm{GeV}$ muon and a $2.6 \mathrm{GeV}$ hadronic shower is selected for analysis. (Figure taken from [9]) 

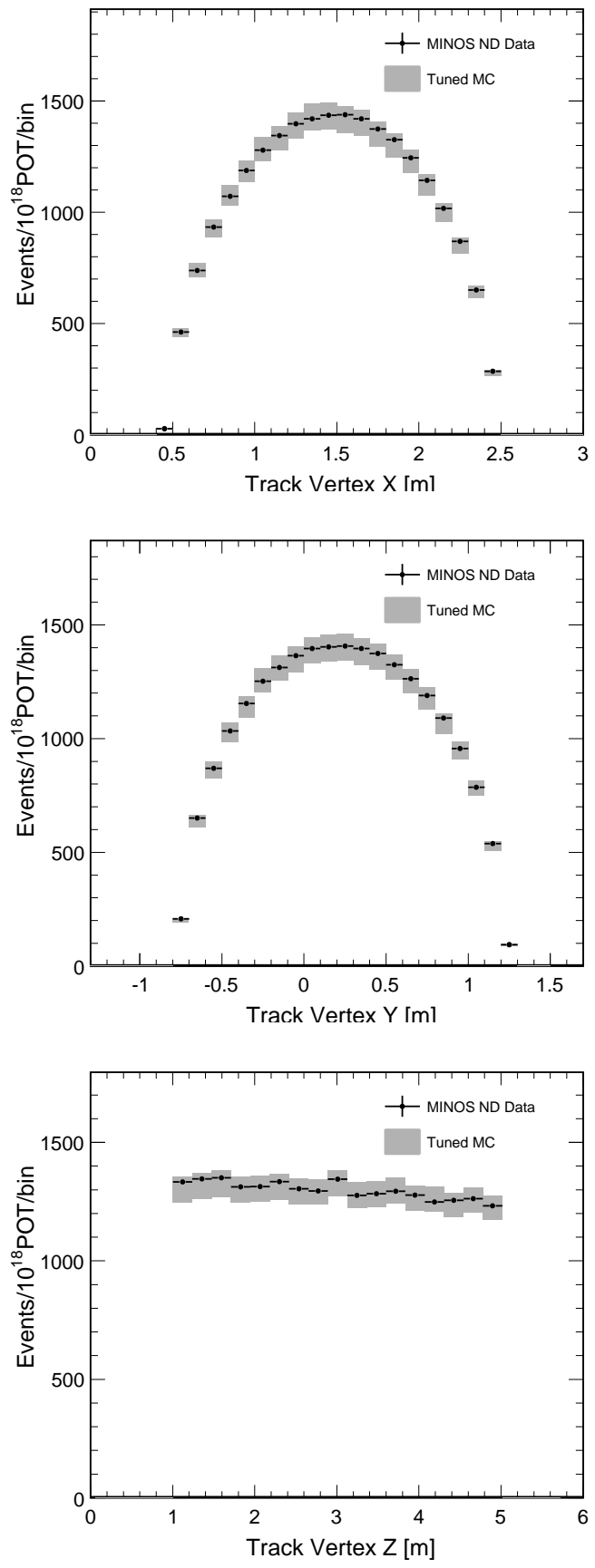

Figure 2.12: The reconstructed $\mathrm{x}, \mathrm{y}$ and $\mathrm{z}$ distributions of interaction points for the selected $\nu_{\mu}$ Charged-Current events in MINOS Near Detector. The shaded envelope represents the MC prediction and the systematic error associated with the calculation of the neutrino flux (Chapters 3 and 6). Courtesy M. Ishitsuka. 


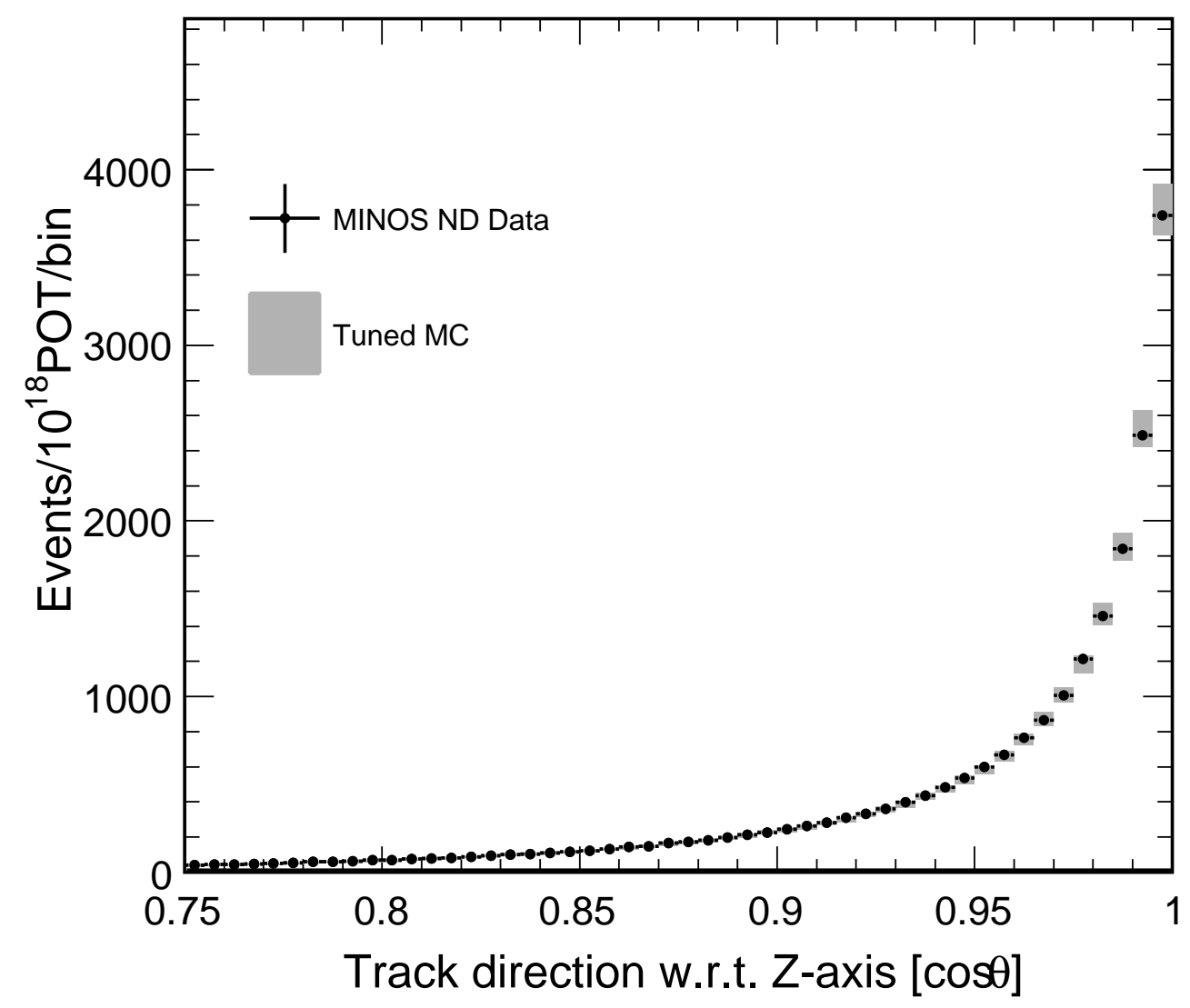

Figure 2.13: The distribution of the reconstructed track direction relative to the incident neutrino beam for the selected Charged-Current (CC) $\nu_{\mu}$ sample. The muon created in the $\mathrm{CC}$ interaction preferentially has a same direction as an incoming neutrino $(\cos \theta=1)$. Courtesy M. Ishitsuka. 

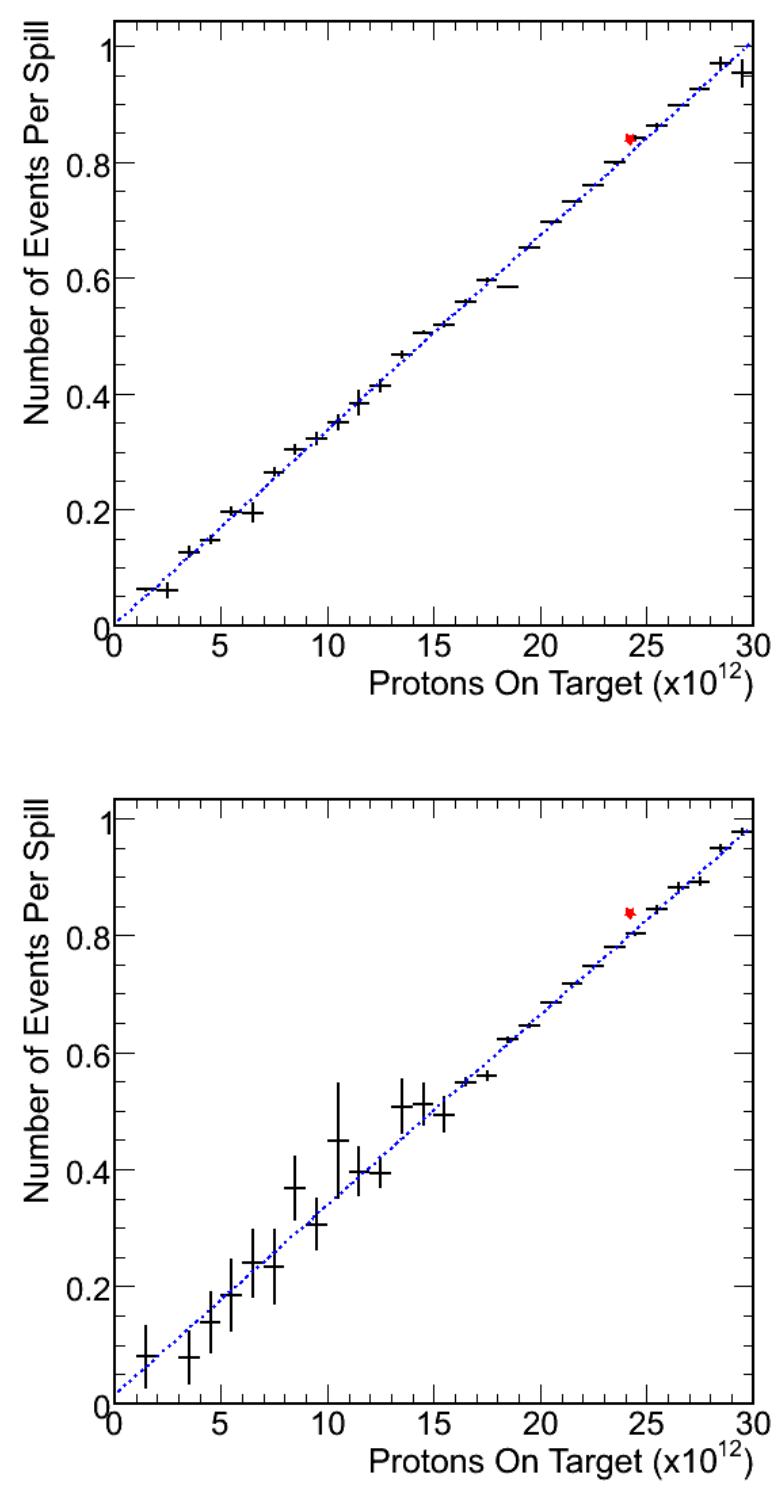

Figure 2.14: Mean number of selected events in the Near Detector per spill as a function of spill intensity. Shown are the events whose interaction vertex is within the fiducial volume for Run I period (top) and Run II period (bottom). The selection algorithm does not show any bias due to the proton beam intensity and as expected the number of selected events is directly proportional to the proton beam intensity. (Figures courtesy B. Armstrong). 
hadronic system. The muon energy is measured either by range or through curvature measurement, depending on whether the muon stops in the detector or escapes.

\subsubsection{Event Classification and Selection}

The $\nu_{\mu} \mathrm{CC}$ event candidates are preselected in both detectors by requiring that the vertex falls in the fiducial volume ensuring that the hadronic energy of the event is contained within the volume of the detector. The events have to have a track and the timing of the event required to be consistent with the beam spill time.

The fiducial volume in ND is a cylinder with $1 \mathrm{~m}$ radius, $4 \mathrm{~m}$ long starting $1 \mathrm{~m}$ downstream of the first plane. The cylinder follows the beam direction. The FD fiducial volume is a cylinder with $3.7 \mathrm{~m}$ radius. The vertices are required to be at least $50 \mathrm{~cm}$ away from the first and last plane in each of the two modules.

One of the possible backgrounds are cosmic muons. Since the beam is delivered in short spills, in the ND the event rate due to cosmic muons is negligible compared to the event rate due to the beam neutrinos. In the FD, on the other hand, the rates are comparable. Therefore, it is required that the muon track direction is consistent with beam direction, ie it lies inside in a $53^{\circ}$ cone. The majority of cosmic muons enter the FD at steep angles with respect to the beam direction.

The other significant background is coming from neutral current (NC) events. The difference between $\mathrm{NC}$ and $\mathrm{CC}$ events is that the $\mathrm{CC}$ events have a muon track. The typical background comes from the proton and pion tracks 

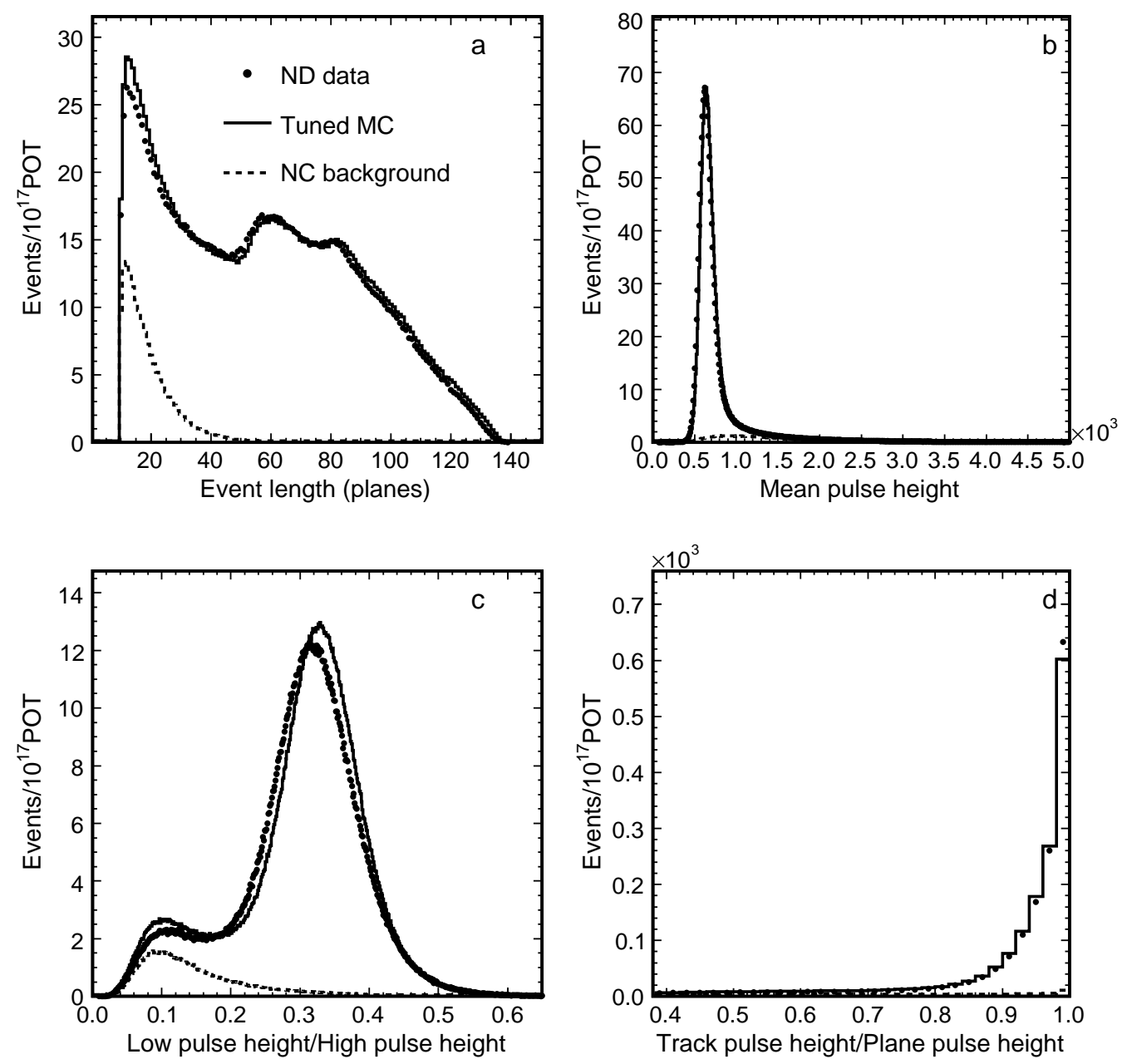

Figure 2.15: The 4 variables used to distinguish between $\mathrm{CC}$ and NC events. Each variable uses some aspect of the muon track that separates it from the misidentified non-muon tracks. (a) The length of the event, (b) The light yield (equivalent to $\mathrm{dE} / \mathrm{dx}$ ), (c) The ratio of pulse height from a set of strips along the track with lowest and highest pulse heights, (d) fraction of activity in the plane that belongs to the track. The muons have a low $\mathrm{dE} / \mathrm{dx}$ that is fairly constant for broad range of muon momenta. Consequently muons leave long tracks with fairly constant and low energy deposit in each strip. The muon tracks are usually sharp with very little activity in the surrounding strips. Courtesy R. Ospanov. 
which are created in NC events. Four variables are being used to separate the NC background. The variables are picked on their ability to separate muon tracks from the misidentified tracks. Figure 2.15 shows the 4 variables used to improve the purity of the selected CC candidate events [46].

The first variable is the event length. The muons often times leave a long track in the detector because of their low rate of energy loss $(\mathrm{dE} / \mathrm{dx})$. The longer events are more likely to be CC than NC.

Hadrons lose much more energy than muons when going through the scintillator. Depositing more energy results in a higher light yield from the strip. The low $\mathrm{dE} / \mathrm{dx}$ for the muons given by the Bethe-Bloch formula (see for example [47] Ch. 2) only weakly depends on muon momentum for wide range of muon momenta. The muons therefore tend to deposit similar lower amounts of energy in each scintillator strip. The light yield per strip in the selected track is used as a second variable.

Hadronic showers have larger fluctuations along the track in the deposited energy. A third variable compares the mean energy deposited in two subsets of strips along the track. The first subset is comprised of strips with most activity and the other of strips with least activity.

The last variable compares the activity in the neighboring strips transverse to the track, to the strip through which the track goes. The muon leaves a sharp track with very little transverse activity, while hadrons tend to have broader transverse activity.

The four variables are an input to a k-nearest neighbors $(\mathrm{kNN})$ algorithm [48] which is used to separate the $\nu_{\mu}$ CC events from the background of NC events. The 4 variables span a 4-dimensional space and each event can be represented by a point in this space. The kNN algorithm uses a training 
sample to find the local densities of $\mathrm{CC}$ and $\mathrm{NC}$ events in any point in space. The parameter $\mathrm{k}$ determines how many neighbors from the training sample will be used to calculate these densities.

Figure 2.16 shows the distribution in the $\mathrm{kNN}$ parameter for the events in the ND. The parameter shows clear separation of $\mathrm{CC}$ events from the NC events. The $\mathrm{CC}$ events are selected by requiring that this $\mathrm{kNN}$ parameter is greater than 0.3. The same cut is used in both the Near Detector and the Far Detector.

Generally the four variables used to build the event separation parameter agree well between data and MC as can be seen in Figure 2.15. The worse agreement can be seen in low pulse height/high pulse height variable. This variable is sensitive to simulating the noise in the detector. A better agreement can be achieved by considering only hits that are beyond a certain threshold. Introducing such a threshold does not affect the selection in any way and it was shown that the same events get selected regardless of having or not having the threshold. [49]

Figure 2.17 shows the efficiency with which the CC neutrino events are selected and the purity of the selected sample. Both the efficiency and the purity are high for higher energy events that are typically identified by a long muon. In lower energy bins the efficiency and the purity drop as it becomes harder to identify the muon track.

For our oscillation analysis the neutrino events are separated from antineutrino events by requiring that the curvature of the muon track is consistent with a negative charge. However, in Chapter 6 we will use the anti-neutrino sample as well to correctly predict flux from all neutrino flavors. 


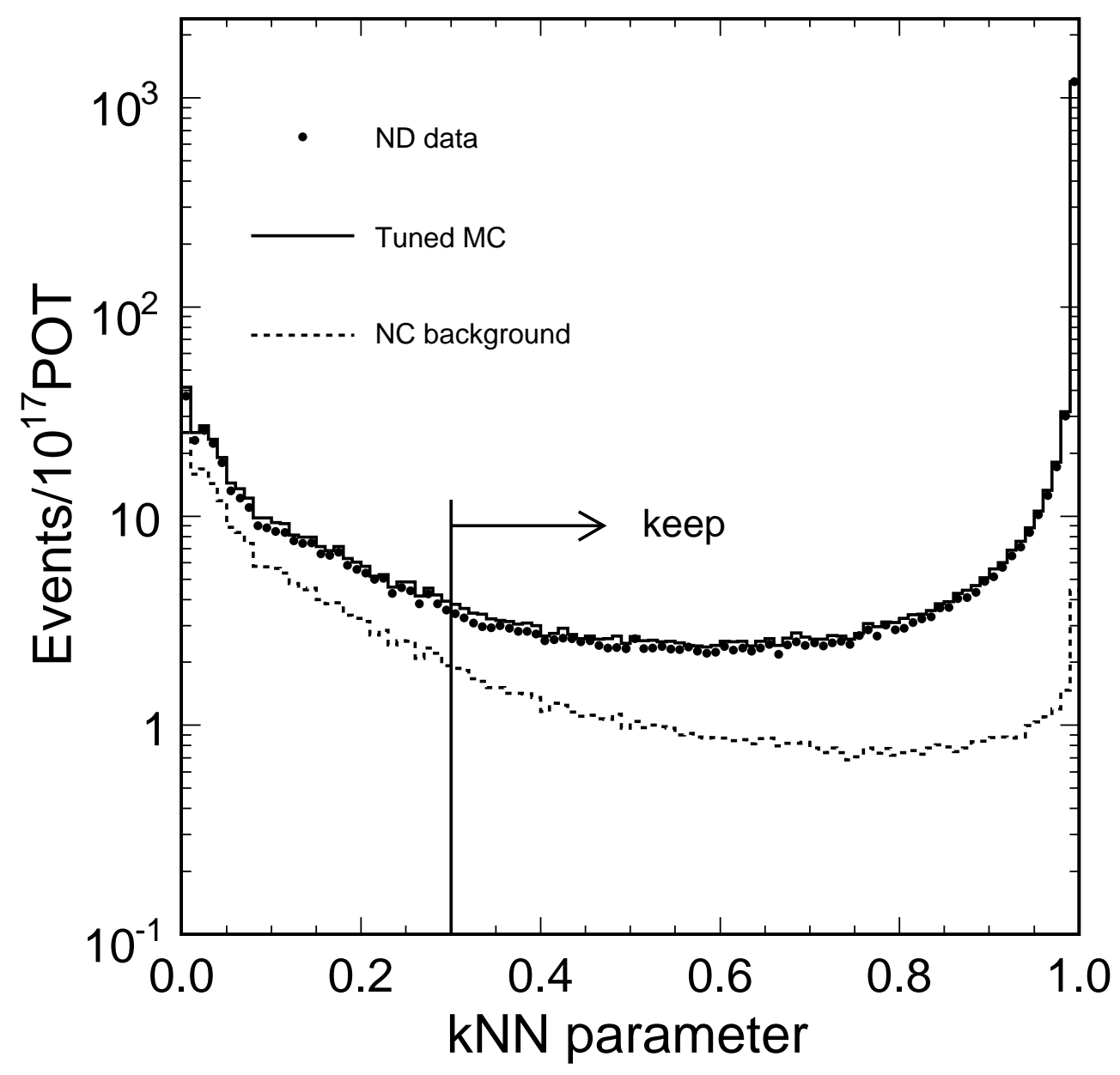

Figure 2.16: The distribution of kNN parameter for the ND events. The parameter is calculated using the $\mathrm{kNN}$ algorithm based on 4 input variables (Figure 2.15) that characterize the $\mathrm{CC}$ events. The parameter provides a way to reduce the NC background when selecting CC events. Courtesy R. Ospanov. 


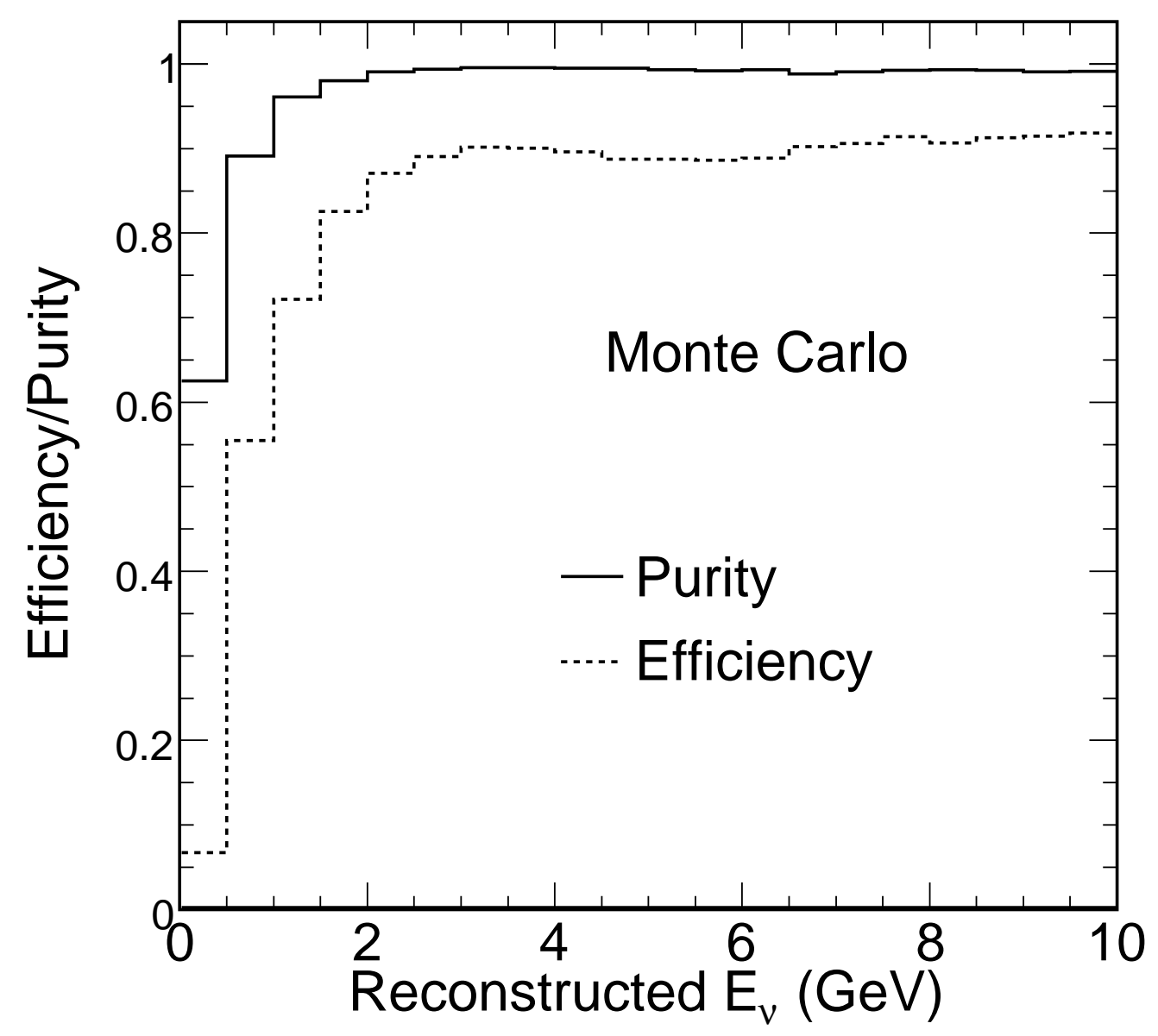

Figure 2.17: The efficiency and the purity of the selected $\nu_{\mu}$ Charged-Current data sample as a function of the reconstructed neutrino energy. The data was selected using kNN algorithm (Figure 2.16). Both the efficiency and the purity are high for higher energy events and drop for lower energy events where finding a muon track becomes harder. 


\subsubsection{Near Detector Charged Current Spectrum}

Figures 2.18 and 2.19 show the neutrino energy spectrum for selected CC events for the two run periods used in this analysis. As mentioned before, the target position was slightly different in the two run periods, causing a small difference between the two spectra. Also shown is the Monte Carlo (MC) calculation of the spectra.

The MC calculation of the spectrum starts with a calculation of the neutrino flux (Chapter 3). The NEUGEN [50] event generator is then used to model the neutrino interactions within MINOS detectors. The NEUGEN model summarizes the current knowledge of cross sections for neutrino interactions. A GEANT [51] based simulation of the detector is used to simulate hadronic interactions in the detector and model the energy deposition in scintillator strips. Subsequently the transmission of the light through WLS fibers is modeled as well as the response of the electronics which includes simulation of effects such as noise, non-linearities, etc.

The MC simulated events are reconstructed using the same reconstruction algorithm used for data (§2.2.3). The $\mathrm{CC}$ events are then selected again using the same selection procedure as used for data (§2.2.4).

The data is shown separately for each month of running. Data shows good agreement with MC simulation in both run periods. It can also be seen that the data is stable suggesting that both the beam and detector calibration were stable over the data taking period. 


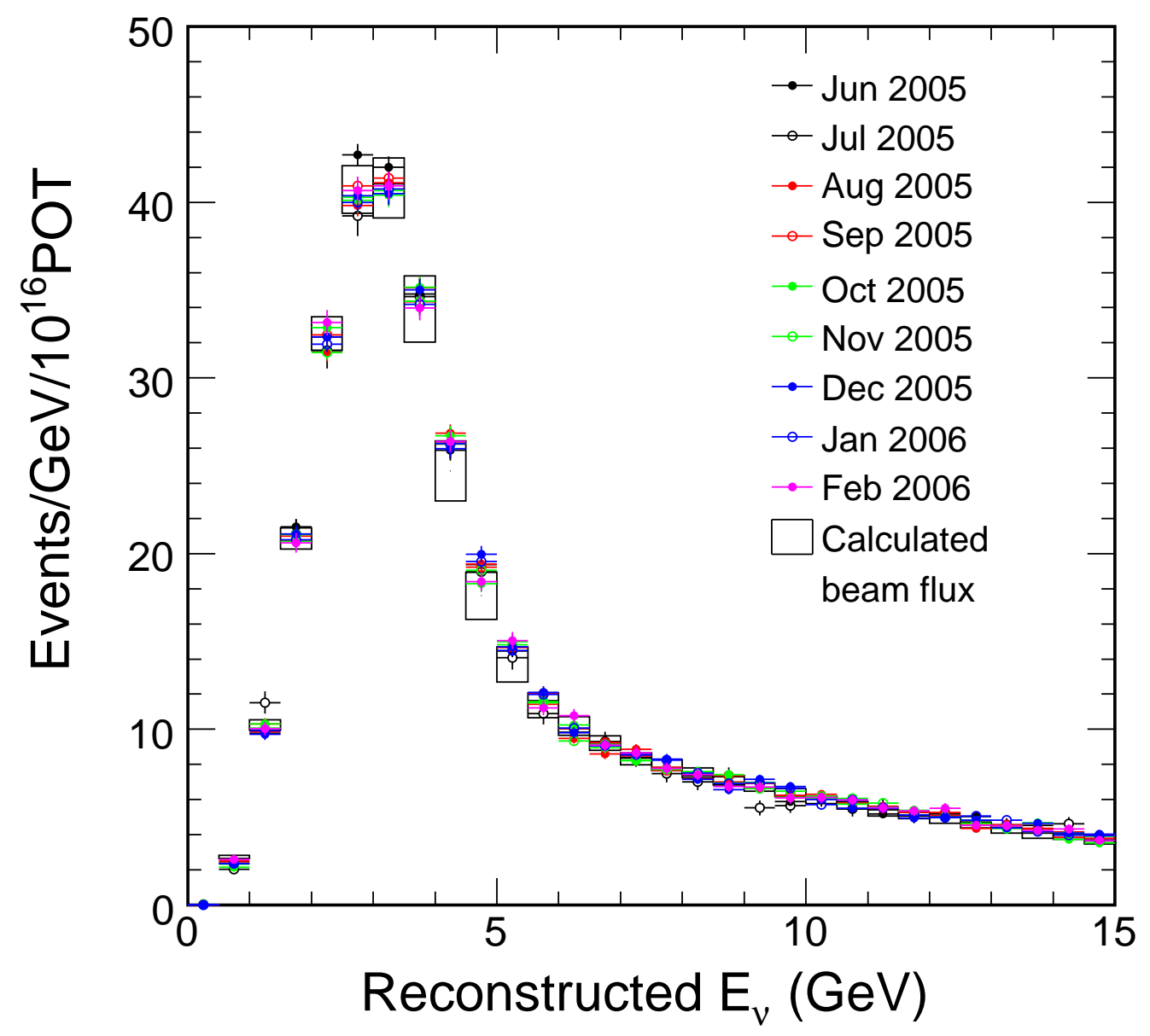

Figure 2.18: The reconstructed neutrino energy distributions for selected Charged-Current events in LE010/185kA beam configuration. The spectrum is plotted for each month of the Run I period (Table 2.1). The envelope represents the MC expectation and the systematic error associated with flux calculation. Differences between the data and beam flux calculation are discussed in Chapter 5. Courtesy M. Ishitsuka. 


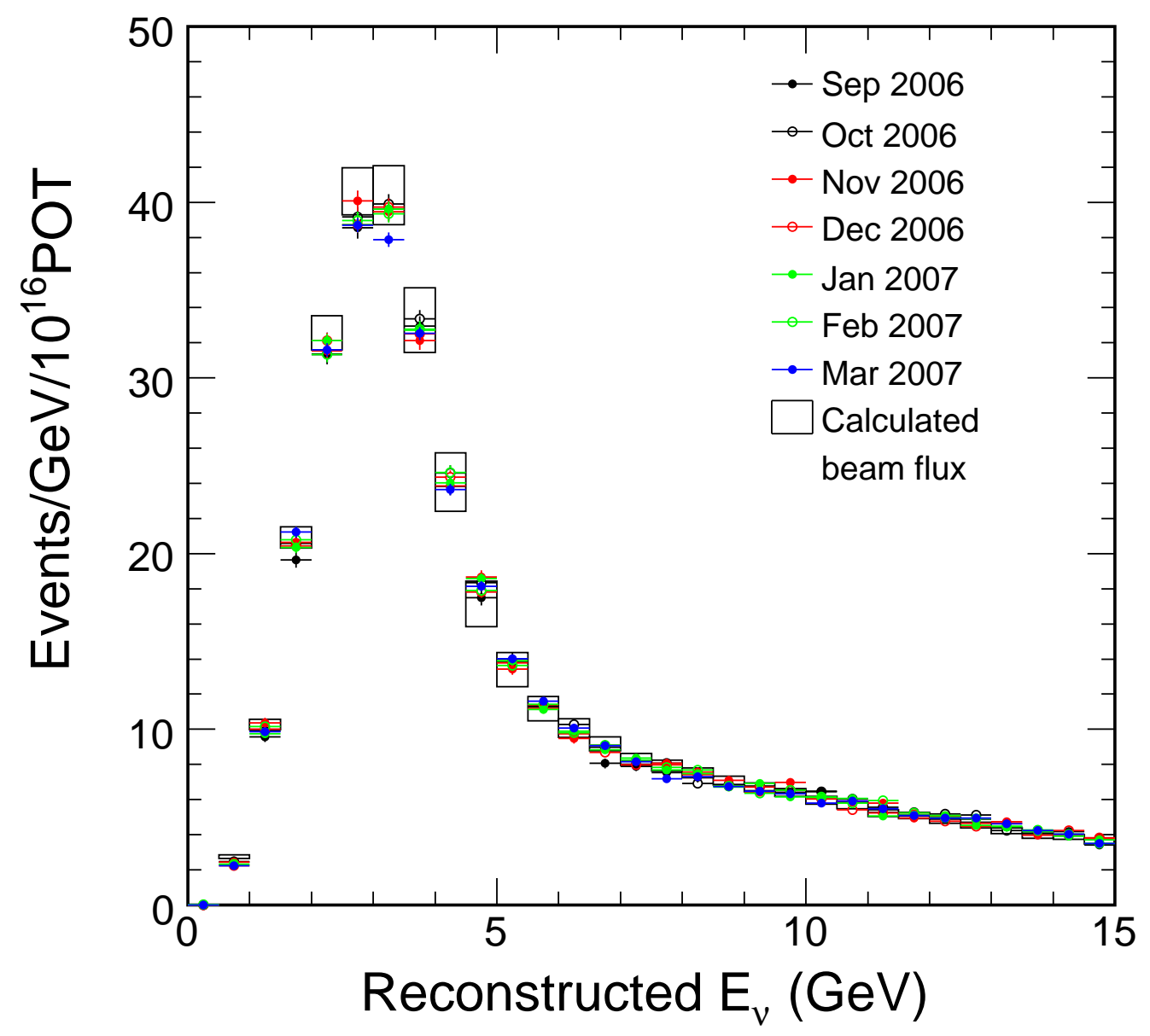

Figure 2.19: The reconstructed neutrino energy distributions for selected Charged-Current events in LE010/185kA beam configuration. The spectrum is plotted for each month of the Run II period (Table 2.1). The envelope represents the MC expectation and the systematic error associated with flux calculation. Differences between the data and beam flux calculation are discussed in Chapter 5. Courtesy M. Ishitsuka. 


\section{Chapter 3}

\section{Calculation of Neutrino Flux}

The neutrino flux from the NuMI beam can be calculated in principle. The calculation requires the knowledge of the flux and energy spectrum of hadrons produced by the proton beam in the target, the tracing of the hadrons through various elements of the beamline, including the magnetic focusing system, and finally the probability that a hadron will decay to neutrinos whose trajectories are in the direction of the MINOS detectors. The analytic calculation would require multidimensional integration over all the afore-mentioned probabilities, making it practically impossible. It is therefore necessary to rely on the Monte Carlo (MC) method [17] to calculate the neutrino flux.

Three different MC computer codes have been written to calculate the neutrino flux from NuMI beam-line. They vary in their modeling of the production of secondaries in the target, reinteractions of particles downstream of target, and in the level of details used to describe the geometry of the beam-line. The more detailed simulations come at the expense of additional computer processing time needed to converge. The two more sophisticated MC codes are based on version 3 and 4 of GEANT [51, 52] code. GEANT 
provides the means to describe the beam-line geometry and handles the tracking of particles going through this geometry. The third code, called PBEAM, is a fast $\mathrm{MC}$ used primarily for optimizing the beam-line and studying the effects of misalignments of focusing elements on the neutrino flux; rather than detailed simulations of particle interactions, it makes use of simple empirical functions to approximate more detailed calculations.

In this chapter we discuss the MC calculation of particle trajectories through the beamline and of particle decays. Additionally, we discuss data

from other experiments which help constrain poorly understood aspects of the MC simulation. The figures of beam-line shown throughout the chapter represent the beam-line geometry as it is described in GEANT4 based MC.

\subsection{Meson Decay to Neutrinos}

The neutrino beam is mostly a result of decays of secondary particles created by proton interactions within the target. We focus our discussion on production of pions and kaons since these are most abundantly created among the particles that decay into neutrinos. The dominant sources of neutrinos are:

$$
\begin{aligned}
& \pi^{ \pm} \rightarrow \mu+\nu_{\mu} \\
& \mu \rightarrow e+\nu_{\mu}+\nu_{e} \\
& K^{ \pm} \rightarrow \mu+\nu_{\mu} \\
& K_{L}^{0} \rightarrow \pi+\mu+\nu_{\mu} \\
& K_{L}^{0} \rightarrow \pi+e+\nu_{e}
\end{aligned}
$$

The energy of the neutrino may be calculated from the energy of the 
CM Frame

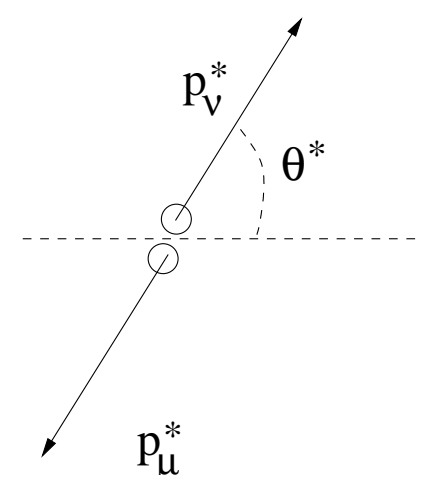

Lab Frame

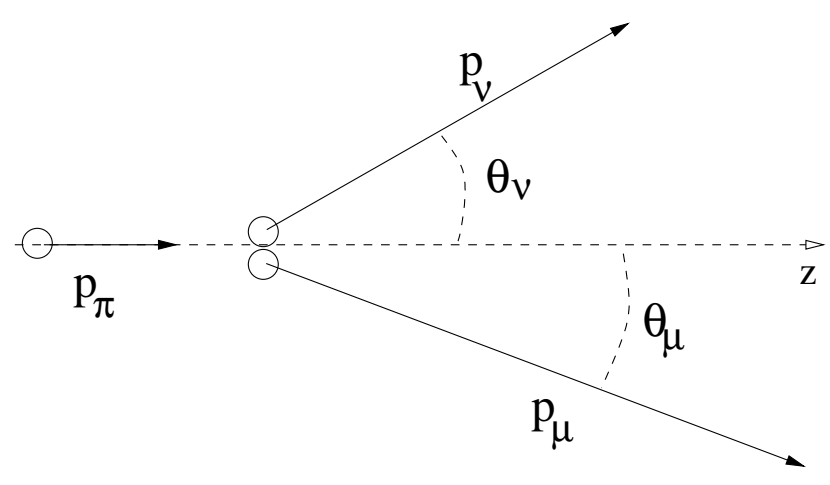

Figure 3.1: View of a $\pi \rightarrow \mu+\nu_{\mu}$ decay in (left) the center of mass (CM) and (right) laboratory frame. The $\pi$ is assumed to travel along the z-axis.

meson parent. Figure 3.1 shows a schematic view of pion decay in the center of mass and laboratory frame. We first consider the pion decay in the center of mass system $S^{*}$. Without loss of generality we can consider decay in x-z plane, such that decay products have y component of momentum equal 0 . The momenta of pion, muon and a neutrino can be written as:

$$
\begin{aligned}
& p_{\pi}^{*} \equiv\left(m_{\pi}, 0,0,0\right) \\
& p_{\nu}^{*} \equiv\left(E_{\nu}^{*}, E_{\nu}^{*} \sin \theta^{*}, 0, E_{\nu}^{*} \cos \theta^{*}\right) \\
& p_{\mu}^{*} \equiv\left(E_{\mu}^{*},-E_{\nu}^{*} \sin \theta^{*}, 0,-E_{\nu}^{*} \cos \theta^{*}\right)
\end{aligned}
$$

From conservation of energy it follows $E_{\mu}^{*}=m_{\pi}-E_{\nu}^{*}$. Now using $p_{\mu}^{2}=m_{\mu}^{2}=$ $E_{\mu}^{* 2}-E_{\nu}^{* 2}$ we can find the neutrino energy in the pion rest frame:

$$
E_{\nu}^{*}=\frac{m_{\pi}^{2}-m_{\mu}^{2}}{2 m_{\pi}}
$$

The similar formula can be derived for the case of two body kaon decays. 
We now perform a boost along z axis to frame $S$ in which the momenta are given with:

$$
\begin{aligned}
& p_{\pi} \equiv\left(E_{\pi}, 0,0, p_{\pi}\right) \\
& p_{\nu} \equiv\left(E_{\nu}, E_{\nu} \sin \theta, 0, E_{\nu} \cos \theta\right) \\
& p_{\mu} \equiv\left(E_{\mu},-E_{\nu} \sin \theta, 0, p_{z \mu}\right)
\end{aligned}
$$

The Lorentz transformations give us relation between the neutrino 4-momentum in the two frames:

$$
\begin{aligned}
& p_{x}=p_{x}^{*} \\
& p_{z}=\gamma\left(p_{z}^{*}+\beta E^{*}\right) \\
& E_{\nu}=\gamma\left(E_{\nu}^{*}+\beta p_{z}^{*}\right)
\end{aligned}
$$

where $\beta=V / c$ and $\gamma=\sqrt{1-\beta^{2}}=\frac{E_{\pi}}{m_{\pi}}$ is a Lorentz boost factor. Using Equations 3.1, 3.3 and 3.4 we find $p_{x}=E \sin \theta, p_{x}^{*}=E^{*} \sin \theta^{*}, p_{z}=E \cos \theta$ and $p_{z}^{*}=E^{*} \cos \theta^{*}$. It is useful to define:

$$
M=\frac{E}{E^{*}}
$$

the ratio of neutrino energy in laboratory and its energy in the center-ofmass frame of the parent hadron. Using this ratio we can rewrite the Lorentz 
transformations given in Equation 3.4:

$$
\begin{aligned}
& M \sin \theta=\sin \theta^{*} \\
& M \cos \theta=\gamma\left(\cos \theta^{*}+\beta\right) \\
& M=\gamma\left(1+\beta \cos \theta^{*}\right)
\end{aligned}
$$

From Equation 3.6 we can eliminate $\cos \theta^{*}$ to find:

$$
M=\frac{1}{\gamma(1-\beta \cos \theta)}
$$

which together with Equation 3.5 and 3.2 gives the formula for neutrino energy in the beam:

$$
E_{\nu}=\frac{m_{\pi}^{2}-m_{\mu}^{2}}{2 \gamma m_{\pi}(1-\beta \cos \theta)}
$$

Assuming that $E_{\pi} \gg m_{\pi}$, the following approximation can be made $\beta=$ $\sqrt{1-\gamma^{2}} \approx 1-\frac{1}{2 \gamma^{2}}$. We can further assume that the angle $\theta$ is small so that $\cos \theta \approx 1-\frac{\theta^{2}}{2}$. In principle, $\theta$ can be any angle up to $180^{\circ}$, however due to the relativistic boost most of the neutrinos will have small angles. Also from Equation 3.8 it can be seen that the energy of neutrino is much smaller for wider angles and we are generally interested in higher energy neutrinos. Finally, we find an often used formula for energy of neutrinos produced by a beam of pions as a function of pion boost and neutrino angle with respect to pion momentum:

$$
E_{\nu}=\frac{\left(1-\frac{m_{\mu}^{2}}{m_{\pi, K}^{2}}\right) E_{\pi, K}}{1+\gamma^{2} \theta^{2}}
$$

As indicated by subscripts, the equation is equally valid for 2 body kaon decays.

To find the angular distribution of neutrinos we use the fact that the 
neutrinos are created isotropically in meson center of mass frame. The probability that the neutrino will be emitted into solid angle $\Delta \Omega^{*}$ is given with:

$$
\frac{d P}{d \Omega^{*}}=\frac{1}{4 \pi}
$$

Therefore, to find the probability that a neutrino is emitted into solid angle $\Delta \Omega$ in a lab frame, we just need to find the transformation of the solid angles between the two reference frames.

$$
\frac{\Delta \Omega^{*}}{\Delta \Omega}=\frac{\sin \theta^{*} d \theta^{*} d \phi^{*}}{\sin \theta d \theta d \phi}=\frac{\sin \theta^{*} d \theta^{*}}{\sin \theta d \theta}
$$

where we have used the fact that the azimuthal angle $\phi$ is unaffected by Lorentz boost. From Equation 3.6 we see that $\sin \theta^{*} / \sin \theta=M$, but one can also show that $d \theta^{*} / d \theta=M$ as well. This gives the transformation of solid angles:

$$
\frac{\Delta \Omega^{*}}{\Delta \Omega}=\left(\frac{1}{\gamma(1-\beta \cos \theta)}\right)^{2}
$$

We can again assume that $E_{\pi} \gg m_{\pi}$ and consider the limit $\theta \ll 1$ in which case we find:

$$
\frac{d P}{d \Omega} \approx \frac{1}{4 \pi}\left(\frac{2 \gamma}{1+\gamma^{2} \theta^{2}}\right)^{2}
$$

\subsection{Meson Production in Nuclear Targets}

The kinematics of meson decay to neutrinos presented in the previous section, and in particular Equations 3.9 and 3.13, require us to know two important facts about how mesons are produced in a nuclear target in order to calculate a neutrino flux. First, by Equation 3.9, $E_{\nu} \propto E_{\pi}$, so to produce a neutrino beam 
of a given energy it is necessary to produce a pion beam of some energy. To calculate the flux, it is therefore required to know the differential yield of pions from a target with respect to energy, $d N / d E_{\pi}$. Second, by Equation 3.13, the typical angular divergence of neutrinos from very relativistic pions is $\theta_{\nu} \sim 1 / \gamma$. As will be shown this divergence is small in comparison to the divergence of pions off the target $\theta_{\pi} \sim 2 / \gamma$. As a result, it is required to know the double differential yield of pions off the target, $d^{2} N / d E_{\pi} d \Omega_{\pi}$. In the literature this double differential yield is sometimes cast as $d^{2} N / d p_{T} d p_{z}$ or $d^{2} N / d p_{T} d x_{F}$, where $x_{F}$ is a scaling variable defined below.

Several experiments have measured the yields of mesons in $\mathrm{p}+\mathrm{A}$ collisions that are relevant for NuMI beam $[54,55,56,57]$. However, the data from these experiments require some corrections or extrapolations to the NuMI case. Firstly, these experiments measured the particle production in discrete points in production phase space, not covering the whole production spectra. Secondly, none of these experiments were performed at $p_{\text {beam }}=120 \mathrm{GeV} / c$. Thirdly, the experiments varied in the target material. Lastly, all of the experiments were performed with very thin targets, and consequently their data will fail to represent geometric effects which would be expected to alter the meson spectra from the NuMI target. Hence, it is necessary both to interpolate and extrapolate these data in order to simulate $120 \mathrm{GeV}$ protons interacting in carbon target.

The Main Injector Particle Production experiment (MIPP) performed at Fermilab collected the hadron production data using the same $120 \mathrm{GeV}$ proton beam from Main Injector and the same target as is being used in NuMI beamline [58]. At the time of writing this thesis the MIPP data has not yet been fully analyzed and therefore is not used. 
There are two approaches how to use the external data. One can either build a model to describe the data $[59,60,61]$ or parametrize the data $[66$, 67]. Both approaches depend on several features of hadron production that allow the combining of different data samples, ie they make the mentioned extrapolations and interpolations possible. We discuss these features now.

Feynman hypothesized that the energy spectrum of the secondaries should show some scaling properties with respect to the energy of collision[68] and introduced a scaling variable $x_{F}=2 p_{L} / \sqrt{s}$, where $p_{L}$ and $\sqrt{s}$ are the longitudinal momentum of the detected particle and the total energy in the center of momentum frame. Figure 3.2 shows the distribution of longitudinal momentum of secondaries and the $x_{F}$ distribution for several different incident momenta of primary proton beam. It can be seen that while the $p_{z}$ distributions differ significantly both in the cutoff momentum (the momentum of a secondary is always less than $p_{0}$ ) and the number of pions produced in each $p_{z}$ bin, the $x_{F}$ distributions look very similar. The difference at low momentum is mostly due to the threshold used in the simulation; it was required that a secondary has $p_{z}>0.5 \mathrm{GeV}$. Such approximate scaling permits data sets acquired at different energies to be combined.

Several other scaling variables are commonly used in literature in addition to $x_{F}$. The $x_{R}=E^{\prime} / E_{\text {max }}^{\prime}$, defined as the ratio of the energy of the detected particle in the center of mass frame and the maximum energy kinematically available extends the range of validity of scaling [69, 70]. For our purposes even the variable $x_{l a b}=p_{L} / p_{0}$ where $p_{L}$ is the longitudinal component of the momentum of the secondary and $p_{0}$ is the momentum of the primary beam is sufficiently good scaling variable. In fact, it can be shown that for $p_{0} \gg M_{p}$ and $p_{z} \gg m_{\pi}$, where $p_{0}$ and $p_{z}$ are primary proton and sec- 

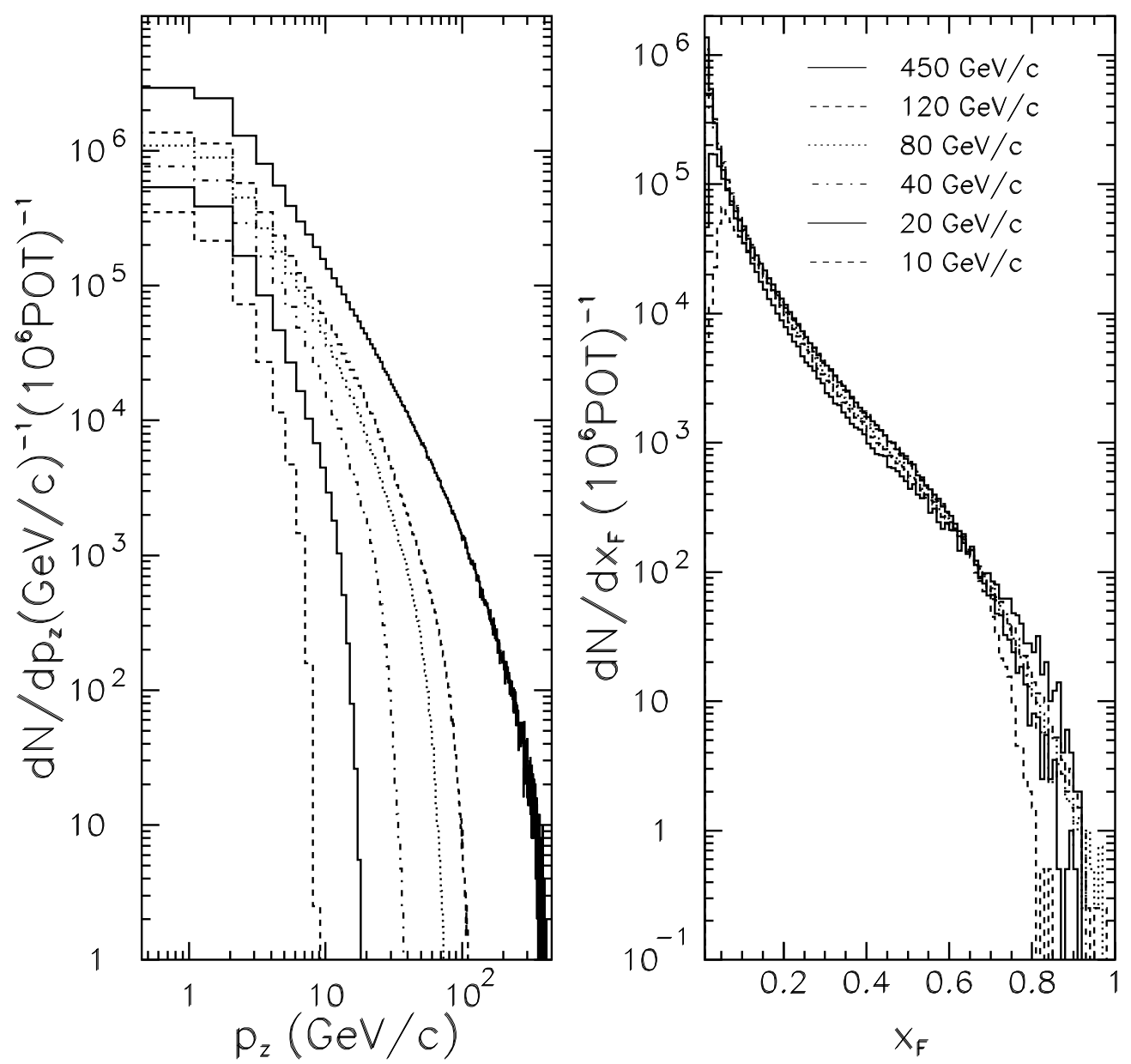

Figure 3.2: Calculation of $p_{z}$ (left) and $x_{F}$ (right) distributions of $\pi^{+}$in p+C collisions at incident momentum $p_{0}=10,20,40,80,120,450 \mathrm{GeV} / c$ and $p_{z}>$ $0.5 \mathrm{GeV} / c$. A $94 \mathrm{~cm}$ long graphite target was assumed. The FLUKA [60] hadron production model was used in a simulation. 
ondary pion momenta and $M_{p}$ and $m_{\pi}$ are proton and pion masses respectively, $x_{l a b} \approx x_{F}$.

Another important feature of hadron production is related to transverse momentum of the secondaries. In a naive model we can assume that partons in the nucleon have a Fermi momentum of

$$
p_{F} \approx \frac{\hbar c}{\Delta x} \approx \frac{\hbar c}{1 \mathrm{fm}} \approx 200 \mathrm{MeV}
$$

The component of this momentum transverse to the beam is not affected by the boost. This would imply that the production spectra in transverse momentum $p_{T}$ should be independent of $x_{F}$.

Figure 3.3 shows the distributions of secondary pion transverse momentum produced in $\mathrm{p}+\mathrm{C}$ collisions for several different energies of proton beam. Indeed, the $p_{T}$ distributions look similar, peaking at around 200-300 MeV. Alternatively, one can look at the $p_{T}$ distributions at different $x_{F}$ for a given energy of primary proton beam (Figure 3.4). Again it can be seen that these $p_{T}$ distributions have similar shape. The $p_{T}$ distributions for $\pi^{-}, K^{+}$and $K^{-}$ can be seen in Figures 3.4 and 3.5. These show the same features, as the corresponding $\pi^{+}$distributions showing only slow evolution.

The Fermi momentum shown in Figure 3.3 results in a typical angular divergence of the pion beam off the target given by

$$
\tan \theta_{\pi}=\frac{\left\langle p_{T}\right\rangle}{p_{z}} \approx \frac{280 \mathrm{MeV}}{E_{\pi}}=\frac{2}{\gamma_{\pi}}
$$

where $E_{\pi} \approx p_{\pi} \approx p_{z}$ is the energy of the very relativistic pion. Thus the angular spread of the pions off the target exceeds the angular spread of neutrinos 


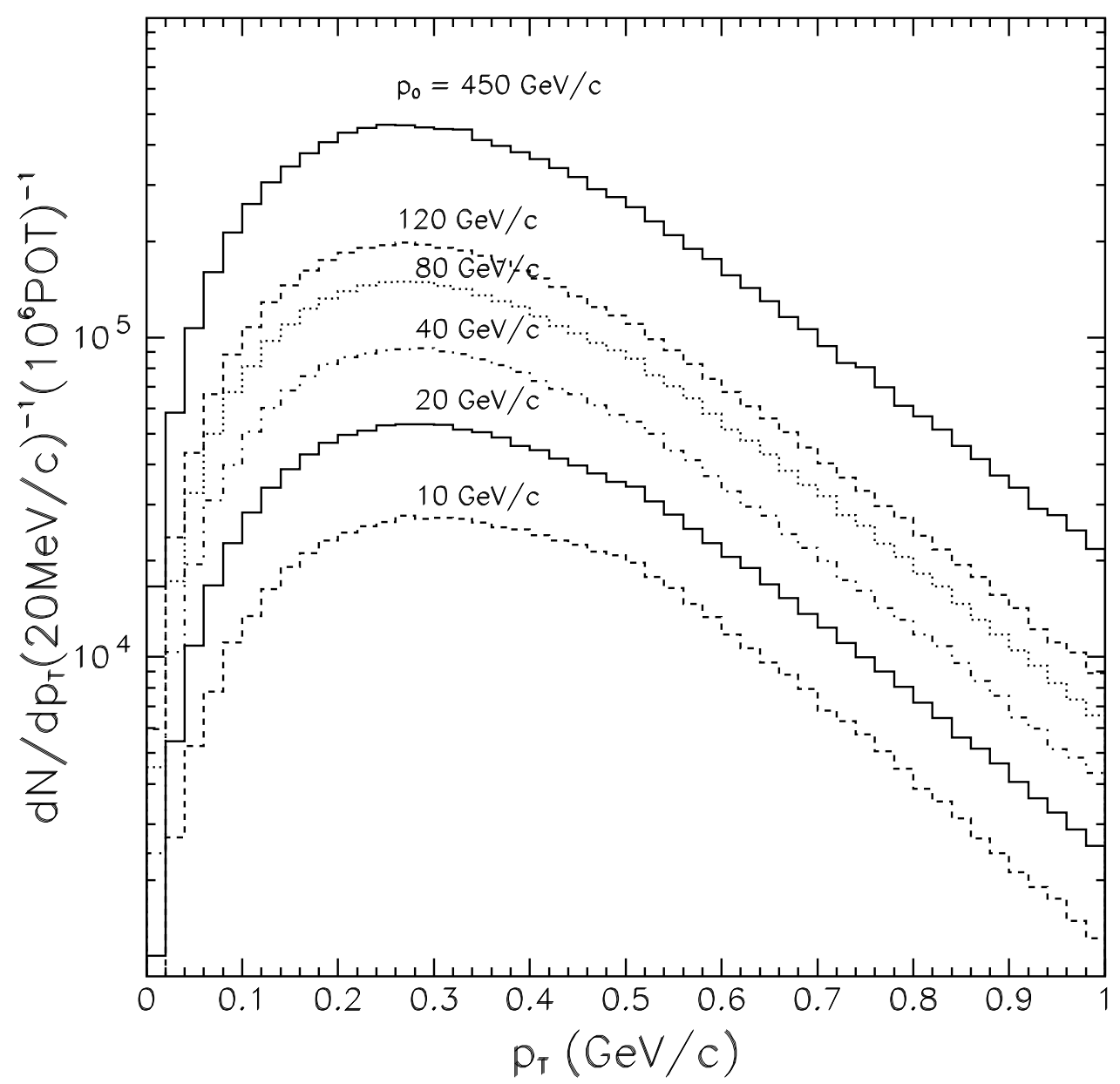

Figure 3.3: The calculation of $p_{T}$ distributions of $\pi^{+}$produced in $\mathrm{p}+\mathrm{C}$ collisions at incident momentum $p_{0}=10,20,40,80,120,450 \mathrm{GeV} / c$. A $94 \mathrm{~cm}$ long graphite target was assumed. FLUKA [60] hadron production model was used in a simulation. 


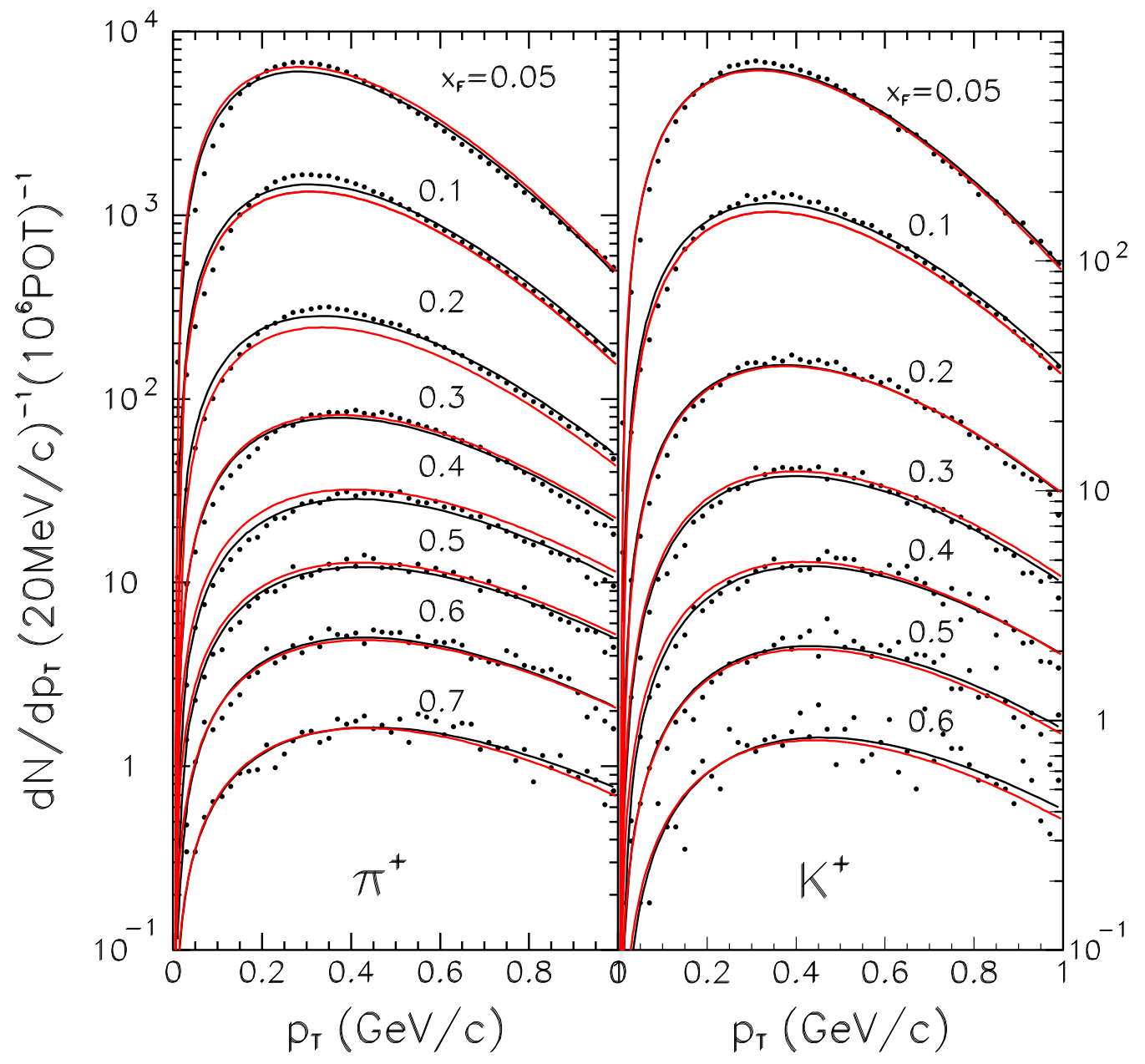

Figure 3.4: The calculation of $p_{T}$ distributions for $\pi^{+}$and $K^{+}$in several $x_{F}$ slices using FLUKA hadron production model and $94 \mathrm{~cm}$ long graphite target target (black dots). Black solid line is a fit to a particular $x_{F}$ slice using Equation 6.1 and red line is the overall parametrization using Equation 6.2. 


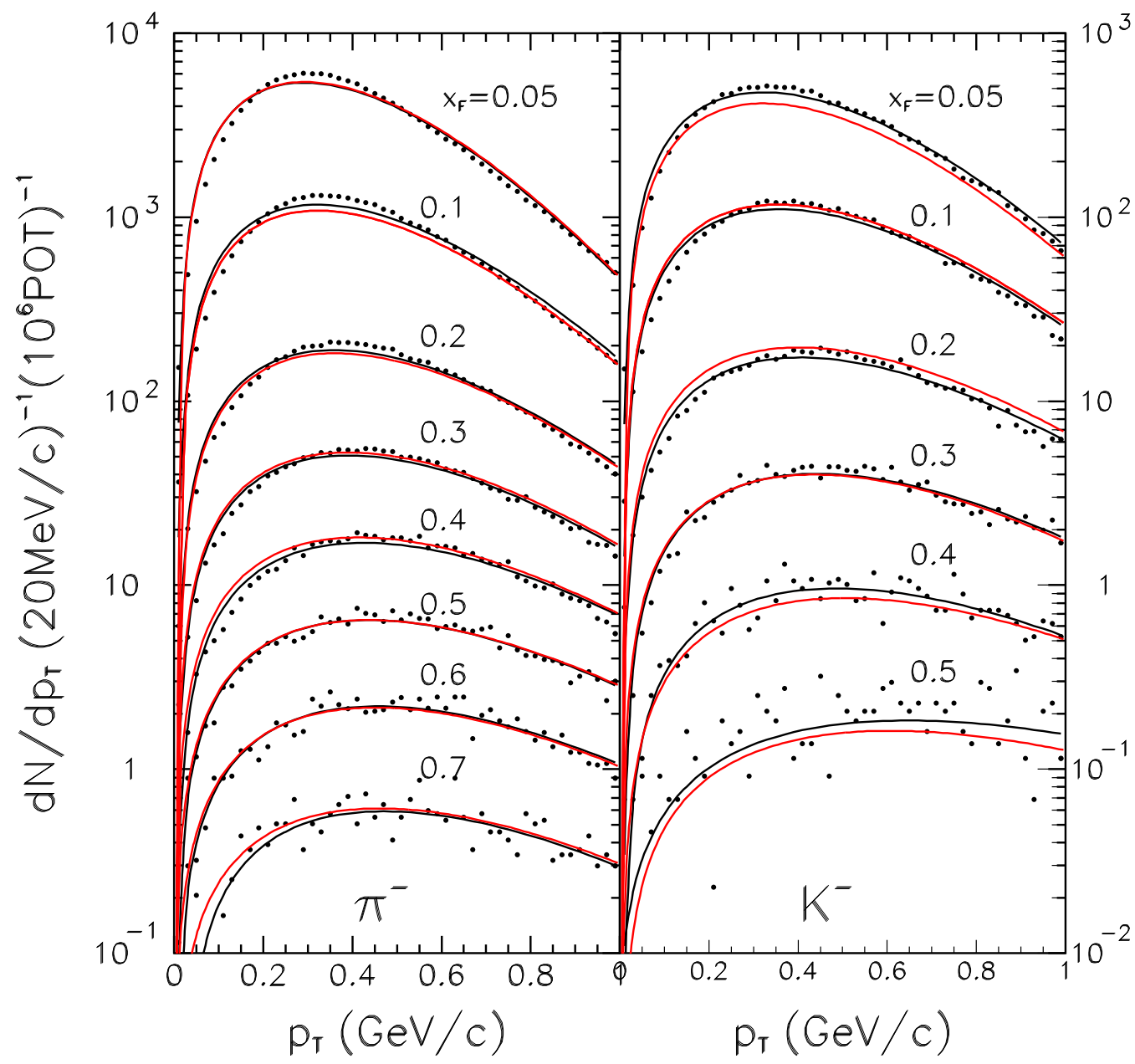

Figure 3.5: The calculation of $p_{T}$ distributions for $\pi^{-}$and $K^{-}$in several $x_{F}$ slices using FLUKA hadron production model and $94 \mathrm{~cm}$ graphite target (black dots). Black solid line is a fit to a particular $x_{F}$ slice using Equation 6.1 and red line is the overall parametrization using Equation 6.2. 
from pion decay.

Several experiments explored the dependence of particle production on the target material (see [55]). It was found that the data shows fairly simple scaling which could be described with one parameter.

Figure 3.6 shows the distribution of transverse and longitudinal momenta of pions that produce neutrino events in the MINOS Near Detector. Overlaid are the points that indicate the parts of the phase space measured in three hadron production experiments $[56,55,54]$. As discussed, these experiments measured yields in discrete points of the phase space and were done at various energies. Also indicated are the target materials used in the experiments.

\section{Parametrized Particle Production Yields}

The features of hadron production discussed so far make it possible to combine the different hadron production data sets and either build a model or parametrize the data to get a prediction of particle yields of the NuMI target. An example of parametrization developed in Chapter 6 is shown in Figures 3.4 and 3.5. The parametrization is compared to the yields as predicted by the FLUKA hadron model [60]. The black curve shows the fits of a simple three parameter function (see Equation 6.1) to the $p_{T}$ distributions for charged pions and kaons. The three parameters, thus become functions of $x_{F}$ and can themselves be fit to a suitable functional form. The red curve shows such a fit using the functions shown in Equation 6.2. In fact, this parametrization will be used later in Chapter 6 to adjust the FLUKA hadron production model to better agree with the neutrino flux observed in the MINOS detectors. 


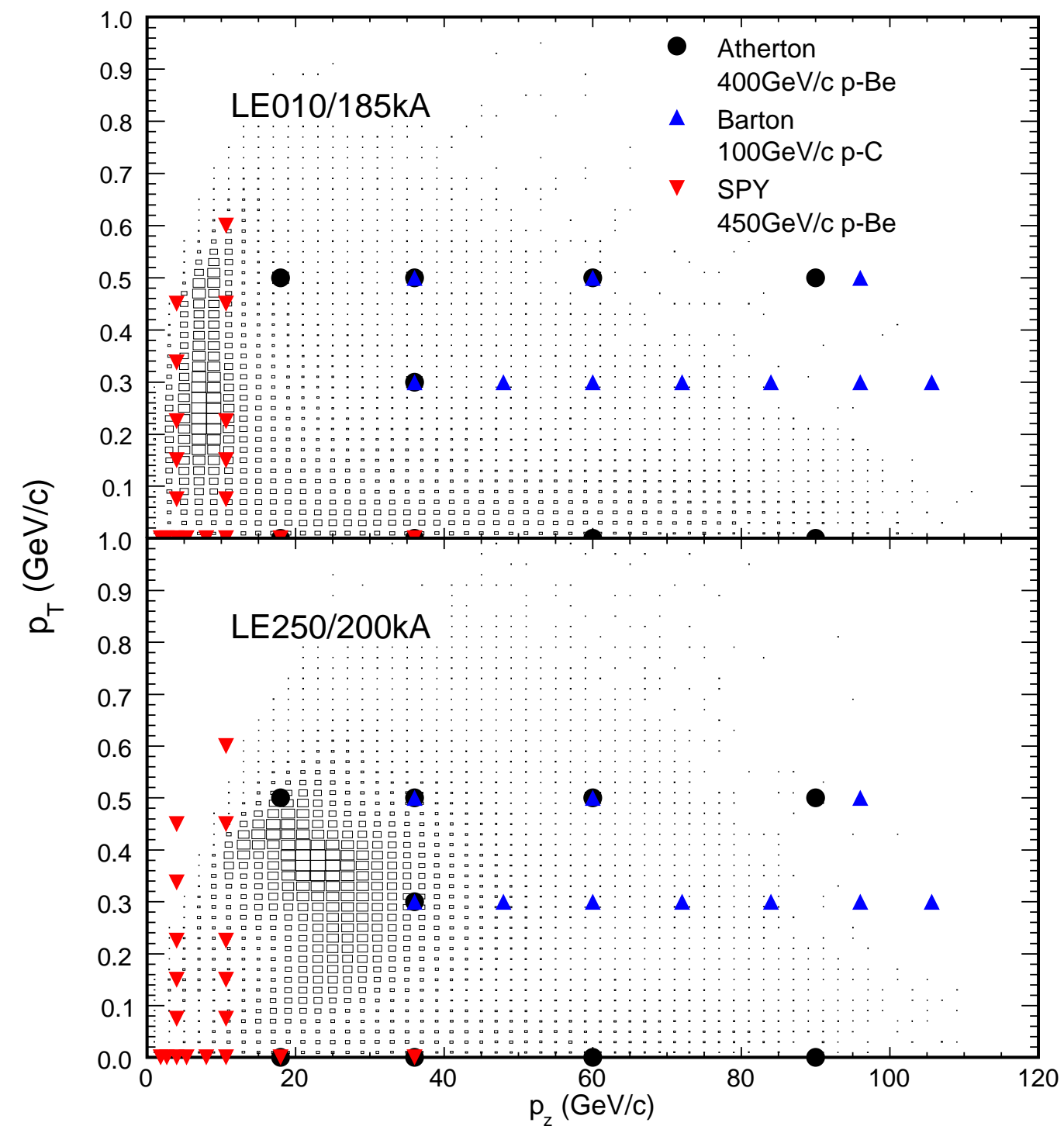

Figure 3.6: The distribution of transverse and longitudinal momentum of $\pi^{+}$ that contribute to neutrinos at Near Detector for two different beam configurations. The size of the box is proportional to the number of Charged Current neutrino events in the MINOS Near Detector that come from $\pi^{+}$momentum $p=\left(p_{z}, p_{T}\right)$. Overlaid are the points that indicate the part of the phase space measured by the three relevant hadron production experiments $([54,55,56])$. 

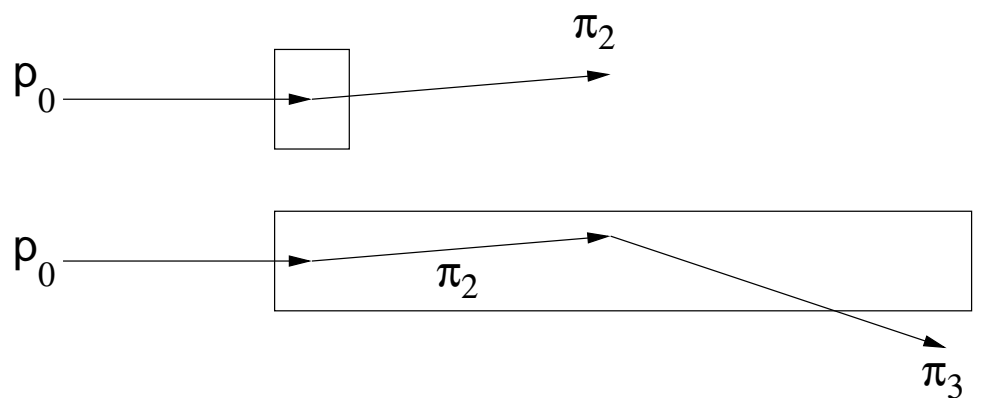

Figure 3.7: Pions created in primary proton interactions can escape out of the thin target without reinteraction. For a thick target there is a high probability that a pion will reinteract.

\section{Cascade Models}

The experiments that measure the production of secondaries in $\mathrm{p}+\mathrm{A}$ collisions typically use thin targets whose thickness is only few \% of a nuclear interaction length. By contrast, the NuMI target is 2 interaction lengths long. For such long targets the produced secondaries can reinteract within the target, as indicate in Figure 3.7. Several cascade models [59, 60, 61] exist that simulate these reinteractions.

Figure 3.8 shows the prediction of the fraction of pions created in reinteractions in the target as a function of primary proton energy. This fraction of course depends on the shape of the target. The longer the target, the bigger this fraction is expected to be. This is confirmed in MC simulations, as seen in the same figure. It can be seen that for $120 \mathrm{GeV}$ protons used in NuMI it is expected that $\sim 30 \%$ of pions with momentum above $5 \mathrm{GeV}$ come from reinteractions.

Since a significant number of pions coming of the target are a product of reinteractions, the predicted yields of pions depends greatly on the cascade model. Figure 3.9 shows few distributions of transverse momenta of pions 


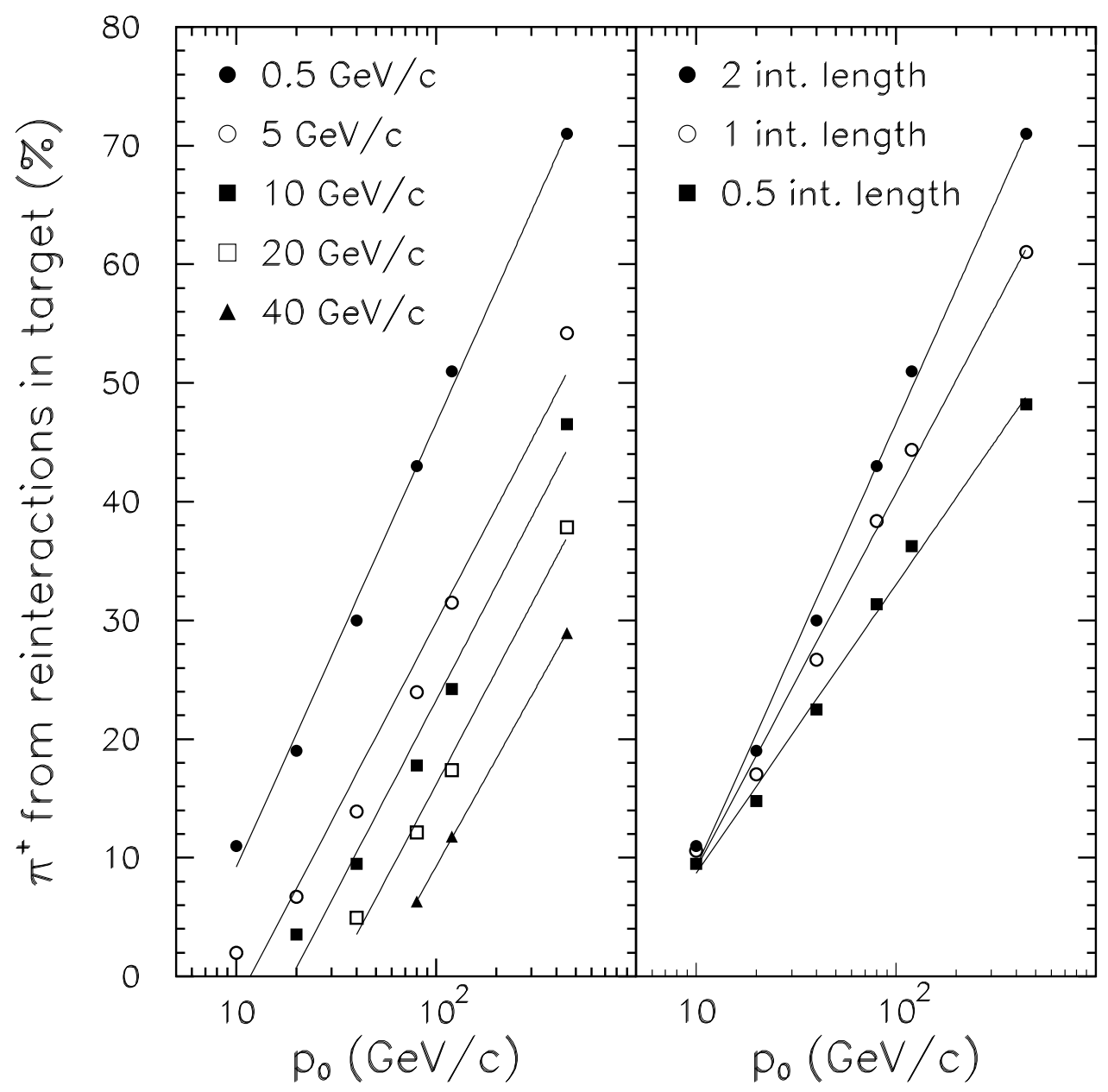

Figure 3.8: The calculation of the fraction of tertiary $\pi^{+}$production from reinteractions in a graphite target as a function of primary beam momentum $p_{0}$. The calculation was done using FLUKA cascade model [60]. The left plot shows the reinteraction fraction for 2 interaction lengths long target with several pion momentum $\left(p_{z}\right)$ thresholds. The right plot shows the reinteraction fraction for three different lengths of the target, ie 0.5, 1 and 2 interaction lengths. The NuMI target is 2 interaction lengths long. 


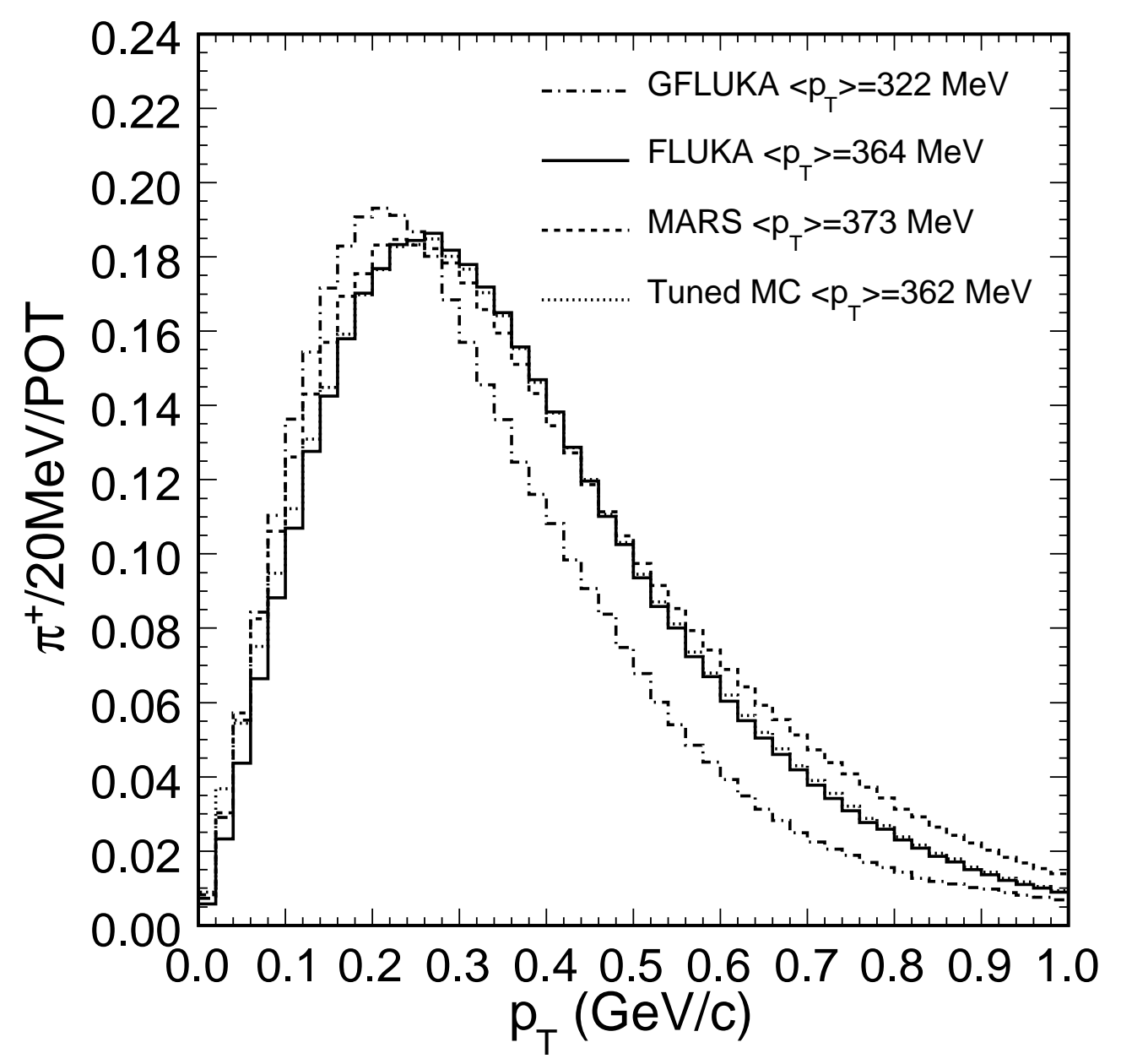

Figure 3.9: The $p_{T}$ distribution of $\pi^{+}$secondaries with $p_{z}>0.5 \mathrm{GeV} / c$ produced in a NuMI graphite target by a $120 \mathrm{GeV}$ proton beam as predicted by various hadron cascade models $[59,60,61]$. The Tuned MC corresponds to the fit to MINOS Near Detector data (see Chapter 6). 
coming off the target as predicted by different cascade models. Indeed the distributions vary significantly between the models. Such variations ultimately result in a very different prediction of neutrino energy spectrum, as can be seen in Figure 3.10.

\subsection{The NuMI Target}

The NuMI target is shown in Figure 3.11. It consists of 47 consecutive graphite segments. Each segment is $6.4 \mathrm{~mm}$ wide, $18 \mathrm{~mm}$ high and $20 \mathrm{~mm}$-long the beam direction. The length of the target corresponds approximately to 2 interaction lengths, ensuring that most of the protons will interact within it, while the relative narrow transverse dimensions allow the secondaries to escape without reinteractions. The spacing between the segments is $0.3 \mathrm{~mm}$, giving a total length of $95.38 \mathrm{~cm}$.

The Main Injector proton beam sent to the NuMI beam-line deposits large amounts of energy in the target. To reduce the stresses induced by proton beam heating, the target is segmented. In addition, the target is water cooled using pipes that run above and below the segments. The graphite segments are soldered to the cooling pipes. To avoid a direct hit from the proton beam which would vaporize the cooling water, the top and the bottom pipes are connected through a ring at the end of the target. The protons that don't interact within the target pass through this ring without directly hitting.

An additional 48th graphite fin is located upstream of the first of the 47 target fins. The so called horizontal fin is rotated by $90^{\circ}$ with respect to the remaining 47 vertical fins. Its center is located $15.73 \mathrm{~cm}$ upstream of the upstream edge of the main target. This additional fin provides a means to 


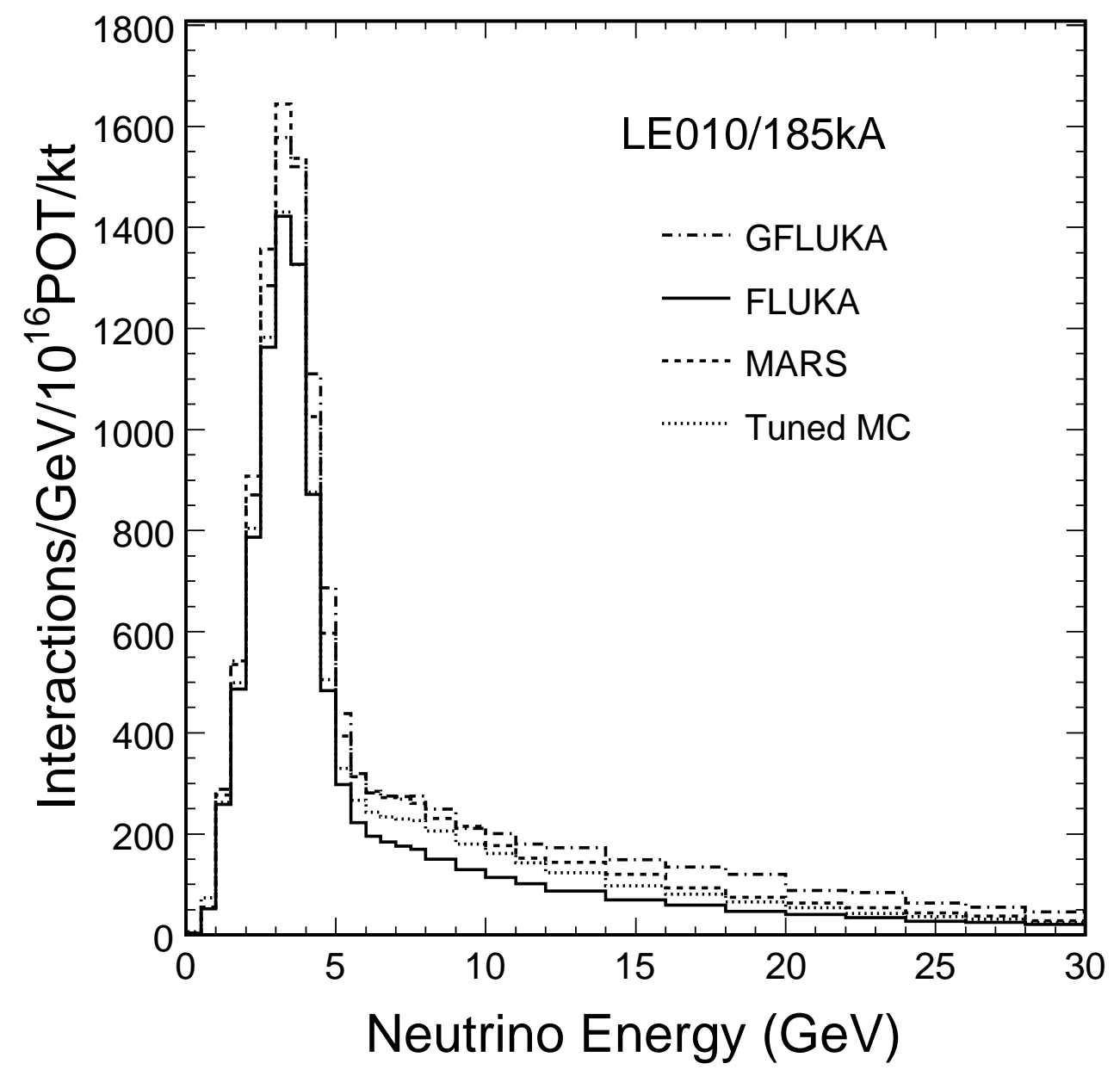

Figure 3.10: Energy spectrum of Charged Current neutrino interactions at the MINOS Near Detector as predicted by different calculations of particle yields off the NuMI graphite target $[59,60,61]$ The above calculations of particle yields are all consistent with experimental production data $[54,55,56]$. The spectrum is for the LE10/185kA configuration (Table 2.1). The Tuned MC corresponds to the fit to MINOS Near Detector data (see Chapter 6). 


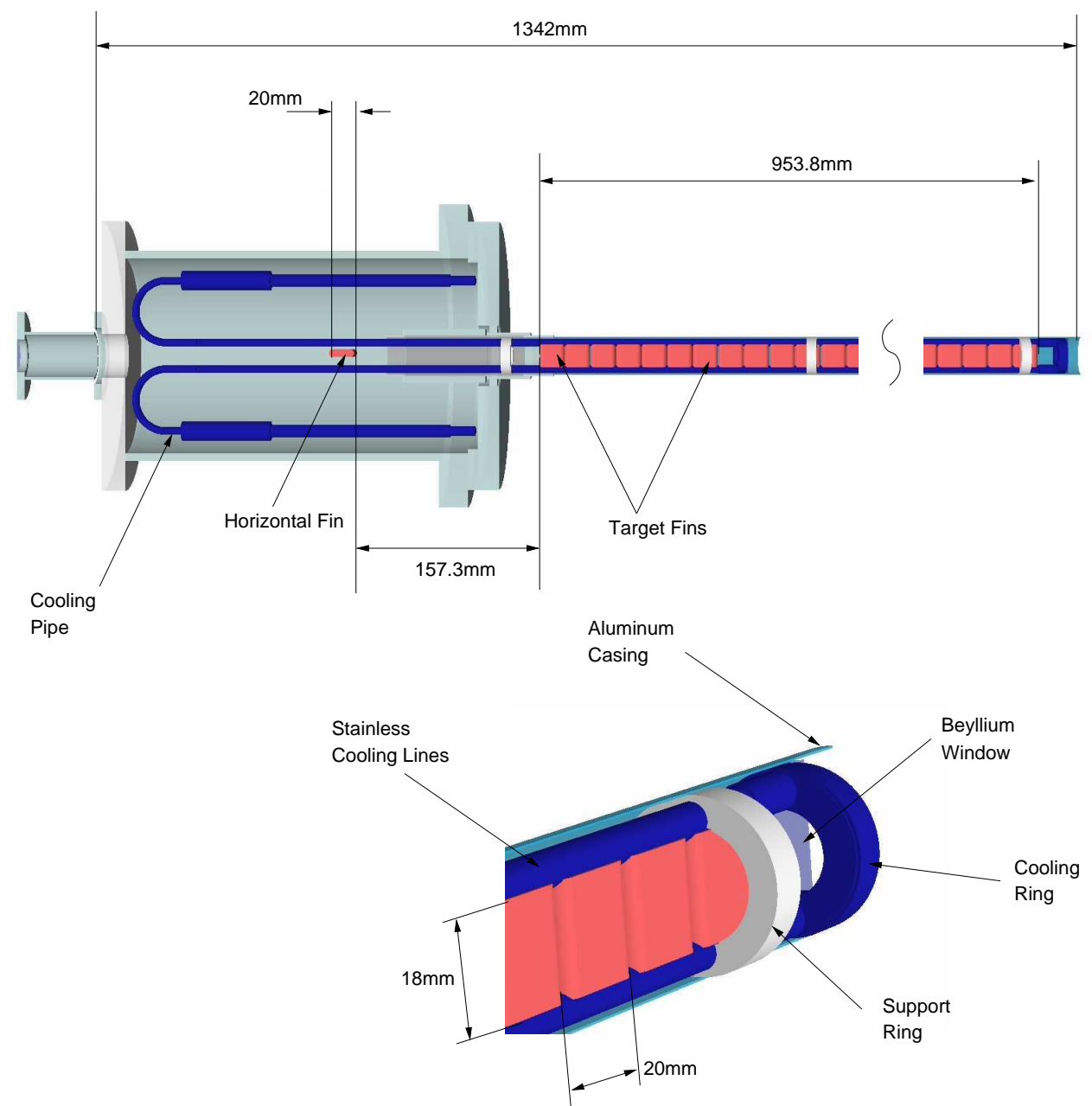

Figure 3.11: The NuMI neutrino target which is used to generate a beam of mesons that give rise to a neutrino beam. The target is exposed to $120 \mathrm{GeV}$ proton beam. The active part of the target is made out of $20 \times 18 \times 6.4 \mathrm{~mm}$ graphite segments which are contained in the Aluminum vessel. The segments are cooled by circulating water in the lines above and below the graphite segments. The upstream and downstream window of the vessel (the parts that are exposed to direct hits from the proton beam) are made out of Beryllium reducing the interaction with proton beam. One graphite segment, used for alignment, has horizontal orientation (opposed to the 47 vertical segments) and is located further upstream. 
check the alignment of the target position [41]. By design this horizontal fin is centered on the beam axis, however the target used during the first run period (Table 2.1) had it offset by $2.2 \mathrm{~mm}$ in the vertical direction. This misalignment is modeled in the MC.

The target is contained within an Aluminum casing with Beryllium windows at the points where the proton beam enters and exits the target. The casing consists of a canister and a meter long tube in which the 47 vertical fins are enclosed. The tube has an outer diameter of $30 \mathrm{~mm}$ and is $0.4 \mathrm{~mm}$ thick. It is Helium filled to prevent the corrosion of the target.

\subsection{Particle Focusing}

The particles emerging from the target have small, but finite divergence. This divergence reduces the neutrino flux for two reasons. Firstly, as can be seen from Equation 3.13, the flux is the greatest along the direction of the parent. It would be, therefore, ideal if all of the secondaries would go directly toward the neutrino detector. From Equation 3.14 the divergence of pions emanating from the target is $\theta_{\pi} \sim 2 / \gamma$ which is large compared to the angular divergence $\theta_{\nu} \sim 1 / \gamma$ of neutrinos from the decays of relativistic pions (Equation 3.13). Equation 3.13 suggests that removing the $2 / \gamma$ divergence of the pions would improve the neutrino flux by a factor of 25 :

$$
\frac{\phi_{\text {ideal }}}{\phi_{\text {smeared }}}=\frac{\left(1+\gamma^{2} \theta_{\text {smeared }}^{2}\right)^{2}}{\left(1+\gamma^{2} \theta_{\text {ideal }}^{2}\right)^{2}}=\frac{\left(1+\gamma^{2}(3 / \gamma)^{2}\right)^{2}}{\left(1+\gamma^{2}(1 / \gamma)^{2}\right)^{2}}=\frac{100}{4}=25
$$

where $\theta_{\text {ideal }}=1 / \gamma$ is the smallest angular divergence one can obtain just from

decay kinematics and $\theta_{\text {smeared }}=3 / \gamma$ combines the effects of decay kinematics 
and pion angular divergence. Secondly, due to divergence, the particles drift radially outward. Since the decay volume has finite transverse size, some of the particles hit the shielding before being able to decay.

The NuMI beam-line uses two magnetic horns to focus the secondary particles. We now discuss the principles of horn focusing and the geometry of NuMI horns.

\subsubsection{Horn Focusing}

Tho horn acts as a lens that bends the secondaries back to the primary proton direction [71]. The horn consists of two conductors, the inner and the outer conductor, that are symmetric along the beam axis (see Figure 3.12). The current $I$ is pulsed down the inner conductor and returned through the outer conductor producing the toroidal magnetic field between the conductors $B(r)=\mu_{0} I / 2 \pi r$, where $r$ is the radial distance from the beam axis. Ideally,

for the horn with perfect axial symmetry, the field outside of the horns would vanish.

The inner conductor of NuMI horns has a parabolic shape that follows the curve $z=a r^{2}$ where $\mathrm{z}$ is the coordinate along the beam (or horn) axis. The amount of $p_{T}$ kick that parabolic horn gives to a particle, ie the amount by which it changes the transverse momentum of the particle, can be estimated by approximating the path of the particle inside the horn with a straight line (as in Figure 3.12). Charged particles moving through the magnetic field inside the horn feel the force $\vec{F}=q(\vec{v} \times \vec{B})$. The change in the momentum of the 


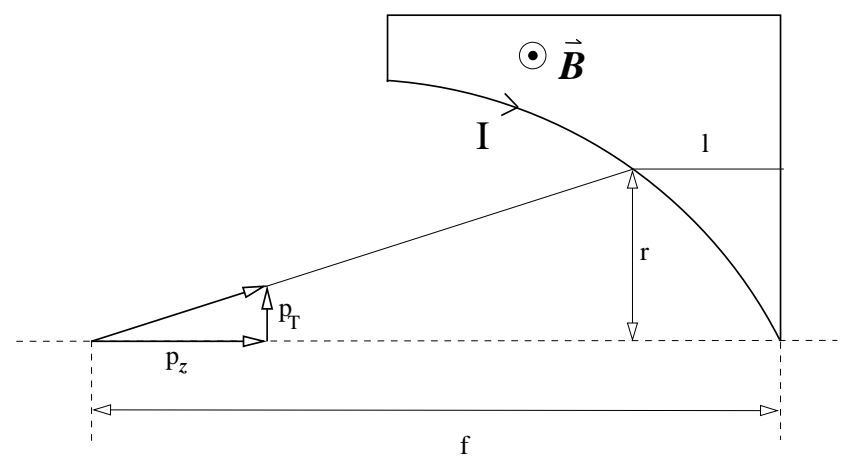

Figure 3.12: Schematic view of particles going through a focusing horn with parabolic shape of inner conductor. Due to the shape of the conductor, the particles entering at bigger radius will see greater $\int B d \ell$ resulting in a greater change in transverse momentum.

particle is equal to the impulse:

$$
|\vec{J}|=\left|\Delta p_{T}\right|=\int \vec{B}(r) d \ell \approx \frac{\mu_{0} I}{2 \pi r} \cdot a r^{2}
$$

The focal length of the horn can be then found assuming the source is far away from the horn $r / f \approx p_{T} / p$. For perfectly focused particle, the horn would remove its transverse momentum, so that $\Delta p_{T}=p_{T}$ :

$$
f \approx \frac{r}{p_{T}} p=\frac{2 \pi}{\mu_{0} I a} p
$$

From this we see that parabolic horns act like lens whose focal length is proportional to particle momentum $p$ [72].

The NuMI horns have a different parabolic shape for the left and right part of the horn. The total focal length of the horn can be estimated by combining the two parts in the thin lens approximation. However, the thin lens approximation can only be used as a very rough approximation of horn focus- 
ing. Several effects need to be considered when calculating the trajectories of particles through the horns. First, the target is an extended object located very close to the first horn, breaking the point source approximation. Second, the horns themselves are large objects extending over $3 \mathrm{~m}$ along the beam axis. Finally, the particles have to pass through the horn conductor, experiencing multiple scattering in the process.

Again, we rely on the MC calculation in order to account for these effects, tracing individual particles through the magnetic fields in infinitesimal steps, deflecting particle trajectories by Equation 3.16 or introducing scattering in the horn material.

\subsubsection{NuMI Horns Geometry}

The NuMI horns are shown in Figures 3.13 and 3.14. The inner and outer conductors of the horns are made out of Aluminum. While the outer conductor is $2.54 \mathrm{~cm}$ thick, the inner conductor is only few millimeter thick in order to reduce the scattering of pions when they enter into the region with magnetic field. On the other hand horn needs to be sturdy enough which prevents making inner conductor any thinner.

Even though the walls of the horns are relatively thin, most of the particles move parallel to the axis of the horn and not perpendicular to the walls of the horn making the travel path through the walls much longer. The nuclear interaction length for Aluminum is $\lambda_{I}=39.4 \mathrm{~cm}[17]$, so the absorption of pions in the horn walls ranges from 20\%-40\% depending on the incidence angle. The focusing of the horns is also affected by multiple scattering of pions 
in the inner conductor. The typical scattering angle is given with [17]:

$$
\theta_{0}=\frac{13.6 \mathrm{MeV}}{p} \sqrt{\frac{x}{X_{0}}}\left[1+0.038 \ln \left(\frac{x}{X_{0}}\right)\right]
$$

where $p$ is the pion momentum and $X_{0}$ is the radiation length. Using $X_{0}=$ $8.9 \mathrm{~cm}$ for Aluminum we find for a $5 \mathrm{GeV}$ pion $\theta_{0} \sim 3 \mathrm{mrad}$.

Since the horn heats up due to the pulsed current and radiation, during running the inner conductor is being cooled with nozzles that spray water on it. The horns have a small field free region, called the neck of the horn. The particles can pass through that region without being affected by horns. The neck of second horn is such that it allows the particles focused by first horn to go through it without being further focused. These necks also allow the proton beam to go through without hitting the horns.

The NuMI horns were designed to sustain currents of up to $200 \mathrm{kA}$ producing the field of up to $\sim 3 \mathrm{~T}$ in the narrowest part, inside the horn field region. The current in the inner conductor of NuMI horns during the normal running flows along the beam axis creating the magnetic field that focuses positive particles and at the same time defocuses negatively charged particles. The positive pions give rise to neutrinos and negative to anti-neutrinos. Figure 3.15 shows the measured magnetic field inside the first NuMI horn. The measurement shows good agreement with $1 / \mathrm{r}$ drop-off of the field. The field outside of the horn at radial values smaller than the radius of the inner conductor was measured to be negligibly small. At most it reached the $\sim 10 \mathrm{mT}$ in horn neck region, and typically is less than few $\mathrm{mT}$ [73].

The field inside of the inner conductor is modeled assuming the homogeneous distribution of current throughout the conductor cross section. The 

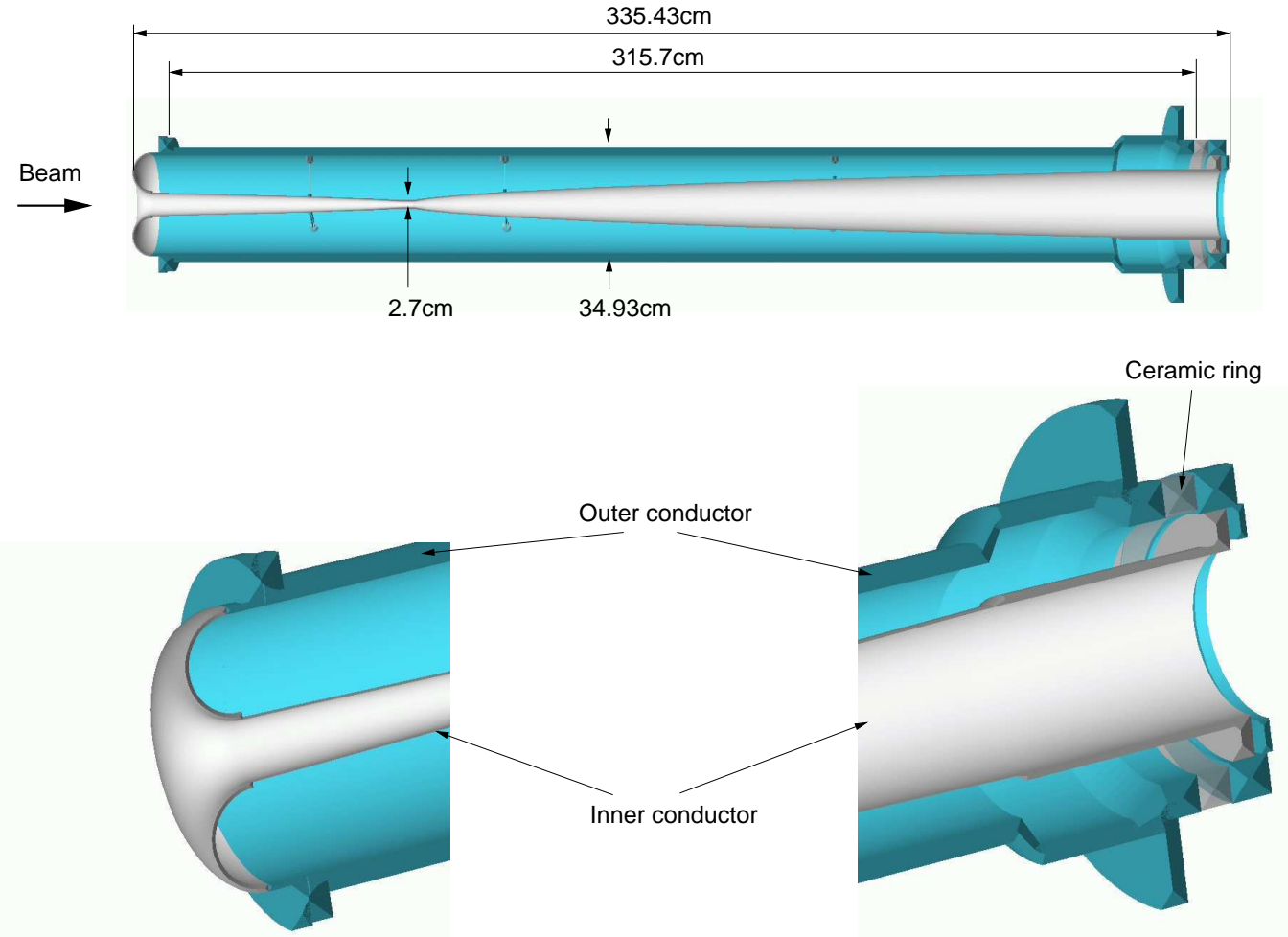

Figure 3.13: The first of the pair of NuMI horns used to focus charged secondaries. The horn is made out of aluminum. During beam operation the current is pulsed down the inner conductor creating a toroidal magnetic field in the region between conductors (Figure 3.15). The inner conductor is made thin to reduce possible scattering of particles passing through it. The thickness of the inner conductor is $4.5 \mathrm{~mm}$ where the conductor has smallest radius (horn neck) and $2 \mathrm{~mm}$ elsewhere. The outer conductor is $2.54 \mathrm{~cm}$ thick. 

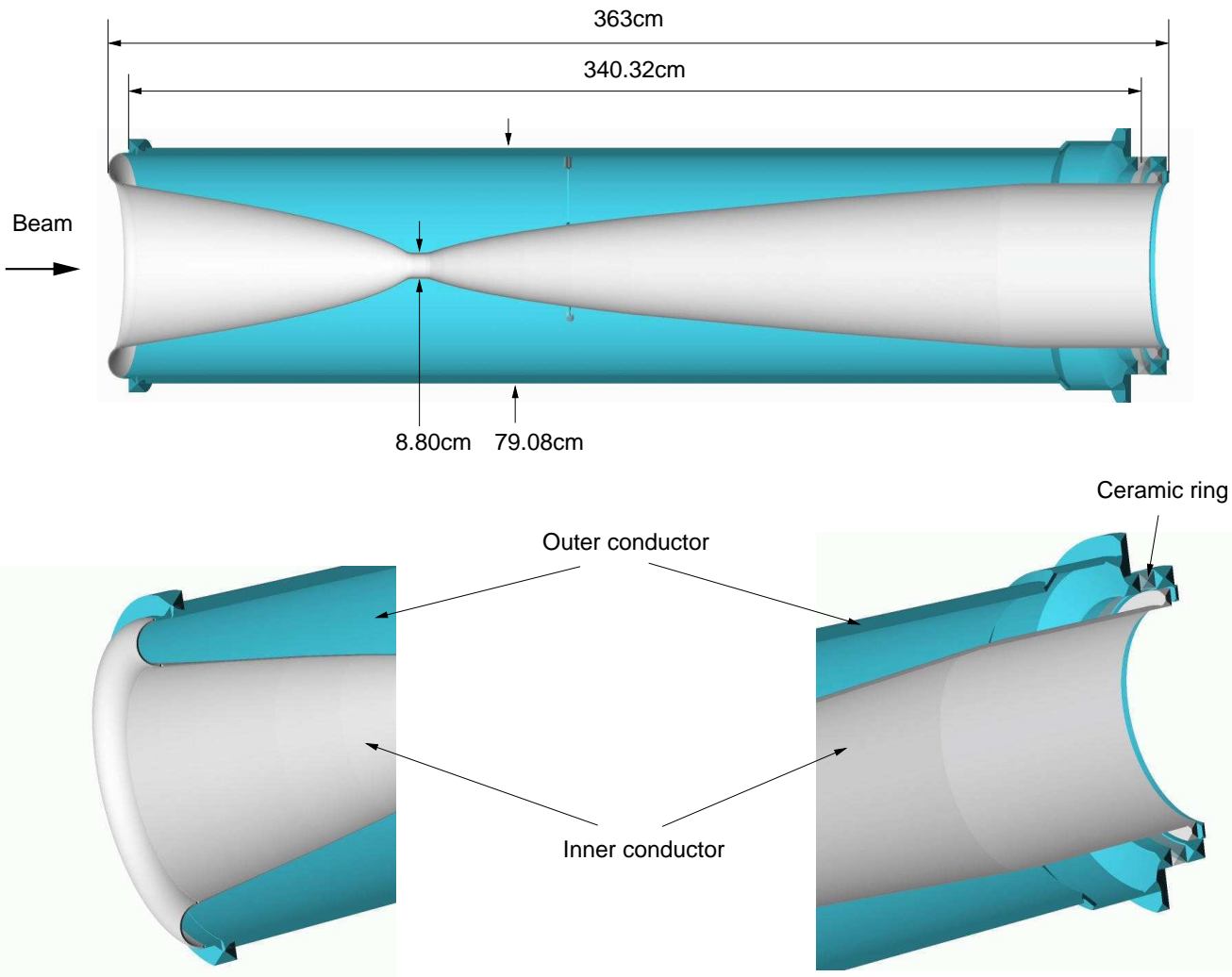

Figure 3.14: The second of the pair of NuMI horns used to focus charged secondaries. The horn is made out of aluminum. During beam operation the current is pulsed down the inner conductor creating a toroidal magnetic field in the region between conductors (Figure 3.15). The inner conductor is made thin to reduce possible scattering of particles passing through it. The thickness of the inner conductor is $5 \mathrm{~mm}$ where the conductor has smallest radius (horn neck) and $3 \mathrm{~mm}$ elsewhere. The outer conductor is $2.54 \mathrm{~cm}$ thick. 


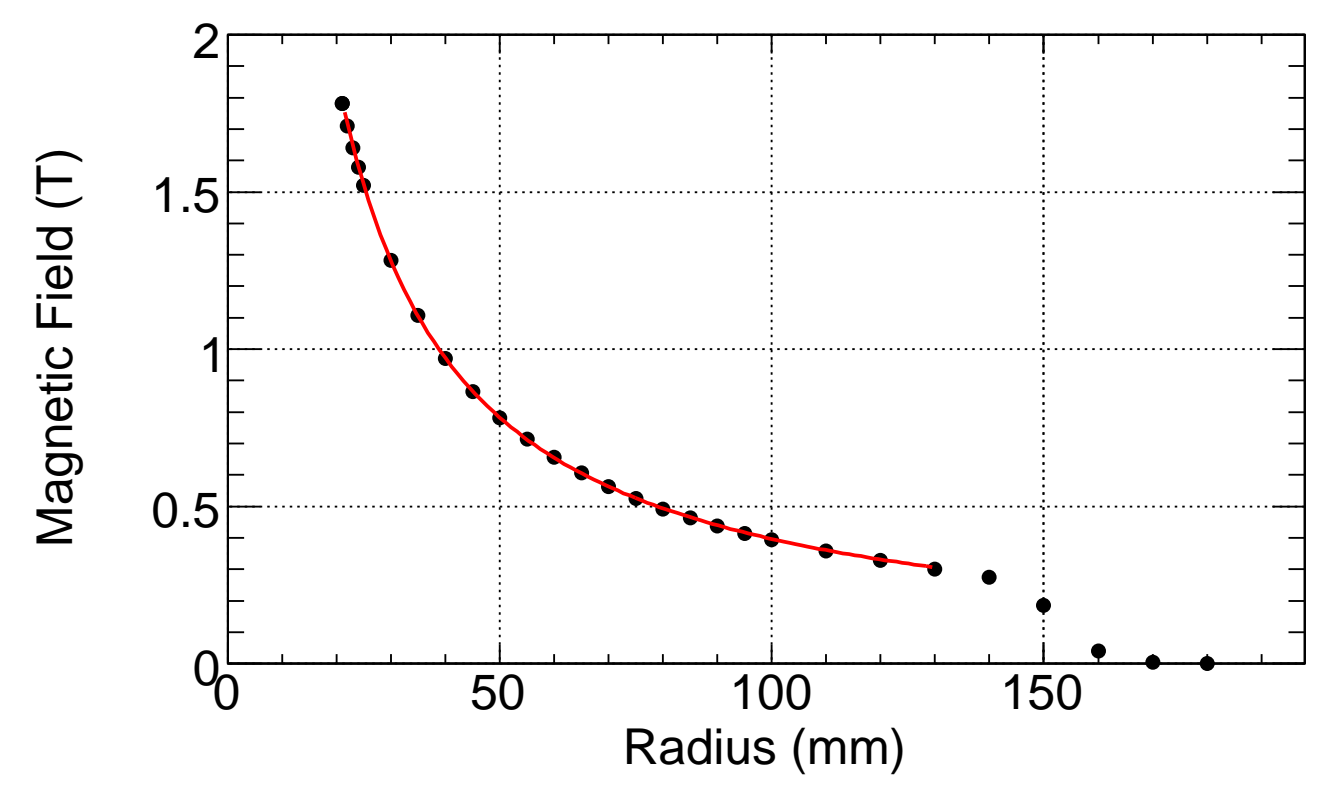

Figure 3.15: The magnetic field between the inner and outer conductor of the first NuMI horn. The data (dots) shows the expected $1 / \mathrm{r}$ dependence (curve). (Figure courtesy L. Loiacono)

assumption stems from the relative thinness of the inner conductor as compared to the skin depth of the current. The validity of the assumption will be discussed in $§ 5.2 .4$.

The second horn is used to capture the particles that were improperly focused by the first horn extending the focusing capabilities. Recalling that the mean transverse momentum $\left\langle p_{T}\right\rangle$ of particles off the target is approximately independent of their longitudinal momentum (Figure 3.4)we find that the angle at which the secondary emerges from the target $\theta \approx\left\langle p_{T}\right\rangle / p=f(1 / p)$. Figure 3.16 shows the schematic view of target and horns and 5 possible trajectories. The softest pions will come at the widest angle and will therefore be over-focused by the first horn, but they will then get focused by the second horn. Similarly, the pions that haven't received enough focusing by first horn 


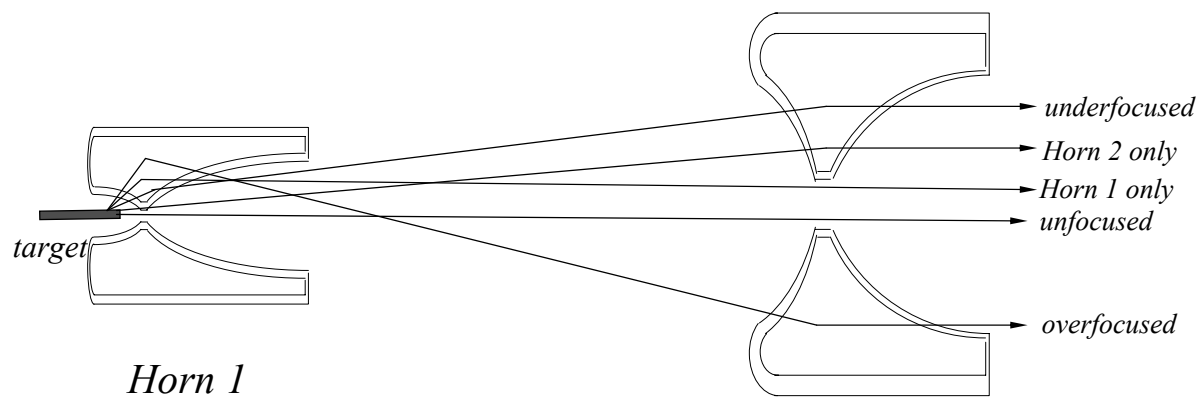

Horn 2

Figure 3.16: Five possible trajectories of particles emanating from target and going through the focusing horns. The label "unfocused" refers to small angle pions that travels through the necks of horns. The labels "Horn 1" and "Horn 2 only" refer to pions that are focused exclusively by one of the two horns and go through the neck of the other horn. The label "underfocused" refers to pions that receive insufficient kick from Horn 1, while the label "overfocused" refers to pions that receive too great a kick from Horn 1 and must be restored by Horn 2 . 
receive additional focusing by second horn. Obviously a two horn system is capable of focusing a wider range of secondary momenta.

The five components contribute to different regions of neutrino energy spectrum as can be seen in Figure 3.17. Indeed we can see that it is the softest pions that contribute to lowest energy bins that get over-focused with first horn and are recaptured by the second horn. The stiffest pions that contribute to higher energy neutrinos go straight through the necks of both horns. The majority of the neutrinos in the focusing peak are under-focused by first horn and properly focused by second horn. The remaining two components are those being focused with exclusively horn 1 or horn 2 .

\subsection{Target Hall}

The NuMI target and the focusing horns are located in a Target Hall which extends to the entrance to the Decay pipe. The Target Hall is filled with steel shielding that surrounds the horns and the target. The particles that stray radially outward are stopped within this shielding.

Figure 3.18 shows the expected radial distribution of pions at the end of the Target Hall if no shielding was present. Also indicated in the figure is the aperture of the shielding blocks. Clearly, many of the pions will be stopped in the shielding. However, the trajectory of these pions is such, that it would require an unfeasible size of the decay volume in order to allow them to decay in flight. The end of the target hall is $\sim 45 \mathrm{~m}$ downstream of target while it is expected that the pions that contribute to the neutrino flux will decay several hundreds of meters downstream of target. 


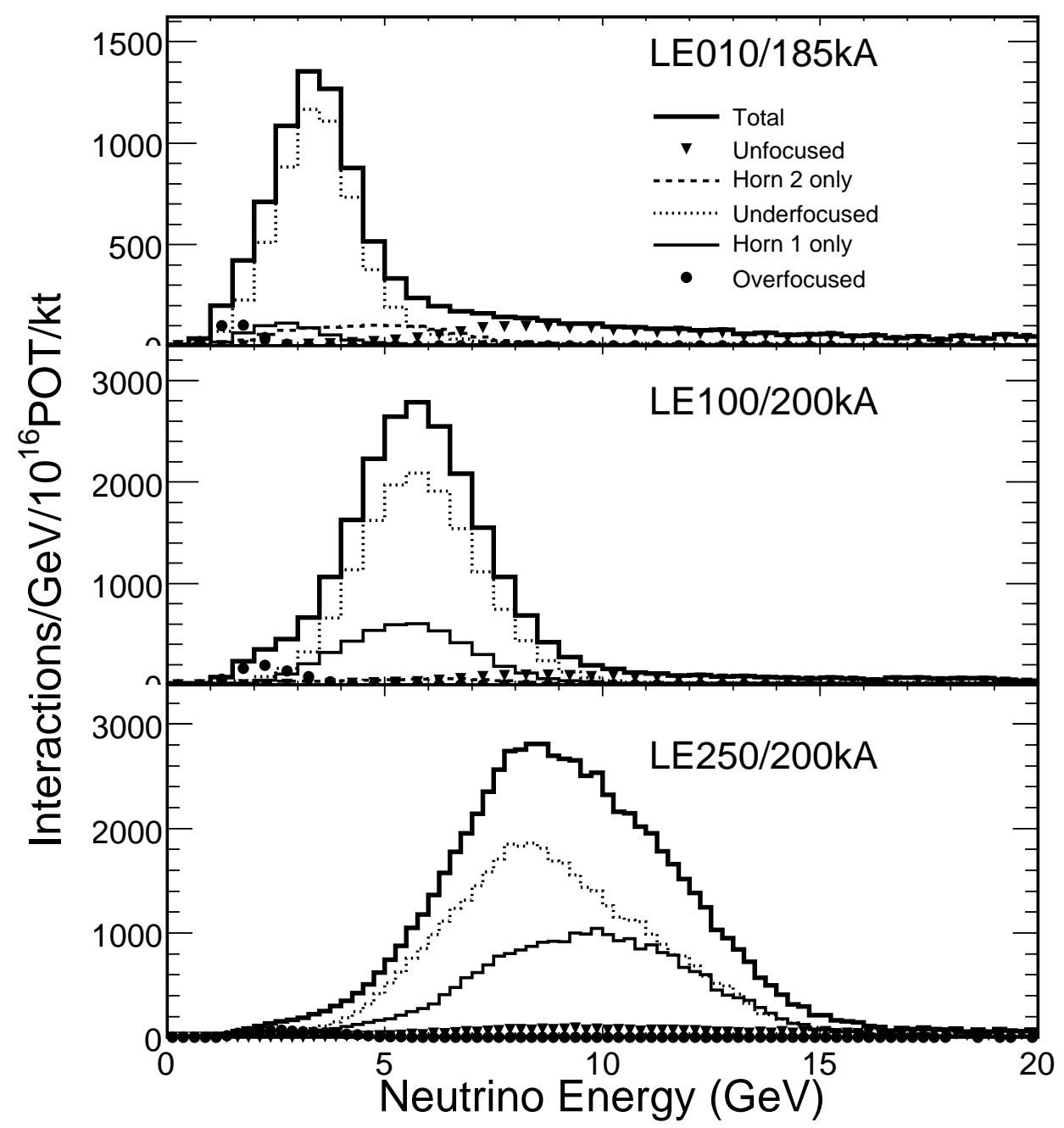

Figure 3.17: The calculated energy spectrum of $\nu_{\mu}$ charged current interactions in the MINOS Near Detector. The components of the spectrum corresponding to 5 possible trajectories of neutrino parents through the focusing horns (Figure 3.16) are indicated in the plot. Shown are the spectra for LE010/185kA, LE100/200kA and LE250/200kA beams (see Table 2.1). 


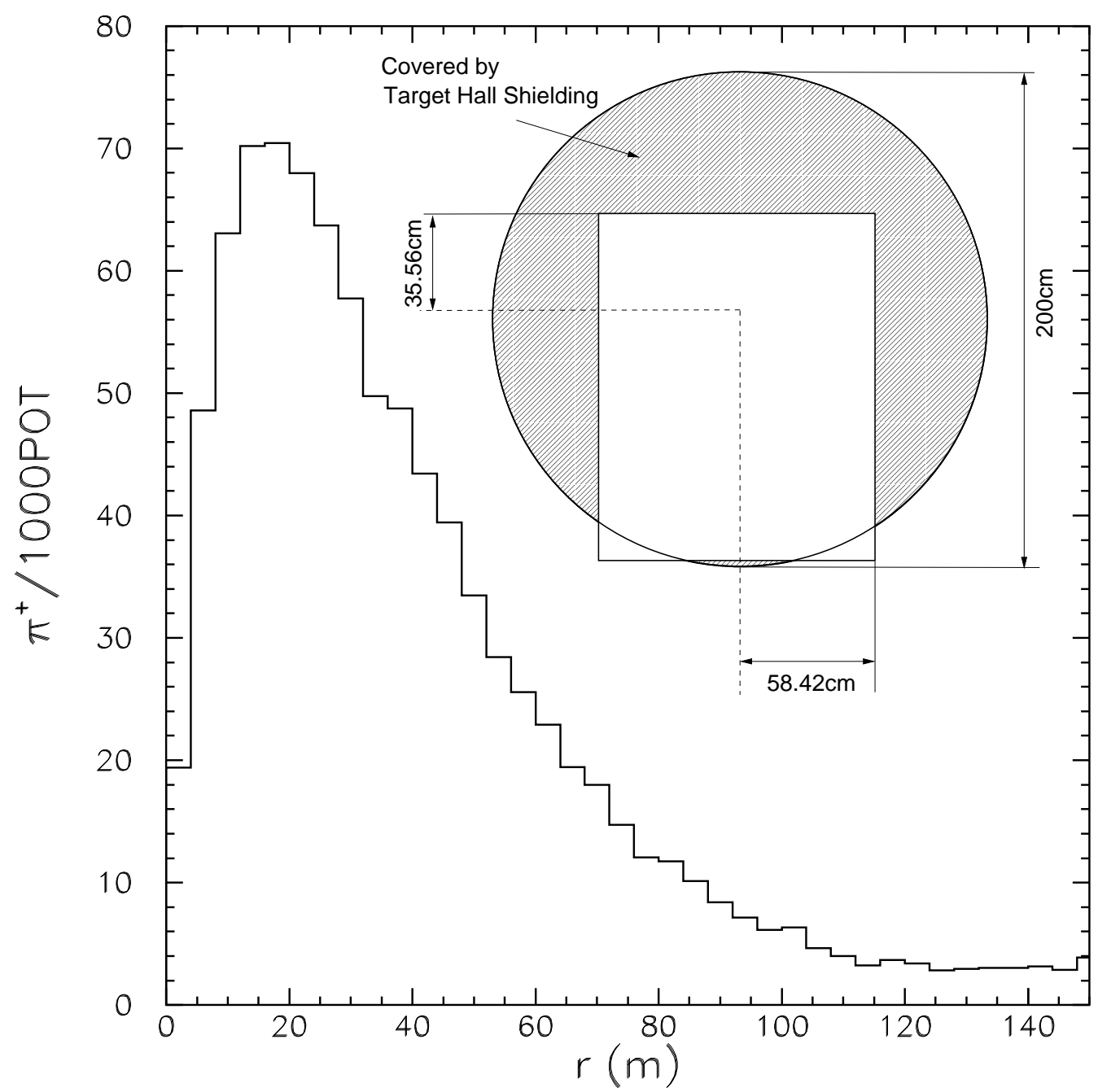

Figure 3.18: The expected radial distribution of pions at the location of the upstream window ( $\sim 45 \mathrm{~m}$ away from target) if no shielding was present in the target hall. The inset shows the beam view of the entrance to the decay pipe from the target hall. The shaded region indicates is the aperture of the shielding in the Target Hall. The decay pipe is centered on the beam axis. 


\subsection{Decay Pipe and Hadron Absorber}

The particles emanating from target hall area enter the $675 \mathrm{~m}$ long evacuated steel pipe. The inner radius of the pipe is $1 \mathrm{~m}$ and the walls of the pipe are $1 \mathrm{~cm}$ thick. The pipe is embedded into concrete shielding, so the particles moving in the outward direction are stopped either in the walls of the pipe or in the surrounding shielding. The temperature of the shielding is maintained by water cooling.

The choice of transverse size and the length of decay volume is a balance between practical issues, and neutrino flux. The gains in the flux are significant as the decay pipe radius is increased up to $1 \mathrm{~m}$, however they become modest beyond that point [32]. The scale for the length of the decay pipe is set by the decay length of a pion. For $\nu_{\mu}$ disappearance measurement in MINOS the neutrinos of most interest come from pions with momentum of up to $5-10 \mathrm{GeV}$. The decay length for a $10 \mathrm{GeV}$ pion is $\gamma \beta c \tau=560 \mathrm{~m}$.

The decay pipe is evacuated in order to reduce the reinteraction of $\pi$ while flying through it. The upstream and downstream end of the pipe are sealed with so-called windows. The downstream window is made out of $4.76 \mathrm{~mm}$ thick steel. The design of the upstream window is more complicated in order to reduce the reinteraction of particles entering the decay pipe, while at the same time maintaining the vacuum seal.

The inner part of the upstream window is made out of $1.6 \mathrm{~mm}$ thick aluminum, while the outer part is made of $9.525 \mathrm{~mm}$ thick steel. The radius of the inner part is $53.34 \mathrm{~cm}$ and it mostly overlaps with the aperture of the shielding in the Target Hall (see Figure 3.18).

Particles that go beyond the end of the decay pipe are stopped within 


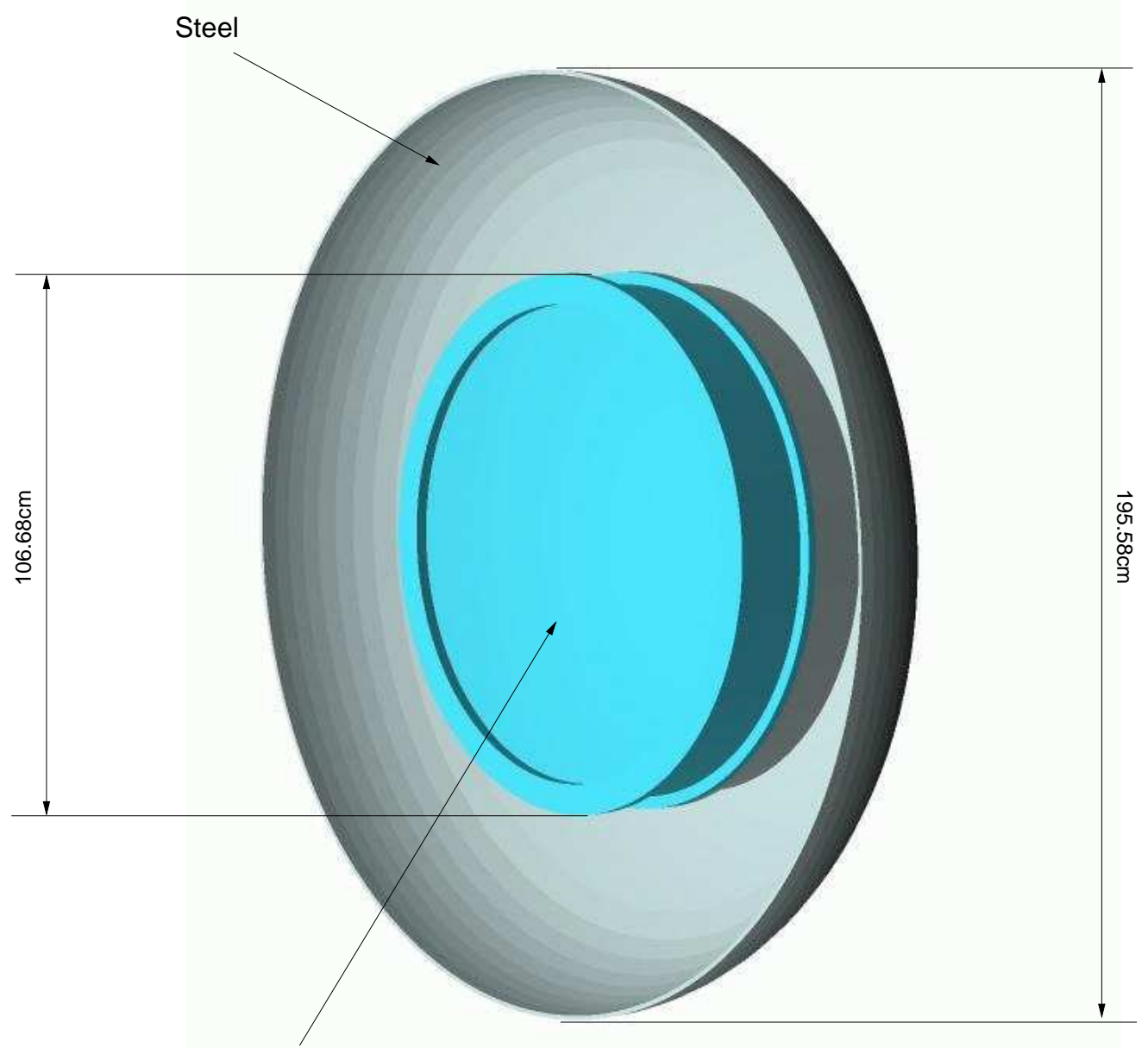

Aluminum

Figure 3.19: The 3d view of the upstream Decay Pipe Window. The window keeps the vacuum seal in the decay pipe. The inner part of the window, which mostly overlaps with the aperture of the Target Hall (see Figure 3.18), is made out of Aluminum in order to reduce the reinteraction of particles as they pass through the window. 
the hadron absorber (Figure 3.20). The absorber consists of $2.4 \mathrm{~m}$ long, water cooled, aluminum core. The core is surrounded by layer of steel blocks and a layer of concrete shielding.

The absorber stops the hadron component of the beam, while neutrinos and some of the muons pass through it. The threshold in momentum for muons is 3-4 GeV. These muons that pass through the absorber are later stopped in the $\sim 300 \mathrm{~m}$ of rock that separate the absorber and the Near Detector.

\subsection{Monte Carlo Biasing Techniques}

In this section we discuss two techniques for biasing the $\mathrm{MC}$ that are used in neutrino flux calculation. The first technique drastically improves the efficiency of the MC, while the other makes the statistical significance of the calculated flux more evenly distributed over different regions of neutrino energy spectrum.

\subsubsection{Forcing Neutrinos to Strike ND/FD}

The two MINOS detectors have relatively small transverse size compared to how far they are from the decay pipe (ie the source of neutrinos). As a result the ND, and in particular the FD, subtends only a small solid angle. Many of the decays in the decay pipe will therefore yield neutrinos that miss the MINOS detectors. The simulation of protons interacting in the target and tracking the secondaries through focusing horns and decay pipe is a very CPU intensive process and it would be very inefficient if decays that don't produce neutrinos going in the direction of one of the detectors would be discarded. 


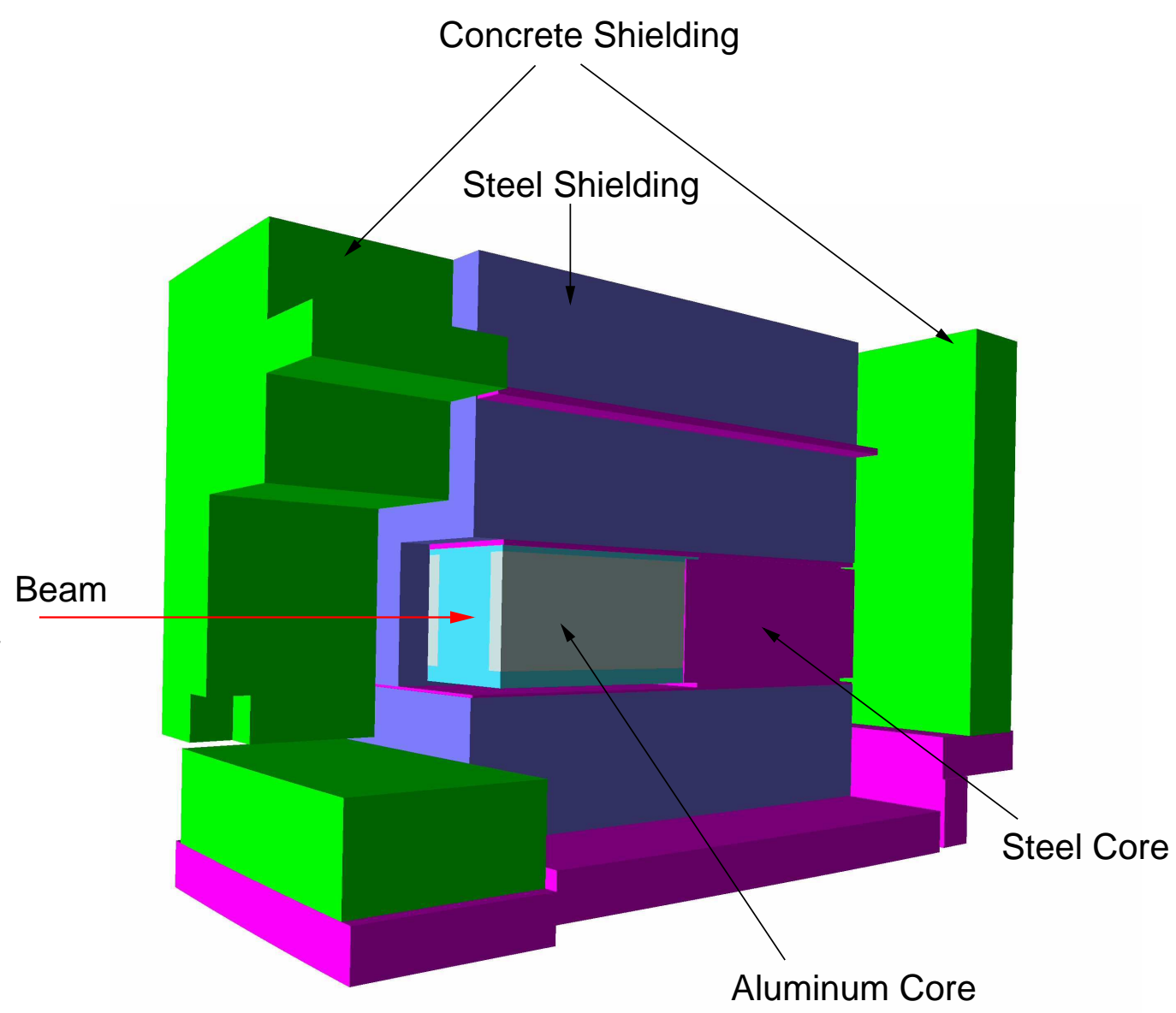

Figure 3.20: Breakaway view of the Hadron Absorber located at the end of decay pipe. The absorber stops the remnant hadrons that reach the end of the decay pipe. The Aluminum core is $2.4 \mathrm{~mm}$ long and the following steel core is $2.3 \mathrm{~m}$ long. 


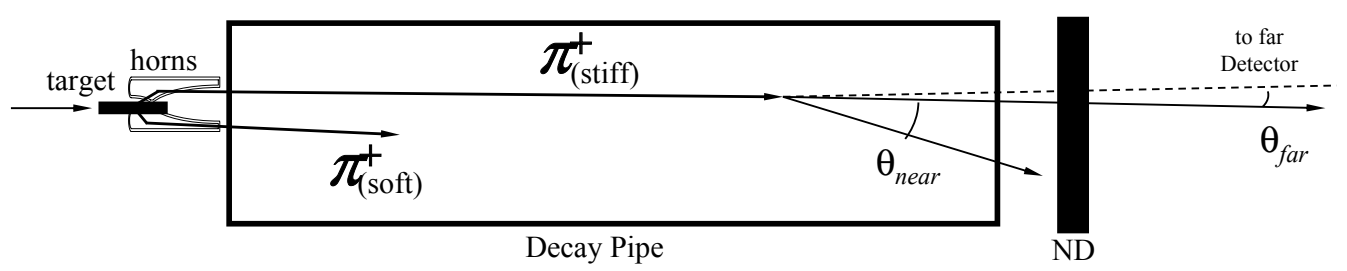

Figure 3.21: The probability that a neutrino coming from a certain decay in a beam-line will be observed in MINOS detector, as well as its energy depend on the angle between the parent momentum and neutrino momentum $\theta$. Schematic view of the NuMI beam-line showing a pion and the angle toward MINOS Near and Far detectors.

The way that we can use all of the decays for flux calculation is to calculate for each decay what is the probability that a neutrino that hits MINOS detector will be produced. The neutrino is then counted fractionally, with the fraction being equal to the calculated probability. Sometimes this fraction is also called a "weight". This procedure is equivalent to analytic calculation of neutrino spectrum using the distributions of neutrino parents in the decay pipe as determined by the MC simulation.

Figure 3.21 shows the beam-line schematic and illustrates the fact that the ND, being so much closer than the FD, subtends much bigger solid angle. To calculate the probability that a neutrino will be produced in a certain solid angle we need to integrate the angular distribution of daughter neutrinos $d N / d \Omega$ over the solid angle subtended by the detector.

Equation 3.12 gives the probability that a neutrino will be emitted in a solid angle $d \Omega$. The solid angle subtended by the detector is given with:

$$
d \Omega_{d e t}=\frac{1}{4 \pi} \frac{R_{D e t}^{2} \pi}{\left(\vec{r}_{\text {decay }}-\vec{r}_{D e t}\right)^{2}}
$$


where $R_{\text {det }}^{2}$ is the radius of the detector, $\vec{r}_{\text {decay }}$ is the position where the neutrino parent decayed and $\vec{r}_{D e t}$ is the radius vector of the detector. For ND $r_{D} e t=$ $1040 \mathrm{~m}$, while for FD it is $735 \mathrm{~km}$.

Choosing the direction of the neutrino affects its energy in the lab frame. The neutrino energy is calculated using Equation 3.9.

The muon decays $\mu^{ \pm} \rightarrow e^{ \pm}+\nu_{e}\left(\bar{\nu}_{e}\right)+\bar{\nu}_{\mu}\left(\nu_{\mu}\right)$ have to be treated more carefully. Muons are spin $1 / 2$ particles and the decays in the rest frame are not spherically symmetric. The distribution of muon antineutrinos (neutrinos) is given with:

$$
\frac{d^{2} N}{d x d \Omega}=\frac{2 x^{2}}{4 \pi}[(3-2 x) \mp(1-2 x) \cos \theta]
$$

where $x \equiv 2 E_{\nu} / m_{\mu}$ and $\theta$ is the angle between the muon polarization and neutrino direction. Similarly for electron neutrinos (antineutrinos) the distribution is given with:

$$
\frac{d^{2} N}{d x d \Omega}=\frac{12 x^{2}}{4 \pi}[(1-x) \mp(1-x) \cos \theta]
$$

These muons are predominantly created in two body decays of pions and kaons. Since, in those decays, the accompanying neutrino has fixed helicity, the muon polarization is determined as well. To calculate the probability for a neutrino decay, we first find the polarization of the muon in the muon rest frame and then calculate the probability using Equations 3.17 and 3.18. Again, the neutrino is counted fractionally according to calculated probability. 


\subsubsection{Weighting Secondaries}

The secondaries produced in the target are largely low energy particles giving rise to lower energy neutrinos. As seen in Figure 3.2 the distribution falls off sharply with momentum of the secondary. For our simulation we wish to produce the neutrino energy spectrum all the way to the highest energies. However, due to the momentum distribution in Figure 3.3 of secondaries, it requires simulating many more protons on target to smooth the statistical fluctuations for high energy part of the neutrino spectrum than it is required for the low energy part of the spectrum.

To enhance statistics in this region of interest we bias the simulation to preferentially track higher energy particles. This is done by selectively choosing which secondaries are tracked and assigning a weight to a neutrino that corrects for the bias. The probability which is used to determine whether a certain particle should be tracked is calculated in the following way:

$$
P=\left\{\begin{aligned}
E /(30 \mathrm{GeV}) & \text { if } E<30 \mathrm{GeV} \\
1 . & \text { if } E \geq 30 \mathrm{GeV}
\end{aligned}\right.
$$

This results in tracking only every other $15 \mathrm{GeV}$ or every 5 th $6 \mathrm{GeV}$ secondary. The weight assigned to neutrinos coming from these lower energy particles in this example would be equal 2 and 5 respectively.

\subsection{Predicted Neutrino Flux at the ND}

The beam MC simulation gives us a list of decay points for various types of particles that decay into neutrinos along with the momentum and type of that 
particle. Using this information we can calculate the flux of neutrinos at both the ND and the FD using formulas from $§ 3.7 .1$.

Figures 3.22-3.25 show the calculated neutrino energy spectrum of $\nu_{\mu}$ and $\bar{\nu}_{\mu}, \nu_{e}$ and $\bar{\nu}_{e}$ neutrinos at the ND in different beam configurations. The distributions showing contributions from different neutrino parents are shown as well. Tables 3.1-3.4 give the integrated number of events for 5 different energy ranges. Also shown is the contribution from each neutrino parent type.

The $\nu_{\mu}$ and $\bar{\nu}_{\mu}$ predominantly come from decays of pions and kaons with pions contributing to the lower and kaons to higher energy part of the spectrum. A large number of $\nu_{e}$ come from muon decays. Since these muons are created in decays of pions along with muon neutrinos, this part of the $\nu_{e}$ spectrum is very correlated with $\nu_{\mu}$ spectrum. Even more direct is the correlation in the spectrum coming from charged kaons. The same kaons that give rise to $\nu_{\mu}\left(\bar{\nu}_{\mu}\right)$ through 2 body decays, give rise to $\nu_{e}\left(\bar{\nu}_{e}\right)$ through $K_{e 3}$ decays.

It can be seen that the flux of $\nu_{\mu}$ is drastically increased once the horns are pulsed and $\pi^{+}$get focused. Some positive kaons get focused as well, which can be seen by increased flux of $\nu_{\mu}$ coming from kaon decays.

The $\bar{\nu}_{\mu}$ energy spectrum looks similar in all beam configurations, regardless of the position of the target. It can be noticed that when horns are pulsed, the flux is somewhat reduced in lower energy bins since horns defocus negative pions. Comparing the $\bar{\nu}_{\mu}$ spectrum in beam configurations where horns are pulsed also shows a change in the flux of $\bar{\nu}_{\mu}$ coming from muon decays. These muons are created in decays of $\pi^{+}$and therefore their flux is significantly increased when positive pions are focused. 


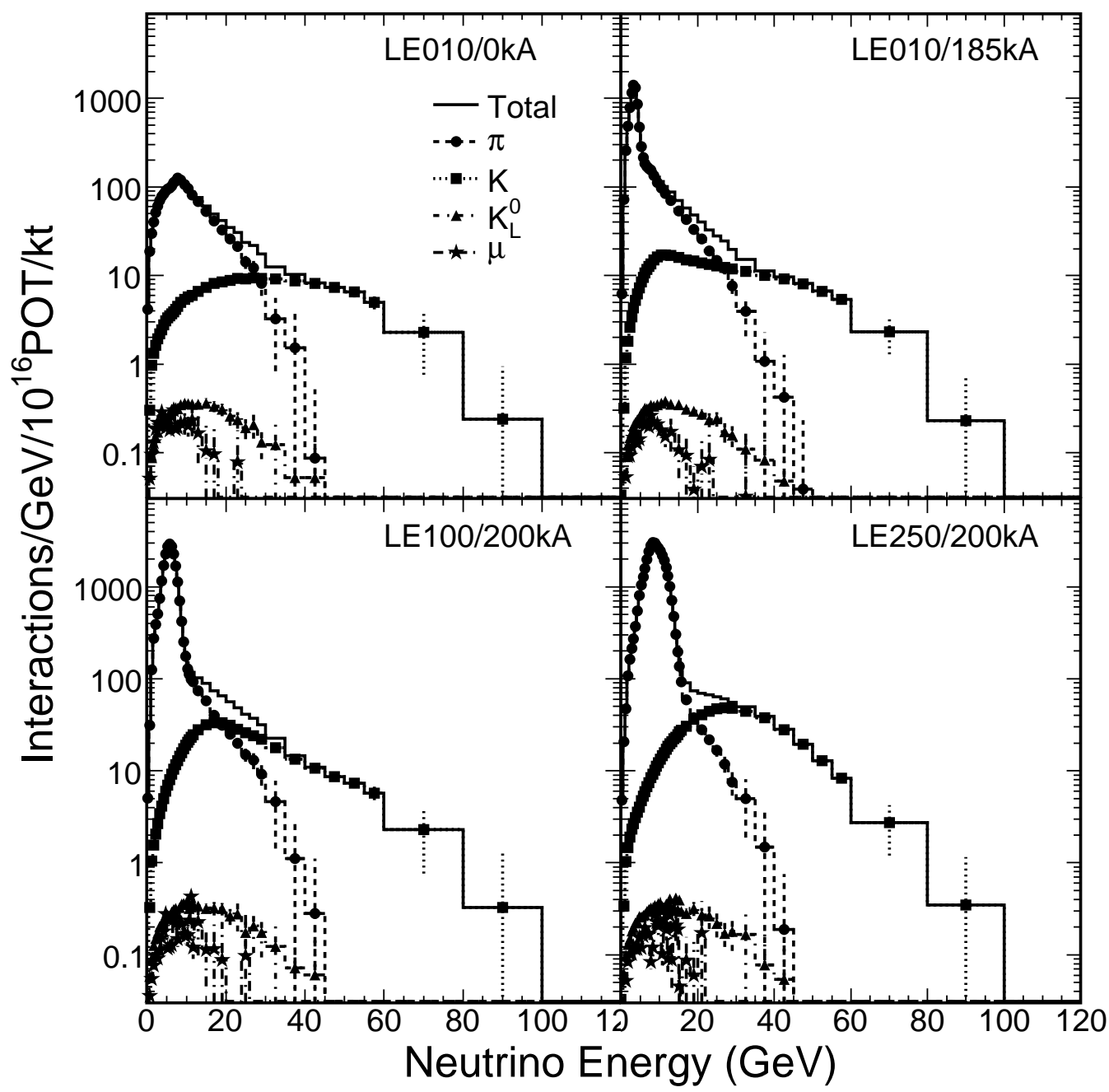

Figure 3.22: The calculation of the energy spectrum of Charged Current $\nu_{\mu}$ interactions in the MINOS Near Detector for 4 different beam configurations (Table 2.1). Also shown are the contributions from each type of neutrino parent particle. Table 3.1 gives the integrated number of events for 5 different energy ranges. 


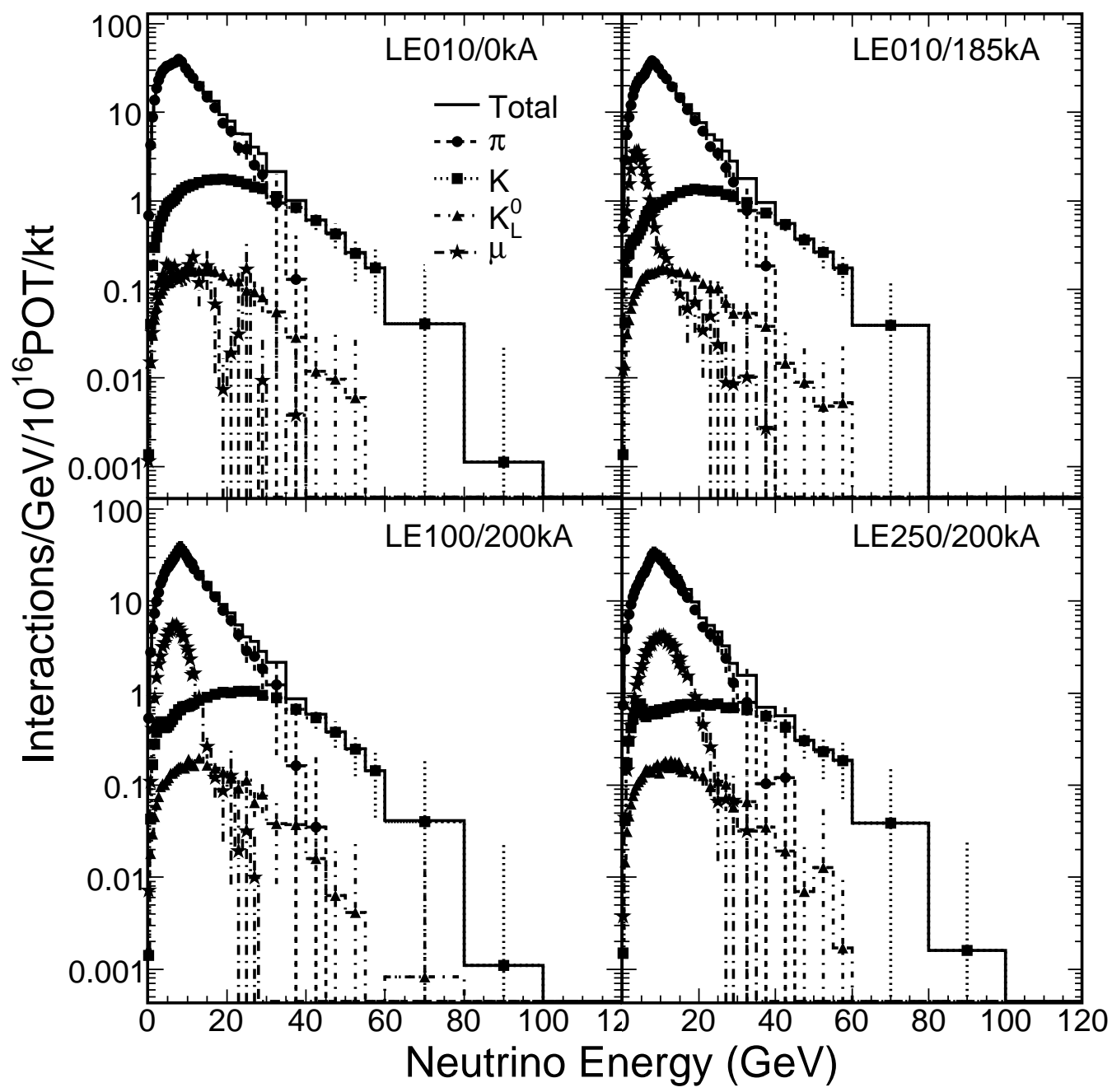

Figure 3.23: The calculation of the energy spectrum of Charged Current $\bar{\nu}_{\mu}$ interactions in the MINOS Near Detector for 4 different beam configurations (Table 2.1). Also shown are the contributions from each type of neutrino parent particle. Table 3.2 gives the integrated number of events for 5 different energy ranges. 


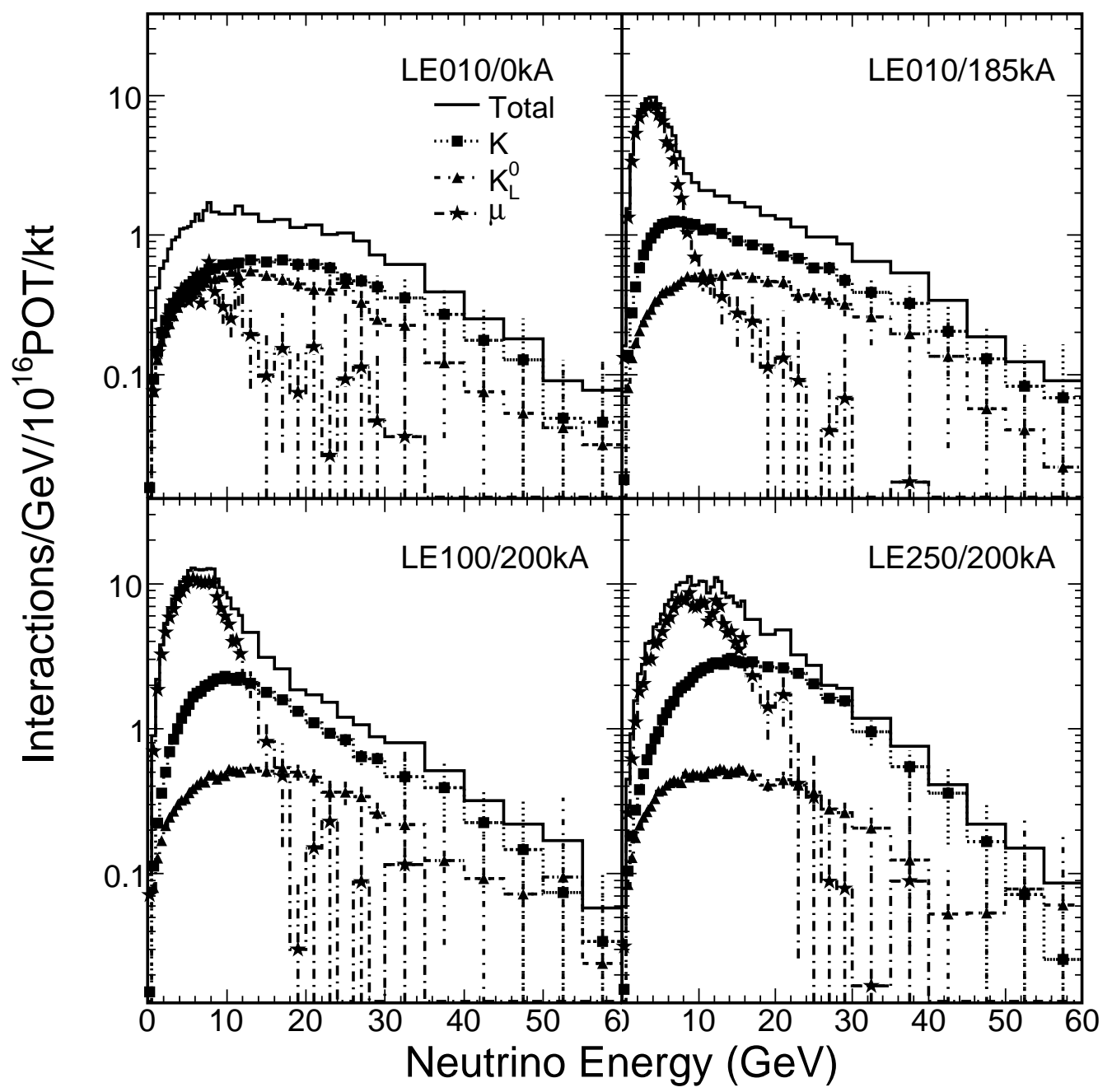

Figure 3.24: The calculation of the energy spectrum of Charged Current $\nu_{e}$ interactions in the MINOS Near Detector for 4 different beam configurations (Table 2.1). Also shown are the contributions from each type of neutrino parent particle. Table 3.3 gives the integrated number of events for 5 different energy ranges. 


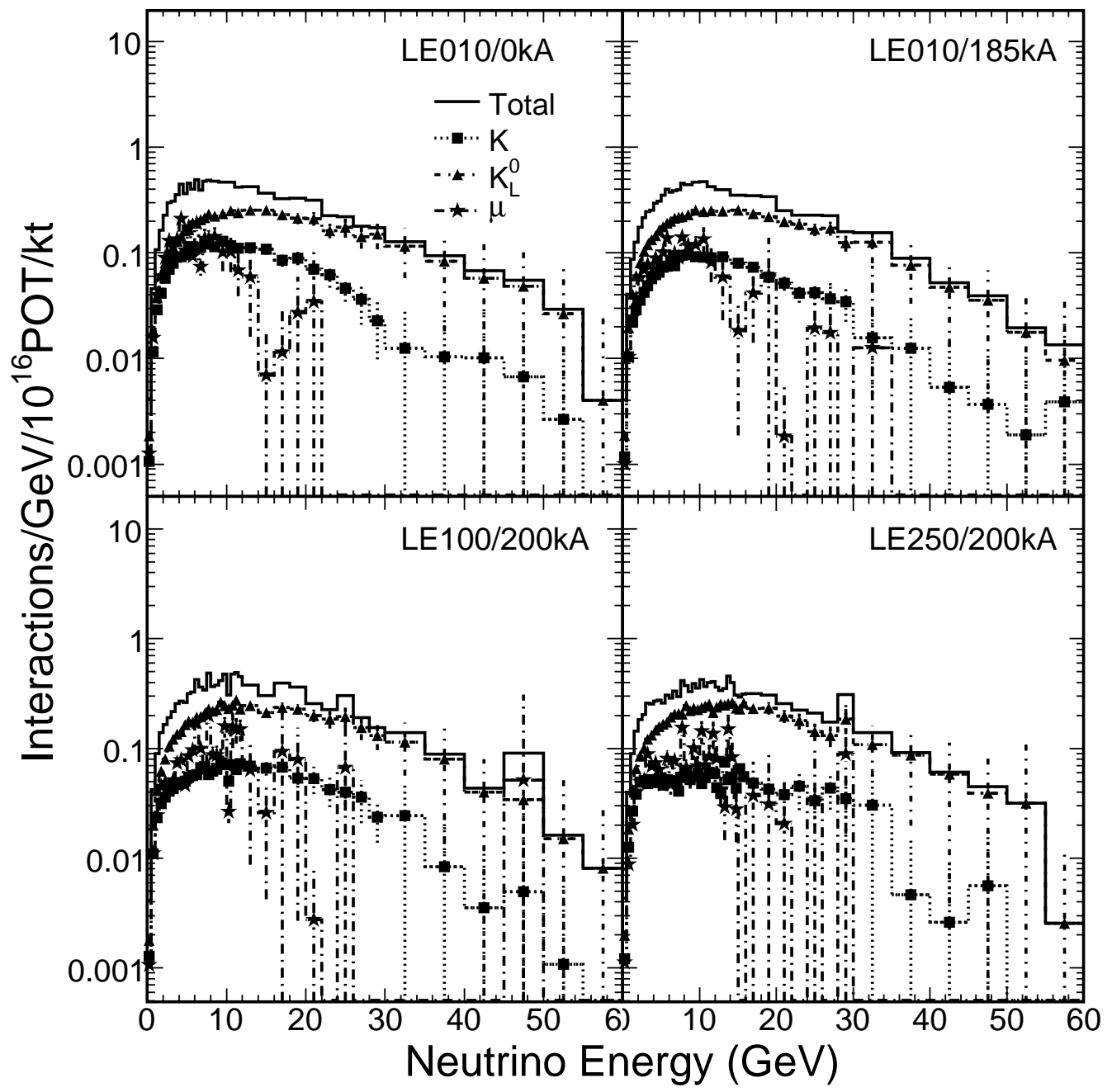

Figure 3.25: The calculation of the energy spectrum of Charged Current $\bar{\nu}_{e}$ interactions in the MINOS Near Detector for 4 different beam configurations (Table 2.1). Also shown are the contributions from each type of neutrino parent particle. Table 3.4 gives the integrated number of events for 5 different energy ranges. 


\begin{tabular}{llccccc} 
& $E_{\nu}<$ & $3 \mathrm{GeV}$ & $5 \mathrm{GeV}$ & $10 \mathrm{GeV}$ & $20 \mathrm{GeV}$ & $120 \mathrm{GeV}$ \\
\hline LE010/0kA & $\pi$ & 205.34 & 525.66 & 1400.93 & 1773.17 & 1859.51 \\
& $K$ & 6.15 & 16.6 & 49.95 & 92.2 & 185.51 \\
& $K_{L}^{0}$ & 0.59 & 1.48 & 3.99 & 6.05 & 7.33 \\
& $\mu$ & 0.69 & 1.61 & 3.36 & 4.17 & 4.25 \\
& Total & 212.76 & 545.34 & 1458.22 & 1875.59 & 2056.59 \\
\hline LE010/185kA & $\pi$ & 2760.58 & 6831.76 & 8248.78 & 8629.06 & 8713.91 \\
& $K$ & 9.33 & 32.42 & 130.29 & 226.72 & 344.13 \\
& $K_{L}^{0}$ & 0.59 & 1.49 & 4.03 & 6.07 & 7.41 \\
& $\mu$ & 0.39 & 0.97 & 2.71 & 3.45 & 3.64 \\
& Total & 2770.89 & 6866.63 & 8385.81 & 8865.3 & 9069.09 \\
\hline LE100/200kA & $\pi$ & 1335.73 & 7214.66 & 22205.1 & 22839.6 & 22927.6 \\
& $K$ & 7.68 & 26.24 & 151.66 & 367.62 & 565.44 \\
& $K_{L}^{0}$ & 0.61 & 1.52 & 4.65 & 7.35 & 8.71 \\
& $\mu$ & 0.36 & 1.08 & 2.83 & 4.33 & 4.48 \\
& Total & 1344.38 & 7243.5 & 22364.2 & 23218.9 & 23506.2 \\
\hline LE250/200kA & $\pi$ & 556.5 & 2544.74 & 25067.3 & 37552.9 & 37645.4 \\
& $K$ & 7.06 & 20.45 & 101.75 & 412.74 & 795.08 \\
& $K_{L}^{0}$ & 0.63 & 1.54 & 4.59 & 9.3 & 10.71 \\
& $\mu$ & 0.37 & 0.88 & 2.6 & 4.71 & 4.94 \\
& Total & 564.56 & 2567.61 & 25176.2 & 37979.6 & 38456.1
\end{tabular}

Table 3.1: The calculation of the rate of Charged Current $\nu_{\mu}$ interactions in the MINOS Near Detector for 4 different beam configurations (Table 2.1). Shown is the number of interactions in units of Interactions $/\left(10^{16} \mathrm{POT} / \mathrm{kt}\right)$ for 5 energy ranges. Also shown are the contributions from each type of neutrino parent particle (see Figure 3.22). 


\begin{tabular}{llccccc} 
& $E_{\nu}<$ & $3 \mathrm{GeV}$ & $5 \mathrm{GeV}$ & $10 \mathrm{GeV}$ & $20 \mathrm{GeV}$ & $120 \mathrm{GeV}$ \\
\hline LE010/0kA & $\pi$ & 69.15 & 189.18 & 474.7 & 579.74 & 599.21 \\
& $K$ & 1.42 & 4.33 & 13.38 & 23.19 & 34.35 \\
& $K_{L}^{0}$ & 0.23 & 0.65 & 1.78 & 2.73 & 3.37 \\
& $\mu$ & 0.3 & 0.89 & 2.12 & 2.92 & 3.15 \\
& Total & 71.11 & 195.06 & 491.98 & 608.58 & 640.07 \\
\hline LE010/185kA & $\pi$ & 45.21 & 130.29 & 383.65 & 486.92 & 505.55 \\
& $K$ & 1.13 & 2.76 & 8.66 & 15.66 & 24.91 \\
& $K_{L}^{0}$ & 0.23 & 0.65 & 1.84 & 2.79 & 3.36 \\
& $\mu$ & 7.71 & 21.44 & 32.42 & 33.27 & 33.41 \\
& Total & 54.28 & 155.14 & 426.56 & 538.65 & 567.24 \\
\hline LE100/200kA & $\pi$ & 38.16 & 113.8 & 424.89 & 579.43 & 598.6 \\
& $K$ & 1.33 & 3.12 & 9.22 & 15.93 & 23.93 \\
& $K_{L}^{0}$ & 0.23 & 0.65 & 2.13 & 3.47 & 4.04 \\
& $\mu$ & 4.94 & 18.35 & 65.13 & 74.86 & 75.04 \\
& Total & 44.66 & 135.91 & 501.36 & 673.69 & 701.61 \\
\hline LE250/200kA & $\pi$ & 36.3 & 96.3 & 367.9 & 626.14 & 644.2 \\
& $K$ & 1.46 & 4.29 & 10.43 & 20.21 & 26.26 \\
& $K_{L}^{0}$ & 0.23 & 0.65 & 2.11 & 4.36 & 5 \\
& $\mu$ & 1.61 & 6.92 & 39.6 & 80.14 & 81.09 \\
& Total & 39.59 & 108.17 & 420.04 & 730.86 & 756.55
\end{tabular}

Table 3.2: The calculation of the rate of Charged Current $\bar{\nu}_{\mu}$ interactions in the MINOS Near Detector for 4 different beam configurations (Table 2.1). Shown is the number of interactions in units of Interactions $/\left(10^{16} \mathrm{POT} / \mathrm{kt}\right)$ for 5 energy ranges. Also shown are the contributions from each type of neutrino parent particle (see Figure 3.23). 


\begin{tabular}{llccccc} 
& $E_{\nu}<$ & $3 \mathrm{GeV}$ & $5 \mathrm{GeV}$ & $10 \mathrm{GeV}$ & $20 \mathrm{GeV}$ & $120 \mathrm{GeV}$ \\
\hline LE010/0kA & $K$ & 1 & 2.46 & 6.66 & 10.48 & 14.09 \\
& $K_{L}^{0}$ & 0.82 & 2.07 & 5.61 & 8.68 & 11.07 \\
& $\mu$ & 0.91 & 2.31 & 5.75 & 6.98 & 7.45 \\
& Total & 2.73 & 6.85 & 18.02 & 26.15 & 32.61 \\
\hline LE010/185kA & $K$ & 2.16 & 6.11 & 15.87 & 21.64 & 25.86 \\
& $K_{L}^{0}$ & 0.84 & 2.08 & 5.61 & 8.66 & 11.24 \\
& $\mu$ & 24.89 & 57.43 & 82.19 & 84.12 & 84.47 \\
& Total & 27.89 & 65.62 & 103.67 & 114.41 & 121.57 \\
\hline LE100/200kA & $K$ & 1.91 & 6.25 & 25.77 & 41.37 & 46.84 \\
& $K_{L}^{0}$ & 0.85 & 2.07 & 6.45 & 10.58 & 13 \\
& $\mu$ & 16.36 & 49.49 & 142.64 & 162.54 & 163.12 \\
& Total & 19.12 & 57.81 & 174.86 & 214.49 & 222.97 \\
\hline LE250/200kA A & $K$ & 1.44 & 4.58 & 22.53 & 61.88 & 74.27 \\
& $K_{L}^{0}$ & 0.86 & 2.13 & 6.56 & 13.51 & 15.86 \\
& $\mu$ & 5.85 & 19.66 & 88.15 & 159.42 & 162.17 \\
& Total & 8.16 & 26.37 & 117.24 & 234.81 & 252.3
\end{tabular}

Table 3.3: The calculation of the rate of Charged Current $\nu_{e}$ interactions in the MINOS Near Detector for 4 different beam configurations (Table 2.1). Shown is the number of interactions in units of Interactions $/\left(10^{16} \mathrm{POT} / \mathrm{kt}\right)$ for 5 energy ranges. Also shown are the contributions from each type of neutrino parent particle (see Figure 3.24). 


\begin{tabular}{llccccc} 
& $E_{\nu}<$ & $3 \mathrm{GeV}$ & $5 \mathrm{GeV}$ & $10 \mathrm{GeV}$ & $20 \mathrm{GeV}$ & $120 \mathrm{GeV}$ \\
\hline LE010/0kA & $K$ & 0.21 & 0.57 & 1.51 & 2.13 & 2.41 \\
& $K_{L}^{0}$ & 0.3 & 0.85 & 2.51 & 3.95 & 5.13 \\
& $\mu$ & 0.34 & 0.9 & 1.95 & 2.23 & 2.26 \\
& Total & 0.85 & 2.32 & 5.98 & 8.32 & 9.81 \\
\hline LE010/185kA & $K$ & 0.14 & 0.37 & 1.06 & 1.54 & 1.79 \\
& $K_{L}^{0}$ & 0.31 & 0.87 & 2.57 & 4.03 & 5.19 \\
& $\mu$ & 0.2 & 0.51 & 1.4 & 1.8 & 1.85 \\
& Total & 0.65 & 1.75 & 5.03 & 7.37 & 8.83 \\
\hline LE100/200kA & $K$ & 0.14 & 0.33 & 0.97 & 1.48 & 1.72 \\
& $K_{L}^{0}$ & 0.31 & 0.88 & 3.01 & 4.92 & 6.07 \\
& $\mu$ & 0.18 & 0.43 & 1.56 & 2.3 & 2.42 \\
& Total & 0.63 & 1.64 & 5.53 & 8.7 & 10.22 \\
\hline LE250/200kA A & $K$ & 0.18 & 0.38 & 0.9 & 1.65 & 1.88 \\
& $K_{L}^{0}$ & 0.31 & 0.87 & 2.95 & 6.34 & 7.51 \\
& $\mu$ & 0.17 & 0.47 & 1.22 & 2.14 & 2.28 \\
& Total & 0.66 & 1.72 & 5.07 & 10.12 & 11.67
\end{tabular}

Table 3.4: The calculation of the rate of Charged Current $\bar{\nu}_{e}$ interactions in the MINOS Near Detector for 4 different beam configurations (Table 2.1). Shown is the number of interactions in units of Interactions $/\left(10^{16} \mathrm{POT} / \mathrm{kt}\right)$ for 5 energy ranges. Also shown are the contributions from each type of neutrino parent particle (see Figure 3.25).. 


\section{Chapter 4}

\section{Predicted Neutrino Flux at the Far Detector}

In a two-detector experiment, the Near Detector (ND) provides a direct measurement of the flux produced by the beam. In the absence of neutrino oscillations, the same $\nu_{\mu}$ flux, in principle, is incident at the Far Detector (FD) (modulo the difference in flux due to the $r^{-2}$ fall off). Any difference in flux or energy spectrum between the two detectors is taken as evidence for $\nu_{\mu}$ disappearance or oscillations. Thus, the principle of the two-detector experiment is to provide a direct precise measurement of the flux.

In practice, the flux at the ND is slightly different than that at the FD, and cannot be used as a direct measure of the FD flux. Therefore, it must be corrected by a small amount to predict the FD flux. In this chapter we will discuss the origin of the differences between the flux at the ND and at the FD. We will also show how the Monte Carlo (MC) calculation of Chapter 3 is used to derive this correction factor between the flux at the ND and FD. 
While the uncertainties in the direct calculation of the flux itself are large (see Chapter 5) the uncertainty in the correction factor is relatively small. We leave the discussion of uncertainties in the calculation of the flux for the following chapters and here, we discuss the calculation of the correction factor.

\subsection{Neutrino Flux at ND and FD}

To study the differences between the ND and FD fluxes it is useful to look at the ratio between neutrino energy spectrum at the ND and FD. We often refer to this ratio as far over near $(\mathrm{F} / \mathrm{N})$ ratio.

It is, of course, expected that a much smaller flux of neutrinos will be observed at the FD when compared to the ND due to a huge difference between the solid angle that the two detectors subtend. The ND is located $1040 \mathrm{~m}$ $\left(z_{N D}\right)$ downstream of the meson production target, compared to $735.4 \mathrm{~km}$

$\left(z_{F D}\right)$ in case of the FD. A reduction in flux of the order of $z_{N D}^{2} / z_{F D}^{2} \approx 2 \cdot 10^{-6}$ is therefore expected.

Figure 4.1 shows the MC calculation of the expected number of $\nu_{\mu}$ charged current (CC) interactions at the two detectors. The ND spectrum was scaled down in order to show both spectra on the same plot. The applied scaling would assume that neutrinos are created on the average around the first quarter of the decay pipe. Not only do the two spectra have very different normalizations, they also show significant differences in their shape.

The origin of the differences seen in Figure 4.1 is a geometric acceptance difference between the ND and the FD which has a kinematic dependence. As seen in Figure 3.21 the mesons created in a target fly through a decay region which is comparable to the distance $z_{N D}$. The hadron absorber which is at the 


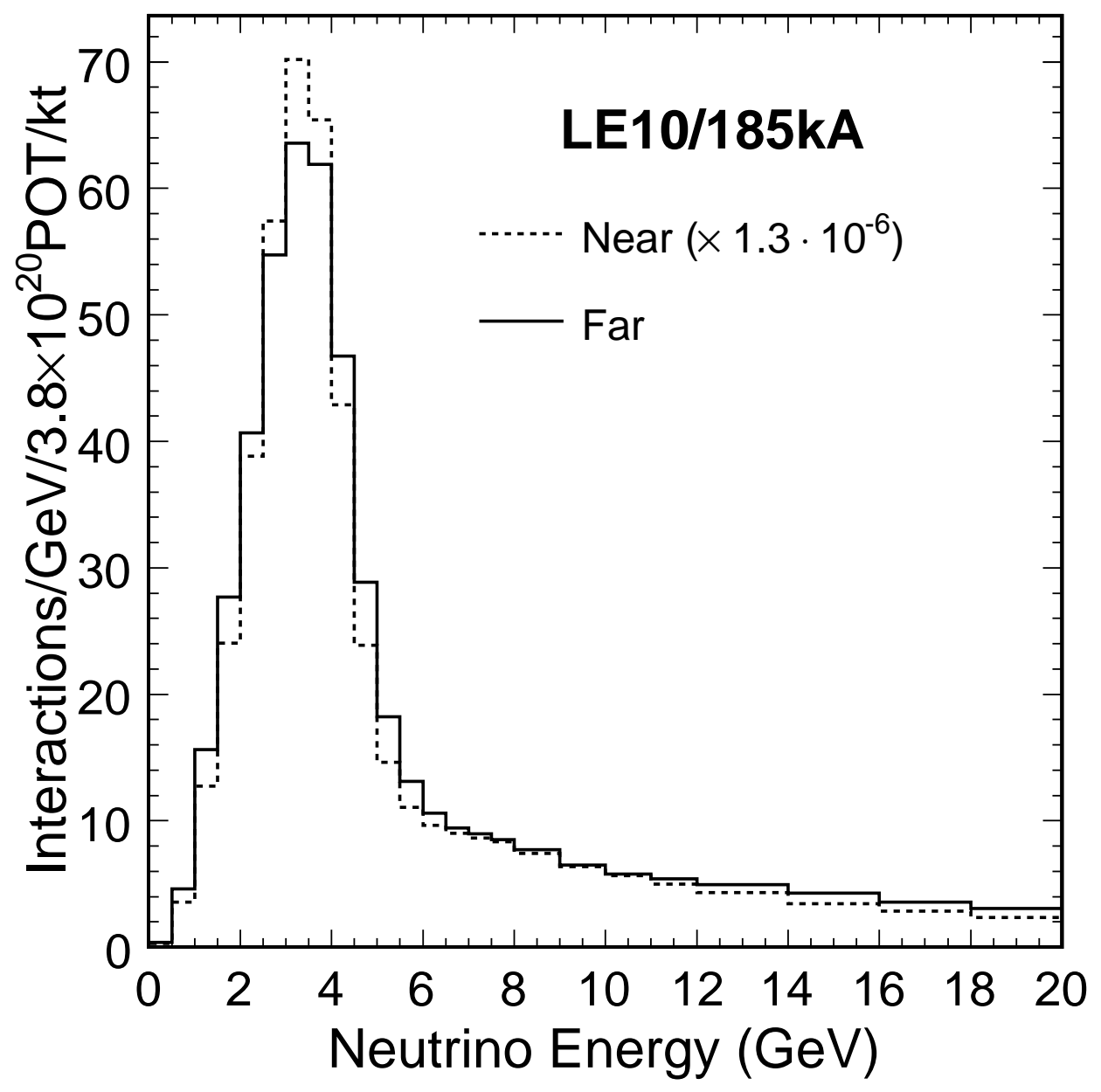

Figure 4.1: Comparison of the neutrino energy spectrum at the Near and the Far detector. The FD subtends much smaller solid angle compared to the ND. As a result the flux at the FD is several orders of magnitude smaller than the flux at ND. Due to the proximity to the neutrino source, the ND sees the extended source of neutrinos, while the FD sees the point source making the shape of the two spectra different. 
end of decay pipe is located $724 \mathrm{~m}\left(z_{A b s}\right)$ downstream of target. The neutrino parents can in principle decay anywhere in that region. We need to account for this since the solid angle subtended by ND is very different depending on where in decay pipe are neutrinos created. If neutrinos are on the average created closer to the downstream end of the decay pipe and hence closer to the ND, that would result in an increase of the flux at the ND and unobservable change in the flux at the FD. In terms of the $\mathrm{F} / \mathrm{N}$ ratio, this results in a smaller ratio.

Figure 4.2 demonstrates the kinematic difference in the solid angle acceptance of the ND and the FD. Shown is the difference in the energy of a neutrino that comes from a pion decay and is emitted toward the ND and the energy of a neutrino that is emitted toward the FD.

The mesons that give rise to neutrinos have a finite lifetime. On the average, the location where a particular particle will decay depends on its mean lifetime $\tau$ and Lorentz boost factor $\gamma=E / m c^{2}$. In general, lower energy particles will decay further upstream in the decay pipe when compared to the higher energy mesons. Considering for the moment only the neutrinos coming from pions we can calculate the $\mathrm{F} / \mathrm{N}$ ratio:

$$
\mathcal{R}_{F N}=\frac{\int_{z=0}^{z_{A b s}} \frac{e^{-\frac{z}{\gamma c \tau}}}{\left(z_{F D}-z\right)^{2}} d z}{\int_{z=0}^{z_{A b s}} \frac{e^{-\frac{z}{\gamma c \tau}}}{\left(z_{N D}-z\right)^{2}} d z}=\frac{\int_{z=0}^{z_{A b s}} \frac{e^{-\frac{0.43 m_{\pi} z}{E_{\nu} c \tau}}}{\left(z_{F D}-z\right)^{2}} d z}{\int_{z=0}^{z_{A b s}} \frac{e^{-\frac{0.43 m_{\pi} z}{E_{\nu} c \tau}}}{\left(z_{N D}-z\right)^{2}} d z}
$$

where we have used Equation 3.9 to relate the pion and neutrino energy. Since $z_{F D} \gg z_{A b s}$, the numerator simplifies to $\sim 1 / z_{F D}^{2}$. This suggests that a $\mathrm{F} / \mathrm{N}$ ratio should drop off exponentially as the mean location of decay points shifts toward ND for higher energy pions. The calculated curve using Equation 4.1 
is shown in Figure 4.3.

Equation 4.1 is an approximation because it assumes all neutrinos are emitted at $\theta=0$ with respect to the pion direction, ie $E_{\nu}=0.43 E_{\pi}$ in Equation 3.9. In reality, the proximity of the ND to the meson decay region causes the transverse size of the ND to become important. This is illustrated in Figure 3.21. The angle of a neutrino with respect to its parent's direction can be, in general, wider for the neutrino that hits the ND compared to the angle for the neutrino that hits the FD. Since the energy of a neutrino depends on this angle (Eq. 3.9) the decay of an individual pion will always yield systematically lower neutrino energy at the ND as compared to the FD. Such a difference will be exaggerated for high energy $\pi$ 's which decay further down the decay pipe because of their longer flight path $\beta \gamma c \tau$ in the lab frame, as indicated schematically in Figure 3.21.

Another approximation of Equation 4.1 is that it assumes the pions are moving colinear with the beam axis, while in reality the pion beam has some divergence. Since the decay region has finite size, due to this divergence some of the pions will strike the shielding, and are attenuated before decaying. This attenuation effectively shifts the distribution of decay points away from the ND, resulting in a lower flux in the $\mathrm{ND}$ and higher $\mathrm{F} / \mathrm{N}$ ratio. The horns focus pions of certain momentum, so the divergence of the pion beam depends on pion momentum. Since neutrino energy is proportional to the parent momentum, this varying divergence should be observable in the $\mathrm{F} / \mathrm{N}$ ratio.

Figure 4.3 shows the $\mathrm{F} / \mathrm{N}$ ratio as calculated by the beam-line simulation. The ratio shows a complicated energy dependence and is different than the simple pion lifetime prediction of Equation 4.1 due to the effects mentioned 


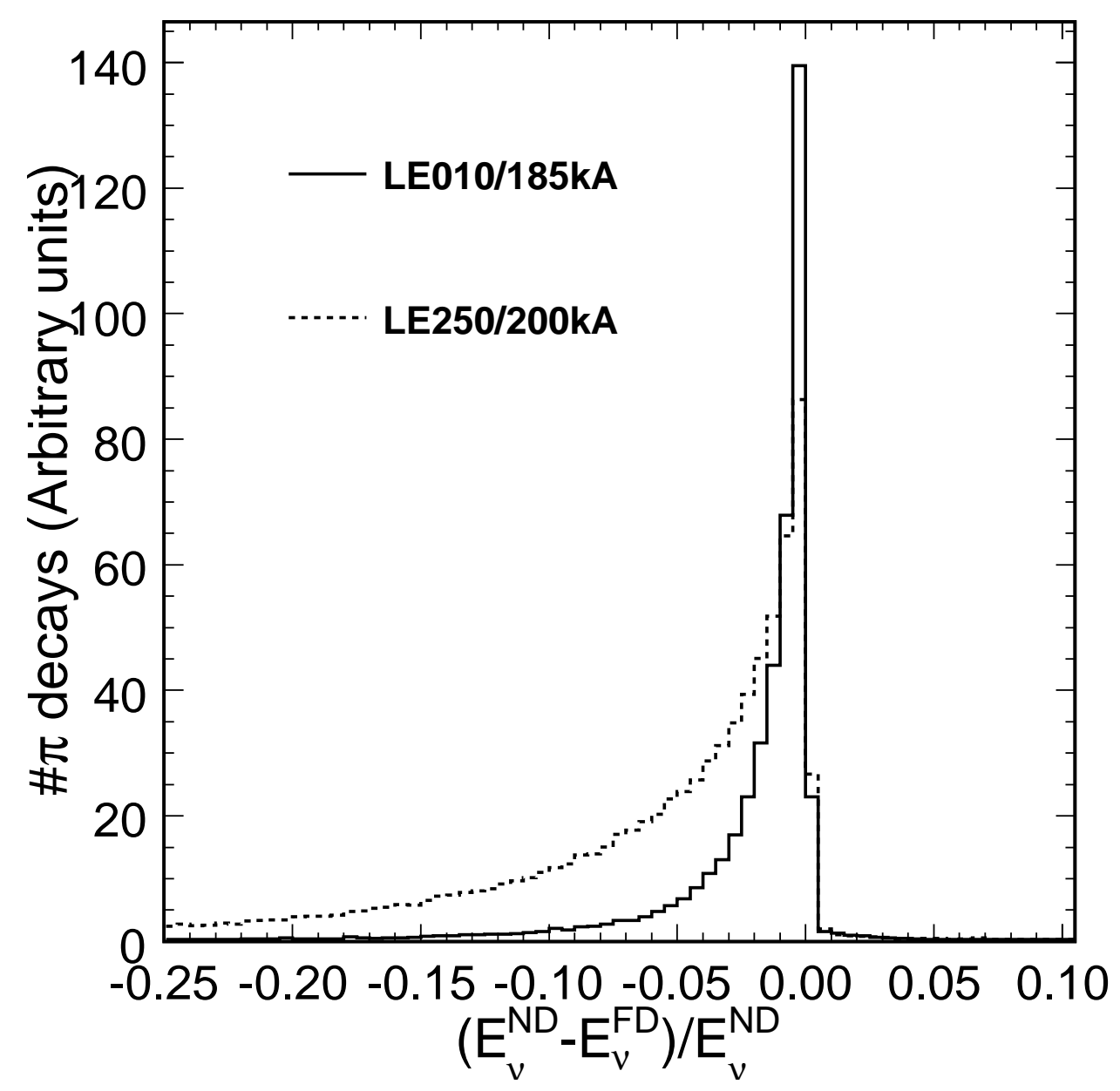

Figure 4.2: The difference in the energy of the neutrino that a given pion would emit toward the Far Detector to that it would emit toward the Near Detector. The neutrinos on the average need to be created at slightly wider angles in order to hit the Near Detector, while the angle toward the Far Detector is smaller. As a result, the energy at the ND is systematically lower contributing to the difference in shape between the neutrino energy spectrum at the ND and FD (Figure 4.1). The bigger effect can be observed in higher energy beam configuration (LE250/200kA) due to stronger dependence of neutrino energy on the angle between the neutrino and the hadron parent. 
above. Pions contributing to the focusing peak part of the neutrino spectrum have the smallest divergence and therefore the $\mathrm{F} / \mathrm{N}$ ratio in that region of the spectrum tends to approach the pion lifetime curve. We see that at the edge of the peak, as the divergence increases, the $\mathrm{F} / \mathrm{N}$ ratio starts to grow and move away from the pion lifetime curve. The same behavior can be seen at $8 \mathrm{GeV}$ where the upper threshold for pions focused by the second horn is.

We have discussed several reasons why the shapes of the neutrino energy spectra are different at the two location. All of the reasons stem from the fact that the FD sees a point source and ND sees an extended source of neutrinos. It is therefore expected that if we could move the ND away, the two spectra would become more similar. Indeed this is observed if we compare the $\mathrm{F} / \mathrm{N}$ ratios for few different locations of the ND (Figure 4.4$)^{1}$. In order to reduce the shape differences to less than $\pm 2 \%$ the ND would have to be placed $7 \mathrm{~km}$ away from the production target. However, since the neutrino beam is steered downward by $3.3 \mathrm{deg}$, the further away the ND, the deeper it has to be making it practicaly impossible to place a detector in such location. Choosing a location further away would also require using bigger detector to aquire the necessary statistics, since the neutrino flux drops off with the distance.

\subsection{Prediction of the FD Flux}

The neutrino energy spectra at the ND and the FD, as we have seen, are not the same. Nevertheless, the two are correlated since they originate from decays of the same hadron beam. We now look at the two methods that use the MC

\footnotetext{
${ }^{1}$ Moving the ND away also reduces the solid angle for that detector and causes the overall shift of the far over near ratio toward higher values
} 


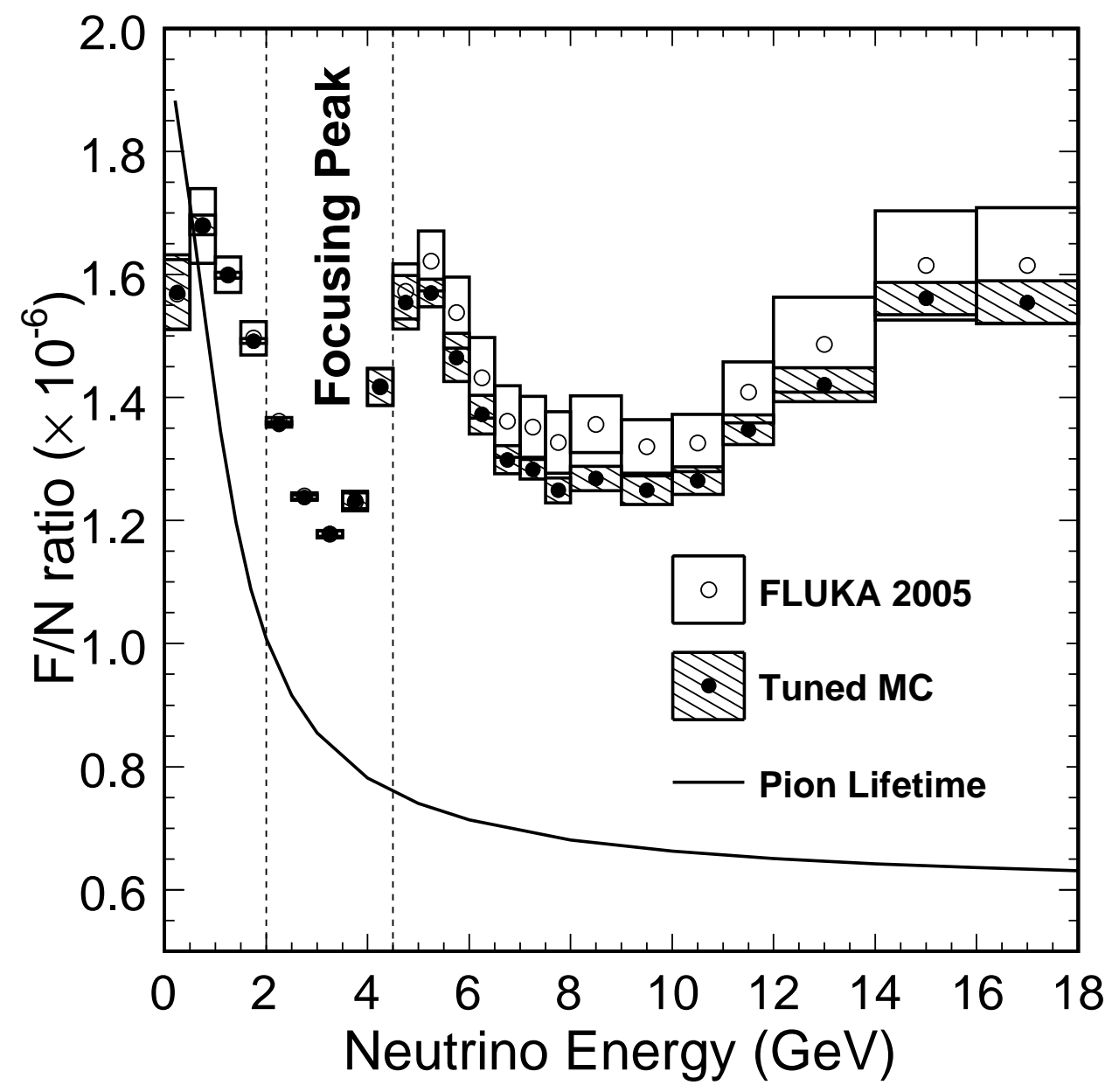

Figure 4.3: The ratio of the neutrino flux at the Far Detector and at the Near Detector for LE010/185kA beam. The ratio quantifies the expected differences between the the neutrino energy spectra at the FD and the ND in the absence of oscillations. Due to finite $\pi$ lifetime, the ratio is expected to change as a function of neutrino energy (Figure 3.21). The complex shape of the ratio as predicted by full beam-line simulation is due to focusing of pions of certain momentum and beam-line acceptance. The curve is the calculation of Equation 4.1. The empty circles are Monte Carlo calculation using Fluka hadron model [60] and full circles correspond to MC tuned to MINOS Near Detector data. The boxes represent the MC systematic errors discussed in Chapters 5 through 7. 


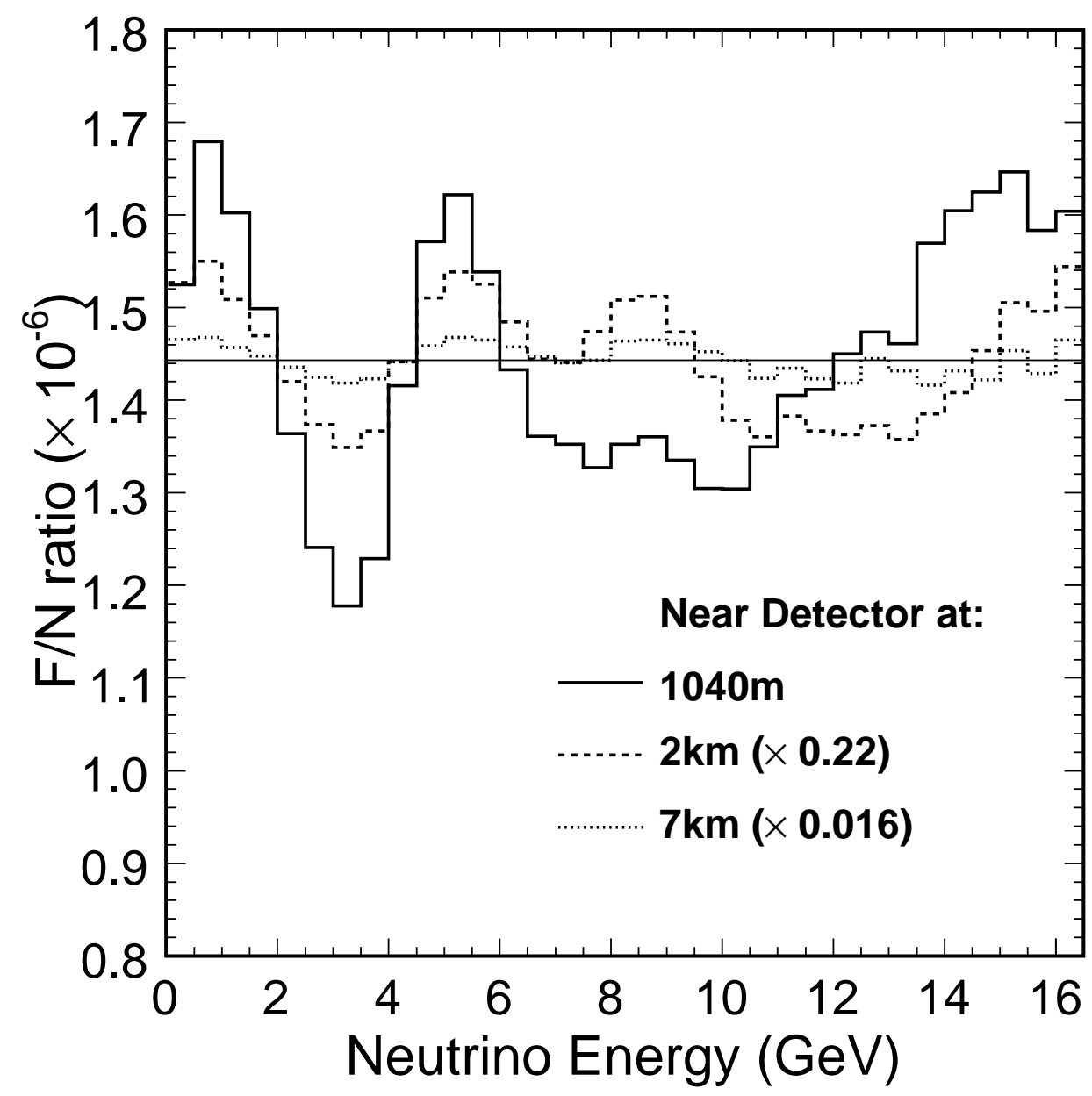

Figure 4.4: The ratio of the neutrino flux at the Far Detector and at the Near Detector in LE010/185kA beam (Figure 4.3) for few different locations of the ND. Due to the proximity of the ND to the source of neutrinos the shapes of the neutrino energy spectra are different at ND and FD. With ND at $1 \mathrm{~km} \sim \pm 20 \%$ differences are expected. The ND would need to be located approximately $7 \mathrm{~km}$ downstream of target in order for the differences to be less than $2 \%$. Moving the ND away also reduces the flux due to the smaller solid angle, making the far over near ratio bigger. For comparison the ratios for two locations further away of the meson production target were scaled down. 


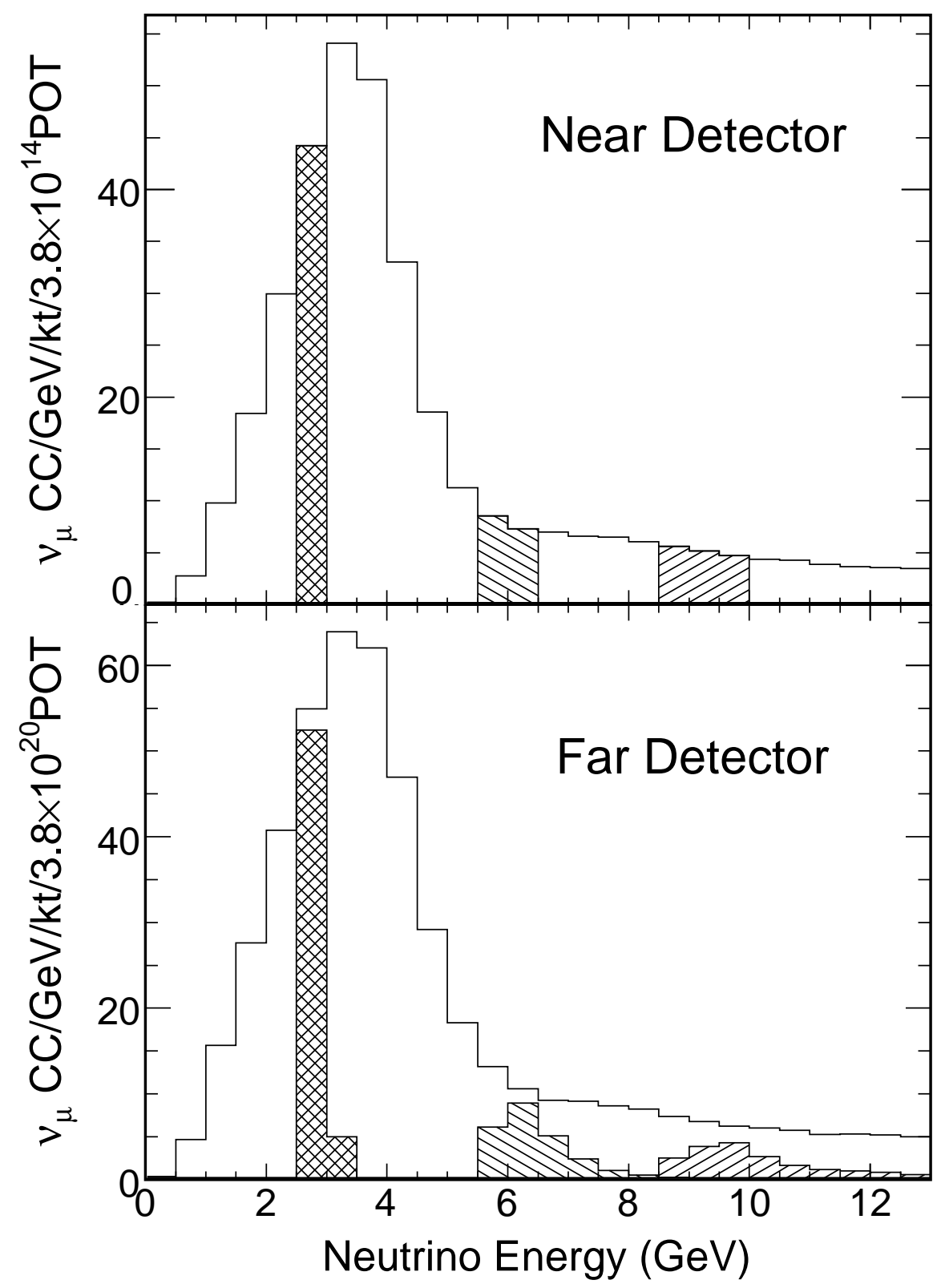

Figure 4.5: Decays producing neutrinos with a given energy in the Near Detector produce a range of energies in the Far Detector, yielding the energy smearing seen here. The neutrinos at the FD have systematically higher energies as seen in Figure 4.2. 
to calculate the correlations and then predict the flux at the FD by providing a correction to the measured spectrum at the ND. Both methods minimize the dependence on the beam-line simulation and use the ND data as a $1^{\text {st }}$-order measurement of the flux spectrum.

\subsubsection{Far Over Near Ratio}

We have seen in $\S 4.1$ how the ratio of energy spectrum at the FD and ND was used to quantify the differences between the two (Figure 4.3). As discussed, the complicated energy dependence is due to the beam-line geometry, focusing, decay kinematics and the acceptances of the two MINOS detectors. As we will see in Chapter 5 the dominant uncertainty in neutrino flux calculation comes from the uncertainty in the production of mesons in NuMI target. The F/N ratio however, is less susceptible to this uncertainty, and can be predicted more accurately than the neutrino flux.

The $\mathrm{F} / \mathrm{N}$ ratio can therefore be used to predict the neutrino flux at the FD based on the measured flux at the ND:

$$
F_{i}^{\text {predicted }}=N_{i} \frac{f_{i}}{n_{i}}
$$

where $f_{i}$ and $n_{i}$ are MC predictions of the flux in the $i$-th energy bin for FD

and ND respectively. The $N_{i}$ is the measured flux in the ND and the $F_{i}^{\text {predicted }}$ is the predicted flux at the FD. 


\subsubsection{Beam Matrix Method}

Figure 4.5 shows the neutrino energy spectrum at the ND and FD. Parents of neutrinos from three different energy bins in the ND were selected and their contribution to the spectrum at the FD is shown. It can be seen that parents that contribute to a single energy bin in ND contribute to a range of energy bins in the FD. The observed energy smearing suggests that it is possible to construct a matrix that would relate the events observed in one energy bin $j$ in the $\mathrm{ND}\left(N_{j}\right)$ with a distribution of events at the FD.

$$
F_{i}^{\text {predicted }}=\sum_{j} B_{i j} N_{j}
$$

Using the NuMI beam-line simulation it is indeed possible to construct such a matrix (see Appendix A). Figure 4.6 shows the calculated beam matrix. It is almost diagonal, especially at lower neutrino energies, where the focusing peak is. Similarly as for the $\mathrm{F} / \mathrm{N}$ ratio, the elements of the matrix are typically of the order of $10^{-6}$. It becomes most off-diagonal at larger $E_{\nu}$, a kinematic region stemming from energetic pions which decay further downstream in the decay pipe.

\subsection{Error on the Predicted Flux at FD}

We need a MC simulation of the NuMI beam-line to predict the flux at the FD even though we measure the flux at the ND. The simulation is used to find the expected correlations between the neutrino energy spectra at the ND and the FD. The uncertainties in beam modeling will be discussed in detail in following chapters. We will summarize here what the net result is on the 


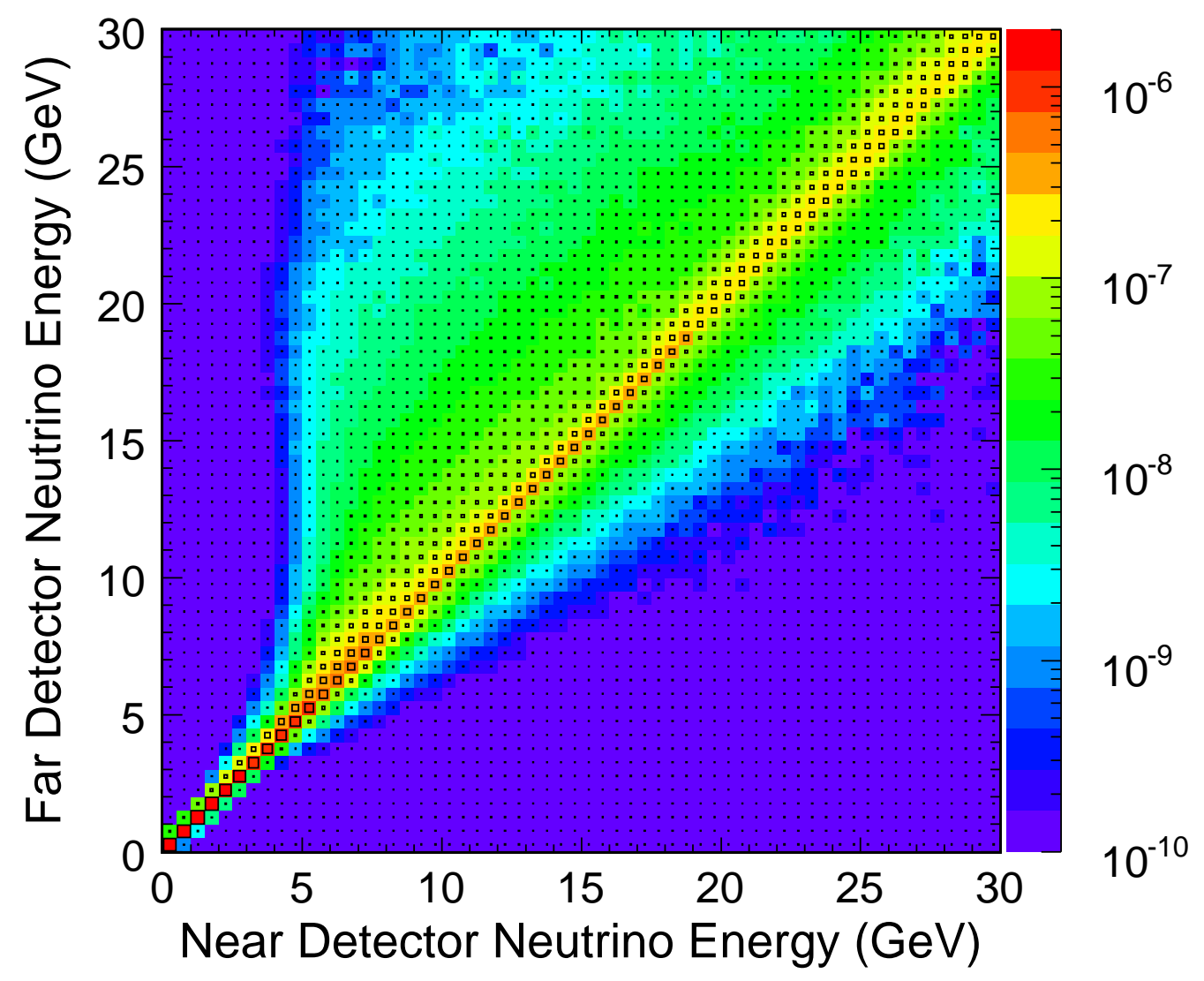

Figure 4.6: The Beam Matrix that can be used to obtain the flux at the Far Detector given the flux at the Near Detector. The matrix element $M_{i j}$ gives the number of neutrinos of energy $E_{j}$ expected in the FD for every observed neutrino in the ND of energy $E_{i}$. 
predicted flux at FD.

As it will be shown, the calculation of correlations between the spectra is more robust than the calculation of neutrino flux and less susceptible to the various uncertainties in modeling of the NuMI beam-line. Figure 4.3 shows the $\mathrm{F} / \mathrm{N}$ ratio and the associated uncertainties. Of the two ratios that are shown, one is calculated using the beam-line MC based upon the FLUKA hadron production model and the other using the beam MC that was tuned to better agree with ND data (the tuning procedure will be described in Chapter 6). The uncertainties in the $\mathrm{F} / \mathrm{N}$ ratio as predicted using FLUKA model are already relatively small, especially in the focusing peak region of neutrino spectrum. After the tuning procedure, the uncertainty in the ratio and consequently in the predicted flux at the FD is less than $2 \%$. 


\section{Chapter 5}

\section{Uncertainties in the Neutrino Flux Calculation}

The Monte Carlo (MC) calculation described in Chapter 3 represents a somewhat idealized situation. In reality, for example, the target and the horns may be slightly misaligned, while in the MC the alignment is perfect. Such effects cause a systematic difference between the predicted neutrino flux and the true neutrino flux. The MC calculation also relies on a hadron cascade model used to predict how many pions with certain momentum are emerging from the NuMI target (§3.2). Different models yield significantly different predictions of neutrino flux. Detailed analysis of these systematic effects is necessary in order to determine the uncertainties in predicted flux and make quantitative statements about neutrino disappearance in the NuMI beam. In this chapter we study possible sources of systematic effects in modeling of the NuMI beam-line and resulting uncertainty in the calculation of neutrino flux.

Throughout this chapter we present only the calculated derivatives for 
the neutrino energy spectrum at the ND. The corresponding plots for the far over near ratio can be found in Appendix E. The distortion of the far over near ratio is always few times smaller than the corresponding distortion of the neutrino spectra at the ND. The calculated uncertainties on the far over near ratio are shown at the end of the chapter.

An interesting feature of beam related uncertainties is that they manifest differently in the different beam configurations listed in Table 2.1. In this chapter we present the uncertainties for three configurations, namely LE010/185kA, LE100/200kA and LE250/200kA. The uncertainties for other beam configurations in which NuMI was operated (Table 2.1) were calculated as well using the same procedure [74].

\section{$5.1 \quad$ Methodology}

The neutrino energy depends on both the parent energy and decay angle between the pion direction and the direction for the neutrino to reach the near or the far detector (see Equation 3.9). The uncertainty in the neutrino flux therefore comes from the uncertainty in knowing the underlying distributions of parent hadrons.

The method we utilize to evaluate the uncertainties in neutrino flux is to make a calculation of the flux with certain systematic shift applied to $\mathrm{MC}$ simulation and then compare it to the results of the calculations done in absence of the systematic shift. In particular we look at the ratio of the two. This method essentially calculates the derivatives of neutrino flux with respect to the magnitude of a particular systematic effect as a function of neutrino energy. For example Figure 5.1 shows a calculated neutrino flux for 
few different magnitudes of geometric misalignment of the first NuMI horn. It can be seen that the ratio is less than one around $4 \mathrm{GeV}$ and greater than one at $6 \mathrm{GeV}$ indicating that the flux will be reduced at $4 \mathrm{GeV}$ and increased at $6 \mathrm{GeV}$ when first horn is misaligned. These derivatives tell us how neutrino flux is changing due to some systematic effect and also show us the correlations between different neutrino energy bins.

Some of the systematic effects have a nonlinear effect on neutrino energy spectrum. In this case knowing just the first derivative is not enough. Misalignment of a horn is one such effect. As can be seen in Figure 5.1, misalignment of first NuMI horn by $2 \mathrm{~mm}$ does not produce half of the distortion that a $4 \mathrm{~mm}$ misalignment does. To account for this nonlinearity we repeat flux calculations a number of times with different magnitudes of systematic shifts and look how the flux changes in each neutrino energy bin. We then fit each energy bin with a second order polynomial that relates the distortion of the neutrino flux to the magnitude of systematic effect. In Figure 5.2 the misalignment of first NuMI horn is again used as an example. The figure shows the result of the fit for two different bins. Once the fitted polynomial is found we can evaluate the distortion of the spectrum for any given magnitude of systematic effect. As will be discussed, in case of the alignment of the horn this is $1 \mathrm{~mm}$.

The use of these derivatives is twofold. Firstly, they are being used to estimate the uncertainty in neutrino flux. The magnitude of each systematic effect can be constrained using the data from beam-line instrumentation. This magnitude and the derivatives yield the uncertainty. Secondly, they are used to correct the MC predicted neutrino flux for specific runs when running conditions differed significantly from those used in simulation. 


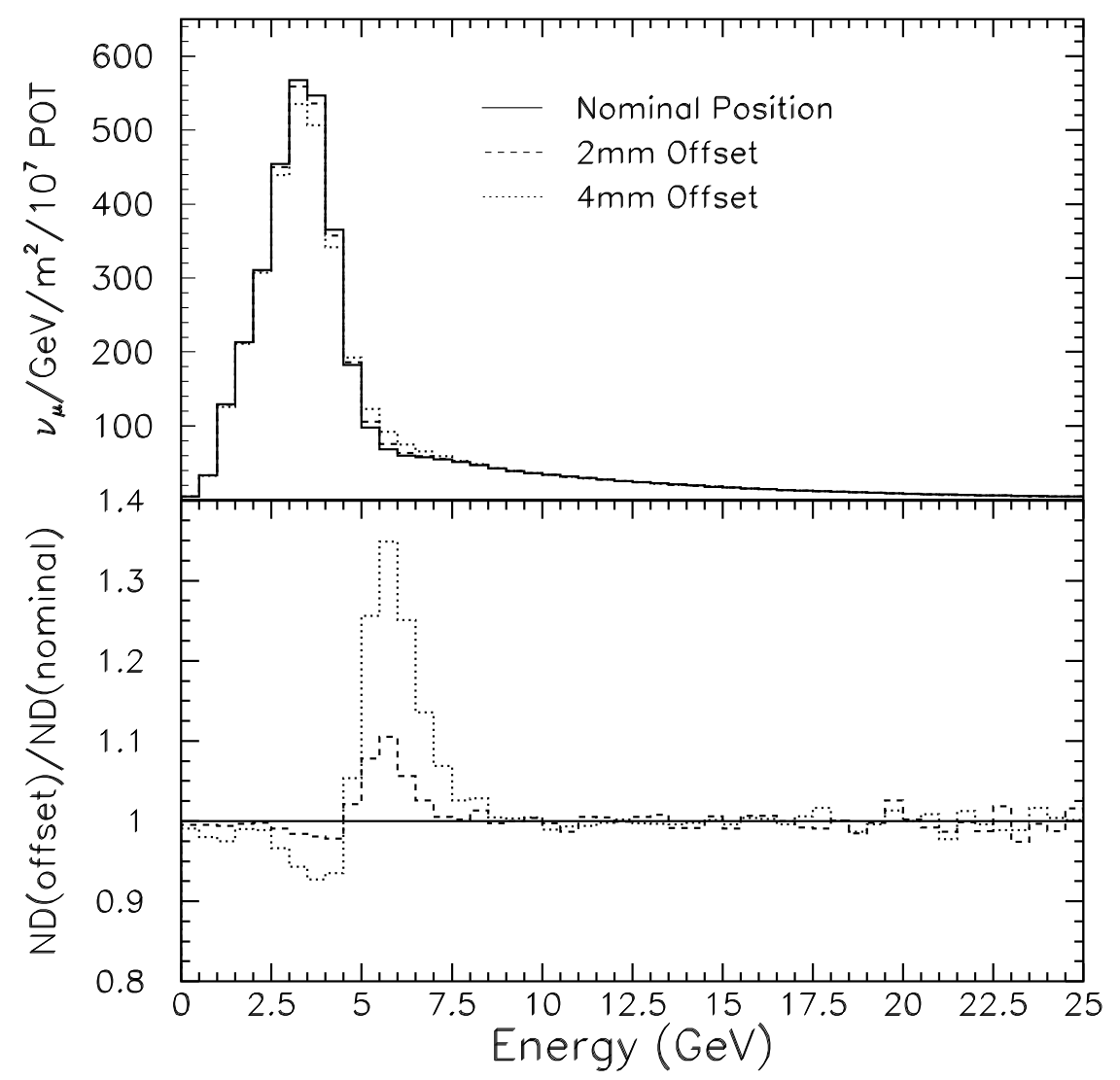

Figure 5.1: (top) The calculated fluxes for few different transverse misalignments of the first NuMI horn with respect to the beam axis. (bottom) Ratios of the predicted flux with misaligned horn to the flux with horn perfectly aligned. 

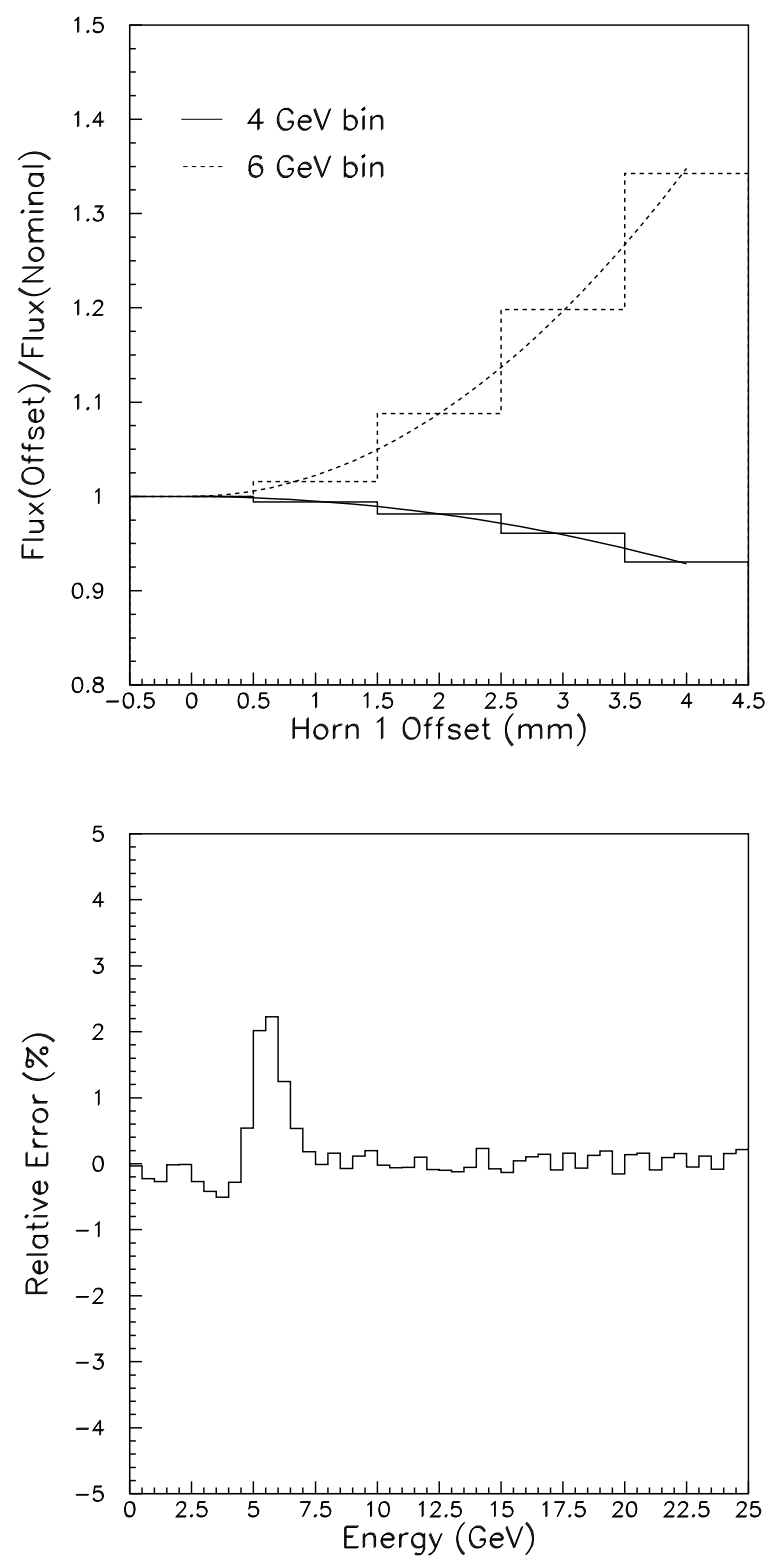

Figure 5.2: Top plot shows only $4 \mathrm{GeV}$ and $6 \mathrm{GeV}$ bins of Figure 5.1 plotted versus horn offset. A second order polynomial was used to fit the fractional change of the flux as a function of horn offset transversely to the beam axis. Bottom plot shows calculated error bar in this case using known uncertainty in the position of the horn of $1 \mathrm{~mm}$ [41] and a polynomial fit for each bin. 
As discussed in Chapter 4 MINOS benefits from having two neutrino detectors. Most of the systematic uncertainties are greatly reduced when Near Detector (ND) data is used to predict the un-oscillated neutrino flux at the Far Detector (FD). The size of the remaining uncertainty becomes obvious when we look at how the far over near ratio $(\S 4.2)$ changes with a certain systematic shift applied to MC simulation. The procedure described above was applied both in calculating the uncertainties in the flux at the ND and in the far over near ratio.

\subsection{Alignment and Focusing Uncertainties}

\subsubsection{Proton Beam}

The proton beam properties were discussed in Chapter 2. Here we will study the precision and the accuracy with which the primary proton beam intensity is measured as well as how the neutrino flux is affected when the beam position or size changes.

\section{Integrated Intensity}

The overall normalization of the neutrino flux depends on the total integrated intensity of the proton beam. The number of protons delivered on the target is determined not only by the number of protons in the beam but it also depends on what fraction of these protons are actually incident on the target.

The NuMI toroids were calibrated using a precision current source. As an additional check the toroids are compared to the Main Injector (MI) toroid during running. The MI toroid is a very accurate and precise device constantly 
being calibrated to measure the proton intensity to better than $1 \%$ [75].

The resolution of NuMI toroids is revealed when we compare how the two toroids track each other. A comparison of the 2 toroids is shown in Figure 5.3. Across the span of proton spills with different intensities it was found that the difference in the readout from two toroids is smaller than $0.2 \%$, or $10^{11}$ protons at $2 \times 10^{13} \mathrm{ppp}$. The NuMI toroids show slight nonlinearity at low intensity as can be seen when toroids are compared to Main Injector toroid [76]. The vast majority of data was taken at intensities of $\sim 2.5 \times 10^{13}$ protons per spill where nonlinearity is expected to contribute less than $\pm 0.4 \%$ in the total uncertainty.

The electronic pedestals for the NuMI toroids are observed to drift over time and vary separately for each toroid. During trigger in which no beam is present, we studied these pedestal variations and found that they are equivalent to $\pm 5 \times 10^{9} \mathrm{ppp}$, or $0.1 \%$ at nominal signal at full intensity. Figure 5.4 shows the effect of these pedestal variations during normal running: shown is the ratio of readings from the two toroids over a 40 day period. The variation of the order of $0.2 \%$ is observed consistent with observed drift of pedestals.

The toroids integrate the signal within some time window surrounding the beam pulse. In particular the gate opens just before the beam spill and remains open for $13.1 \mu \mathrm{s}$. The beam spill length varies and it can fall within different portions of the electronic gate depending on the running mode ( $\S 2.1 .1)$. This slightly affects the toroid readout. Figures 5.5 shows the shift in the ratio between the two toroids as NuMI beam is operated in different modes. The shift is of the order of $0.2 \%$.

The proton beam profile at the target is of the order $1.1 \times 1.2 \mathrm{~mm}^{2}$ 


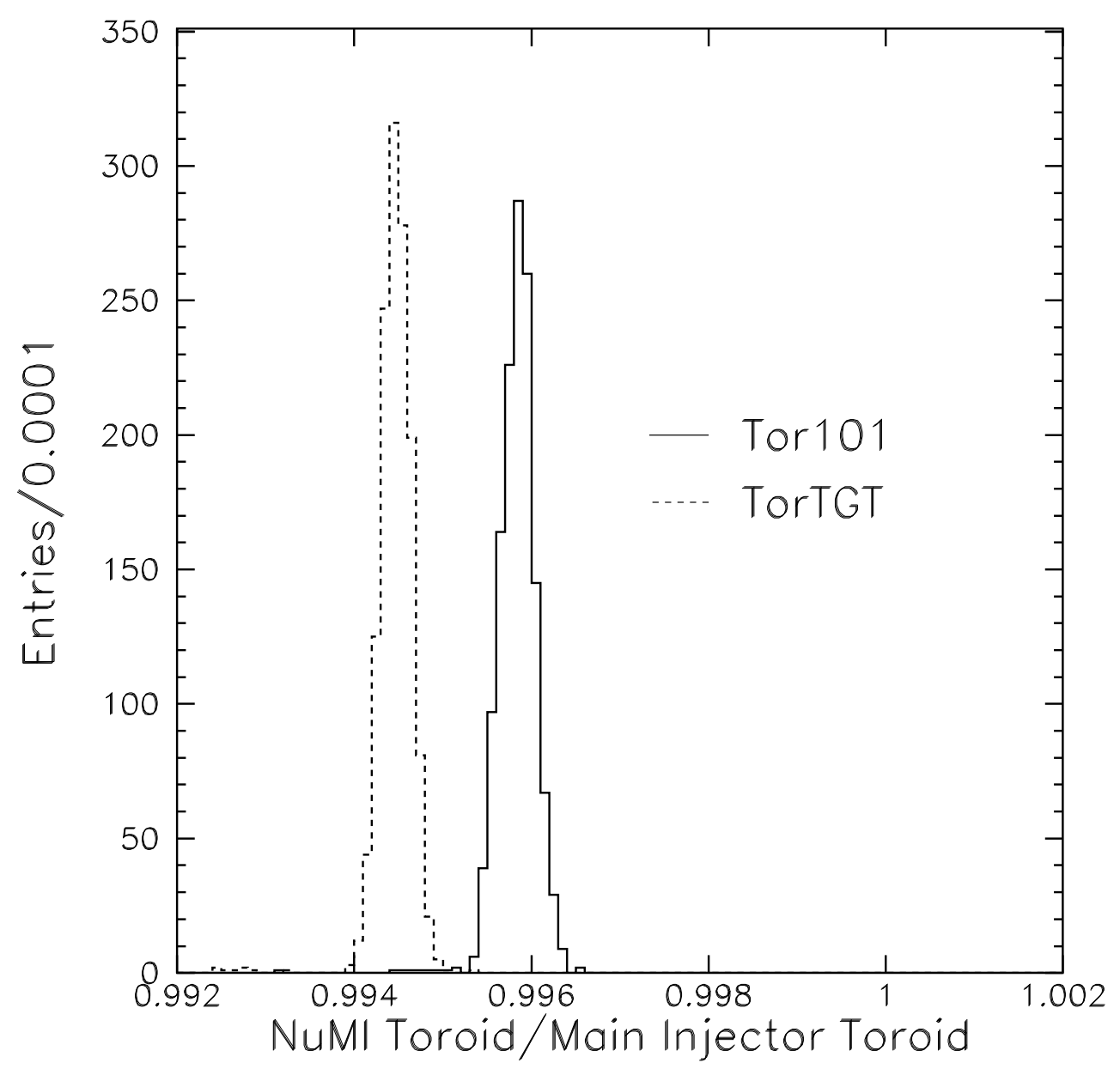

Figure 5.3: Shown are the ratios of NuMI toroid currents compared to the Main Injector toroid current for several NuMI-only accelerator cycles (ie all the MI beam was delivered to NuMI). The Main Injector toroid is regularly being calibrated and has an accuracy of better than $1 \%$ [75]. Two NuMI toroids agree to within $\mathrm{a} \sim 0.5 \%$. 


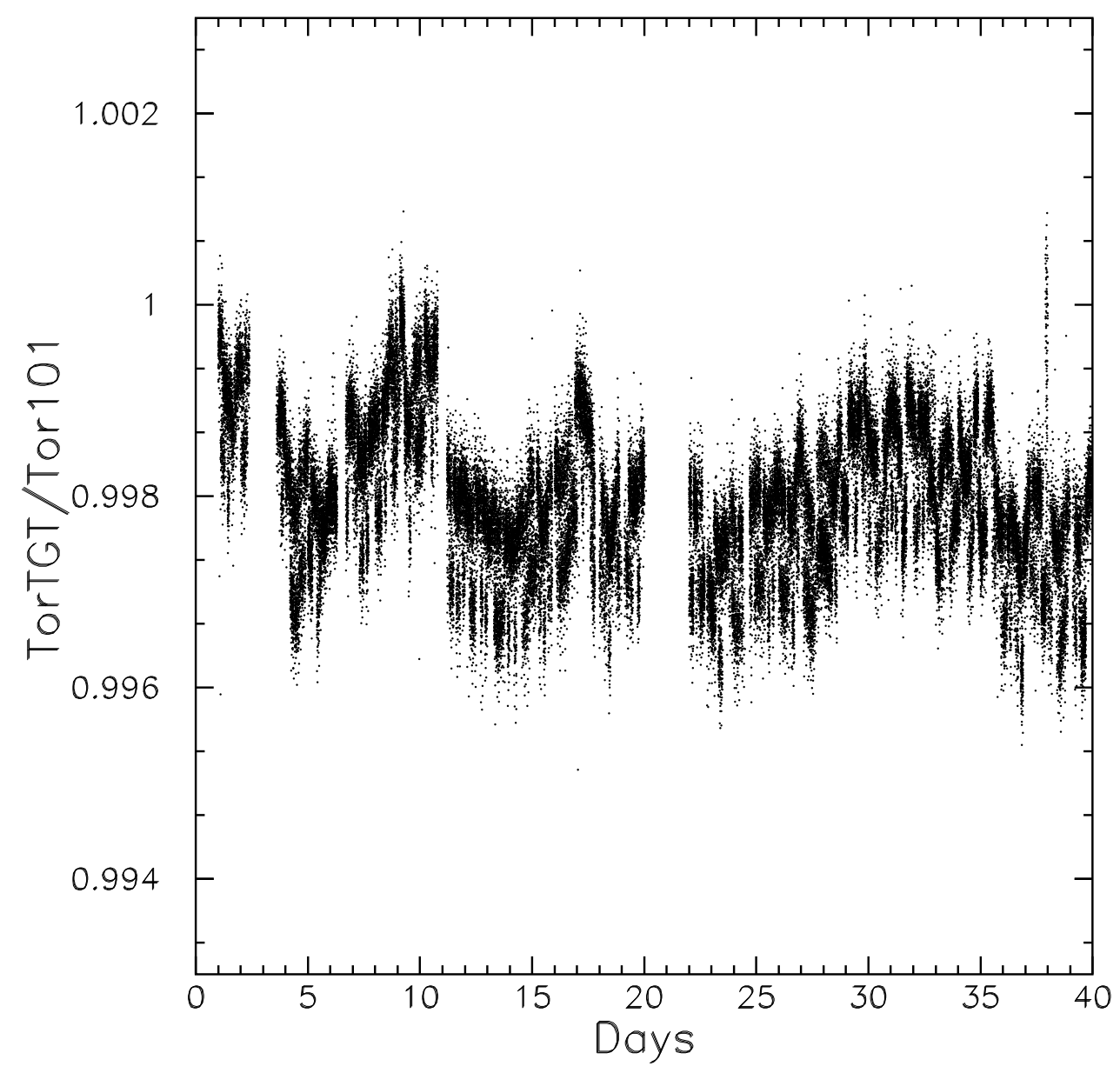

Figure 5.4: Plot of the ratio of two NuMI toroids, TorTGT and Tor101, plotted over a long period of time (Data from August 10th - September 20th 2005). The drift in this ratio by $\pm 0.2 \%$ is similar to the magnitude of the drift observed in the toroids' pedestal values. 


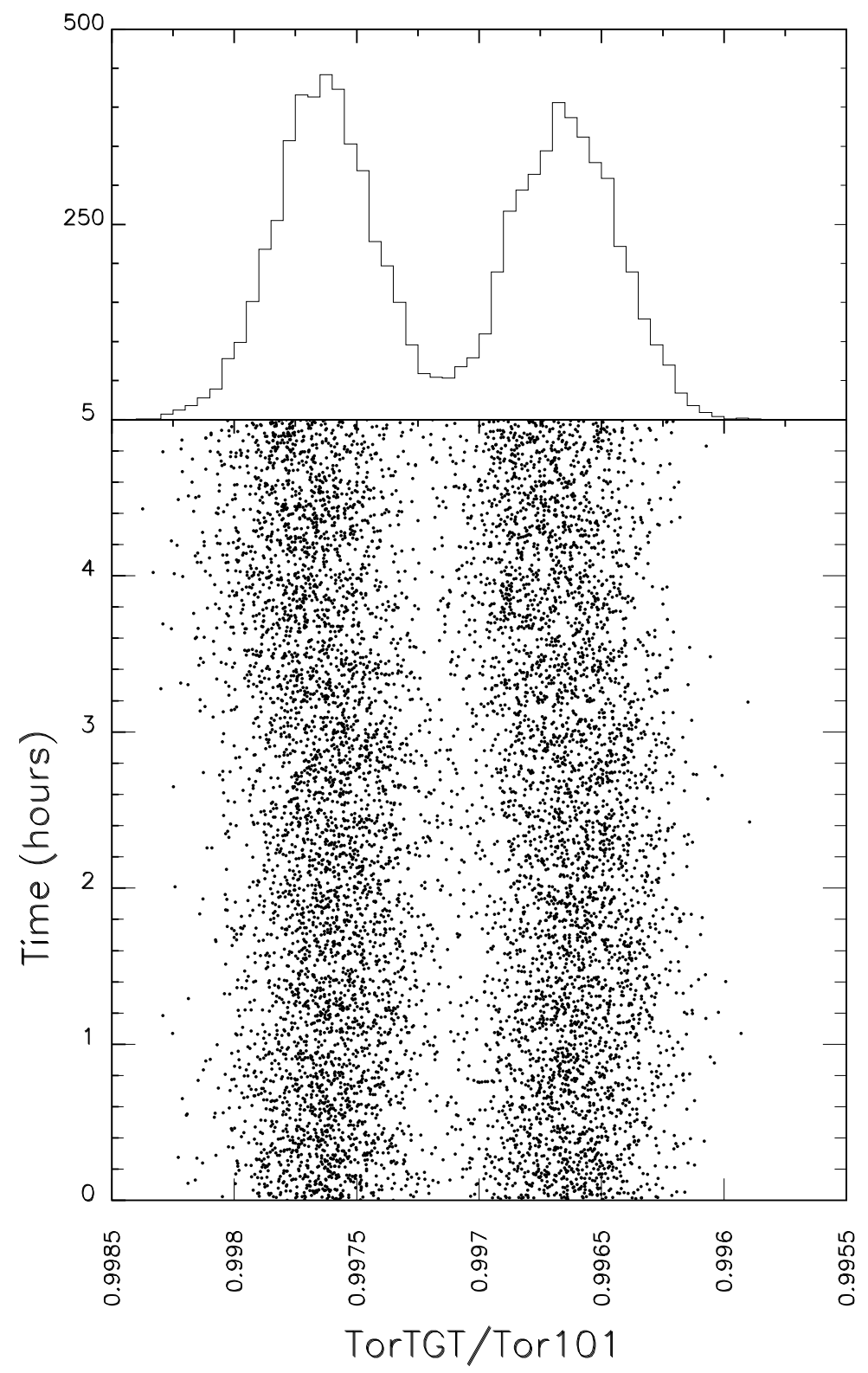

Figure 5.5: Double-valuedness of one of the NuMI toroids depending on which portion of the proton beam spill from the Main Injector gets delivered to NuMI $\S 2.1 .1$. The ratio of TorTGT to Tor101 versus time for few hours of running in that mode is shown. The shifts suggest the uncertainty at the level of $0.2 \%$. 
which is comparable with target size, causing some of the beam to miss the target altogether. The spot size at the NuMI target is not exactly measured, but needs to be extrapolated from the profile monitor which is $6 \mathrm{~m}$ upstream of the target. This results in the error in spot size of order $50-100 \mu \mathrm{m}$. Figure 5.6 shows the fraction of the beam that missed the target over a period of few months. Less than $0.5 \%$ of the beam misses the target during normal running [77].

The total uncertainty in the number of protons delivered on the target, when all of the contributions discussed above are summed, is estimated to be $2 \%$.

\section{Beam Fraction Hitting Protective Baffle}

The large beam spot size also causes a small fraction of the protons to hit the horn protection baffle (§2.1.1). The baffle is made of graphite and acts as another target; since this baffle is further upstream of the real target, it produces a higher energy neutrino beam, as for example we would expect from the variable neutrino beam concept discussed in $§ 2.1 .2$. The baffle is not as efficient as the target when it comes to producing neutrinos (because its outer radius is large, the pions can escape only through one side).

The number of protons hitting the baffle can be estimated in two ways. First, the beam width measurement (§2.1.1) in front of the baffle and target assembly can be extrapolated (gaussian approximation) to the $5.5 \mathrm{~mm}$ radius of the baffle. Figure 5.7 shows the estimated fraction of the beam on baffle per spill for few months of accumulated data. The mean is less than $0.015 \%$. Second, one can measure the temperature of the baffle. Protons hitting the baffle deposit energy, causing it to heat up. The deposited energy is propor- 


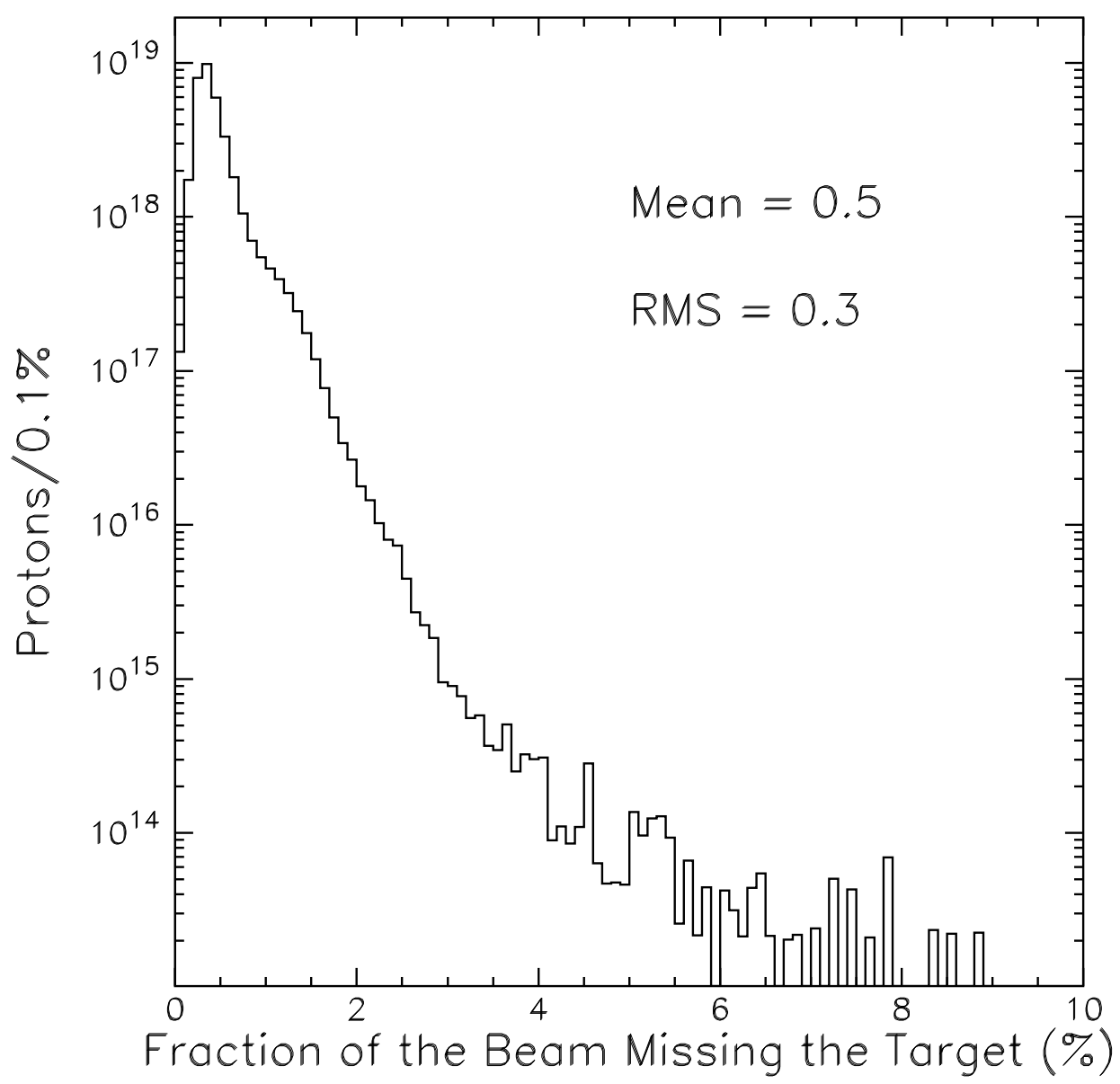

Figure 5.6: The proton beam has a spot size of $\sigma_{x} \times \sigma_{y} \approx 1.1 \times 1.2 \mathrm{~mm}^{2}$ which is comparable to the target width of $6.4 \mathrm{~mm}$ (see Figure 2.6). A certain number of protons misses the target depending on the beam position and width. Shown is the fraction of the beam missing the target over a period of few months of normal running. 
tional to the proton beam power. Two thermocouples attached to the baffle monitor the temperature ( $\S 2.1 .1$ ), and the data indicate that less than $\sim 0.2 \%$ of the proton beam scrapes on the baffle.

Figure 5.8 shows the comparison of MC calculation of neutrino flux with the proton beam centered on the target and with the beam hitting directly on the baffle. Adding the spectra in proportion yields the estimate of the error due to baffle scraping. Figure 5.9 shows the far over near ratio for the two considered configurations and the fractional change in the far over near ratio for different levels of scraping on the baffle.

As the running progresses the beam size changes, mainly as a side effect of intensity improvements. This means that the level of baffle scraping can change from one time period to another. The uncertainty in the number of protons hitting the baffle over the entire run using both the beam width and baffle temperature measurement was estimated to be of the order of $0.25 \%$ [77]. 


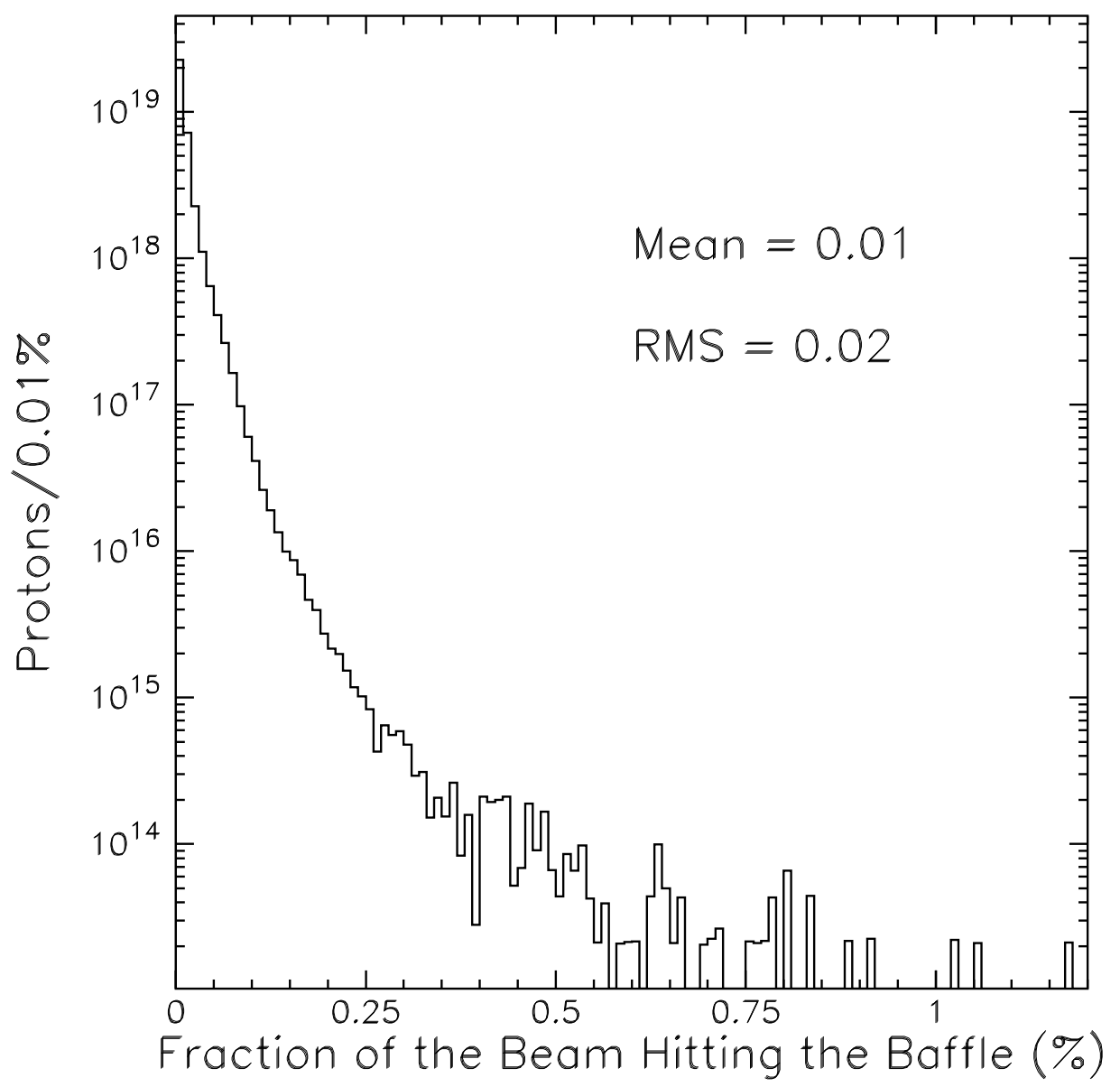

Figure 5.7: A collimating baffle located just upstream of the target protects the horns from being directly hit by a stray proton spill (§2.1.1). Even when the beam is centered on the target some of the protons can hit the baffle depending on the profile of the proton beam (see Figure 2.6). Shown is the fraction of the beam hitting the baffle determined using the beam profile in front of the target as measured by a Secondary Emission Monitor (§2.1.1). Data was accumulated over a period of few months of normal running. 


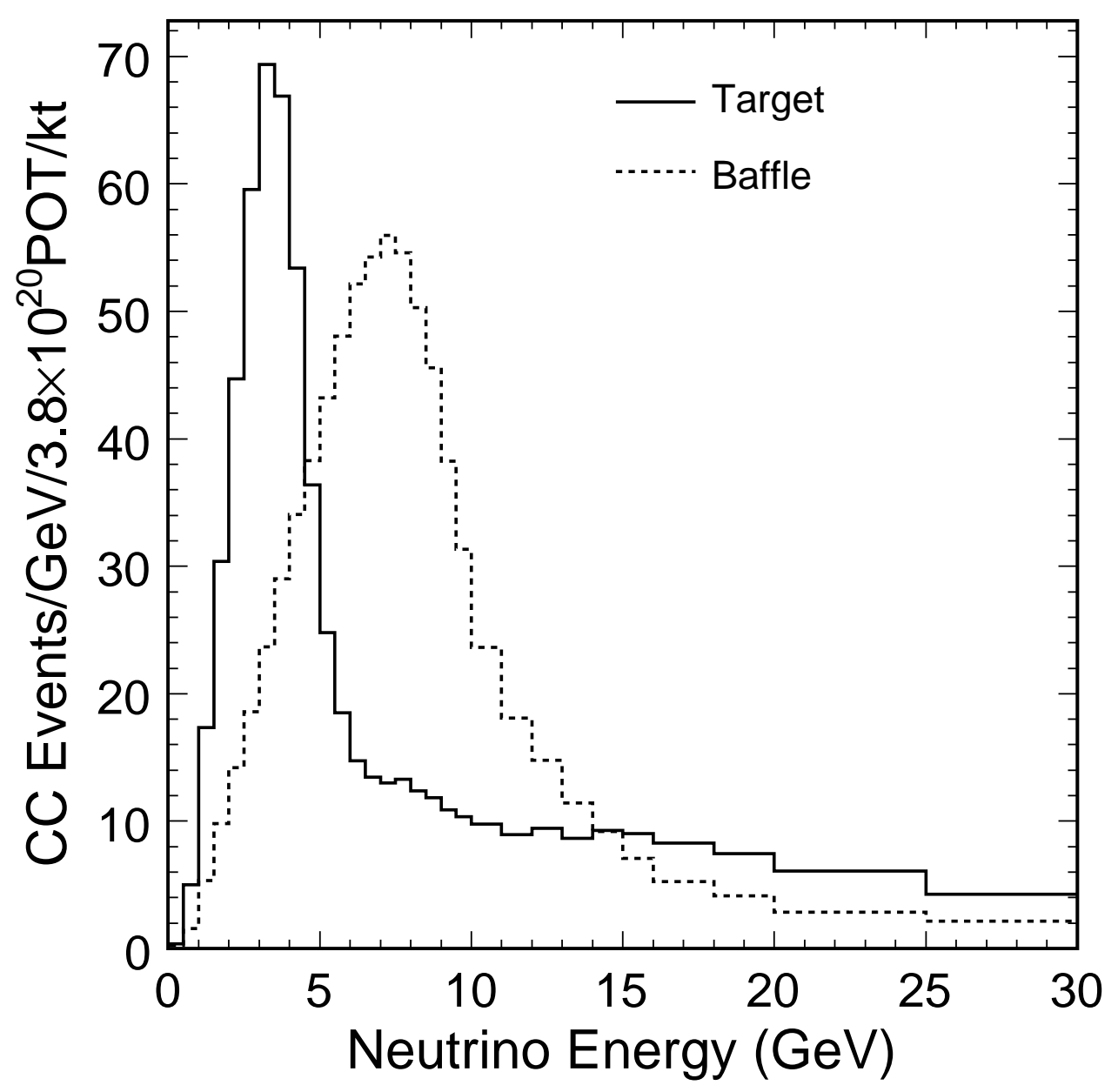

Figure 5.8: The collimating baffle located upstream of target is made of graphite and can act as another target. As per variable energy beam concept (§2.1.2) this produces higher energy neutrino spectrum. Shown is the neutrino spectra at the far detector corresponding to beam centered on target and beam hitting directly on baffle. 

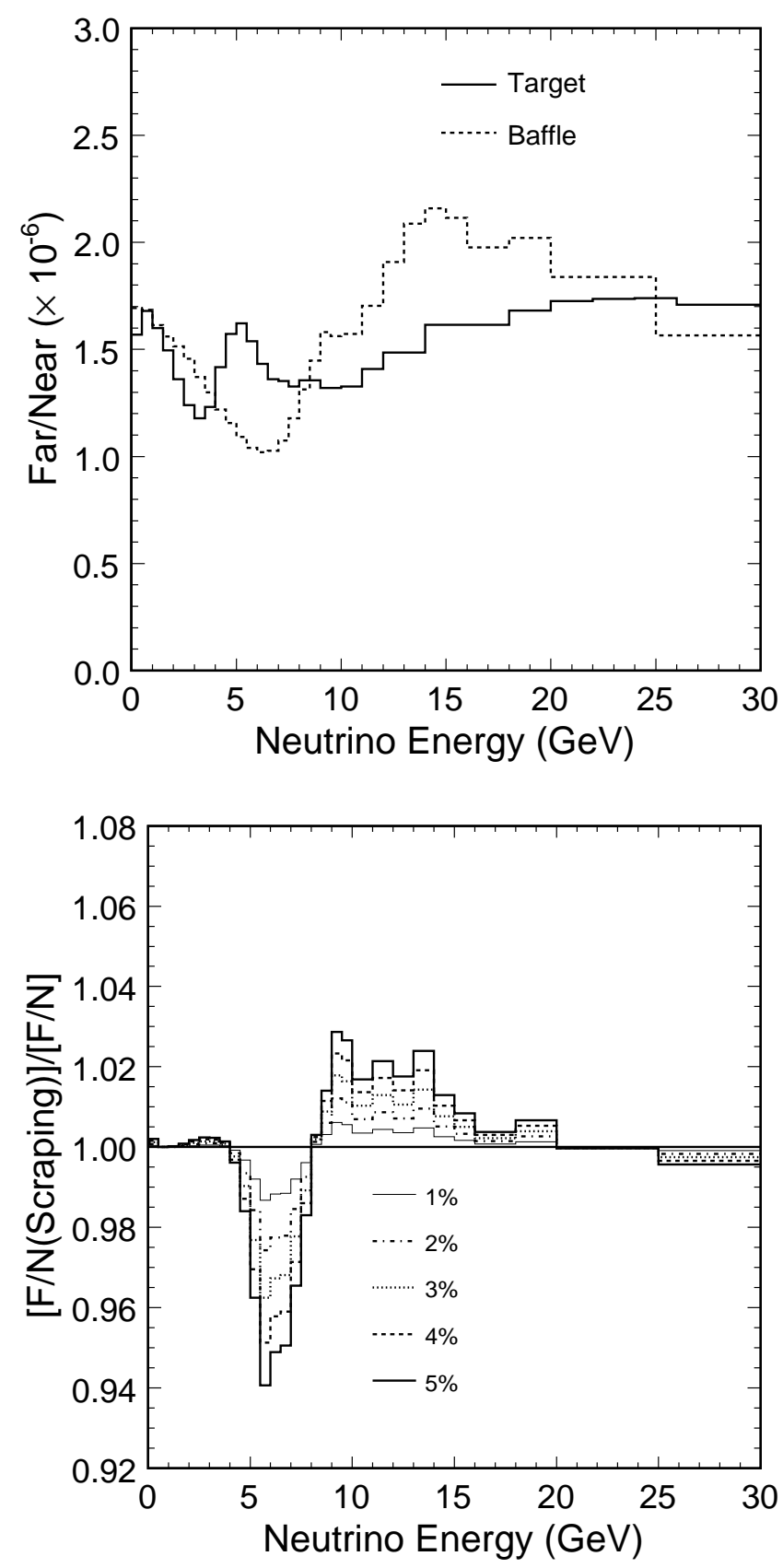

Figure 5.9: Top plot shows comparison of the far over near ratio when beam is centered on the target and when it hits directly into baffle. Bottom plot shows the change in far over near ratio for different levels of baffle scraping. 


\section{Beam Position and Width}

The protons hitting different portions of the target produce the same spectrum of pions. However, pions created near the edge of a target fin are more likely to exit the target without any reinteraction compared to the pions created in center. Reinteractions in the target cause the pions to loose energy. Since the $E_{\nu} \propto E_{\pi}$ it is expected that the neutrino spectrum shifts toward higher energy as the protons hit the edge of the target. While the beam is normally centered on the target its position can vary spill-to-spill. Figure 2.7 shows the beam position over few months of running. The fitted widths of these distributions are $90 \mu \mathrm{m}$ and $84 \mu \mathrm{m}$ in the horizontal and vertical respectively, indicating that the proton beam position is quite stable over time.

Variations in the beam's spot size at the target, like variations in the beam's position, can change the neutrino energy spectrum. The proton beam width varies over time because it is correlated with beam intensity. Some of the runs were done with very low proton beam intensity having significantly different beam width. The beam MC was generated with $\sigma_{x} \times \sigma_{y}=1.1 \times 1.2 \mathrm{~mm}^{2}$, most common beam width during normal running, requiring a correction when comparing to data from runs with smaller or larger widths. The width in horizontal plane is more relevant due to the narrow shape of the target. In Figure 5.10 we compare the expected ND flux at several proton beam widths.

The overall effect on the neutrino flux from proton beam position is also reflected in a flux of muons that are created along with muon neutrinos. The yield of muons in the secondary beam monitors is observed to be stable when the beam is centered on the target. The final neutrino data sample is selected with the requirement that the muon flux does not change by more than $2 \%$. 


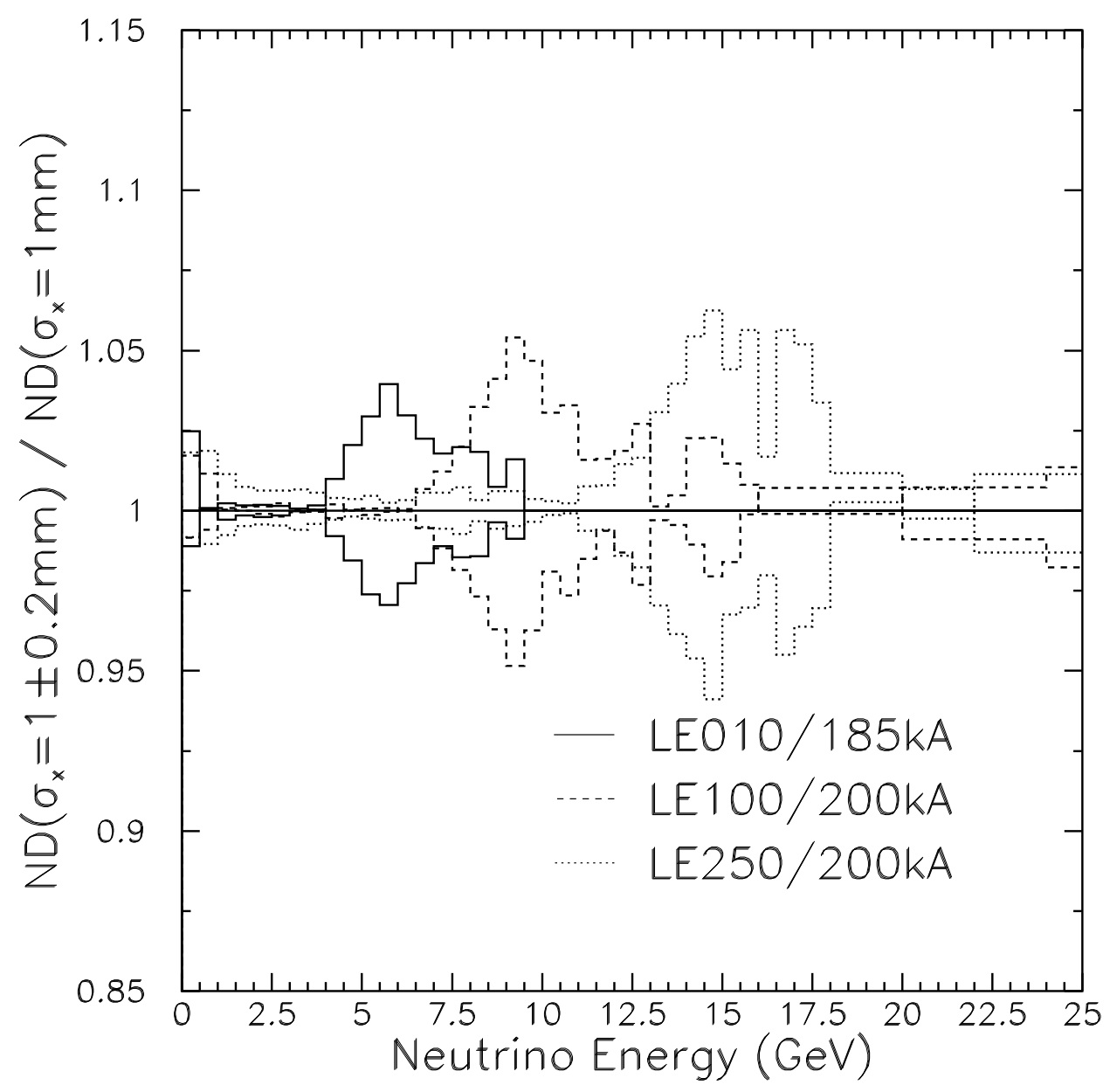

Figure 5.10: The curves represent the distortions in the neutrino flux for $\pm 20 \%$ excursions in the proton beam width $\sigma_{x}$. Excursions by $\pm 10 \%$ produce approximately half as large of an effect on the spectrum. 


\subsubsection{Target Geometry}

The target description in the $\mathrm{MC}$ reproduces the important features of the target. We consider here a couple of possible differences with the real target that could affect the neutrino flux.

The target has 47 vertical fins and 1 horizontal fin which is further upstream (§3.3). Since the width of the beam in vertical direction is small compared to the height of the vertical fins, the beam is always hitting the 47 vertical fins regardless of its vertical position. This is not necessarily true for the horizontal fin and some fraction of the beam might miss it.

The target that was used for the first year of running had the horizontal fin offset by $2.2 \mathrm{~mm}$ as was determined by optical survey of the target. Figure 5.11 shows the expected fraction of the beam hitting the horizontal fin depending on the vertical position of the proton beam. It can be seen that with $2.2 \mathrm{~mm}$ offset, less than $80 \%$ of the beam would hit the horizontal fin. During normal running the beam position in vertical direction was set to $1.1 \mathrm{~mm}$ (Figure 2.7) in order to compensate for the misalignment of the horizontal fin.

The fraction of the beam hitting the horizontal fin can be calculated for different periods of running since the proton beam width is being measured on a spill by spill basis( $(2.1 .1)$. To estimate the effect on the neutrino spectrum we compare the simulation of the NuMI target and resulting neutrino flux with and without the horizontal fin. Comparison of the two spectra gives us the maximal size of the distortion (Figure 5.12) if the beam would be completely steered away from the horizontal fin.

The second effect that we consider here is the target density. The 


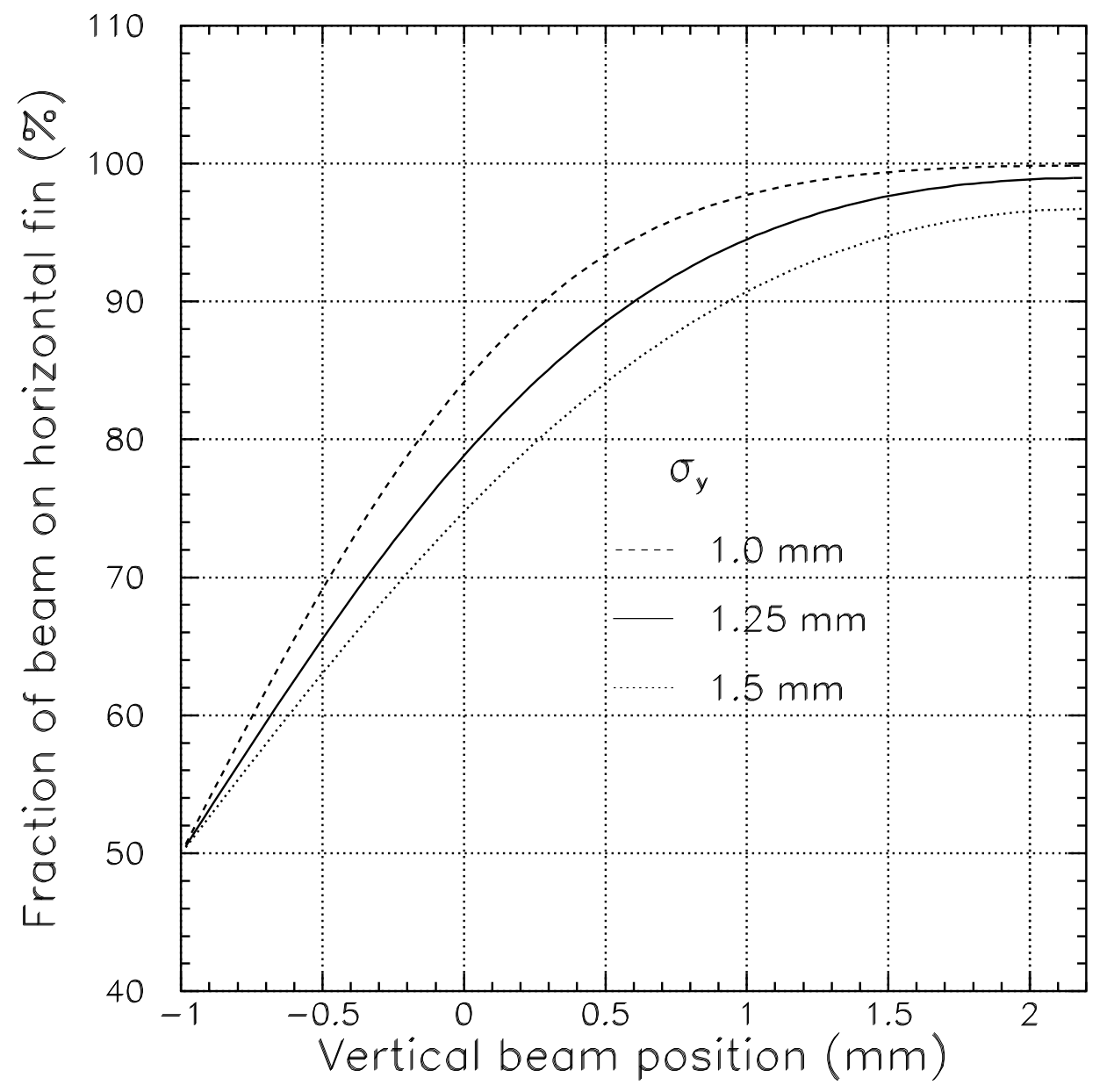

Figure 5.11: The fraction of the proton beam which strikes the first horizontal fin of the 48 fin target (see Figure 3.11). Due to the misalignment of this first fin, the fraction of the beam which strikes varied over the course of running period, depending on the vertical beam location and spot size $\left(\sigma_{y}\right)$. For the NuMI target used during first year of running the center of the fin was at $2.2 \mathrm{~mm}$ and most beam running was at $1.1 \mathrm{~mm}$ (Figure 2.7). 


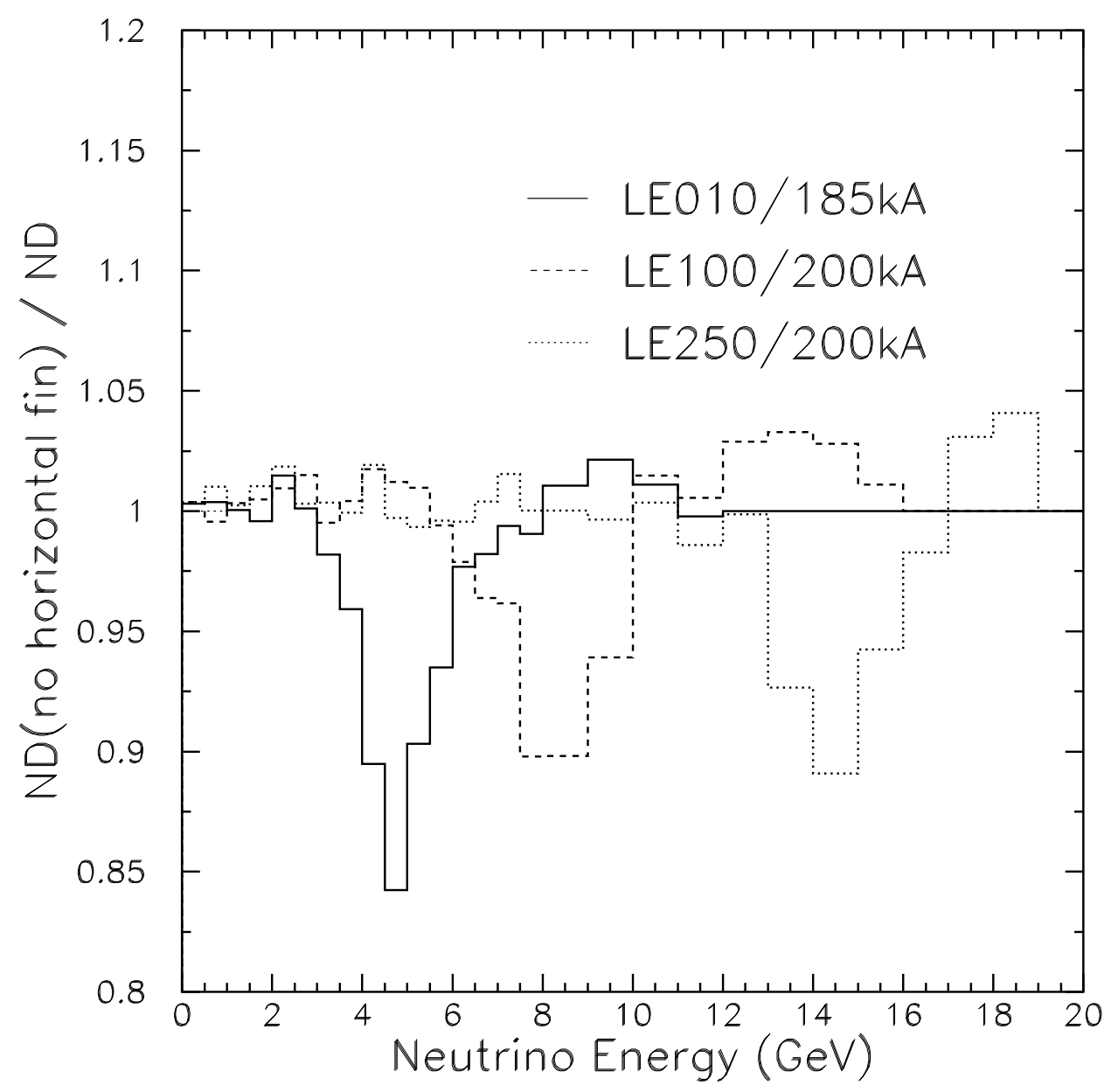

Figure 5.12: The curves represent the distortion of the neutrino flux when the proton beam misses the horizontal fin. 
number of pions created in the target and how much they reinteract depends on the density of target medium. As mentioned NuMI target is made of graphite whose density was measured to within $2 \%$. We looked at how the neutrino flux is affected if target density is varied, but no significant distortions of the spectrum were observed for such small density variations.

\subsubsection{Misalignment Errors}

Relative misalignment of magnetic horns with respect to the target affects the focusing of neutrino parents. In calculations of the beam flux we assumed that the target and the horns are perfectly aligned, however in reality some misalignments are present [41]. As was discussed in Chapter 3 and is indicated in Figure 3.12 the soft pions, that give rise to lower energy neutrinos come of the target at wider angles, while the higher momentum pions that contribute to the higher energy portion of the neutrino flux come at small angles ${ }^{1}$. A horn misalignment, which moves the horn neck toward the beam axis, has the effect of increasing the focusing for high energy pions.

The neck of the first NuMI horn has a radius $r=9 \mathrm{~mm}$ and is located approximately $60 \mathrm{~cm}$ downstream of the target in the LE010 configuration. This suggests that in this configuration first horn focuses particles down to $\tan \theta=0.9 \mathrm{~cm} / 60 \mathrm{~cm} \approx 0.02$ which corresponds to pion momentum of $p \approx$ $(250 \mathrm{MeV} / c) / 0.02=13 \mathrm{GeV} / c$. Such a pion gives rise to neutrino of energy $E_{\nu} \approx 5.5 \mathrm{GeV}$ (Equation 3.9). It is therefore, around that neutrino energy in LE010 beam that we expect a distortion could arise due to misalignment of

\footnotetext{
${ }^{1}$ As discussed in $\S 3.4$ there is an inversely proportional relation between the angle at which a pion leaves the target and its longitudinal momentum $p_{z} \approx\left\langle p_{T}\right\rangle / \theta$. The distribution of transverse momentum $\left(p_{T}\right)$ of pions peaks at $\sim 250 \mathrm{MeV} / c$ (see Figure 3.3).
} 
first horn.

Figure 5.13, like Figure 3.17 is again a breakdown of the ND neutrino spectrum into the 5 possible trajectories of pions through the focusing horns. In Figure 5.13, however, Horn 1 has been shifted transverse to the beam line by $4 \mathrm{~mm}$ (a somewhat exaggerated amount). Comparison of Figure 5.13 and Figure 3.17 reveals that the neutrino spectrum is indeed affected, and in fact the component most significantly modified are the pion trajectories focused entirely by Horn 1 .

We consider the following misalignments of beam-line elements and their effect on neutrino flux:

- Horn 1 and Horn 2 transverse misalignment

- Horn 1 and Horn 2 angular misalignment with respect to the beam axis

- Misalignment of the shielding blocks surrounding the Target and the Horns

- Longitudinal misalignment of the Target

Figures 5.14 and 5.15 show the effect of Horn 1 and Horn 2 transverse misalignment on the neutrino flux at the ND. We see that the effect of Horn 1 misalignment indeed appears to be the greatest in the expected $5 \mathrm{GeV}$ region for the LE010 beam. A similar set of plots corresponding to Horn 1 and Horn 2 angular misalignment are shown in Figures 5.16 and 5.17. The location of the horns and the angle of the horns with respect to the beam axis was determined [41] with uncertainty of $1 \mathrm{~mm}$ and $0.2 \mathrm{mrad}$ respectively.

The distance between the target and the focusing horns determines the energy of the neutrino beam (§2.1.2). Small shifts in target position along 


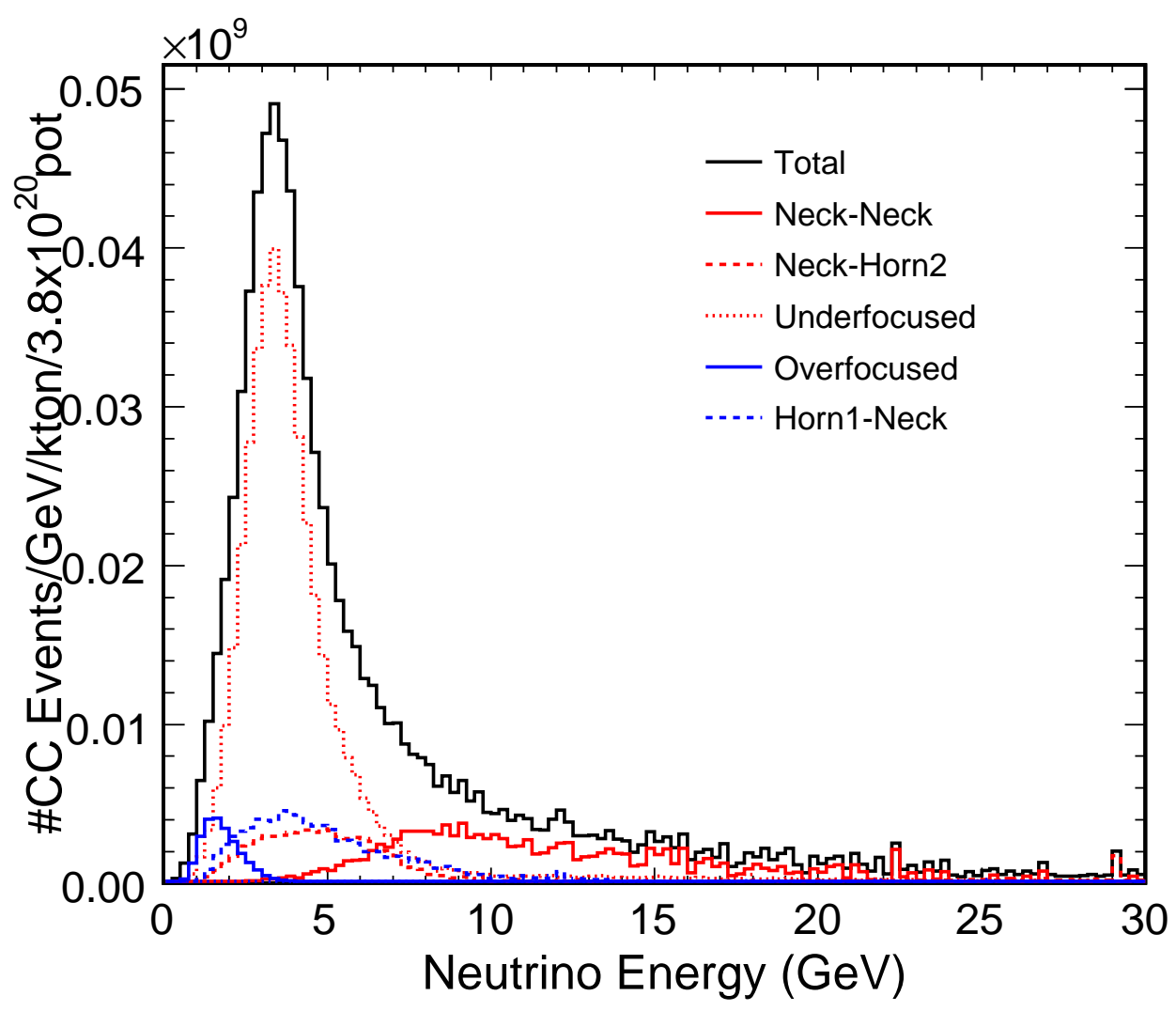

Figure 5.13: The neutrino flux at the ND calculated with a $4 \mathrm{~mm}$ transverse shift of first NuMI horn. The spectrum changed compared with that of Figure 3.17 mostly in the 5-6 GeV region. The component that was affected the most is the one focused entirely by first horn. 
the beam axis result in shifting of the neutrino peak. Figure 5.18 shows this effect of longitudinal misplacement of the target. The longitudinal position of the second NuMI target which was installed in September 2006 was measured using optical survey to within few millimeters. However, the position of the target used during the first run period is known less precisely. The impact of this on the neutrino flux calculation is further discussed in Chapter 7. 


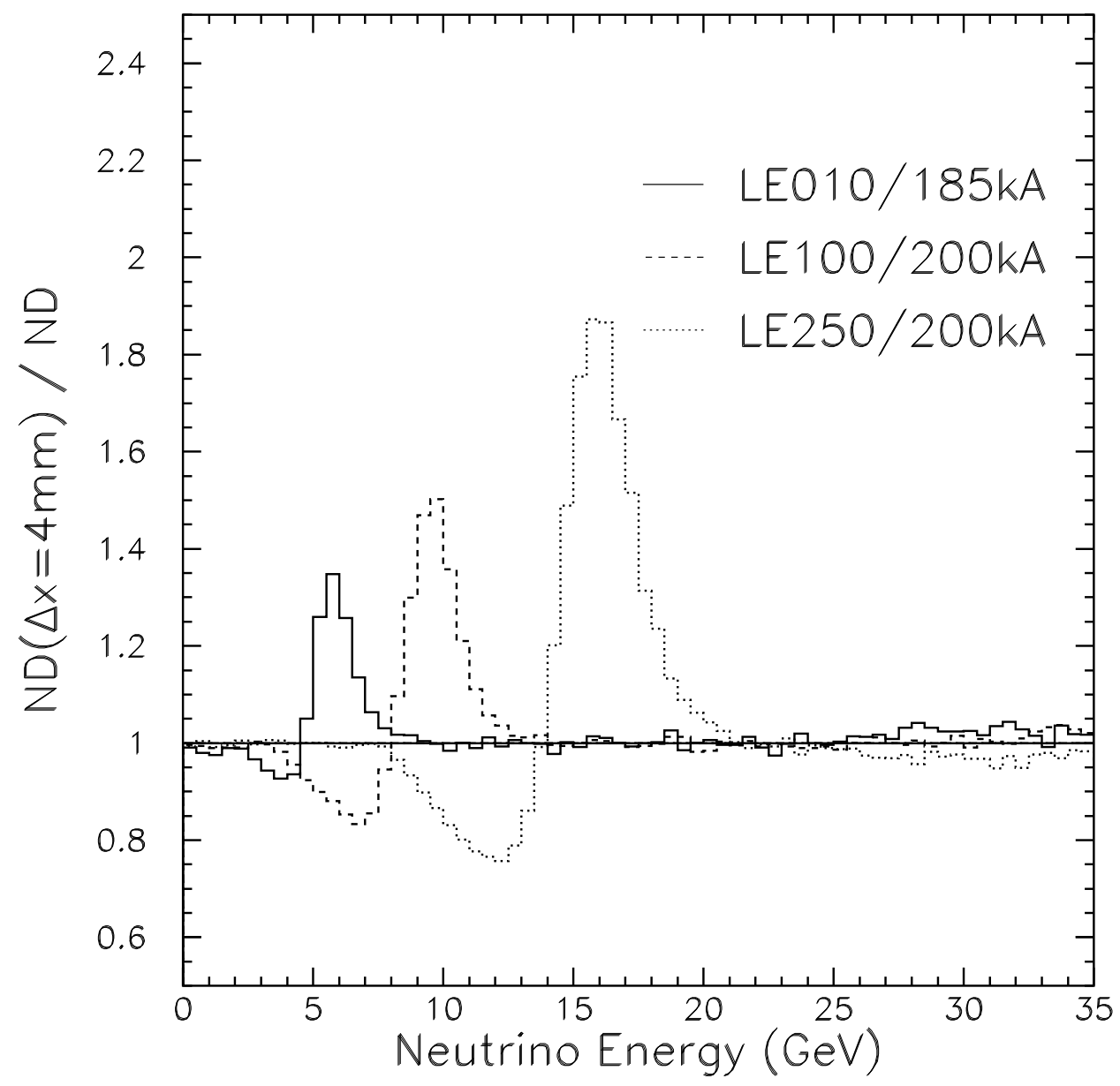

Figure 5.14: The fractional change of the neutrino energy spectrum at the Near Detector due to transverse misalignment of first NuMI horn. The curve corresponds to Horn 1 offset by $4 \mathrm{~mm}$ with respect to the beam axis. 


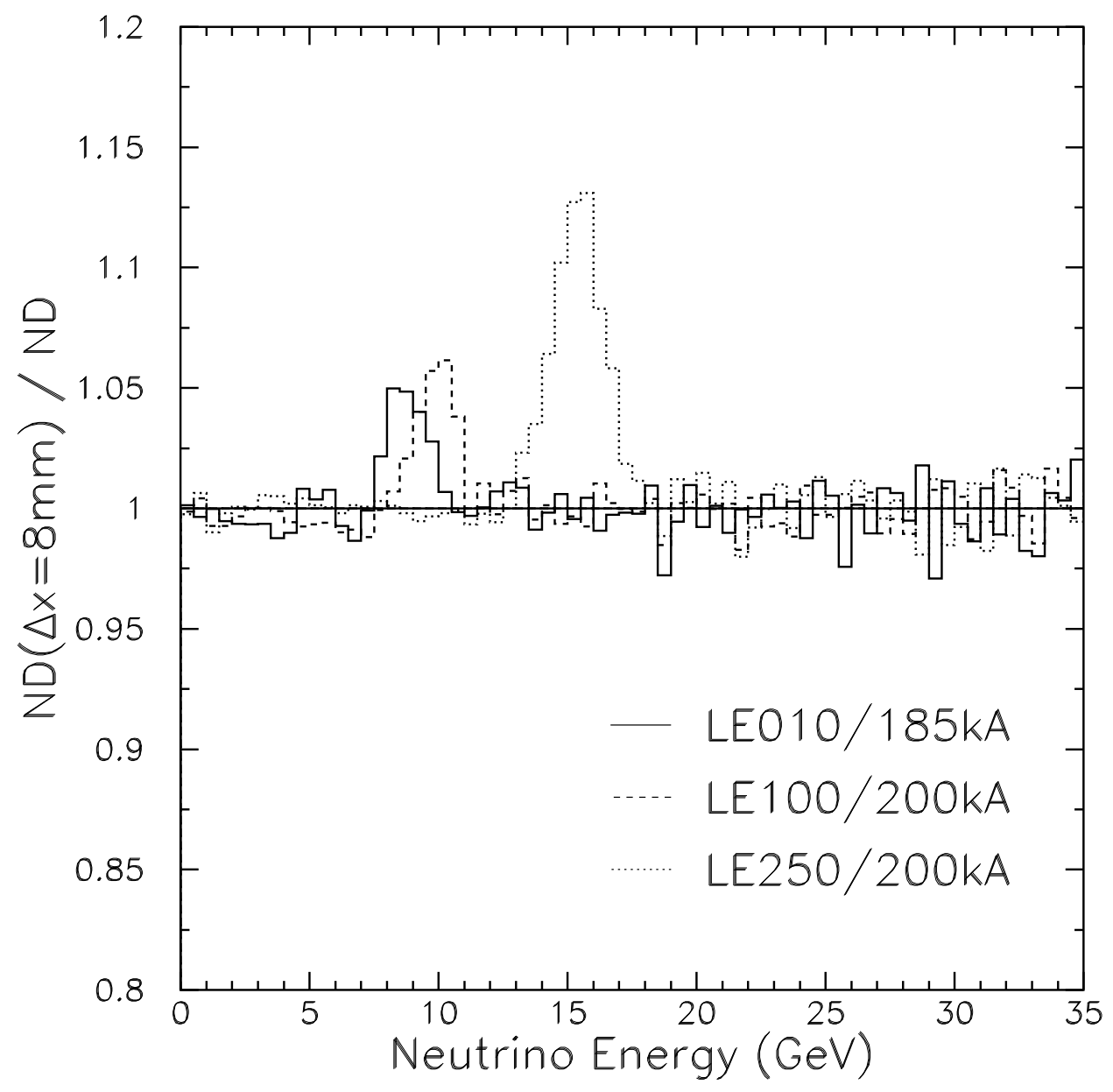

Figure 5.15: The fractional change of the neutrino energy spectrum at the Near Detector due to transverse misalignment of second NuMI horn. The curve corresponds to Horn 2 offset by $8 \mathrm{~mm}$ with respect to the beam axis. 


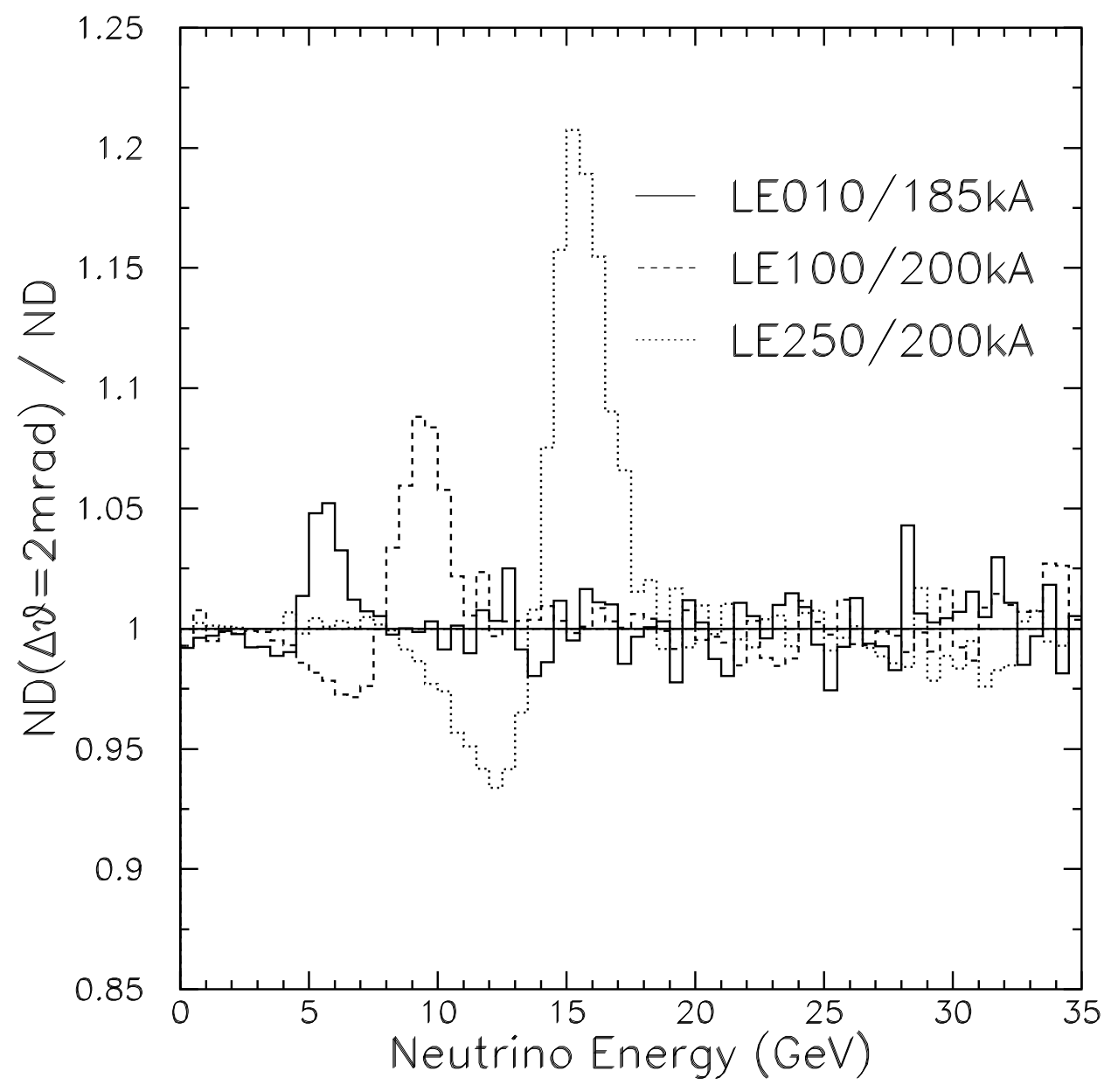

Figure 5.16: The fractional change of the neutrino energy spectrum at the Near Detector due to angular misalignment of first NuMI horn. The curve corresponds to Horn 1 tilted by 2 mrad with respect to the beam axis. 


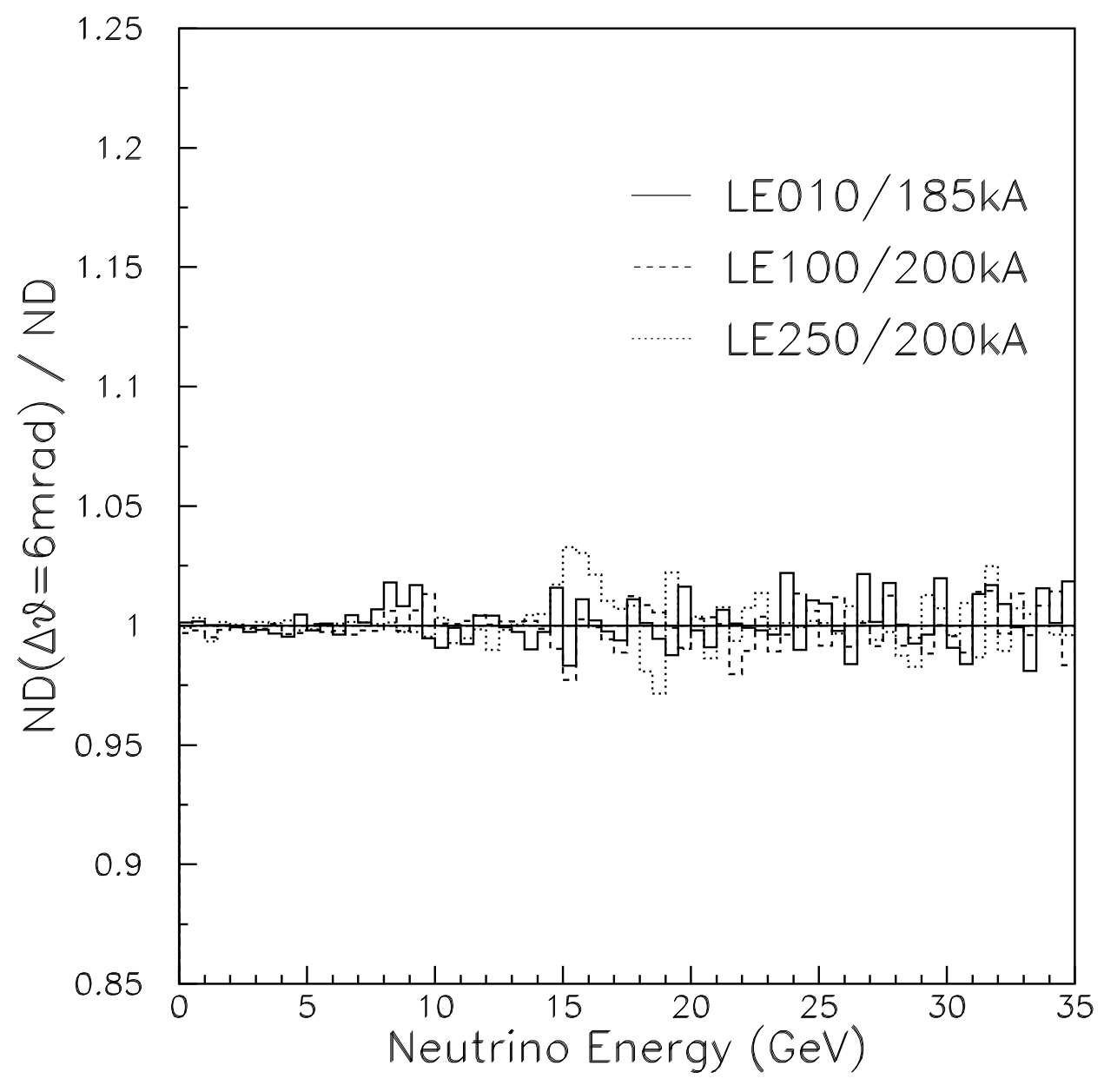

Figure 5.17: The fractional change of the neutrino energy spectrum at the Near Detector due to angular misalignment of second NuMI horn. The curve corresponds to Horn 2 tilted by 6 mrad with respect to the beam axis. 


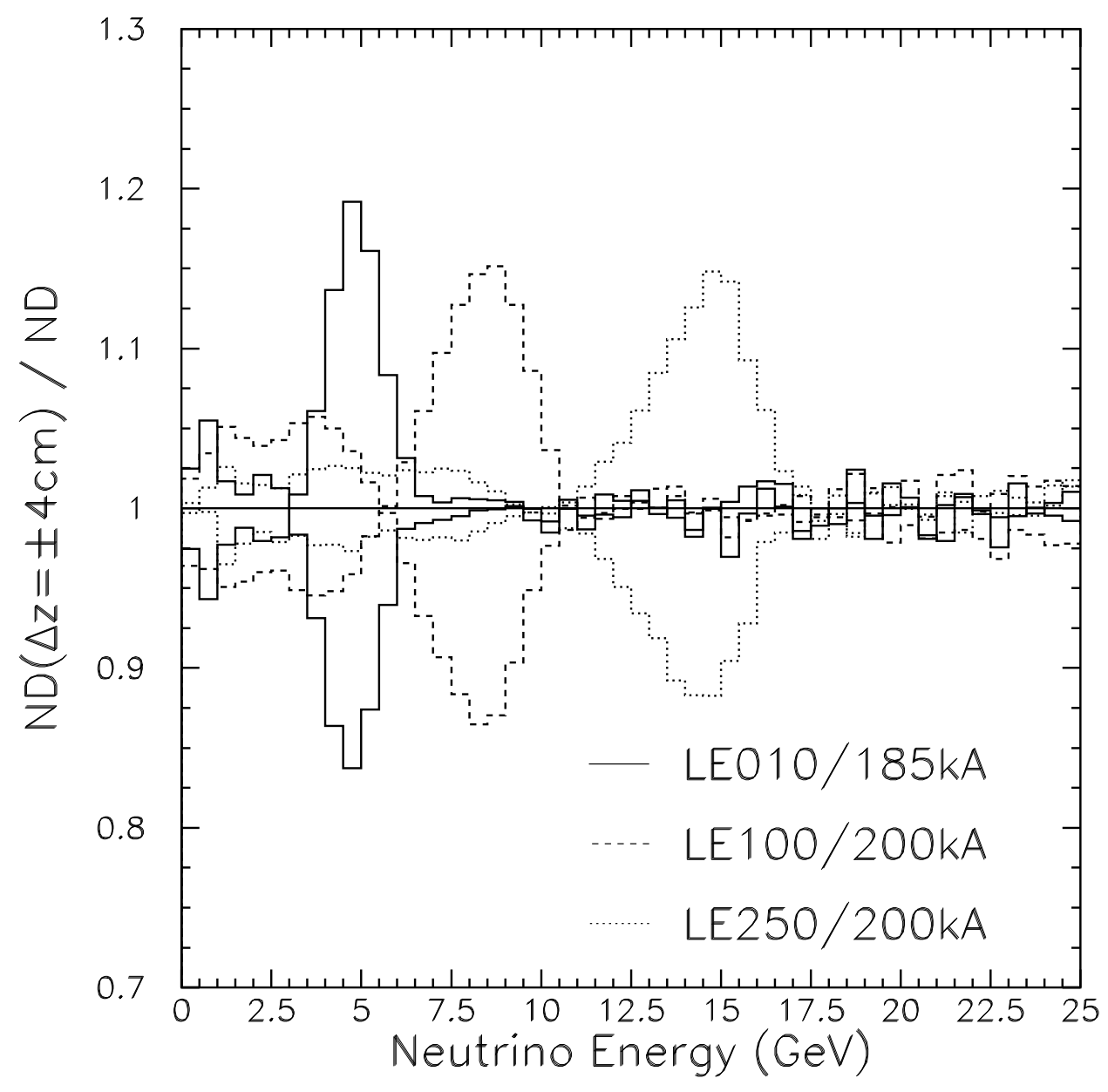

Figure 5.18: The fractional change of the neutrino energy spectrum at the Near Detector due to target displacement along the beam axis. The curves correspond to the $\pm 4 \mathrm{~cm}$ displacements of the target. Note the inflection points on the curves. The flux is increased on one side of inflection point and decreased on the other. For example a positive target displacement in LE250/200kA beam causes increase of the flux above $\sim 10 \mathrm{GeV}$ and decrease below.. The reverse is true for negative target displacement. 


\subsubsection{Horn Magnetic Field}

There are two uncertainties related to the current flowing through the focusing horns. The first is the absolute value of the horn current which affects the focusing of all particles. The second comes from the uncertainty in the skin depth, resulting in the uncertainty in the distribution of the current in the horn conductors. We discuss each in turn.

\section{Horn Current Scale}

The nominal horn current setting for each beam configuration is listed in Table 2.1. These currents are obtained in practice by discharging a set of capacitor banks into the horn circuit (which is largely an RL circuit). A set of calibration constants for the NuMI horn current readout was derived [78]. When a direct measurement of the current was performed, it was found that the actual horn current was off by a scale factor $(0.984 \pm 0.005)$. The beam $\mathrm{MC}$ used throughout this thesis was in fact generated with these corrected values. The remaining uncertainty in the horn current was estimated to be 1\%. Figure 5.19 shows the effect of horn current miscalibration on the neutrino spectrum at the ND.

\section{Skin Depth Effect}

The field inside the horn is given by $B=2 I / R c$, where $I$ is the current enclosed in the radius $R$. When a particle passes through the air volume between the inner and outer conductor of the horn, $I$ is clearly given by the nominally $185 \mathrm{kA}$ for the LE10 beam ${ }^{2}$. For a particle traversing the inner conductor of

\footnotetext{
${ }^{2}$ Actual value is $182.1 \mathrm{kA}$ as discussed above
} 


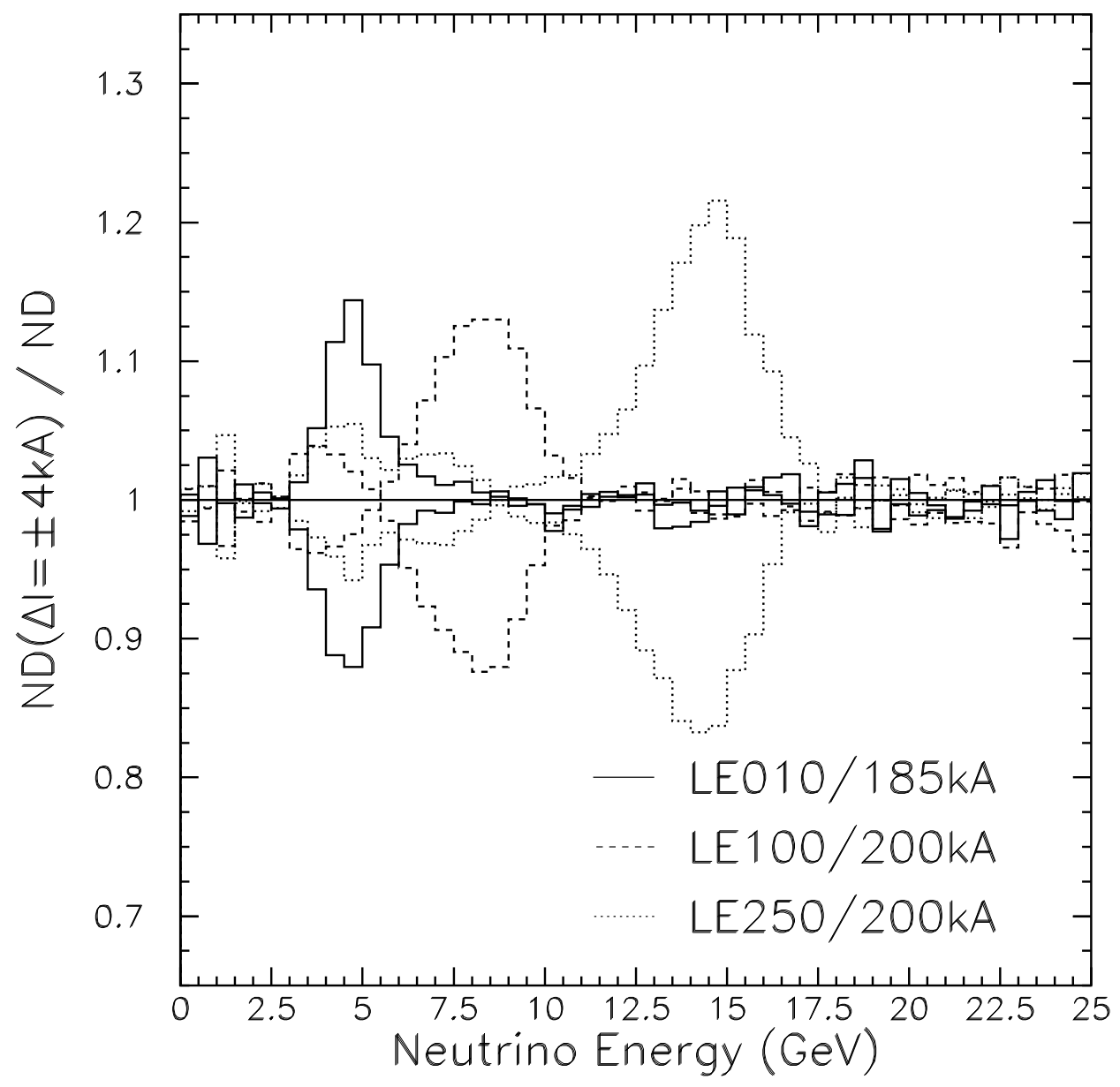

Figure 5.19: The ratio of neutrino flux at the Near Detector with modified horn current to the flux with nominal horn current for a given beam configuration (Table 2.1). The curves correspond to $\pm 4 \mathrm{kA}$ variation of horn current. Note the inflection points on the curves. The flux is increased on one side of inflection point and decreased on the other. For example a positive change in horn current in LE250/200kA beam causes increase of the flux above $8 \mathrm{GeV}$ and decrease below $\sim 8 \mathrm{GeV}$. The reverse is true for negative change in horn current. 
the horn, however the correct value of current to be used in modelling the horn field is a non-trivial matter. For an ideal conductor, all current would flow on the outer surface of the horn, by Gauss' Law. For a real conductor with finite conductivity, this current penetrates into the bulk of the horn conductors over a distance scale given by the skin depth $\delta$. The uncertainty in the current distribution within the horn conductor results in uncertainty of the magnetic field distribution in the conductor.

The proper modelling of this region of the magnetic field would be expected to affect a very specific portion of the neutrino spectrum, namely that portion coming from pions which spend a great deal of time traversing horn's inner conductor. As was noted earlier, those are the pions at the smallest possible angles that receive horn focusing and are at the upper momentum limit of the horn focusing. It is thus expected that the horn current modelling affects the falling edge of the neutrino peak for each beam configuration.

The skin depth for a conductor of semi-infinite extent is given by [79]:

$$
\delta=\frac{c}{\sqrt{2 \pi \sigma \mu \omega}}
$$

where $\sigma$ is the conductivity, $\mu$ is the permeability and $\omega$ is the frequency of the current. The frequency of relevance for the NuMI horns comes from the fact that the horn current comes in a pulse which is roughly a half sine wave with $1.0 \mathrm{~ms}$ rise time and $4.6 \mathrm{~ms}$ period. For these parameters, the skin depth is approximately $7.7 \mathrm{~mm}$.

The well-known exponential description of the current density penetrating a conductor's thickness is only an idealization for a conductor of semiinfinite extent. For the NuMI horns, the conductor thickness is of order $3 \mathrm{~mm}$, 

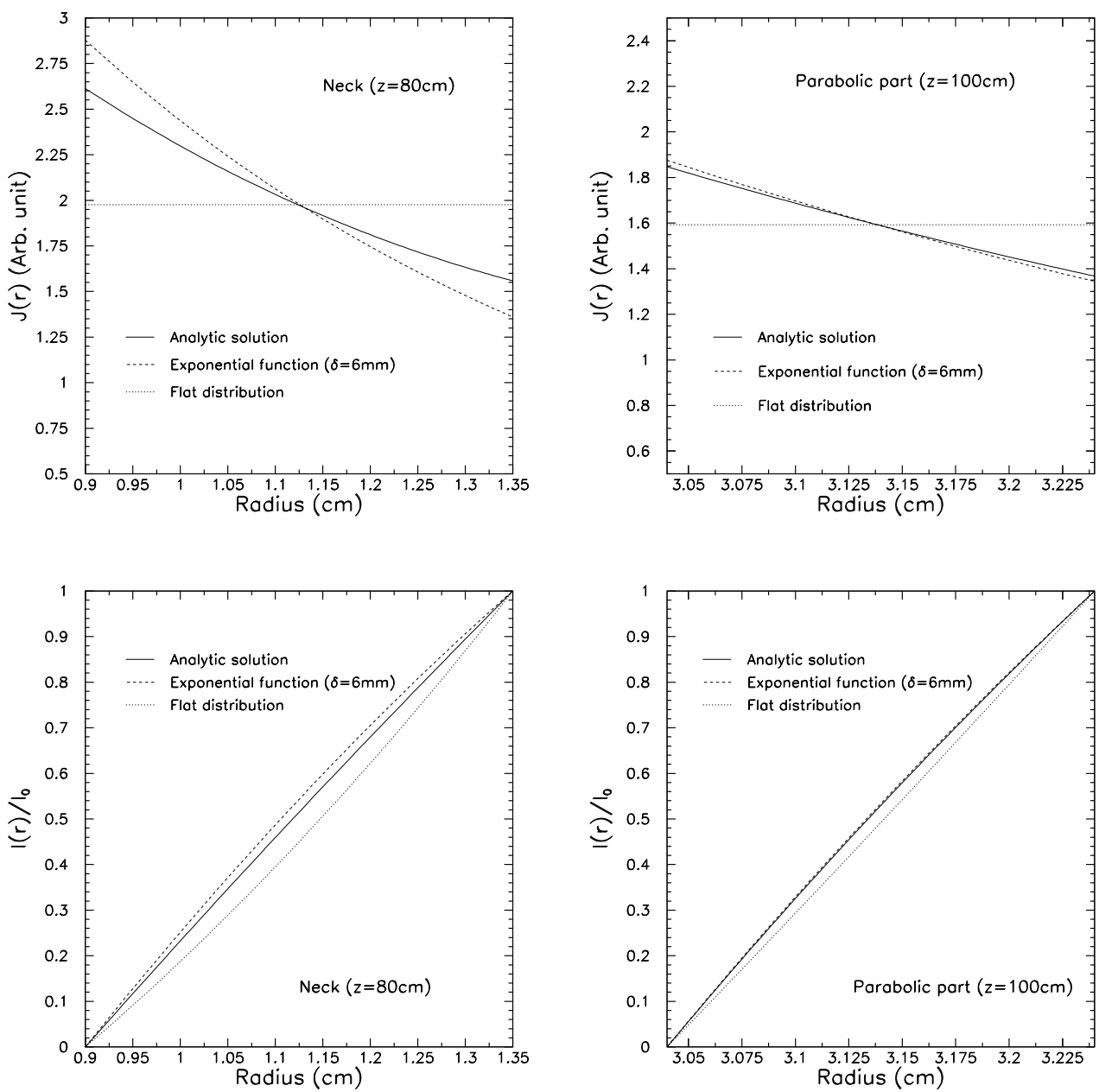

Figure 5.20: Comparison of three possible distributions of the horn current in the inner conductor: uniform current density (corresponding to an infinite skin depth $\delta=\infty$, an exponentially falling current distribution with $\delta=6 \mathrm{~mm}$, and the analytical expression for a round conductor from [80]. Top plots show the current $I$ as a function of radius $r$ from the beam line axis. Bottom plots show the enclosed current $I_{\text {enc }}$ inside a radius $r$ (which is proportional to the magnetic field $B(r)$ ). Left plots are for the horn neck, right plots show a location along the parabolic conductor at $z=100 \mathrm{~cm}$. 


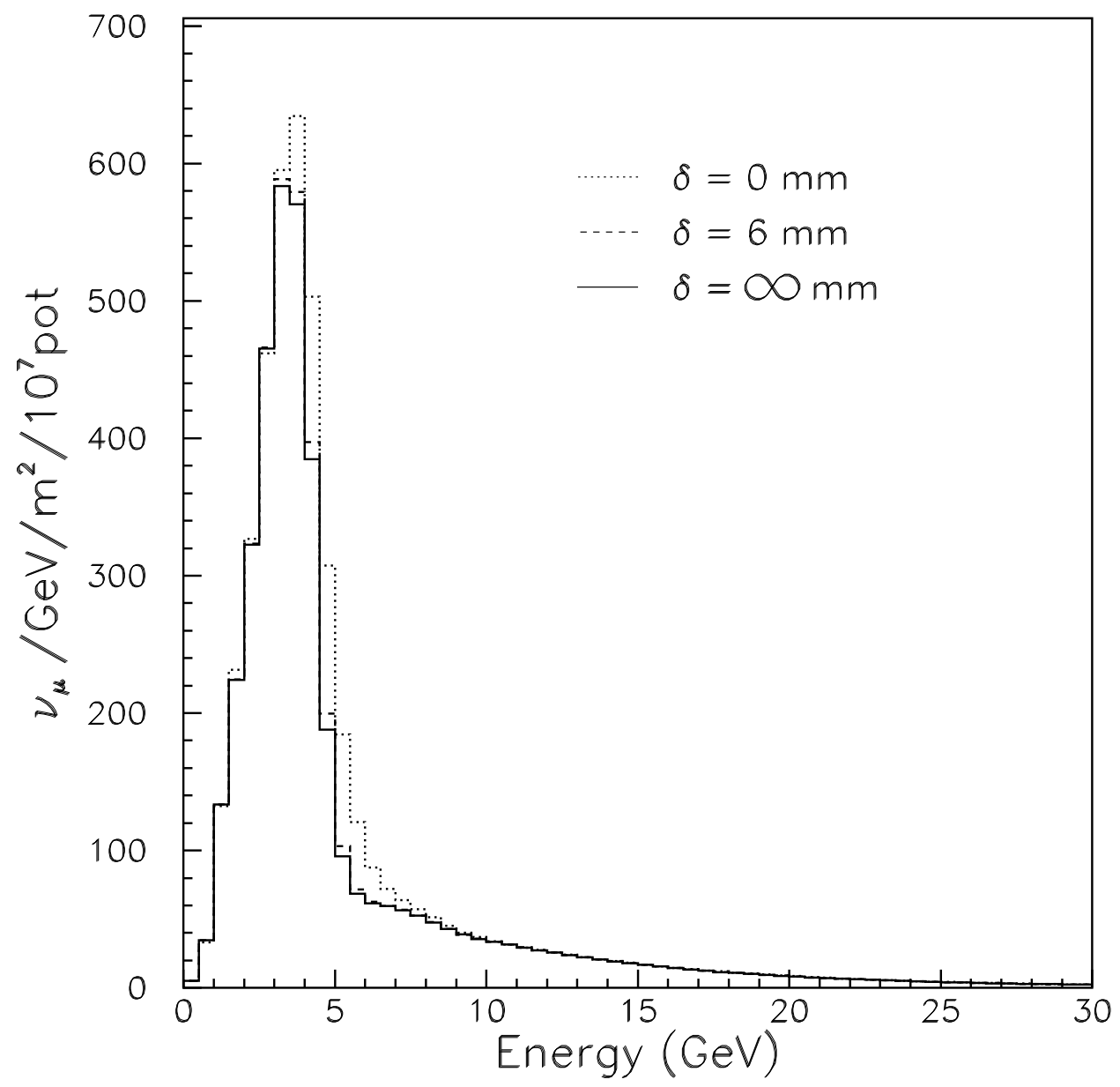

Figure 5.21: The neutrino flux at Near Detector for two extremal distributions of current in horn inner conductor. Three current distributions correspond to different skin depths $\delta=0,6 \mathrm{~mm}$, and $\delta=\infty$. 
comparable to the skin depth estimated above. Therefore the exponential is only an approximation to the real distribution. To find the current distribution we can look at the analytical solution for a current distribution in a cylindrical conductor. This solution has the following form ([80]):

$$
J_{z}(r, t)=A\left[\operatorname{ber}\left(\frac{\sqrt{2}}{\delta} r\right)-i \operatorname{bei}\left(\frac{\sqrt{2}}{\delta} r\right)\right] e^{-i \omega t}
$$

where ber and bei are "Bessel real" and "Bessel imaginary" functions, or "Kelvin" functions. The proton beam comes when the horn current is at the peak, so using the solution for the distribution we can find current density relative to surface current density at that moment:

$$
\left|\frac{J_{z}(r)}{J_{z}\left(R_{\text {out }}\right)}\right|=\left[\frac{\operatorname{ber}^{2}\left(\frac{\sqrt{2} r}{\delta}\right)+b e i^{2}\left(\frac{\sqrt{2} r}{\delta}\right)}{b e r^{2}\left(\frac{\sqrt{2} R_{\text {out }}}{\delta}\right)+b e i^{2}\left(\frac{\sqrt{2} R_{\text {out }}}{\delta}\right)}\right]^{1 / 2}
$$

Using this analytical solution we find that the distribution varies along the horn and its different at its neck then it is at its ends. Interestingly, this functional form deviates somewhat from a simple exponential of $\delta=7.7 \mathrm{~mm}$. Figure 5.20 shows the enclosed current $I(r)$ within a radius $r$ for each of these estimations of the horn current - the enclosed current being of course proportional to the magnetic field strength in the inner conductor.

In the beam MC simulation the horn current is uniformly distributed throughout the conductor and is the same along the horn. This corresponds to the case of infinite skin depth $\delta=\infty$, which is roughly true since inner horn conductor is $3 \mathrm{~mm}$ thick and skin depth is $7.7 \mathrm{~mm}$ as was previously noted. To estimate the error coming from this choice of modelling we ran a beam simulation with different values of skin depth $\delta$, shown for the LE10 beam 
in Figure 5.21. As might be expected, the different descriptions of the horn current mostly affect the upper edge of the focusing peak. We used the difference between $\delta=6 \mathrm{~mm}$, and $\delta=\infty$ to find the error bar. In the figure, the idealization of an ideal conductor $(\delta=0 \mathrm{~mm})$ is also shown for reference, but is not expected to be a realistic model of our horns' current distribution. 


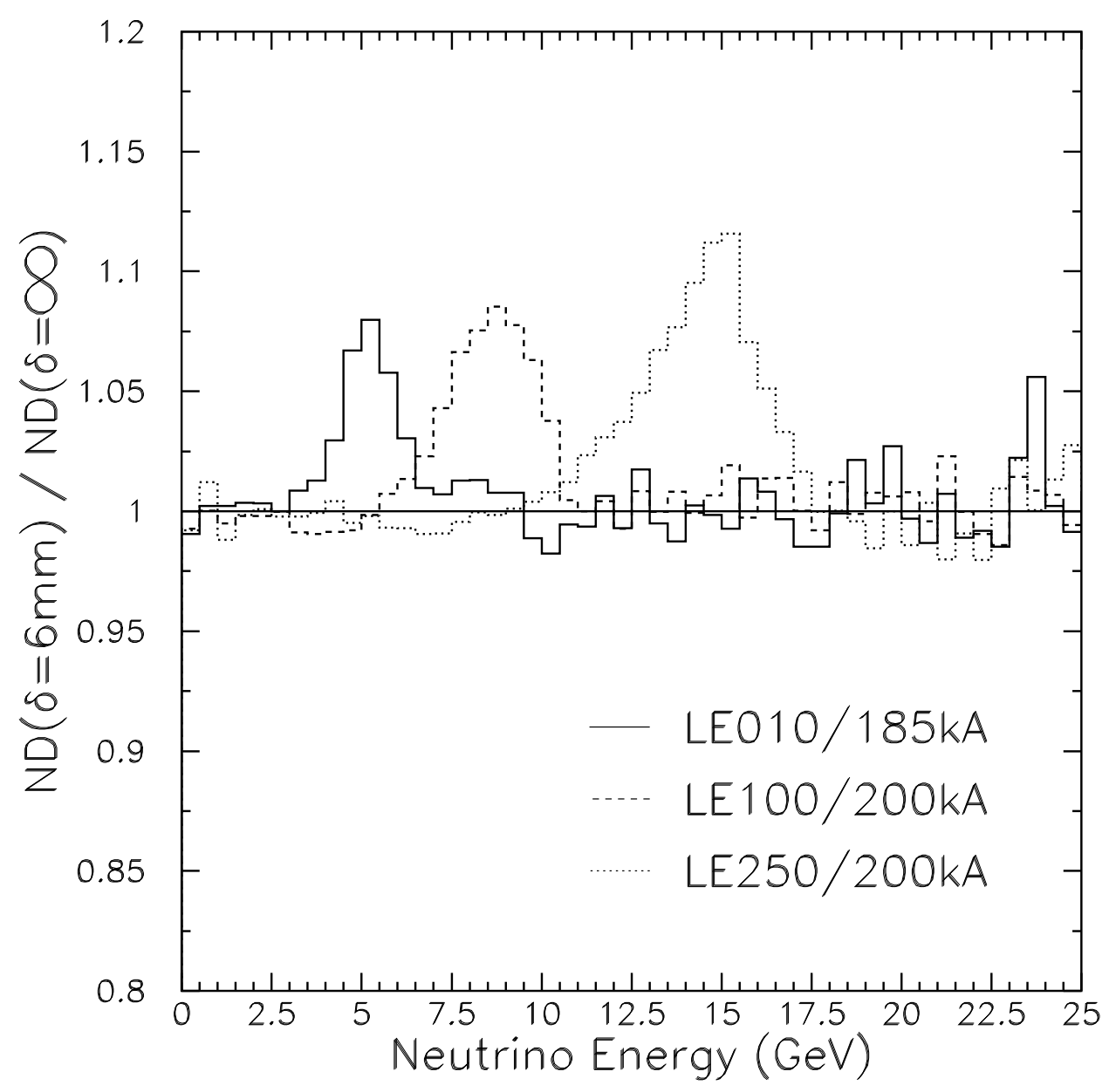

Figure 5.22: The ratio of neutrino fluxes at the Near Detector where different distributions of horn current inside the inner conductor were used to calculate the fluxes. The current distribution affects the distribution of the magnetic field and consequently what is the highest momentum of the particles being captured by a horn. Shown is the difference between the skin depth $\delta=6 \mathrm{~mm}$ and $\delta=\infty$. 


\subsubsection{Summary}

Figures 5.23-5.25 and 5.26-5.28 show the calculated uncertainties in the neutrino flux at the ND and in the far over near ratio respectively. These uncertainty bands were calculated using the derivatives found in Section 5.2 and the uncertainties in Table 5.1. Some of the beam systematics whose effect on the flux was found to be negligible are not shown.

\begin{tabular}{lc} 
Source & Uncertainty \\
\hline Number of protons on target & $2.0 \%$ \\
Horn transverse misalignment & $1.0 \mathrm{~mm}$ \\
Horn tilt & $0.2 \mathrm{mrad}$ \\
Horn current miscalibration & $1.0 \%$ \\
Horn current distribution & $\delta=6 \mathrm{~mm} / \delta=\infty$ \\
Baffle scraping & $0.25 \%$ \\
Misalignment of shielding blocks & $1.0 \mathrm{~cm}$ \\
Target density & $2 \%$
\end{tabular}

Table 5.1: Summary of uncertainties used to find the error bars in Figures 5.235.28 (Systematics whose effect on the flux was found to be negligible are not listed).

\subsection{Hadron Production Uncertainty}

The biggest uncertainty in the calculation of the ratio of near and far neutrino flux comes from an imperfect knowledge of particle yields off the target. As was discussed in Chapter 3 various models and parameterisations have been developed $[60,61,59,66,67]$ that fit the measurements of these yields $[54,55,56,57]$. The uncertainty in the neutrino flux arises not only due to uncertainties in measured yields of secondaries by various hadron pro- 


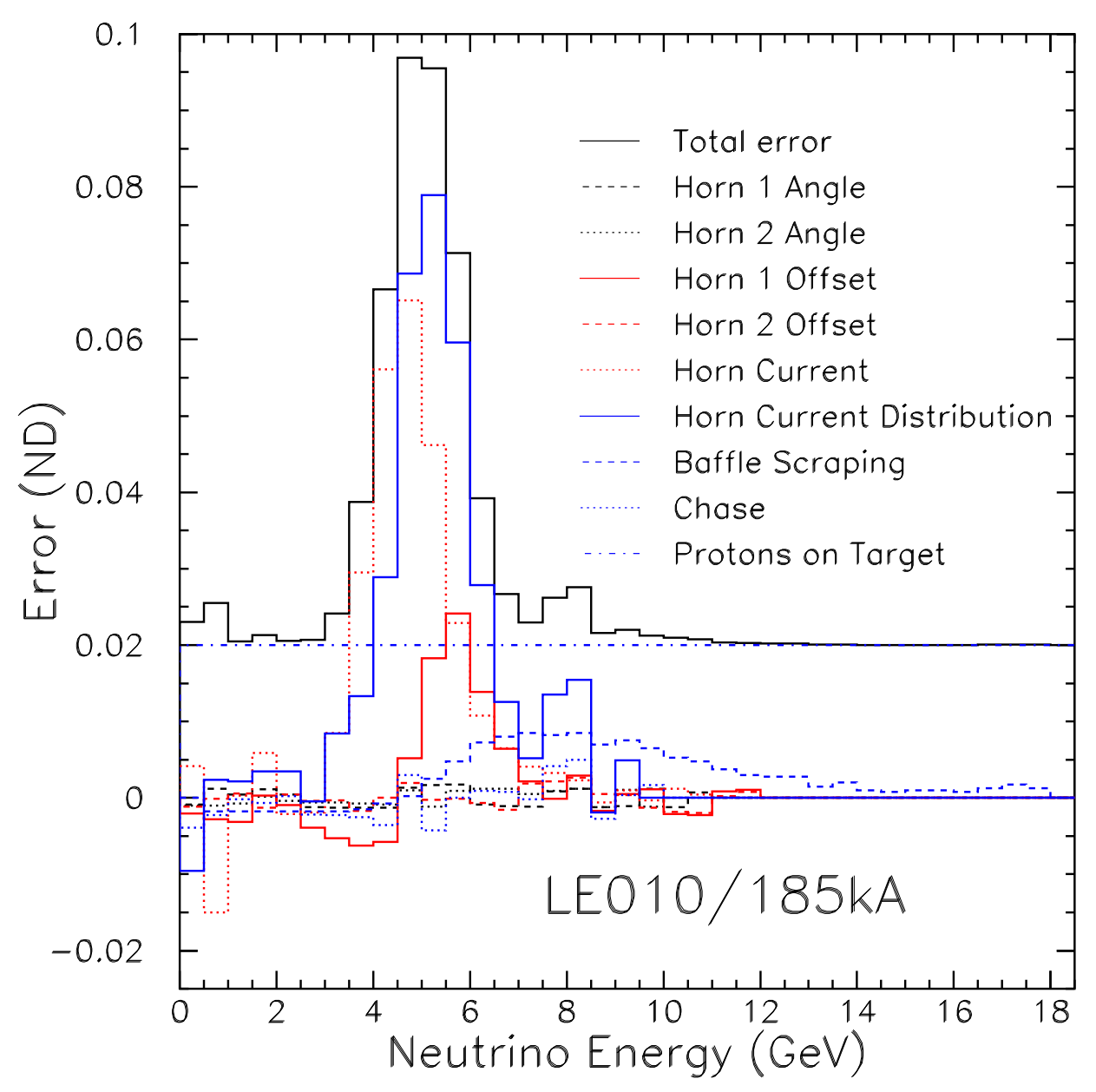

Figure 5.23: The summary of neutrino energy spectrum uncertainties at the ND due to proton beam and focusing uncertainties for the LE010/185kA beam. The uncertainties were calculated using distortion functions that were found as described in Sections 5.2.1-5.2.4 and an estimate of the size of each uncertainty based upon NuMI beam-line instrumentation data (Table 5.1). The total uncertainty is given as a sum of all uncertainties in quadrature. 


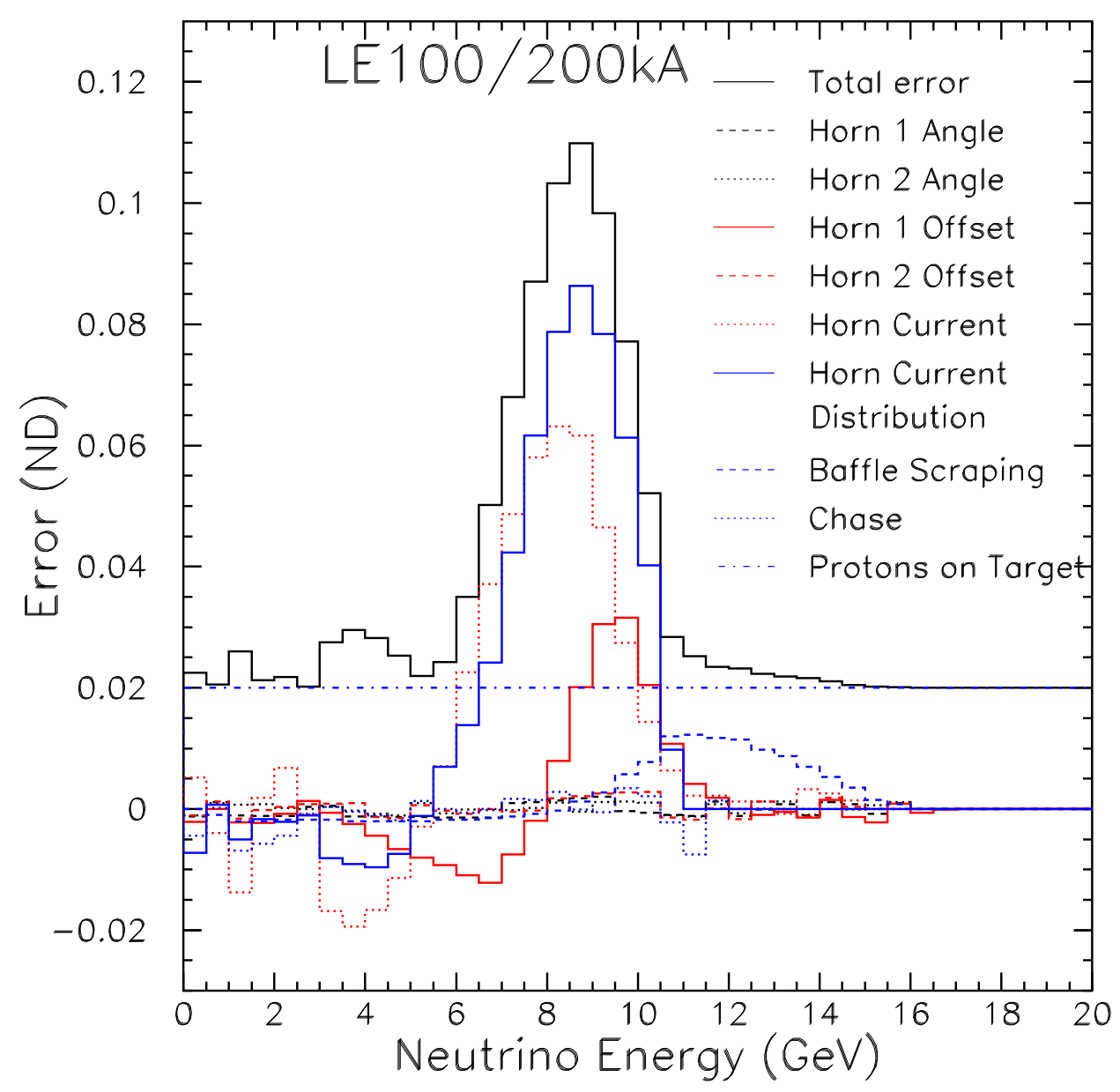

Figure 5.24: The summary of neutrino energy spectrum uncertainties at the ND due to proton beam and focusing uncertainties for the LE100/200kA beam. The uncertainties were calculated using distortion functions that were found as described in Sections 5.2.1-5.2.4 and an estimate of the size of each uncertainty based upon NuMI beam-line instrumentation data (Table 5.1). The total uncertainty is given as a sum of all uncertainties in quadrature. 


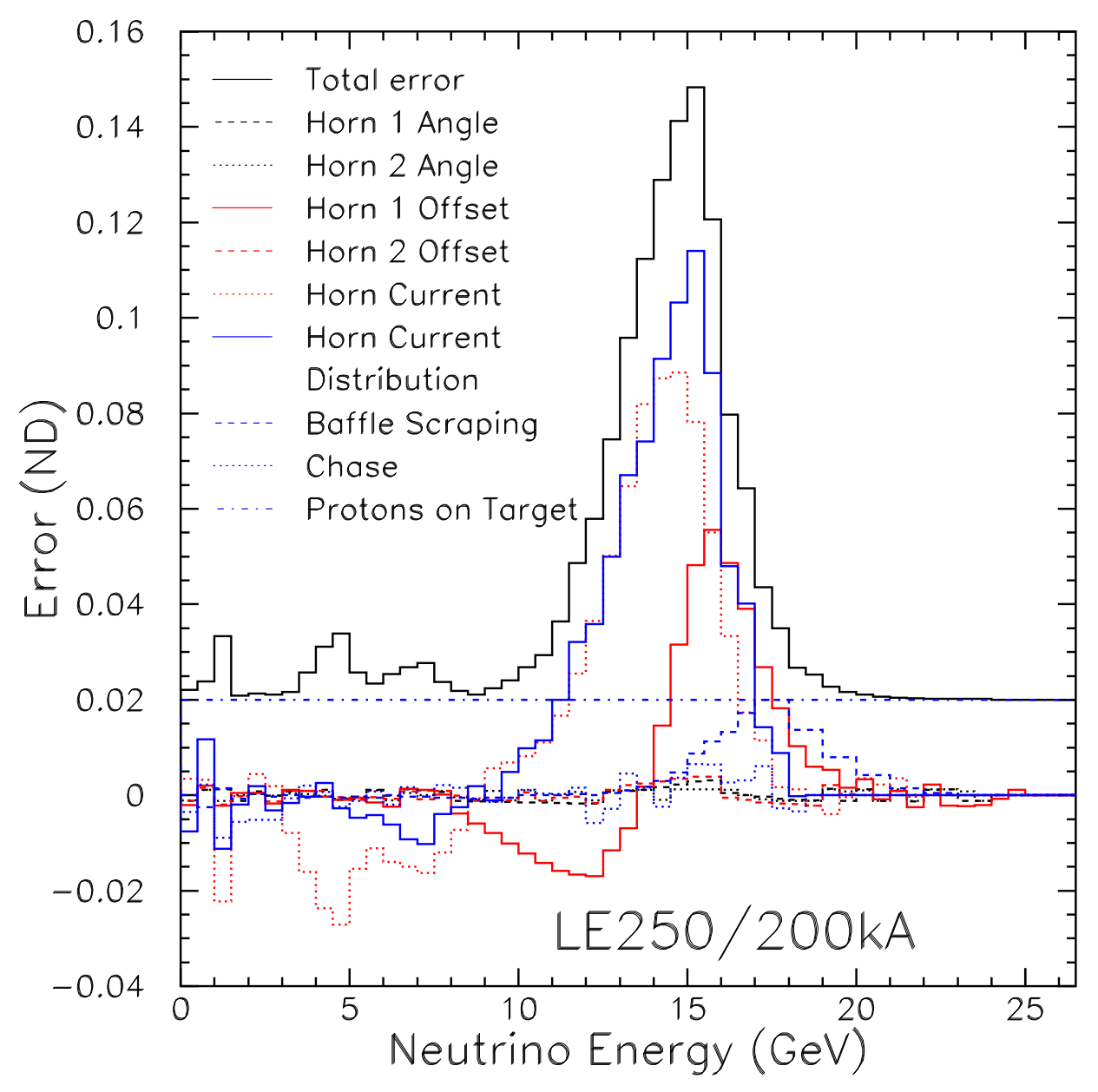

Figure 5.25: The summary of neutrino energy spectrum uncertainties at the ND due to proton beam and focusing uncertainties for the LE250/200kA beam. The uncertainties were calculated using distortion functions that were found as described in Sections 5.2.1-5.2.4 and an estimate of the size of each uncertainty based upon NuMI beam-line instrumentation data (Table 5.1). The total uncertainty is given as a sum of all uncertainties in quadrature. 


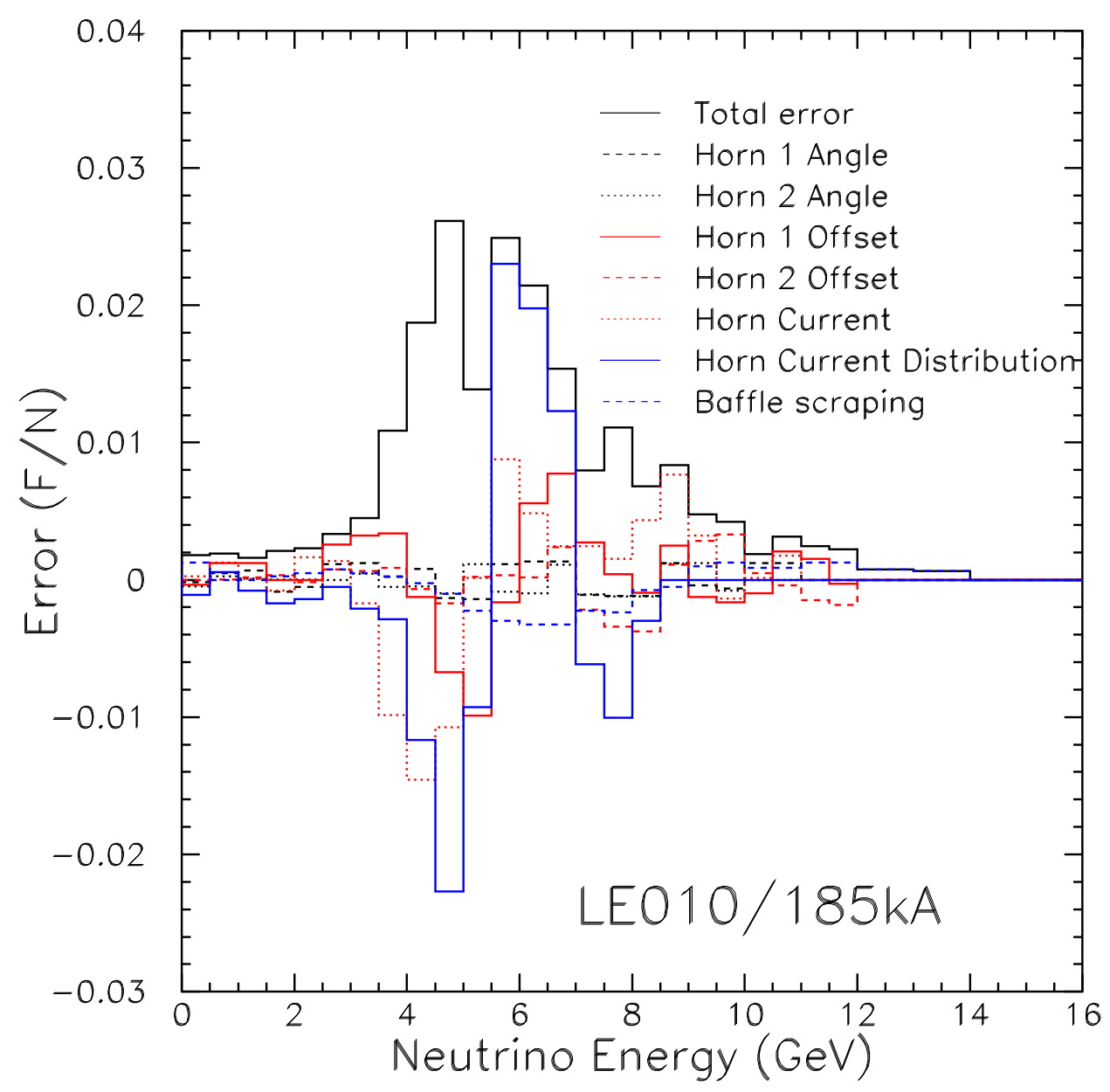

Figure 5.26: The summary of far over near ratio uncertainties due to proton beam and focusing uncertainties for the LE010/185kA beam. The uncertainties were calculated using distortion functions that were found as described in Sections 5.2.1-5.2.4 and an estimate of the size of each uncertainty based upon NuMI beam-line instrumentation data (Table 5.1). The total uncertainty is given as a sum of all uncertainties in quadrature. 


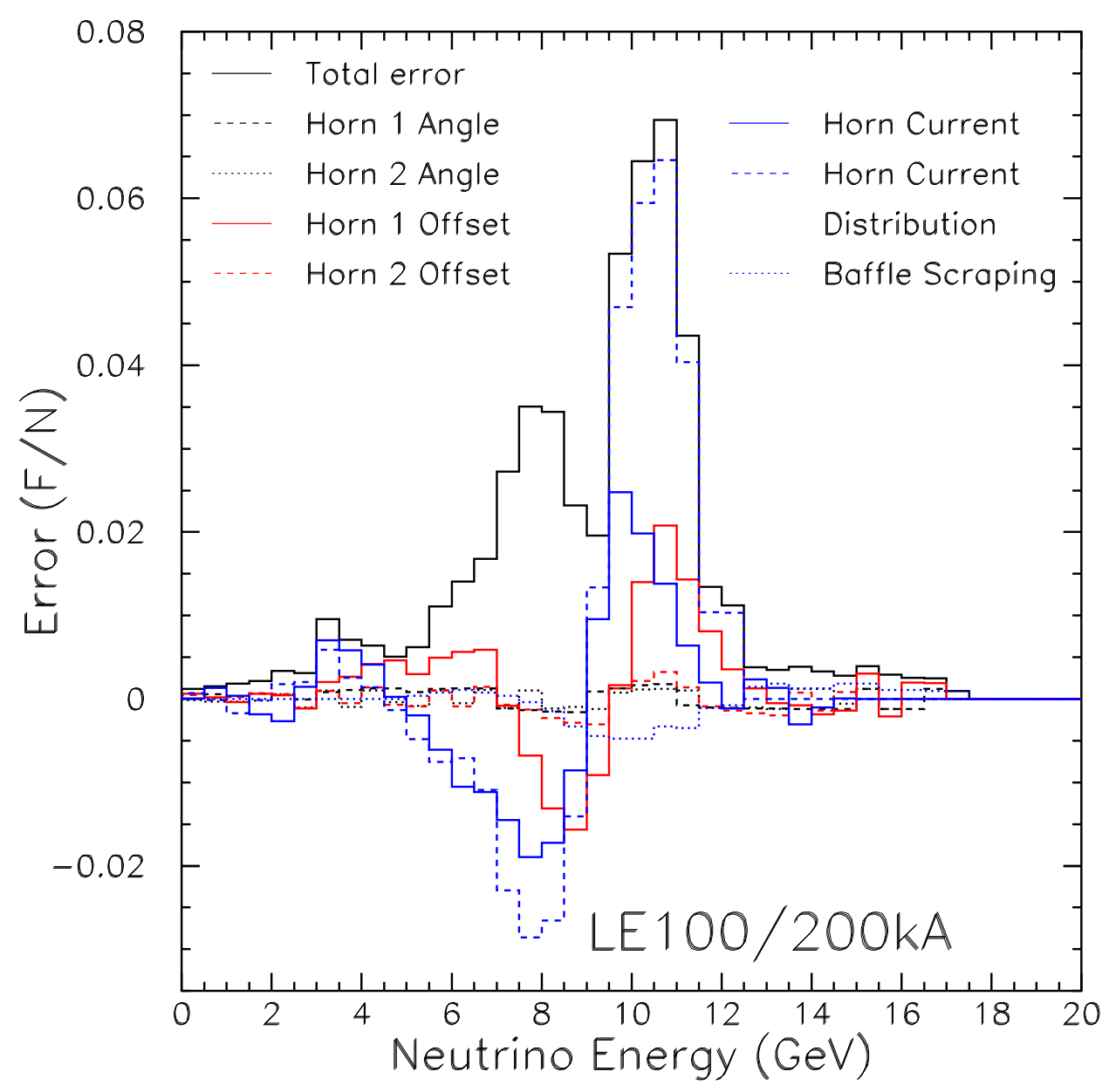

Figure 5.27: The summary of far over near ratio uncertainties due to proton beam and focusing uncertainties for the LE100/200kA beam. The uncertainties were calculated using distortion functions that were found as described in Sections 5.2.1-5.2.4 and an estimate of the size of each uncertainty based upon NuMI beam-line instrumentation data (Table 5.1). The total uncertainty is given as a sum of all uncertainties in quadrature. 


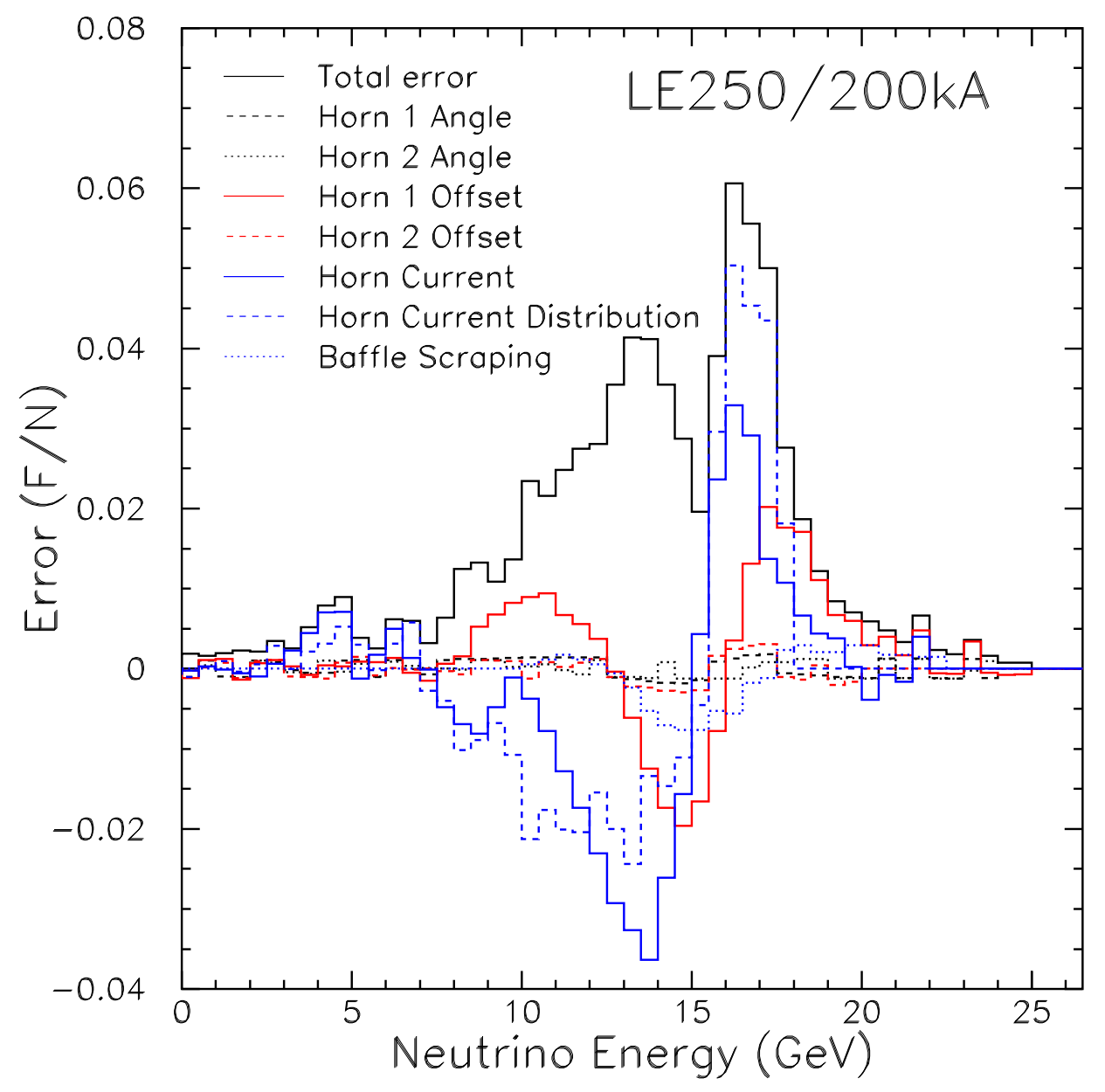

Figure 5.28: The summary of far over near ratio uncertainties due to proton beam and focusing uncertainties for the LE250/200kA beam. The uncertainties were calculated using distortion functions that were found as described in Sections 5.2.1-5.2.4 and an estimate of the size of each uncertainty based upon NuMI beam-line instrumentation data (Table 5.1). The total uncertainty is given as a sum of all uncertainties in quadrature. 
duction experiments, but also because the existing hadron production data in many cases was acquired under conditions that don't exactly match the NuMI beam conditions.

We have developed two techniques to estimate a neutrino flux uncertainty due to particle production uncertainties. These techniques are described in the next 2 sections. The two techniques are a crude estimate of the uncertainty, but both indicate that hadron production is the dominant uncertainty in the predicted neutrino flux. In Chapter 6 we describe a method which is used to reduce and better estimate these uncertainties using the measured neutrino energy spectrum in the MINOS Near Detector.

\subsubsection{Choosing Different Cascade Model}

Variations in the neutrino flux prediction using different cascade models suggest what is the effect of uncertainty in yield of secondaries off the NuMI target on the neutrino flux. However, some of the models are correlated, for example two of the models that we considered, FLUKA and MARS, appear to have very similar description of kaon production. Using the differences between these models would under-estimate the uncertainty. On the other hand some of the models have known flaws in certain kinematic regions [81]. These models, if included into consideration, over-estimate the uncertainty. Figure 3.10 shows the neutrino flux in LE010/185kA beam as predicted by three different

hadron production models. Similar plots for other beam configurations can be found in Appendix E.

With these caveats, the uncertainty in neutrino flux due to model uncertainty appears to be $\sim 8 \%$ in the focusing peak and $\sim 15 \%$ in the high 
energy part of the neutrino spectrum. As expected, the uncertainty in far over near ratio is much smaller (Figure 5.29).

\subsubsection{Skewing Pion $p_{T}$ Distribution}

Various cascade models predict different distributions of transverse momenta $p_{T}$ of secondary particles created in the target (see §3.2). Because the NuMI horns focus particles most efficiently in a window centered at $p_{T} \sim 250 \mathrm{MeV} / c$, different shapes of $p_{T}$ distributions result in different flux predictions. We applied different skewing functions to obtain $p_{T}$ distributions whose mean $\left\langle p_{T}\right\rangle$ values varied as shown in Figure 5.30. The limits chosen for the variation in $\left\langle p_{T}\right\rangle$ were determined using the different cascade models (see Figure 3.9). The method we used was somewhat crude because the skewing function didn't depend on Feynman $x_{F}$. We skewed only the pion distributions, so the resulting uncertainty applies only in the parts of the spectra where neutrinos are mostly coming from pions, ie for neutrinos with energy of up to $20-25 \mathrm{GeV}$. Figures 5.31 and 5.32 show the effect on the neutrino flux at the ND and on the far over near ratio. The uncertainty is similar as the uncertainty derived from looking at different cascade models.

\subsection{Summary}

In this chapter we looked at the various sources of uncertainties in the calculation of neutrino flux. These uncertainties can be divided into two categories, the focusing uncertainties and those arising from the uncertainty in the hadron production in the target. Here we have quantified the focusing uncertainties 


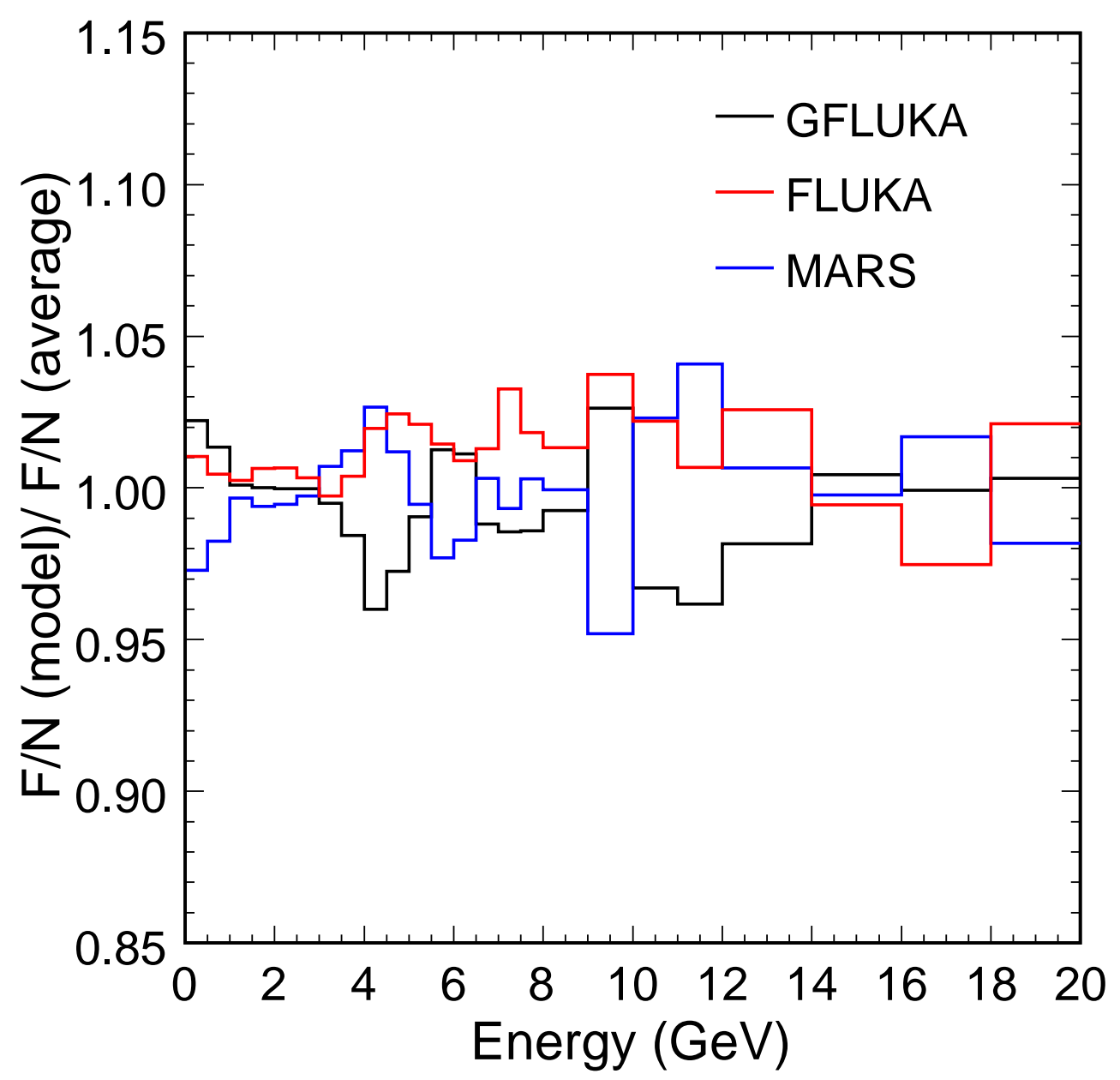

Figure 5.29: Different hadron production models exist that fit the existing experimental data (§3.2). Shown are the far over near ratios as predicted by different hadron production models divided by the average far over near ratio. Excursions reflect the uncertainties in the far over near ratio due to hadron production uncertainty. 


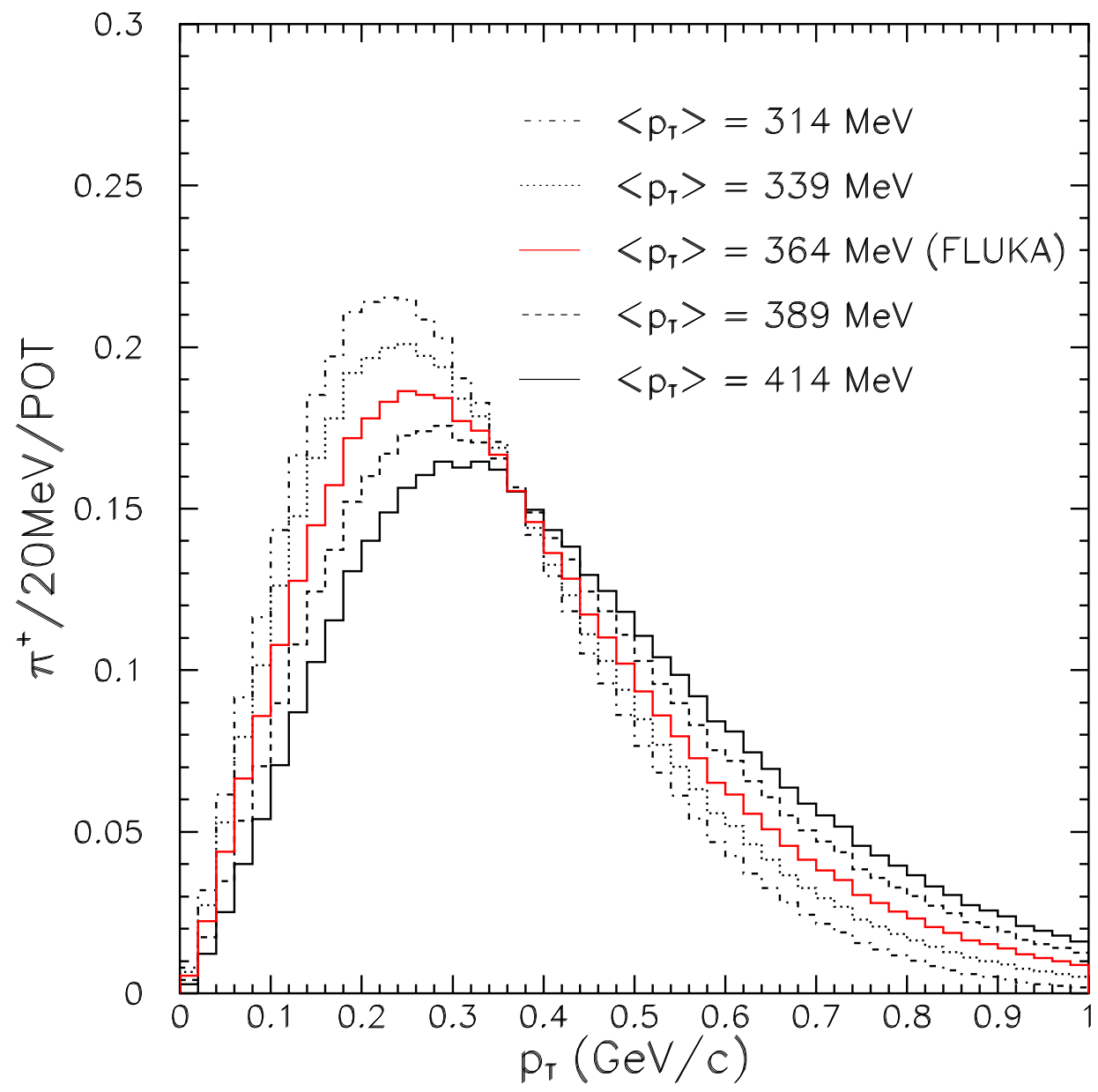

Figure 5.30: Transverse momentum of pions created in a graphite target by a proton beam of $120 \mathrm{GeV}$, as predicted by FLUKA hadron production model (red). Also shown are several skewed distributions with shifted $\left\langle p_{T}\right\rangle$. Various hadron production models predict different $p_{T}$ distributions with $\left\langle p_{T}\right\rangle$ value varying by $\pm 50 \mathrm{MeV} / c$ between models. We skewed the FLUKA prediction to simulate this model variation seen in Figure 3.9. 


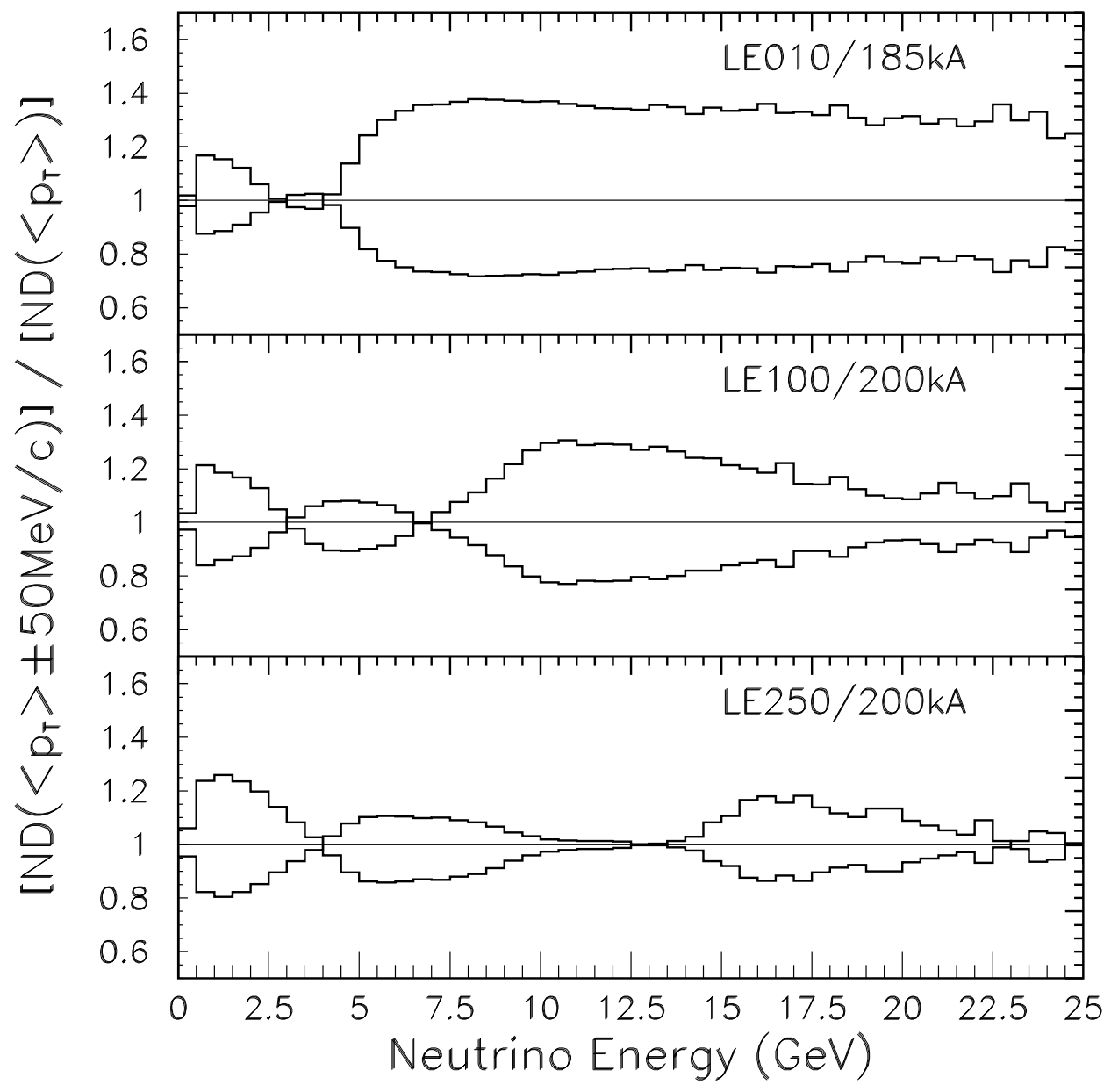

Figure 5.31: Ratio of neutrino spectra in the ND as predicted from the skewed $p_{T}$ spectra to the one predicted using FLUKA hadron production model (Figure 5.30). The curves correspond to the pion $p_{T}$ distributions shifted by $\pm 50 \mathrm{MeV} / c$. 


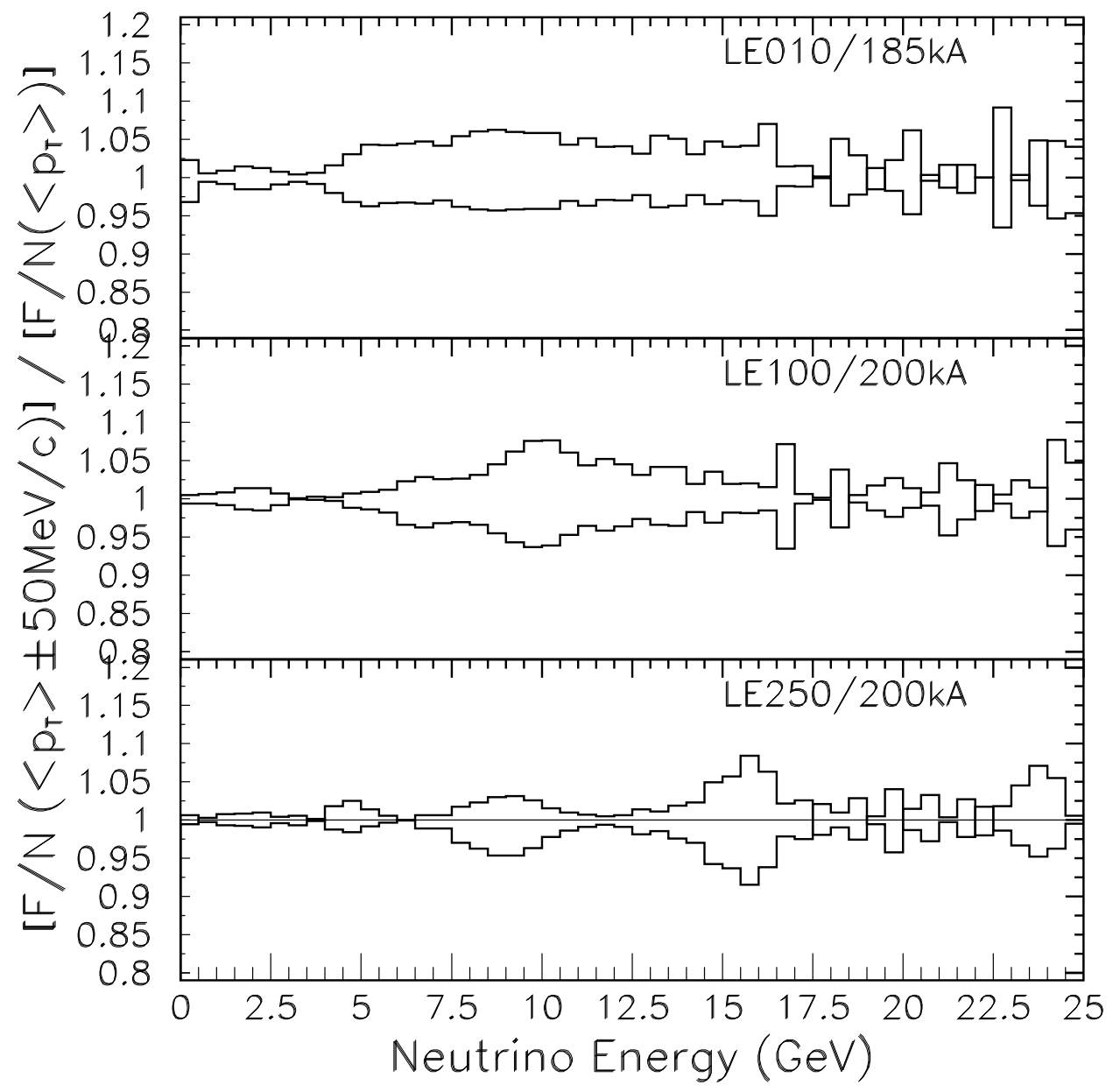

Figure 5.32: Ratio of a far over near ratio as predicted from the skewed $p_{T}$ spectra to the one predicted using FLUKA hadron production model (Figure 5.30). The curves correspond to the pion $p_{T}$ distributions shifted by $\pm 50 \mathrm{MeV} / c$. 
and found the distortions of the neutrino spectrum for several beam configurations in which NuMI was operated.

The hadron production uncertainty was found to be difficult to estimate, but it is clearly large and dominant contribution to the flux uncertainty. Therefore, we need a better in situ technique to estimate and reduce this uncertainty. The next chapter discusses the method of using ND data to reduce the flux uncertainties. 


\section{Chapter 6}

\section{Constraint on Flux uncertainties Using Near Detector Data}

The major contribution to the uncertainty in neutrino flux prediction comes from the uncertainty in hadron production (see Chapter 5). This uncertainty can be reduced by using the Near Detector (ND) data to constrain the calculated flux. Several previous experiments used their neutrino data to constrain the yield of hadrons off the target $[82,83,84,85]$, but the variable beam design of NuMI allows this to be accomplished with fewer assumptions, eg. about neutrino interaction cross sections. In this chapter we discuss a method that uses $\nu_{\mu}$ and $\bar{\nu}_{\mu}$ ND data acquired in different beam configurations to constrain the flux uncertainty.

\subsection{Introduction}

The neutrino event rate measured in the ND depends on the product of the neutrino flux, the cross sections for neutrino interaction within the detector 
and the efficiency of the detector. Given that the uncertainties in the cross sections and detector modeling are not too big ${ }^{1}$, the neutrino data can be used to constrain the hadron production uncertainty.

Beam-related systematic effects affect different regions of neutrino spectra in different beam configurations (see for example Figure 5.14 or 5.31). This allows us to deconvolve beam-related uncertainties and uncertainties that affect the same energy bin in all beam configurations in a same manner (for example neutrino cross section uncertainties).

The 7 beam configurations (Table 2.1) in which NuMI has been operated probe different regions of the production spectra of $\pi$ and $K$ yields off the target. Figures 6.1 and 6.2 show the distribution of longitudinal $p_{z}$ and transverse momenta $p_{T}$ of $\pi^{+}$and $K^{+}$off the NuMI target for those hadrons that give rise to a $\nu_{\mu}$ that interacts in the ND via charged current (CC) interaction. Figures 6.3 and 6.4 show corresponding spectra for $\bar{\nu}_{\mu} \mathrm{CC}$ interactions in the ND. These plots are a convolution of hadron production yields, probability that a meson will be focused, probability that it will decay into a $\nu$ going in the direction of the ND and a $\nu$ CC cross section. It should be noted that different beam configurations focus particles from different regions of $\left(p_{z}, p_{T}\right)$.

By comparing the level of agreement between the ND data and the beam $\mathrm{MC}$ in these 7 configurations, it is possible to "map out" the $\pi / K$ production in $\left(p_{z}, p_{T}\right)$, effectively disentangling hadron production uncertainties from other systematic effects. To constrain the neutrino flux prediction we will modify the $\left(p_{z}, p_{T}\right)$ production in the beam $\mathrm{MC}$ in order to achieve a better fit to the ND data. Additionally, we have used the methods of the present chapter,

\footnotetext{
${ }^{1}$ Even in the limit of large cross section uncertainties, these tend to cancle in a twodetector oscillation experiment such as MINOS
} 
along with $\nu$ data from the NuMI beam recorded in the nearby MiniBooNE detector, as a check of our analysis (Appendix B). 


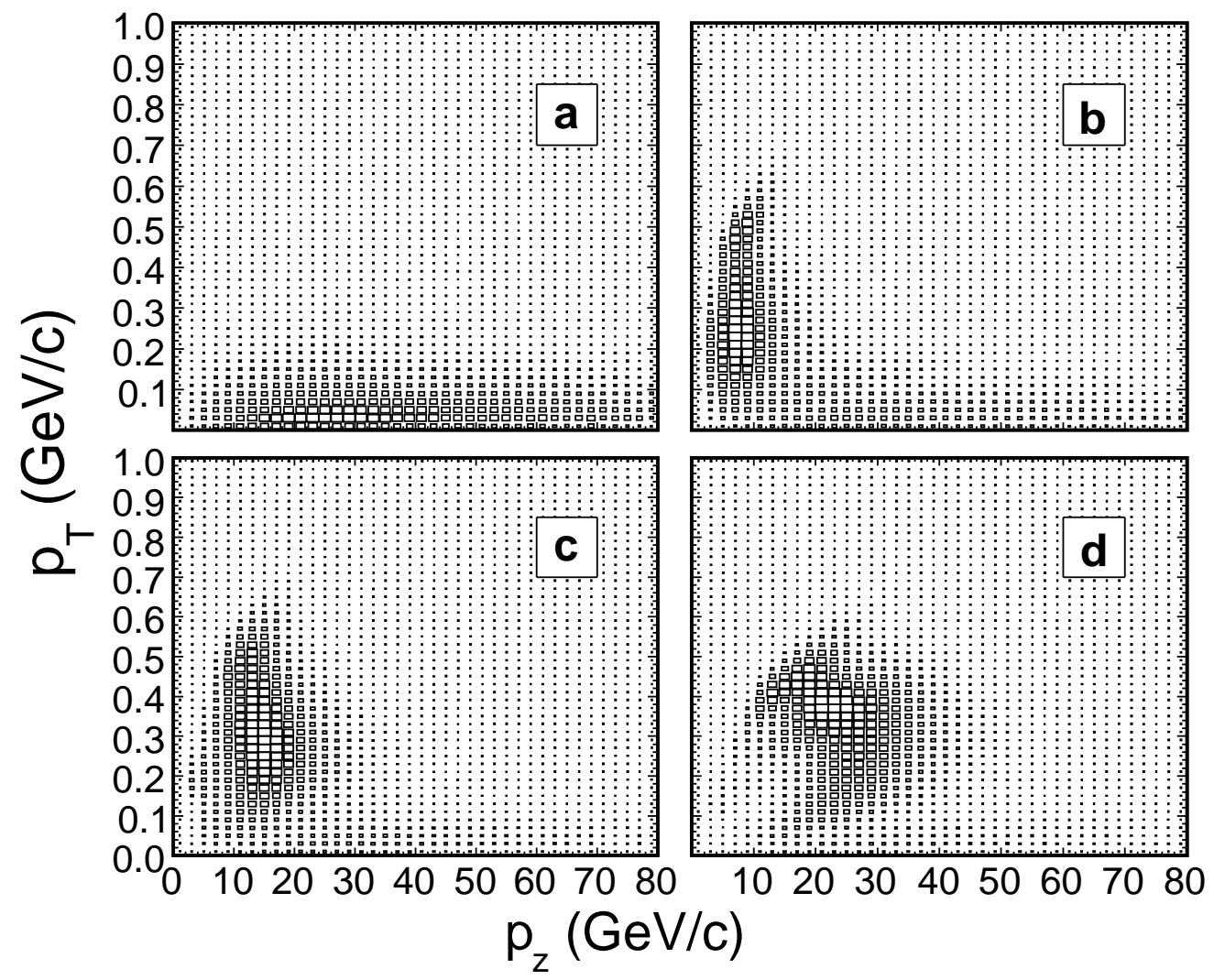

Figure 6.1: Distribution of transverse $\left(p_{T}\right)$ and longitudinal $\left(p_{z}\right)$ momenta of secondary $\pi^{+}$that contribute to the the $\nu_{\mu}$ CC event rate at the Near Detector. Shown are the momentum components at the production target. Plots correspond to different beam configurations (a) LE010z000i, (b) LE010z185i, (c) LE100z200i and (d) LE250z200i (see Table 2.1). As is evident, each beam configuration samples different region of $\left(p_{z}, p_{T}\right)$. 


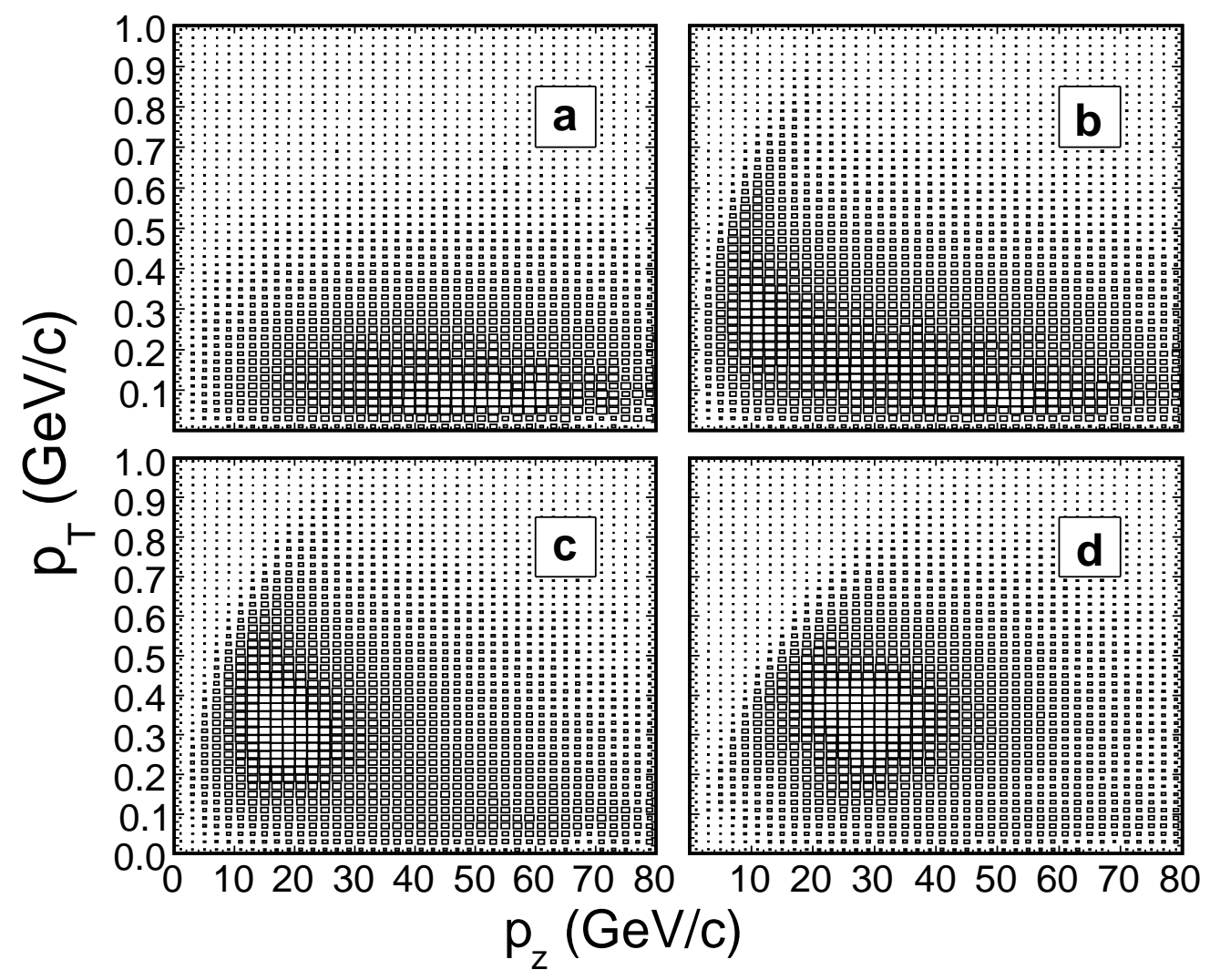

Figure 6.2: Distribution of transverse $\left(p_{T}\right)$ and longitudinal $\left(p_{z}\right)$ momenta of secondary $K^{+}$that contribute to the the $\nu_{\mu}$ CC event rate at the Near Detector. Shown are the momentum components at the production target. Plots correspond to different beam configurations (a) LE010z000i, (b) LE010z185i, (c) LE100z200i and (d) LE250z200i (see Table 2.1). Different beam configurations sample different regions of $\left(p_{z}, p_{T}\right)$. 


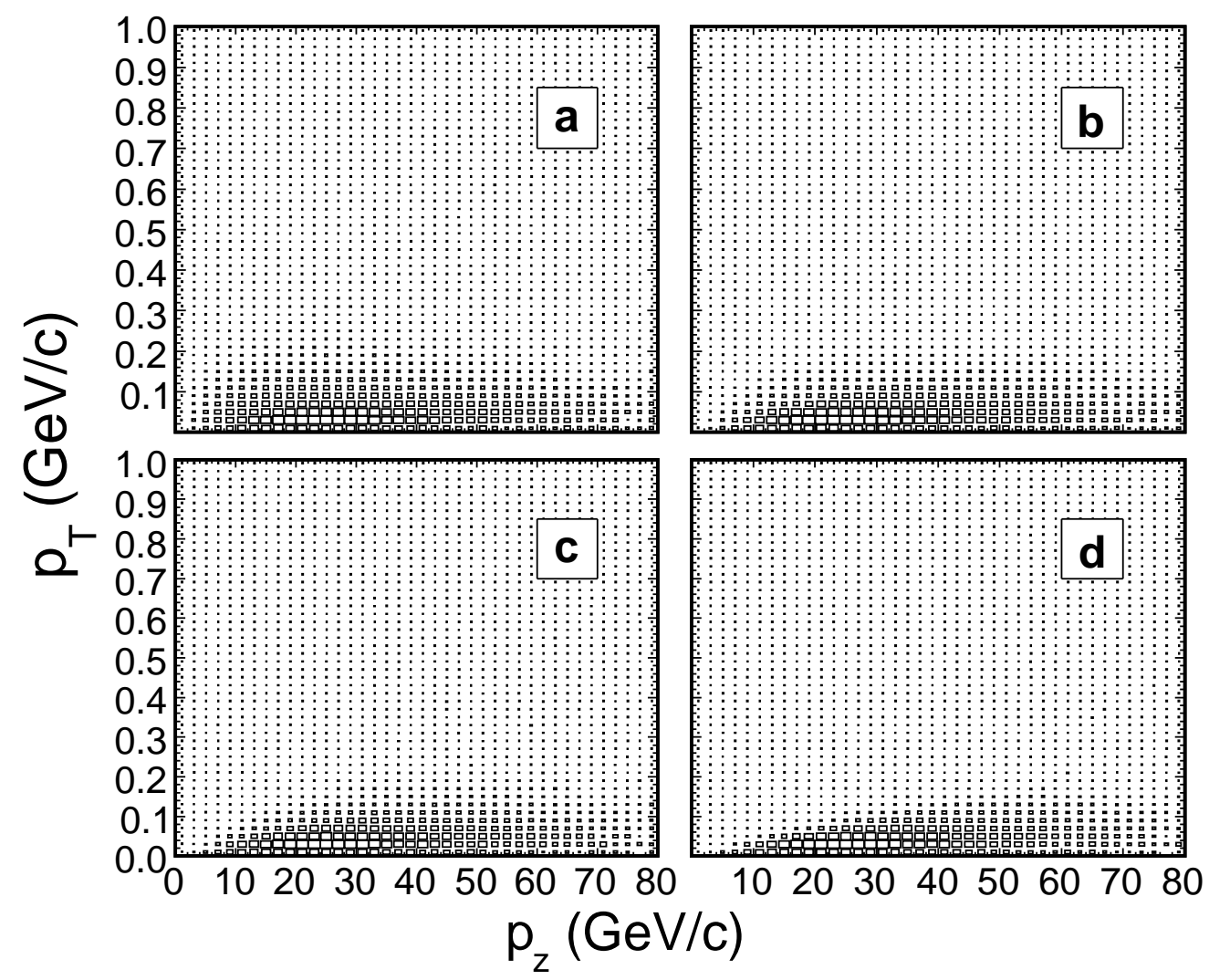

Figure 6.3: Distribution of transverse $\left(p_{T}\right)$ and longitudinal $\left(p_{z}\right)$ momenta of secondary $\pi^{-}$that contribute to the the $\bar{\nu}_{\mu}$ CC event rate at the Near Detector. Shown are the momentum components at the production target. Plots correspond to different beam configurations (a) LE010z000i, (b) LE010z185i, (c) LE100z200i and (d) LE250z200i (see Table 2.1). Unlike for $\pi^{+}$(Figure 6.1), different beam configurations in case of $\pi^{-}$sample only the region of $\left(p_{z}, p_{T}\right)$ for which $\pi^{-}$are transmitted through the field-free apertures in the horn. 


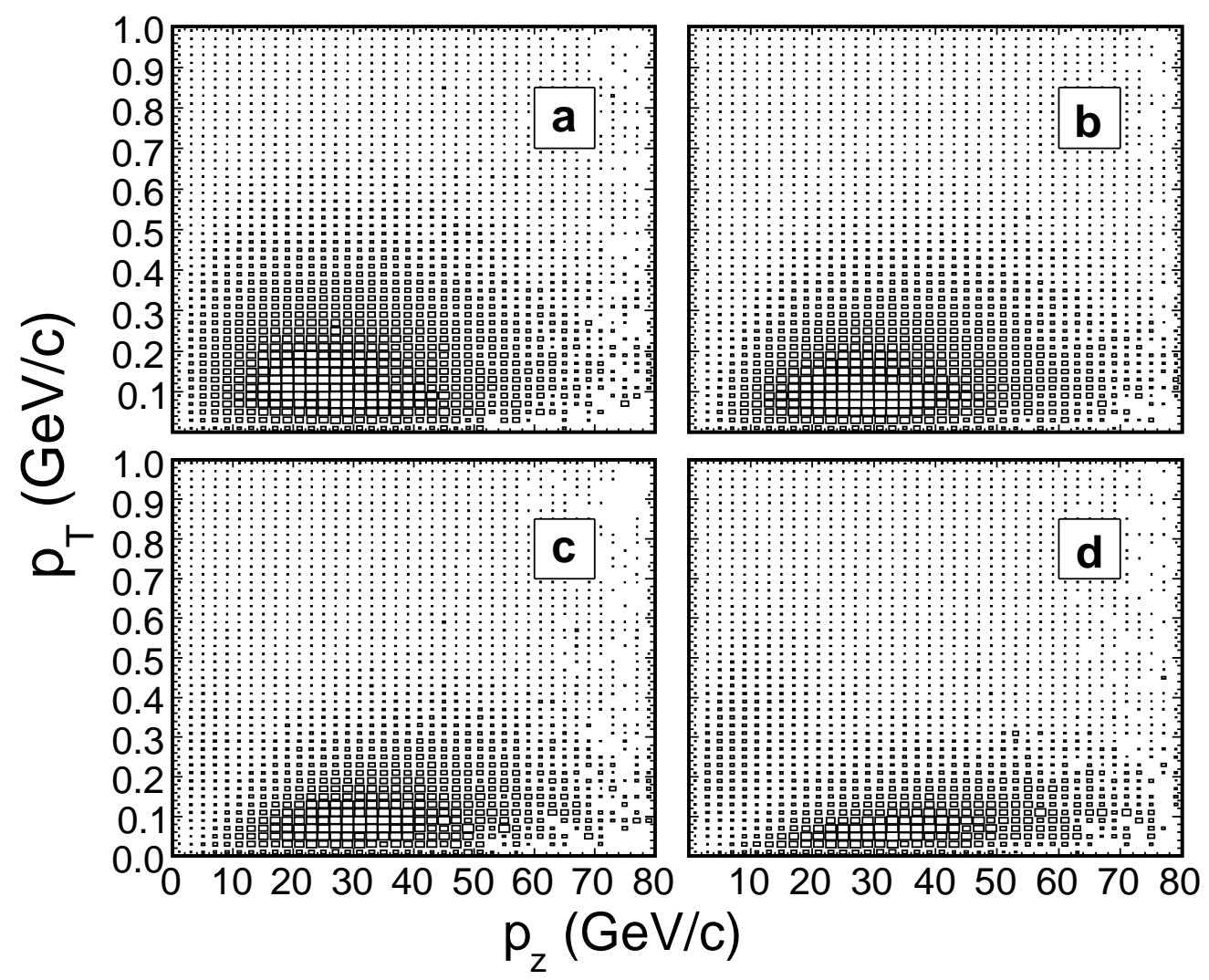

Figure 6.4: Distribution of transverse $\left(p_{T}\right)$ and longitudinal $\left(p_{z}\right)$ momenta of $K^{-}$that contribute to the the $\bar{\nu}_{\mu}$ CC event rate at the Near Detector. Shown are the momentum components at the production target. Plots correspond to different beam configurations (a) LE010z000i, (b) LE010z185i, (c) LE100z200i and (d) LE250z200i (see Table 2.1). Unlike for $\pi^{+}$(Figure 6.1), different beam configurations in case of $K^{-}$sample only the region of $\left(p_{z}, p_{T}\right)$ for which $\pi^{-}$ are transmitted through the field-free apertures in the horn. 


\subsection{Parameterizing Hadron Production}

Before we proceed to fitting the ND data for hadron production, we develop a simple empirical function to describe the yields of secondaries. We parameterize the $p_{T}-p_{z}$ distributions of pions and kaons coming off the target as predicted by the Fluka cascade model ${ }^{2}$. The following functional form is used to fit the $p_{T}$ distributions in different $x_{F}$ bins.

$$
\frac{d^{2} N}{d x_{F} d p_{T}}=\left[A+B p_{T}\right] * \exp \left(-C p_{T}^{3 / 2}\right)
$$

$\mathrm{A}, \mathrm{B}$ and $\mathrm{C}$ are the parameters in the fit. Fitting $p_{T}$ distributions in different $p_{z}$ bins gives us those three parameters as function of $x_{F}$. Parameter A determines the yields at low $p_{T}$, B determines how fast the distribution rises and $\mathrm{C}$ determines the fall-off at high $p_{T}$. With these parameters we can change the position of the peak of the distribution, its width and the total area under the curve (which is proportional to the total number of particles in a particular $x_{F}$ bin). These are exactly the knobs that we will need when we fit the ND data.

Figures 3.4 and 3.5 show the $p_{T}$ distributions of positive and negative pions and kaons from the NuMI target as given by FLUKA cascade model, for several bins of $x_{F}$. Also shown as black curves in these Figures are the fits to the $p_{T}$ distributions using Equation 6.1.

The parameters $A, B$, and $C$ from these fits are plotted in Figure 6.5.

\footnotetext{
${ }^{2}$ Alternatively, instead of $p_{z}$ Feynman variable $x_{F}=2 p_{L}^{*} / \sqrt{s}$, where $p_{L}^{*}$ is the longitudinal momentum of secondary and $\sqrt{s}$ is the total energy in center of mass, can be used. At large $x_{F}$ the approximate relation holds $x_{F} \approx p_{z} / p_{0}$ where $p_{0}$ is incident proton momentum. Since secondaries that are relevant for MINOS are of relatively high momenta, we use $p_{z}$ and $x_{F}$ interchangeably.
} 
We then fit the points in Figure 6.5 to the following functions $A\left(x_{F}\right), B\left(x_{F}\right)$ and $C\left(x_{F}\right)$ to give parametric expressions as a function of $x_{F}$ :

$$
\begin{aligned}
A\left(x_{F}\right) & =a_{1} *\left(1 .-x_{F}\right)^{a_{2}} *\left(1 .+a_{3} * x_{F}\right) * x_{F}^{-a_{4}} \\
B\left(x_{F}\right) & =b_{1} *\left(1 .-x_{F}\right)^{b_{2}} *\left(1 .+b_{3} * x_{F}\right) * x_{F}^{-b_{4}} \\
C\left(x_{F}\right) & =c_{1} / x_{F}^{c_{2}}+c_{3} \quad\left(\text { for } x_{F}<0.22\right) \\
& =c_{1} / e^{\left(x_{F}-c_{3}\right) c_{2}}+\left(c_{4} x_{F}\right)+c_{5} \quad\left(\text { for } x_{F}>0.22\right)
\end{aligned}
$$

This functional form loosely follows the BMPT [66] parameterization. The $\left(1-x_{F}\right)^{\alpha}$ term in $A$ and $B$ is expected from theoretical considerations ${ }^{3}$ [86, 87], while the terms $\left(1+\beta x_{F}\right)$ and $x_{F}^{\gamma}$ are empirically motivated [88]. The fitted functions from Equation 6.2, are shown for the $\pi^{+}$yields in Figure 6.5. The coefficients $a_{i}, b_{i}$, and $c_{i}$ for the functions $A\left(x_{F}\right), B\left(x_{F}\right)$ and $C\left(x_{F}\right)$ are given in Table 6.1, and the parametric function for $d^{2} N / d x_{F} d p_{T}$ using Equations 6.1 and 6.2 are shown as the red curves in Figures 3.4 and 3.5. If the parameterization was perfect, the black and red curves would coincide, the small discrepancies arise from the imperfect parameterizations in Equations 6.2. Nonetheless, this parameterization shows satisfactory agreement with FLUKA predictions. As it will become obvious in the Section 6.3, it is not necessary to get the perfect agreement here since the aim of this procedure is to obtain empirical parameters that can be adjusted to modify the resulting neutrino spectra.

\footnotetext{
${ }^{3}$ Such behavior at large $x_{F}$ is expected on basis of quark counting.
} 

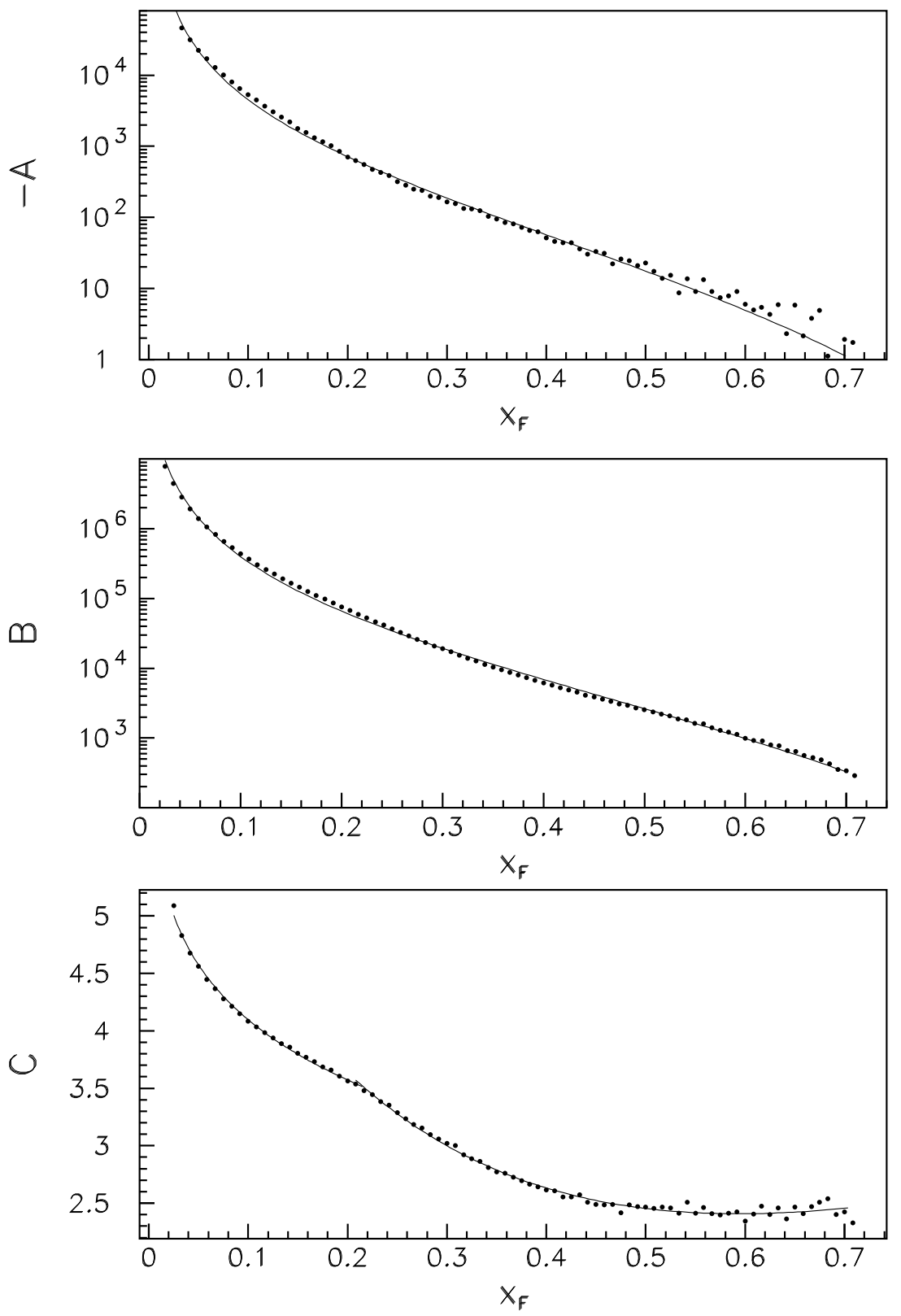

Figure 6.5: The points are the coefficients $A, B$, and $C$ obtained when fitting Figure 3.4 to Equation 6.1. The curves are the fit of these points to the functions $A\left(x_{F}\right), B\left(x_{F}\right)$, and $C\left(x_{F}\right)$ from Equation 6.2. 


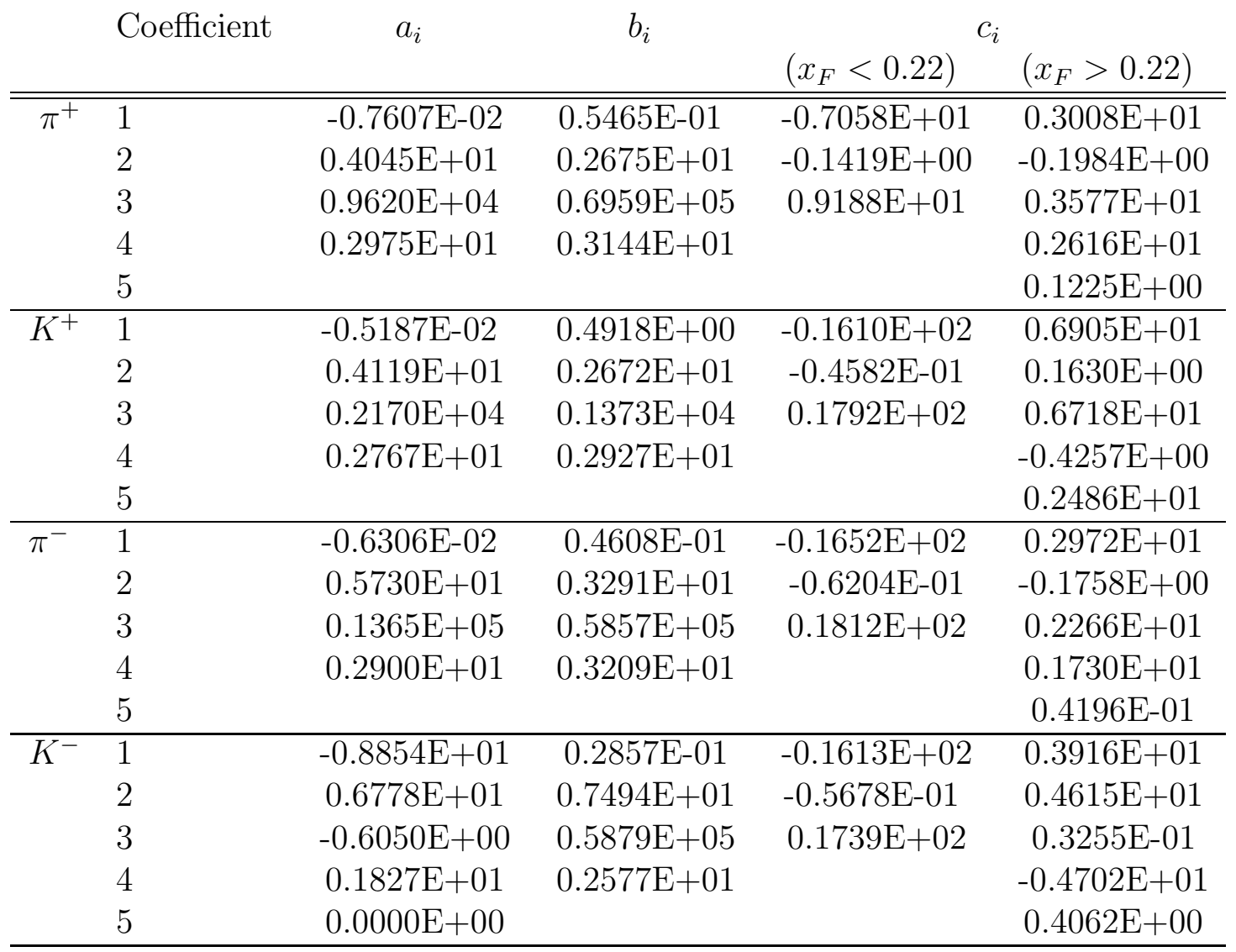

Table 6.1: Coefficients of the fits of Figures 3.4 and 3.5 to Equations 6.1 and 6.2 . 


\subsection{Fit Technique}

The goal of the fit is to minimize the differences between the beam MC prediction and the measured event rate at the ND. This was achieved by adjusting the $\mathrm{MC} \pi / K$ prediction of Equations 6.1 and 6.2 and also a set of $\nu$ cross section and event selection parameters that account for the uncertainty in the measured $\mathrm{CC}$ event rate in the ND. Data from all 7 different beam configurations in which the NuMI beam was operated (see Table 2.1) are used in the fit because each beam configuration samples a different region of $\left(p_{T}, p_{z}\right)$ (see Figures 6.1-6.4),. The data is binned in energy with 70 bins used for $\nu_{\mu}$ spectra and LE010/185kA $\bar{\nu}_{\mu}$, while 28 bins are used for the rest of $\bar{\nu}_{\mu}$ data. Finer binning was used if there was enough data statistics.

The data in LE100/200kA and LE250/200kA (from 2005) beam configurations was taken with low proton beam intensity. This also means that the proton beam width at the target was much narrower during those runs than what it is in the beam MC. We apply a correction to the MC to account for that. The second run in LE250/200kA was done at normal intensities. Because the running conditions between the two LE250/200kA runs were different the two data sets were included in the fit separately.

We use MINUIT [89] to find the set of parameters that minimizes the $\chi^{2}$ function defined in the following way:

$$
\chi^{2}=\sum_{\nu_{\mu}, \bar{\nu}_{\mu}} \sum_{b} \sum_{i} \frac{\left(e_{i}-o_{i}\right)^{2}}{\sigma_{e_{i}}^{2}+\sigma_{o_{i}}^{2}}+\sum_{j} \frac{p_{j}^{2}}{\sigma_{p_{j}}^{2}}
$$

where $b$ runs over all beam configurations, $i$ runs over the bins in data histograms and $j$ runs over the penalty terms. The $e_{i}$ and $o_{i}$ are expected and 
observed number of events in $\mathrm{i}$-th bin respectively. The expectations $e_{i}$ are functions of the inputs to the $\mathrm{MC}$, such as the $\pi / K$ yields off the target, horn current, $\nu$ cross sections, event selection efficiency, etc. The $\sigma_{e_{i}}$ and $\sigma_{o_{i}}$ are corresponding statistical errors.

Some of the parameters in the fit are constrained to particular values that are known with some certainty (eg one of the parameters effectively changes transverse position of NuMI horn in the MC whose real position is known to within $1 \mathrm{~mm}$ ). To use the information we have about some of the parameters we introduce penalty terms that are added to the function that is being minimized in the fit. The $p_{j}$ is the value of certain parameter and $\sigma_{p_{j}}$ is the penalty term for that parameter.

\subsubsection{Hadron Production Parameters}

We use 16 parameters to modify the yields of charged pions and kaons. Six parameters were used for positive pions, while only two were used for negative. Similarly $6+2$ parameters were used for positive and negative kaons. The smaller number of parameters for negative particles is motivated by the smaller size of the $\pi^{-}$and $K^{-}\left(p_{T}, p_{z}\right)$ space sampled by MINOS Near Detector and by the available external constraint from particle production experiments like NA49 [57], which generally measure the ratio of $\pi^{+} / \pi^{-}$yields more accurately than the individual $\pi^{+}$and $\pi^{-}$yields. This adds an additional constraint on negative particles in addition to the fit to MINOS ND data.

There are couple of reasons why less parameters were used for negative particles. The first one is that the size of the phase space of the secondary particles that contribute to neutrino flux at the ND is much bigger for positive 
parents than it is for negative (see Figures 6.1-6.4). It is thus desirable to have more parameters and more flexibility in tuning production yields of positive particles. The second reason is that, as discussed in $\S 3.2$ the ratios in yields of positive to negative pions (kaons) are somewhat better determined by hadron production experiments than individual yields. This adds an additional constraint on negative particles, ones the positives are fixed.

The 6 parameters for $\pi^{+}$rescale the $A\left(x_{F}\right), B\left(x_{F}\right)$ and $C\left(x_{F}\right)$ (defined in Equation 6.2) in the following way:

$$
\begin{aligned}
& A^{\prime}\left(x_{F}\right)=\left(p_{1}+p_{2} x_{F}\right) A\left(x_{F}\right) \\
& B^{\prime}\left(x_{F}\right)=\left(p_{3}+p_{4} x_{F}\right) B\left(x_{F}\right) \\
& C^{\prime}\left(x_{F}\right)=\left(p_{5}+p_{6} x_{F}\right) C\left(x_{F}\right)
\end{aligned}
$$

Similarly 6 parameters are used to rescale the $A\left(x_{F}\right), B\left(x_{F}\right)$ and $C\left(x_{F}\right)$ parameters for $K^{+}$.

Given a set of parameters $p_{i}$ we calculate by what fraction should the yields of pions or kaons be scaled up or down given their transverse and longitudinal momentum.

$$
W\left(\pi^{+} / K^{+}\right)=\frac{\left[A^{\prime}+B^{\prime} p_{T}\right] \times \exp \left(-C^{\prime} p_{T}^{3 / 2}\right)}{\left[A+B p_{T}\right] \times \exp \left(-C p_{T}^{3 / 2}\right)}
$$

It should be noted that because we use this ratio to modify the FLUKA prediction, the method does not critically depend on how well the parameterization using Equations 6.1 and 6.2 describes the FLUKA distributions of Figures 3.4 and 3.5 . 
For negative pions and kaons we adjust the $\pi^{+} / \pi^{-}$or $K^{+} / K^{-}$ratio using a linear function in $x_{F}$.

$$
W\left(\pi^{-}\right)=\left(p_{13}+p_{14} x_{F}\right) W\left(\pi^{+}\right)
$$

If the yields of $\pi^{-}$change by the same fraction as yields of $\pi^{+}$, the $\pi^{+} / \pi^{-}$ ratio is not changed to the first order.

It is not entirely possible to attribute physical meaning to individual hadron production parameters used in the fit. Very different values of parameters can yield similar net effect on yields of secondaries that give rise to neutrinos in the NuMI beam. For this reason, in case of hadron production we don't constrain individual parameters by introducing penalty terms on them. However, the external hadron production data does constrain the yields. To account for this we look at $p_{T}$ distribution of secondaries. Different hadron production models that fit external data differ in the predicted mean transverse momentum $\left\langle p_{T}\right\rangle$ of secondary pions on the average at the order of $\pm 15 \mathrm{MeV} / c(\S 3.2)$. We use that as a penalty term on the shift in the $\left\langle p_{T}\right\rangle$.

\subsubsection{Beam Parameters}

In addition to parameters used to modify the production of secondaries, we adjust the beam MC to better agree with the ND data by use of several parameters associated with the simulation of the beam focusing. These additional parameters are used to account for the proton beam and focusing systematic effects that have the biggest impact on neutrino flux (see Figures 5.23-5.28). In particular, one parameter was used for each of the 5 following systematic effects: transverse misalignment of Horn 1, proton beam scraping the baffle, 
total integrated proton intensity, horn current miscalibration and horn current distribution.

It was sufficient to use one parameter per systematic effect, since it is expected that these miscalibrations or misalignments are common to all the 7 different beam configurations in which data was acquired. For example, the relative transverse misalignment of the target and the horns didn't change when target position along the beam axis was changed.

The derivatives calculated in Chapter 5 were used to find the effective change in the neutrino flux for any given values of these parameters.

The beam instrumentation constrains what the size of any of these miscalibrations and misalignments can be as was discussed in $§ 5.2$. Hence, the penalty terms are assigned for these parameters (the values summarized in Table 5.1).

\subsubsection{Other Parameters}

The measured CC event rate in ND of course depends on the neutrino flux, but it also depends on CC cross sections and on detector properties. The predicted event rate in the ND using the calculated neutrino flux assumes certain cross sections for neutrino interaction and it assumes certain detector response. We are going to do a fit in which we force our predicted neutrino spectrum to look like the data by adjusting the yields of pions of the target and consequently the neutrino flux. If our assumptions about the cross sections and detector response are wrong than we will end up with incorrect neutrino flux.

The Near Detector measures the product of neutrino flux $\phi_{\nu}$ and cross sections $\sigma_{\nu}$. Therefore, the two quantities are degenerate and it is necessary 
to know one of the two in order to find the other. This is not an issue for this analysis since the FD also measures the product $\phi_{\nu} \times \sigma_{\nu}$. However we will perform a fit to both the $\nu_{\mu}$ and $\bar{\nu}_{\mu}$ data. Since we use some constraints on relative production of positive and negative pions, ie the $\pi^{+} / \pi^{-}$ratio we are sensitive to the ratio of cross sections $\sigma\left(\nu_{\mu}\right) / \sigma\left(\bar{\nu}_{\mu}\right)$. We use one parameter to adjust the neutrino cross section ratio below $25 \mathrm{GeV}$ where neutrino cross sections are less known and measured to within $\sim 20 \%$.

The neutrino energy in MINOS detectors is reconstructed by summing the muon momentum and the visible shower energy near the interaction vertex. We use one parameter to adjust the reconstructed muon momentum and one to adjust the reconstructed shower energy.

The purity of the CC data sample is estimated by detector MC and from MINOS data [90]. Some disagreement between the two estimates at the level of $30 \%$ was found. To allow the NC contamination in $\nu_{\mu}$ and $\bar{\nu}_{\mu}$ energy spectra to change we use two parameters that can scale the contamination up or down.

\subsection{Results of the Fit}

Tables 6.2-6.4 show the best fit values for the 26 parameters used in the fit. The values of the parameters appear to be reasonable and the parameters don't assume either unphysical or improbable values. Figure 6.6 shows the resulting change in the $\bar{\nu}_{\mu}$ cross section.

In the following sections we will examine what the effect of the fit is on the predicted neutrino flux and also how it changes the hadron production at the target. 


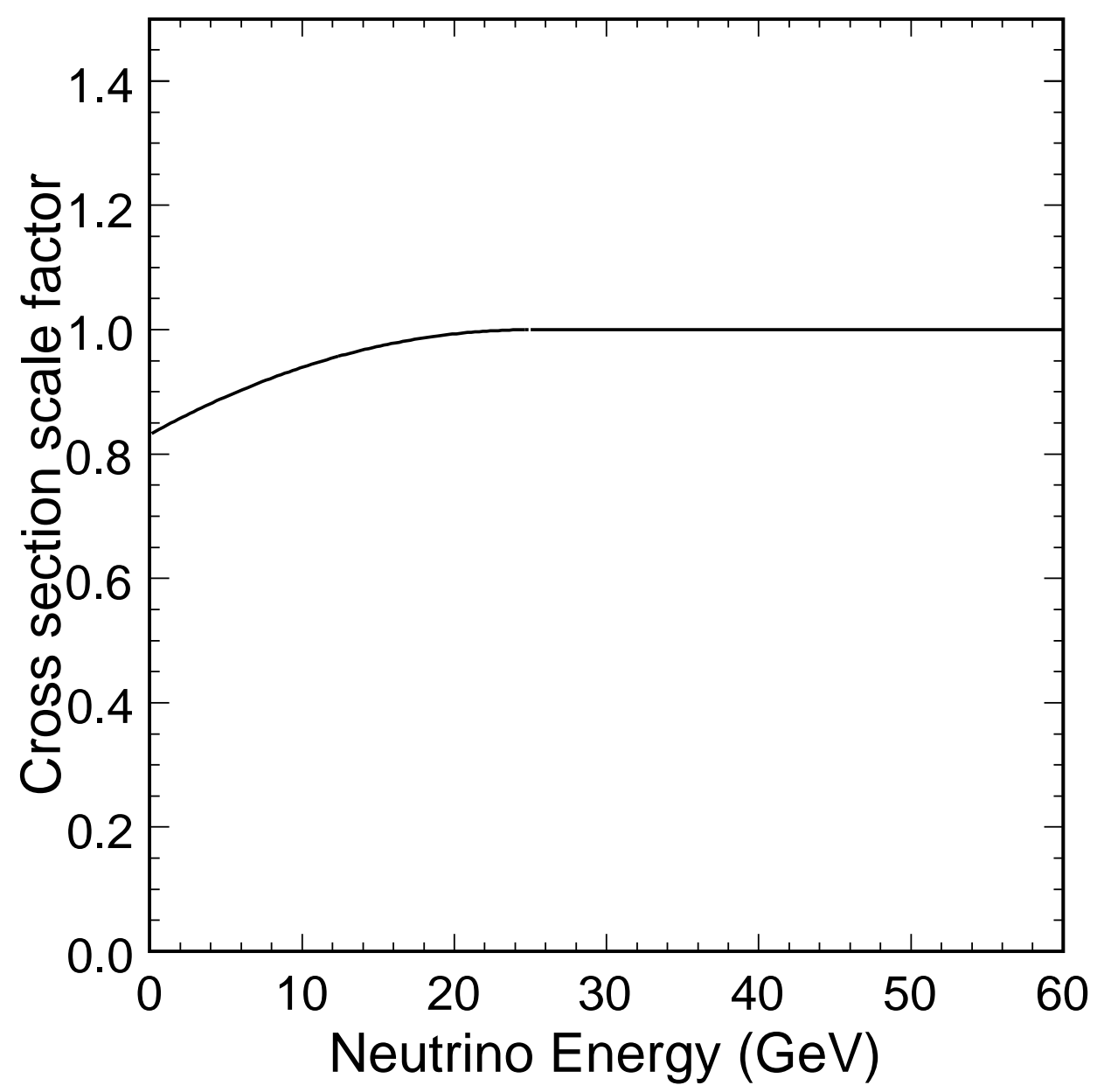

Figure 6.6: The $\bar{\nu}_{\mu}$ cross section was allowed to change for neutrinos with energy below $25 \mathrm{GeV}$. The plot shows the preferred change in the cross section[50] that comes out of the fit depending on the $\bar{\nu}_{\mu}$ energy. 


\begin{tabular}{lcc} 
& Parameter & Error \\
\hline \hline Horn 1 Offset & -0.58 & 0.22 \\
Baffle Scraping & 0.29 & 0.24 \\
POT & 0.02 & 0.20 \\
Horn Current Miscalibration & -0.24 & 0.15 \\
Horn Current Distribution & -0.26 & 0.15
\end{tabular}

Table 6.2: The best fit values for 5 beam parameters that were used in the fit to tune the $\mathrm{MC}$ for proton beam and focusing uncertainties.

\begin{tabular}{lcc} 
& Parameter & Error \\
\hline \hline Neutrino Energy Miscalibration / \% & 5.7 & 0. \\
Reconstructed Shower Energy Offset / MeV & 11 & 0. \\
$\nu_{\mu}$ Neutral Current Contamination / \% & 2 & 7 \\
$\bar{\nu}_{\mu}$ Neutral Current Contamination / \% & 5 & 7 \\
$\bar{\nu}_{\mu}$ Cross Section Parameter & 0.83 & 0.02
\end{tabular}

Table 6.3: The best fit values for 5 non-beam related parameters. In total 26 parameters were used to fit the $\nu_{\mu}$ and $\bar{\nu}_{\mu}$ ND data.

\subsubsection{Impact on $\nu_{\mu}$ and $\bar{\nu}_{\mu}$ Flux}

Table 6.5 shows the break down of the $\chi^{2}$ (Equation 6.3) contributions from each data histogram used in the fit along with the part coming from penalty terms. The total $\chi^{2}$ per degree of freedom was reduced from $8847 / 816=10.8$ to $1342 / 790=1.7$. This is a huge improvement in the agreement. It can also be seen that $\chi^{2}$ is significantly reduced for all the beam configurations simultaneously and this is true for both the $\nu_{\mu}$ and $\bar{\nu}_{\mu}$ data.

Figures 6.7-6.10 show the $\nu_{\mu}$ data along with the MC prediction based on FLUKA hadron production and tuned MC for LE010/0kA, LE010/185kA, LE100/200kA and LE250/200kA. The tuned MC agrees well with data in all the beams at the level of $4 \%$, while initially in some beam configurations at 


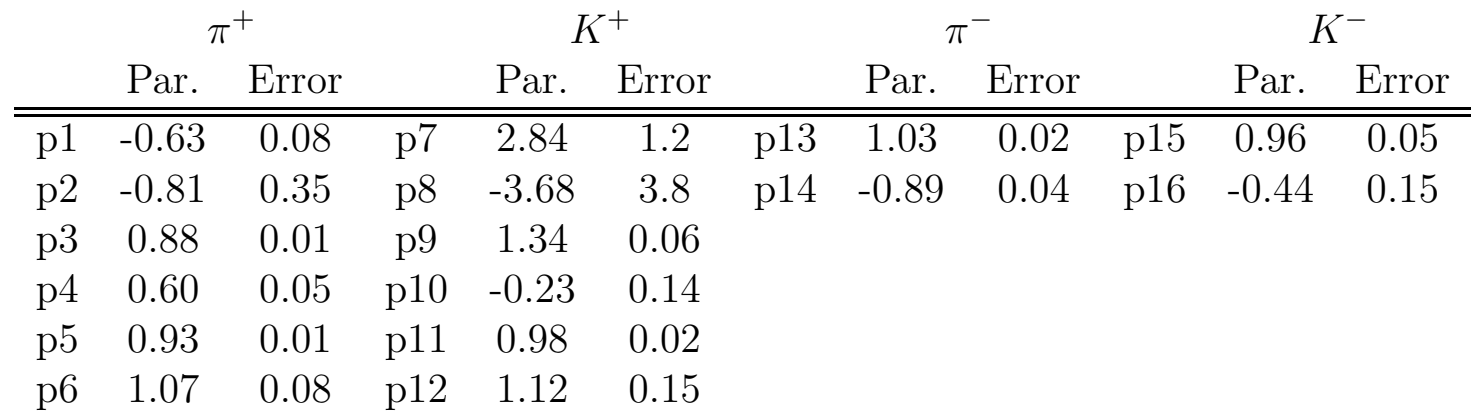

Table 6.4: The best fit values for 16 parameters that were used to modify the yields of secondaries off the target. In total 26 parameters were used to fit the $\nu_{\mu}$ and $\bar{\nu}_{\mu}$ Near Detector CC data sample.

certain energies the disagreement was $30 \%$ or more.

The FLUKA based MC prediction in LE010/0kA beam configuration hugely underestimated the data. After tuning, the agreement is improved, however the shape of the spectra still does not perfectly agree. The reason for this is that we tune the yields of secondaries off the target. The relative contribution to neutrino flux of the particles created in the target compared to the contribution of particles created in material downstream of target, although still dominant, is smaller for LE010/0kA beam. Thus, the correction is not as big for this beam configuration as it is for the others.

Figure 6.11 shows the $\bar{\nu}_{\mu}$ data compared to FLUKA and tuned MC for the LE010/185kA beam. The agreement is similar in other beam configurations (Figures for other beam configurations can be found in Appendix E). This is expected since $\pi^{-}$and $K^{-}$secondaries from practically the same region in phase space in all beam configurations (see Figures 6.3 and 6.4) contribute to $\bar{\nu}_{\mu}$ flux. Similarly as discussed for $\nu_{\mu}$ flux in LE010/0kA beam configuration, for $\bar{\nu}_{\mu}$ flux in all configurations the relative contribution to neutrino flux 
of particles off the target compared to those created in downstream material is somewhat smaller. As a result the overall agreement between tuned MC and $\bar{\nu}_{\mu}$ data is slightly worse than overall agreement for $\nu_{\mu}$ data.

The worse agreement between tuned $\mathrm{MC}$ and data is found in the LE100/200kA beam configuration. It is not entirely understood what is the cause of that. One possibility is that the target is longitudinally misaligned. The position in that configuration is known with least certainty. This is further discussed in next chapter.

\subsubsection{Impact on $\nu_{e}$ Flux}

The same hadrons whose decays lead to $\nu_{\mu}$ and $\bar{\nu}_{\mu}$ events also lead to $\nu_{e}$ and $\bar{\nu}_{e}$. Tuning the yields of pions and kaons affects the $\nu_{e}$ and $\bar{\nu}_{e}$ flux.

Unlike the muon neutrino spectrum, electron neutrino spectrum has also a significant component coming from $K_{L}^{0}$. These neutral kaons give rise to $\nu_{e}$ and $\bar{\nu}_{e}$ through $K_{L}^{0} \rightarrow \pi e \nu\left(K_{e 3}^{0}\right)$ decays ${ }^{4}$. By fitting muon neutrinos we don't have any sensitivity to $K_{L}^{0}$, however the number of produced neutral kaons can be correlated with number of charged kaons in the simple parton model [66]. Taking into consideration valence quarks $q_{v}$ and sea quarks $q_{s}$ and assuming the isospin symmetry we find $u_{s}=\bar{u}_{s}=d_{s}=\bar{d}_{s}$ and $s_{s}=\bar{s}_{s}$. For structure functions of the valence quarks inside a projectile proton we assume $u_{v} / d_{v}=n$. In such model the production of neutral and charged kaons is

\footnotetext{
${ }^{4} K_{L}^{0}$ also decay into $\nu_{\mu}\left(\bar{\nu}_{\mu}\right)$ through $K_{L}^{0} \rightarrow \pi \mu \nu\left(K_{\mu 3}^{0}\right)$ decays. The $K_{e 3}^{0}$ and $K_{\mu 3}^{0}$ modes have similar branching ratio, but the fractional contribution to muon neutrino flux is negligible because of large flux coming from pions.
} 


\begin{tabular}{lcc} 
Beam & \multicolumn{2}{c}{ Fit $\chi^{2}$ (statistical errors only)/NDF } \\
Configuration & No Tuning & Tuned MC \\
\hline \hline$\nu_{\mu}$ LE10/000kA & $1042 / 70$ & 146 \\
$\nu_{\mu}$ LE10/170kA & $588 / 70$ & 84 \\
$\nu_{\mu}$ LE10/185kA & $1440 / 70$ & 84 \\
$\nu_{\mu}$ LE10/200kA & $587 / 70$ & 64 \\
$\nu_{\mu}$ LE100/200kA(ME) & $1441 / 70$ & 199 \\
$\nu_{\mu}$ LE150/200kA(ME) & $1205 / 70$ & 109 \\
$\nu_{\mu}$ LE250/200kA(HE) (2005) & $699 / 70$ & 93 \\
$\nu_{\mu}$ LE250/200kA(HE) (2007) & $778 / 70$ & 50 \\
$\bar{\nu}_{\mu}$ LE10/000kA & $119 / 28$ & 50 \\
$\bar{\nu}_{\mu}$ LE10/170kA & $107 / 28$ & 47 \\
$\bar{\nu}_{\mu}$ LE10/185kA & $459 / 68$ & 90 \\
$\bar{\nu}_{\mu}$ LE10/200kA & $58 / 28$ & 32 \\
$\bar{\nu}_{\mu}$ LE100/200kA(ME) & $114 / 27$ & 73 \\
$\bar{\nu}_{\mu}$ LE150/200kA(ME) & $72 / 28$ & 29 \\
$\bar{\nu}_{\mu}$ LE250/200kA(HE) (2005) & $67 / 28$ & 43 \\
$\bar{\nu}_{\mu}$ LE250/200kA(HE) (2007) & $70 / 28$ & 39 \\
Penalty terms & & 109 \\
\hline Total (all beams) & $8847 / 816$ & $1342 / 790=1.7$
\end{tabular}

Table 6.5: The $\chi^{2}$ values when comparing the neutrino energy spectrum between data and MC before and after tuning. The tuning procedure improves the agreement significantly. 


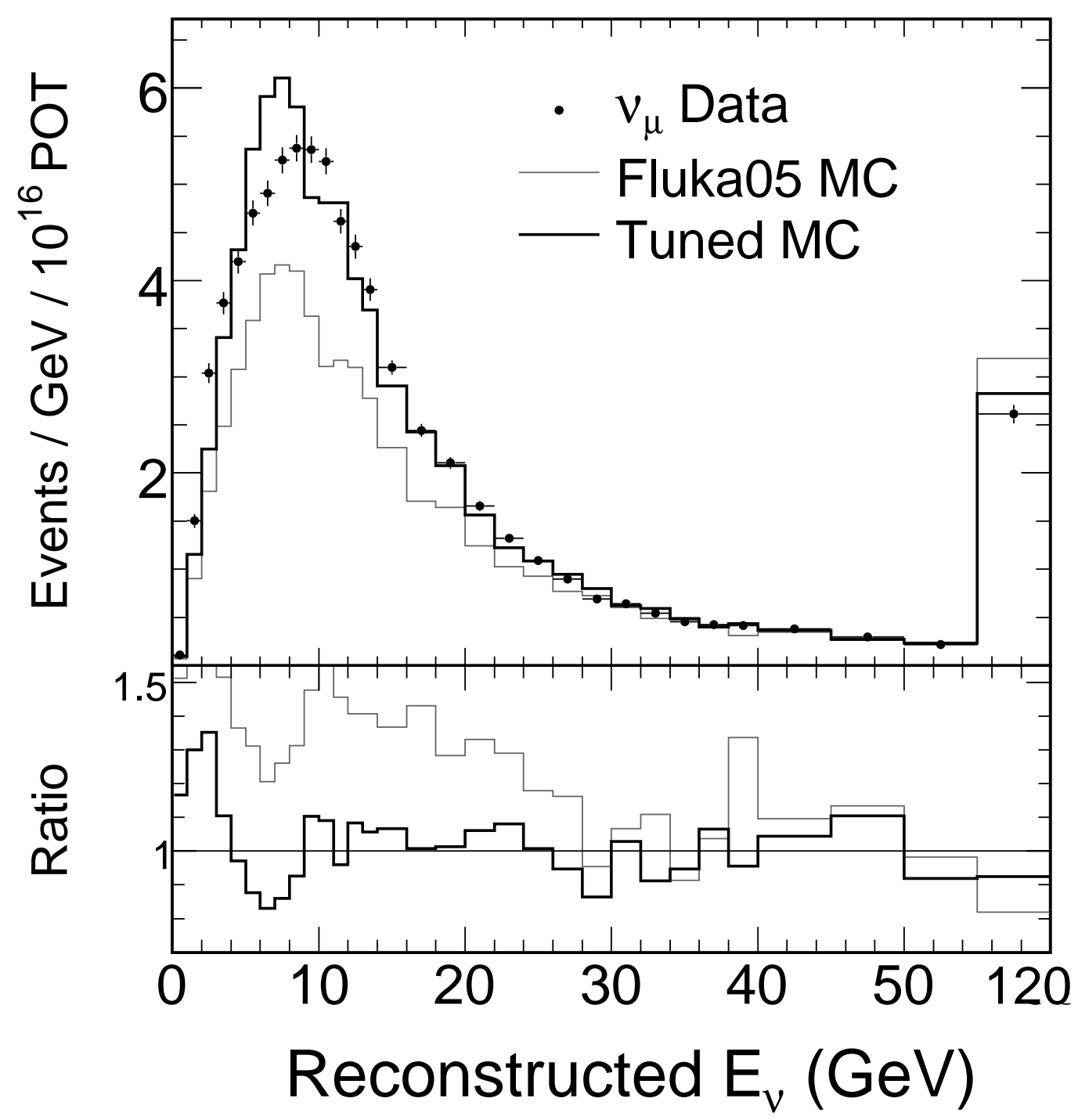

Figure 6.7: The MC flux prediction based on FLUKA cascade model (light gray curve) was tuned (black curve) to better agree with the measured $\nu_{\mu}$ CC energy spectrum at the ND (black points). Data from all of the beam configurations was used in a fit. Here shown is LE010/0kA. 


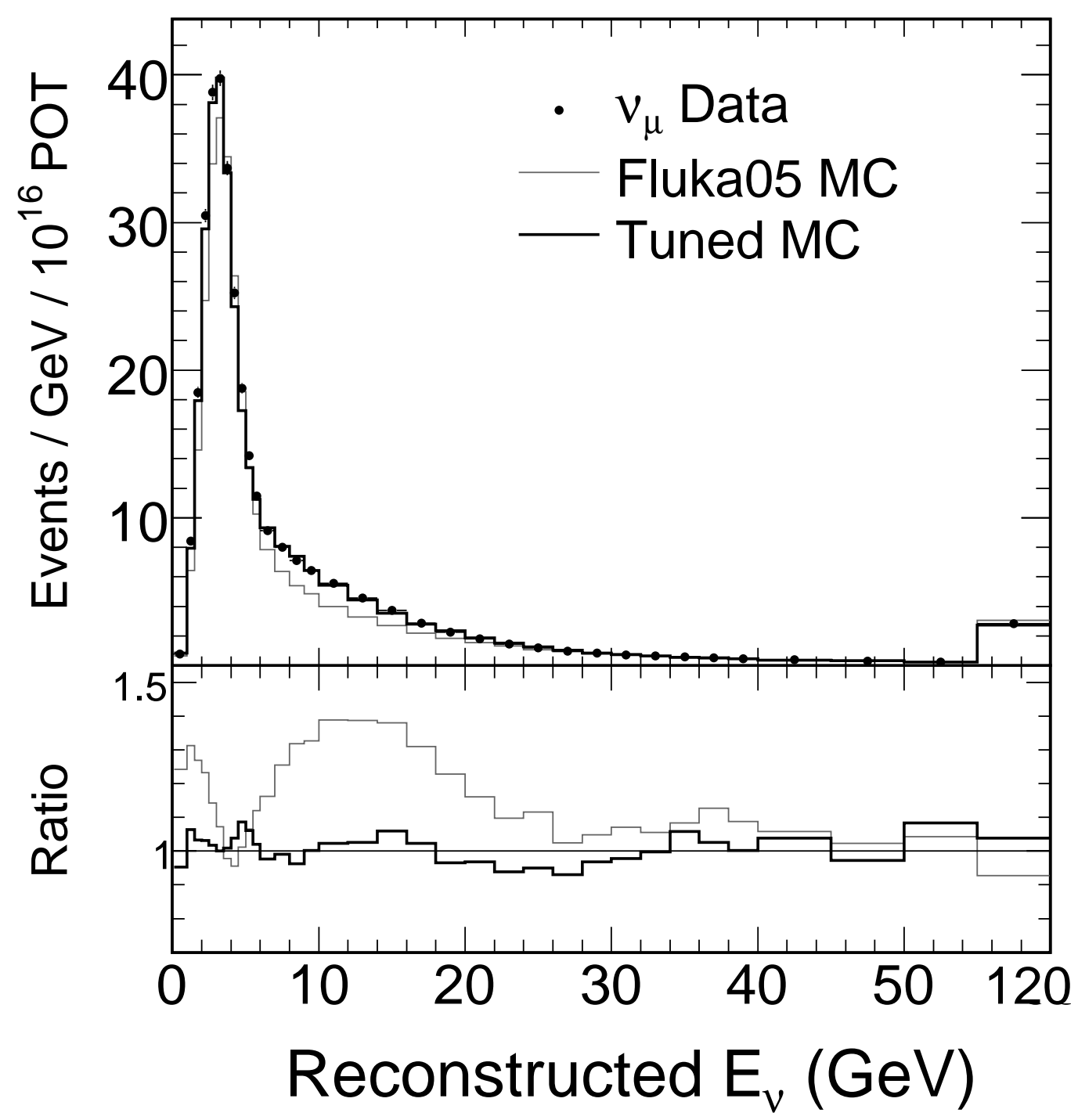

Figure 6.8: The MC flux prediction based on FLUKA cascade model (light gray curve) was tuned (black curve) to better agree with the measured $\nu_{\mu}$ CC energy spectrum at the ND (black points). Data from all of the beam configurations was used in a fit. Here shown is LE010/185kA. 


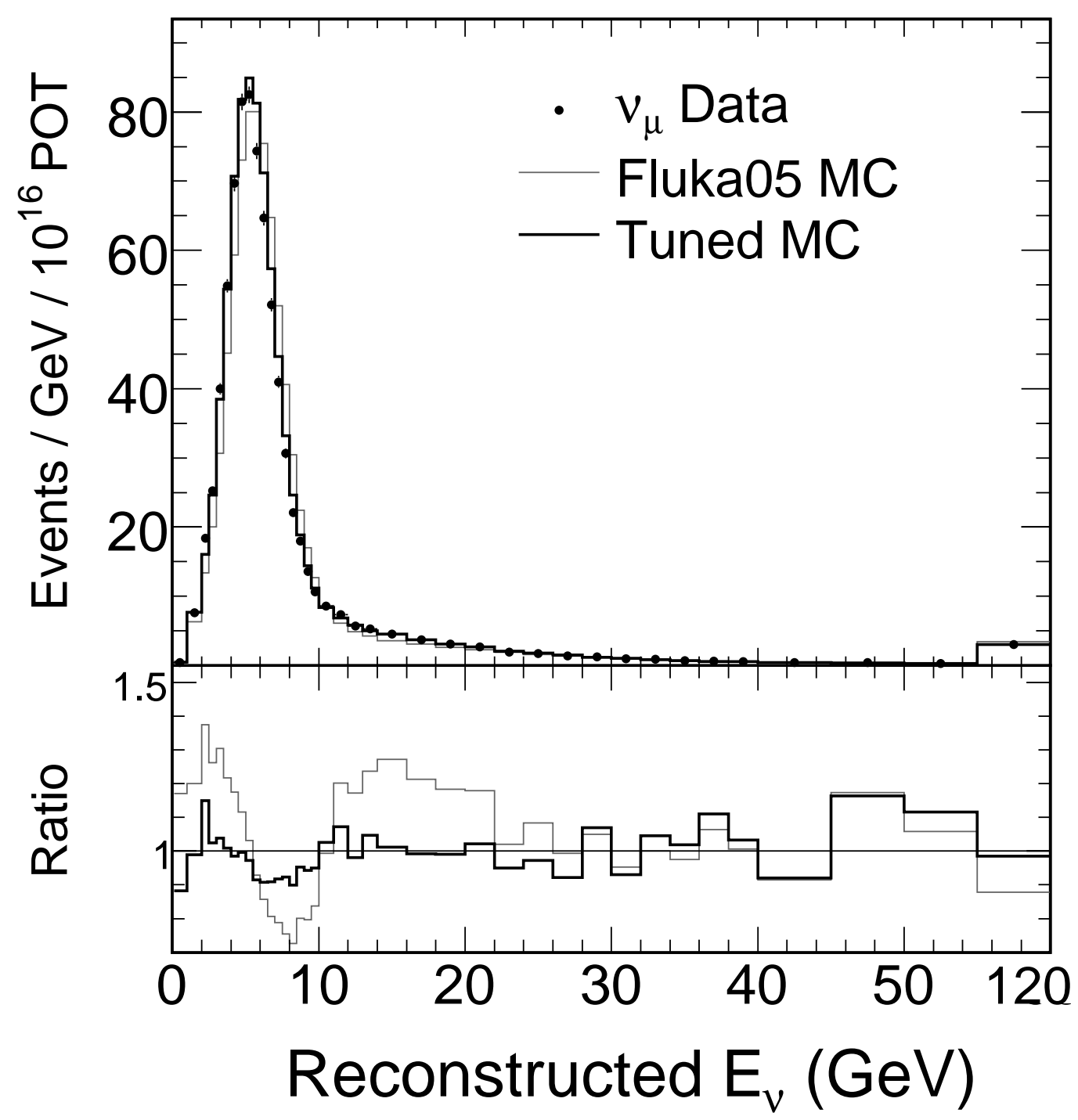

Figure 6.9: The MC flux prediction based on FLUKA cascade model (light gray curve) was tuned (black curve) to better agree with the measured $\nu_{\mu}$ CC energy spectrum at the ND (black points). Data from all of the beam configurations was used in a fit. Here shown is LE100/200kA. 


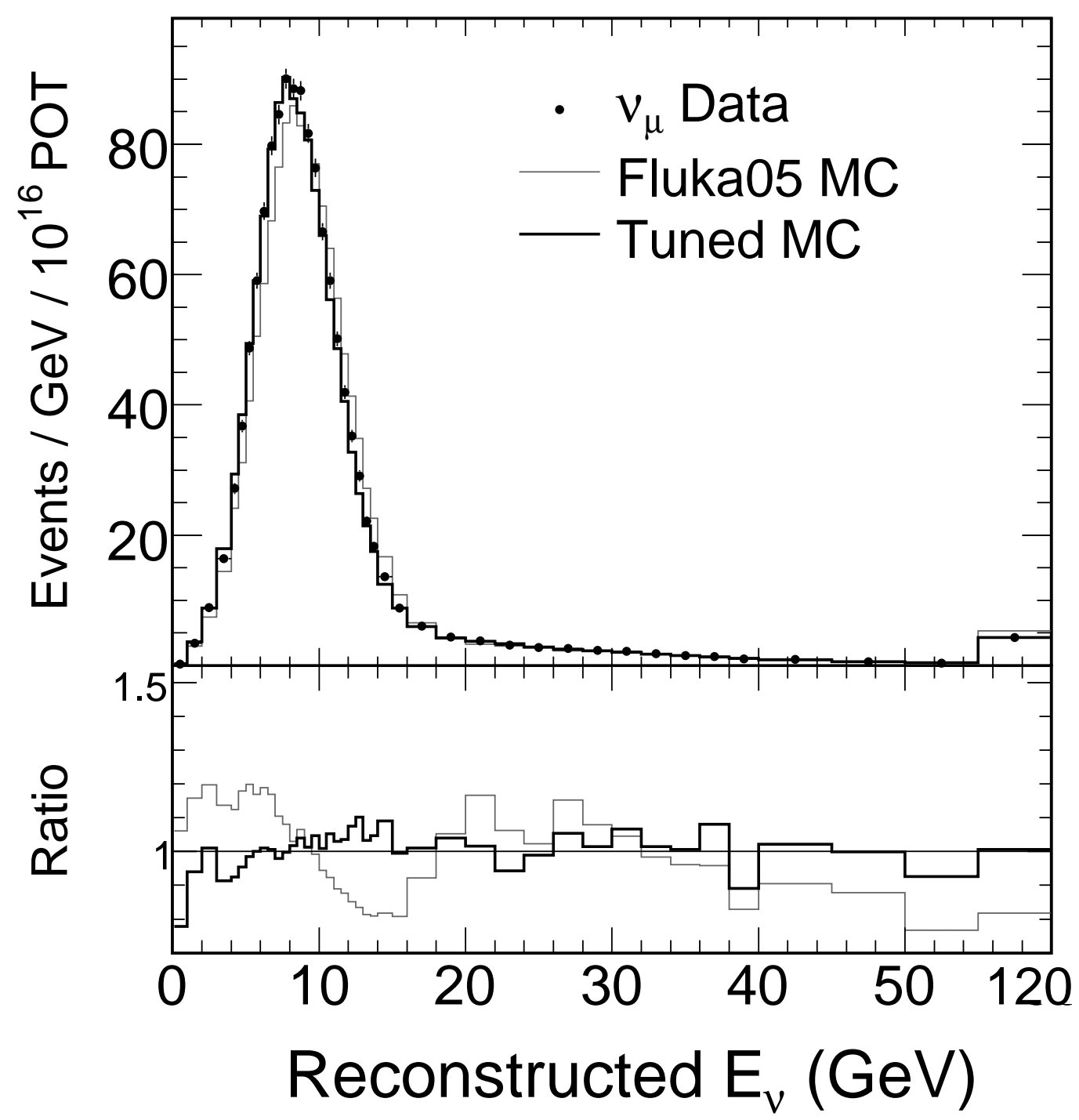

Figure 6.10: The MC flux prediction based on FLUKA cascade model (light gray curve) was tuned (black curve) to better agree with the measured $\nu_{\mu}$ CC energy spectrum at the ND (black points). Data from all of the beam configurations was used in a fit. Here shown is LE250/200kA. 


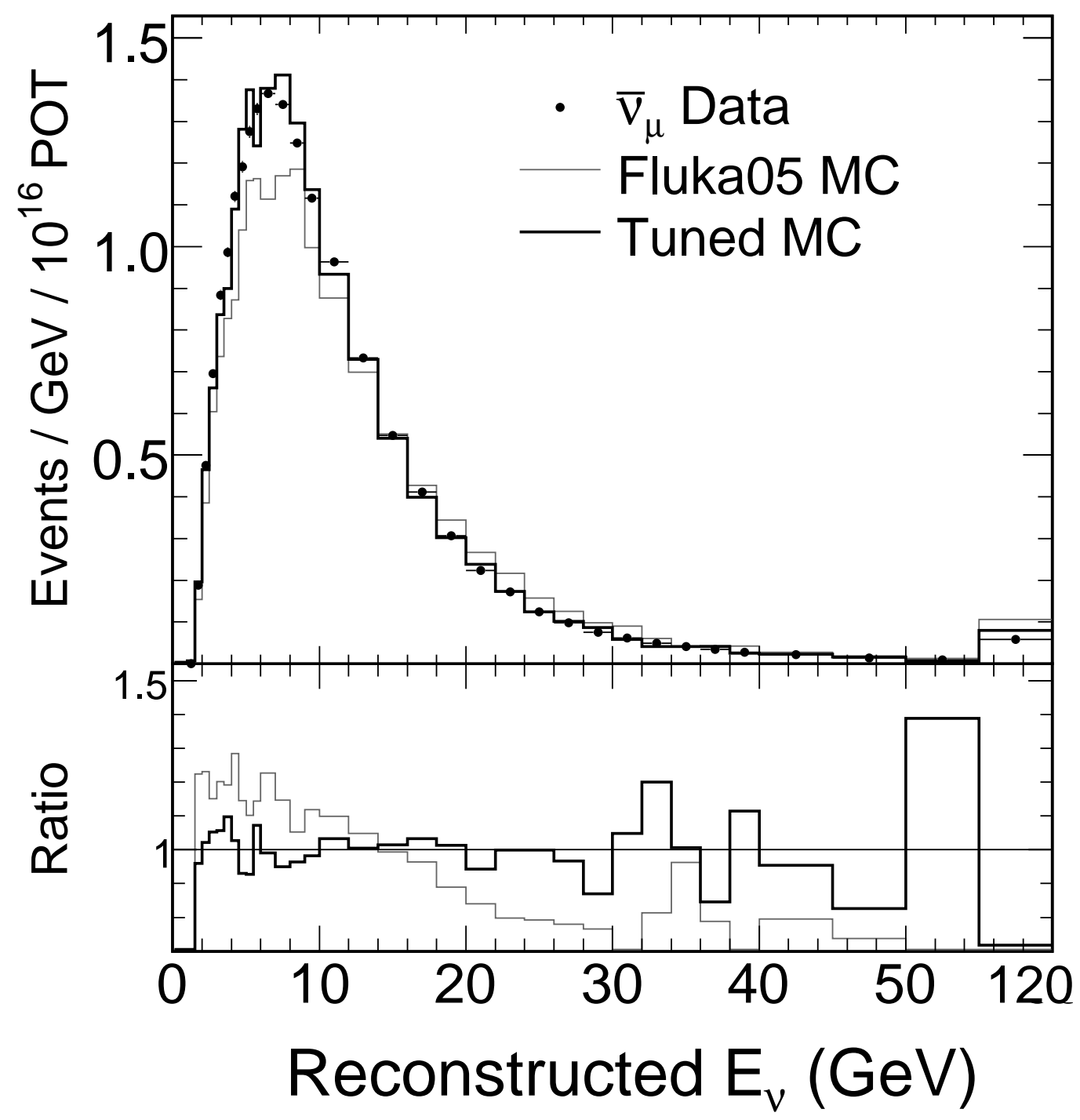

Figure 6.11: The MC flux prediction based on FLUKA cascade model (blue curve) was tuned (red curve) to better agree with the measured $\bar{\nu}_{\mu} \mathrm{CC}$ energy spectrum at the ND (black points). Shown is only the LE010/185kA beam. Data from all of the beam configurations was used in a fit. 
related through:

$$
N\left(K_{L}^{0}\right)=N\left(K_{S}^{0}\right)=\frac{N\left(K^{+}\right)+(2 n-1) N\left(K^{-}\right)}{2 n}
$$

in simplest model we can assume $\mathrm{n}=2$, so we have:

$$
N\left(K_{L}^{0}\right)=\frac{N\left(K^{+}\right)+3 N\left(K^{-}\right)}{4}
$$

This model agrees with $K_{S}^{0}$ production within $15 \%$ up to $x_{F}=0.5$ [66]. At higher $x_{F}$, the dependence of $\mathrm{n}$ on $x_{F}$ becomes important.

Figure 6.12 shows the predicted electron neutrino flux. It can be seen that due to increase in pion yield, the muon yield increased as well resulting in more neutrino flux. Increase in kaon yield increases electron neutrino flux directly, but also through $K_{L}^{0}$ component. 


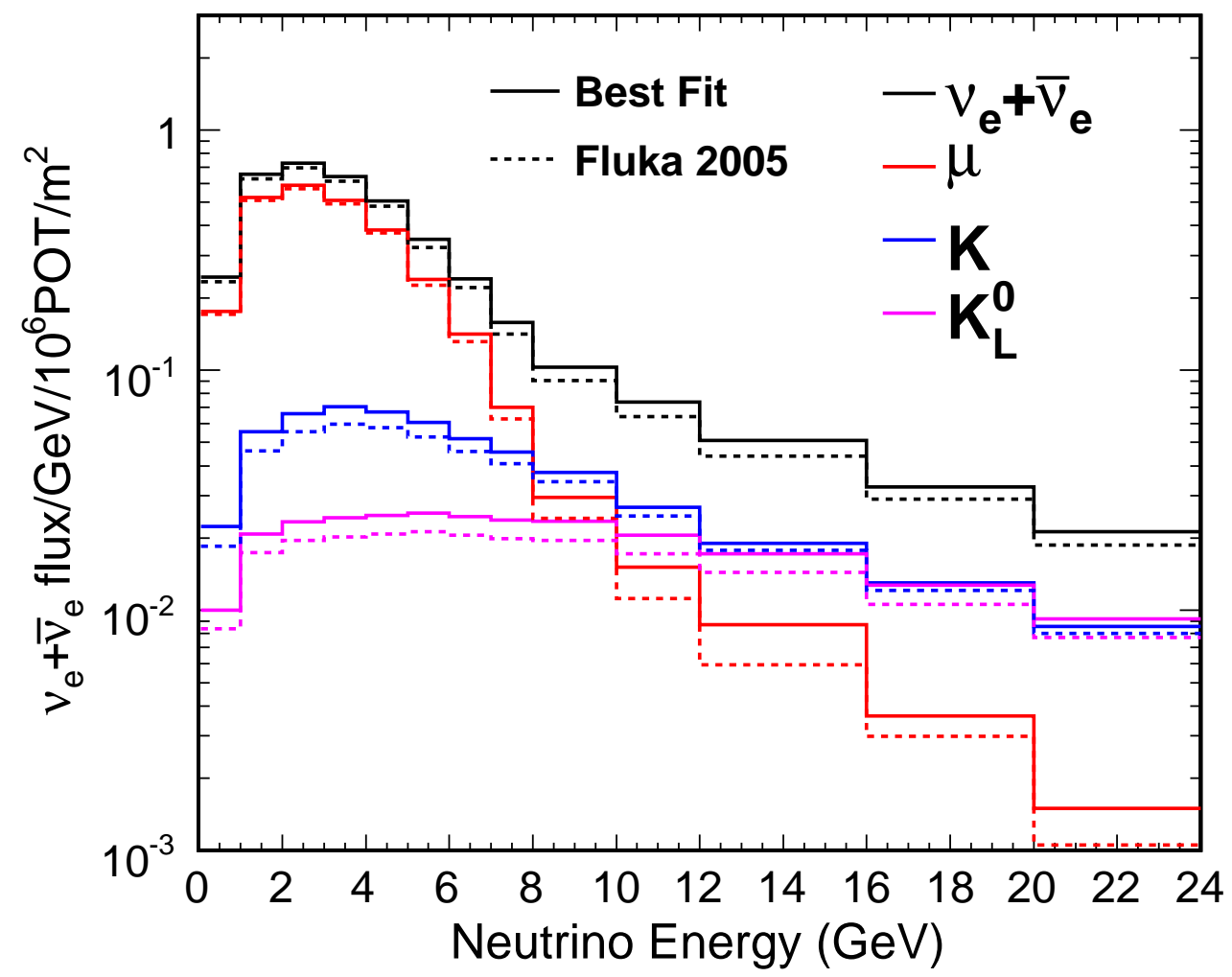

Figure 6.12: The predicted $\nu_{e}+\bar{\nu}_{e}$ energy spectrum at Near Detector. The electron neutrinos share the parents with muon neutrinos. Shown is the prediction using FLUKA cascade model and the prediction after the MC was tuned to measured $\nu_{\mu}$ and $\bar{\nu}_{\mu}$ energy spectra at Near Detector. 


\subsubsection{Impact on Hadron Production}

Already by looking at the disagreement between data and FLUKA based MC in different beam configurations it is possible to predict what some of the effects of the fit on the yields of secondaries is going to be. The $\nu_{\mu}$ data shows significant excess at neutrino energies beyond the focusing peak. This is the region where high energy $\pi^{+}$that go through the necks of the horns (ie have low transverse momentum $p_{T}$ ) contribute most. The same pions contribute to neutrino flux in the LE010/0kA beam. We expect that as a result of the fit the yields of those pions would be increased.

The same argument applies for $\pi^{-}$with low transverse momenta. The $\bar{\nu}_{\mu}$ data when compared to MC prediction also shows the excess in the region of neutrino energy spectrum where these pions contribute (Figure 6.11).

At higher energies the $\bar{\nu}_{\mu}$ data is over-estimated by MC. This suggests that the yields of very high energy $\pi^{-}$secondaries and also the yields of higher energy $K^{-}$may be over-estimated in the MC.

These predictions are confirmed in Figure 6.13. The figure shows the scale factors that should be applied to yields of pions and kaons coming off the target depending on their transverse and longitudinal momentum to get the best agreement between data and MC. It should be noted that these scale factors have meaning only in the regions of phase space which is sampled by ND neutrino data. The overall shift in mean $p_{T}$ from FLUKA prediction was small and equal $2 \mathrm{MeV} / c$, so is well within acceptable shifts compared to other models [59, 60, 61] (see Figure 3.9).

Figure 6.14 shows the ratios of yields of positive pions to negative pions for the tuned MC along with the predictions from several other hadron 


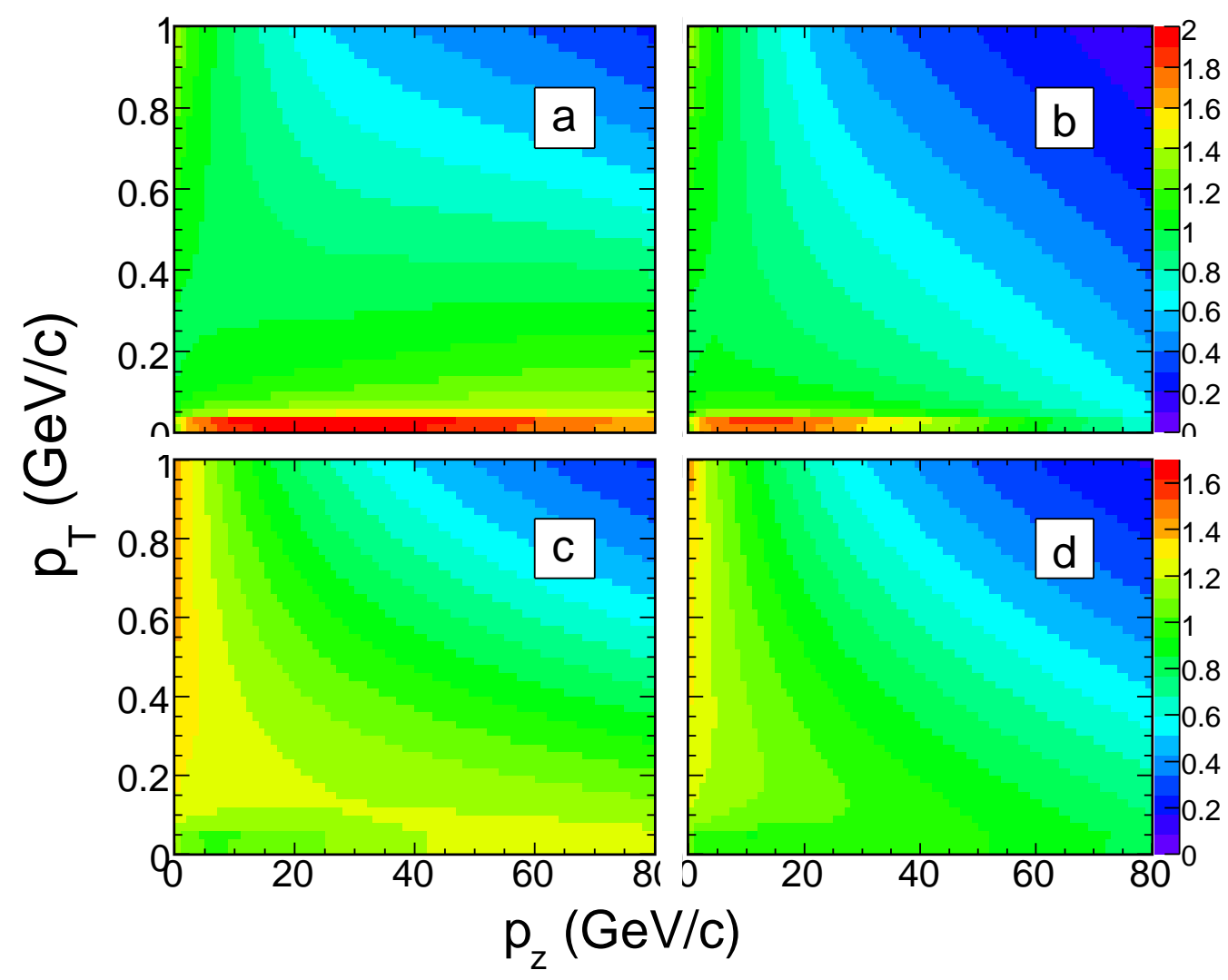

Figure 6.13: The weights that should be applied to secondaries leaving the target depending on their longitudinal $\left(p_{z}\right)$ and transverse momentum $\left(p_{T}\right)$ to achieve better agreement between $\mathrm{MC}$ and Near Detector data. (a) $\pi^{+}$, (b) $K^{+}$, (c) $\pi^{-}$and (d) $K^{-}$. 
production models. The data from NA49 experiment [57] is also shown. This data became available only after the present analysis was completed and it wasn't used in any way in the fit. It is interesting to note that our tuned MC agrees well with the NA49 data.

Figures $6.15,6.16$ and 6.17 show the $K^{+} / K^{-}, K^{+} / \pi^{+}$and $K^{-} / \pi^{-}$ ratios for the tuned MC compared to several other hadron production models. The tuned MC distorts these ratios slightly, however these ratios are not as constrained by data as the $\pi^{+} / \pi^{-}$ratio and these distortions don't appear to be unreasonable.

The qualitative behavior of the ratios as a function of $x_{F}$ can be understood in terms of valence quark structure. The $\pi^{+} / \pi^{-}$ratio rises at high $x_{F}$ similarly as the ratio of valence quark $u_{v} / d_{v}$ of projectile proton. The quark content of $\pi+$ is $(u \bar{d})$ and of $\pi^{-}(d \bar{u})$. The $K^{+} / K^{-}$ratio diverges at large $x_{F}$ due to the fact that $K^{-}(s \bar{u})$ does not contain any valence quark from projectile, while $K^{+}(u \bar{s})$ does. The $K^{+} / \pi^{+}$ratio is fairly constant since both $\pi^{+}$ and $K^{+}$contain $u_{v}$-quark from the proton projectile. Finally the $K^{-} / \pi^{-}$ratio falls off at high $x_{F}$ since $\pi^{-}$contains the valence $d_{v}$-quark from the projectile, while $K^{-}$must come from projectile sea quarks. 


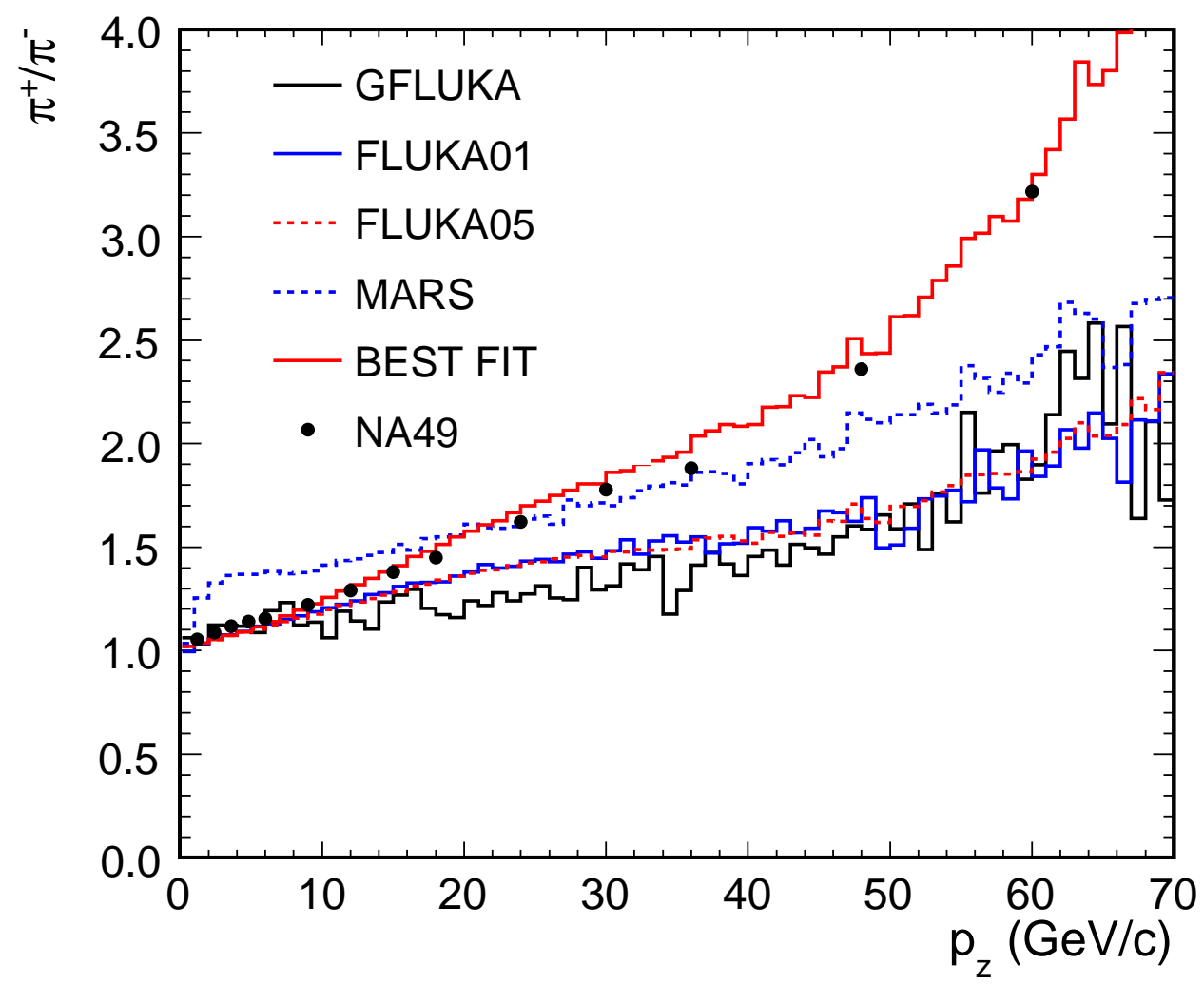

Figure 6.14: The ratio of $\pi^{+}$and $\pi^{-}$yields as a function of longitudinal momentum $\left(p_{z}\right)$ of secondary pions at production target. Shown are the ratios before and after MC tuning along with a prediction from few other hadron production models and NA49 data [57]. Tuned MC prefers higher ratio at larger momentum $p_{z}$ than the predicted ratio using FLUKA hadron production model. This agrees well with NA49 data. 


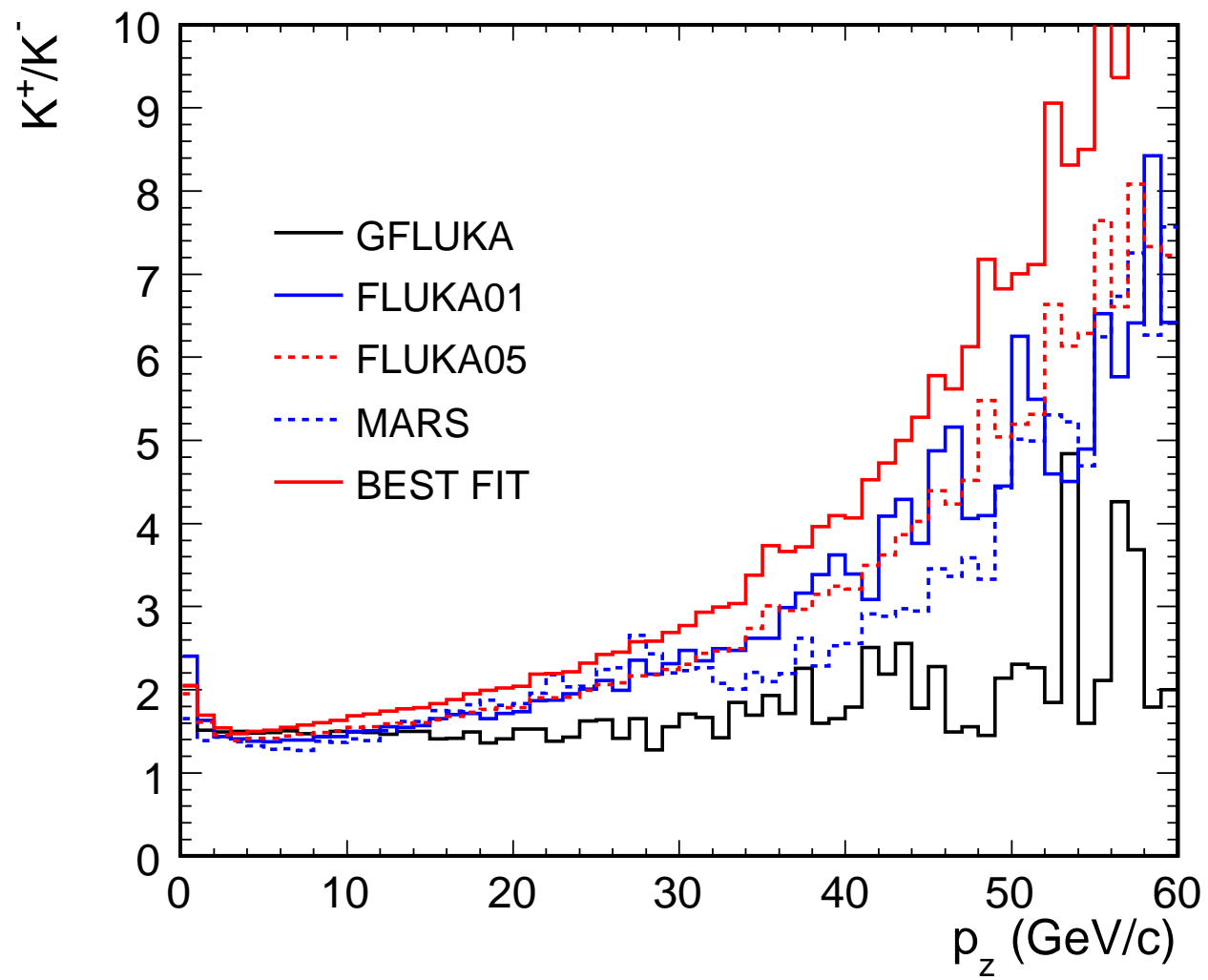

Figure 6.15: The ratio of $K^{+}$and $K^{-}$yields as a function of longitudinal momentum $\left(p_{z}\right)$ of secondary kaons at production target. Shown are the ratios before and after MC tuning. Prediction from few other hadron production models are shown for comparison. 


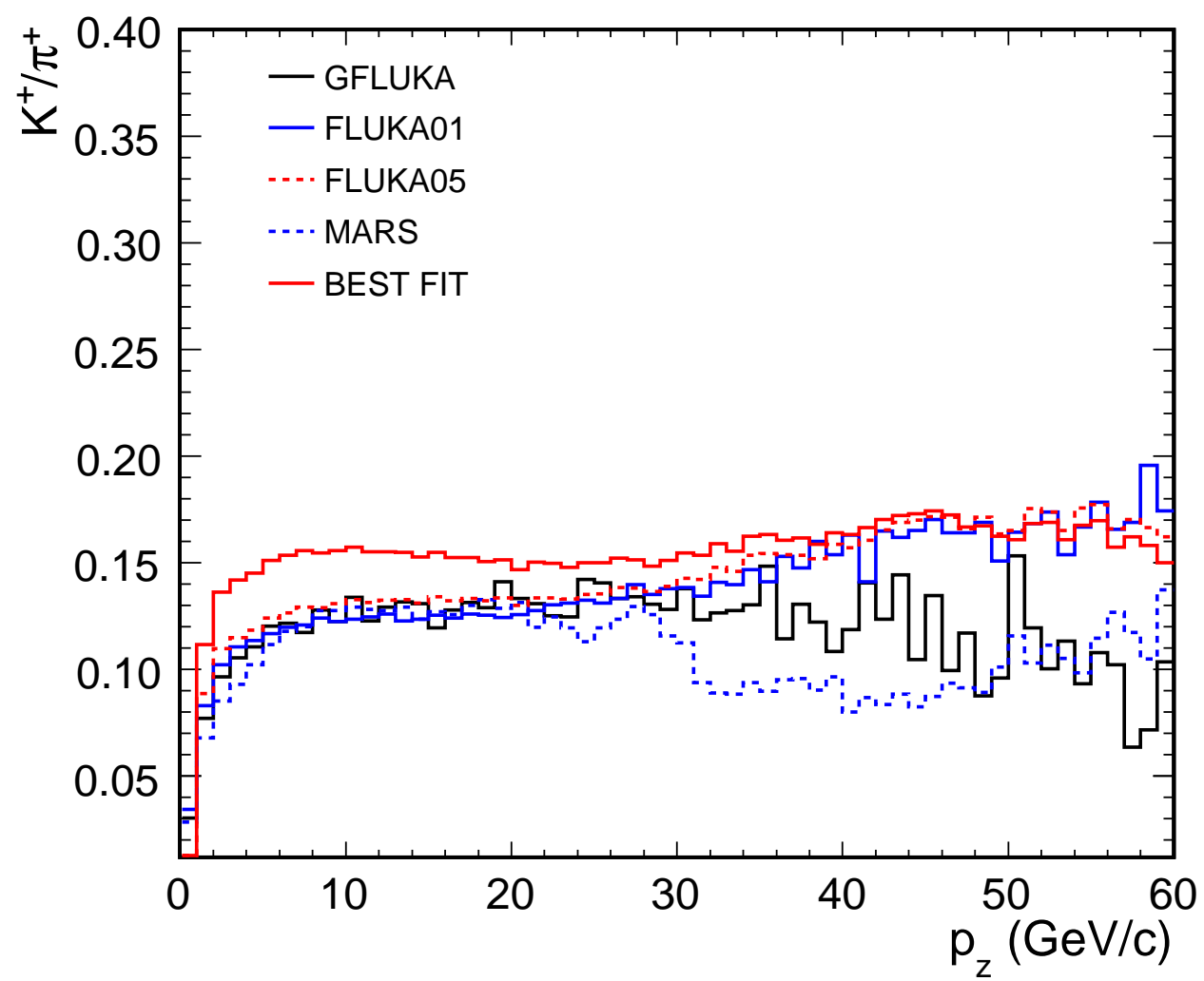

Figure 6.16: The ratio of $K^{+}$and $\pi^{+}$yields as a function of longitudinal momentum $\left(p_{z}\right)$ of secondaries at production target. Shown are the ratios for before and after the MC tuning. For comparison ratios from different hadron production models are also shown. 


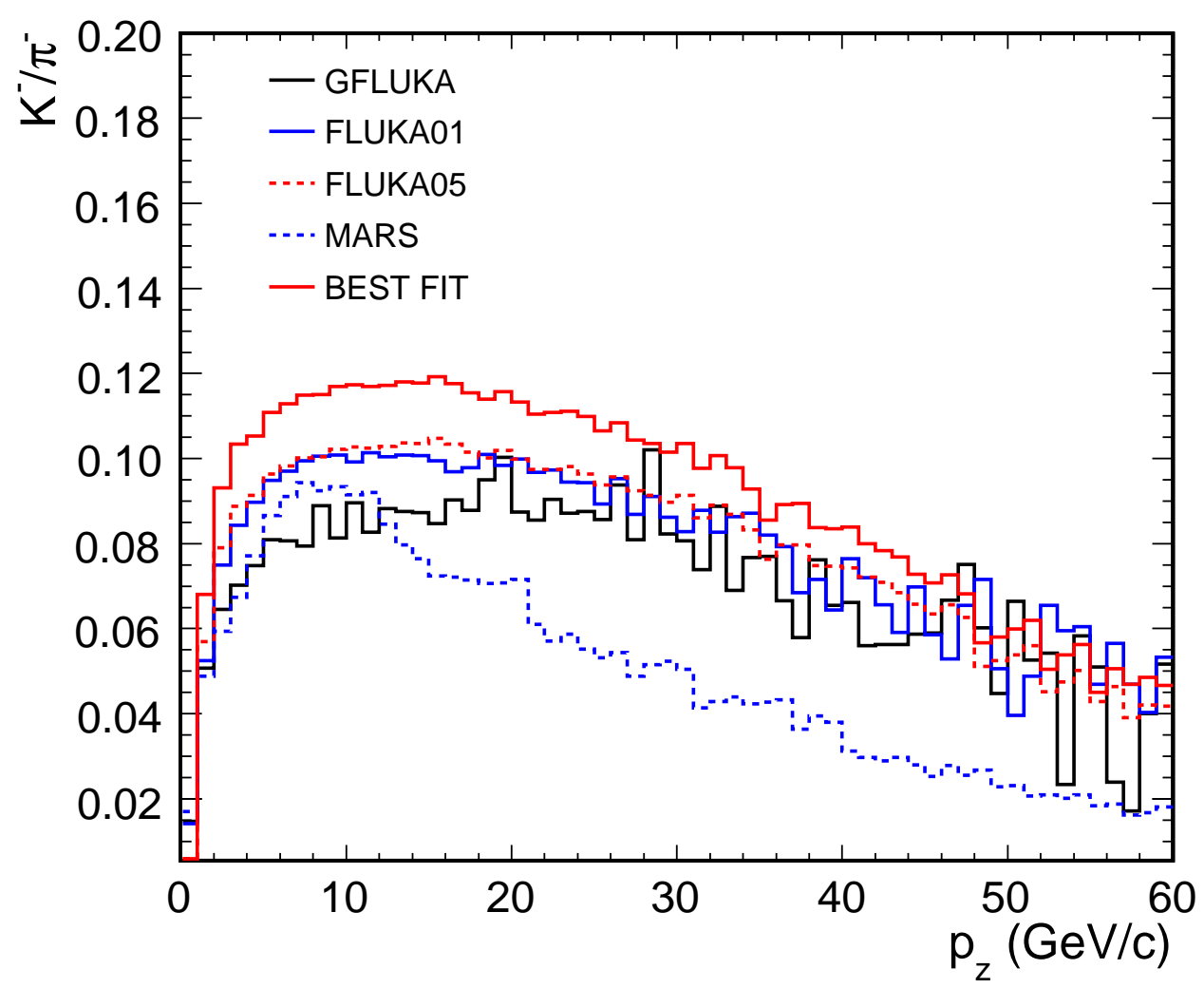

Figure 6.17: The $K^{-}$and $\pi^{-}$yields as a function of longitudinal momentum $\left(p_{z}\right)$ of secondaries at production target. Shown are the ratios for before and after the MC tuning. For comparison ratios from different hadron production models are also shown. 


\subsection{Neutrino Flux Uncertainty After Tuning}

The ND data adds an additional constraint on the neutrino flux prediction. The procedure described in this chapter tunes the MC to better describe MINOS neutrino data. While, in principle, the observed event rate in the MINOS ND is the product of the $\nu$ flux and the interaction cross section, each of which have substantial uncertainties, the variable beam design of NuMI helps break the energy dependence of the degeneracy between neutrino cross sections $\sigma_{\nu}$ and neutrino flux $\phi_{\nu}$.

The constraint from the ND data reduces the uncertainty in the flux prediction, though some residual uncertainty remains due to the statistical uncertainty on the parameters used in the fit. How this uncertainty propagates into the neutrino flux can be estimated by looking at how varying the parameters of the fit affects the neutrino flux. The best fit values for hadron production parameters along with the errors on the parameters were given in Table 6.4. Varying all the parameters simultaneously within $1 \sigma$ away from their best-fit value would over-estimate the error. Some of the parameters are correlated and this has to be taken into account to properly calculate the uncertainty.

We generate 750 sets of parameters, taking into account the correlations between them, and use them to generate neutrino flux predictions. For each neutrino energy bin we take the maximal deviation from the best fit result as the uncertainty. The result for $\nu_{\mu}$ flux is $\sim 2 \%$ uncertainty in focusing peak region of the spectra and $\sim 3 \%$ uncertainty at higher energies. The uncertainty in far over near ratio is very small in the peak and generally less than $1-2 \%$. Figure 4.3 shows the remaining uncertainty due to beam uncertainties. The 
$\bar{\nu}_{\mu}$ flux is less constrained and the uncertainties range from $4-8 \%$. The $\bar{\nu}_{\mu}$ far over near ratio is constrained to $1-4 \%$.

As discussed above, fitting pions and kaons also affects the $\nu_{e}$ and $\bar{\nu}_{e}$ flux prediction. It is expected that the uncertainty in this prediction is also reduced. However, $\nu_{e}$ and especially $\bar{\nu}_{e}$ spectra have a component coming from $K_{L}^{0}$. This neutral kaon component is not very well constrained by fitting charged pions and kaons. It is, therefore expected that the uncertainty in $\nu_{e}$ and $\bar{\nu}_{e}$ flux would not be reduced as much. The assumed uncertainty in the $K_{L}^{0}$ component was $50 \%$. The resulting uncertainty in $\nu_{e}$ flux rises from $2 \%$ at low energies up to $10 \%$ around $20 \mathrm{GeV}$, while for $\bar{\nu}_{e}$ the uncertainty rises from 10 to $30 \%$. The uncertainty in far over near ratio is approximately two times smaller. Figures showing this uncertainty can be found in Appendix E. 


\section{Chapter 7}

\section{Changes in Running Conditions}

Figure 7.1 shows the neutrino energy spectrum in the Near Detector for two run periods, "Run I" which spans the period March, 2005-February, 2006 and "Run II" which spans September, 2006-March, 2007 (see Table 2.1). The two spectra show a statistically significant difference which varies as a function of $\nu$ energy. The calibration procedure of MINOS detectors accounts for the temporal changes in detector response $(\S 2.2 .2)$, so this suggests that the root cause of the change is a change in neutrino flux.

The first run period ended in February 2006 when the Fermilab accelerator complex went into a several months long shutdown period. During this period various maintenance operations were performed. When operations resumed in June 2006, marking the start of run II period, MINOS started acquiring data in the LE150/200kA configuration and then switched to the LE250/200kA configuration. After two months of running, the NuMI target had to be replaced. The NuMI beam started running again in September, 2006 in the LE010/185kA configuration. Hence, between the two runs shown in Figure 7.1 the shielding in the target hall was moved as well as both fo- 
cusing horns and the target was replaced. Replacing or moving any of the components could possibly result in a systematic change of neutrino energy spectrum as misplacement of any element can affect beam focusing.

In this chapter we examine the possible sources of the discrepancy. We discuss its the impact on the predicted flux at the Far Detector (FD).

\subsection{Possible Explanations of Differences Be- tween Two LE010/185kA Data Sets}

The two neutrino spectra from two run periods differ mostly in the $5 \mathrm{GeV}$ region of the spectrum. This is the falling edge of the focusing peak in the LE010/185kA beam configuration and many effects related to focusing could cause such distortion (Figure 5.23). Significantly a separate Near Detector data set acquired without pulsing the horns showed no discrepancy between the run I and run II period suggesting that the focusing is indeed the source of the discrepancy.

The size of the observed distortion is $\sim 5 \%$ in the reconstructed neutrino energy spectrum. This means that true neutrino flux is distorted even more in certain energy bins, but due to finite resolution of the MINOS detectors it gets smoothed out. We therefore look for an effect which can produce a sizeble distortion of the spectrum in the $5 \mathrm{GeV}$ region.

We first consider transverse alignment of the target or horns. The misalignment of the second horn produces a relatively small effect at higher energies, and it would require a few millimeter misalignment of the first horn (Figures 5.14 and 5.15) to explain the observed discrepancy. Such a big mis- 


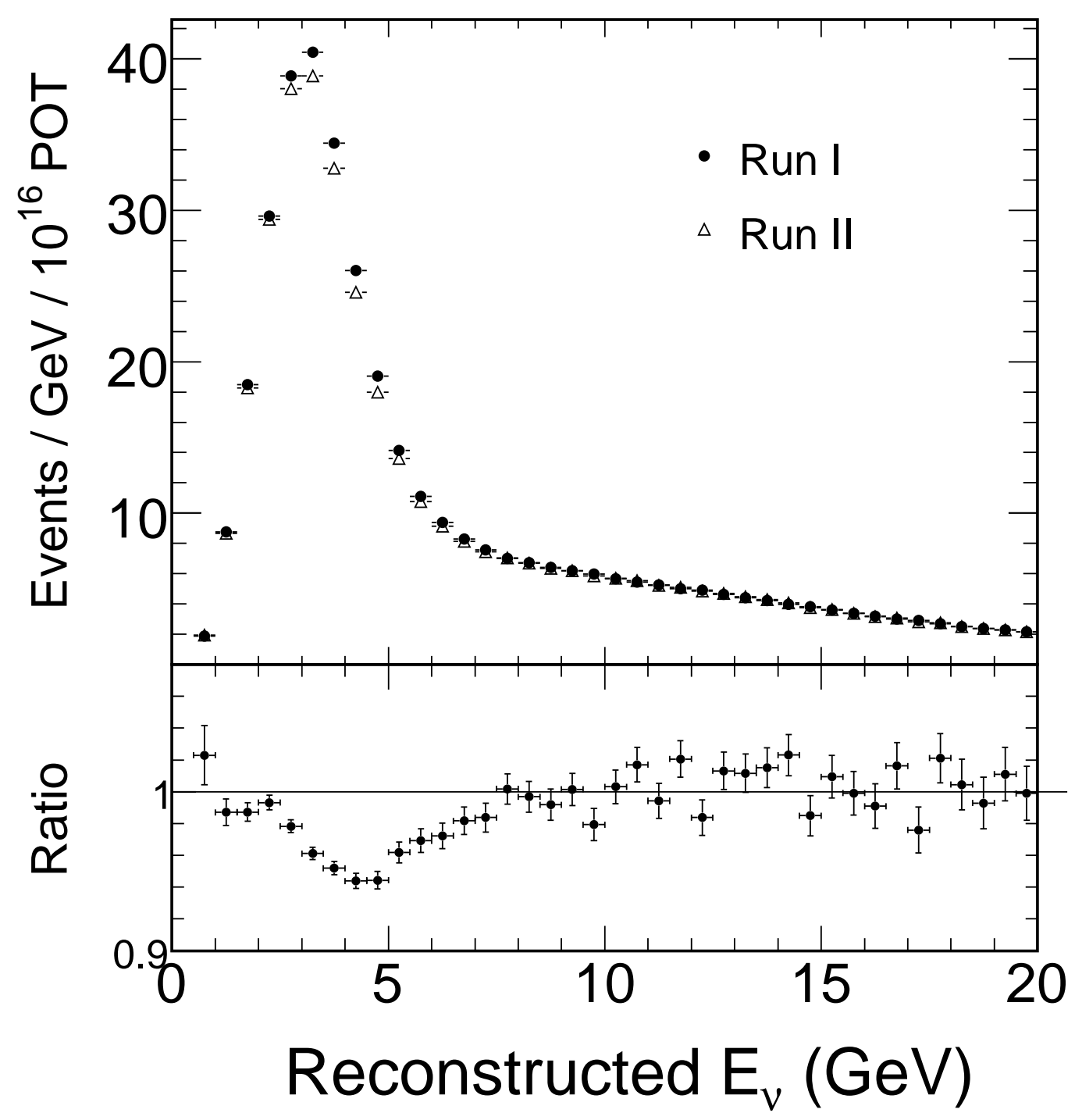

Figure 7.1: The measured $\nu_{\mu}$ Charged Current spectrum in the Near Detector. Shown is the data taken in LE010/185kA beam configuration (Table 2.1) obtained during two run periods. A $5 \%$ drop in the flux is observed at the falling edge of the peak. Several systematic effects related to the neutrino beam can produce such distortion as can be seen in Figure 5.23. 
alignment of first horn is highly unlikely and it wasn't supported by beam based alignment which was used to check the transverse alignment [41]. Similar distortion would be produced by transverse misalignment of the target. Beam based alignment is used to center the proton beam on the target. For that reason the transverse misalignment of the target becomes equivalent to transverse misalignment of the horns and equally unlikely to explain the disagreement.

The change in the horn magnetic field is another candidate. The field can change if the current flowing through the inner conductor changes in either its magnitude or its distribution. By Figure 5.23, a $\sim 3 \mathrm{kA}$ change would be required to explain the discrepancy. The instrumentation monitoring the current flowing through horn conductors is able to measure relative changes to better than $0.2 \%$ and showed no evidence for a change between Run I and Run II (Figure 5.19). The distribution of the current could possibly change due to the corrosion of the inner conductor. The corrosion is a result of humid and radioactive environment and it was in fact observed when the horn was removed from the beam-line and inspected. The effect of corrosion would be similar like the one shown in Figure 5.21 where the effect of different distribution of current in the inner conductor was calculated. There are couple of reasons why this seemed to be unlikely source of disagreement. First, it seems that the effect is going in the opposite direction, ie the distribution of the current would need to get broader due to corrosion. Second, it would be expected that this effect would be more gradual. Figure 7.2 compares the LE250/200kA data taken during run I and run II. The two data sets are consistent, suggesting that at the beginning of run II, the horns performed the same as in run I. 


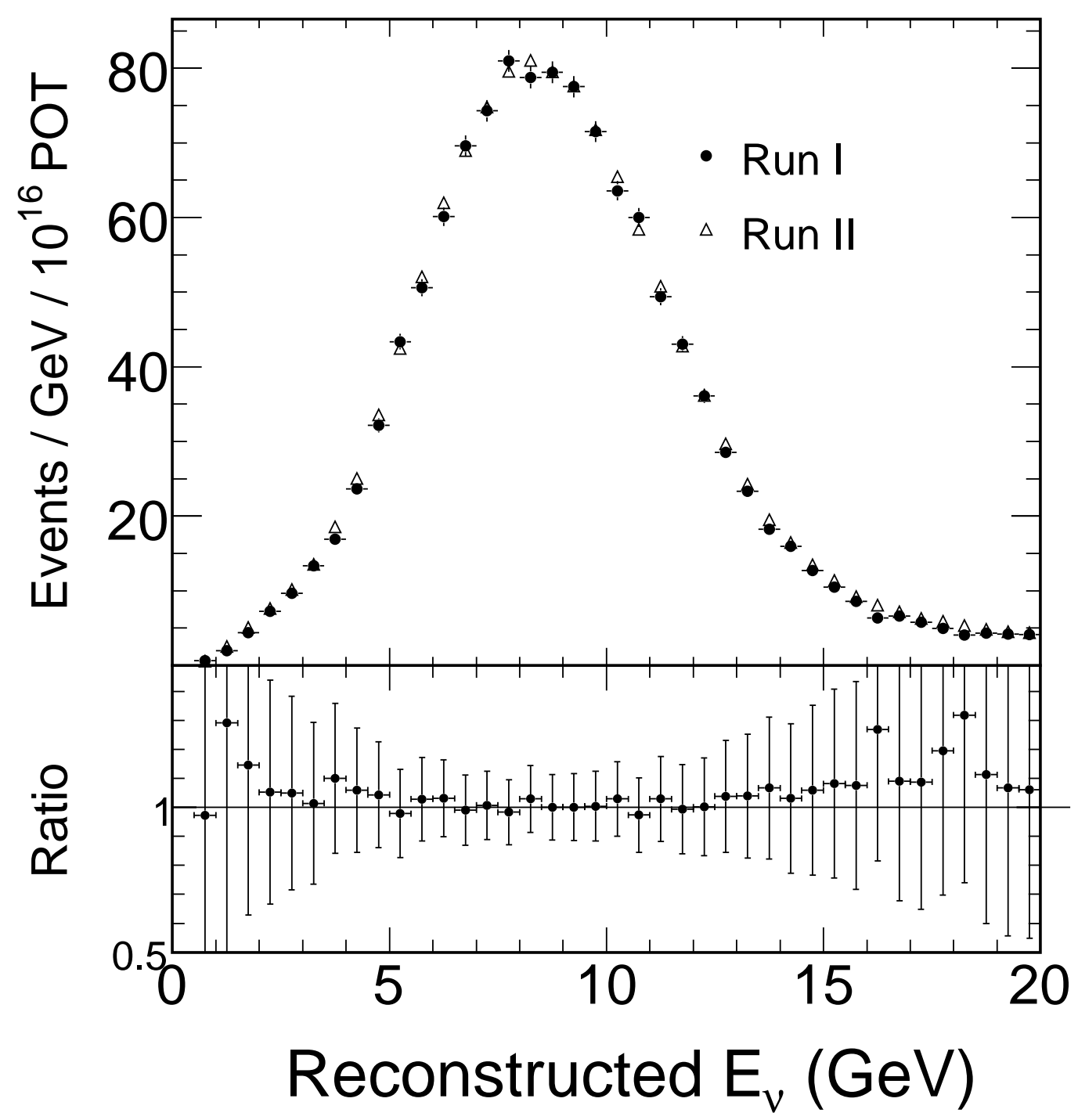

Figure 7.2: The measured $\nu_{\mu}$ Charged Current spectrum in the Near Detector. Shown is the data taken in LE250/200kA beam configuration (Table 2.1) obtained during two run periods. Unlike for LE010/185kA data (Figure 7.1), the two data sets appear to be consistent. 
The agreement between the data taken in LE250/200kA configurations during run I and run II period further suggests that the change occurred when the spare target was installed. This happened just before MINOS started acquiring the data in LE010/185kA configuration in run II period and after LE250/200kA data was taken.

The fraction of the beam hitting the baffle is another possible reason for discrepancy (see Figure 5.8). However the number of protons hitting the baffle is measured by directly measuring the transverse distribution of protons in the final SEM chamber and also by monitoring the temperature of the baffle (see $\S 2.1 .3)$. Both methods suggest that during the two periods fraction of protons hitting the baffle did not change appreciably and the fraction of the beam hitting the baffle was at the level of $0.6-0.7 \%$ during both run periods [77].

In $§ 5.2 .2$ it was discussed that the target used during the first run period had the first, horizontal graphite fin misaligned. As a result some of the protons were missing it. The horizontal fin in the target, which was used in run II period, was aligned on the beam axis so all of the protons struck it. A $\mathrm{MC}$ calculation of the effect of completely removing the horizontal fin is shown in Figure 5.12. Scaling down the size of the effect using the measured fraction of the beam that was hitting the horizontal fin suggests that this could cause only a small $\sim 1 \%$ effect, insufficient to explain the discrepancy in Figure 7.1.

The final effect that we consider is the target longitudinal position, which is established by optical survey of the baffle-target assembly once the target is installed and covered with shielding. Regardless if the assembly is placed in exact same location, the longitudinal position of target itself can change depending how exactly it was mounted on a carrying module. The effect of such longitudinal misalignment, shown in Figure 5.18, is roughly linear 
for few centimeter displacement of the target and is the best candidate to explain the discrepancy observed in Figure 7.1 because the new target was installed just prior to the Run II data collection.

\subsection{Determination of Target Position}

We now attempt to use the ND data taken in LE010/185kA configuration from Run I and Run II to determine target's longitudinal position. We performed two different fits using the procedure described in Chapter 6. The first fit (Fit \#1) was just like the fit presented in Chapter 6 with the addition of the data taken in LE010/185kA configuration during Run II period. Additionally, one more parameter was added to the MC tuning procedure to allow for the position of the target in the Monte Carlo simulation of Run II data. In the second fit (Fit \#2) we allowed the longitudinal position of the target to vary for $\mathrm{MC}$ simulations of all the beam configurations included in the fit. However, since the data in LE010/170kA, LE010/185kA and LE010/200kA configuration during Run I period were taken without moving the target we constrain the target position to be the same for those configurations. In total 5 more fit parameters were added in Fit \#2.

Table 7.1 summarizes the $\chi^{2}$ values for all ND data under the two Fits described above. Both fits show significant improvement in the $\chi^{2}$ when compared to the raw Monte Carlo calculation. Including additional parameters for the target longitudinal position yields an overall better fit and further reduction of $\chi^{2} / N D F$ by $\sim 0.2$ when compared to Fit $\# 1$. It can be seen that the improvement is achieved primarily in agreement with the $\nu_{\mu}$ data, while $\bar{\nu}_{\mu}$ data shows similar agreement in both fits. This is expected since anti 
neutrinos come from unfocused particles and are not affected by changes in focusing.

Table 7.2 lists the fitted target positions. Both fits yield a similar shift $\Delta z=-1 \mathrm{~cm}$ in target position for the Run II LE010/185kA data. Fit \#2 also predicts that the target was shifted in the opposite direction for LE010 beam configurations taken during Run I period.

Figure 7.3 shows the ratio of run I and run II data compared to MC prediction using the two fits described above. As mentioned the result of the first fit (Fit $\# 1$ ) is a $1 \mathrm{~cm}$ relative shift in target longitudinal position between the two runs. Such shift appears to distort the spectrum in a correct way, but it does not explain the whole discrepancy. In the second fit (Fit \# 2) when target longitudinal position was allowed to change for all data sets, the relative difference in target position for the two runs came out to be $2.9 \mathrm{~cm}$ (see Table 7.2). This was enough to accommodate all the discrepancy.

A shift from the nominal target position was subsequently confirmed for Run II data using optical survey. It was determined that the target was displaced by $1.3 \mathrm{~cm}$ in the direction of the first horn, agreeing with the results of the fits to the neutrino data. The survey data on target position during Run I period is not available and therefore cannot be checked. Likewise, while the fitted z positions of the LE250 beams are correct within errors, an uncomfortable shift in the LE100 and LE150 beams is suggested by Fit \#2 (see Table 7.2). 


\begin{tabular}{lccc} 
Beam & \multicolumn{2}{c}{ Fit $\chi^{2}$} & (statistical errors only) \\
Configuration & FLUKA & Fit \#1 & Fit \#2 \\
\hline \hline$\nu_{\mu}$ LE10/000kA & 1042 & 151 & 159 \\
$\nu_{\mu}$ LE10/170kA & 579 & 86 & 64 \\
$\nu_{\mu}$ LE10/185kA (Run I) & 1477 & 55 & 47 \\
$\nu_{\mu}$ LE10/185kA (Run II) & 1494 & 60 & 52 \\
$\nu_{\mu}$ LE10/200kA & 554 & 78 & 73 \\
$\nu_{\mu}$ LE100/200kA & 1114 & 175 & 119 \\
$\nu_{\mu}$ LE150/200kA & 866 & 107 & 95 \\
$\nu_{\mu}$ LE250/200kA (Run I) & 515 & 92 & 69 \\
$\nu_{\mu}$ LE250/200kA (Run II & 562 & 75 & 52 \\
$\bar{\nu}_{\mu}$ LE10/000kA & 122 & 50 & 46 \\
$\bar{\nu}_{\mu}$ LE10/170kA & 101 & 52 & 53 \\
$\bar{\nu}_{\mu}$ LE10/185kA (Run I) & 489 & 94 & 88 \\
$\bar{\nu}_{\mu}$ LE10/185kA (Run II) & 460 & 74 & 70 \\
$\bar{\nu}_{\mu}$ LE10/200kA & 66 & 48 & 49 \\
$\bar{\nu}_{\mu}$ LE100/200kA & 121 & 83 & 80 \\
$\bar{\nu}_{\mu}$ LE150/200kA & 75 & 33 & 35 \\
$\bar{\nu}_{\mu}$ LE250/200kA (Run I) & 76 & 44 & 44 \\
$\bar{\nu}_{\mu}$ LE250/200kA (Run II) & 70 & 59 & 56 \\
\hline Total (all beams)/NDF & $9783 / 952=10.3$ & $1415 / 926=1.54$ & $1250 / 921=1.36$ \\
\end{tabular}

Table 7.1: The $\chi^{2}$ values when comparing the neutrino energy spectrum between data and MC. The first column compares raw Monte Carlo calculation based on Fluka cascade model. The other two columns compare the tuned MC using two different fit models. Fit \#1 is just like the fit described in Chapter 6 with the addition of Run II data taken in LE010/185kA beam configuration and an additional parameter used to effectively modify target position in the Monte Carlo simulation of the added beam. In Fit \#2 the position of the target in all of the beam configurations was allowed to change. 


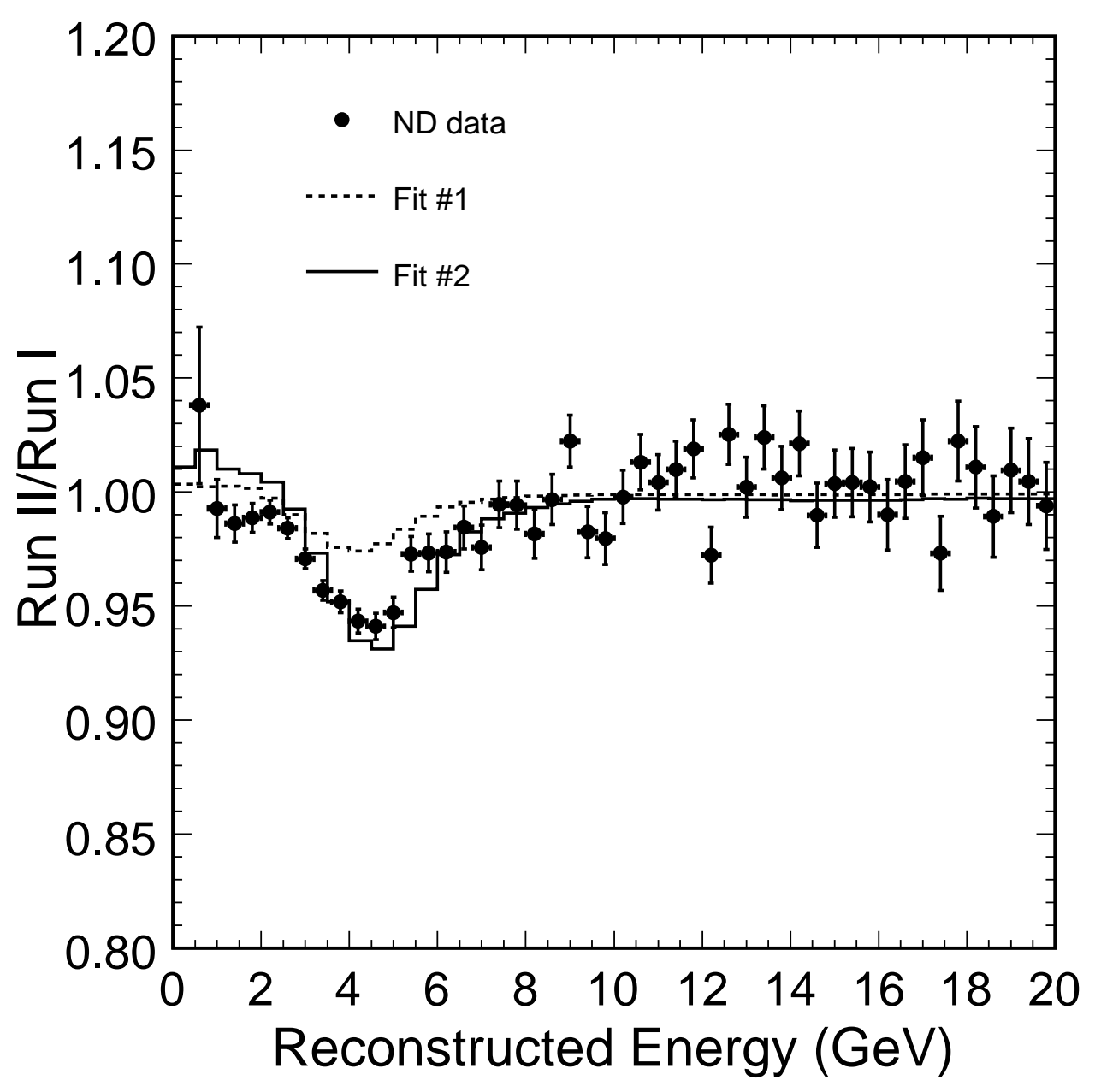

Figure 7.3: The ratio of run I and run II Near Detector data from Figure 7.1 (black points) is compared to different tunings of Monte Carlo, Fit \#1 and Fit \#2. In Fit \#1 the longitudinal position of the target in Run II period was adjusted to achieve best fit (dashed line), while in Fit \#2 the longitudinal position of the target was adjusted for all beam configurations included in the fit. Both fitting procedures suggested that the target was shifted by $\Delta z \sim$ $-1 \mathrm{~cm}$ during Run II period, however that does not explain the discrepancy fully. Fit \#2 suggests that the target position was different than what was used to calculate the flux even for Run I period by $\Delta z \sim+2 \mathrm{~cm}$. 


\begin{tabular}{lcc}
$\begin{array}{l}\text { Beam } \\
\text { Configuration }\end{array}$ & $\Delta z$ & $\Delta z$ \\
$($ Fit \#1) & $($ Fit \#2) \\
\hline \hline LE10/185kA (Run I) & 0. & $1.9 \pm 0.5$ \\
LE10/185kA (Run II) & $-1.0 \pm 0.4$ & $-1.0 \pm 0.5$ \\
LE100/200kA (Run I) & 0. & $-4.1 \pm 0.6$ \\
LE150/200kA (Run II) & 0. & $-3.1 \pm 0.8$ \\
LE250/200kA (Run I) & 0. & $1.8 \pm 1.3$ \\
LE250/200kA (Run II) & 0. & $0.2 \pm 1.3$
\end{tabular}

Table 7.2: The shifts in the target longitudinal positions as a result of the two fitting methods. The shift in target location for LE10/170kA and LE10/200kA beams was constrained to be the same as for LE10/185kA, since that data was taken without moving the target between the runs.

\subsection{Impact on Oscillation Analysis}

Figure 7.4 shows the calculated far over near ratio for the two run periods using two fit models described above. Both fits predict a slightly different ratio for two run periods due to the relative shift in the longitudinal position in two runs. Comparing the calculated ratio using the two different fit models (ie Fit \#1 and Fit \#2) for a particular run period reveals a small change in the ratio. In the $5 \mathrm{GeV}$ region of the spectrum a $\sim 3 \%$ change can be observed for Run I period (solid versus dot curve). This is expected since in one fit model the longitudinal position of the target is fixed while in the other it is allowed to float for that run period. The ratio predicted by both fit models for Run II period agrees well in the $5 \mathrm{GeV}$ region (dash versus dash-dot) since both fit models achieve best fit for the $\Delta z=-1 \mathrm{~cm}$ shift in target longitudinal position.

It is interesting to note that in Figure 7.4 the two fits differ at the $2 \%$ level in the $\mathrm{F} / \mathrm{N}$ ratio calculated at the higher energy part of the neu- 


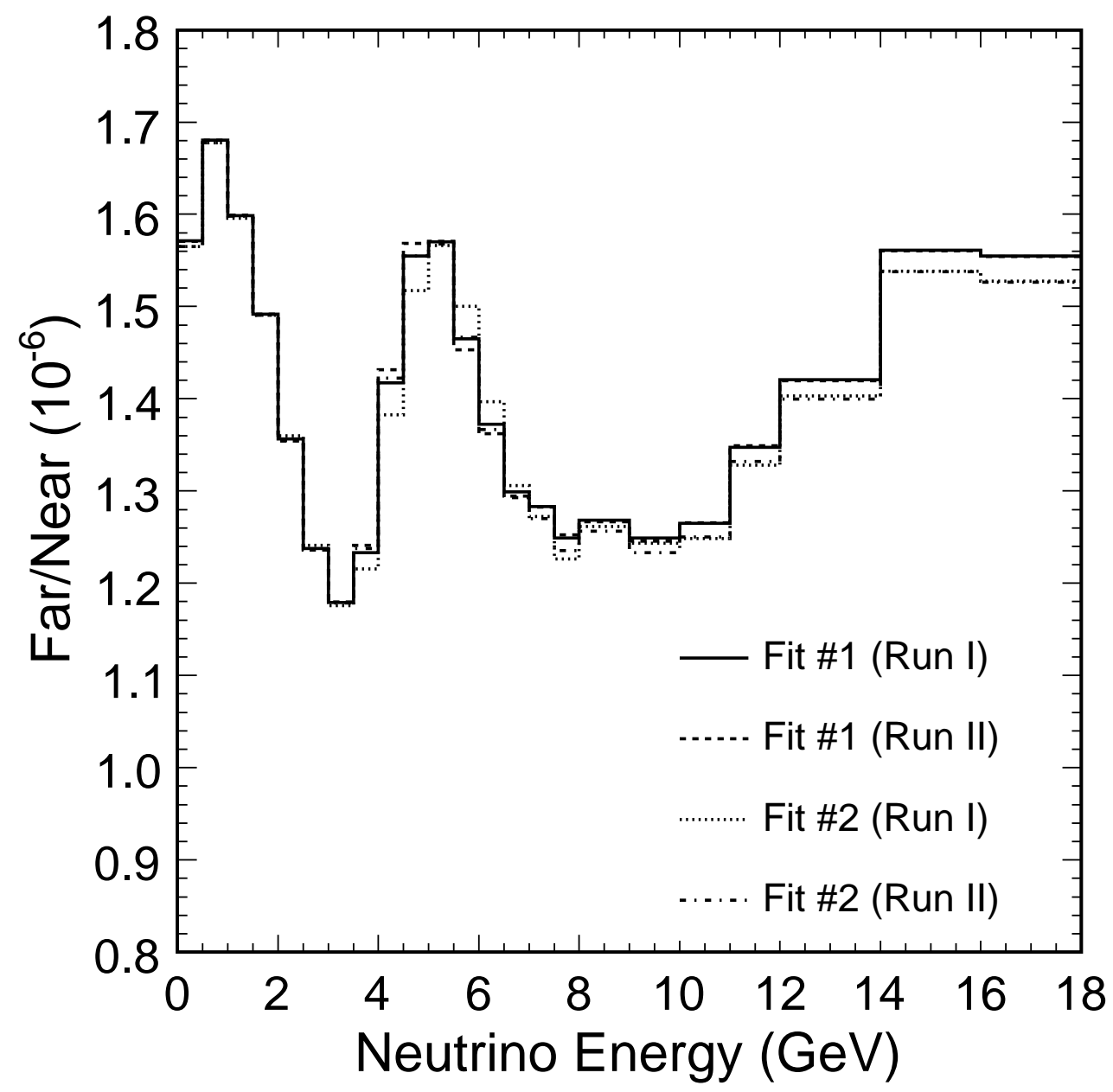

Figure 7.4: Far/Near ratio in the LE010/185kA configuration for two run periods calculated using Monte Carlo simulation which was tuned to Near Detector data. Prediction from two different fits are shown (Figure 7.3). The difference in the ratio between Run I and Run II period is due to the different position of the target. The ratio varies for the two fit models by $\sim 3 \%$ in $5 \mathrm{GeV}$ region and less than $2 \%$ in the high energy tail. 
trino spectrum. This part of the spectrum comes from the particles which are not focused. These particles go through the field free part of the focusing horns and therefore are not affected by changes in beam focusing. The difference arises because in Fit \#2 the target position was allowed to change in all beam configurations that were fitted. Some of the discrepancy between the ND data and raw Monte Carlo calculation (which uses FLUKA prediction of particle yields off the target) in higher energy beam configurations, in particular LE100/200kA and LE150/200kA, was absorbed by shifting the target longitudinal position. In Fit \#1 the target position was allowed to change only for Run II LE010/185kA beam, and the discrepancy in LE100/200kA and LE150/200kA beam configurations was reduced by adjusting the yields of secondary particles in the neutrino target. Therefore, using a different model when fitting the ND data can introduce a small systematic change in the far over near ratio. Becuase we cannot mechanically verify the placement of the target during Run I as we could in Run II, the large fitted displacement of the target $\mathrm{z}$ position in Fit \#2 cannot be confirmed. We, therefore add the difference between Fit \#1 and Fit \#2 to the uncertainty in calculated far over near ratio shown in Chapter 5 . 


\section{Chapter 8}

\section{Observation of Neutrino}

\section{Disappearance}

The Near Detector (ND) measures the $\nu_{\mu}$ energy spectrum before the oscillations have occurred. To estimate the neutrino flux at the FD, we multiply the flux spectrum observed at the ND by the "F/N ratio" from Chapter 4 . We calculate this ratio using the Monte Carlo simulation (Chapter 3) which was tuned to ND data (Chapter 6). The uncertainty in the calculated far over near ratio and therefore in predicted FD neutrino energy spectrum was discussed in Chapters 5 and 7 . In this chapter we analyze the FD data and compare it to our prediction.

\subsection{Far Detector Data}

In order to avoid any possible bias when analyzing the data, the MINOS experiment performed a "blind analysis". The possible bias could arise from knowing the results of Super-Kamiokande [22, 23] and K2K [28] experiments 
and expecting that the MINOS data should exhibit same oscillation features. The procedure for the blind analysis was to store the FD events into two "boxes". One box contained the open data set which was available for analysis at all times. The other box was closed until the analysis procedures were defined. Each event was stored either into an opened or closed box. Furthermore, the events were sorted in such a way that it was impossible to extract the oscillation parameters from the open data set. However, using the open data set it was possible to test the calibration of the detector, reconstruction code as well as monitor that the detector is fully operational over the course of the data collection period.

Figure 8.1 shows, for example, the distribution of reconstructed neutrino interaction vertices in the FD. The figure was made using the open box data set and the predicted spectrum was scaled to the same number of events. The events are evenly distributed throughout the detector. The distribution of vertices along the $\mathrm{z}$ axis reflects the geometry of the FD which consists of two "super-modules" which are separated by an air gap. Although some unknown portion of the data was hidden and presumably some of the neutrinos oscillated, the data in the open box was sufficient to test that the detector was indeed functioning properly.

The full data set using the data from both boxes was analyzed once all the steps of the analysis were defined and tested. No changes to the analysis procedure were done after unblinding the data.

Before looking at the neutrino energy spectrum, however, various distributions were checked to test the integrity of the data.

Figure 8.2 shows the timing of all neutrino events in the FD relative to the time of the nearest beam spill. The events fall in a $\sim 10 \mu s$ window 

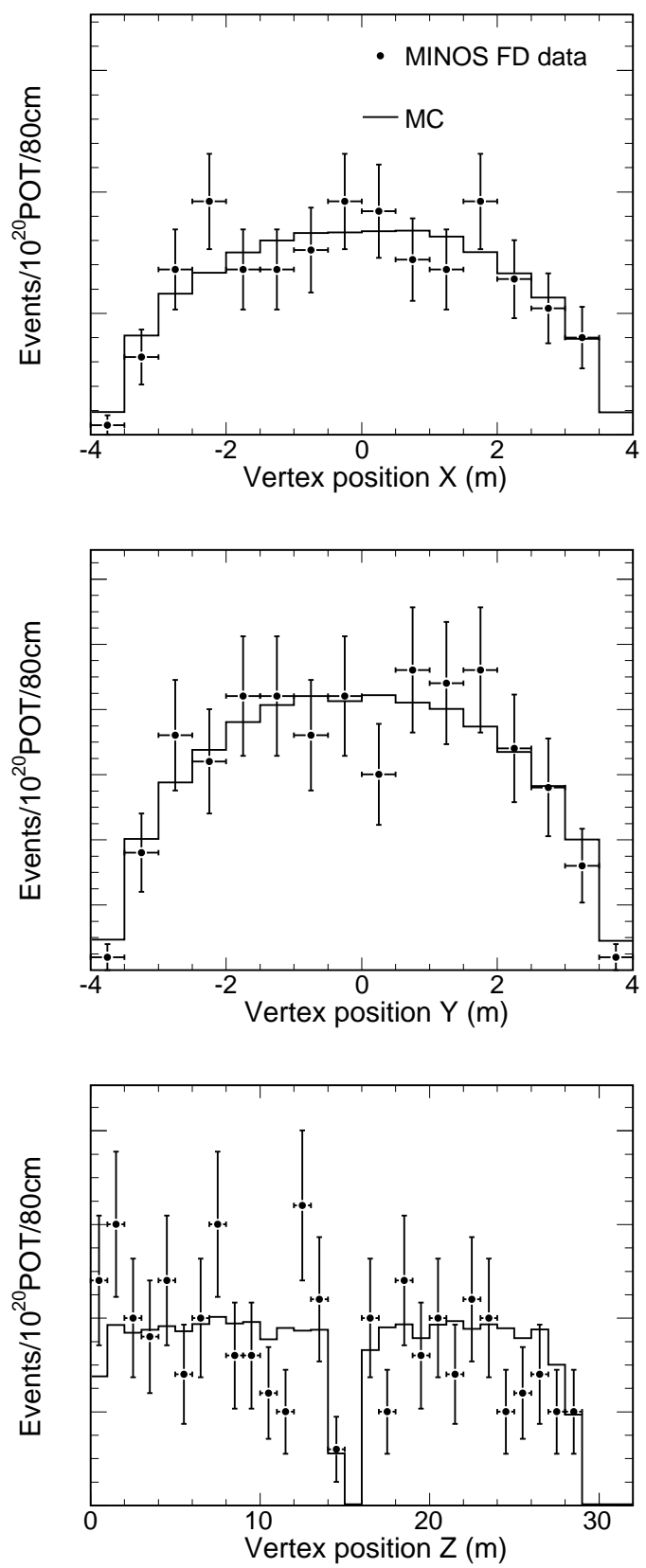

Figure 8.1: The distribution of reconstructed neutrino vertices for $\nu_{\mu}$ ChargedCurrent event candidates in the "open box" (black dots). The Monte Carlo prediction (solid line) was scaled to same total number of events as data. 
which is equal to the length of the proton beam spill from the Main Injector. Furthermore, it can be noticed that the shape of the distribution reflects the expected batch structure of the proton beam (§2.1.1). The time it takes for relativistic neutrinos to reach the $\mathrm{FD}(\approx 2.45 \mathrm{~ms})$ was subtracted.

Figure 8.3 shows the number of neutrino events per POT observed in the FD as a function of time. Only the neutrino events acquired in LE10/185kA configuration (Table 2.1) are shown. The gap between March and September, 2006, corresponds to the accelerator shut-down period and running in LE150/200kA and LE250/200kA configurations. The number of events per POT was constant in time over the data taking period. The rate is slightly different in two run periods due to slightly different target position (Chapter 7).

Figure 8.4 again shows the $\mathrm{x}-\mathrm{y}$ distributions of reconstructed interaction points of the selected FD neutrino events, but now for the all FD data. Again the events appear to be, as expected, evenly distributed throughout the detector. The distributions don't show any asymmetries or clustering.

Figure 8.5 shows the distribution of track directions relative to the incident neutrino beam direction. Interestingly, at the $\mathrm{FD}$, this is $\langle\theta\rangle=+3^{\circ}$, while at the ND it is $\langle\theta\rangle=-3^{\circ}$ due to Earth's curvature and the fact that the beam is steered through the Earth crust (see Figure 2.1). Two predictions are shown, one in which it is assumed that neutrinos don't oscillate and one in which some of $\nu_{\mu} \mathrm{s}$ are oscillated using Equation 1.2. The $\Delta m_{23}^{2}$ and $\sin ^{2}\left(\theta_{23}\right)$ parameters used in the calculation, as well as in figures in the remainder of this chapter, are coming from the best fit to MINOS data which will be discussed in next chapter. It can be seen that the data agrees well with oscillated MC prediction. As mentioned in $\S 2.2 .4$ the selected events in the FD are required to 


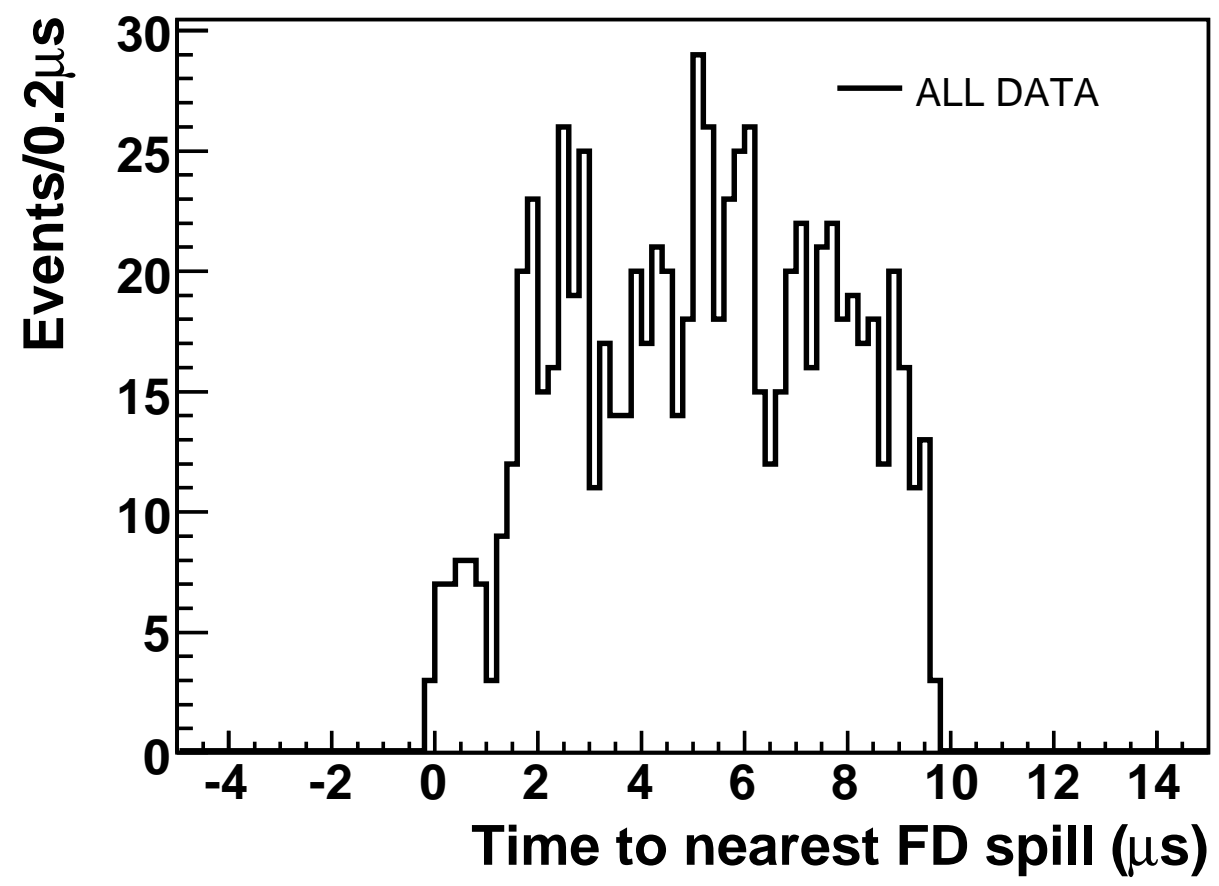

Figure 8.2: Timing of all neutrino events in the FD relative to the nearest beam spill. The timing distribution is consistent with $\sim 10 \mu s$ long proton beam spill. Figure courtesy D. Petyt. 


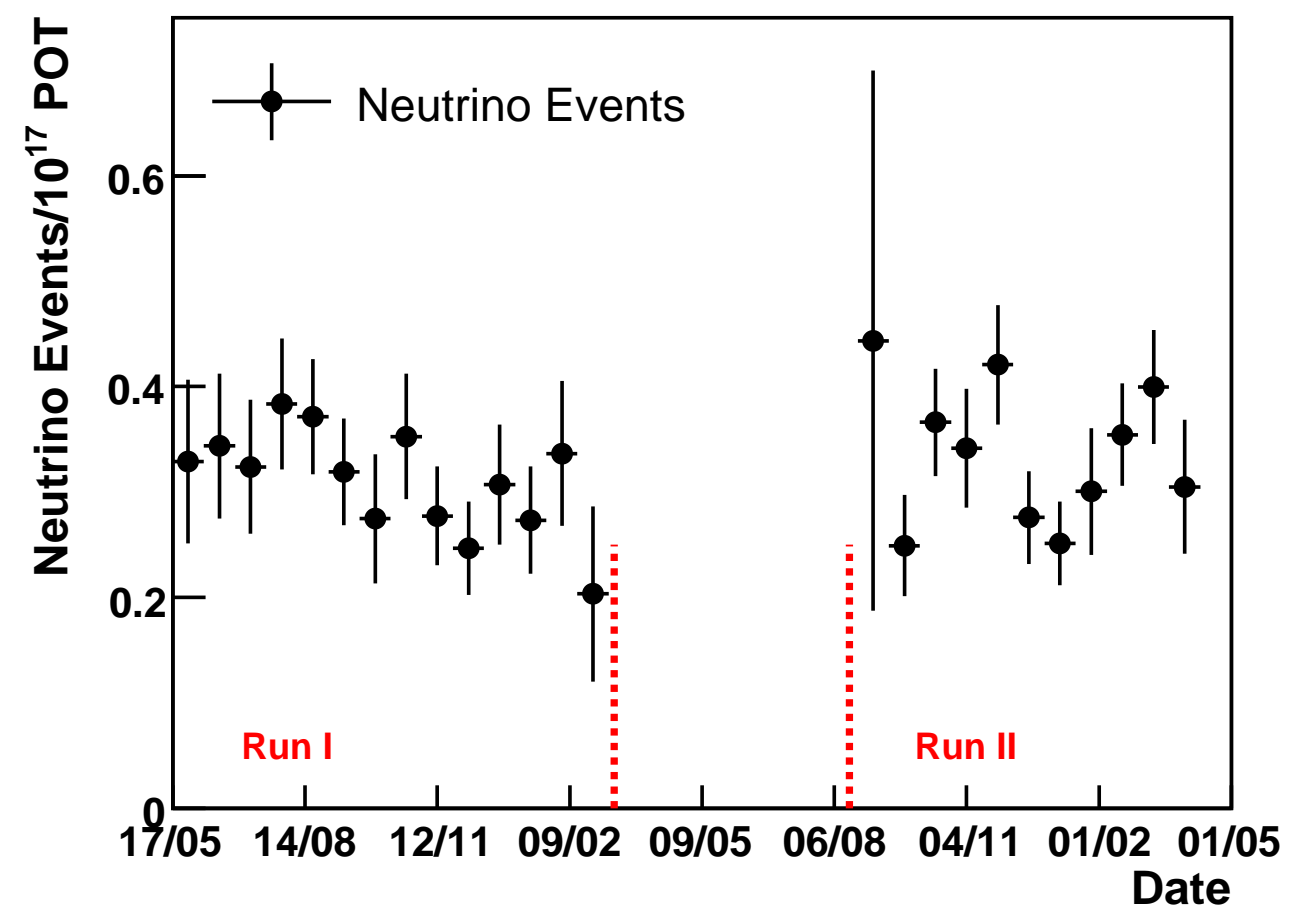

Figure 8.3: Number of neutrino events in the Far Detector normalized to delivered number of proton on target (POT). The event rate was constant in time over the periods of time when the beam was on. Slight difference between the rate during the Run I and Run II periods is observed due to different target positions 7. Figure courtesy D. Petyt. 


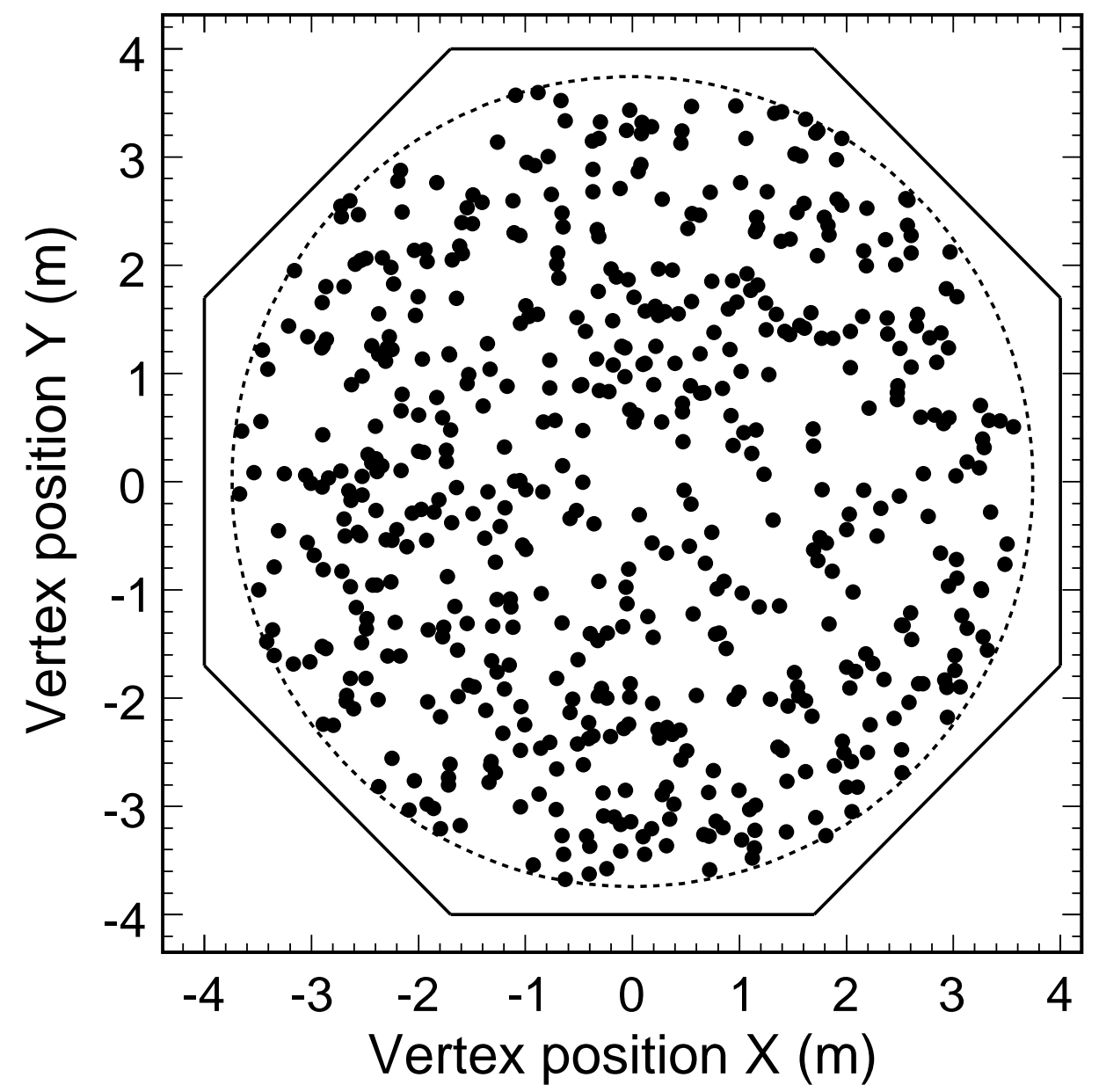

Figure 8.4: The distribution of reconstructed neutrino vertices for all $\nu_{\mu}$ Charged-Current event candidates in the Far Detector. The events are evenly distributed throughout the detector. The solid line traces the shape of the detector, while the dotted line indicates the fiducial volume used for analysis. 
have track direction consistent with neutrino beam direction to further reduce cosmic ray background.

Figure 8.6 shows the distributions of 4 variables used to separate the Neutral Current (NC) events from the Charged Current (CC) events as discussed in $\S 2.2 .4$. The distribution of neutrino events in the $\mathrm{kNN}$ parameter which is calculated based on those four variables and used for event separation is shown in Figure 8.7.

The reconstructed y $\left(y \equiv E_{s h w} / E_{\nu}\right)$ distribution of selected FD neutrino events is shown in Figure 8.8. The distribution shows good agreement with the prediction which assumes neutrino oscillations. Furthermore, the contamination of $\mathrm{NC}$ events in the $\mathrm{CC}$ sample, collected at high $y$, is small.

\subsection{Background}

The Neutral Current (NC) background in the Far Detector is important as it appears in the lower neutrino energy bins where we expect the oscillation signal. The event classification using the $\mathrm{kNN}$ parameter described in Chapter 2 drastically improves the purity of the selected CC events, however, some NC events pass the selection cuts. The Monte Carlo simulation is used to estimate the NC background. The number of true NC events that are classified as $\mathrm{CC}$ events indicates that this background contributes to less than $1 \%$ of events in the unoscillated $\nu_{\mu} \mathrm{CC}$ sample. The uncertainty in the MC prediction was found using MINOS ND data [91] to be $\sim 25 \%$. The uncertainty arises due to uncertainty in reconstruction of a track in hadronic shower and due to uncertainty in the total NC cross-section.

The other potential background in the FD comes from Cosmic rays. 


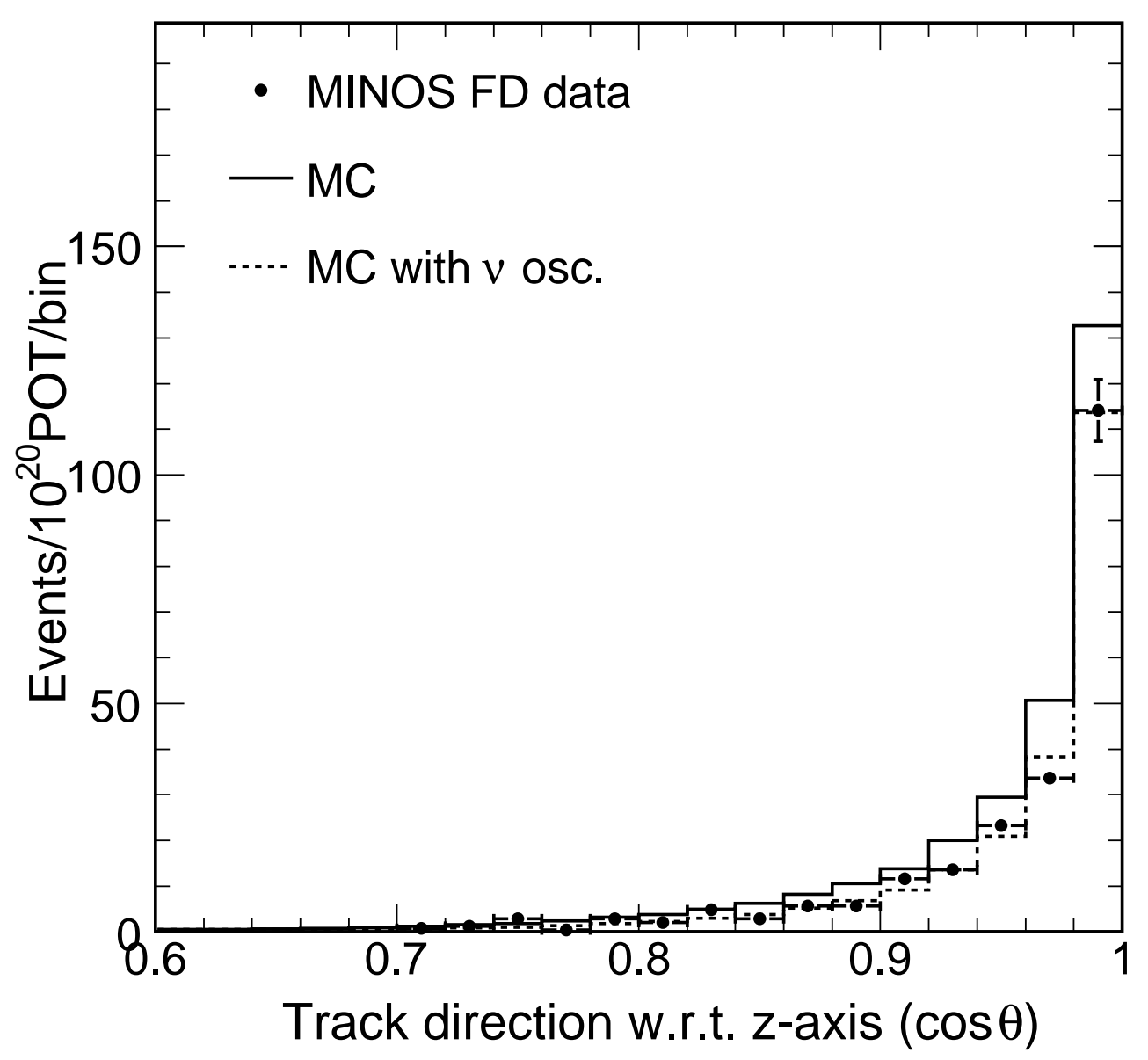

Figure 8.5: The distribution of reconstructed neutrino track directions relative to incident neutrino beam for the selected $\nu_{\mu}$ Charged-Current event candidates (black dots). Predicted distributions assuming that neutrinos do oscillate (dashed line) and don't oscillate (solid line) are shown as well. The oscillated distribution was generated using oscillation parameters $\Delta m^{2}=$ $2.38 \times 10^{-3} \mathrm{eV}^{2} / c^{4}$ and $\sin ^{2} 2 \theta=1$. 

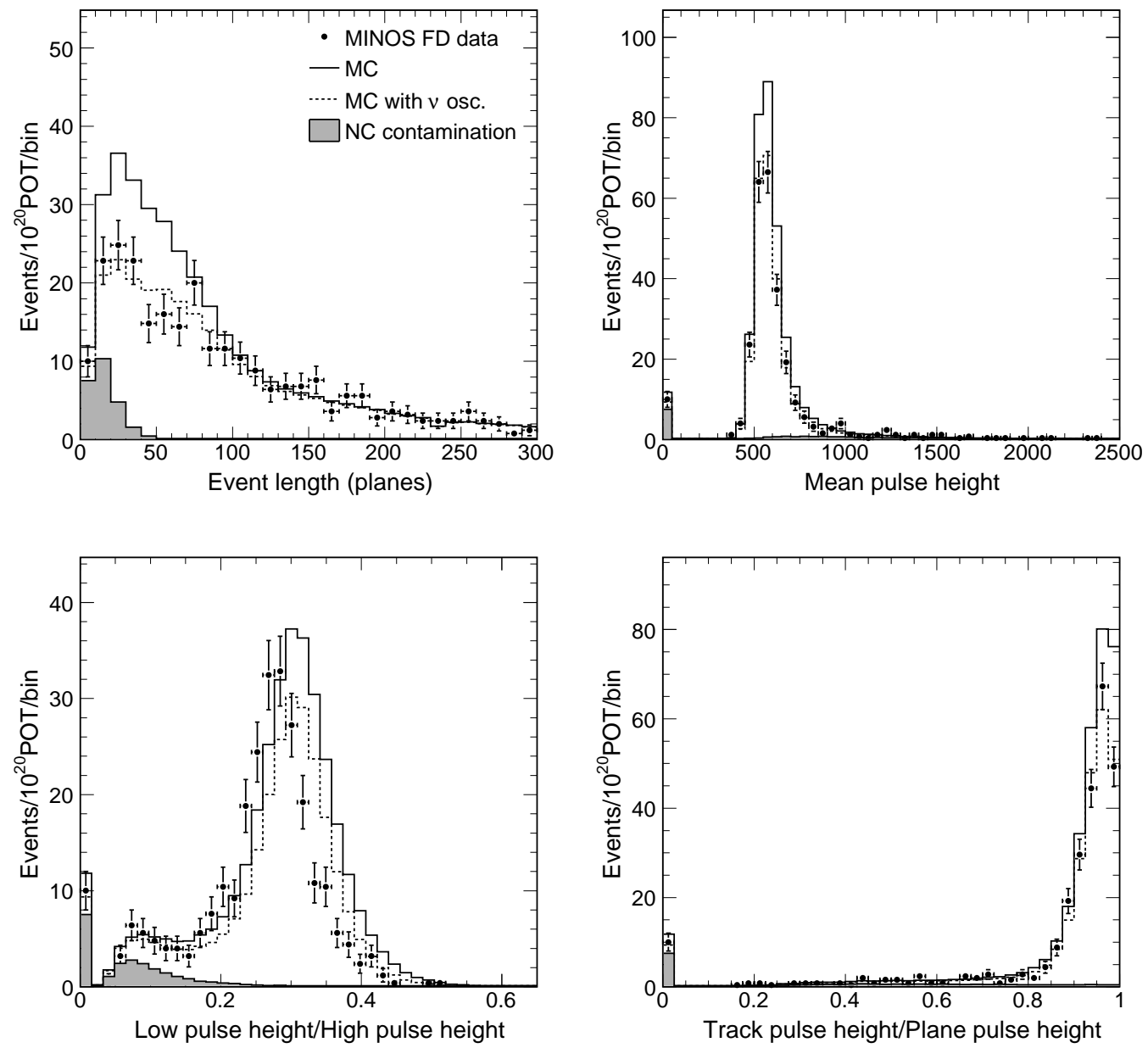

Figure 8.6: The 4 variables used to distinguish between $\mathrm{CC}$ and $\mathrm{NC}$ events (§2.2.4). Each variable uses some aspect of the muon track that separates it from the misidentified non-muon tracks. (a) The length of the event, (b) The light yield (equivalent to $\mathrm{dE} / \mathrm{dx}$ ), (c) The ratio of pulse height from a set of strips along the track with lowest and highest pulse heights, (d) fraction of activity in the plane that belongs to the track. Predicted distributions assuming that neutrinos do oscillate (dashed line) and don't oscillate (solid line) are shown as well. The oscillated distribution was generated using oscillation parameters $\Delta m^{2}=2.38 \times 10^{-3} \mathrm{eV}^{2} / c^{4}$ and $\sin ^{2} 2 \theta=1$. 


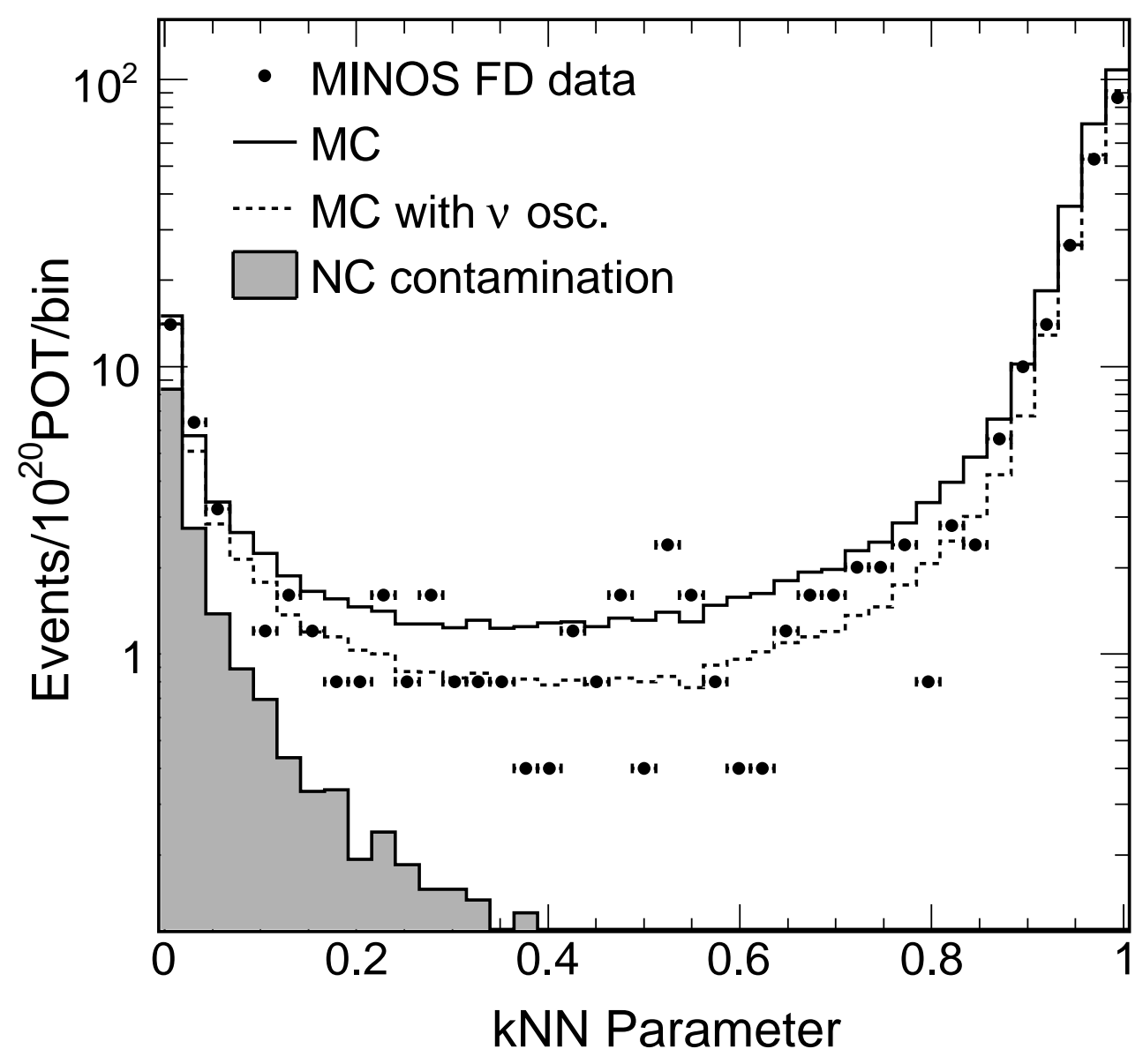

Figure 8.7: The distribution of kNN parameter for the FD events. The parameter is calculated using the $\mathrm{kNN}$ algorithm (§2.2.4) based on 4 input variables shown in Figure 2.15. The oscillated distribution was generated using oscillation parameters $\Delta m^{2}=2.38 \times 10^{-3} \mathrm{eV}^{2} / c^{4}$ and $\sin ^{2} 2 \theta=1$. 


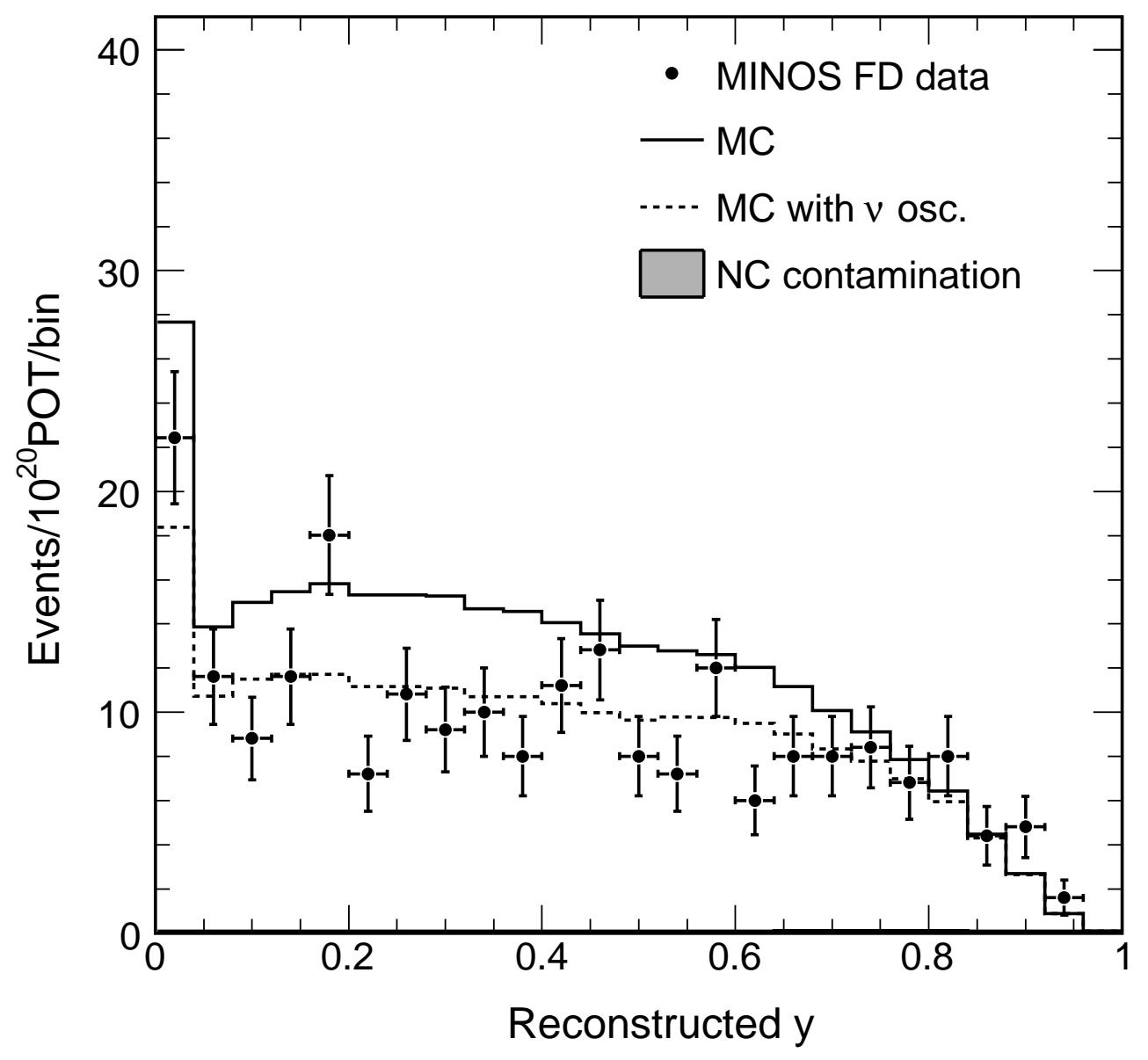

Figure 8.8: The reconstructed y $\left(y \equiv E_{s h w} / E_{\nu}\right)$ distribution of CC neutrino event candidates in the Far Detector. The predicted distribution assuming no oscillations (solid line) and assuming neutrinos do oscillate (dashed line) are shown for comparison. The oscillated distribution was generated using oscillation parameters $\Delta m^{2}=2.38 \times 10^{-3} \mathrm{eV}^{2} / c^{4}$ and $\sin ^{2} 2 \theta=1$. 
This background was estimated using two different techniques. The data in the FD is recorded in a $50 \mu$ s window surrounding the actual beam spill. The cosmic ray background is estimated by looking at the number of selected events that are not within the expected $10 \mu$ s spill. The other technique uses fake triggers that are taken in anti-coincidence with the beam-spill. Again looking at the number of events that pass the selection, yields the estimate on the cosmic ray background. Both techniques suggest an upper limit of 0.8 events for the current data set.

\subsection{Observed Energy Spectrum}

We have seen already in previous section that FD data agrees well with the prediction of neutrino flux that takes into account neutrino oscillations. However, the most convincing proof for neutrino oscillations is found when the neutrino energy spectrum is considered. The oscillation hypothesis predicts a specific energy dependent distortion of neutrino energy spectrum (Equation 1.2).

As mentioned, we use far over near ratio to predict the flux at the FD. In Chapter 4 this ratio was found in terms of neutrino energy (Figure 4.3). In practice, however, both Near and Far Detector have a finite resolution. To take that into account we use the MC simulation of MINOS detectors to simulate the detector response and find the smearing in energy. We then find the far over near ratio in terms of the reconstructed neutrino energy in the MINOS detectors [92]. Since we use events that are selected as $\nu_{\mu} \mathrm{CC}$ event candidates to construct the far over near ratio, the efficiency of MINOS detectors gets folded into the ratio.

The near detector data extrapolated using this ratio, predicts the re- 
constructed energy spectrum in the Far Detector. To predict the oscillated neutrino spectrum we need to find the true neutrino energy to use in Equation 1.2. Therefore we need to somehow convert the reconstructed neutrino energy spectrum into a true $E_{\nu}$ spectrum. In practice this is done by constructing a matrix $f_{i j}$ using the $\mathrm{MC}$ simulation of the $\mathrm{FD}$ response to the neutrino flux calculated in Chapters 3 and 6 . The indices $i$ and $j$ run over reconstructed and true neutrino energy respectively. The events that are selected as neutrino $\mathrm{CC}$ candidates are used to construct the matrix. The matrix element $(i, j)$ gives the expected number of neutrinos with energy $E_{j}$ that will be reconstructed as having energy $E_{i}$. The predicted oscillated spectrum in reconstructed neutrino energy bin $E_{i}$ is then calculated using:

$$
F_{i}^{\text {predicted }}=\frac{N_{i}^{\text {data }}}{n_{i}^{M C}} \sum_{j}\left(f_{i j}^{M C} P\left(\nu_{\mu} \rightarrow \nu_{\mu}, E_{j}\right)\right)
$$

where $P\left(\nu_{\mu} \rightarrow \nu_{\mu}, E_{j}\right)$ is the survival probability of Equation 1.3. This simplifies to Equation 4.1 if neutrinos wouldn't oscillate and the probability $P\left(\nu_{\mu} \rightarrow \nu_{\mu}\right)$ would be 1 .

To account for the appearance of $\nu_{\tau} \mathrm{s}$, an additional FD MC is generated in which all of the $\nu_{\mu} \mathrm{s}$ are oscillated into $\nu_{\tau} \mathrm{s}$. This MC is used to construct a similar $f_{i j}^{\nu_{\tau}}$ matrix and the predicted spectrum is found in a same way using the events that are selected as $\nu_{\mu} \mathrm{CC}$ candidates, but now the probability $P\left(\nu_{\mu} \rightarrow \nu_{\tau}, E_{j}\right)$ for appearance of $\nu_{\tau}$ is used. The two predicted spectra, $F_{i}^{\nu^{\mu}}$ and $F_{i}^{\nu_{\tau}}$, are added to find the final oscillated spectrum.

The neutral current events arise from interactions of all neutrino types, so when applying the Equation 8.1 we would ideally have pure sample of CC $\nu_{\mu}$ events in the ND. Since some of the NC events enter the selected CC 
candidates and get oscillated when using Equation 8.1 we need to account for that. However, this gets accounted for when $F_{i}^{\nu_{\tau}}$ is added.

The total number of events observed in the FD, corresponding to the exposure of $2.5 \times 10^{20} \mathrm{POT}$, was 562 , while the expectation was 740 events, indicating a significant disappearance of $\nu_{\mu}$ s. Below $5 \mathrm{GeV}$ even more significant disappearance was observed. The data below $5 \mathrm{GeV}$ contained 194 events, while the expectation was 336 events.

Figure 8.9 and Figure 8.10 show the comparison of predicted FD spectrum and the measured FD spectrum during Run I and Run II periods, while Figure 8.11 shows all the data. It can be seen that a significant number of lower energy neutrinos have disappeared and that the prediction of $\nu_{\mu}$ flux made under assumption that neutrinos don't oscillate is disfavored. On the other hand the prediction which assumes neutrino oscillations agrees well with data. 


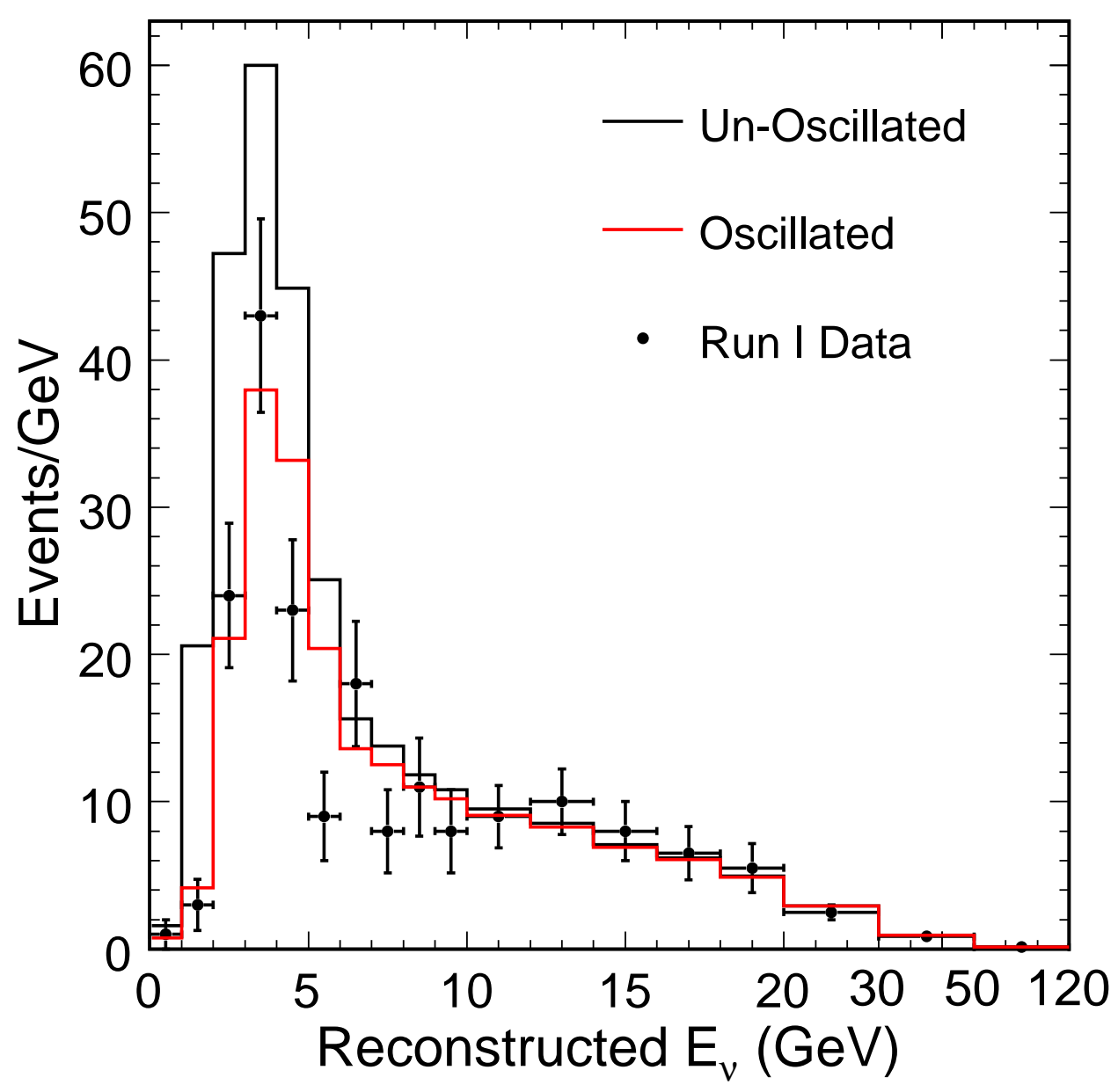

Figure 8.9: The reconstructed energy spectrum of muon neutrinos measured at the Far Detector (black dots) compared to the prediction made under assumption that neutrinos don't oscillate (solid line) and that neutrinos do oscillate (dashed line). The oscillated distribution was generated using oscillation parameters $\Delta m^{2}=2.38 \times 10^{-3} \mathrm{eV}^{2} / c^{4}$ and $\sin ^{2} 2 \theta=1$. Shown is the data acquired during Run I period (Table 2.1). 


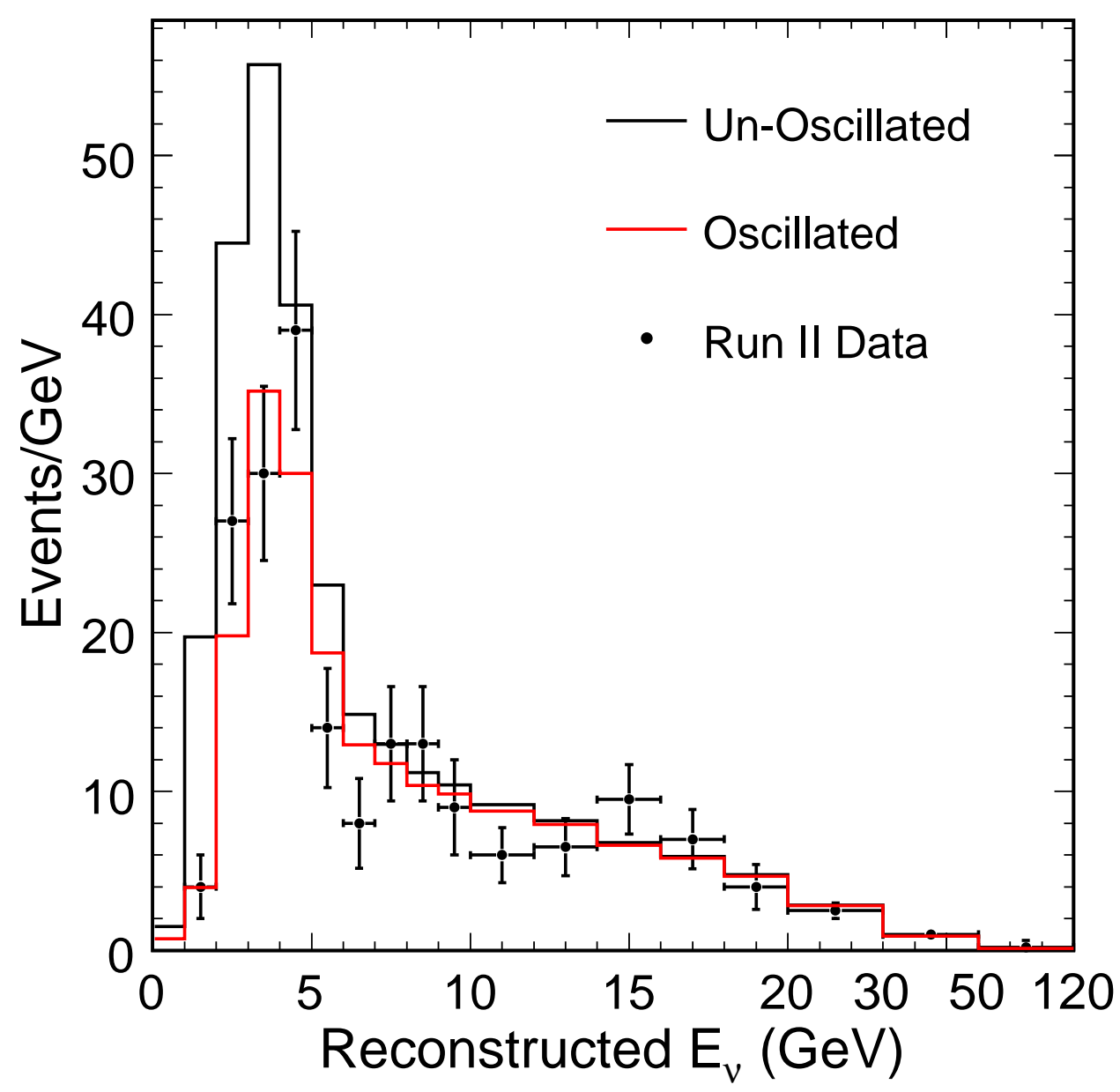

Figure 8.10: The reconstructed energy spectrum of muon neutrinos measured at the Far Detector (black dots) compared to the prediction made under assumption that neutrinos don't oscillate (solid line) and that neutrinos do oscillate (dashed line). The oscillated distribution was generated using oscillation parameters $\Delta m^{2}=2.38 \times 10^{-3} \mathrm{eV}^{2} / c^{4}$ and $\sin ^{2} 2 \theta=1$. Shown is the data acquired during Run II period (Table 2.1). 


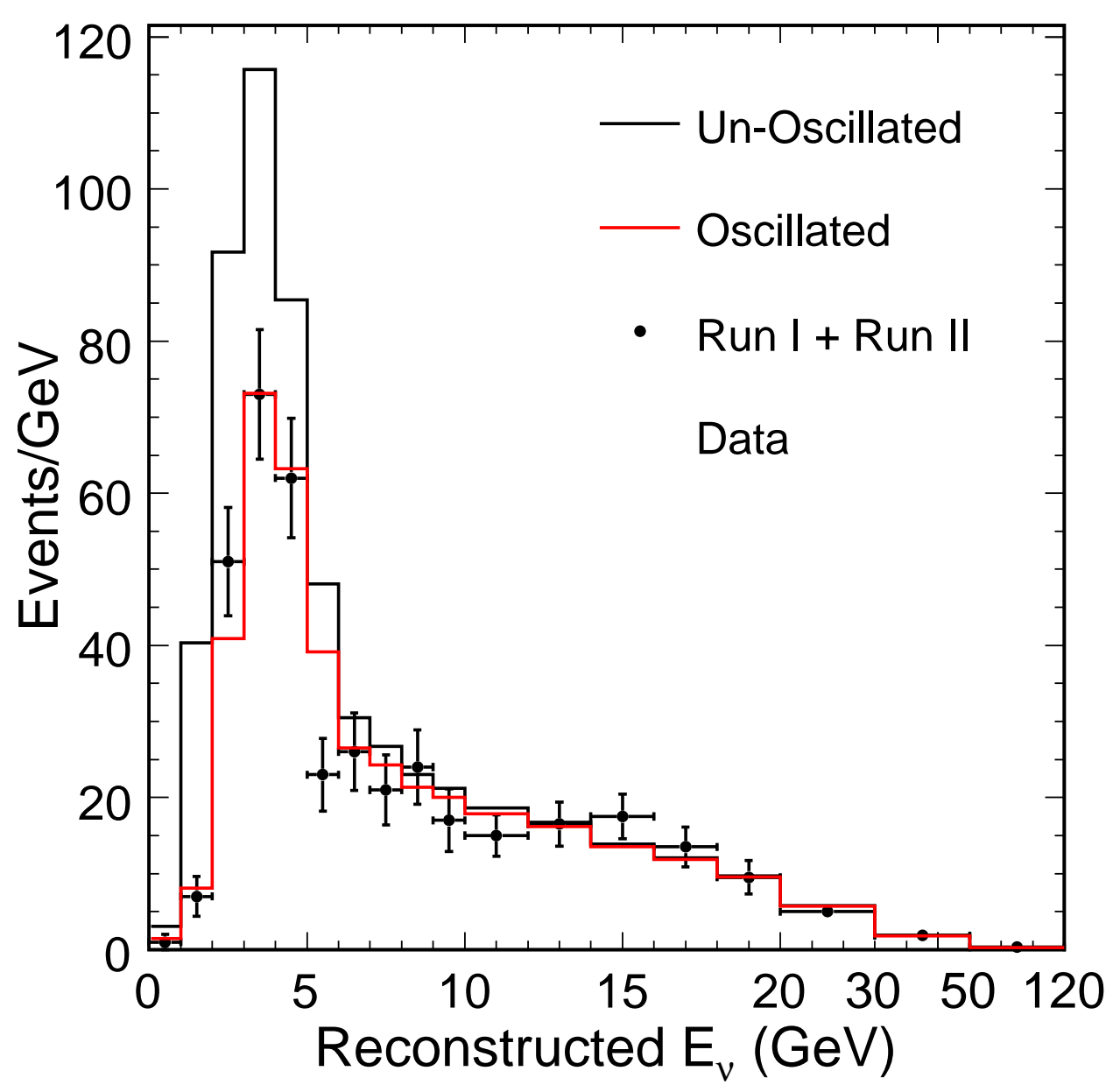

Figure 8.11: The reconstructed energy spectrum of muon neutrinos measured at the Far Detector (black dots) compared to the prediction made under assumption that neutrinos don't oscillate (solid line) and that neutrinos do oscillate (red line). The oscillated distribution was generated using oscillation parameters $\Delta m^{2}=2.38 \times 10^{-3} \mathrm{eV}^{2} / c^{4}$ and $\sin ^{2} 2 \theta=1$. Shown is the total data corresponding to $2.5 \times 10^{20} \mathrm{POT}$. 


\section{Chapter 9}

\section{Summary and Outlook}

This thesis presented how MINOS makes an accurate prediction of the neutrino flux at the Far Detector (FD). In a two-detector experiment, the Near Detector (ND) measures the flux from the $\nu$ beam at first-order. However, corrections have to be applied to the neutrino spectrum measured in the ND in order to predict the FD spectrum. This thesis details how these corrections are calculated. The uncertainty in these corrections as well as the technique that uses ND data to reduce these uncertainties were described. The predicted $\nu_{\mu}$ energy spectrum was then compared to the FD data which revealed a clear energy dependent distortion of the data.

The final step of the analysis is to find the oscillation parameters $\Delta m_{23}^{2}$ and $\sin ^{2} 2 \theta_{23}$ that best fit the FD data. The first MINOS result on $\nu_{\mu}$ disappearance utilized a data set consisting of $1.27 \times 10^{20}$ POT [24]. This chapter summarizes results using $2.5 \times 10^{20}$ POT reported in [93]. 


\subsection{Fit to Oscillation Hypothesis}

The position in energy spectrum where the oscillation effect is the strongest depends on the $\Delta m_{23}^{2}$. On the other hand, the $\sin ^{2} 2 \theta$ parameter determines how strong is the oscillation. To extract the oscillation parameters we look for the oscillation parameters that best describe the $\nu_{\mu}$ energy spectrum distortion seen in the FD data.

The oscillation analysis presented here was done using the data acquired in the LE010/185kA beam configuration only (Table 2.1). The fit was performed on the $E_{\nu}$ spectra for Run I and II simultaneously. However, the data from two runs was treated separately since the target longitudinal position was slightly different between the runs (Chapter 7 ) resulting in a $\sim 5 \%$ difference in the peak region of energy spectrum. A two flavor $\nu_{\mu} \rightarrow \nu_{\tau}$ oscillation scenario was assumed.

In the fit, a quantity $\chi^{2}=-2 \ln \mathcal{L}$, where $\mathcal{L}$ is the likelihood function, was minimized with respect to the oscillation parameters $\Delta m^{2}$ and $\sin ^{2} 2 \theta$ as well as nuisance parameters $\boldsymbol{\alpha}$ which incorporated the most significant sources of systematic uncertainty:

$$
\chi^{2}\left(\Delta m^{2}, \sin ^{2} 2 \theta, \boldsymbol{\alpha}\right)=\sum_{i}\left\{2\left(e_{i}-o_{i}\right)+2 o_{i} \ln \left(o_{i} / e_{i}\right)\right\}+\sum_{j} \frac{\Delta \alpha_{j}^{2}}{\sigma_{\alpha_{j}}^{2}}
$$

where $o_{i}$ represents the observed number of events in the $i^{\text {th }}$ energy bin and $e_{i}$ represents the corresponding oscillation weighted expectation. Only physical values of $\sin ^{2} 2 \theta \leq 1$ were considered in the fit. Three nuisance parameters included as penalty terms were: relative FD/ND normalization, the hadronic shower energy scale and the NC background. 
The best fit oscillation parameter were found to be:

$$
\begin{aligned}
\Delta m_{23}^{2} & =\left(2.38_{-0.16}^{+0.20}\right) \times 10^{-3} \mathrm{eV}^{2} / c^{4} \\
\sin ^{2} 2 \theta_{23} & =1.00_{-0.08}
\end{aligned}
$$

The $\chi^{2}$ after the fit was 41.2 for 34 degrees of freedom.

When the $\sin ^{2} 2 \theta \leq 1$ condition is relaxed, the fit yields the result in unphysical region with $\Delta m_{23}^{2}=2.26 \times 10^{-3} \mathrm{eV}^{2} / c^{4}$ and $\sin ^{2} 2 \theta_{23}=1.07$ and corresponding $\chi^{2}=40.9$.

Figure 9.1 shows the $68 \%$ and $90 \%$ confidence level (CL) contours for $\Delta m^{2}$ and $\sin ^{2} 2 \theta$. The contours correspond to $\Delta \chi^{2}=2.30$ and 4.61 respectively. For comparison the results for several other experiments are shown as well.

\subsection{Systematic Errors}

To evaluate the systematic errors, the MC simulation was utilized to generate fake "data sets" which had a $\pm 1 \sigma$ shift in a given systematic parameter. These fake data sets were generated assuming the best fit oscillation parameters $\Delta m^{2}$ and $\sin ^{2} 2 \theta$. The full analysis was then performed and shifts in the fitted oscillation parameters were recorded. Table 9.1 lists all the systematic effects considered.

The absolute hadronic energy scale uncertainty comes from the uncertainty in MC simulation of energy deposition and in the hadronic response of the detector. The uncertainty affects the reconstructed energy of a neutrino and therefore as expected produces a shift in measured $\Delta m^{2}$. 


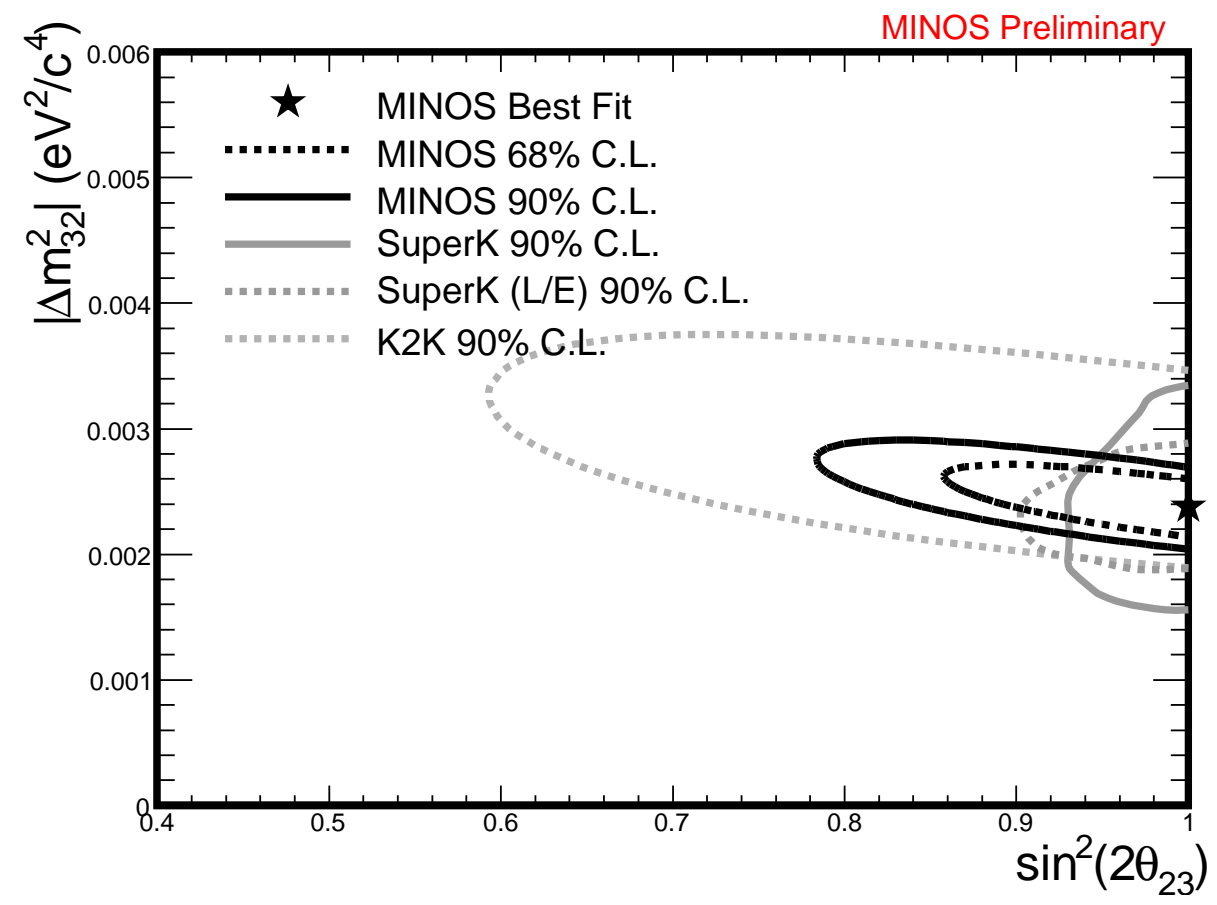

Figure 9.1: The best fit point to MINOS Far Detector data corresponding to exposure of $2.5 \times 10^{20} \mathrm{POT}$. The $68 \%$ and $90 \% \mathrm{CL}$ contours were determined according to $\Delta \chi^{2}=2.30$ and 4.61 respectively. Overlaid are the $90 \% \mathrm{CL}$ contours from the Super-Kamiokande zenith angle [23] and L/E [22], and from K2K experiment [28]. 


\begin{tabular}{lcc}
\hline \hline Source & $\begin{array}{c}\delta\left(\Delta m^{2}\right) \\
{\left[10^{-3} \mathrm{eV}^{2} / c^{4}\right]}\end{array}$ & $\delta\left(\sin ^{2} 2 \theta\right)$ \\
\hline Absolute hadronic energy scale $(10 \%)$ & 0.075 & $<0.005$ \\
Normalization $(4 \%)$ & 0.065 & $<0.005$ \\
NC background $(50 \%)$ & 0.010 & 0.0075 \\
\hline$M_{A}^{Q E}(15 \%)$ & 0.005 & $<0.005$ \\
$M_{A}^{R e s}(15 \%)$ & $<0.010$ & $<0.005$ \\
KNO $(20 \%)$ & 0.005 & $<0.005$ \\
$\bar{\nu}_{\mu}$ cross-sections $(20 \%)$ & $<0.010$ & $<0.005$ \\
\hline Beam flux/hadron production models & 0.015 & $<0.005$ \\
$\mu$ momentum scale-contained tracks $(2 \%)$ & 0.035 & $<0.005$ \\
$\mu$ momentum scale-non-contained tracks $(6.9 \%)$ & 0.015 & $<0.005$ \\
\hline Sum (in quadrature) & $\mathbf{0 . 1 0 8}$ & $\mathbf{0 . 0 0 7 5}$ \\
\hline Statistical uncertainty & 0.18 & 0.08 \\
\hline \hline
\end{tabular}

Table 9.1: Summary of systematic uncertainties on best fit values for oscillation parameters. Statistical uncertainty corresponding to exposure of $2.5 \times 10^{20} \mathrm{POT}$ is given for comparison.

The normalization is due to the uncertainty in the ratio of fiducial masses $\mathrm{FD} / \mathrm{ND}(2 \%)$, relative $\mathrm{FD} / \mathrm{ND}$ event reconstruction uncertainty (2$3 \%$ ) and a $1 \%$ uncertainty in counting the protons delivered on the target. A total uncertainty of $4 \%$ in normalization was assumed. Naively one would assume that normalization affects the measurement of $\sin ^{2} 2 \theta$ more than $\Delta m^{2}$, however the high energy tail in some sense fixes the normalization for the measurement of the mixing angle. On the other hand, the measured $\Delta m^{2}$ appears to be low, so the re-appearance of the muon neutrinos below $E_{\nu} \sim$ 1.5 GeV predicted by neutrino oscillations has not yet been observed in MINOS with statistical significance. For that reason, the normalization uncertainty affects $\Delta m^{2}$ strongly.

As mentioned in previous chapter, the NC background was determined to within $25 \%$ using data driven studies. It was also found that MC over- 
estimates the NC background by $\sim 10 \%$. Since the NC background appears in lower energy bins where the oscillation signal is, it affects how well we can measure the strength of the oscillations and therefore it mostly causes a shift in $\sin ^{2} 2 \theta$.

Several parameters $\left(M_{A}^{Q E}, M_{A}^{R e s}, \mathrm{KNO}\right.$ and $\bar{\nu}_{\mu}$ cross section parameter $)$ were used to study the effect of neutrino cross section uncertainties on the measured oscillation parameters [94]. The $M_{A}^{Q E}$ and $M_{A}^{\text {Res }}$, the so called axialvector masses, are two parameters that are used to describe the neutrino cross section in the few $\mathrm{GeV}$ region. The KNO parameter, is used to modify the cross section in a transition region between resonance and deep inelastic scattering. The last parameter, $\bar{\nu}_{\mu}$ cross section parameter, in fact adjusted the ratio of cross sections $\sigma\left(\nu_{\mu}\right) / \sigma\left(\bar{\nu}_{\mu}\right)$. It was found that cross sections don't produce significant systematic error.

The muon momentum is measured either through range of the muon in the MINOS detector or using its curvature. Similarly as for absolute hadronic energy scale this affects the reconstructed energy and therefore causes a shift in measured $\Delta m^{2}$.

Finally, this thesis discussed in detail the uncertainties in the neutrino flux. A technique was developed that greatly reduces this uncertainty. As a result the effect of beam uncertainty on the measured oscillation parameters is small compared to other systematic effects. For many other neutrino oscillation experiments the systematic errors related to neutrino flux were typically large. This is especially true for one detector experiments such as [95, 96, 84]. These experiments were able to calculate the neutrino flux only at the $\sim 10 \%$ level or in some cases much worse. Perhaps, the NOMAD experiment [84] was most succesful determining the flux error to $6-7 \%$. 
To reduce the uncertainties in neutrino flux many experiments use two detectors. However, even for two detector experiment like $\mathrm{K} 2 \mathrm{~K}$, the flux uncertainty was a significant systematic uncertainty. The experiment used the far over near ratio method, similar to the one described in this thesis (Chapter 4), to predict the neutrino spectrum at the Super-Kamiokande. In case of K2K, the neutrino spectrum peaked at around $1 \mathrm{GeV}$. The uncertainty in Far/Near was $\sim 2-3 \%$ below $1 \mathrm{GeV}$ and $4-9 \%$ above $1 \mathrm{GeV}[28]$.

Several two detector neutrino experiments performed at CERN, like CHARM [97] and CDHS [98], decided to use "bare target" beam to reduce the uncertainty in neutrino flux. The "bare target" beam uses no focusing for the mesons produced in the target. The benefit was that a corrections needed to be applied to the $\nu$ energy spectrum measured in the near detector in order to predict the far spectrum deviated less than $5 \%$ from the simple $L^{-2}$ drop-off in flux, allowing them to predict the total flux at the far detector at the $0.5-1 \%$ level. This came at the expense of the statistics, requiring longer running. The two experiments, CHARM and CDHS, had a far detector at a distance of less than 900 m away from target, closer than MINOS' near detector, so they could afford such a trade-off. Furthermore, the $L^{-2}$ fall off is better approximated at high $E_{\nu}$ since the divergence of the pion beam is $\theta_{\pi} \sim 2 / \gamma$, and the divergence of the neutrinos produced in pion decays is $\theta_{\nu} \sim 1 / \gamma$, and consequently the detectors subtend large solid angles for high energy beams.

MINOS uses horn focusing to increase the number of events in a $735 \mathrm{~km}$ distant FD. The corrections that need to be applied to near detector spectrum are of the order of $\pm 20 \%$ (see Figure 4.3 ) on top of the $L^{-2}$ drop-off. Nevertheless, we have shown that this correction can be calculated to within $0.5-2 \%$, depending on the neutrino energy, resulting in a better than a $2 \%$ prediction 
of the integrated flux at the FD.

\subsection{Outlook}

The MINOS experiment completed the analysis of the data corresponding to an exposure of $2.5 \times 10^{20}$ POT. MINOS' current result is the world's best measurement of $\Delta m_{23}^{2}$. Table 9.1 listed the statistical error along with the total systematic error. As can be seen, the total error is still dominated by statistical error. Furthermore, some of the systematic errors will be reduced with more statistics and some will get reduced with further analysis, allowing MINOS to measure the oscillation parameters more precisely. With more data MINOS will also be able to rule out models competing with neutrino oscillations. These models predict a different energy dependence of the distortion of the $E_{\nu}$ spectrum $[99,100]$.

Figure 9.2 shows MINOS sensitivity as a function of number of protons delivered on target. The results presented in this thesis correspond to the exposure of $2.5 \times 10^{20}$ POT. Currently, MINOS is in process of analyzing data corresponding to exposure of $3.25 \times 10^{20}$ POT. The total number of protons delivered up to date is $4.4 \times 10^{20}$. By the end of FY2010 it is expected that MINOS will pass $1.0 \times 10^{21}$ POT.

This thesis discussed how the neutrino flux can be accurately predicted at the FD and we focused on study of disappearance of $\nu_{\mu} \mathrm{s}$. MINOS will perform several other studies. Here we mention just a few that benefit from the accurate prediction of the neutrino flux at the FD.

The magnetized detectors enable MINOS to look at the disappearance of $\nu_{\mu} \mathrm{s}$ and $\bar{\nu}_{\mu} \mathrm{s}$ separately. The sensitivity to anti-neutrino oscillation param- 


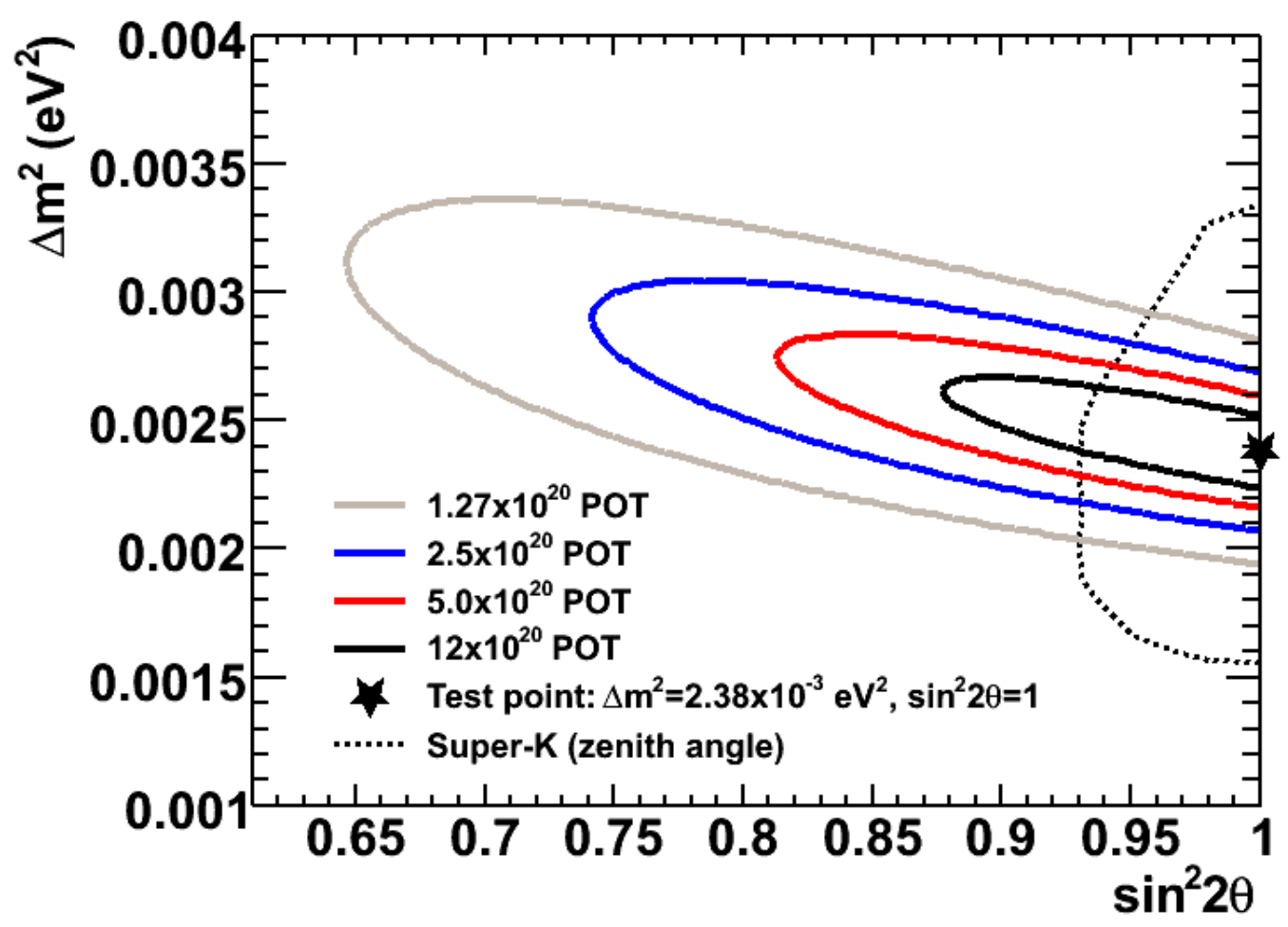

Figure 9.2: MINOS sensitivity to oscillation parameters $\Delta m_{23}^{2}$ and $\sin ^{2} 2 \theta_{23}$ for different exposures. The contours represent $90 \%$ CL based upon statistical errors only. First MINOS result on $\nu_{\mu}$ disappearance used $1.27 \times 10^{20}$ POT. This thesis used a data set corresponding to $2.5 \times 10^{20}$ POT. Up to date MINOS accumulated $4.4 \times 10^{20}$. 
eters could even be improved by running with reversed horn field. In such configuration negative mesons off the target would be focused giving rise to anti-neutrino beam.

MINOS will also look at the appearance of $\nu_{e}$ at the FD. The procedure described in Chapter 6 constrains the electron neutrino as well as muon neutrino flux. With current exposure, MINOS will be able to push the limit on $\sin ^{2} 2 \theta_{13}$ or perhaps even make the first measurement.

By looking at the $\mathrm{NC}$ interactions a total neutrino flux at the FD can be measured. Comparison with the predicted rate will set a limit on $\nu_{\mu} \rightarrow \nu_{\text {sterile }}$ mixing. 


\section{Appendix A}

\section{Calculating The Beam Matrix}

To calculate the beam matrix which can be used to predict the expected neutrino energy spectrum in the Far Detector (FD) given the measured energy spectrum in the Near Detector (ND) we use the same simulation of the neutrino beamline that was used to calculate the neutrino flux in Chapter 3. The simulation is used to track the neutrino parents up to their decay points $\left(\vec{r}_{l}\right)$. The matrix elements are then calculated as follows:

$$
B_{i j}=\frac{\int_{E_{j}}^{E_{j}+\Delta E_{n}} \int_{E_{i}}^{E_{i}+\Delta E_{f}} \sum_{l} \delta\left(E^{N D}\left(\vec{r}_{l}, \vec{p}_{l}\right)-E_{2}\right) P_{F D}\left(\vec{r}_{l}, \vec{p}_{l}, E_{1}\right) d E_{1} d E_{2}}{\int_{E_{j}}^{E_{j}+\Delta E_{n}} \sum_{l} P_{N D}\left(\vec{r}_{l}, \vec{p}_{l}, E\right) d E}
$$

where $E^{N D}\left(\vec{r}_{l}, \vec{p}_{l}\right)$ is the energy of a neutrino that emerges from a decay of a particle whose momentum is given with $\vec{p}_{l}$ and goes through the ND. $P_{N D}$ and $P_{F D}$ are probabilities that a neutrino with energy $E$ will be produced in that decay and hit the ND or FD respectively. $\Delta E_{n}$ and $\Delta E_{f}$ are bin sizes for the ND and FD. The sum over $l$ goes over all the simulated neutrino parents. The beam-line simulation provides, through this sum, the distribution 
of hadrons downstream of the target multiplied by the probability that they will decay into a neutrino at a certain point $\vec{r}_{l}$. The beam matrix only weakly depends on these distributions since they appear both in the numerator and the denominator. How much they cancel depends on how different are the energies of neutrinos at the FD coming from a selected group of neutrino parents that produce neutrinos at the ND with energy $E^{N D}$. If those parents produce the neutrinos of same energy at the FD as at the ND, which is the case if those parents are focused particles, the parent distributions become unimportant, ie they cancel out. In that case the matrix also becomes diagonal.

The beam matrix calculated this way can be seen in Figure 4.6. 


\section{Appendix B}

\section{Cross-check of NuMI Flux Using MiniBooNE Detector}

The neutrinos in NuMI beamline are predominantly produced in the direction of the MINOS detectors due to a relativistic boost of neutrino parents along the beam axis. In case of a two body decays of pions and kaons, the angular distribution is given with Equation 3.13. Although the flux is significantly reduced at off-axis angles it is still appreciable given the high intensity of the NuMI beam.

In addition to MINOS, the Fermilab complex at present sites an additional neutrino experiment called MiniBooNE. The MiniBooNE experiment was designed to measure the appearance of electron neutrinos in a muon neutrino beam. The source of neutrinos for MiniBooNE is booster neutrino beamline.

The MiniBooNE detector is located $745 \mathrm{~m}$ downstream of the NuMI target at a 110 mrad angle with respect to the NuMI beam axis. Figure B.1 

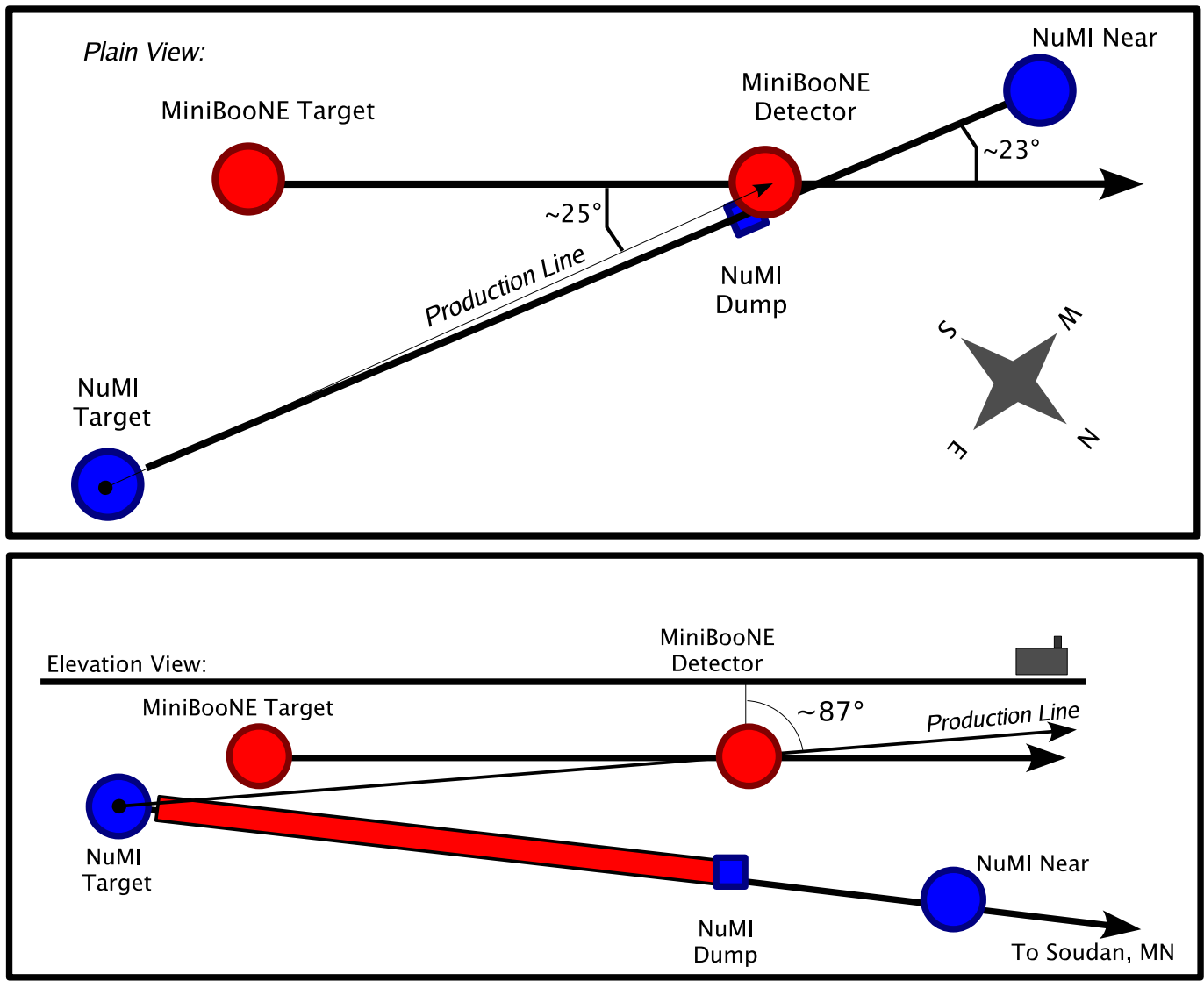

Figure B.1: Plan and elevation view of the NuMI beamline and MiniBooNE detector. The detector is located $745 \mathrm{~m}$ downstream of NuMI target at an angle of $110 \mathrm{mrad}$ with respect to the NuMI beam axis. The MiniBooNE detector sees the NuMI offaxis beam. 
shows the plan and elevation view of the MiniBooNE detector and NuMI beamline.

The neutrino energy as a function of parent energy and an angle between the outgoing neutrino and parent momentum is given in Equation 3.9. The equation predicts that at small angles $\theta$ the neutrino energy is proportional to parent energy $E_{\pi, K}$, however for off-axis decays neutrino energy becomes uncorrelated in energy with their hadron parents ${ }^{1}$. Figure B.2 shows the dependence of neutrino energy on parent energy. At the rather large off-axis angle $\theta=110$ mrad between the NuMI beam line and the MiniBooNE detector, muon neutrinos are nearly monoenergetic in energy, with pions creating $\sim 0.25 \mathrm{GeV}$ neutrinos and kaons $\sim 2.0 \mathrm{GeV}$ neutrinos.

Figure B.3 shows the distributions of transverse and longitudinal momenta of mesons contributing to the neutrino flux at MiniBooNE detector. These are compared to the same distributions for MINOS Near Detector (Figures 6.1-6.4). Clearly the hadrons which contribute to the MINOS flux and those that contribute to the MiniBooNE flux overlap only partially. The segregation in $\left(p_{z}, p_{T}\right)$ has the negative consequence that the MINOS neutrino data does not entirely constrain the flux at the MiniBooNE detector. On the positive side, this segregation implies that the MiniBooNE data provide a nearly independent check of $\pi / K$ production in a new region, and thus constrain cascade models such as FLUKA.

\footnotetext{
${ }^{1}$ This was first realized in BNL-889 proposal
} 

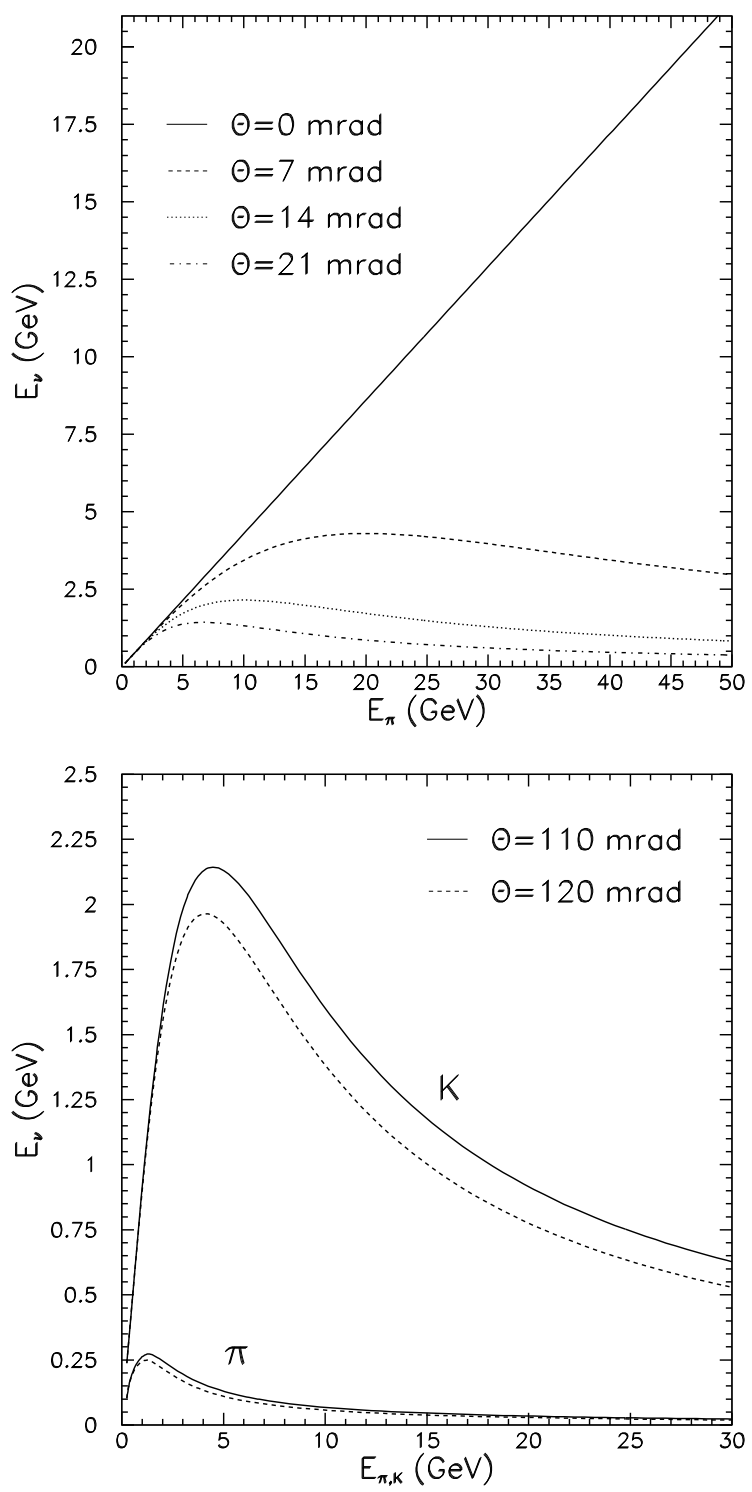

Figure B.2: (top) Energy of the neutrino in pion decay as a function of the pion energy, for several choices of decay angle $\theta$. (bottom) Energy of the neutrino in pion and kaon decay as a function of parent energy at an angle of $110 \mathrm{mrad}$. 

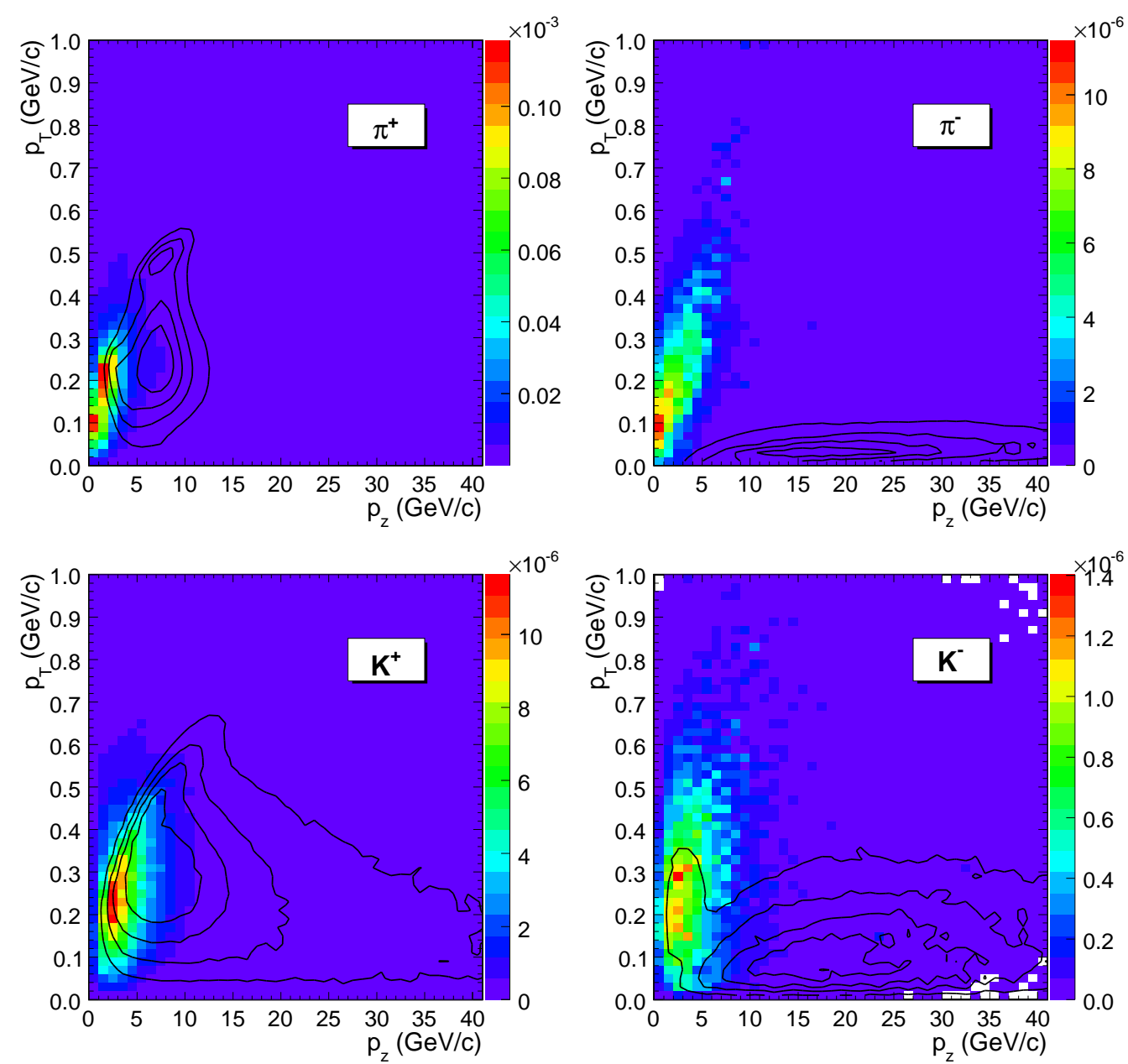

Figure B.3: The $p_{T}$ and $p_{z}$ of $\pi^{+}$and $K^{+}$which contribute to the muon neutrino flux at the MiniBooNE and MINOS Near detectors. The MiniBooNE contribution is shown by the color scale, while the MINOS contribution is shown by the contours (see Figures 6.1-6.4). 


\section{B.1 Calculation of Offaxis Flux}

The calculation of the offaxis flux follows the same steps as the calculation of the on-axis flux described in Chapter 3. The difference is only in the last step where we now calculate the energy of a neutrino at the MiniBooNE detector and the solid angle spanned by the MiniBooNE detector. Figure B.4 shows the calculated flux spectrum at the center of the MiniBooNE detector from NuMI beam line. The $\nu_{\mu}$ flux shows the expected two prominent peaks in the energy spectrum associated with pion and kaon decays Additionally, there are peaks due to meson decay-at-rest, predominantly from mesons stopped in the hadron absorber at the end of the decay region.

The production of particles in the NuMI hadron absorber is important for understanding the neutrino flux at MiniBooNE. These low-energy neutrinos are irrelevant for MINOS, but significant when analyzing the flux in the MiniBooNE detector because of the lower threshold in neutrino energy and because of the close (vertical) proximity of the MiniBooNE. As can be seen in Figure B.1 the MiniBooNE detector is approximately $15 \mathrm{~m}$ west and $80 \mathrm{~m}$ above the NuMI hadron absorber.

The fact that pions of all energies focused into the NuMI decay pipe will yield essentially the same neutrino energy has positive implications for the level of systematic uncertainties in the calculation of the beam flux. Many systematic effects, such as an incorrect alignment of a horn, for example, serve to incorrectly focus smaller-angle pions and enhance higher-energy neutrinos in the MINOS on axis flux. In the off-axis case, however, all such pions may be expected to deposit neutrinos of the same energy, with potentially only the overall rate being affected. As will be demonstrated, most of the systematic 

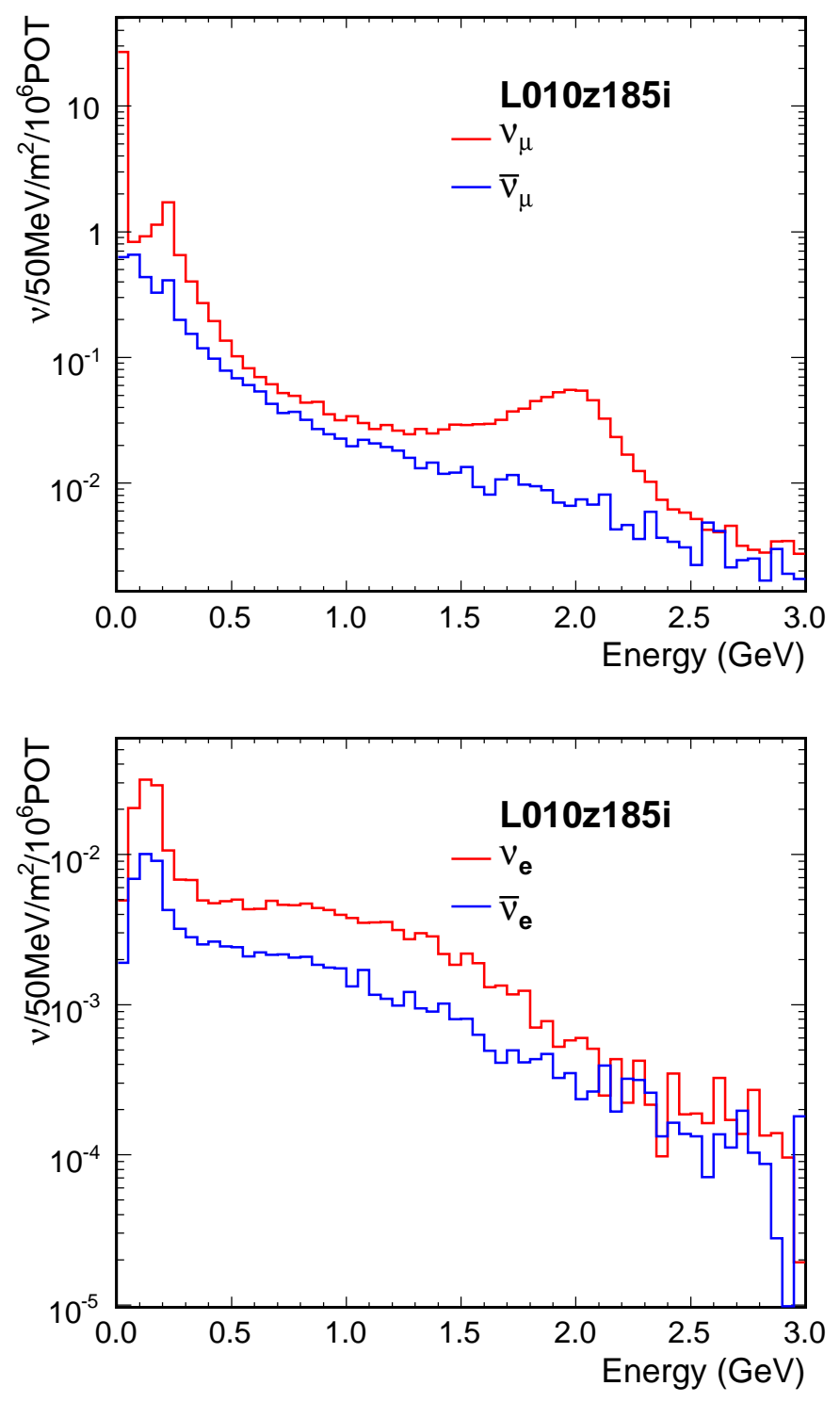

Figure B.4: Simulation of the off-axis flux from the NuMI LE10/185kA beam expected at the center of the MiniBooNE detector as a function of neutrino energy. Fluxes are shown for $\nu_{\mu}, \bar{\nu}_{\mu}, \nu_{e}$, and $\bar{\nu}_{e}$. The two prominent peaks correspond to pion and kaon decays. The very sharp peaks are due to decays of mesons that are stopped in the shielding. 
uncertainties are small for the off-axis flux. The dominant uncertainty is just the MiniBooNE reconstruction of the neutrino interaction point (since this affects the determination of the off-axis angle). Even particle production rates have a lesser effect on the off-axis flux, since the spectrum of particles off the target is less important to know than the overall rate.

In fact, because the neutrinos from pion and kaon decays are wellseparated in energy in the off-axis configuration, the measurement of the neutrino energy spectrum at MiniBooNE could be helpful to determine the overall relative rate of $\pi / K$ off the NuMI target. As mentioned, the portion of the phase space in $\left(x_{F}, p_{T}\right)$ of pions and kaons from the NuMI target that is sampled by MiniBooNE is somewhat different from that sampled by the MINOS detectors, but is potentially of value in constraining particle production models.

Figure B.5 shows the same neutrino fluxes as in Figure B.4, but in addition shows the contributions to these fluxes from $\pi, K, K_{L}$, and muons. The muon neutrinos arise largely from focused $\pi^{+}$and $K^{+}$, although there is a small component from $K_{L}$ decays. The muon antineutrino rates come from unfocused $\pi^{-}$and $K^{-}$decays, with a similar $K_{L}$ component as the neutrinos. The peaks at $\sim 0.2 \mathrm{GeV}$ in the $K$ component is from decay-at-rest in the NuMI beam dump, which lies almost immediately below the MiniBooNE detector.

The $\nu_{e}$ and $\bar{\nu}_{e}$ are similarly separated into muon and kaon parents, with both contributions separated in energy. Interestingly the $K_{L}$ from the beam dump are not-insubstantial component of the low-energy electron neutrinos and antineutrinos. Low energy $\nu_{e}$ also arise from $\mu^{+}$decay, where the $\mu^{+}$in turn come from focused $\pi+$ and from $K_{L}$ decays in the dump. For $\bar{\nu}_{e}$, the defocusing of $\pi^{-}$and $K^{-}$reduces the two dominant parent types to the point 

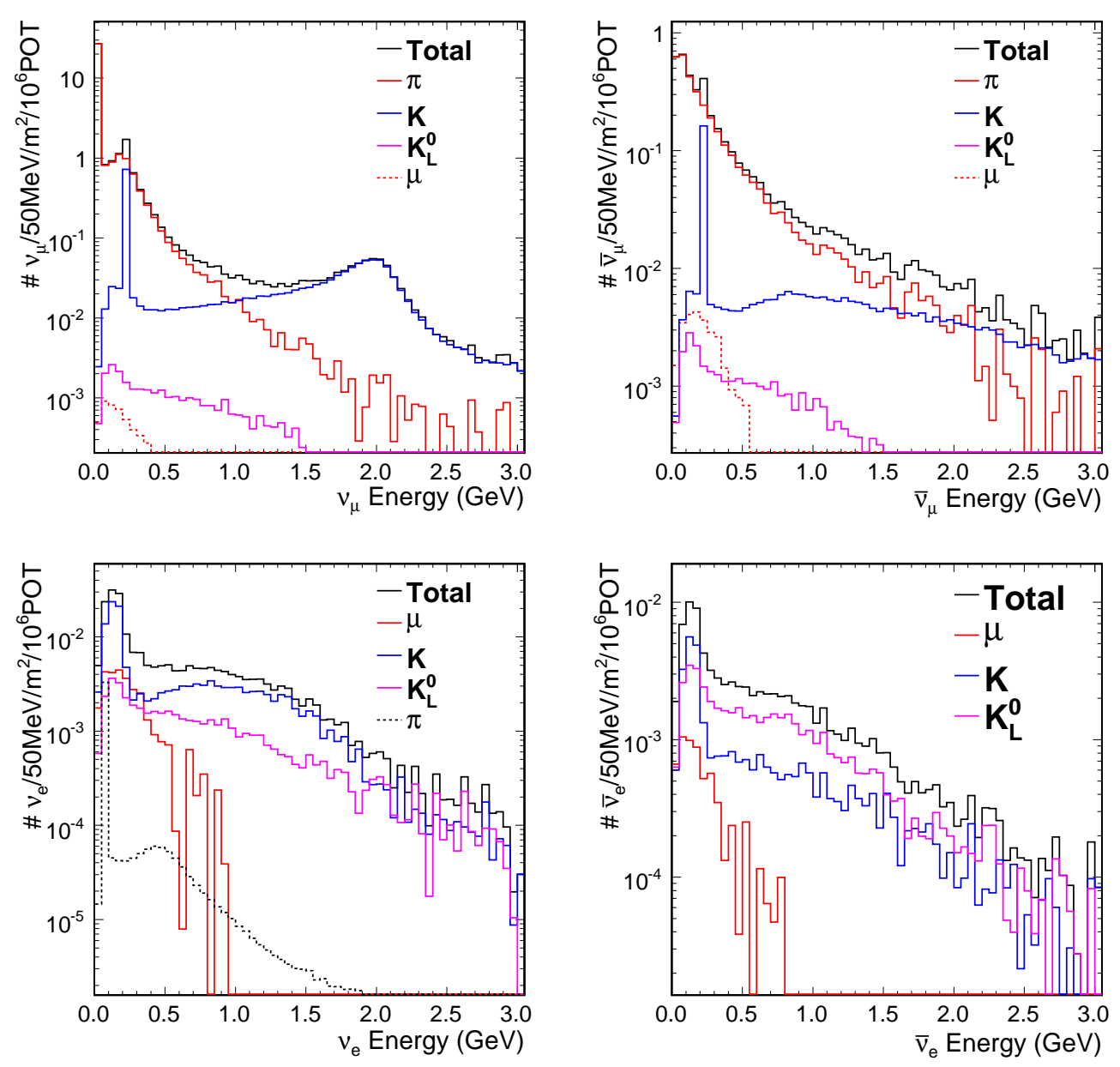

Figure B.5: Simulation of the off-axis flux from the NuMI LE beam expected at the center of the MiniBooNE detector (in units of $\nu / \mathrm{m}^{2} / 10^{6} / \mathrm{POT}$ ) as a function of neutrino energy. Fluxes are shown for $\nu_{\mu}, \bar{\nu}_{\mu}, \nu_{e}$, and $\bar{\nu}_{e}$. The contributions from $\pi, K, K_{L}$, and $\mu$ decays are shown separately. 
where $\bar{\nu}_{e}$ are approximately $60 \%$ due to $K_{L}$ across the entire energy spectrum.

Figures B.6 and B.7 show where the neutrinos' parents are created. In this figure, "Target" implies that the collision which produced the parent hadron took place in the NuMI target (these are conventional "decays in flight"). "Horn Al" implies an interaction in the horns' aluminum produced a cascade shower, one of whose hadrons subsequently decayed to a neutrino reaching MiniBooNE. "Iron" similarly implies that a shower produced in the target hall shielding or the NuMI decay pipe, and "absorber" implies a parent hadron created in the hadron absorber. "Air" implies that the neutrino parent was created in flight through the target hall or NuMI decay pipe (these are nearly entirely due to muon decays, where the muon arises from pion/kaon decays-in-flight).

We have seen in Figure B.3 that very low $p_{z}$ particles contribute to the MiniBoonE flux. These particles are expected to be poorly focused by horn 1 (largely over-focused), thus are heading at large angles with respect to the NuMI beam axis. Since they are soft, they would be expected to decay quickly, and their large angles with respect to the beam axis means that their neutrino daughters can be produced along the hadron axis, heading directly at MiniBooNE. In this respect, the events observed in MiniBooNE are not entirely from a naive off-axis decay-in-flight of a hadron focused by the NuMI beam - they are in some respect the on-axis decays of the poorly-focused tails of the NuMI beam. 

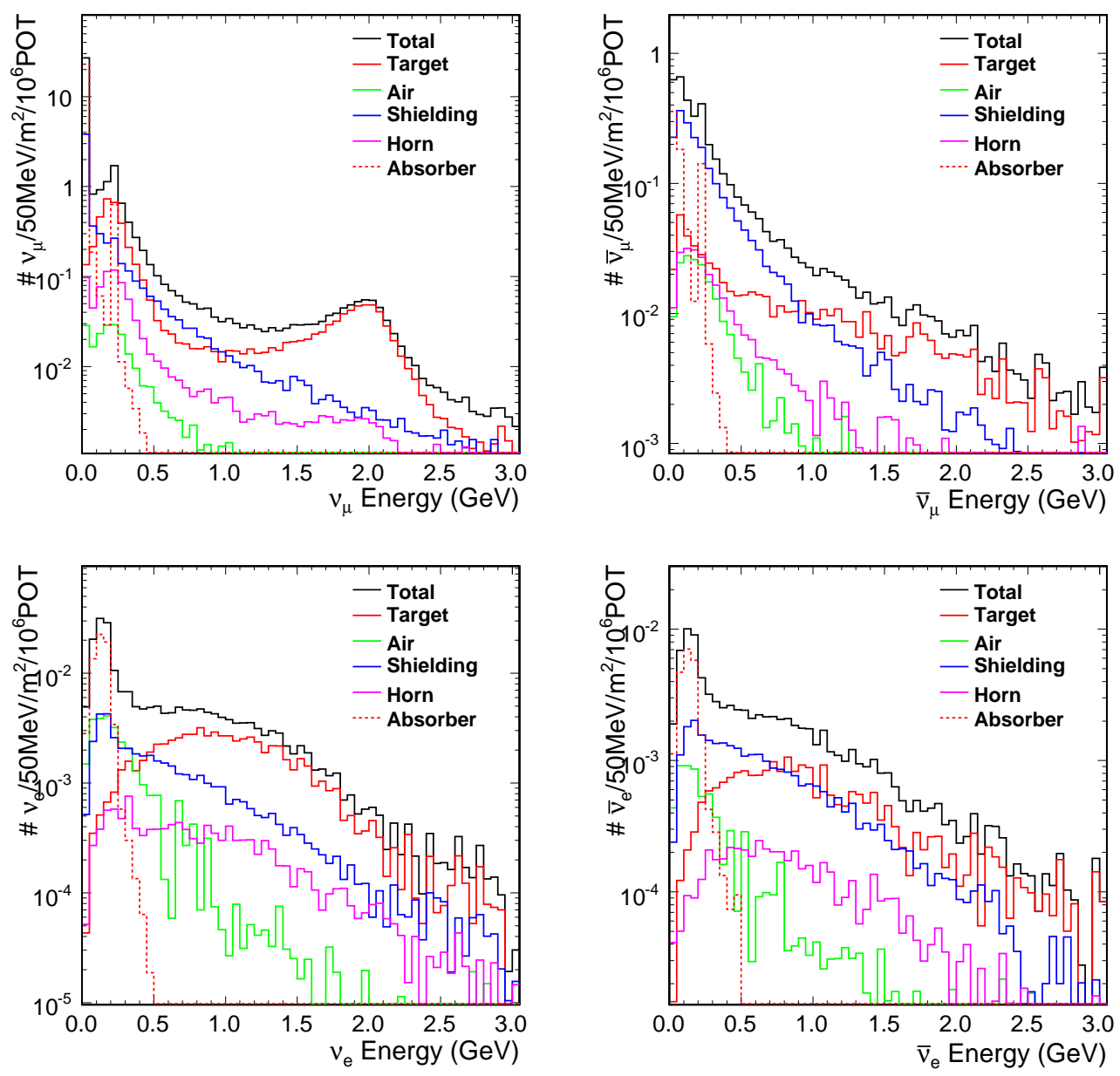

Figure B.6: The energy spectrum of $\nu_{\mu}, \bar{\nu}_{\mu}, \nu_{e}, \bar{\nu}_{e}$, divided by the material type which produced the neutrino's parent hadron or muon. "Target" implies the parent was created in the NuMI target, "Iron" implies the creation in the chase or decay pipe, and "absorber" refers to the beam dump, "Horn" implies the parent hadron was created in a shower cascade in the horns while "Air" is the designation for muons which were created by decays-in-flight of other hadrons. 

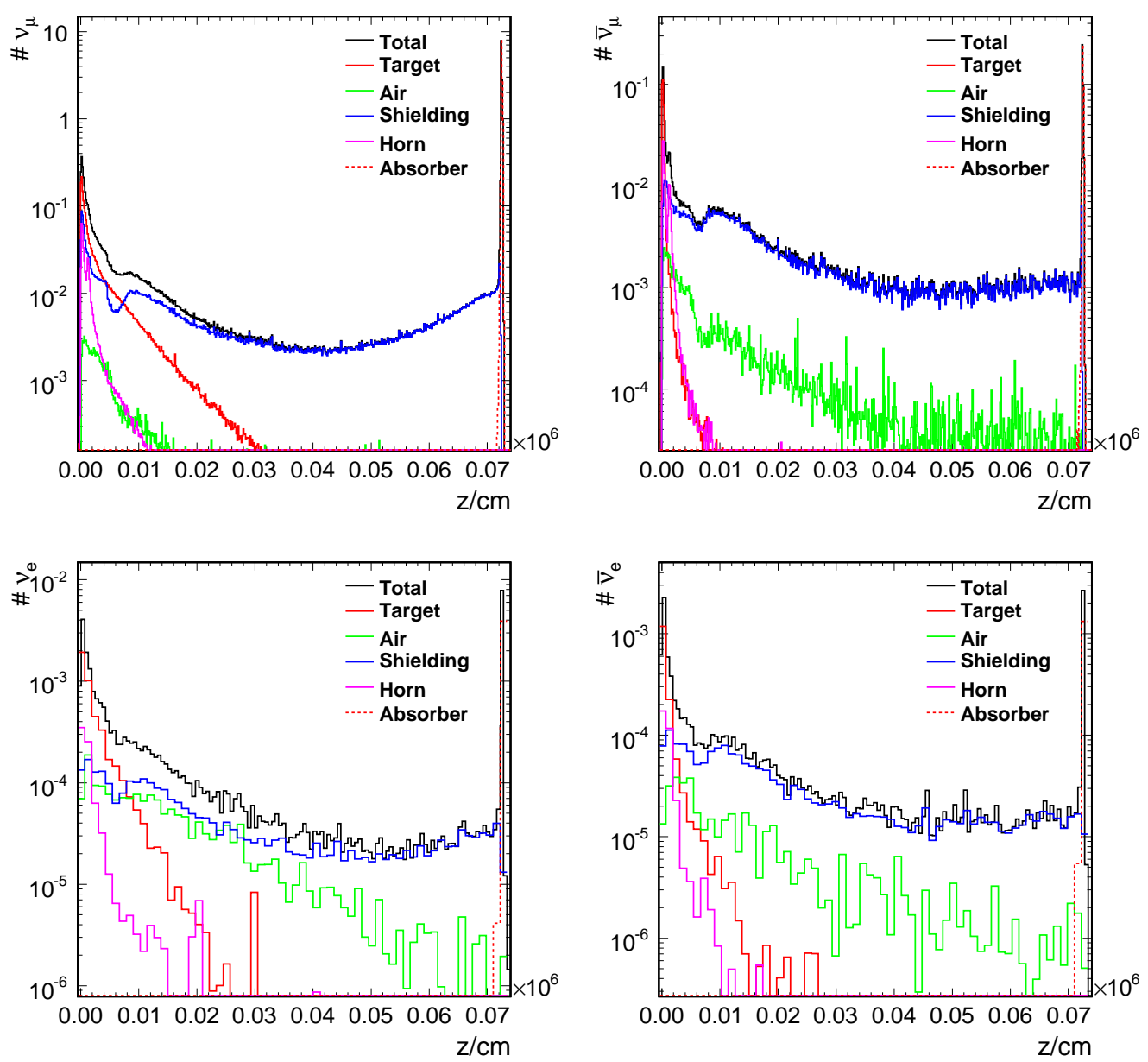

Figure B.7: The distribution in the $z$ (beam) direction at which the parent particle is produced whose daughter neutrino arrives at MiniBooNE. The "Target," "Horn," "Iron," "Absorber" and "Air" labels follow the same convention as in Figure B.6. 


\section{B.2 Hadron Production Uncertainties}

Particle production uncertainties for the off-axis beam considered here have three major components.

The first, the uncertainty in particle production of the NuMI target, has been studied extensively using the MINOS Near Detector data from several NuMI beam configurations. By tuning the Fluka cascade model (Chapter 6), we have been able to tune the yields of pions and kaons to agree well with the MINOS Near Detector data. Thus one might have a relative degree of confidence in the MiniBooNE flux prediction. Even though the relevant pion and kaon $\left(x_{F}, p_{T}\right)$ are different for MiniBooNE and and MINOS, the exact

$\left(x_{F}, p_{T}\right)$ distributions should be expected to be smooth functions, so the tuning performed for MINOS should extrapolate down to the relevant phase space for MiniBooNE. Furthermore, the corrections due to the tuning were modest (at most $\sim 10 \%)$ in the low $x_{F}$ region. Thus, it is not a surprise that there is little difference $(5-10 \%)$ in the Fluka and tuned Fluka flux predictions for MiniBooNE, as shown in Figure B.8. We take the full difference as an uncertainty, however.

The second uncertainty arises because of downstream interactions in the horn aluminum and the beam line shielding. For the MINOS data, such interactions comprise a relatively insignificant portion of the neutrino flux, with the exception of the "horn off" beam configuration, not considered here. For the MiniBooNE flux, however, downstream interactions account for $\sim 20 \%$ of the low energy $\nu_{\mu}$ flux and $30 \%$ of the low energy $\nu_{e}$ flux. Of further concern is that in the NuMI beam Monte Carlo, only the interactions in the target itself are simulated using the well-tuned Fluka cascade model. Interactions in the 
downstream horn or shielding materials are simulated using Geant, which can be configured to call the Geant/Fluka(92) or the GCALOR cascade models.

As an estimate of the flux uncertainty due to downstream interactions, we simulated the MiniBooNE flux using either the GEANT/Fluka(92) or GCALOR models and compared the results. The $\nu_{\mu}$ and $\nu_{e}$ spectra can be seen in Figure B.9. For the $\nu_{\mu}$ 's, the difference between these two predictions is approximately $20 \%$ at $E_{\nu}=500 \mathrm{MeV}$ and grows to $40 \%$ below $E_{\nu}<100 \mathrm{MeV}$, predominantly due to interactions in the iron shielding. At higher neutrino energies the $\nu_{\mu}$ uncertainty is smaller since most of the parents are kaons, which are produced in the target, not in the shielding. For the $\nu_{e}$ 's, the uncertainty for much of the spectrum is limited to $5-10 \%$ due to most of the parent being muons which are the daughters of hadrons coming from decays-in-flight off the target.

The third contribution to the uncertainty is from stopped $K$ decays in the NuMI beam dump, which is particularly important for low-energy $\nu_{e}+\bar{\nu}_{e}$, since a strong $K_{L}$ component exists there. We ascribed $50 \%$ uncertainty to the decays-at-rest component.

The net hadron production uncertainty will be taken as the sum, in quadrature, of the deviations between dashed and solid curves in Figures B.8 and B.9, plus a $50 \%$ uncertainty on the $K_{L}$.

\section{B.3 Focusing Uncertainties}

We have repeated the calculations of uncertainties in the off-axis neutrino flux due to beam focusing uncertainties following the same procedure used for the on-axis neutrino flux described in Chapter 5 In particular, these effects were 

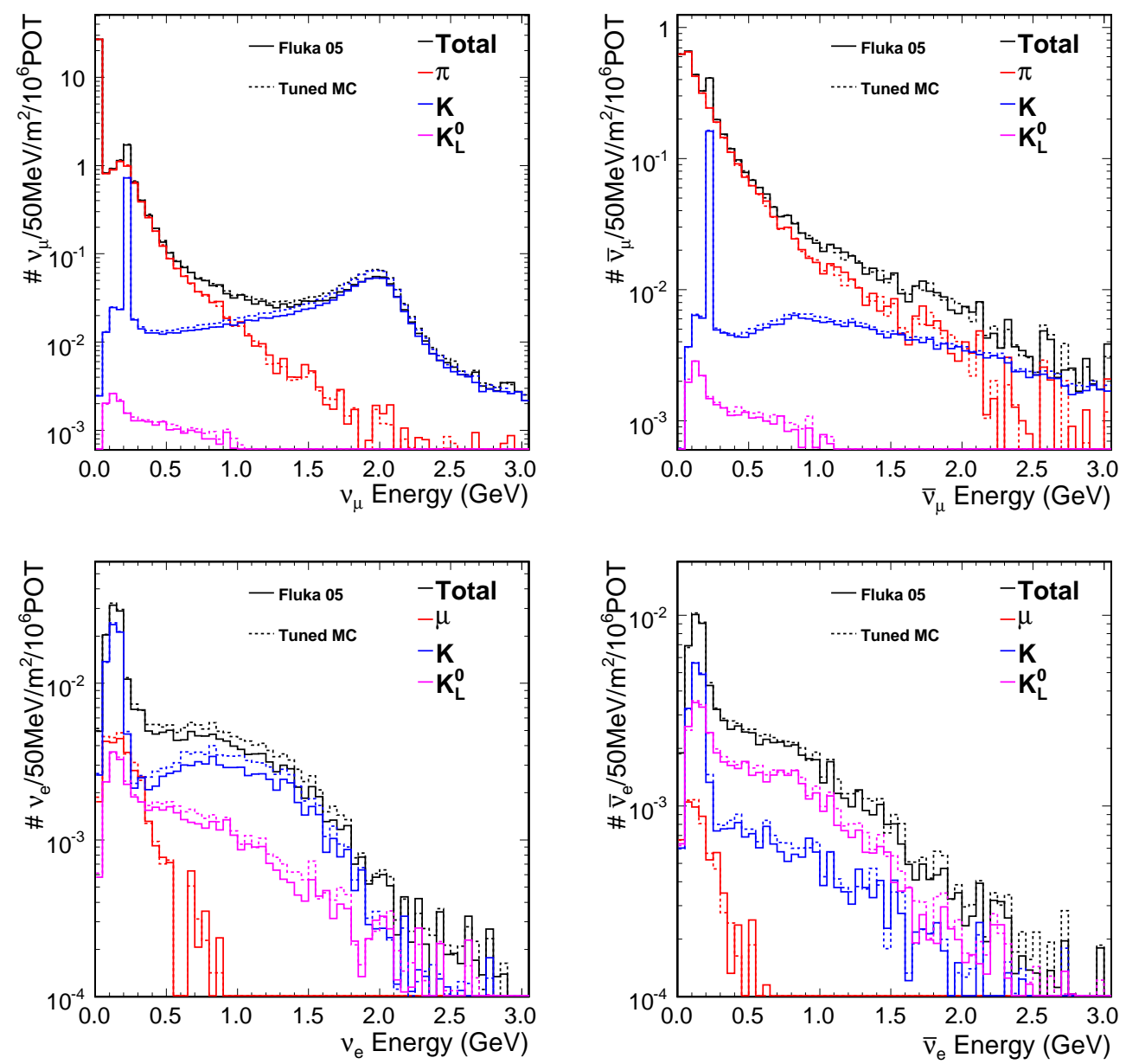

Figure B.8: NuMI fluxes for $\nu_{\mu}, \bar{\nu}_{\mu}, \nu_{e}$, and $\bar{\nu}_{e}$ at the MiniBooNE detector. The contributions from $\pi, K, K_{L}$, and $\mu$ decays are shown separately. The solid curves correspond to the Fluka2005 prediction while the dashed curves show the version of Fluka tuned to agree with the MINOS Near Detector data (Chapter 6). 

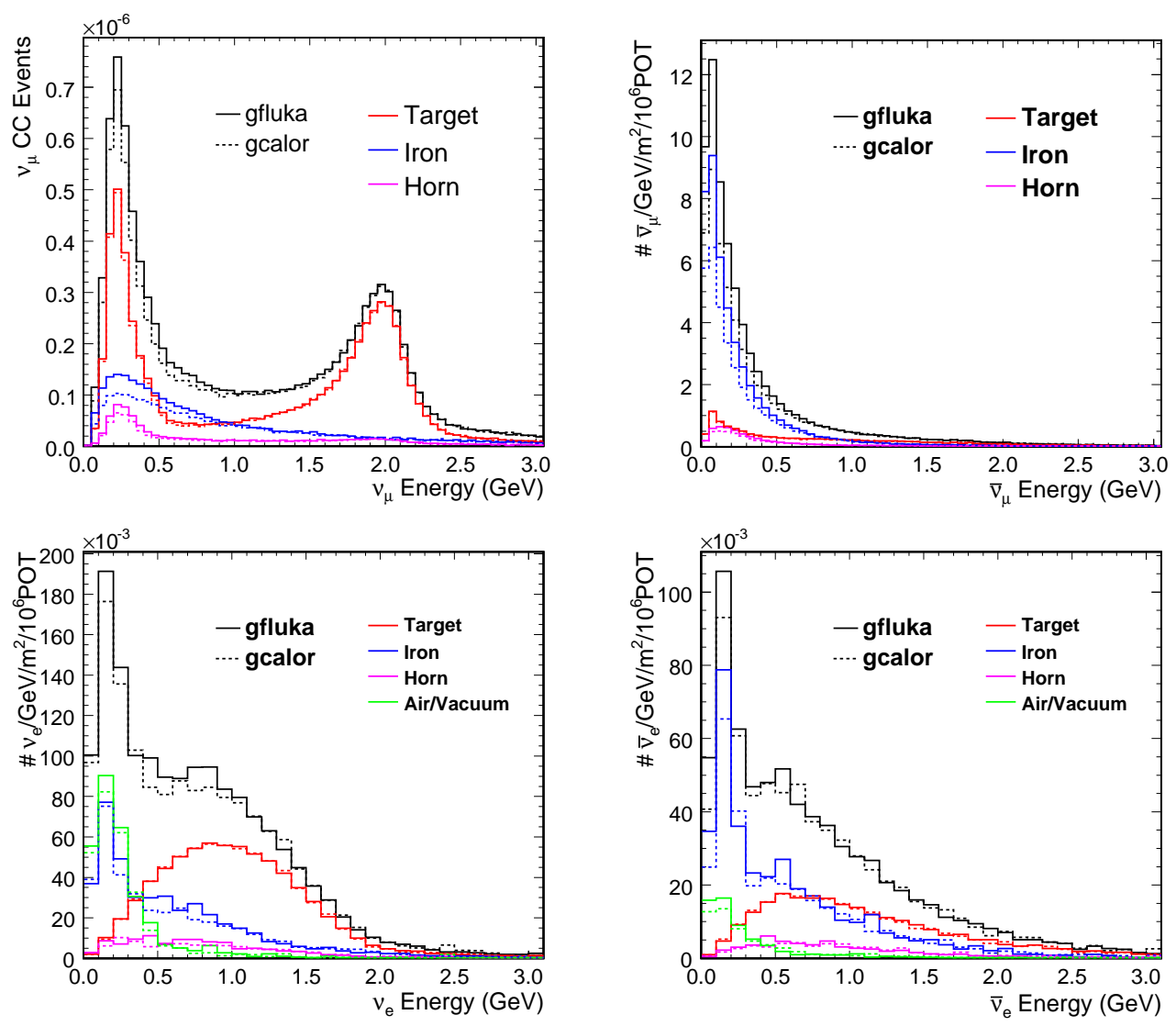

Figure B.9: NuMI fluxes for $\nu_{\mu}, \bar{\nu}_{\mu}, \nu_{e}$, and $\bar{\nu}_{e}$ at the MiniBooNE detector. The contributions from the target and downstream shielding and horn materials are shown separately. Interactions in the NuMI target are simulated by the Fluka2005 Monte Carlo, while interactions in the downstream material are simulated by either the GCALOR or GEANT/FLUKA packages. 
simulated:

- transverse misalignment of horns 1 and/or 2 by $1 \mathrm{~mm}$

- angular misalignment of horns 1 and/or 2 by $1 \mathrm{mrad}$

- uncertainty in the chase wall locations by 0.5 inches

- uncertainty (taken to be 1\%) in the absolute value of the horn current

- uncertainty in the distribution of the horns' current in their inner conductor.

- scraping of $1 \%$ of the proton beam on the collimating baffle

- uncertainty in the angle of the neutrino with respect to the NuMI target.

The uncertainty in the neutrino angle with respect to the NuMI target represents a reconstruction uncertainty. Because MiniBooNE is an optical detector, the neutrino vertex can be reconstructed with $\sim 20-30 \mathrm{~cm}$ resolution. To be conservative, we assumed a $0.5 \mathrm{~m}$ position error, corresponding to a potential angular uncertainty of $0.6 \mathrm{mrad}$, not insignificant compared to the off-axis angle of $\sim 110 \mathrm{mrad}$. That this uncertainty is so large is revisited below.

Because so many of the uncertainties considered in Figure B.10 are quite small, the $\nu_{e}$ flux uncertainties can be expected to be even smaller, arising from either 3-body decays or decays of randomly distributed particles produced in the NuMI shielding. These decays will have smaller dependence upon the focusing or off-axis angle. We therefore only considered the largest of these contributions to the possible $\nu_{e}$ flux error, namely the vertex uncertainty. In 
Figure B.11 we show this error as a function of $\nu_{e}$ energy, and indeed even this distortion is minuscule.

\section{B.4 Angular Error}

It is somewhat surprising that the reconstruction of the neutrino vertex in Section B.3 can have such a large effect on the neutrino flux. The source of this large spread due to the neutrino location is the resulting change in the off-axis angle caused by reconstructing the neutrino in the incorrect location. This means that an incorrect angle systematically shifts the peak of the offaxis kaon and pion spectra. Keeping in mind that the flux grows strongly as the off-axis angle is decreased, such a smearing results in a systematic shift. The Monte Carlo results properly calculate this shift in the peak of the fluxes across the MiniBooNE detector and the decreasing flux as the off-axis angle increases.

It is of interest to see if the MC-based result tracks our intuitive understanding. To approach this analytically, one may take the derivative

$$
\frac{d E_{\nu}}{d \theta}=-\frac{1-\frac{m_{\mu}^{2}}{m_{K}^{2}} E_{K}}{\left(1+\gamma^{2} \theta^{2}\right)^{2}} 2 \gamma^{2} \theta=-E_{\nu} \frac{2 \gamma^{2} \theta}{1+\gamma^{2} \theta^{2}}
$$

Kaon Peak: Figure B.3 indicates that the prominent kaon momentum relevant for MiniBooNE is $p_{K} \approx 3.5 \mathrm{GeV} / c$, or $\gamma \approx 7$. Further, $\theta \sim 0.11$. This gives

$$
\frac{d E_{\nu}}{d \theta} \approx-\left(0.0064 \text { milliradian }^{-1}\right) \times E_{\nu}
$$

This is the shift of the peak of the neutrino spectrum from kaons. From 


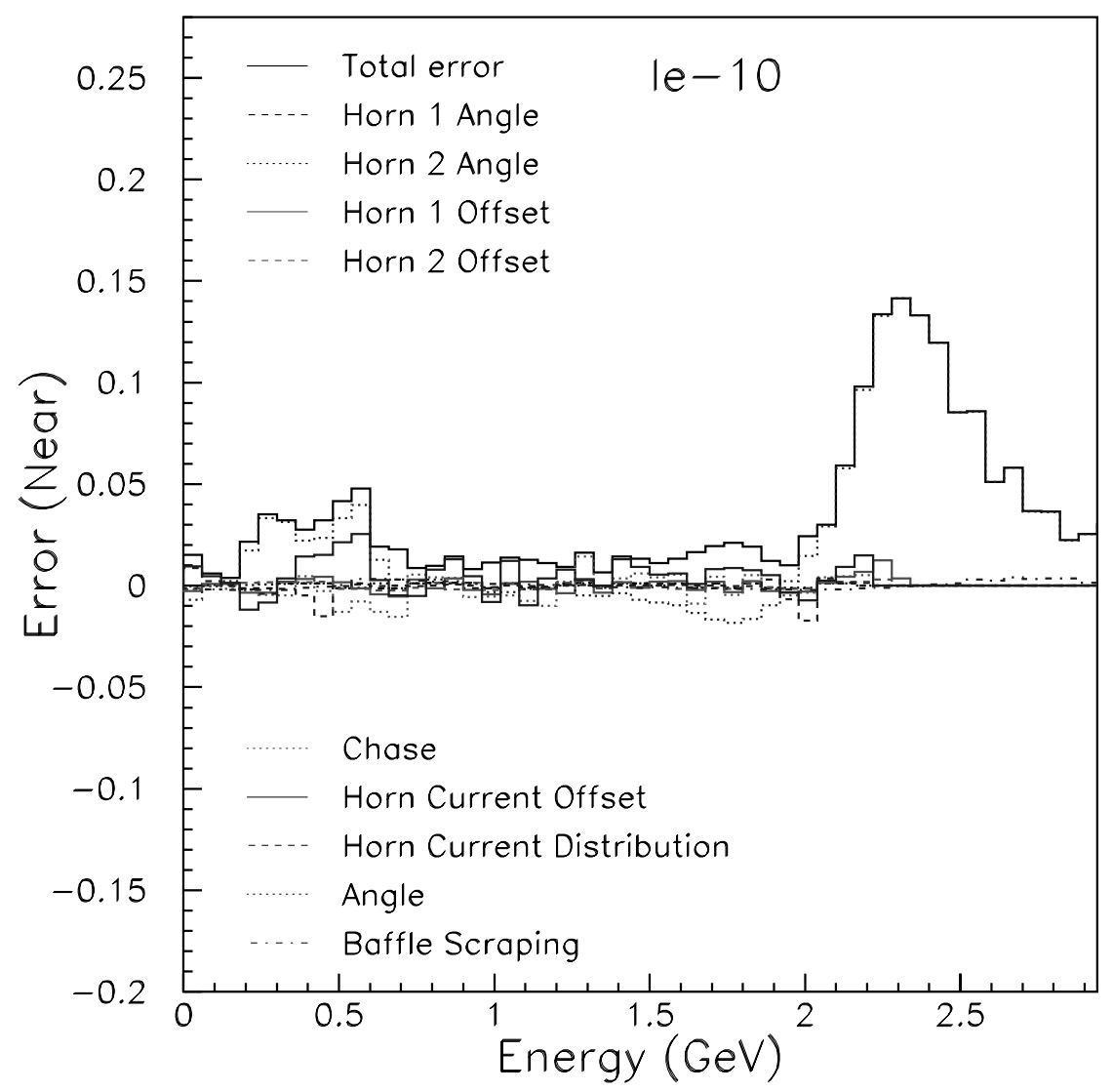

Figure B.10: Fractional uncertainty on the muon neutrino flux at the MiniBooNE detector from the NuMI LE-10 beam due to focusing effects. The uncertainty quoted above does not include the uncertainty from the pion and kaon yields. 


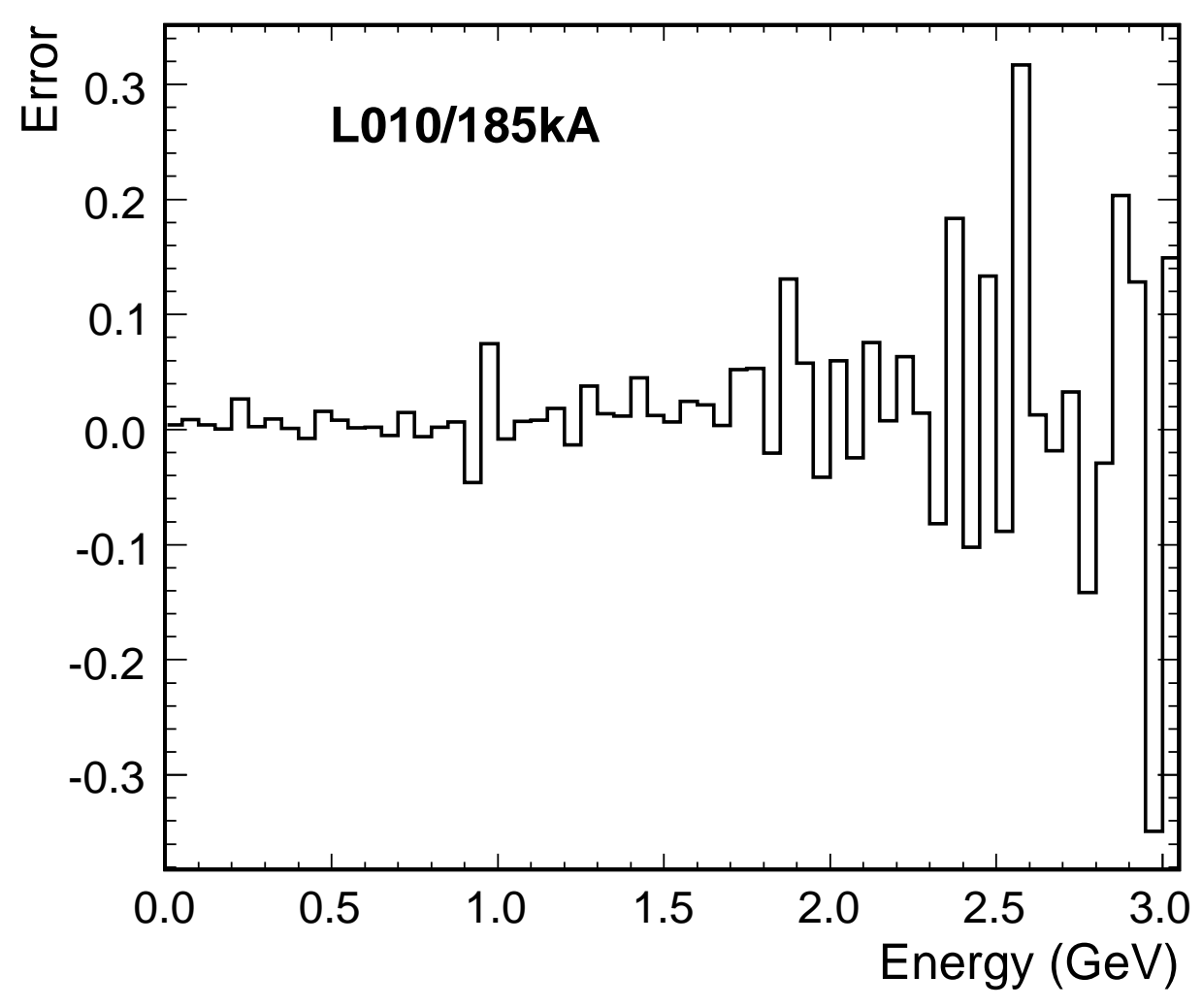

Figure B.11: Fractional uncertainty on the electron neutrino flux at the MiniBooNE detector from the NuMI LE-10 beam due to focusing effects. The uncertainty quoted above does not include the uncertainty from the pion and kaon yields. 
Figure B.4, the kaon peak is at about $E_{\nu} \approx 2 \mathrm{GeV}$, indicating a shift of $d E_{\nu} / d \theta \approx 13 \mathrm{MeV} /$ milliradian for the kaon peak.

Pion Peak: From Figure B.3 the dominant pion momentum of relevance is $p_{\pi} \approx 1.5 \mathrm{GeV} / c$, or $\gamma \approx 11$. This gives

$$
\frac{d E_{\nu}}{d \theta} \approx-\left(0.008 \text { milliradian }{ }^{-1}\right) \times E_{\nu}
$$

The pion peak is at about $E_{\nu} \approx 0.25 \mathrm{GeV}$, so $d E_{\nu} / d \theta \approx 2.0 \mathrm{MeV} /$ milliradian for the pion peak.

Figures B.12 and B.13 show the MC calculation estimates for the effect of angular offsets on the flux spectrum. The simulation roughly confirms the analytical estimates given above. The differences from the above calculations can be ascribed to the simplifications made above of selecting a unique pion or kaon momentum and unique decay angle in performing the computation. In reality, a broad band of pion and kaon momenta contribute to the decayin-flight beam, so $\gamma$ varies, and in reality such decays occur $\sim 50 \mathrm{~m}$ down the decay pipe (for $K$ 's with $\gamma=14$ ), giving a spread in decay angles.

It may therefore be concluded that the uncertainty in the MiniBooNE flux which diverges at large neutrino energies, as shown in Figure B.10, is in reasonable agreement with our expectations. 

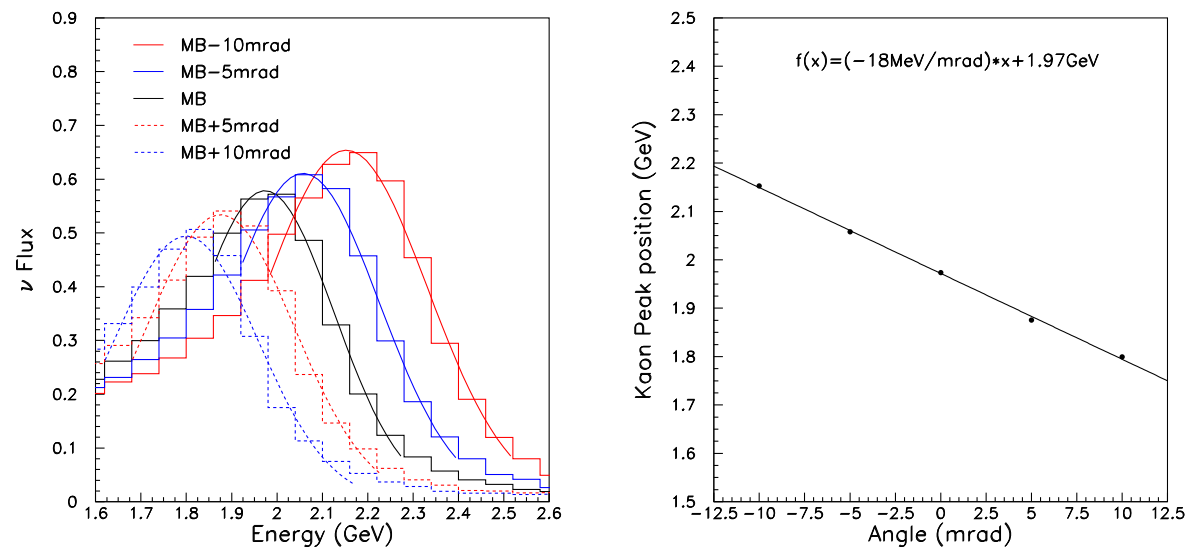

Figure B.12: (left) Monte Carlo calculation of the neutrino flux peak from kaon decays at the nominal MiniBooNE off-axis angle, as well as angles $\pm 5 \mathrm{mrad}$ and $\pm 10 \mathrm{mrad}$ from the nominal location. (right) Energy of the neutrino flux peak from kaon parents as a function of angular offset from the center of the MiniBooNE detector.
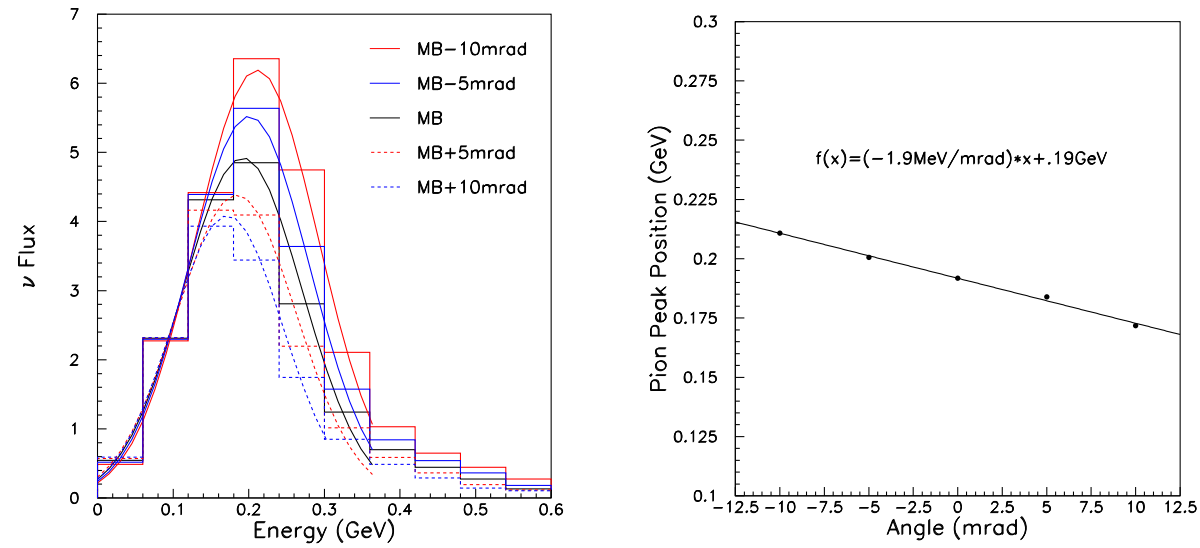

Figure B.13: (left) Monte Carlo calculation of the neutrino flux peak from pion decays at the nominal MiniBooNE off-axis angle, as well as angles $\pm 5 \mathrm{mrad}$ and $\pm 10 \mathrm{mrad}$ from the nominal location. (right) Energy of the neutrino flux peak from pion parents as a function of angular offset from the center of the MiniBooNE detector. 


\section{B.5 Summary of Off-axis $\nu$ Beam Uncertain- ties}

The 110 mrad off-axis flux is relatively easy to simulate, given our knowledge

of the off-axis flux and constraints from the MINOS data. However, a number of systematics are particular to the off-axis prediction at MiniBooNE, exacerbated by its close proximity to our beamline. Secondary interactions in the NuMI shielding material comprise a non-negligible portion of the neutrino flux at MiniBooNE (larger than would be expected at MINOS or an long-baseline off-axis experiment like NOvA).

Figure B.4 shows the expected neutrino fluxes at MiniBooNE. Figure B.14 shows the expected systematic uncertainty, adding in quadrature all the focusing and hadron production effects discussed previously.

\section{B.6 MiniBoone Data Analysis}

The MiniBooNE detector is a $12.2 \mathrm{~m}$ spherical tank filled with 800 tons of pure mineral oil. The detector consists of two parts, the inner part which has a radius of $5.5 \mathrm{~m}$ and acts as target and the outer part which forms a veto region. Two parts are optically separated by a support structure which holds 1280 8-inch Photo Multiplier Tubes (PMTs) that point inward and 240 PMTs pointed outward. The detector can reconstruct neutrino events with visible muon energy up to $2.2 \mathrm{GeV}$ and somewhat higher electron energy.

The observed neutrino interactions in the detector can be classified depending on the particles present in the final state. 39\% of events are ChargedCurrent Quasi-Elastic (CCQE) scatterings. The charged-current (CC) inter- 

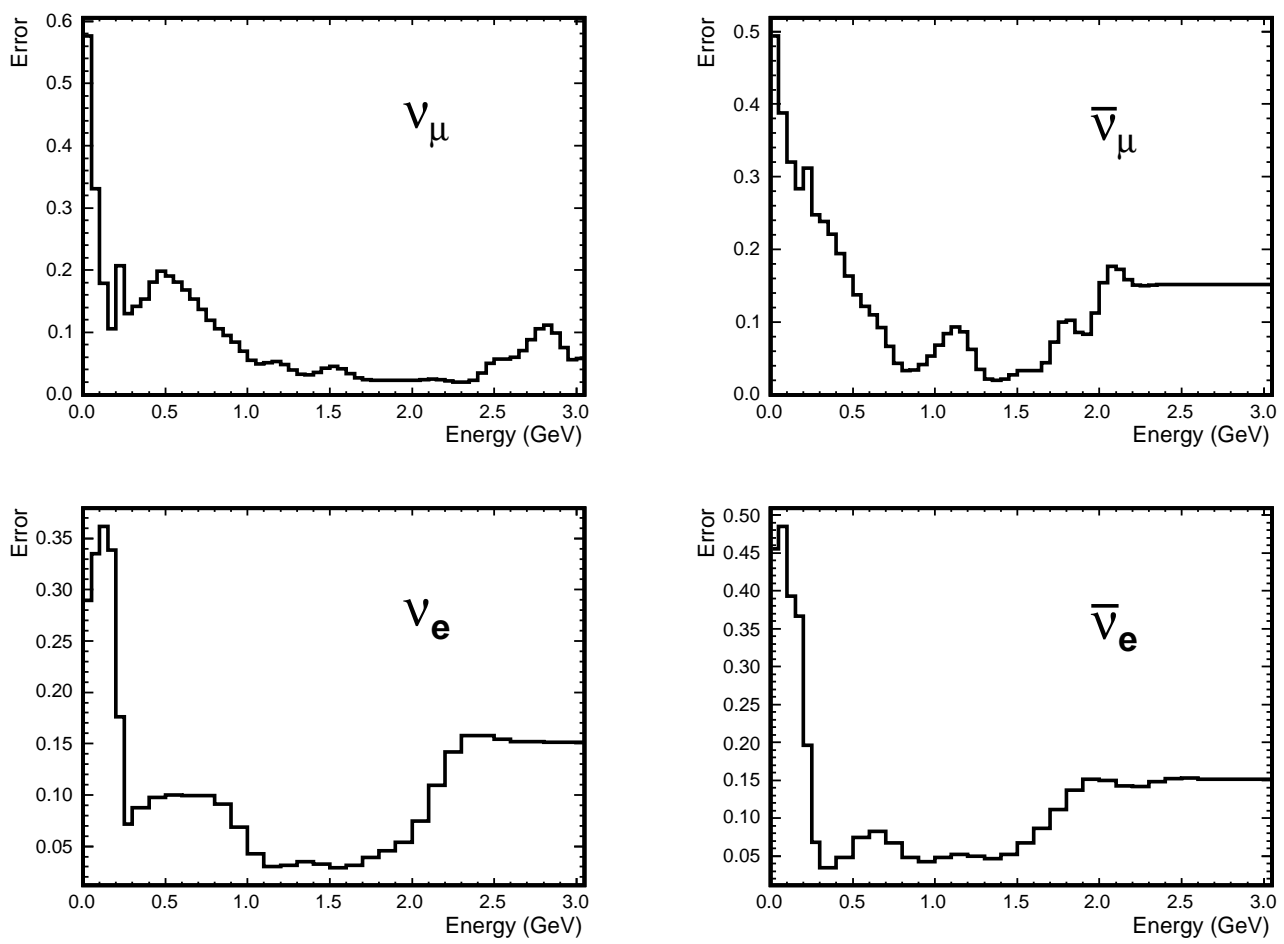

Figure B.14: The relative uncertainty in the neutrino flux at the MiniBooNE detector, plotted as a function of the true neutrino energy. The four plots are the uncertainty for $\nu_{\mu}$ (upper left), $\bar{\nu}_{\mu}$ (upper right), $\nu_{e}$ (lower left), and $\bar{\nu}_{e}$ (lower right). 
action in which a single pion is produced form $31 \%$ of events. The neutralcurrent (NC) single pion production makes $14 \%$ of events. Charged current multi-pion production contributes to $5 \%$ of events and deep inelastice scattering contributes to $4 \%$ of events.

The particle identification primarily uses Cherenkov radiation. The muons produce a sharp Cherenkov ring. The thickness of the ring is related to the travel path of the muon. The neutral pions produce two Cherenkov rings. Finally electrons produce one fuzzy ring due to multiple scattering. Additionally scintillation light is used to improve particle identification.

A likelihood based reconstruction algorithm is used to classify events. The events are fit under the single muon and single electron hypotheses. The event that favors the electron hypotheses is then fit with two ring fits, with the mass both unconstrained and constrained to the $\pi_{0}$ mass. Using the likelihood ratio for electron and $\pi_{0}$ as well as reconstructed $\pi_{0}$ mass, the events can then be classified as $\pi_{0}$-like or $\nu_{e}$ CCQE-like.

The systematic errors in the predicted energy spectrum are due to the neutrino flux uncertainty (as discussed in Section B.5) and also due to cross section and detector modeling uncertainties. Both external data and MiniBooNE data is used to constrain the cross section and detector modeling uncertainties.

We have seen in Section B.2 that the biggest uncertainty in neutrino flux is coming from the stopped mesons in the NuMI beam absorber. In order to remove this component it was required that the visible energy had to be above $200 \mathrm{MeV}$.

For this analysis we have used the data acquired in LE10/185kA beam configuration (Table 2.1) that corresponds to $1.42 \times 10^{20}$ protons on target. 


\section{B.6.1 $\nu_{\mu}$ CCQE events}

The identification of the Charged-Current Quasi-Elastic (CCQE) scattering of $\nu_{\mu}$ is based upon the detection of the created muon and the associated creation of electron in $\mu$ decay:

$$
\begin{array}{r}
\nu_{\mu}+n \rightarrow \mu^{-}+p \\
\mu^{-} \rightarrow e^{-}+\nu_{\mu}+\bar{\nu}_{e}
\end{array}
$$

From the reconstructed quantities in the CCQE event it is possible to reconstruct the neutrino energy using the kinematics formula:

$$
E_{\nu}^{Q E}=\frac{1}{2} \frac{2 M_{p} E_{\mu}-m_{\mu}^{2}}{M_{p}-E_{\mu}+\sqrt{\left(E_{\mu}^{2}-m_{\mu}^{2}\right) \cos \theta_{\mu}}}
$$

where $M_{p}$ is the proton mass, $m_{\mu}$ is muon mass, $E_{\mu}$ is muon energy and $\theta_{\mu}$ is the angle of the muon with respect to the incoming neutrino. Since a cut in visible energy removes the neutrinos created in beam absorber and downstream shielding, it can be assumed that the neutrinos originate from the target area. The resolution on $E_{\nu}^{Q E}$ is $\sim 11 \% / \sqrt{E}$.

Figure B.15 shows the reconstructed energy spectrum for $\nu_{\mu}$ CCQE-like events. The data, shown in black points, shows very good agreement with Monte Carlo calculation shown as a red band. The band reflects the total systematic and statistical uncertainty. Also indicated is the component of neutrinos coming from pion and kaon parents. Again it can be seen that the pions contribute to lower energies and kaons to higher energies. The kaon peak however is not obvious any more since higher energy neutrinos are more likely to leave MiniBooNE detector so their energy cannot be reconstructed. 


\section{B.6.2 $\nu_{e}$ CCQE events}

One of the backgrounds to selected $\nu_{e}$ events comes from the $\nu_{\mu}$ CCQE events in which a muon gets captured. This happens in $8 \%$ of events, but since there are many more $\nu_{\mu}$ s than $\nu_{e} \mathrm{~s}$ in the beam, this presents a significant background. To reduce it a cut in a relative electron and muon likelihoods is imposed.

The other significant component to the background comes from the $\mathrm{NC}$ events in which a $\pi_{0}$ is produced. If only one electromagnetic track is reconstructed from the decay of $\pi_{0}$, the event can look like $\nu_{e}$ CCQE. Most of these $\pi_{0} \mathrm{~s}$ are successfully reconstructed using a two ring likelihood fit. As can be seen in Figure B.16 excellent agreement between data (black dots) and Monte Carlo simulation (black line) is achieved. Also indicated in the plot are true $\pi_{0}$ events and the two backgrounds coming from misidentified muon and electron neutrino interactions.

The $\pi_{0}$ background in the $\nu_{e}$ CCQE sample is reduced using reconstructed $\pi_{0}$ mass and the electron and pion likelihood ratio.

Figure B.17 shows the data (black dots) and Monte Carlo (black dashed line) comparison. The total systematic and statistical error associated with Monte Carlo prediction is shown as a red band. A slight excess in data over Monte Carlo prediction at the level of $1.4 \sigma$ can be seen below $0.9 \mathrm{GeV}$. This is of interest as $\nu_{e}$ s largely come from the same kaon parents that also contribute to $\nu_{\mu}$ flux. 


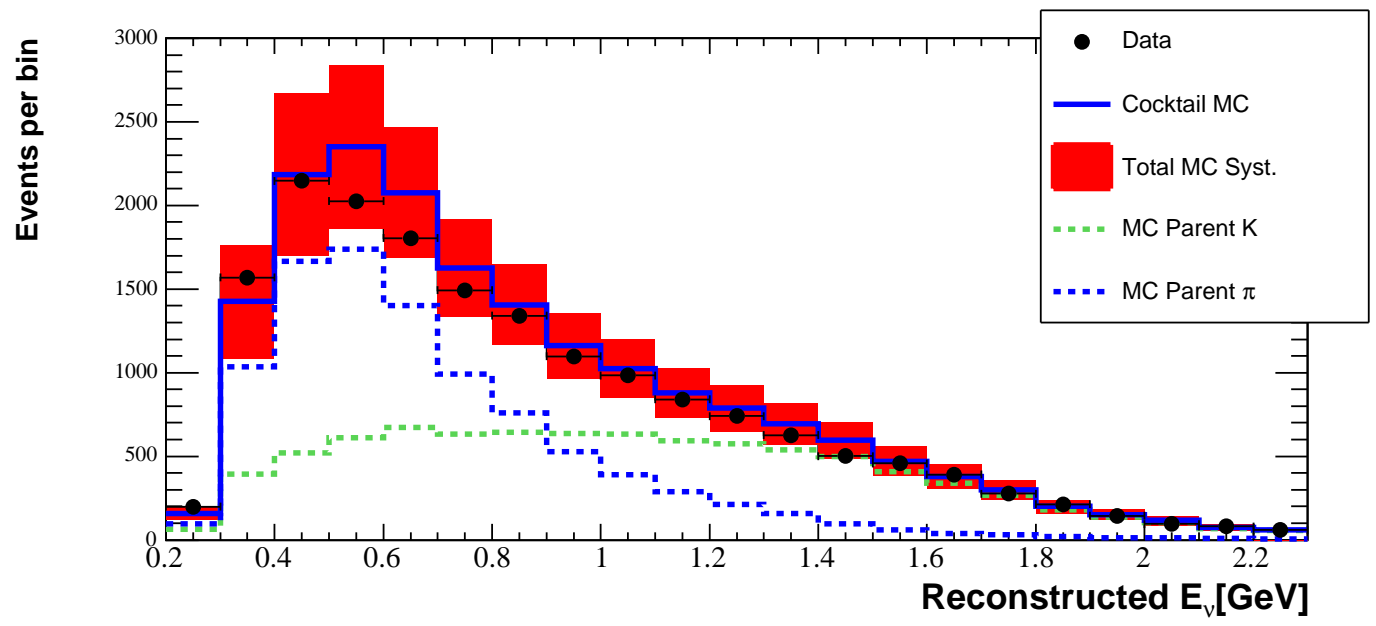

Figure B.15: The reconstructed neutrino energy $E_{\nu}$ for the selected $\nu_{\mu}$ CCQE candidates. The data (black dots) shows excellent agreement with Monte Carlo prediction (red band). The MC band reflects the total systematic and statistical error. Figure courtesy Z. Djurcic.

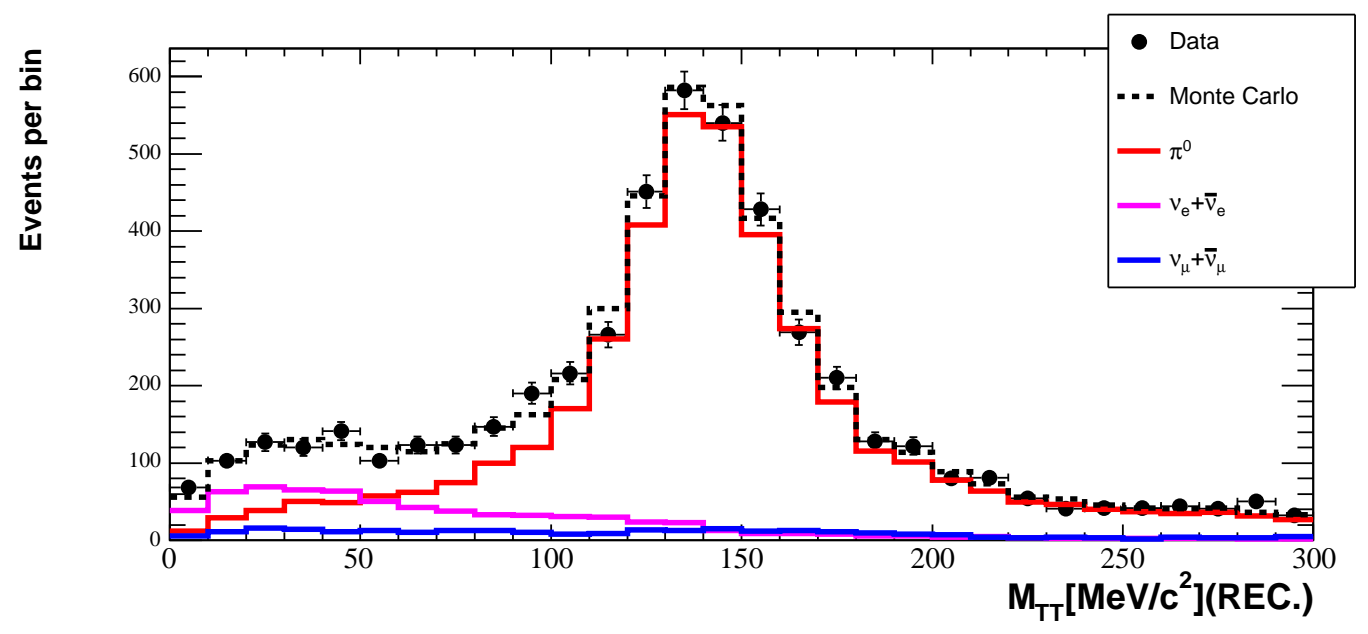

Figure B.16: The mass distribution of $\pi_{0}$ candidates. The data (black dots) shows excellent agreement with Monte Carlo prediction (black dashed line). Figure courtesy Z. Djurcic. 


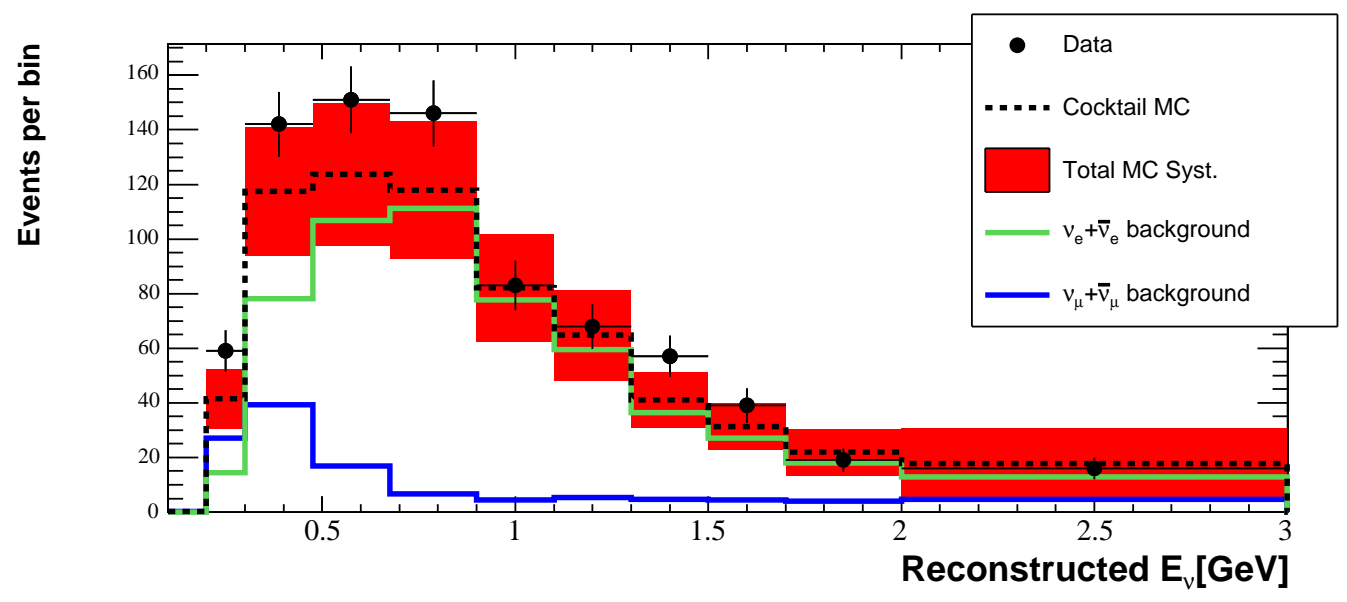

Figure B.17: The reconstructed neutrino energy $E_{\nu}$ for the selected $\nu_{e}$ CCQE candidates. The data (black dots) is compared to Monte Carlo prediction (red band). The MC band reflects the total systematic and statistical error. A small excess in data over Monte Carlo prediction is visible in energy bins below $0.9 \mathrm{GeV}$. The green line indicates the true $\nu_{e}$ events coming from NuMI beamline. The blue line shows the misidentified $\nu_{\mu}$ events. Figure courtesy $\mathrm{Z}$. Djurcic. 


\section{B.7 Summary}

A good agreement between data and Monte Carlo prediction was observed for both $\nu_{\mu} \mathrm{CCQE}$ and $\nu_{e} \mathrm{CCQE}$ samples. This provides an additional cross check and constraint of pion and kaon production in the NuMI target which is somewhat complementary to the constraint provided by MINOS Near Detector (Chapter 6).

This was a successful demonstration of the off-axis neutrino beam. Several future neutrino oscillation experiments will utilize the offaxis concept to perform precision measurements of oscillation parameters [101, 31].

The $\nu_{e}$ CCQE sample showed slight excess in data over the prediction for reconstructed neutrino energies below $0.9 \mathrm{GeV}$. This excess will be further studied as more data is accumulated. With improvements in the analysis it is also possible to reduce the systematic uncertainty in the predicted spectrum. 


\section{Appendix C}

\section{Cross-check of NuMI Flux Using the Accompanying Muon Beam}

In past experiments, muon monitors have been used to infer or constrain the fluxes to the experiment $[102,103,104,105,106,107]$. These past experiments have benefited from 9-16 muon alcoves distributed at various positions within the shielding which can be used to perform integral flux measurements. The large number of alcoves permitted unfolding of the threshold measurements into a differential distribution of muons, and hence neutrinos. Furthermore, these preceding wide-band neutrino lines were rather short (80-140 m long decay pipes) so that the muon monitors were close to the target and could measure the lateral profile of muons; such is beneficial to help constrain the energy spectrum because softer muons (from pions) are emitted at larger angles and large angle muons also constrain the neutrinos from kaon parents. 
The NuMI beam has only three instrumented alcoves (see Figure 2.2), and the thresholds are higher than these past beam lines. This is especially important for the low-energy beam configuration (in Appendix D it will be shown that the effective neutrino energy threshold of Muon Alcove 1 is approximately $1.6 \mathrm{GeV}$ ). Further, the NuMI decay pipe is quite long, so the radial fall-off of the muon beam becomes rather flat at their location $700-800 \mathrm{~m}$ from the target, especially in the low-energy beam configuration.

Despite these limitations, the muon monitors, when studied in the various configurations of the flexible NuMI beam (§2.1.2), do have some sensitivity to the neutrino flux. As will be discussed, measurements of muon fluxes in the three alcoves at various target positions and horn currents can help to place constraints on the $\left(x_{F}, p_{T}\right)$ of parent hadrons which lead to neutrinos in the MINOS detectors. Thus, the two variables of horn current and target position provide somewhat independent constraints, much like the multiple muon thresholds and muon radial distributions provided in past beam lines.

In this appendix we use a weighting scheme described in Appendix D to calculate the muon flux in the alcoves. These Monte Carlo calculations of muon rates will be compared to data collected during special runs of the NuMI beam line [108]. During these studies, 100-500 pulses were acquired at each target location $(0 \mathrm{~cm}, 10 \mathrm{~cm}, 100 \mathrm{~cm}, 150 \mathrm{~cm}, 250 \mathrm{~cm})$ and over a variety of horn currents.

The muon monitors in the NuMI beam are arrays of ion chambers. The total integrated charge collected from the ion chambers is proportional to the muon flux. The response of the chambers was studied using a test beam $[109,110]$. However, these studies were performed at an unknown absolute pressure and temperature, and furthermore had a Helium filling gas whose 
composition/purity may not be identical to that filling the NuMI chambers. Because of that the conversion factor between charge recorded in the muon monitors and number of incident muons is not known. Therefore this study will be limited to a shape analysis of the beam spectrum, i.e. an absolute rate will not be determined. An absolute rate fit to the data is further precluded because a beam muon is often accompanied by $\delta$-electrons which cause additional ionization in the chamber gas $[111,112]$, which requires future study.

\section{C.1 Muon Alcove Acceptances}

Ideally the muon alcoves would have a similar acceptance as the MINOS neutrino detectors, ie the muons seen in the alcoves would come from the same parents that produce neutrinos in MINOS detectors. Especially of interest is the low-energy neutrino beam configuration (LE010) which is used for the oscillation analysis (Table 2.1). Naively, however, one would expect that the acceptance of the muon monitor is rather limited in the low energy neutrino beam. There are two effects that would suggest that:

- The lower-energy muons in the beam have greater probability for ranging out in the beam absorber or inter-alcove rock than do the muons in the higher-energy beam configurations (ie LE100, LE150 and LE250).

- The decays of soft pions, which decay upstream in the NuMI decay pipe, can send their muons at off-angles such that they cannot reach the alcoves.

As will be shown in Appendix D, the above two effects are qualitatively correct but quantitatively do not limit the muon alcoves' acceptance. First, a $4 \mathrm{GeV} / c$ 
pion produces at most a $1.6 \mathrm{GeV}$ neutrino or (at most) a $4 \mathrm{GeV} / c$ muon (though not in the same decay). The $4 \mathrm{GeV} / c$ threshold for a muon at the end of the decay pipe to reach alcove 1, therefore, implies that the alcoves can monitor the parent hadrons down to an equivalent $1.6 \mathrm{GeV}$ neutrino, which is below the peak of the LE10/185kA beam (peak value $\sim 3 \mathrm{GeV}$ ). With regard to the angular acceptance of the muon monitors, the muons in pion decay are more forward-boosted than the neutrinos. The muons are all contained within a cone of $\tan \theta_{\max }=\beta^{\prime} /\left(\gamma \sqrt{\beta^{2}-\left(\beta^{\prime}\right)^{2}}\right)$, where $\beta$ and $\gamma$ describe the pion/kaon velocity and boost factor, and $\beta^{\prime}$ is the muon velocity in the rest frame of the parent meson $(=0.28$ for $\pi$ decays and 0.9 for $K$ decays). This works out to about $10 \mathrm{mrad}(4 \mathrm{mrad})$ for $4 \mathrm{GeV} / c(10 \mathrm{GeV} / c)$ pion decays. Thus, the $\sim 1.4 \mathrm{mrad}$ solid angle acceptance of the monitors is not negligible. To be sure, the lateral profile of the muons at the alcoves will be quite broad for the decays of low-energy pions, but the rate should be still measurable.

Figure C.1 attempts to demonstrate the preceding argument. Shown are the momenta of muons from all $\pi^{ \pm}$and $K^{ \pm}$decays ${ }^{1}$ in the horn off (LE010/0kA), LE010/185kA, LE100/200kA, and LE250/200kA beam configurations. Figure C.1 also shows the momenta of those muons from $\pi^{ \pm}$and $K^{ \pm}$decays which reach the end of the NuMI decay pipe, anywhere around its surface. The "end-of-decay-pipe" muon distribution is further multiplied by the efficiencies calculated in [113] for such muons to penetrate the absorber and rock and not multiple-scatter out of the $2 \times 2 \mathrm{~m}^{2}$ area of the muon monitor arrays. As can be seen, the Muon Alcoves retain a substantial fraction of the muons produced in the focusing peaks of all the beam configurations.

\footnotetext{
${ }^{1} K_{L}$ decays have not been included, but are of order $10 \%$ of the $K^{+}$.
} 

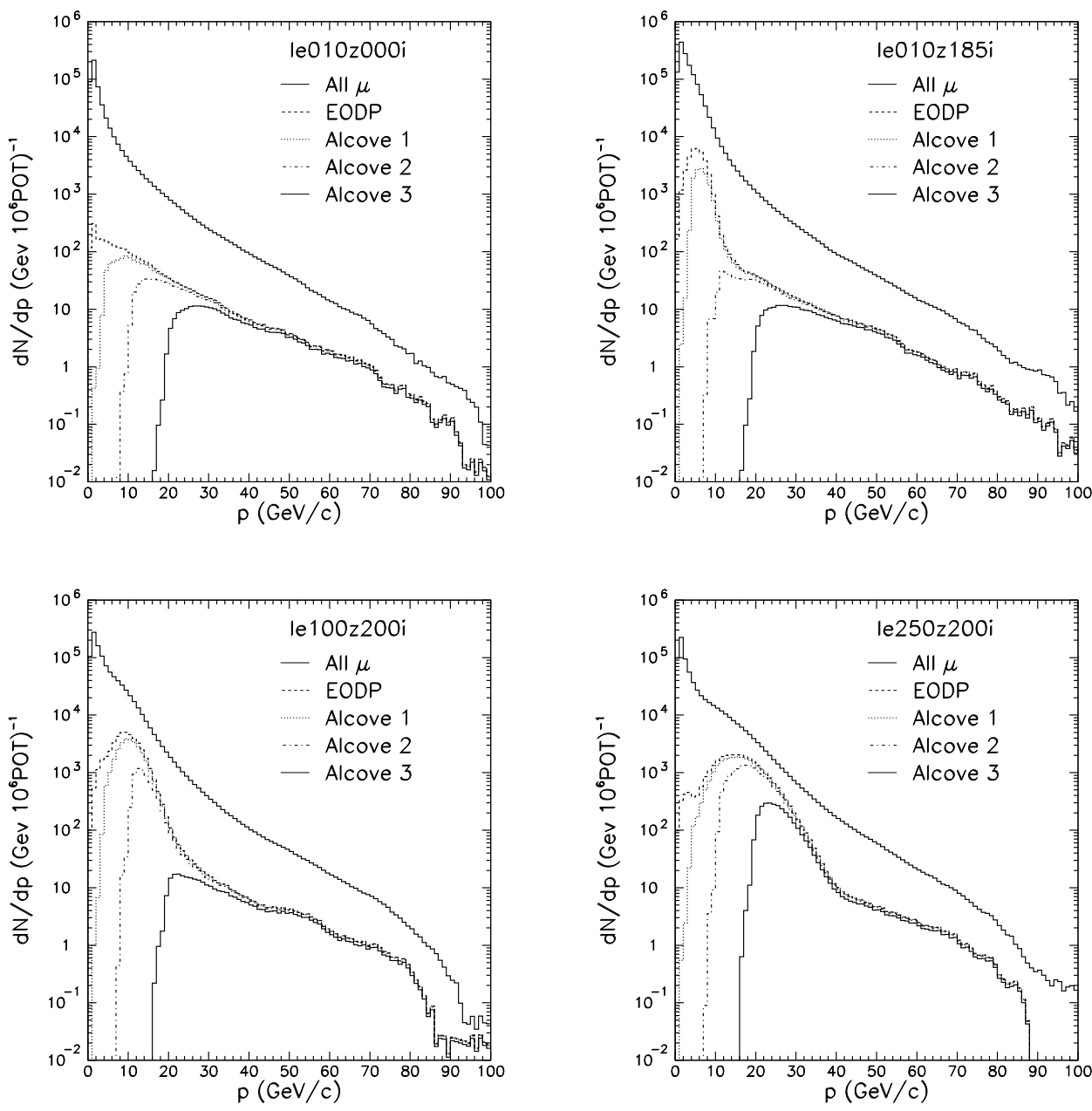

Figure C.1: Distribution of momenta of muons $\left(\mu^{+}\right.$and $\left.\mu^{-}\right)$in the horn off (top left), LE10/185kA (top right), LE100/200kA (bottom left) and LE250/200kA (bottom right) beam configurations. Shown are the distribution of muons created at any angle and anywhere in the decay pipe, the distribution of those muons arriving at the end of the decay pipe (upstream of the hadron absorber, and in each of the 3 alcoves. 

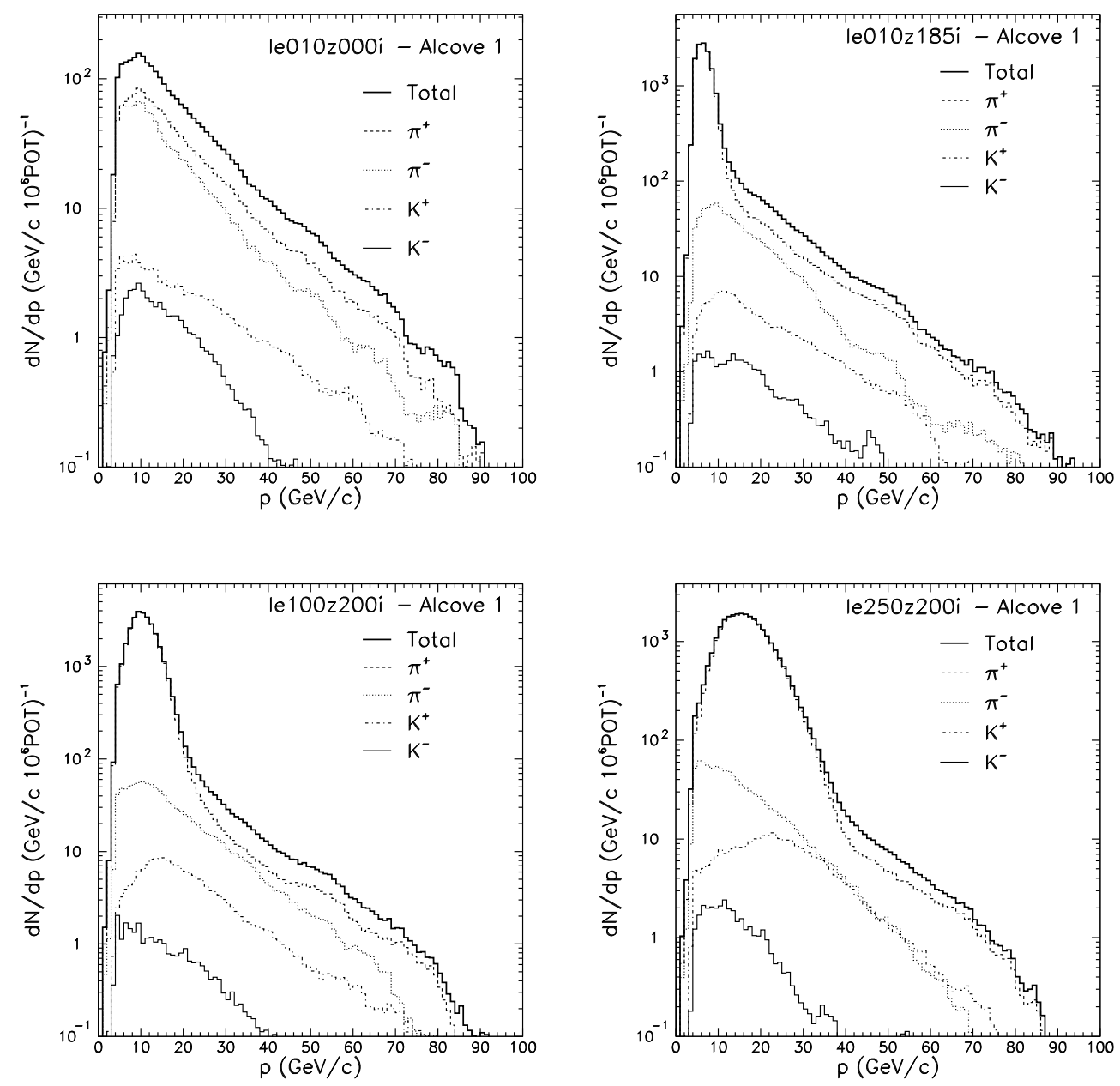

Figure C.2: Distribution of momenta of $\mu^{ \pm}$arriving in Alcove 1 in the horn off (top left), LE10/185kA (top right), LE100/200kA (bottom left) and LE250/200kA (bottom right) beam configurations. The spectra by parent type are also shown. 

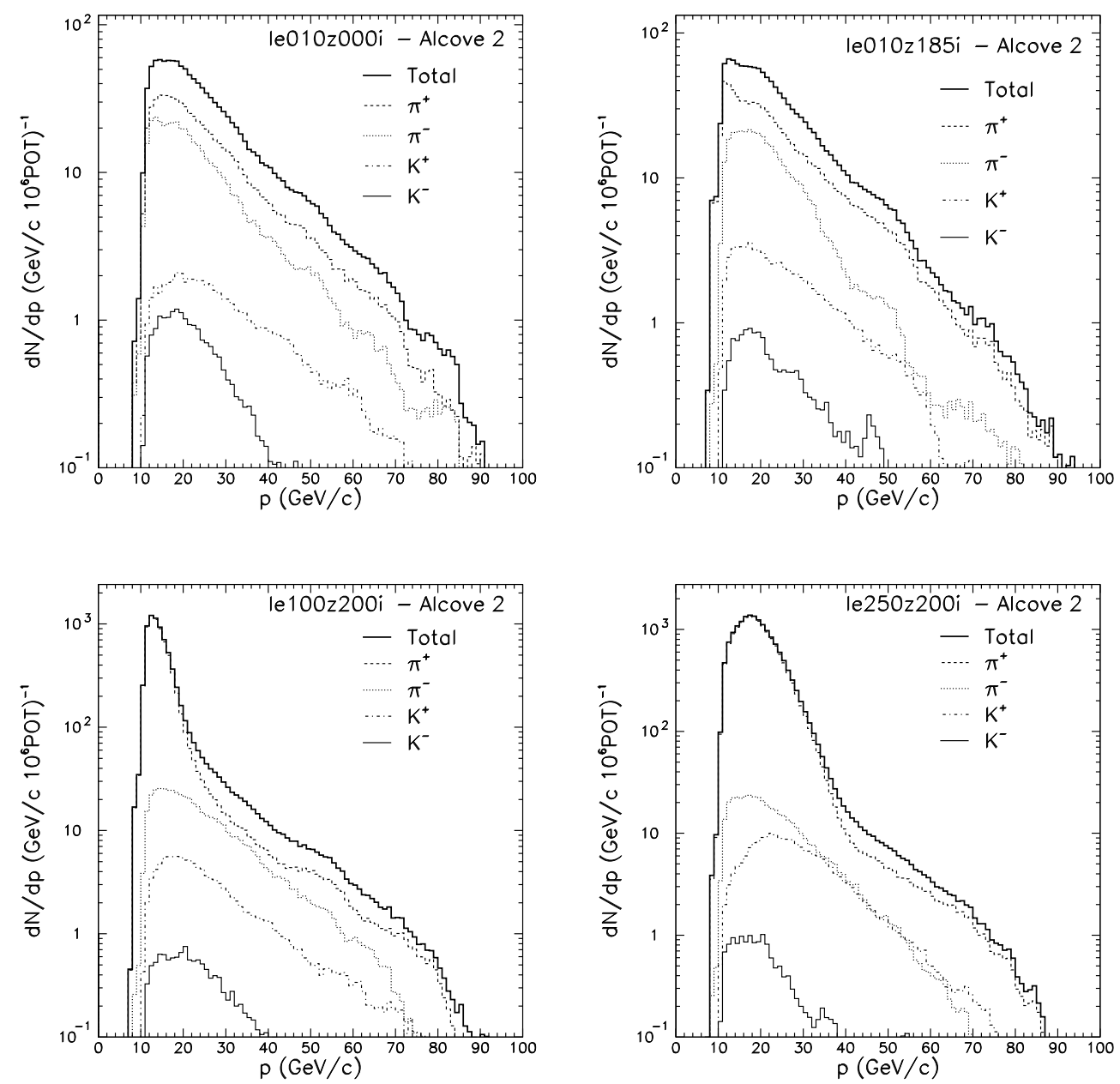

Figure C.3: Distribution of momenta of $\mu^{ \pm}$arriving in Alcove 2 in the horn off (top left), LE10/185kA (top right), LE100/200kA (bottom left) and LE250/200kA (bottom right) beam configurations. The spectra by parent type are also shown. 

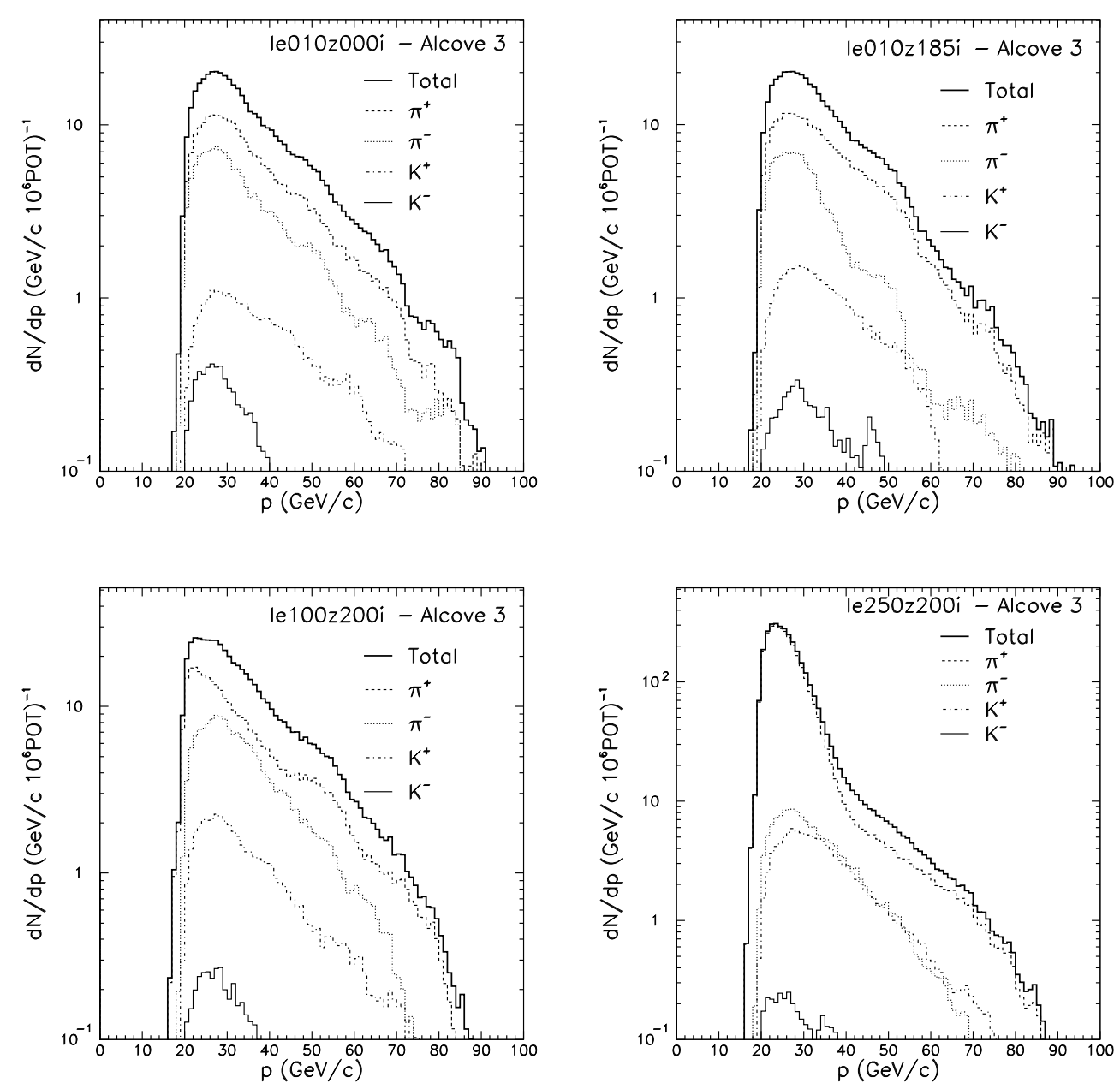

Figure C.4: Distribution of momenta of $\mu^{ \pm}$arriving in Alcove 3 in the horn off (top left), LE10/185kA (top right), LE100/200kA (bottom left) and LE250/200kA (bottom right) beam configurations. The spectra by parent type are also shown. 
Figures C.2 through C.4 show the parentage of the muons that reach the three alcoves in these four beam configurations. As can be seen, the horn off beam contains nearly equal contributions from $\pi^{+}$and $\pi^{-}$decays, with $K^{ \pm}$ less than $3 \%$ (expected because of the broader angular distribution for kaon decays). With the horns on, the $\pi^{+}$and $K^{+}$contributions grow substantially in all three alcoves over the unfocused "background," however. It is encouraging that the muons from both $\pi$ and $K$ decays reach all the alcoves, since we know that the high energy neutrinos, particularly in the LE250/200kA beam, come from kaon decays. Because the flux of muons seen in the muon monitors primarily comes from the pion parents the flux extracted using the muon monitors will best describe neutrino flux below $30 \mathrm{GeV}$ where the neutrinos predominantly come from pions.

Figures C.5 through C.7 show the acceptance for the underlying parent pions. Shown in the upper left of each figure are the $\left(p_{z}, p_{T}\right)$ of parent pions, as they emerge from the target, that contribute to the neutrino flux in the MINOS near detector (same as Figure 6.1). Figure C.5 is for the LE010/185kA beam, Figure C.6 is for the LE100/200kA beam, and Figure C.7 is for the LE250/200kA beam. The upper right plot of each of these figures shows the same information, but for those $\pi^{+}$which contribute a muon which reaches Alcove 1. The distribution looks quite similar to that for the MINOS ND, with the exception of the threshold $p_{z}=4 \mathrm{GeV} / c$ for Alcove 1. The $\left(p_{z}, p_{T}\right)$ sensitivities of Alcoves 2 and 3 are also shown, which have higher effective parent thresholds of $8 \mathrm{GeV} / c$ and $18 \mathrm{GeV} / c$, respectively. That the Alcove 1 acceptance is so similar to the MINOS detector reinforces the expectation that the muon monitors can observe most of the relevant flux muons which contribute to the flux of neutrinos seen in MINOS. 

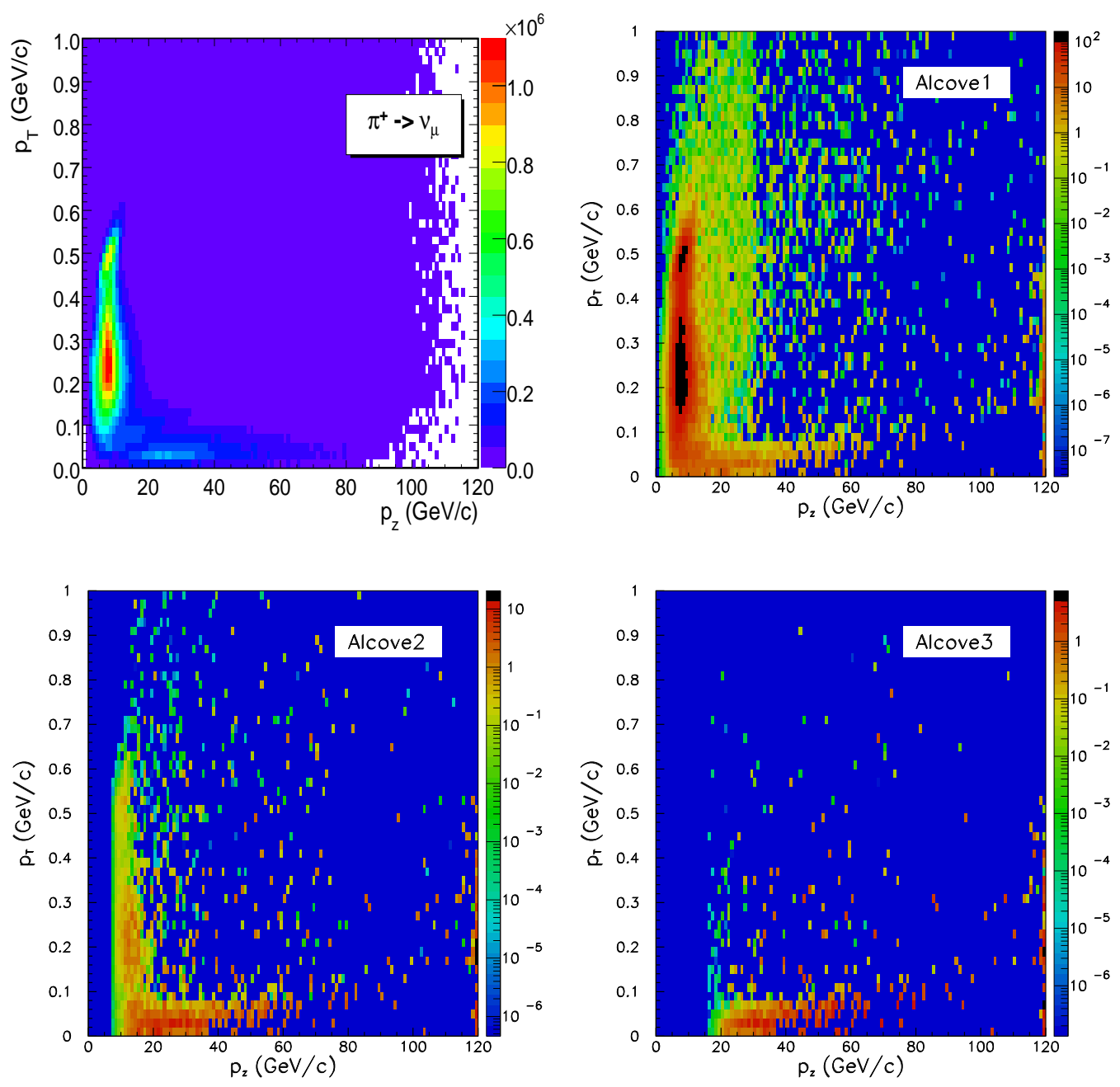

Figure C.5: Fluka 2005 Monte Carlo prediction of the $p_{T}$ and $p_{z}$ of pions which contribute a CC neutrino in the ND (upper left plot), or a muon in Alcoves 1, 2, or 3. Distributions are for the LE010/185kA beam configuration. The distributions for Alcoves 2 and 3 are similar to Alcove 1, but with higher thresholds of $8 \mathrm{GeV} / c$ and $18 \mathrm{GeV} / c$, respectively. 

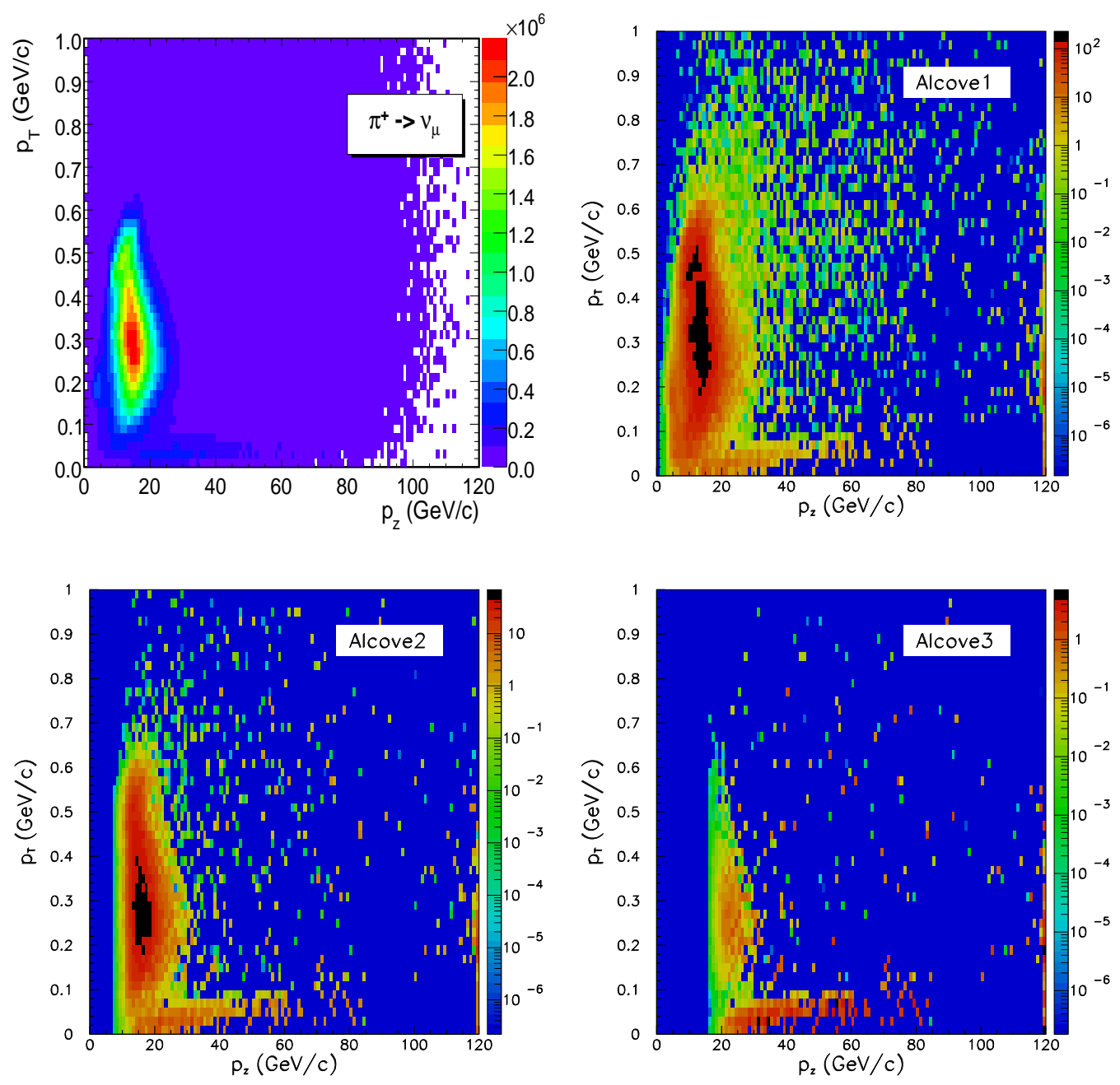

Figure C.6: Fluka 2005 Monte Carlo prediction of the $p_{T}$ and $p_{z}$ of pions which contribute a CC neutrino in the ND (upper left plot), or a muon in Alcoves 1, 2, or 3. Distributions are for the LE100/200kA beam configuration. The distributions for Alcoves 2 and 3 are similar to Alcove 1, but with higher thresholds, but with higher thresholds of $8 \mathrm{GeV} / c$ and $18 \mathrm{GeV} / c$, respectively. 

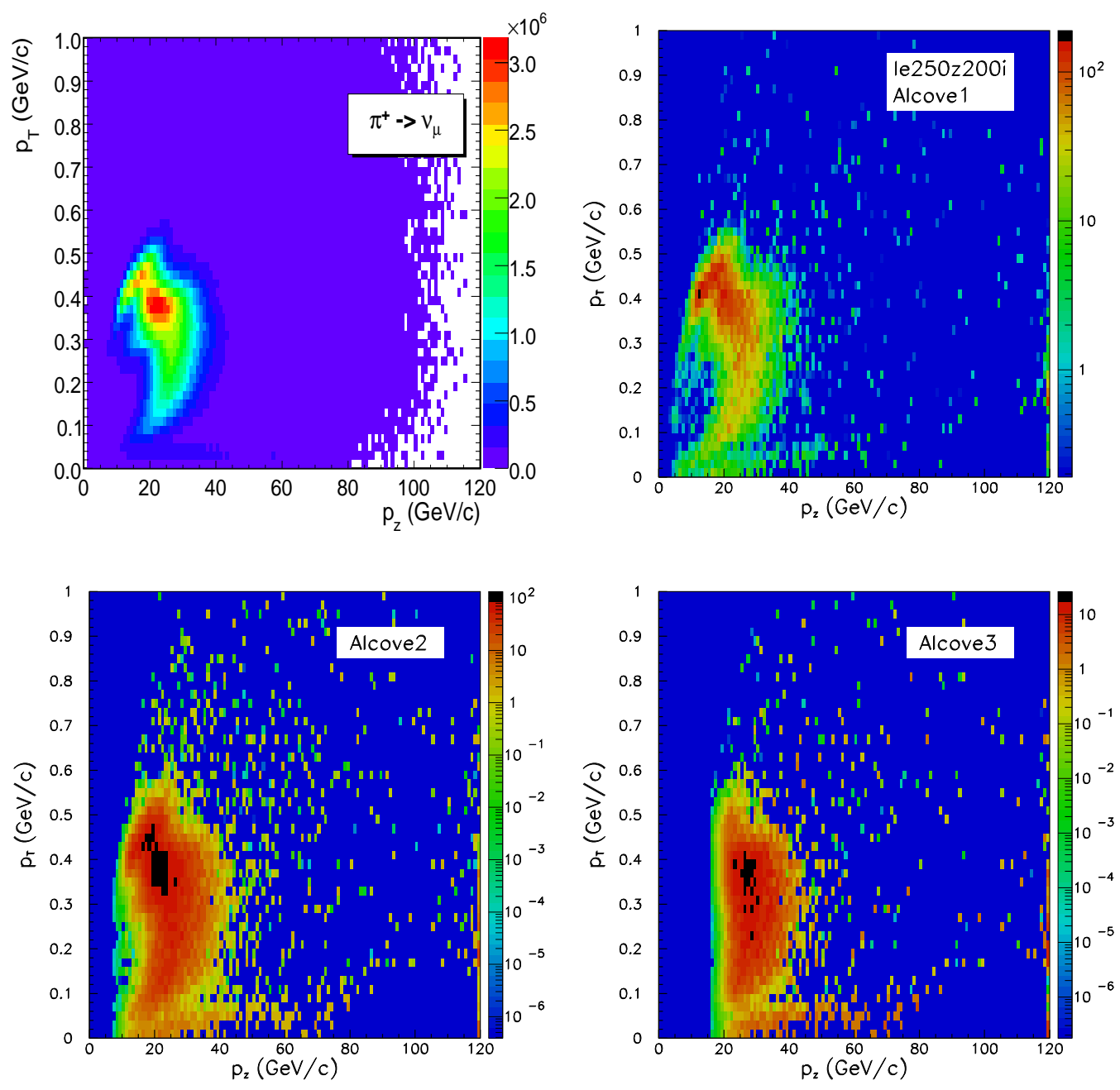

Figure C.7: Fluka 2005 Monte Carlo prediction of the $p_{T}$ and $p_{z}$ of pions which contribute a CC neutrino in the ND (upper left plot), or a muon in Alcoves 1, 2, or 3. Distributions are for the LE250/200kA beam configuration. The distributions for Alcoves 2 and 3 are similar to Alcove 1, but with higher thresholds, but with higher thresholds of $8 \mathrm{GeV} / c$ and $18 \mathrm{GeV} / c$, respectively. 


\section{C.2 Horn Current Scans}

To be useful as a tool to measure the differential flux with respect to neutrino energy, the muon monitors must overcome the fact that they consist of only three arrays, with three very coarse threshold bins in energy (to be contrasted with the 9-16 alcoves in previous beam lines). This is done using the ability in the NuMI beam to vary both the horn current $I_{h o r n}$, which affects the $\left\langle p_{T}\right\rangle$ kick given to particles by the horns, and also vary the position, $z_{t g t}$, of the target, which changes the $\left\langle p_{z}\right\rangle$ of parent mesons focused by the horns (§2.1.2). Dedicated $\sim 1$ hour runs varying both of these parameters were taken and the Muon Monitor data recorded [108]. While such measurements could in principle be taken using neutrinos in the MINOS detector as well, the power of the Muon Monitors is that they can record relatively accurate (integral) fluxes in just a few short spills.

Figures C.8 through C.10 demonstrate the ability to sweep in both $p_{T}$ and $p_{z}$ using $\left(I_{h o r n}, z_{t g t}\right)$. Shown are the $p_{T}$ and $p_{z}$ of $\pi^{+}$that contribute muons to Alcove 1 at a variety of horn currents in the LE010, LE150, and LE250 target configurations. As can be seen in the LE010 beam, variation of the horn current sweeps the $p_{T}$ of focused particles to larger values. In the LE250 configuration, variation of the horn current sweeps in both the $p_{T}$ and $p_{z}$ directions. Alcoves 2 and 3, though not shown in these figures, add the information of independent flux measures above the thresholds of $p_{z}=8$ and $18 \mathrm{GeV} / c$, respectively. Similar information is shown in Figure C.11, which shows the spectra of pion $p_{T}$ in several $\left(I_{h o r n}, z_{t g t}\right)$ configurations. As expected, larger $I_{\text {horn }}$ focuses larger $\left\langle p_{T}\right\rangle$. 


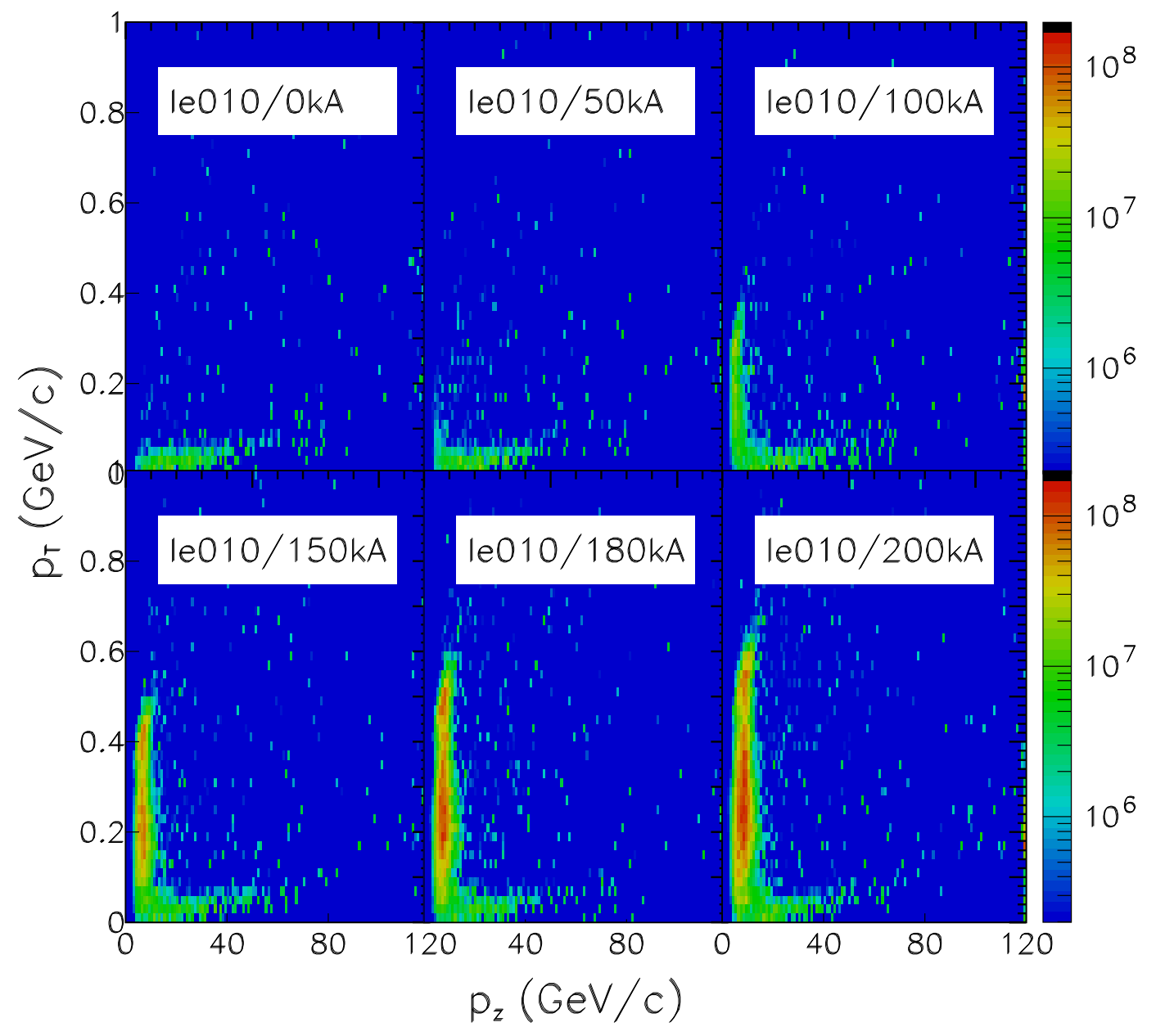

Figure C.8: Fluka 2005 Monte Carlo prediction of the $p_{T}$ and $p_{z}$ of pions which contribute a muon in Alcove 1. Distributions are for the LE010 beam configuration with $0,50,100,150,180$, and $200 \mathrm{kA}$. The distributions for Alcoves 2 and 3 are similar to Alcove 1, but with higher thresholds, but with higher thresholds of $8 \mathrm{GeV} / c$ and $18 \mathrm{GeV} / c$, respectively. 


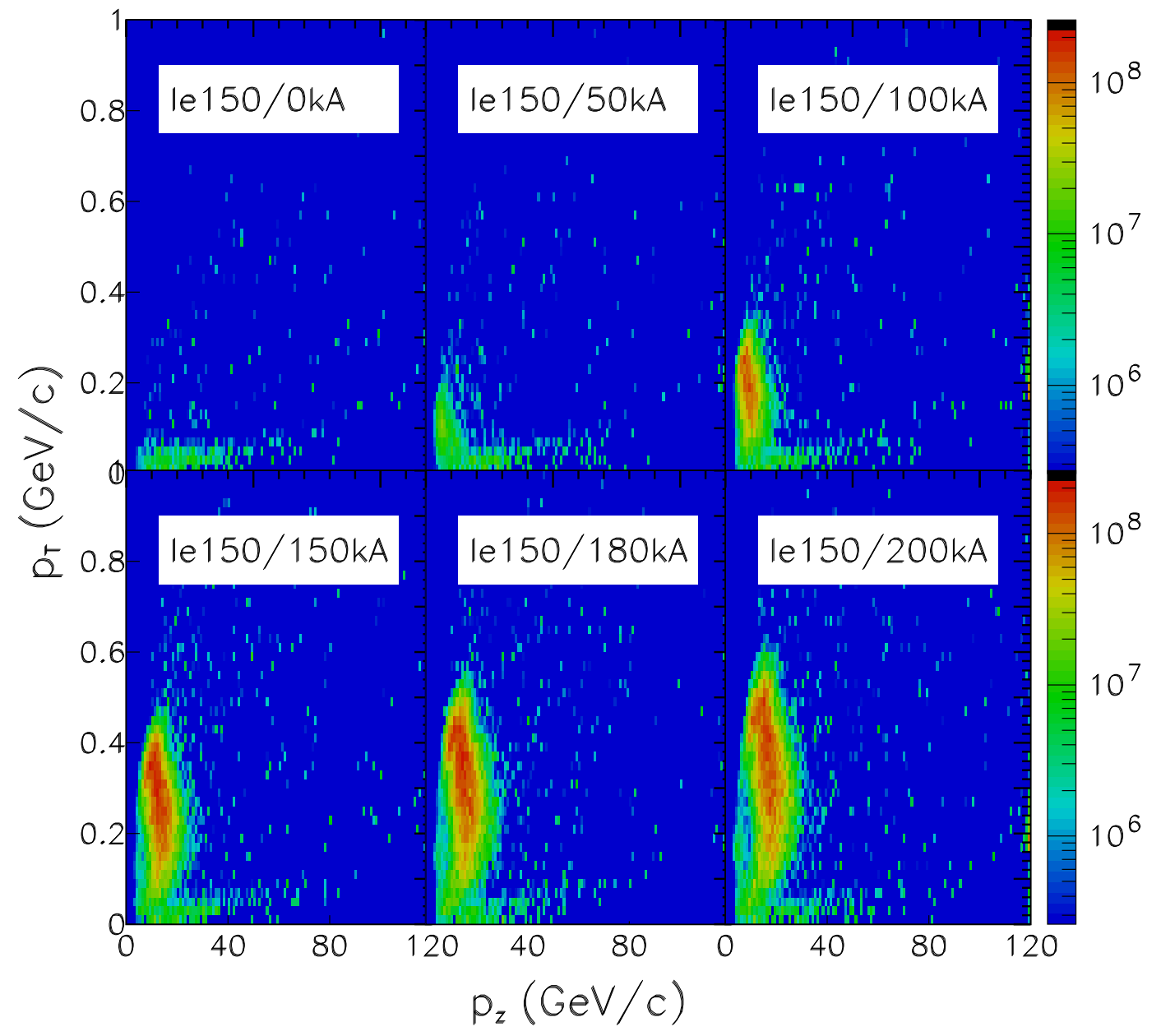

Figure C.9: Fluka 2005 Monte Carlo prediction of the $p_{T}$ and $p_{z}$ of pions which contribute a muon in Alcove 1. Distributions are for the LE150 beam configuration with $0,50,100,150,180$, and $200 \mathrm{kA}$. The distributions for Alcoves 2 and 3 are similar to Alcove 1, but with higher thresholds, but with higher thresholds of $8 \mathrm{GeV} / c$ and $18 \mathrm{GeV} / c$, respectively. 


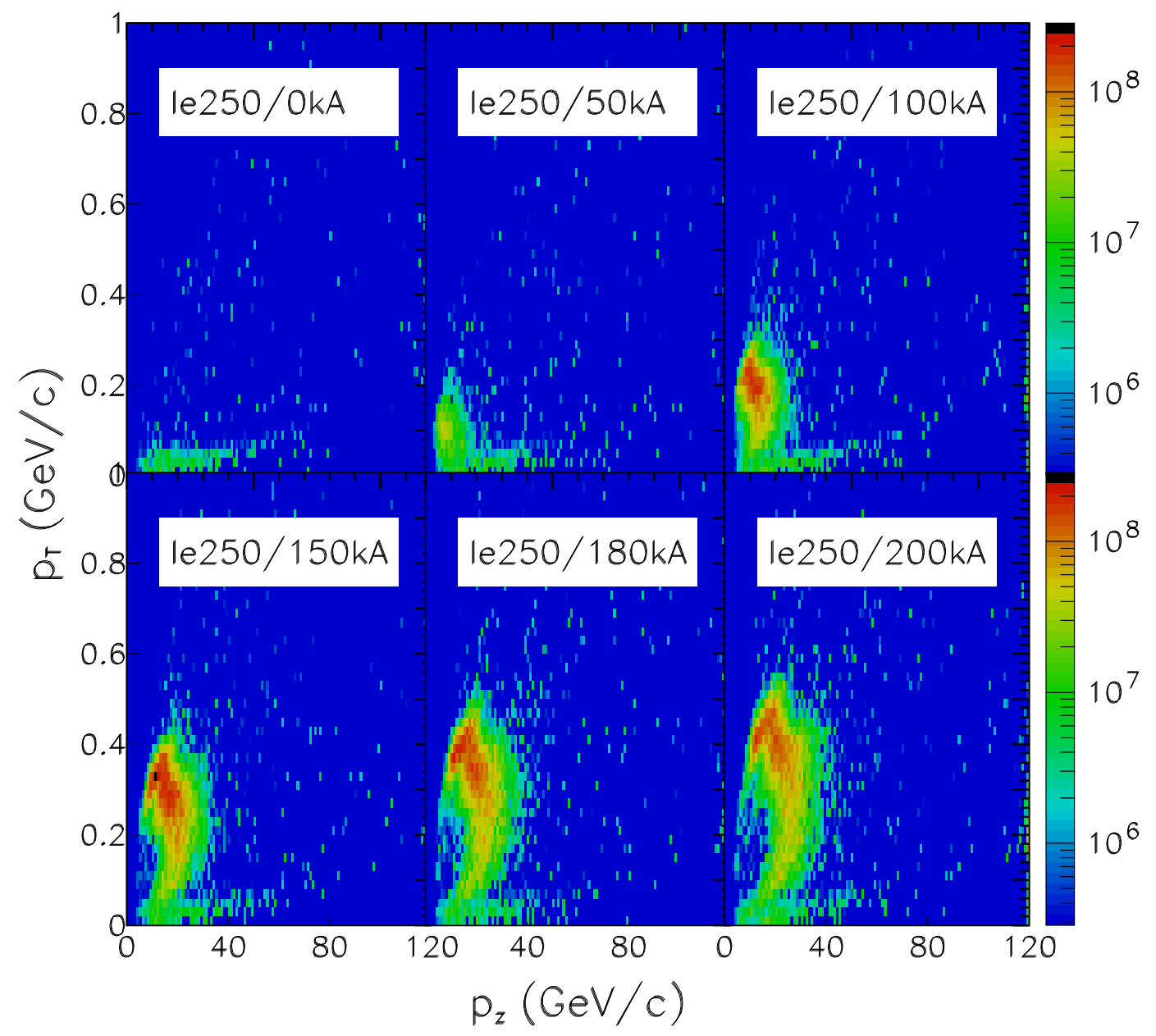

Figure C.10: Fluka 2005 Monte Carlo prediction of the $p_{T}$ and $p_{z}$ of pions which contribute a muon in Alcove 1. Distributions are for the LE250 beam configuration with $0,50,100,150,180$, and $200 \mathrm{kA}$. The distributions for Alcoves 2 and 3 are similar to Alcove 1, but with higher thresholds, but with higher thresholds of $8 \mathrm{GeV} / c$ and $18 \mathrm{GeV} / c$, respectively. 

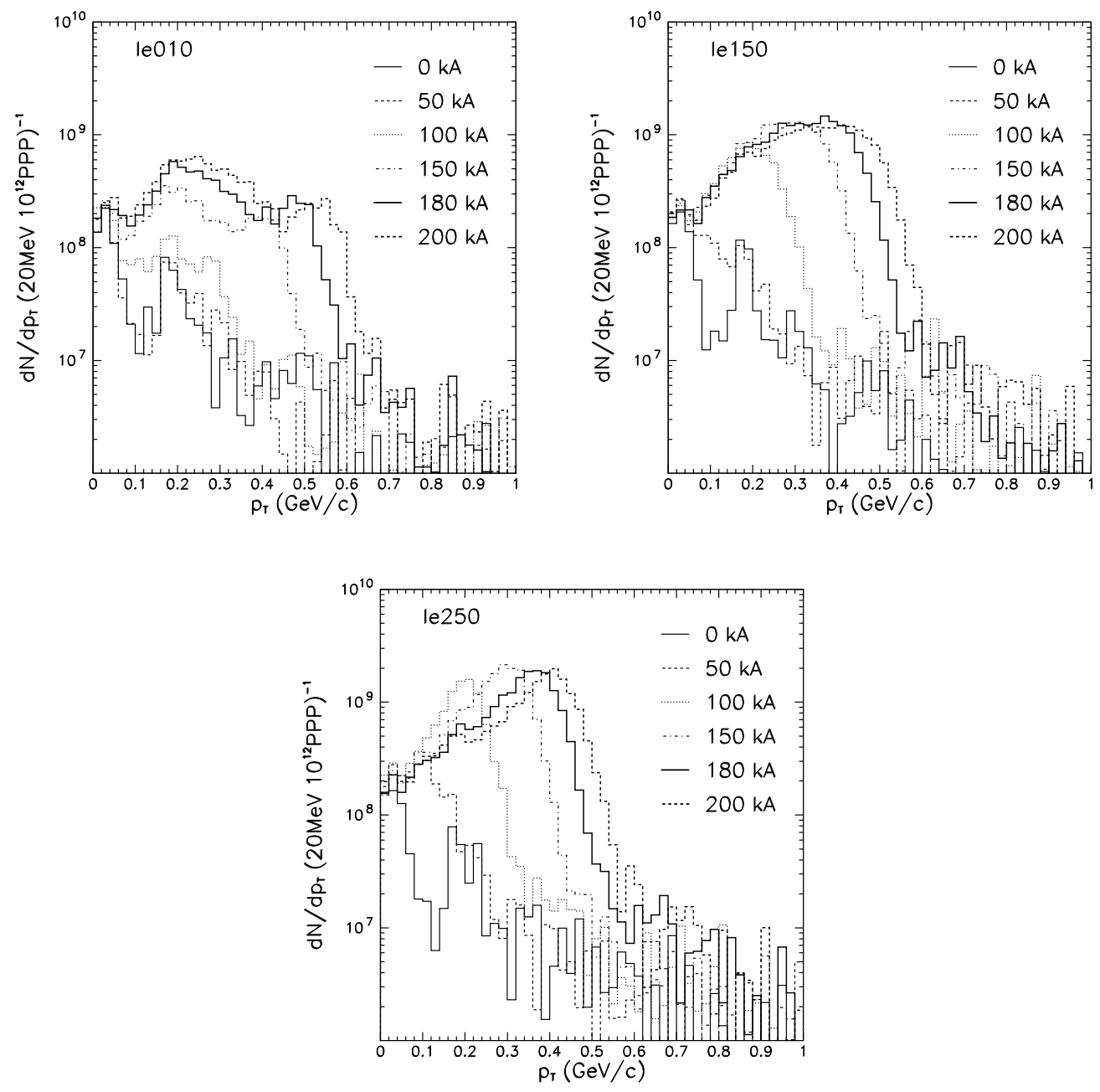

Figure C.11: Fluka 2005 Monte Carlo prediction of the $p_{T}$ pions which contribute a muon in Alcove 1. Distributions are for the LE0010, LE100, and LE250 beam configurations with $0,50,100,150,180$, and $200 \mathrm{kA}$. Increase of the horn current is seen to increase the $\left\langle p_{T}\right\rangle$ of focused particles. 


\section{C.3 Comparison to Muon Monitor Data}

Figures C.12 through C.14 show the data and Monte Carlo prediction of the muon flux in the muon alcoves. The data shows the total collected charge in the muon monitors. As discussed above, the exact conversion factor that would relate this to muon flux is not known precisely. The Monte Carlo prediction on the other hand gives us the muon flux in the alcoves.

The overall shape looks similar in various beam configurations for data and Monte Carlo, however, when the relative fluxes are compared in data and in $\mathrm{MC}$ between different data points the difference between the MC and data is revealed. We can now try to tune the yields of secondaries of the target to achieve better agreement between the muon monitor data and Monte Carlo prediction of the flux.

\section{C.4 Fit to Muon Monitor data}

The muon monitors do not measure directly these underlying $\left(p_{z}, p_{T}\right)$ distributions. All that is seen in the Alcoves is a signal proportional to the total number of muons, in effect the integral under any one of the surface plots in Figures C.8 through C.10. The situation is actually quite similar to the fitting of hadron production models to the MINOS near detector data described in Chapter 6 . The one data point provided by a single muon alcove rate is analogous to a bin of neutrino energy in the MINOS Near Detector. The power of the ND data is that a single data set consists of $\sim 60$ neutrino energy bins, and a few beam configurations were acquired. The power of the muon monitors is not in the number of energy bins (there are just three for the three alcoves), 

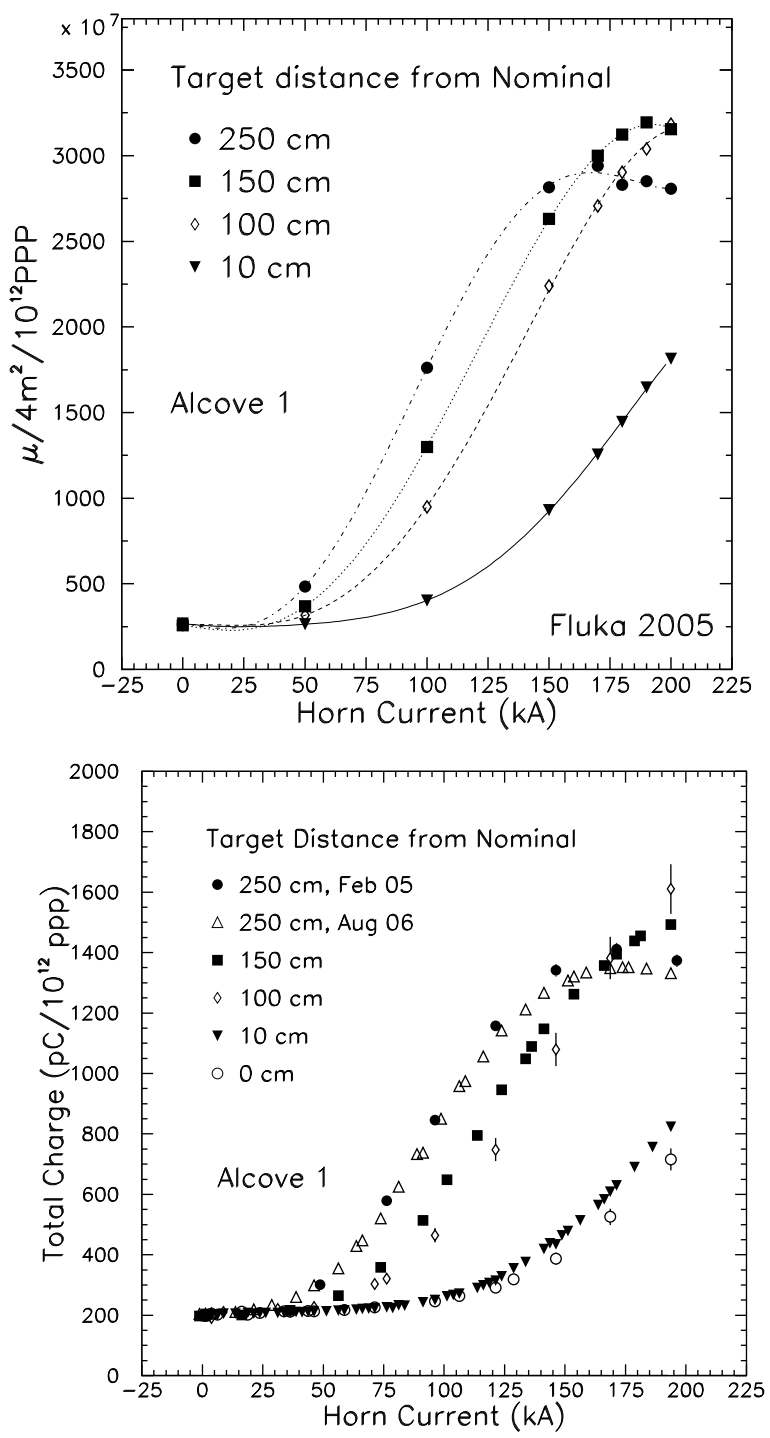

Figure C.12: Muon rates in Alcove 1 as a function of the horn current for several target positions. (top) Prediction from the GNuMI beam MC with Fluka 2005 input hadrons off the target. The lines are polynomial fits to the MC points to guide the eye. (bottom) NuMI data taken in 2005-2006 [108]. A background of $35 \pm 7 \mathrm{pC} / 10^{12} \mathrm{ppp}$, due to particles produced in the beam absorber, expected to be independent of horn current, has not been subtracted from these data. 

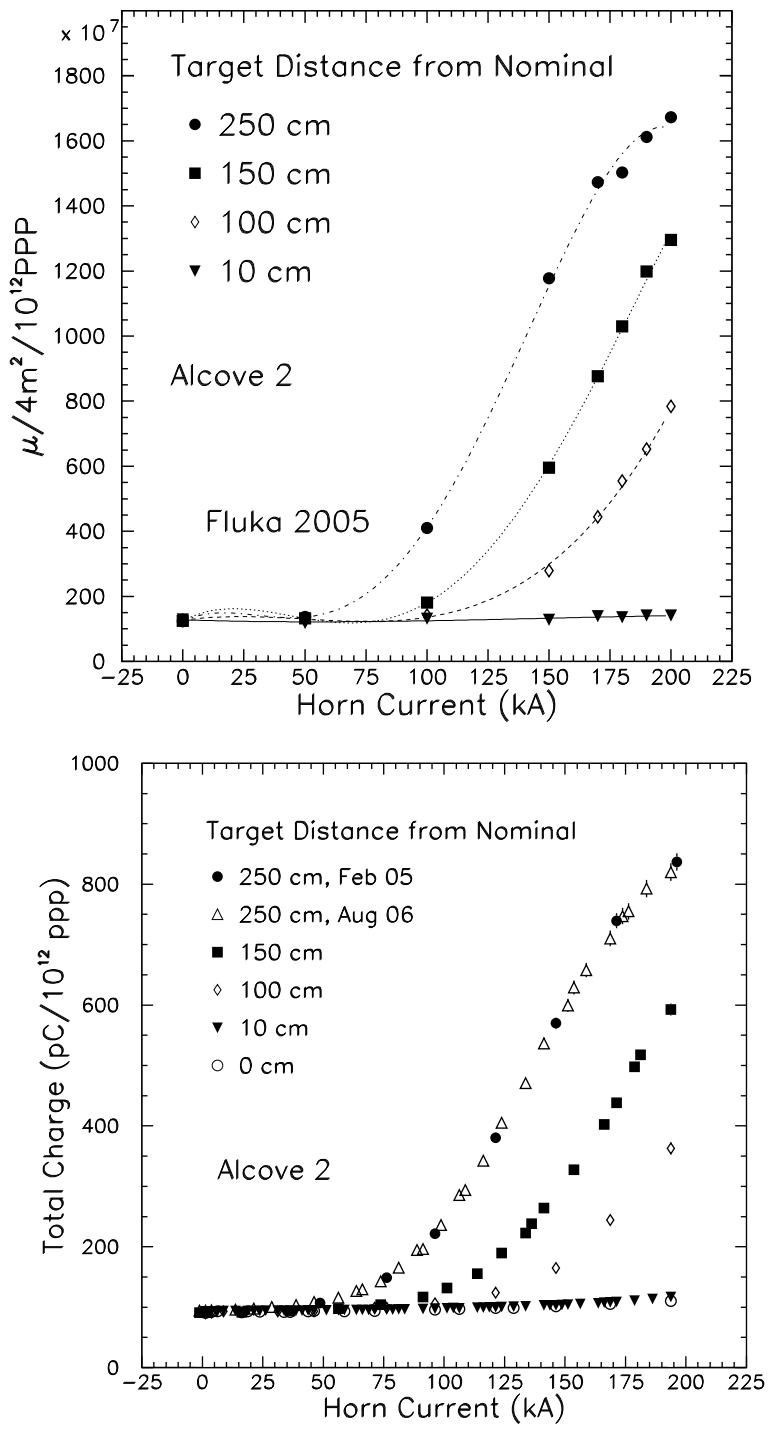

Figure C.13: Muon rates in Alcove 2 as a function of the horn current for several target positions. (top) Prediction from the GNuMI beam MC with Fluka 2005 input hadrons off the target. The lines are polynomial fits to the MC points to guide the eye. (bottom) NuMI data taken in 2005-2006 [108]. A background of $7 \pm 2 \mathrm{pC} / 10^{12} \mathrm{ppp}$, due to particles produced in the beam absorber, expected to be independent of horn current, has not been subtracted from these data. 

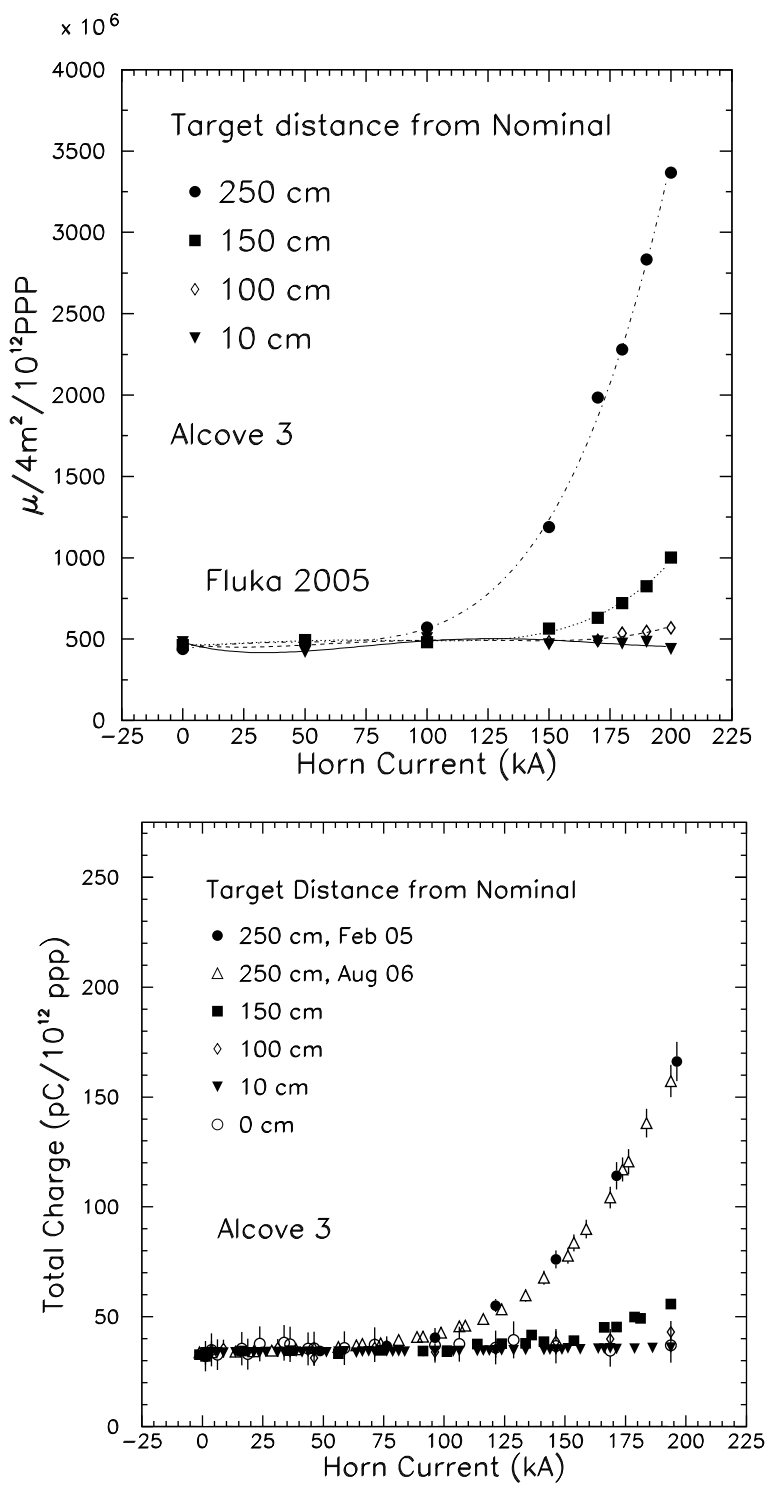

Figure C.14: Muon rates in Alcove 3 as a function of the horn current for several target positions. (top) Prediction from the GNuMI beam MC with Fluka 2005 input hadrons off the target. The lines are polynomial fits to the MC points to guide the eye. (bottom) NuMI data taken in 2005-2006 [108]. A background of $1.4 \pm 1.4 \mathrm{pC} / 10^{12} \mathrm{ppp}$, due to particles produced in the beam absorber, expected to be independent of horn current, has not been subtracted from these data. 
but in the larger number of beam configurations acquired in the $\left(I_{\text {horn }}, z_{t g t}\right)$ data set.

Without knowing the conversion factor between the signal in the muon monitors and muon flux precisely we cannot find the absolute muon (or neutrino) flux. The extracted flux will have the overall normalization uncertainty roughly equal to the uncertainty of the conversion factor. As discussed, the scale factor determined in test beam [109, 110] may not be the correct scale factor for muon monitors, nevertheless we will take the measured value of $9.4 \mathrm{pC} / 10^{7} \mu$ as the correct factor. We allow that the scale factor varies between the alcoves within $5 \%$ due to gas quality.

The background signal in the muon monitors is not negligible compared to the signal especially for low horn current settings. Since the flux will essentially be extracted by using the relative difference in the signal for different $\left(I_{h o r n}, z_{t g t}\right)$ points, it is important to estimate the background correctly.

There are couple of sources of background that need to be considered. The NuMI target is roughly 2 interaction lengths long, so $\sim 13.5 \%$ of proton beam gets to the hadron absorber without interacting in the target. These protons interact in the absorber and among other particles create muons as well. The first muon alcove which is just downstream of hadron absorber also sees the neutrons created in the absorber [114]. To estimate the two sources of background data taken with proton beam steered directly to the hadron absorber is analysed. The measured signal in the muon alcoves is then subtracted from the data shown in Figures C.12 through C.14 
To perform the fit we minimize the $\chi^{2}$ calculated in the following way:

$$
\chi^{2}=\sum_{I_{\text {horn }}, z_{\text {tgt }}} \frac{\left(\phi^{\text {data }}\left(I_{\text {horn }}, z_{t g t}, a, b\right)_{\mu}-\phi_{\mu}^{M C}\left(I_{\text {horn }}, z_{t g t}, x_{F}, p_{T}\right)\right)^{2}}{\sigma_{\text {data }}^{2}+\sigma_{M C}^{2}}
$$

where $\phi_{\mu}^{M C}$ is the predicted flux by the Monte Carlo simulation and $\phi_{\mu}^{\text {data }}$ is the measured muon flux in a beam configuration $\left(I_{h o r n}, z_{t g t}\right)$. The parameters $a$ and $b$ are the scale factor which convert the signal in the muon monitor to number of muons and subtract the background from data, respectively. The Monte Carlo prediction on the other hand depends on the tuned hadron production in terms of parent $x_{F}$ and $p_{T}$.

To fit the data we use the same 6 parameters used in Chapter 6 to modify the yields of pions. As seen in Figure C.2-C.4 negative pions don't contribute significantly to muon flux and therefore muon monitors are not sensitive to them. We use the $\pi^{+} / \pi^{-}$ratio measured in NA49 experiment [57] to constrain the $\pi^{-}$flux. Since the sensitivity to kaons is also relatively small we constrain them using the $K / \pi$ ratio predicted by Fluka MC.

The muon flux in a particular alcove for a given $\left(I_{h o r n}, z_{t g t}\right)$ point can be found by looking at the integral under the surface of the plots shown in Figures C.8 through C.10 (or similar plots for $\left(I_{\text {horn }}, z_{t g t}\right)$ points which are not shown). This way the resulting change in a muon flux due to the change in the flux of secondaries in a particular $\left(x_{F}, p_{T}\right)$ bin can be quickly found.

Two additional parameters are used to change slightly the overall scale factor for Alcoves 2 and 3. The scale factors could in principle be different for the three alcoves due to, for example, variations in gas quality.

The results of the fit are shown in Figure C.15-C.17. The muon monitor data in figures is compared to the Fluka based Monte Carlo and the tuned 
Monte Carlo. Both Monte Carlo predictions use the same conversion factor and assume same backgrounds. The difference in predicted muon flux using Fluka based $\mathrm{MC}$ and tuned $\mathrm{MC}$ can be ascribed to the modified yields of secondaries.

The resulting tuned production of secondaries can now be used to predict the neutrino flux. Figure C.18 shows both the Fluka Monte Carlo prediction as well as the flux prediction using $\mathrm{MC}$ which was tuned to muon monitor data. Just like the neutrino data (Chapter 6), the muon monitor data seems to prefer stronger flux of lower $p_{T}$ particles that increase the neutrino flux in the high energy tail.

\section{C.5 Summary}

In this Appendix we have shown the technique that can be used to find the neutrino flux using the Muon Monitors in the NuMI beamline. The final prediction of the flux has not been done since the method depends on several inputs which are not currently available.

We have assumed that the $K / \pi$ ratio is correctly predicted by Fluka cascade model. This has the impact on how the neutrino flux is normalized at higher energies. Since neutrino cross sections are well known at higher energies the exact $K / \pi$ ratio would allow the determination of cross sections at lower energies.

To be able to extract the fluxes it is necessary to subtract the backgrounds. This was done using the data taken when the proton beam was steered directly to beam absorber. However, there is another source of "background" that was not considered. The muons while passing through the rock 

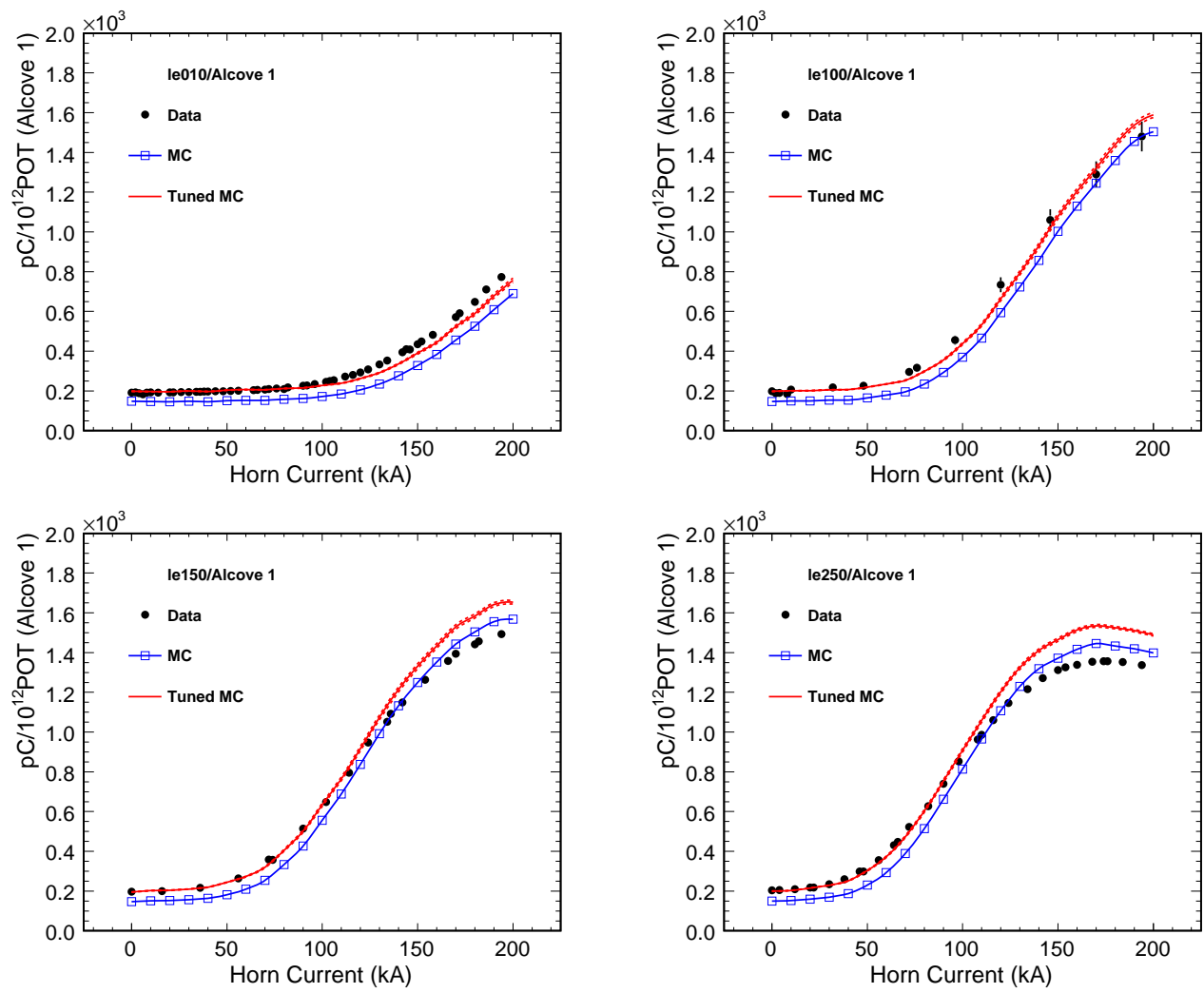

Figure C.15: The muon flux in the first muon Alcove depending on the horn current setting for 4 different target positions (LE010, LE100, LE150 and LE250). The Fluka Monte Carlo prediction (blue line) is tuned to agree with Muon Monitor data (black dots). The tuned Monte Carlo (red line) show better agreement when compared to all the data. The same scale factors that convert the muon monitor signal to muon flux are applied to tuned and Fluka Monte Carlo as well as the same background. 

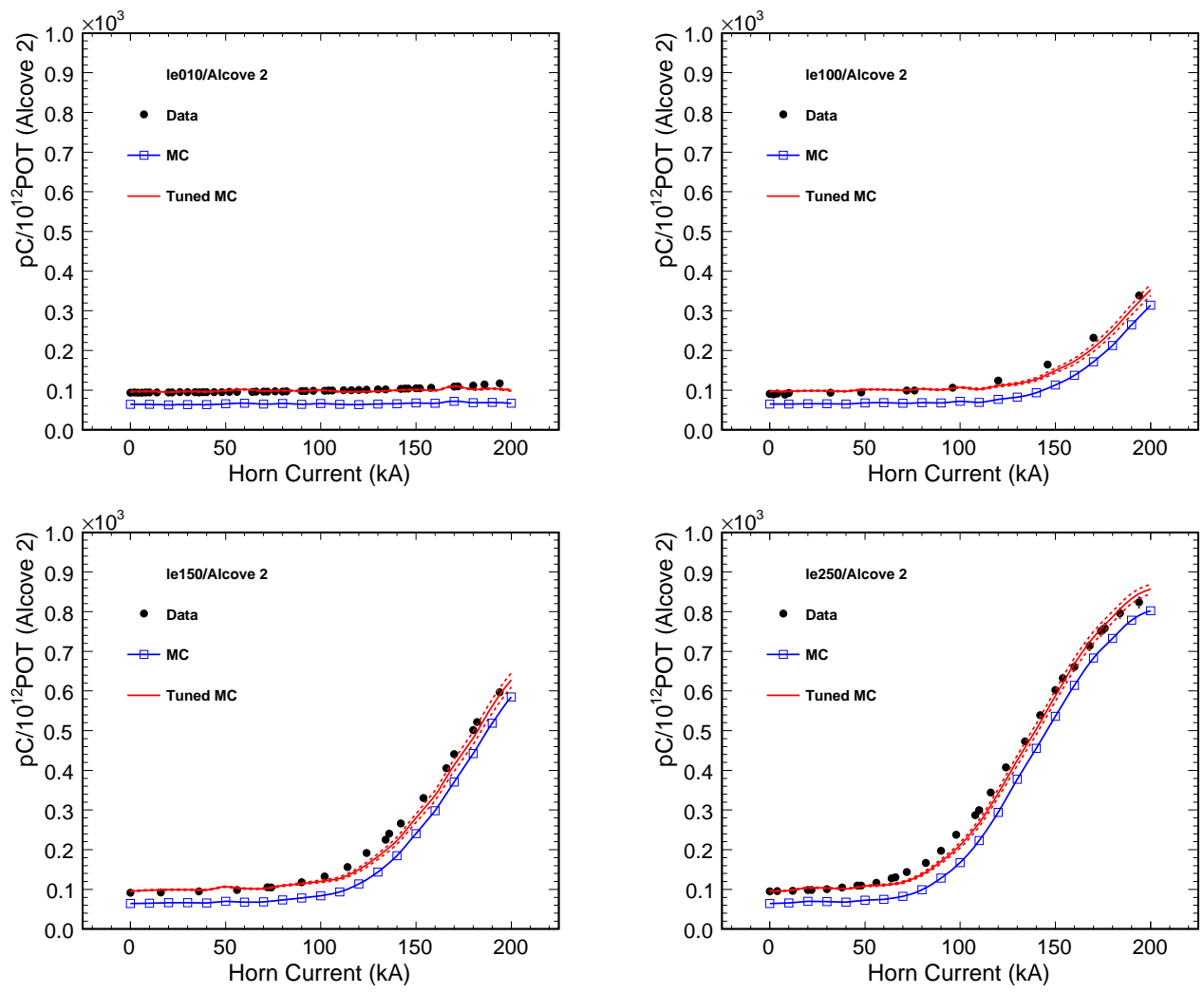

Figure C.16: The muon flux in the first muon Alcove depending on the horn current setting for 4 different target positions (LE010, LE100, LE150 and LE250). The Fluka Monte Carlo prediction (blue line) is tuned to agree with Muon Monitor data (black dots). The tuned Monte Carlo (red line) show better agreement when compared to all the data. The same scale factors that convert the muon monitor signal to muon flux are applied to tuned and Fluka Monte Carlo as well as the same background. 

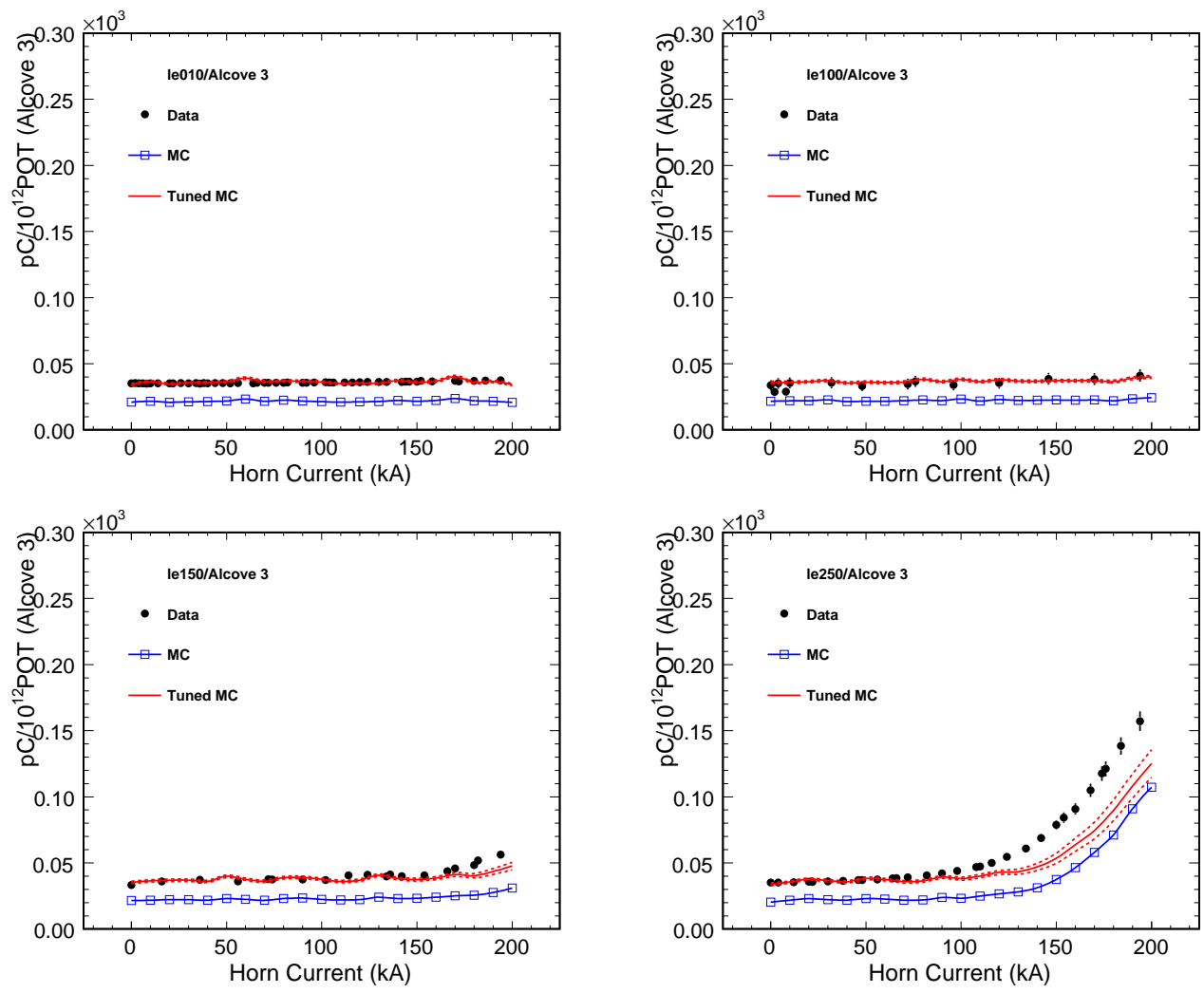

Figure C.17: The muon flux in the first muon Alcove depending on the horn current setting for 4 different target positions (LE010, LE100, LE150 and LE250). The Fluka Monte Carlo prediction (blue line) is tuned to agree with Muon Monitor data (black dots). The tuned Monte Carlo (red line) show better agreement when compared to all the data. The same scale factors that convert the muon monitor signal to muon flux are applied to tuned and Fluka Monte Carlo as well as the same background. 

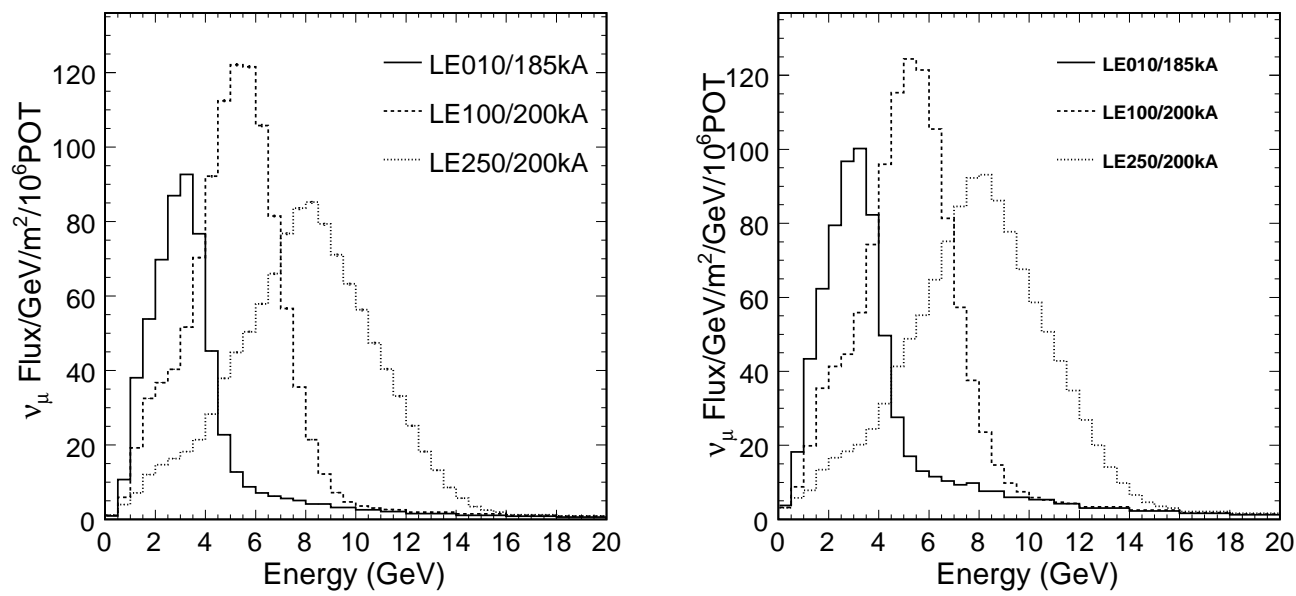

Figure C.18: The neutrino flux at the MINOS Near Detector as predicted by Fluka based Monte Carlo (left) and the Monte Carlo which was tuned to muon monitor data of Figures C.12-C.14 (right). The tuning was achieved by modifying the yields of secondaries of the NuMI target.

and walls of ion chamber create delta rays. Currently this is not simulated. If the production of delta rays would be independent of muon momentum, then "background" due to delta rays could be absorbed in the overall scale factor. However, this is not the case and the fraction of energy deposited in the ion chamber by delta rays produced by incident muon depends on the muon energy. This effect needs to be studied before using the muon monitor data.

Finally the method could be improved by redefining the $\chi^{2}$ defined in Equation C.1. While this $\chi^{2}$ was suitable to demonstrate that the technique is working it does not use the data optimally. It gives less weight to the data points where horn current was sampled more coarsely which is not necessarily correlated with how interesting is the $\left(p_{T}, x_{F}\right)$ region sampled by that point. For future analysis it would be useful to assign the importance to certain points which, for example, would be proportional to the fraction of $\left(p_{T}, x_{F}\right)$ 
sampled at that point which is relevant for the flux in LE010/185kA beam configuration. 


\section{Appendix D}

\section{Using GNuMI Ntuples for Muon Monitor Rates}

The NuMI beam Monte Carlo (GNuMI) described in Chapter 3 was optimized for prediction of neutrino fluxes. A pion or kaon is tracked through the target and horns, and enters the decay pipe. While the meson decays isotropically in the center-of-mass frame, GNuMI forces the meson to decay with the neutrino direction which hits either the MINOS ND or FD. To account for this artificial forcing, the probability for this set of decay angles is calculated and utilized as a weighting probability for the particular neutrino event.

For the calculation of muon rates at the alcoves, the opposite is desired: we wish to force the meson to decay with a forward-going muon, and the neutrino may in general miss the ND and FD. In this Appendix we look at some kinematical relations used to calculate the required muon rates. These kinematical formulae are used to reweight the pions by their probability to produce a muon which passes through the end of the decay pipe, where such 


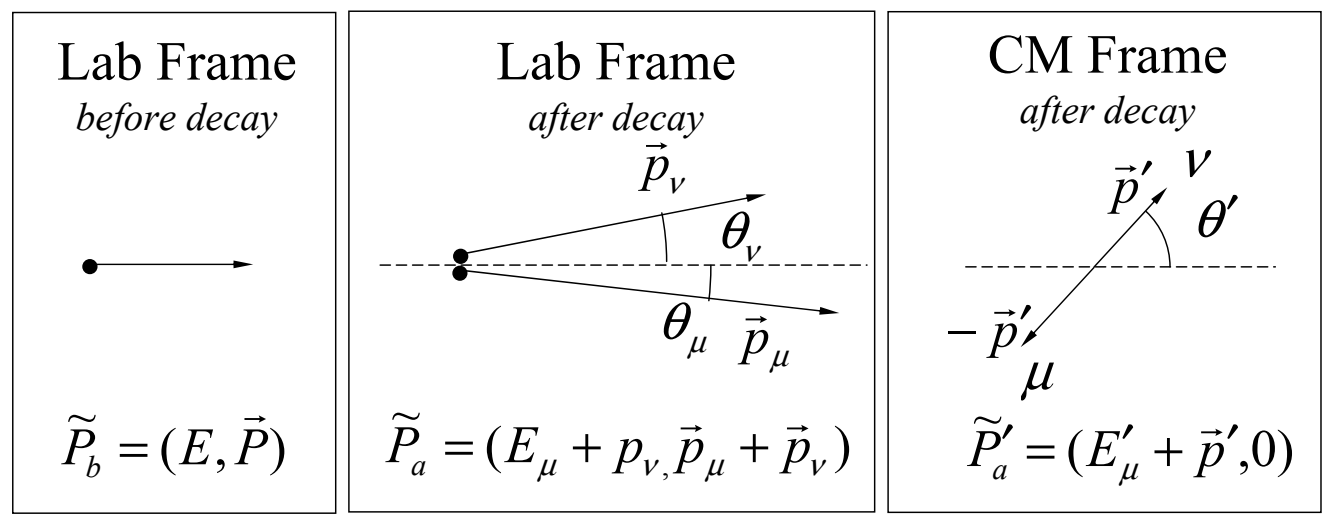

Figure D.1: View of a parent $\pi$ or $K$ meson decay in the laboratory and center-of-mass (CM) frames, defining the momenta, energies, and angles of the parent and daughter particles.

muons might be able to then penetrate the shielding to the muon monitors.

\section{D.1 Definition of Reference Frames}

Figure D.1 defines several momentum vectors and directions for the daughters from a $\pi$ or $K$ decay. Being a two-body decay into a muon and neutrino, the momenta $p^{\prime}$ of the daughters in the center-of-mass frame can be calculated as:

$$
p^{\prime}=\frac{M}{2}\left(1-\frac{m_{\mu}^{2}}{M^{2}}\right)
$$

where $M$ is the mass of the $\pi$ or $K$ parent and $m_{\mu}$ is the muon mass. For the daughters in $\pi(K)$ decay, $p^{\prime}=29.8 \mathrm{MeV}(235.6 \mathrm{MeV})$. For neutrinos, $E^{\prime}=p^{\prime}$; for the muons, $E_{\mu}^{\prime}=\sqrt{\left(p^{\prime}\right)^{2}+m_{\mu}^{2}}=109 \mathrm{MeV}(258 \mathrm{MeV})$ in pion (kaon) decays.

Transformation of the daughter momenta to the lab is done by the 
Lorentz boost:

$$
\begin{gathered}
E=\gamma\left(E^{\prime}+\beta p_{z}^{\prime}\right) \\
p_{z}=\gamma\left(p_{z}^{\prime}+\beta E^{\prime}\right) \\
p_{T}=p_{T}^{\prime}
\end{gathered}
$$

where $\gamma=E / M, \beta=\left(1-1 / \gamma^{2}\right)^{1 / 2}$, and $E$ and $M$ are the energy of the parent meson in the lab and its mass, respectively. The fact that $p_{T}^{\prime 2}+p_{z}^{\prime 2}=p^{\prime 2}$ means that the daughter momentum vectors lie on a circle in the CM frame (see Figure D.2), while this relation, upon substitution of Equations D.3 and D.4 yield that the daughter momentum vectors lie on an ellipse in the laboratory frame:

$$
\frac{\left(p_{z}-\beta \gamma E^{\prime}\right)^{2}}{\gamma^{2} p^{2}}+\frac{p_{T}^{2}}{p^{\prime 2}}=1
$$

in which the ellipse's center is at $\left(\gamma \beta E^{\prime}, 0\right)$ and has semi-major (minor) axes $\gamma p^{\prime}$ $\left(p^{\prime}\right)$. As indicated in Figure D.2, the ellipse for the neutrino momentum vector in the lab approximately intercepts the origin for very relativistic parents $(\beta \approx$ 1) since $E^{\prime}=p^{\prime}$ for the neutrino. Because of the large CM muon energy $E_{\mu}^{\prime}$, the ellipse for the muon is shifted to the right. As indicated in Figure D.2, the neutrino momentum in the lab ranges from 0 to $p_{\nu}^{\max }=2 \gamma p^{\prime}=\left(1-\frac{m_{\mu}^{2}}{M^{2}}\right) E$ (when looking at all possible decay angles), which is $0.43 E$ for $\pi \rightarrow \mu \nu$ decays and $0.96 E$ for $K \rightarrow \mu \nu$ decays. The muon momentum ranges from $0.57 E$ to $E$ in $\pi \rightarrow \mu \nu$ decays and $0.04 E$ to $E$ in $K \rightarrow \mu \nu$ decays. 


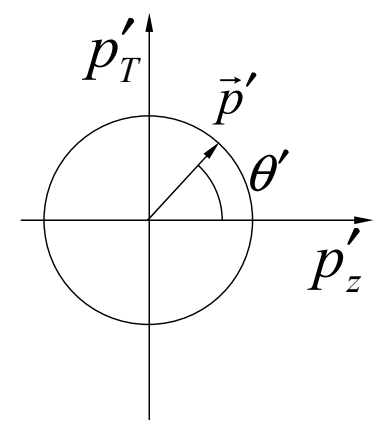

CM Frame

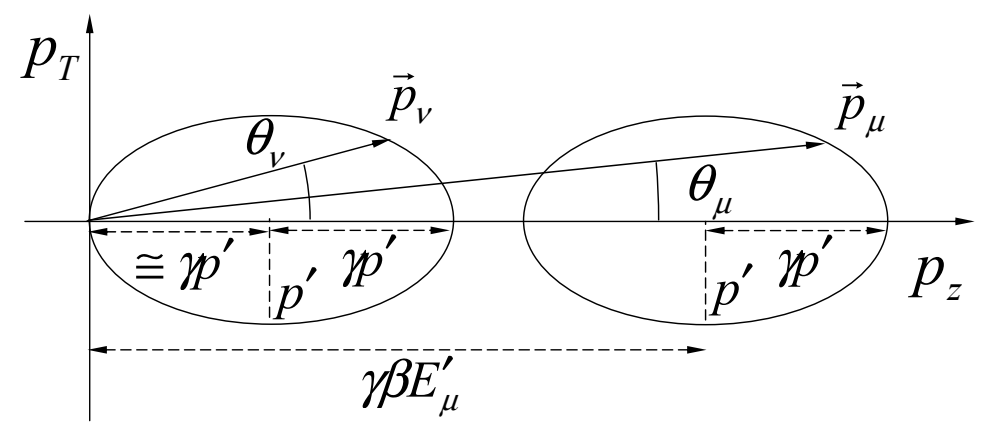

Laboratory Frame

Figure D.2: Lorentz transformation of a momentum 3-vector $p^{\prime}$ from the CM frame to the laboratory frame. In the CM frame, $p^{\prime}$ lies on a circle, while in the lab it is required to lie on an ellipse (see Equation D.5). For $\beta \approx 1$ parents as sketched above, the neutrino ellipse approximately is tangent to the $p_{T}$ axis and the muon ellipse is shifted to the right. In $\pi \rightarrow \mu \nu$ decays, as sketched above, the muon energy in the lab always larger than the neutrino energy, though for $K \rightarrow \mu \nu$ decays these ellipses overlap.

\section{D.2 Angular Distribution of Daughters}

In Section 3.1 we have seen that the angular distribution of neutrino daughters is given with Equation 3.12. We now want to find the angular distribution of muons.

Transformation of the daughter angles is found from Equations D.3 and D.4, noting that $p_{T}=p \sin \theta, p_{T}^{\prime}=p^{\prime} \sin \theta^{\prime}, p_{z}^{\prime}=p^{\prime} \cos \theta^{\prime}$, and $p_{z}=p \cos \theta$ :

$$
\gamma \tan \theta=\frac{\sin \theta^{\prime}}{\cos \theta^{\prime}+\left(\beta / \beta^{\prime}\right)}
$$

where $\beta^{\prime}=p^{\prime} / E^{\prime}$ is the daughter velocity in the CM frame (=1 for the neutrino and $=0.28$ or 0.91 for the muon in $\pi$ or $K$ decays).

The muons are more forward-boosted than the neutrinos. Taking, as an example, the center-of-mass angle $\theta^{\prime}=\pi / 2$, then by Equation D.6, the 

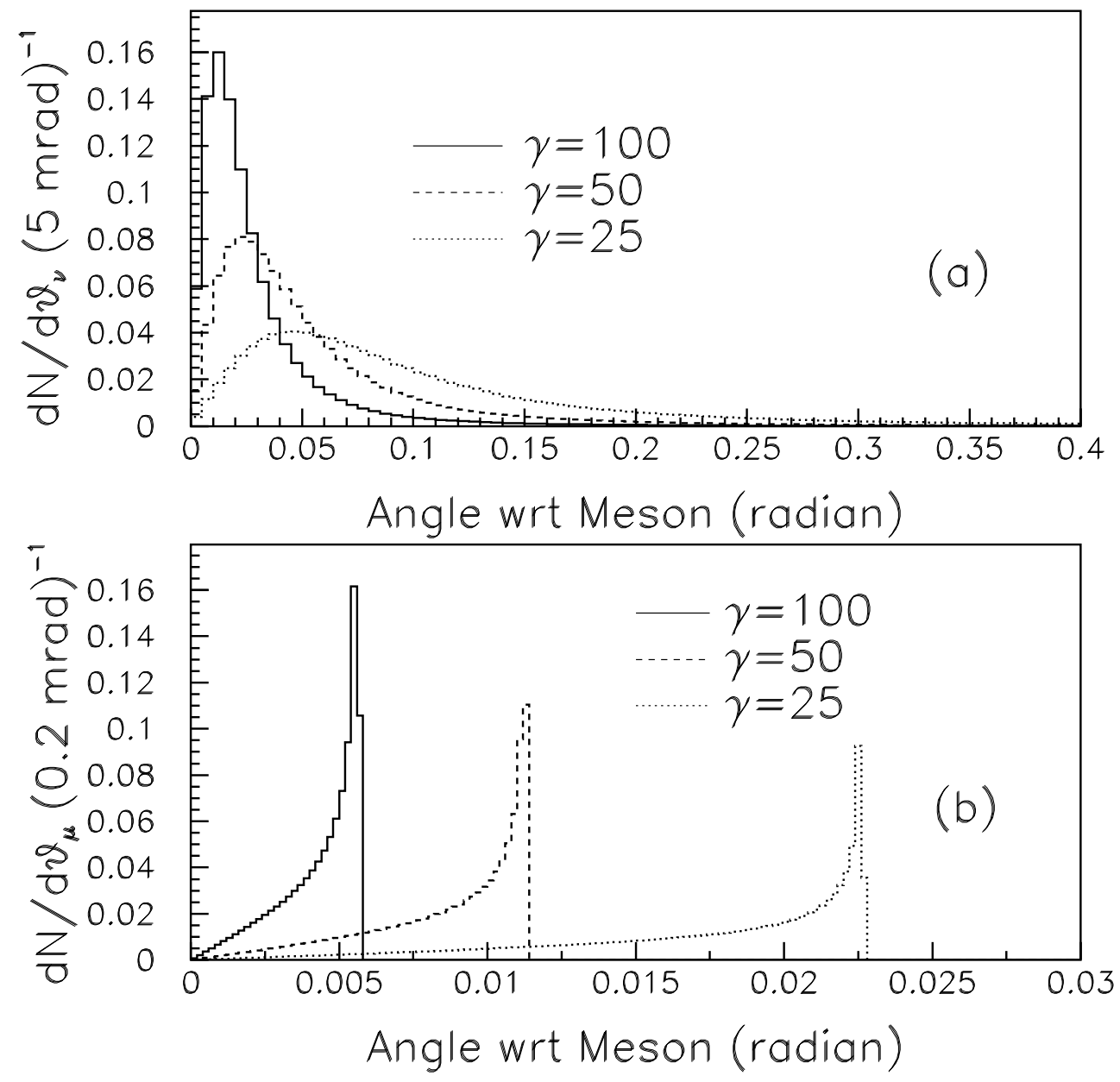

Figure D.3: Angular distribution in the lab frame of (a) neutrinos and (b) muons from $\pi \rightarrow \mu \nu$ decays. Shown are three cases, for pion boosts of $\gamma=$ $100,50,25$. Note that the distribution in the CM frame is flat, and the above distributions arise from the Lorentz boost. 
corresponding muon lab angle is $\tan \theta_{\mu}=\beta^{\prime} / \gamma \beta \approx \beta^{\prime} / \gamma$, while $\theta_{\nu} \sim 1 / \gamma$. While for neutrinos the velocity in center-of-mass system $\beta^{\prime}=1$, for muons it is 0.28 or 0.91 for $\pi$ and $K$ decays respectively. Thus the muons are more forward-boosted.

The maximum daughter angles can be derived using Equation D.5. Noting that $p_{T}=p_{z} \tan \theta$, then the longitudinal momentum of the daughter can be solved for:

$$
p_{z}=\gamma \beta E^{\prime} \frac{1 \pm \sqrt{1-\left[1-\left(\beta^{\prime} / \beta\right)^{2}\right]\left(1+\gamma^{2} \tan ^{2} \theta\right)}}{1+\gamma^{2} \tan ^{2} \theta}
$$

The solution for the daughter longitudinal momentum has two roots because for a given lab angle $\theta$ the momentum ray in Figure D.2 passes through the daughter's ellipse at two locations. The maximum angle $\theta$ occurs when the two solutions for Equation D.7 coincide, ie: the square root is zero. The maximum angle is given by

$$
\gamma^{2} \tan ^{2} \theta_{\max }=\frac{\left(\beta^{\prime}\right)^{2}}{\beta^{2}-\left(\beta^{\prime}\right)^{2}}
$$

Equation D.8 shows that for the neutrino $\left(\beta^{\prime}=1\right)$ from a very relativistic parent $(\beta \approx 1)$, the max angle is $\pi / 2$. Such occurs when the neutrino angle is $\pi$ in the CM frame, and the neutrino ends up on the low edge of the ellipse in Figure D.2. For the muon the maximum angle is of order $0.27 / \gamma$ for muons from pion decays. This is fortunate for the muon monitors: since the muons are more forward-boosted, they are more likely to exit the decay pipe at its downstream end, especially at high momentum.

Figure D. 3 shows the angular distribution of neutrinos and muons from pion decays. Shown are the angular distributions in the laboratory frame, where angles are taken with respect to the pion direction. The distributions 
for three different pion boosts are shown. As can be seen, the neutrino angle peaks at the characteristic $\theta_{\nu} \approx 1 / \gamma$ but a maximum angle which goes out as far as $\theta_{\nu}=\pi / 2$. The muon angle has a hard edge predicted by Equation D.8.

\section{D.3 Energy Distribution of Daughters}

The neutrino energy in the lab is found from Equation D.2. For $\beta \approx 1$, again using $\cos \theta^{\prime} \approx\left(1-\gamma^{2} \tan ^{2} \theta_{\nu}\right) /\left(1+\gamma^{2} \tan ^{2} \theta_{\nu}\right)$ the lab energy is

$$
E_{\nu} \approx \frac{2 \gamma p^{\prime}}{1+\gamma^{2} \tan ^{2} \theta_{\nu}}=\frac{\left(1-\frac{m_{\mu}^{2}}{M^{2}}\right) E}{1+\gamma^{2} \tan ^{2} \theta_{\nu}}
$$

which reduces to familiar equation $E_{\nu} \approx 0.43 E_{\pi} /\left(1+\gamma^{2} \theta_{\nu}^{2}\right)$ for pion decays in the limit that $\theta_{\nu} \ll 1$. Equations D.9 and 3.12 combine to show that the energy distribution of the neutrinos, $d P / d E_{\nu}=\left(d P / d \Omega_{\nu}\right)\left(d \Omega_{\nu} / d E_{\nu}\right)$, is constant

$$
\frac{d P}{d E_{\nu}}=\frac{1}{\left(1-\frac{m_{\mu}^{2}}{M^{2}}\right) E}
$$

which averages over all decay angles $\theta_{\nu}$. This is a restatement of the fact that neutrinos populate all the locations around the ellipse in Figure D.2.

An example of the energy distribution of muons and neutrinos in pion and kaon decays is shown in Figure D.4. Shown is the case for $\gamma=200$ pions and kaons $\left(E_{\pi}=27.9 \mathrm{GeV}\right.$ or $\left.E_{K}=98.7 \mathrm{GeV}\right)$. As expected, the energy of the neutrino in pion decay is constant from zero up to $\left(1-\frac{m_{\mu}^{2}}{M^{2}}\right) E_{\pi} \approx 0.43 E_{\pi}$, and the muon momentum ranges from $0.57 E_{\pi}$ up to $E_{\pi}$. For kaons, the higher kaon mass means that the neutrino carries as much as $0.96 E_{K}$, and the muon momenta range as low as $0.04 E_{K}$. The energy distribution of the muons is 
quite important for the efficiency of the muon monitors. The typical decay leading to a neutrino in the MINOS detectors is that which gives the maximum neutrino energy $E_{\nu}=0.43 E_{\pi}$ and minimum muon momentum $p_{\mu}=0.57 E_{\pi}$. However, just as many decays lead to the maximum muon energy $p_{\mu}=E_{\pi}$ and minimum neutrino energy $E_{\nu}=0$. While the latter do not lead to a neutrino in the MINOS detectors, they result from the same hadrons as give us neutrinos, and they result in a higher-momentum muon which can penetrate the downstream absorber and shielding to get to the muon alcoves. Naively, if the downstream shielding imposes a muon threshold of $p_{\mu}=4 \mathrm{GeV} / c$, this results in a parent hadron threshold of $4 \mathrm{GeV} / c$ and the typical neutrino energy of $1.6 \mathrm{GeV}$.

\section{D.4 Implementation into beam Monte Carlo}

The simulation of muon rates in the alcoves will be broken up into two steps. The first step will be to calculate the muon rates at the end of the decay pipe. The NuMI beam MC (Chapter 3) produces so called "ntuples" which contain the information about neutrino parent type, decay point and momentum. The muon rates at the end of the decay pipe are found using the hadrons in the GNuMI ntuples. We re-decay the hadrons in the ntuples to yield a muon at the end of the decay pipe (see below). The second step is to track the muons through the absorber (Figure 3.20) and the downstream rock until they arrive at the muon alcoves. This second step is CPU-intensive because muons must be tracked down to low energy, and requires an accurate knowledge of the geometry of the absorber and rock, which has been documented in [113].

The efficiencies for a muon at the end of decay pipe to successfully arrive 

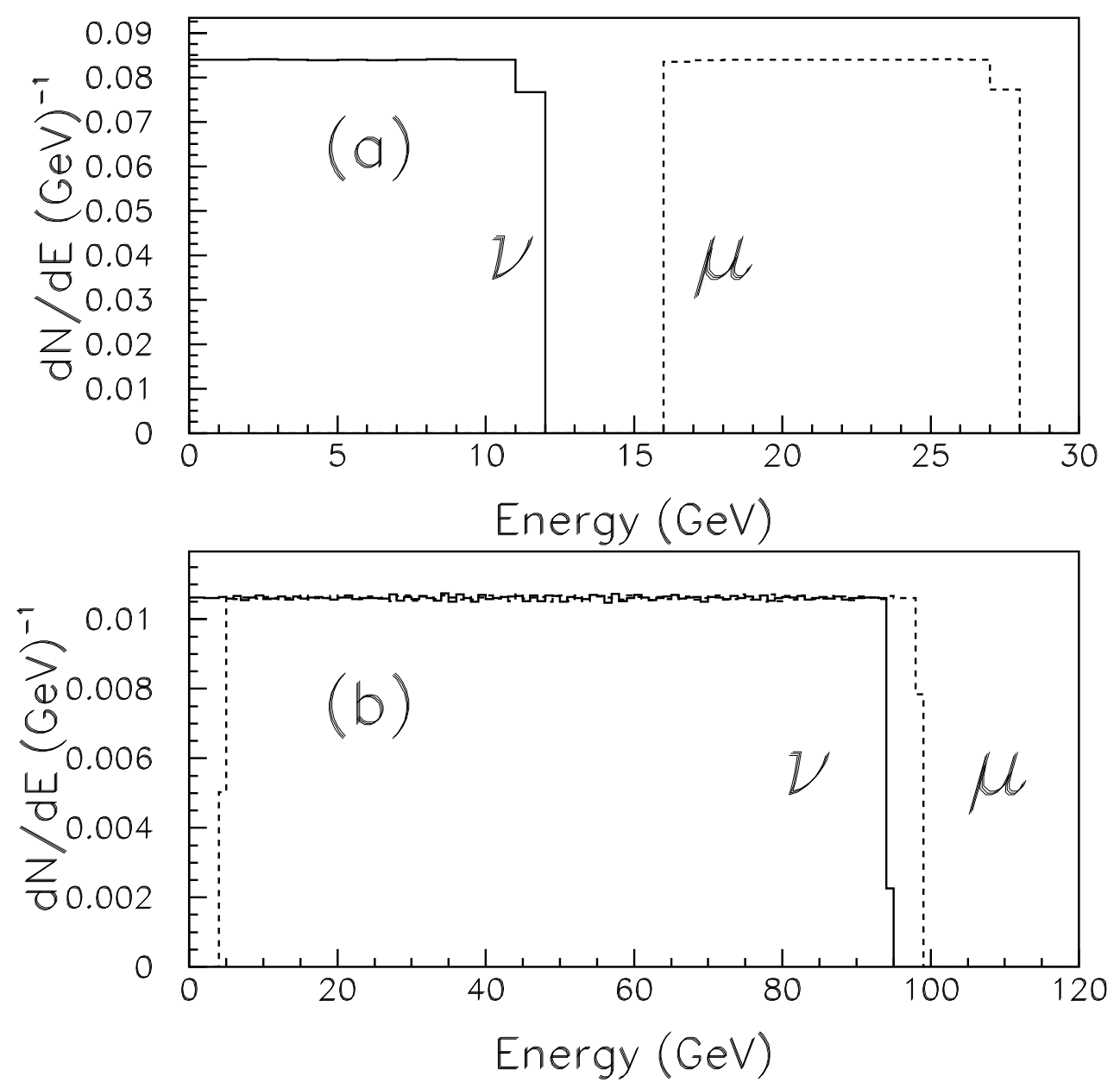

Figure D.4: Momentum distribution of the daughter muon and neutrino in meson decays with $\gamma=200$. (a) $\pi \rightarrow \mu \nu$ (b) $K \rightarrow \mu \nu$. The solid line is the neutrino energy distribution, while the dashed line is the muon energy distribution. 
at one of the alcoves depending on the position of muon at the end of the decay pipe have been found in [113]. In particular the efficiency was calculated for 12 zones in $3 \times 4$ array that spans $1 \times 1 \mathrm{~m}^{2}$ covering the end of the decay pipe. The 12 zones were chosen to roughly match the geometry of the hadron absorber. The 2 zones in the very middle overlap with the aluminum core of the beam absorber. The muons will most easily pass through this section. The outer zones overlap with iron shielding and muons passing through that region will have slightly higher momentum threshold to reach the muon alcoves.

Re-decaying the $\pi^{ \pm}$and $K^{ \pm}$so as to force muons to reach the end of the decay pipe proceeds as follows: each meson is re-decayed 10,000 trials, and for each decay trial we force the meson decay angle in its CM frame through all possible values so as to essentially perform a numerical integral of

$$
\int \frac{d P}{d \Omega^{\prime}} d \Omega^{\prime} \approx \sum_{i=1}^{10,000} \frac{d P}{d \Omega^{\prime}} \Delta \Omega_{i}^{\prime}=1
$$

Each meson gives birth to 10,000 muons, each of fractional weight. Briefly, the algorithm is:

1. Increment the $\mathrm{CM}$ angle $\theta^{\prime}$ from 0 to $\pi$ in steps of $\Delta \theta^{\prime}=\pi / 10000=$ $0.3 \mathrm{mrad}$.

2. For this $\theta_{i}^{\prime}=i \times \Delta \theta^{\prime}$, calculate the muon polar angle $\theta_{\mu}$ in the lab using Equation D.6.

3. For this $\theta^{\prime}$, calculate the muon momentum $p_{\mu}$ in the lab using Equations D.3 and D.4.

4. The meson direction is projected to the plane of the end of the decay 
pipe, as in Figure D.5 to obtain its projected radius of exit from the decay pipe, $r_{\text {meson }}$.

5. The muon decay angle $\theta_{\mu}$ obtained in step 2 defines a cone around the meson direction, as shown in Figure D.5. The azimuthal fraction $\Delta \phi$ of this cone that falls within the decay pipe exit window is determined. We identify three cases:

(a) $r_{\mu}+r_{\text {meson }}<r_{\text {pipe }}$ : in this case $\Delta \phi=2 \pi$ because the entire cone falls within the decay pipe.

(b) $\left|r_{\mu}-r_{\text {meson }}\right|>r_{\text {pipe }}$ : in this case $\Delta \phi=0$ because the entire cone falls outside the decay pipe.

(c) $r_{\mu}+r_{\text {meson }}>r_{\text {pipe }}: \Delta \phi=2 \alpha$, where $\cos \alpha=\left(r_{\mu}^{2}+r_{\text {meson }}^{2}-\right.$ $\left.r_{\text {pipe }}^{2}\right) / 2 r_{\mu} r_{\text {meson }}$.

6. The decay is given a weight from Equation D.11:

$$
\frac{d P}{d \Omega^{\prime}} \Delta \Omega_{i}^{\prime}=(1 / 4 \pi) \Delta \phi\left(\sin \theta_{i}^{\prime} \Delta \theta^{\prime}\right)
$$

Figure C.1 demonstrates the output of the algorithm above applied to a GNuMI file. Plotted are the momenta of muons which arrive at the end of the decay pipe, with weights calculated from above. Many muons do not arrive at the end of the decay pipe, and these would likely be the decays which give a forward neutrino and an off-angle muon. As discussed in the previous sections, however, many pion decays do produce forward muons. As shown in Figure C.1, the energy spectrum of muons reaching the end of the decay 

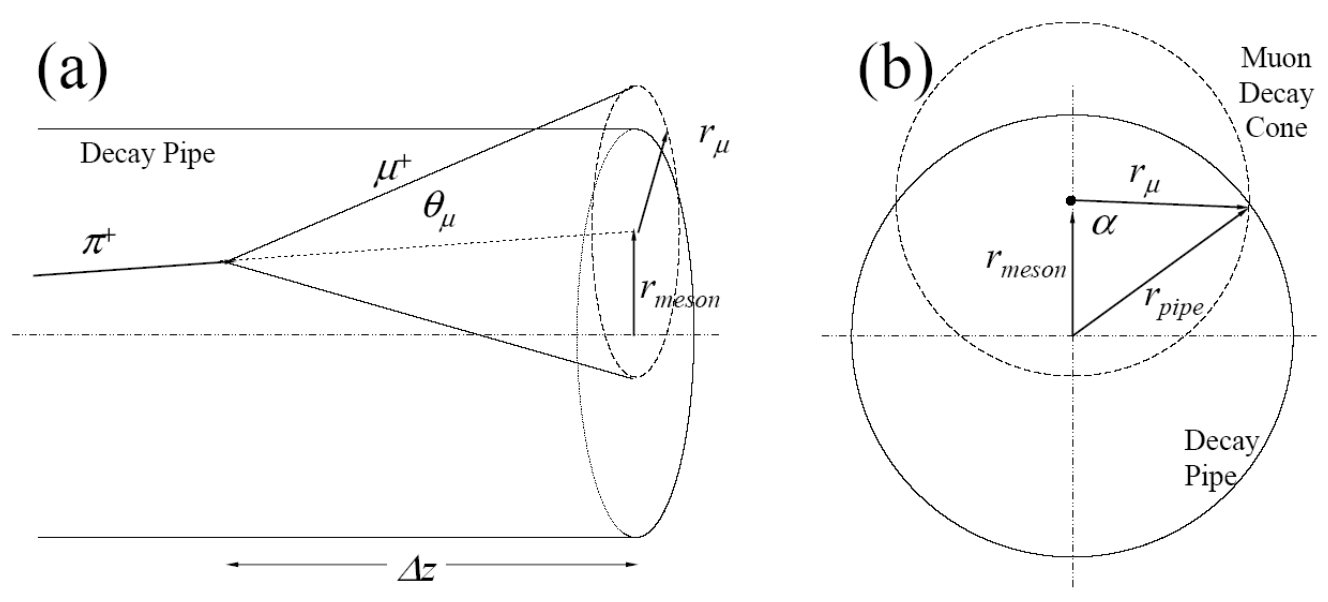

Figure D.5: View of a meson decay and resulting muon in the decay pipe: (a) side view (b) end view looking upstream. The decay occurs a distance $\Delta z$ upstream of the decay pipe end. The meson projects to an exit point a distance $r_{\text {meson }}$ from the decay pipe axis at the end of the decay pipe. The muon decay at an angle $\theta_{\mu}$ defines a cone whose intersection with the plane at the end of the decay pipe is approximately a circle of radius $r_{\mu}=\Delta z \tan \theta_{\mu}$.

pipe displays significant information about the focusing peak as well as the high energy tail of the parent hadron spectrum. Figure C.1 also shows the fractions of the muons which would be expected to reach alcoves 1-3, using the efficiencies from [113]. Note that Alcove 1 sees a reasonable fraction of the muons in the focusing peak. 


\section{Appendix E}

\section{Neutrino Flux Uncertainty}

Here we repeat the calculations of Chapter 5 to find the uncertainties in the far over near ratio (§4.2) predicted by the Monte Carlo calculation of Chapter 3 for several systematic effects. The uncertainty in the ratio reflects the uncertainty in the predicted flux at the far detector.

Tuning the beam Monte Carlo simulation to the Near Detector neutrino spectrum (Chapter 6) reduces the uncertainty in the predicted flux and far over near ratio. The appendix concludes with flux uncertainties after the tuning procedure. 


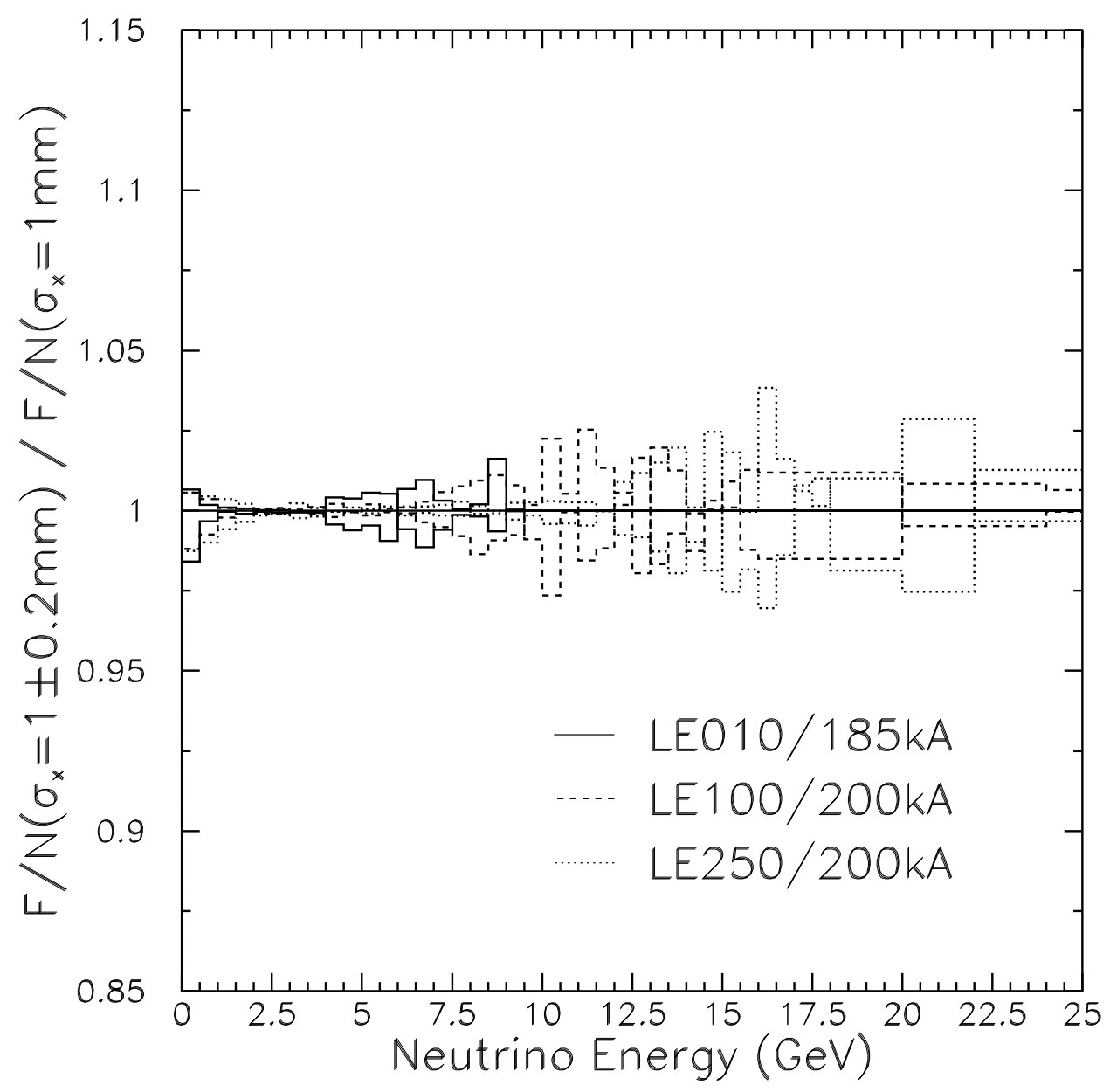

Figure E.1: The curves represent the changes in far over near ratio for $\pm 20 \%$ excursions in the proton beam width $\sigma_{x}$. Excursions by $\pm 10 \%$ produce approximately half as large of an effect on the $\mathrm{F} / \mathrm{N}$ ratio. 


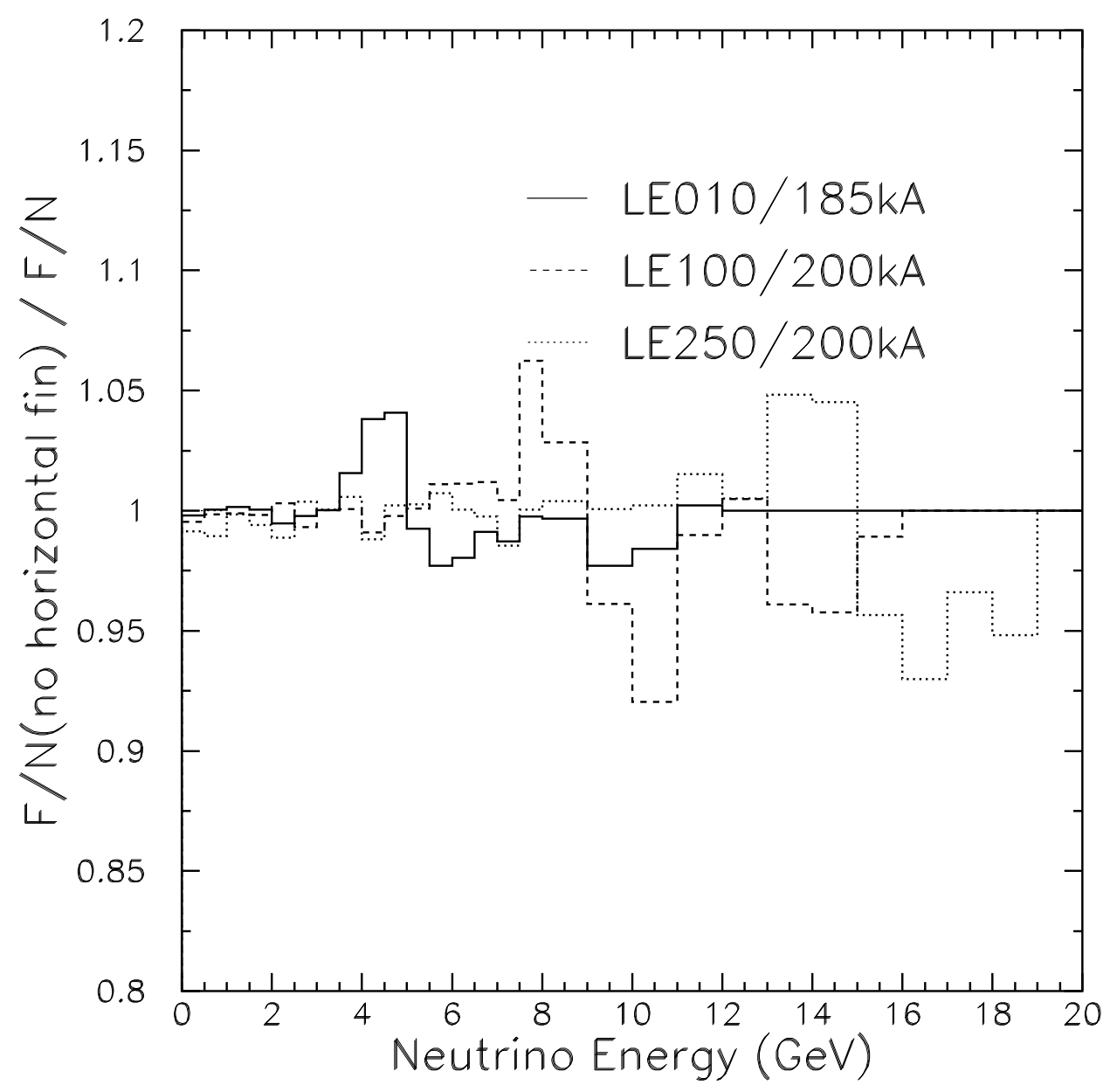

Figure E.2: The target consists of 48 graphite fins (47 vertical and 1 horizontal). The curves represent the fractional change in the far over near ratio when the proton beam misses the horizontal fin (budal monitor). 


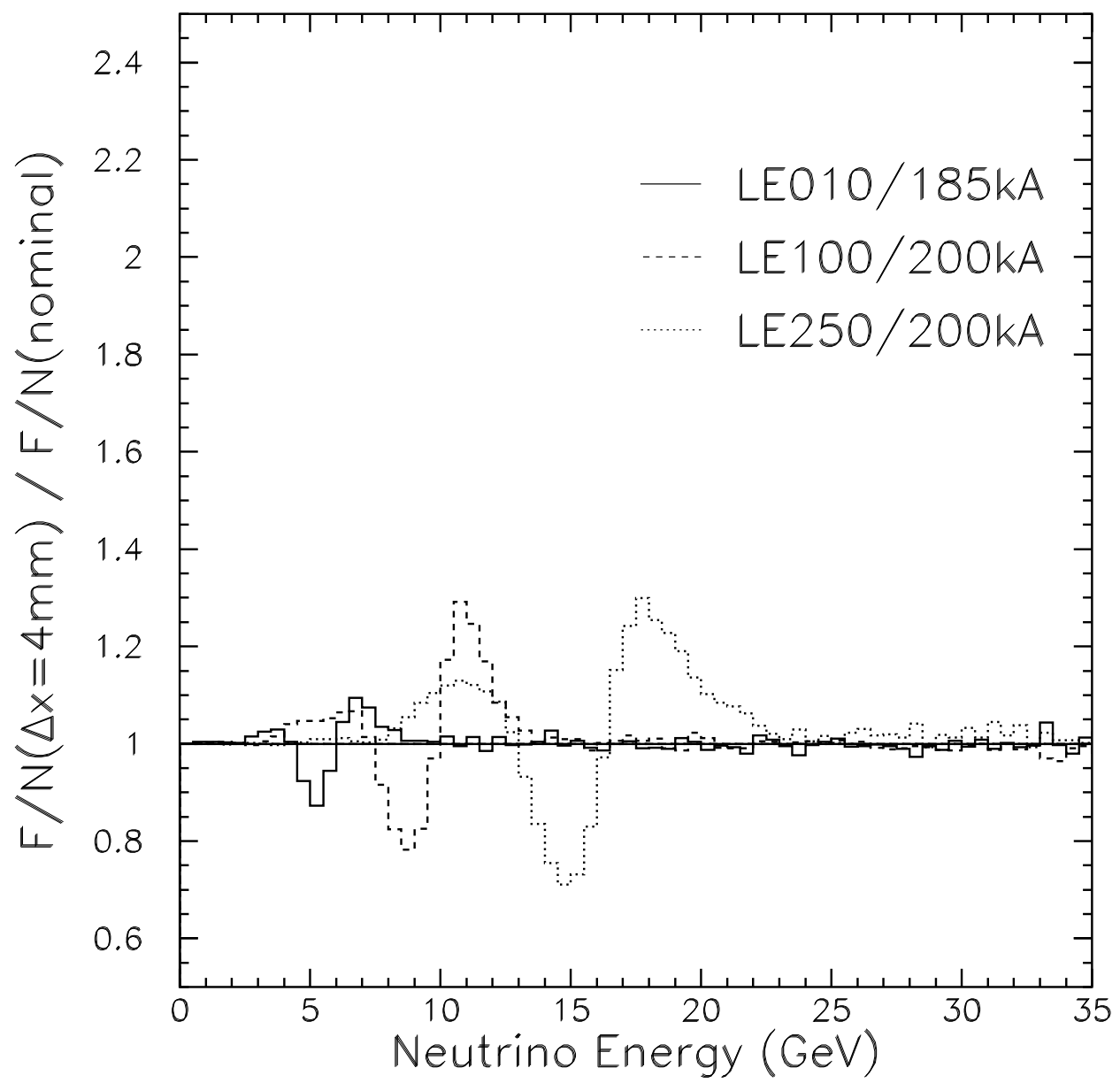

Figure E.3: Far over near ratio fractional change due to Horn 1 misalignment transverse to the beam axis. The curve corresponds to horn offset by $4 \mathrm{~mm}$. 


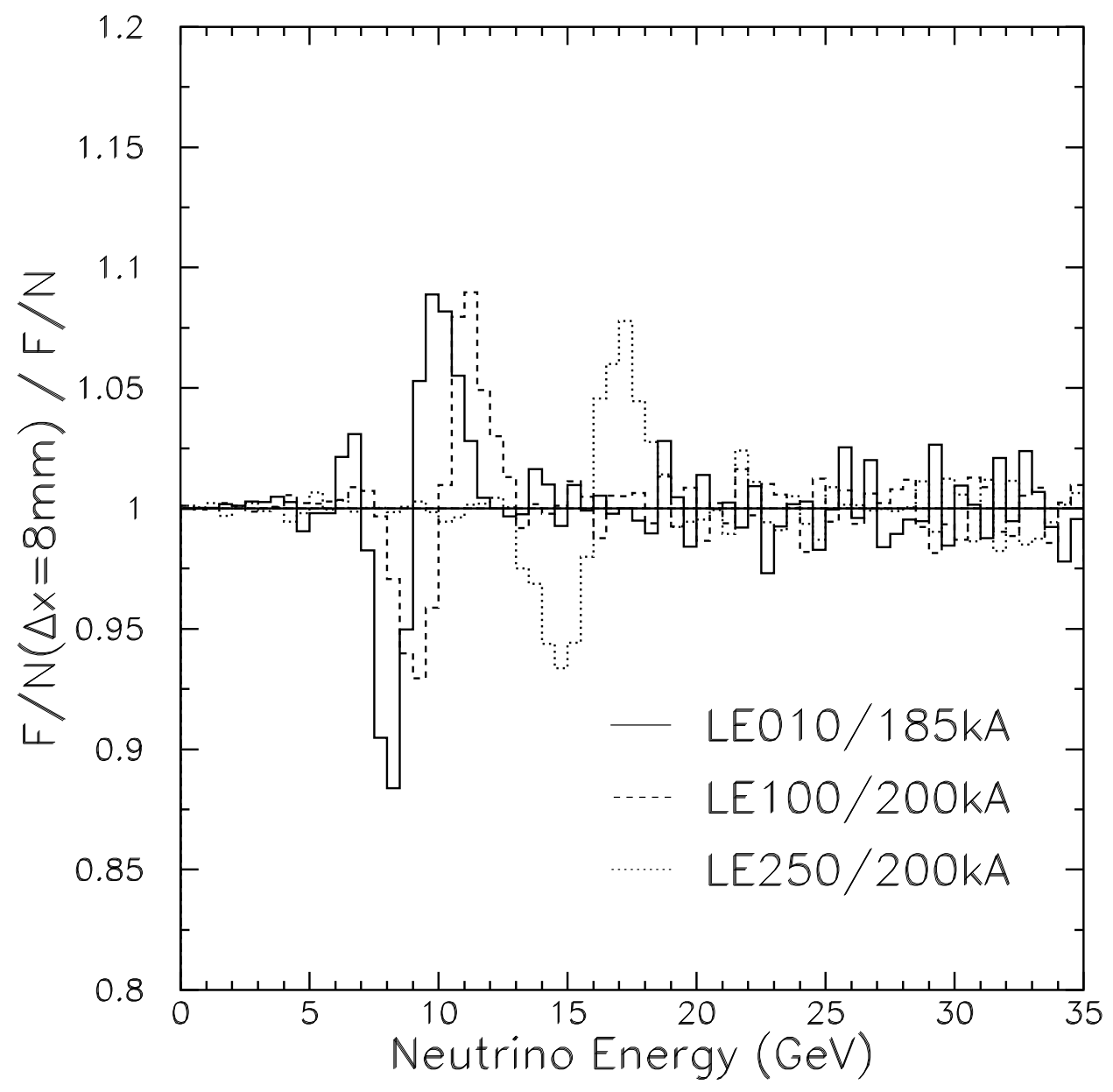

Figure E.4: Far over near ratio fractional change due to Horn 2 misalignment transverse to the beam axis. The curve corresponds to horn offset by $8 \mathrm{~mm}$. 


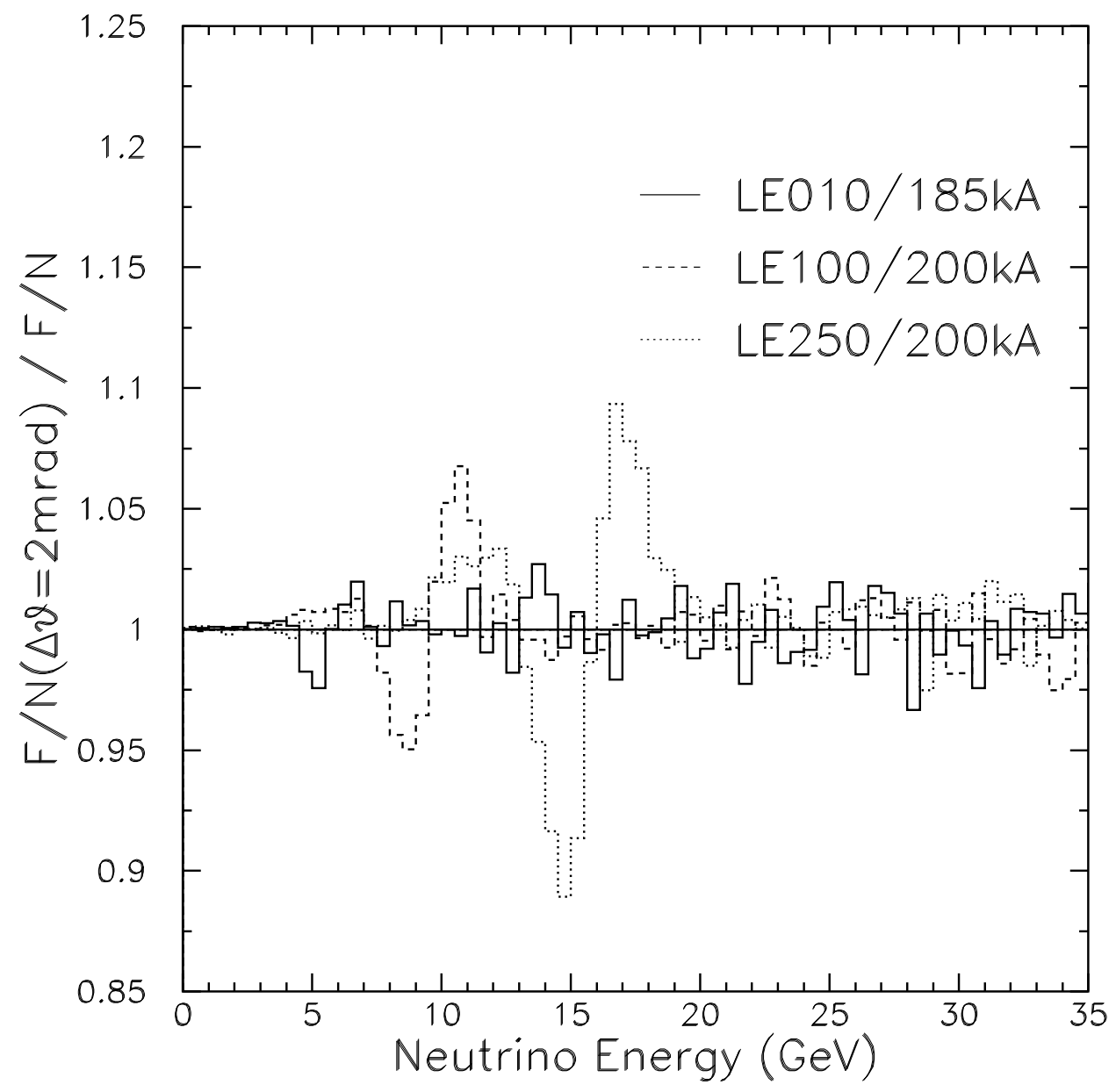

Figure E.5: Far over near ratio fractional change due to Horn 1 Angle Variation. 


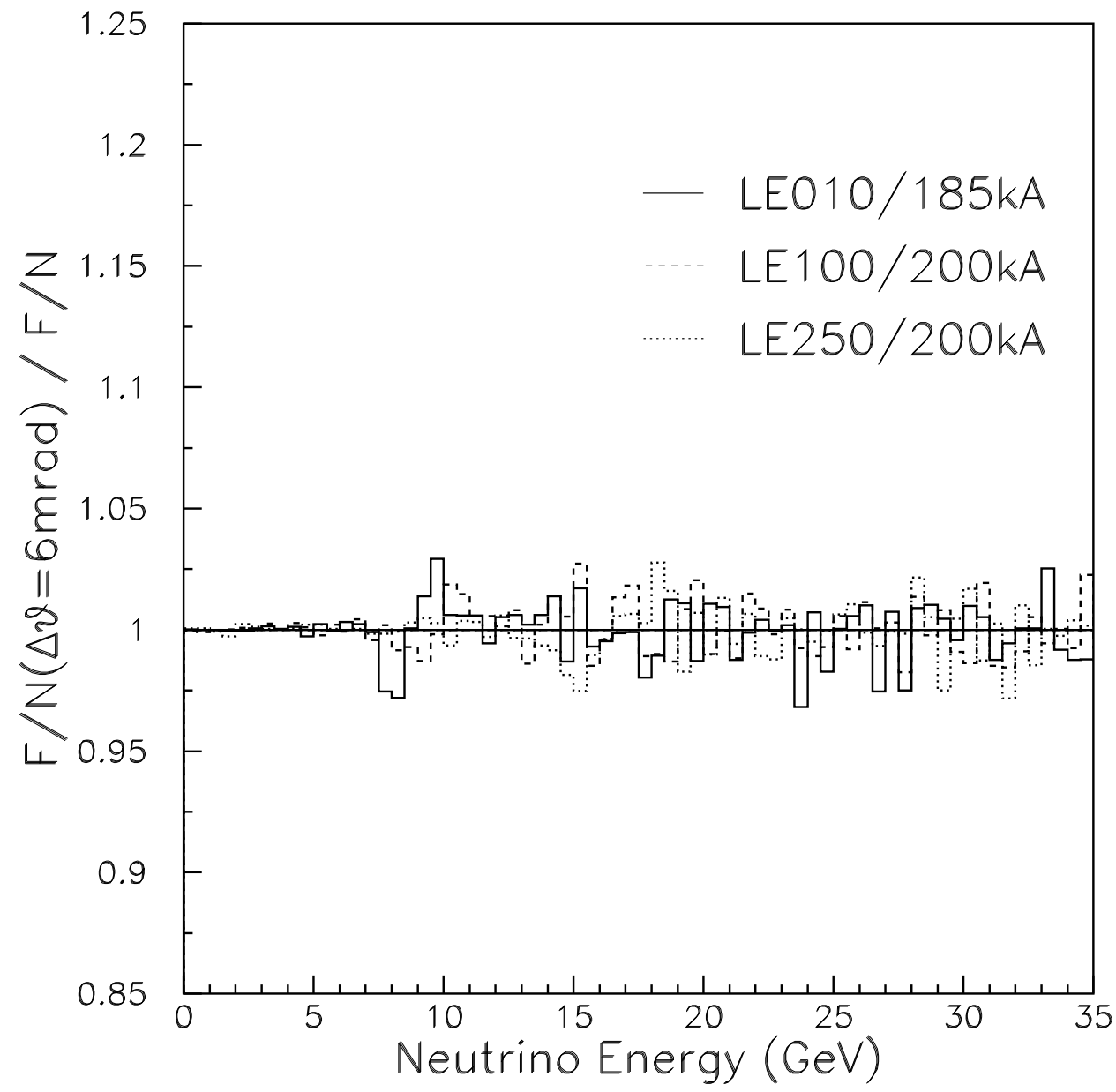

Figure E.6: Far over near ratio fractional change due to Horn 2 Angle Variation. 


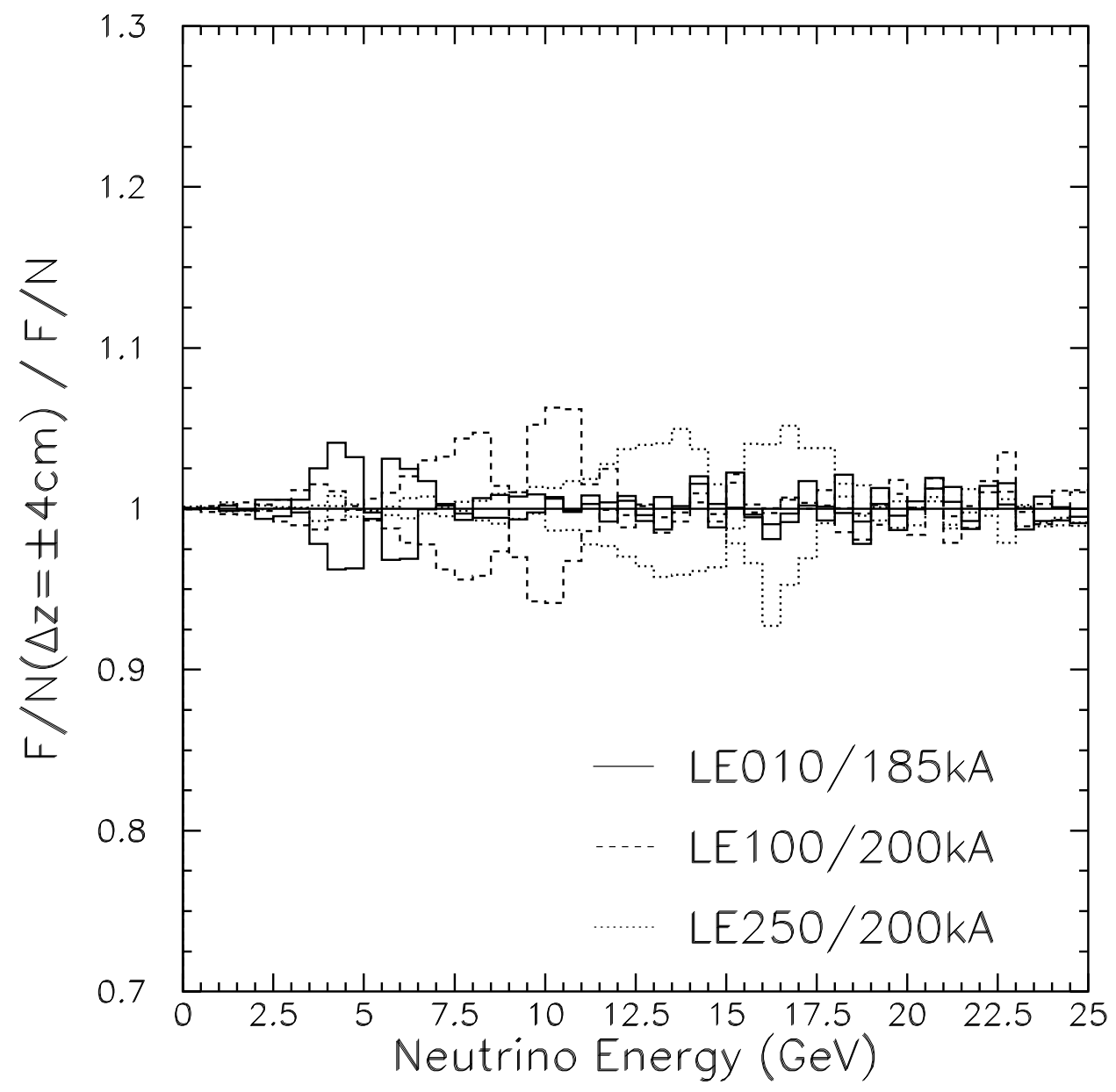

Figure E.7: Far over near ratio fractional change due to target offset in longitudinal direction. 


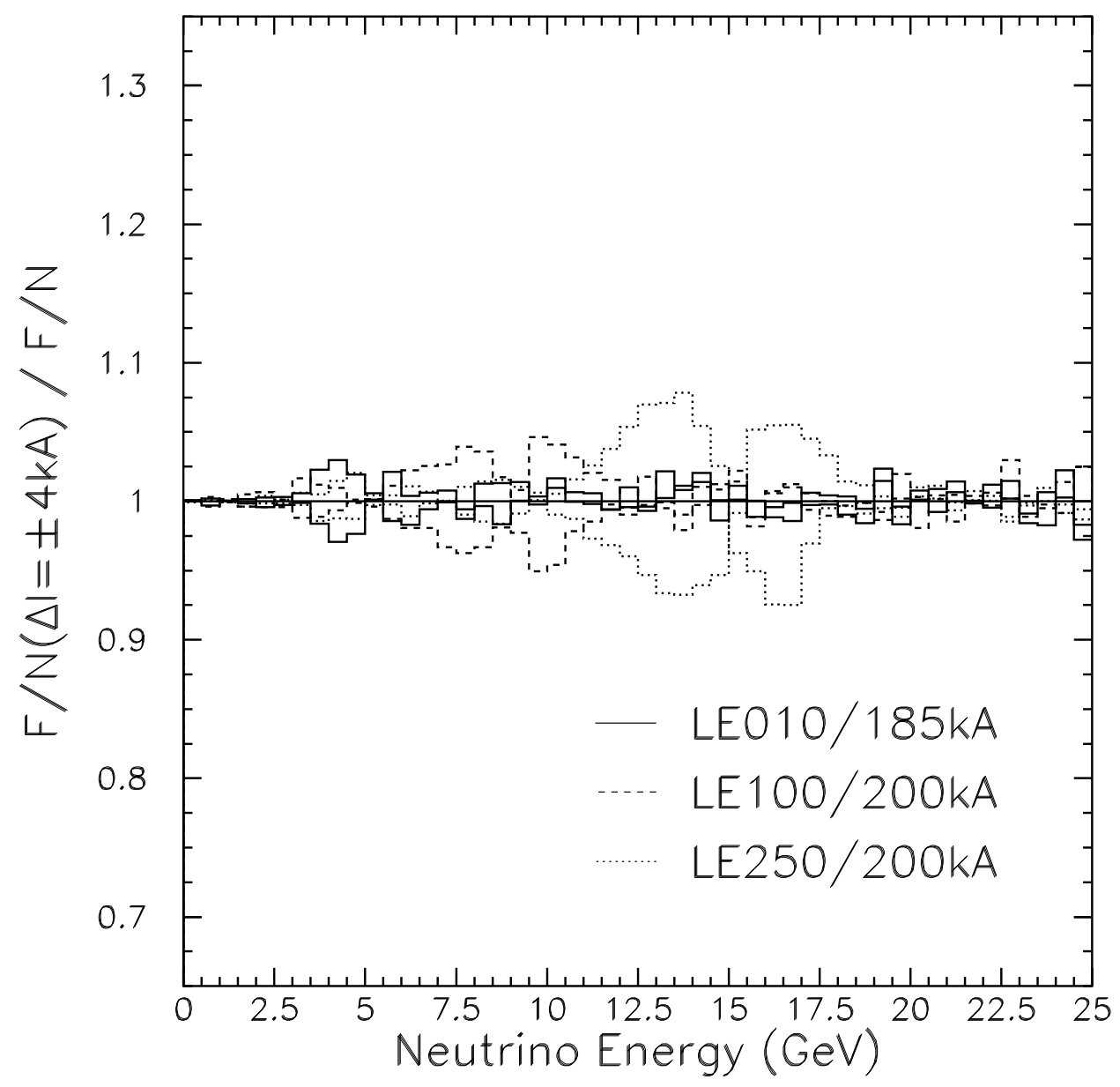

Figure E.8: Far over near ratio fractional change due to Horn Current Miscalibration. 


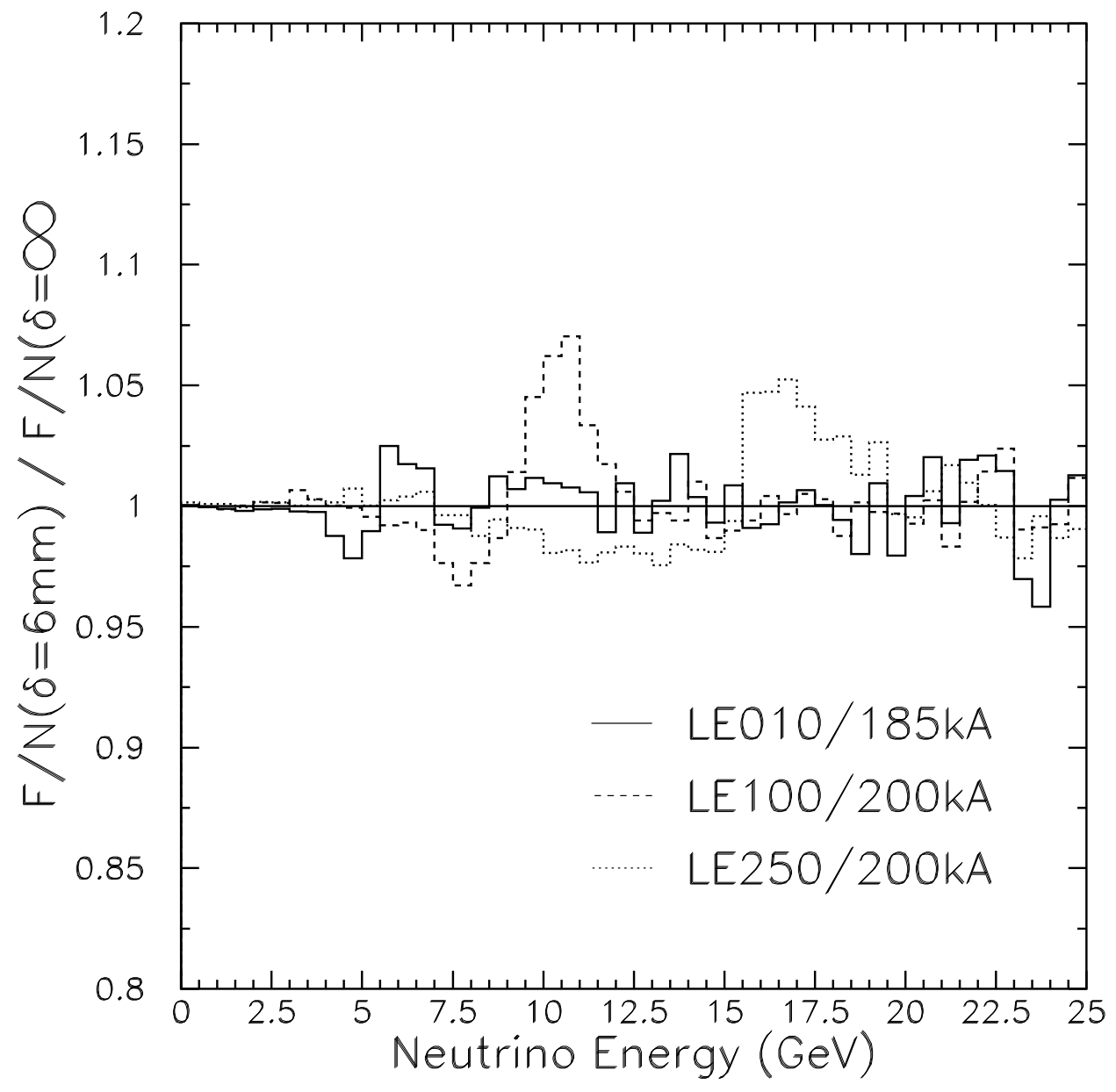

Figure E.9: Far over near ratio fractional change due to Horn Current Distribution uncertainty. 


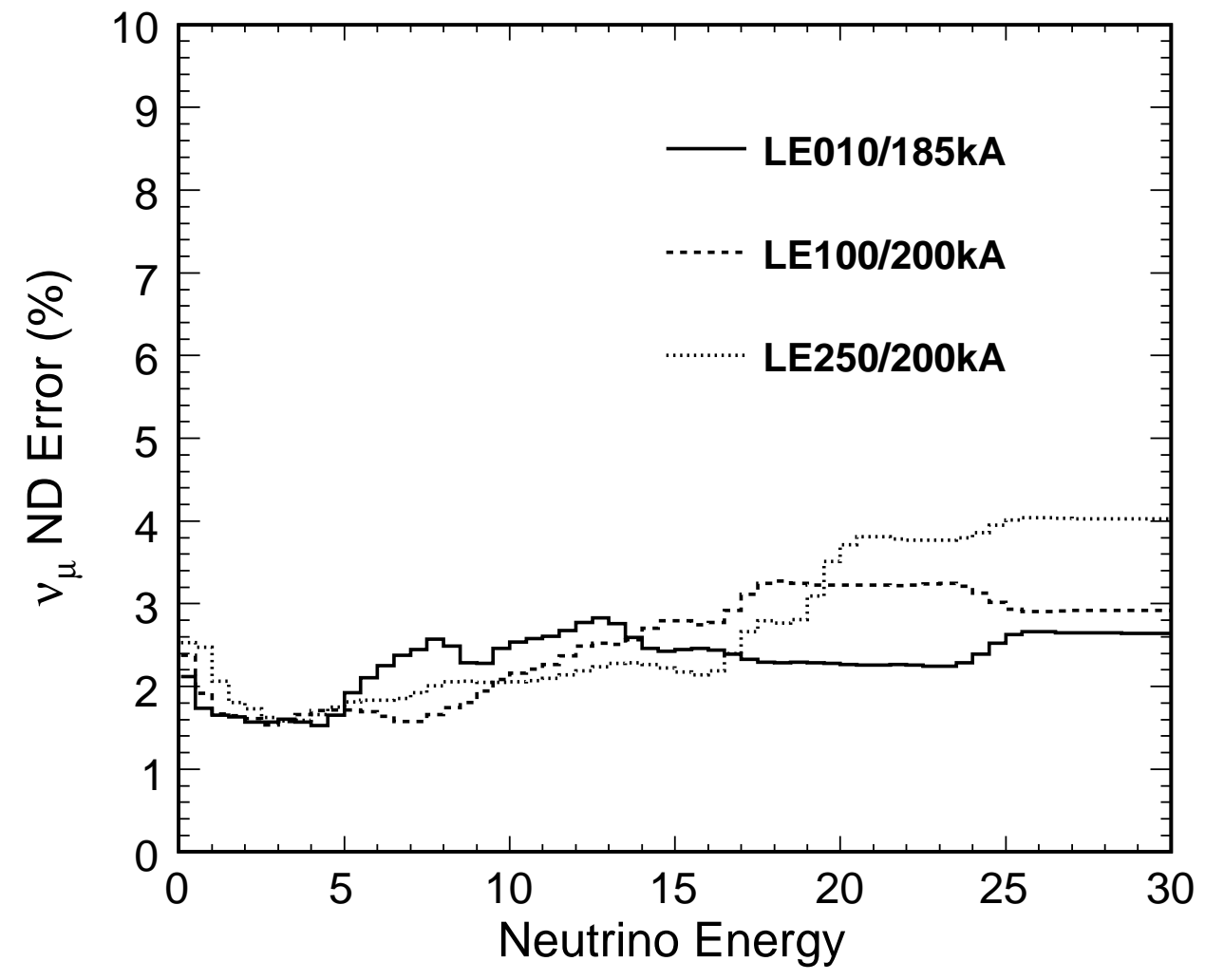

Figure E.10: The uncertainty in $\nu_{\mu}$ energy spectrum at Near Detector due to hadron production uncertainty. Shown is the remaining uncertainty after the MC was fitted to measured $\nu_{\mu}$ and $\bar{\nu}_{\mu}$ energy spectra at Near Detector. 


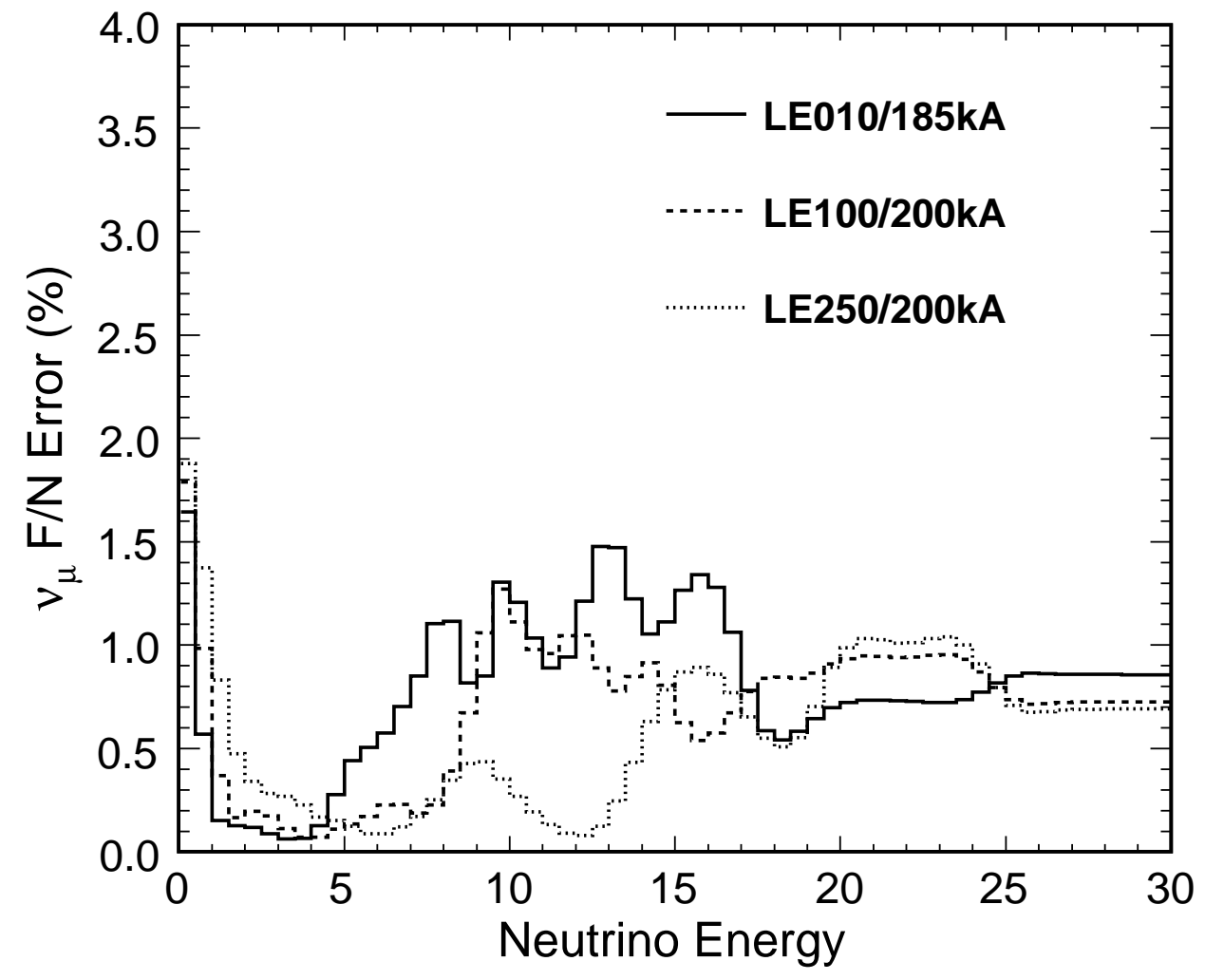

Figure E.11: The uncertainty in $\nu_{\mu}$ far over near ratio due to hadron production uncertainty. Shown is the remaining uncertainty after the MC was fitted to measured $\nu_{\mu}$ and $\bar{\nu}_{\mu}$ energy spectra at Near Detector. 


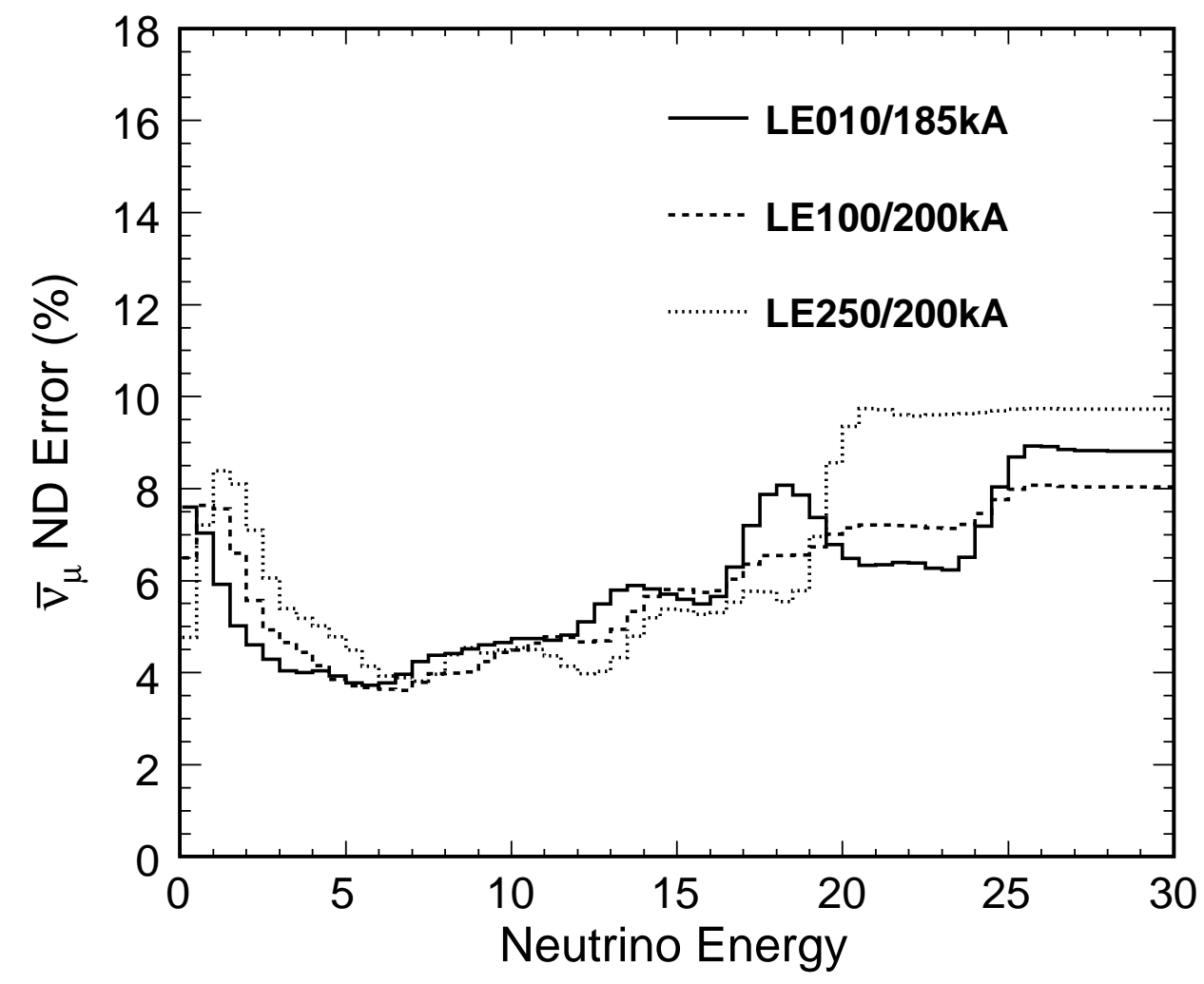

Figure E.12: The uncertainty in $\bar{\nu}_{\mu}$ energy spectrum at Near Detector spectrum due to hadron production uncertainty. Shown is the remaining uncertainty after the MC was fitted to measured $\nu_{\mu}$ and $\bar{\nu}_{\mu}$ energy spectra at Near Detector. 


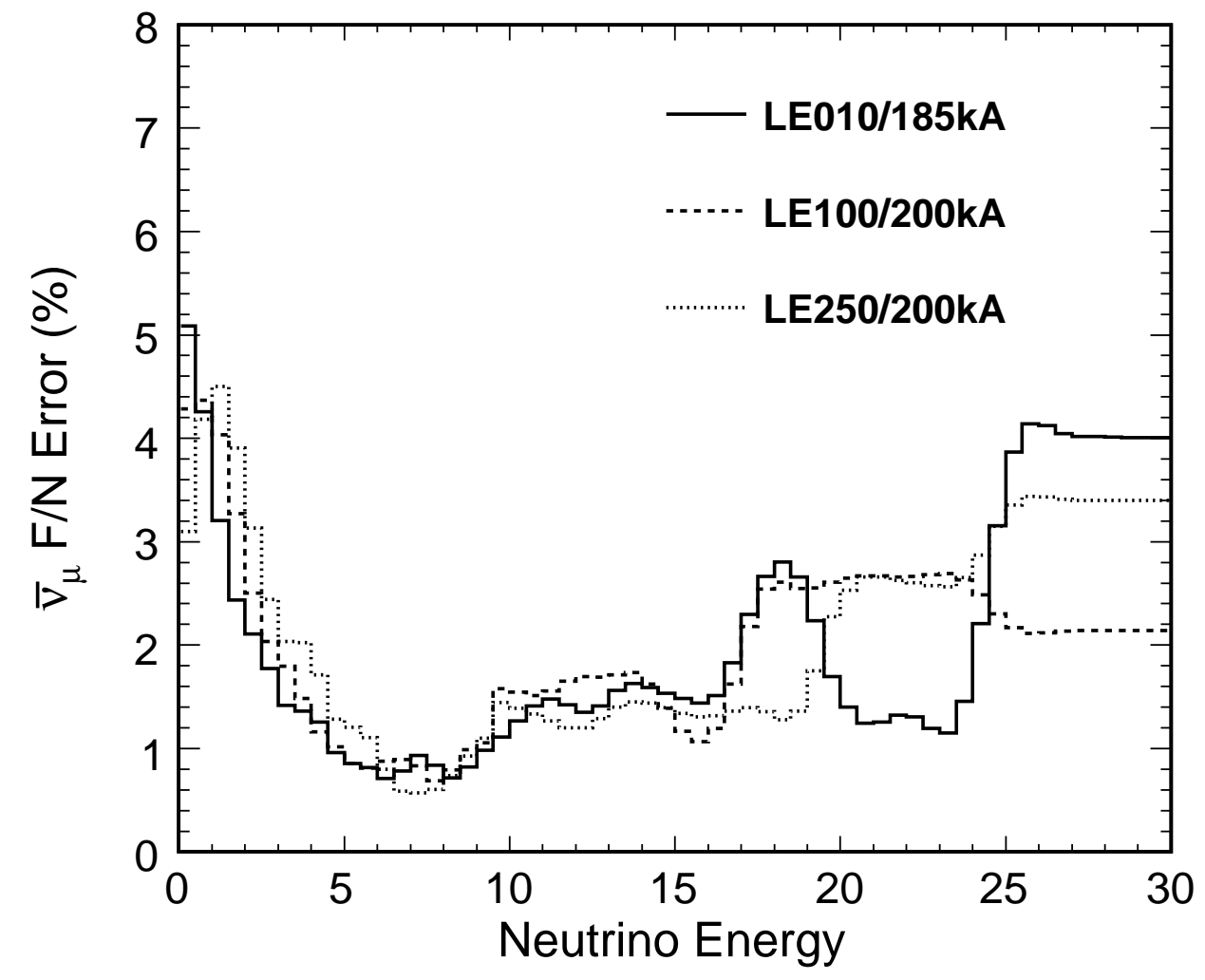

Figure E.13: The uncertainty in $\bar{\nu}_{\mu}$ far over near ratio due to hadron production uncertainty. Shown is the remaining uncertainty after the MC was fitted to measured $\nu_{\mu}$ and $\bar{\nu}_{\mu}$ energy spectra at Near Detector. 


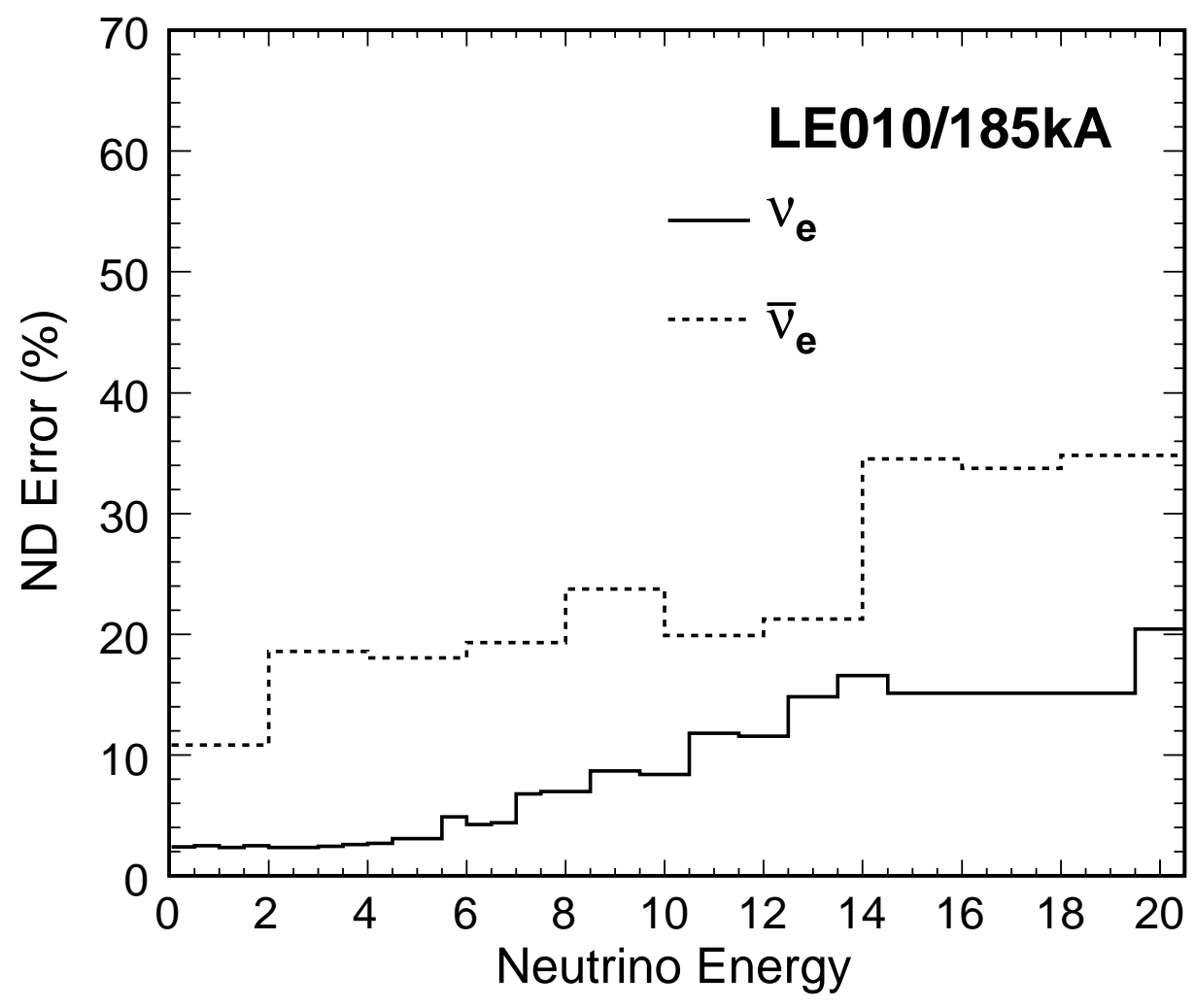

Figure E.14: The uncertainty in $\nu_{e}$ and $\bar{\nu}_{e}$ energy spectrum at Near Detector due to hadron production uncertainty. Shown is the remaining uncertainty after the MC was fitted to measured $\nu_{\mu}$ and $\bar{\nu}_{\mu}$ energy spectra at Near Detector. 


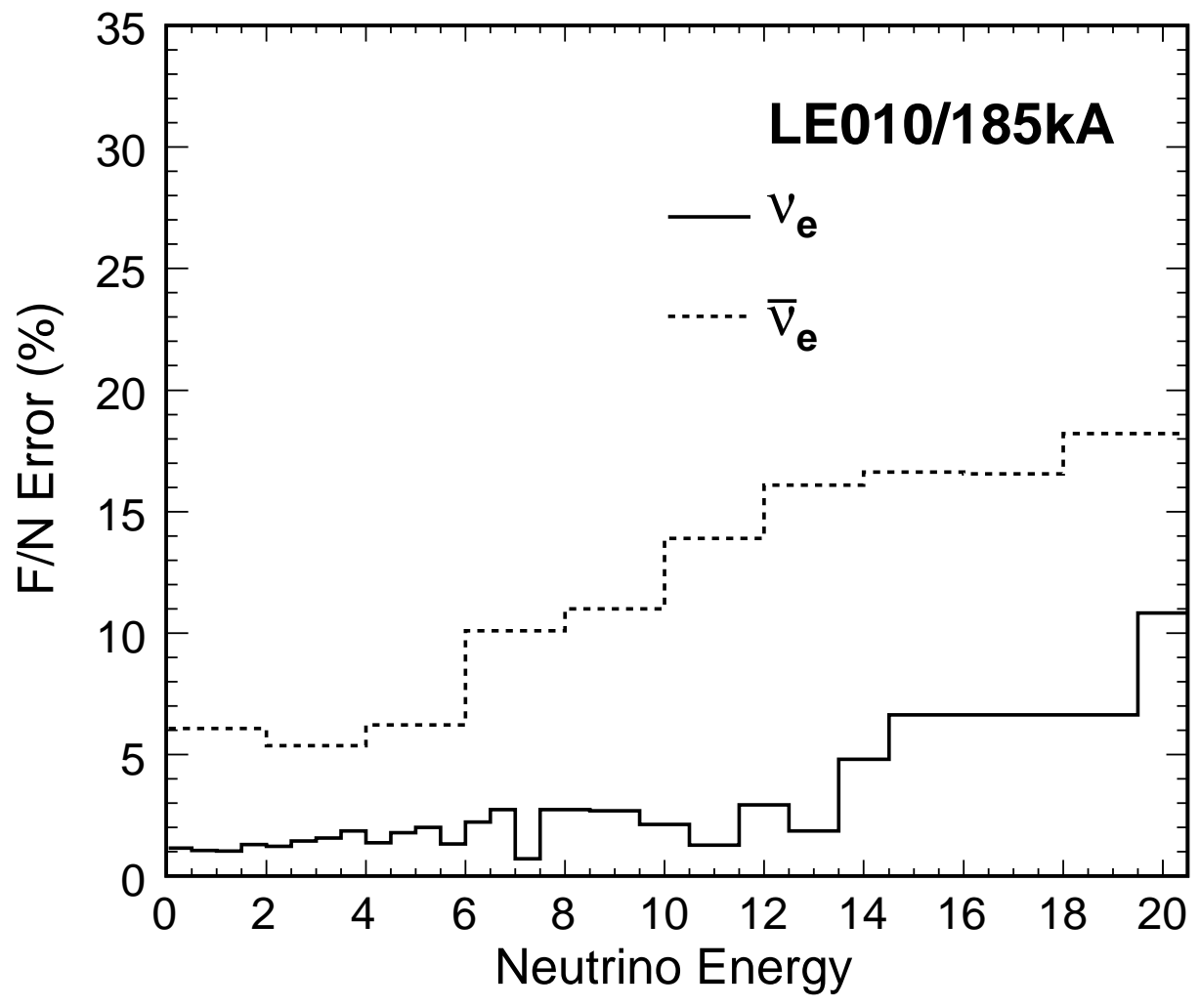

Figure E.15: The uncertainty in far over near ratio for $\nu_{e}$ and $\bar{\nu}_{e}$ due to hadron production uncertainty. Shown is the remaining uncertainty after the MC was fitted to measured $\nu_{\mu}$ and $\bar{\nu}_{\mu}$ energy spectra at Near Detector. 


\section{Bibliography}

[1] E. Fermi, "Theory of beta decay," Z. Physik 88 (1934) no. 12, 161.

[2] H. A. Bethe and R. E. Peierls, "The "Neutrino"," Nature 133 (1934) 532.

[3] F. Reines and C. L. Cowan, "Detection of the free neutrino," Phys. Rev. 92 (1953) 830-831.

[4] G. Danby et al., "Observation of High-Energy Neutrino Reactions and the Existence of Two Kinds of Neutrinos," Phys. Rev. Lett. 9 (Jul, 1962) 36-44.

[5] C. F. Powell et al., "Processes Involving Charged Mesons," Nature 159 (1947) 694-697.

[6] M. L. Perl et al., "Evidence for anomalous lepton production in e+ eannihilation," Phys. Rev. Lett. 35 (1975) 1489-1492.

[7] K. Kodama et al., "Observation of tau neutrino interactions," Phys. Lett. B504 (2001) 218-224.

[8] "The LEP Collaborations and the LEP Electroweak Working Group, 
as reported by J. Dress at the XX International Symposium on Lepton and Photon Interactions at High Energy, Rome, Italy (July 2001).".

[9] MINOS Collaboration, M. Kordosky and D. Petyt, "Study of Muon Neutrino Disappearance Using the Fermilab Main Injector Neutrino Beam," Phys. Rev. D77 (2008) 072002, arXiv:0711.0769 [hep-ex].

[10] C. Kraus et al., "Final results from phase II of the Mainz neutrino mass search in tritium beta decay," Eur. Phys. J. C40 (2005) 447-468, arXiv:hep-ex/0412056.

[11] E. Komatsu et al., "Five-Year Wilkinson Microwave Anisotropy Probe (WMAP) Observations: Cosmological Interpretation," arXiv:0803.0547 [astro-ph].

[12] B. Pontecorvo J. Exptl. Theoret. Phys. (U.S.S.R) 33 (1957) 549.

[13] B. Pontecorvo J. Exptl. Theoret. Phys. (U.S.S.R) 34 (1958) 247.

[14] Z. Maki, M. Nakagawa, and S. Sakata, "Remarks on the Unified Model of Elementary Particles," Progress of Theoretical Physics 28 (1962) no. $5,870-880$.

[15] B. Pontecorvo J. Exptl. Theoret. Phys. (U.S.S.R) 53 (1967) 1717.

[16] V. Gribov and B. Pontecorvo Physics Letters B 28 (1969) 493.

[17] Particle Data Group Collaboration, W. M. Yao et al., "Review of particle physics," J. Phys. G33 (2006) 1-1232.

[18] L. Wolfenstein, "Neutrino oscillations in matter," Phys. Rev. D17 (May, 1978) 2369-2374. 
[19] CHOOZ Collaboration, M. Apollonio et al., "Limits on neutrino oscillations from the CHOOZ experiment," Phys. Lett. B466 (1999) 415-430, arXiv:hep-ex/9907037.

[20] SNO Collaboration, B. Aharmim et al., "Electron energy spectra, fluxes, and day-night asymmetries of B-8 solar neutrinos from the 391-day salt phase SNO data set," Phys. Rev. C72 (2005) 055502, arXiv:nucl-ex/0502021.

[21] KamLAND Collaboration, "Precision Measurement of Neutrino Oscillation Parameters with KamLAND," arXiv:0801.4589 [hep-ex].

[22] Super-Kamiokande Collaboration, "Evidence for an oscillatory signature in atmospheric neutrino oscillation," Phys. Rev. Lett. 93 (2004) 101801, arXiv:hep-ex/0404034.

[23] Super-Kamiokande Collaboration, "A Measurement of Atmospheric Neutrino Oscillation Parameters by Super-Kamiokande I," Phys. Rev. D71 (2005) 112005, arXiv:hep-ex/0501064.

[24] MINOS Collaboration, "Observation of muon neutrino disappearance with the MINOS detectors and the NuMI neutrino beam," Phys. Rev. Lett. 97 (2006) 191801, arXiv:hep-ex/0607088.

[25] MACRO Collaboration, M. Ambrosio et al., "Measurements of atmospheric muon neutrino oscillations, global analysis of the data collected with MACRO detector," Eur. Phys. J. C36 (2004) 323-339. 
[26] Soudan-2 Collaboration, W. W. M. Allison, "Neutrino Oscillation Effects in Soudan-2 Upward-stopping muons," Phys. Rev. D72 (2005) 052005, arXiv:hep-ex/0507068.

[27] Super-Kamiokande Collaboration, "Solar neutrino measurements in Super-Kamiokande-I," Phys. Rev. D73 (2006) 112001, arXiv:hep-ex/0508053.

[28] K2K Collaboration, M. H. Ahn, "Measurement of Neutrino Oscillation by the K2K Experiment," Phys. Rev. D74 (2006) 072003, arXiv:hep-ex/0606032.

[29] C. W. Walter, "The Super-Kamiokande Experiment," arXiv:0802.1041 [hep-ex].

[30] "CNGS project." http://proj-cngs.web.cern.ch/proj-cngs/.

[31] Y. Itow et al., "The JHF-Kamioka neutrino project," arXiv:hep-ex/0106019.

[32] K. Anderson et al., "The NuMI Technical Design Report." Fermi National Accelerator Laboratory, December, 2002.

[33] I. Stancu et al., "Technical Design Report for the MiniBooNe Neutrino Beam." Fermi National Accelerator Laboratory, May, 2001.

[34] K. Hagiwara et al., "Review of Particle Physics," Physical Review D 66 (2002) 010001+. http://pdg.lbl.gov.

[35] A. G. Abramov et al., "Beam optics and target conceptual designs for the NuMI project," Nucl. Instrum. Meth. A485 (2002) 209-227. 
[36] A. Abramov et al., "Conceptual Design of the Narrow Band Beam Absorber and Low Energy Wide Band Beam Calculations for the NuMI Project." MINOS internal note 394, 1998.

[37] A. Abramov et al., "Advanced Conceptual Design of the NuMI Hadron Beam Absorber Core." MINOS internal note 652, 2000.

[38] M. Kostin et al., "Proposal for a continuously-variable beam energy." MINOS internal note 783, 2001.

[39] S. Kopp et al., "Beam Test of a Segmented Foil SEM Grid," Nucl. Instrum. Meth. A554 (2005) 138, arXiv:physics/0507211.

[40] S. Kopp et al., "Secondary beam monitors for the numi facility at fnal," Nucl. Instrum. Meth. A568 (2006) 503, arXiv:physics/0607229.

[41] R. Zwaska et al., "Beam-Based Alignment of the NuMI Target Station Components at FNAL," Nucl. Instrum. Meth. A568 (2006) 548, arXiv:physics/0609106.

[42] M. A. Kordosky, Hadronic interactions in the MINOS detectors. PhD thesis, The University of Texas, 2004. FERMILAB-THESIS-2004-34.

[43] P. L. Vahle, Electromagnetic interactions in the MINOS detectors. PhD thesis, The University of Texas, 2004. FERMILAB-THESIS-2004-35.

[44] C. Zeitnitz and T. A. Gabriel, "The GEANT - CALOR interface and benchmark calculations of ZEUS test calorimeters," Nucl. Instrum. Meth. A349 (1994) 106-111. 
[45] J. J. Hartnell, Measurement of the calorimetric energy scale in MINOS. PhD thesis, The University of Oxford, 2005.

FERMILAB-THESIS-2005-51.

[46] K. Lang and R. Ospanov, "A muon identification technique using the k-nearest neighbor algorithm." MINOS internal note 2737, 2008.

[47] B. Rossi, High Energy Particles. Prentice-Hall, Inc., Englewood Cliffs, NJ, 1952.

[48] T. Hastie, R. Tibshirani, and J. Friedman, The elements of statistical Learning. Springer, 2001.

[49] K. Lang and R. Ospanov, "Selecting charged current $\nu_{\mu}$ events in the data and the simulation." MINOS internal note 4390, 2008.

[50] H. Gallagher, "The NEUGEN neutrino event generator," Nucl. Phys. Proc. Suppl. 112 (2002) 188-194.

[51] R. Brun et al., "GEANT3 manual." CERN Program Library Long Writeup W5013, 1994. CERN Program Library Long Writeup W5013.

[52] GEANT4 Collaboration, S. Agostinelli et al., "GEANT4: A simulation toolkit," Nucl. Instrum. Meth. A506 (2003) 250-303.

[53] R. Milburn, "Neutrino beam simulation using paw with weighted monte carlo." MINOS internal note 109, 1995.

[54] H. W. Atherton et al., "Precise Measurements of Particle Production by $400-\mathrm{GeV} / \mathrm{c}$ Protons on Beryllium Targets," . CERN-80-07. 
[55] D. S. Barton et al., "Experimental Study of the a-Dependence of Inclusive Hadron Fragmentation," Phys. Rev. D27 (1983) 2580.

[56] NA56/SPY Collaboration, G. Ambrosini et al., "Measurement of charged particle production from $450-\mathrm{GeV} / \mathrm{c}$ protons on beryllium," Eur. Phys. J. C10 (1999) 605-627.

[57] NA49 Collaboration, C. Alt et al., "Inclusive production of charged pions in $\mathrm{p}+\mathrm{C}$ collisions at $158-\mathrm{GeV} / \mathrm{c}$ beam momentum," Eur. Phys. J. C49 (2007) 897-917, hep-ex/0606028.

[58] MIPP Collaboration, J. Paley, "First Results from MIPP," AIP Conf. Proc. 981 (2008) 154-156.

[59] A. Fasso, A. Ferrari, J. Ranft, and P. Sala, "FLUKA: Present status and future developments,". Given at 4th International Conference on Calorimetry in High-energy Physics, La Biodola, Italy, 19-25 Sep 1993.

[60] A. Fasso', A. Ferrari, J. Ranft, and P. Sala, "FLUKA: a multi-particle transport code." CERN 2005-10 (2005), INFN/TC_05/11, SLAC-R-773.

[61] N. V. Mokhov, The MARS Code System User's Guide. Fermilab-FN-628 (1995).

[62] A. Fasso' et al., "The physics models of fluka: status and recent developments," . arXiv:hep-ph/0306267.

[63] N. V. Mokhov and O. E. Krivosheev, "MARS Code Status,". Proc. Monte Carlo 2000 Conf., p. 943, Lisbon, October 23-26, 2000; Fermilab-Conf-00/181 (2000). 
[64] N. V. Mokhov, "Status of MARS Code,". Fermilab-Conf-03/053 (2003).

[65] N. V. Mokhov, K. K. Gudima, C. C. James, et al., "Recent Enhancements to the MARS15 Code,". Fermilab-Conf-04/053 (2004); http://www-ap.fnal.gov/MARS/. MARS was developed in part with Government funding provided under DOE Contract DE - AC02 -76CH03000.

[66] M. Bonesini, A. Marchionni, F. Pietropaolo, and T. T. de Fatis, "On particle production for high energy neutrino beams," Eur. Phys. J. C20 (2001) 13-27, hep-ph/0101163.

[67] A. J. Malensek, "Empirical Formula for Thick Target Particle Production,". FERMILAB-FN-0341.

[68] R. P. Feynman, "Very High-Energy Collisions of Hadrons," Phys. Rev. Lett. 23 (Dec, 1969) 1415-1417.

[69] E. Yen, "New scaling variable and early scaling in single-particle inclusive distributions for hadron-hadron collisions," Phys. Rev. D10 (Aug, 1974) 836-843.

[70] F. E. Taylor et al., "Analysis of radial scaling in single-particle inclusive reactions," Phys. Rev. D14 (Sep, 1976) 1217-1242.

[71] S. van der Meer. CERN Report CERN-61-07, 1961.

[72] G. I. Budker. International Conference on Accelerators, Dubna, 1963. 
[73] A. Abramov et al., "Calculations and Mapping od the Magnetic Field in the Prototype Horn 1." MINOS internal note 710, 2000.

[74] D. Indurthy, S. Kopp, and Ž. Pavlović, "Systematic Uncertainties in the NuMI Beam Flux." MINOS internal note 1283, 2007.

[75] A. Ibrahim. FNAL Beam Documents 1507.

[76] P. Adamson et al., "Measuring the Number of Protons-on-Target (POT) in the NuMI Beamline." MINOS internal note 1491, 2006.

[77] S. Kopp and L. Loiacono, "Proton Beam Quality." MINOS internal note 2854, 2007.

[78] J. Hylen, "Calibration of NuMI Horn Current Readout." MINOS internal note 1303, 2006.

[79] J. D. Jackson, Classical Electrodynamics. John Wiley \& Sons, 1999.

[80] M. A. Heald and J. B. Marion, Classical Electromagnetic Radiation. John Wiley \& Sons, 1999.

[81] D. E. Jaffe, K. H. Lo, J. R. Comfort, and M. Sivertz, "Comparison of inclusive particle production in $14.6-\mathrm{GeV} / \mathrm{c}$ proton nucleus collisions with simulation," Nucl. Instrum. Meth. B246 (2006) 309-321, arXiv:hep-ex/0511012.

[82] G. P. Zeller et al., "Precise Determination of Electroweak Parameters in Neutrino-Nucleon Scattering," Phys. Rev. Lett. 88 (Feb, 2002) 091802.

[83] R. C. Allen et al., "Study of electron-neutrino-electron elastic scattering at LAMPF," Phys. Rev. D 47 (Jan, 1993) 11-28. 
[84] NOMAD Collaboration, P. Astier et al., "Prediction of neutrino fluxes in the NOMAD experiment," Nucl. Instr. Meth. A515 (Dec, 2003) 800.

[85] L. A. Ahrens et al., "Determination of the neutrino fluxes in the Brookhaven wide-band beams," Phys. Rev. D 34 (Jul, 1986) 75-84.

[86] R. Blankenbecler and S. J. Brodsky, "Unified description of inclusive and exclusive reactions at all momentum transfers," Phys. Rev. D10 (Nov, 1974) 2973-2992.

[87] J. F. Gunion, "Short distance counting rules for low pT fragmentation," Phys. Lett. B88 (Dec, 1979) 150-156.

[88] J. Kuti and V. F. Weisskopf, "Inelastic Lepton-Nucleon Scattering and Lepton Pair Production in the Relativistic Quark-Parton Model," Phys. Rev. D4 (Dec, 1971) 3418-3439.

[89] F. James, "Function Minimization and Error Analysis." CERN Program Library Long Writeup D506, 1998.

[90] J. Marshal et al., "NC Background Studies." MINOS internal note $3245,2007$.

[91] J. Marshal, "A Data-Driven Correction to the MC NC Background." MINOS internal note 3307, 2007.

[92] C. Smith, J. Thomas, and P. Vahle, "F/N ratio method position paper." MINOS internal note 1545, 2006.

[93] MINOS Collaboration, "Preliminary Results from MINOS on Muon 
Neutrino Disappearance Based on an Exposure of $2.5 \times 10^{20} 120 \mathrm{GeV}$ Protons on the NuMI Target," arXiv:0708.1495 [hep-ex].

[94] C. Andreopoulos et al., "Updated Cross Section Model Uncertainties for the Charged Current Analysis." MINOS internal note 2989, 2007.

[95] MiniBooNE Collaboration, "A Search for Electron Neutrino Appearance at the $\Delta m^{2} \sim 1 \mathrm{eV}^{2}$ Scale," Phys. Rev. Lett. 98 (2007) 231801, arXiv:0704.1500 [hep-ex].

[96] L. A. Ahrens et al., "Comparison of Narrow Band and Wide Band Neutrino Beams in the Search for Muon-Neutrino $\rightarrow$ Electron-Neutrino Oscillations," Phys. Rev. D36 (1987) 702-706.

[97] CHARM Collaboration, F. Bergsma et al., "A Search for Neutrino Oscillations," Z. Phys. C40 (1988) 171.

[98] F. Dydak et al., "A Search for Muon-neutrino Oscillations in the Delta m**2 Range 0.3-eV**2 to 90-eV**2," Phys. Lett. B134 (1984) 281.

[99] V. D. Barger, J. G. Learned, S. Pakvasa, and T. J. Weiler, "Neutrino decay as an explanation of atmospheric neutrino observations," Phys. Rev. Lett. 82 (1999) 2640-2643, arXiv:astro-ph/9810121.

[100] G. L. Fogli, E. Lisi, A. Marrone, and D. Montanino, "Status of atmospheric $\nu_{\mu} \rightarrow \nu_{\tau}$ oscillations and decoherence after the first $\mathrm{K} 2 \mathrm{~K}$ spectral data," Phys. Rev. D67 (2003) 093006, arXiv:hep-ph/0303064. 
[101] NO $\nu$ A Collaboration, "NO $\nu$ A: Proposal to Build an Off-Axis Detector to Study $\nu_{\mu} \rightarrow \nu_{e}$ Oscillations in the NuMI beamline." FERMILAB-PROPOSAL-0929.

[102] R. Burns, "Determination of the neutrino flux." CERN Informal Conference on Experimental Neutrino Physics, CERN Yellow Report 65-32, 1965.

[103] D. Bloess et al., "Determination of the neutrino spectrum in the CERN 1967 neutrino experiment," Nucl. Instrum. Meth. 91 (1971) 605-612.

[104] H. W. Wachsmuth, "The neutrino spectrum for the CERN 1967 neutrino experiment." Proceedings of the CERN Neutrino Meeting, CERN Yellow Report 69-28, 1969.

[105] V. B. Anikeev et al., "Total cross-section measurements for muon-neutrino, anti- muon-neutrino interactions in $3-\mathrm{GeV}-30-\mathrm{GeV}$ energy range with IHEP-JINR neutrino detector," Z. Phys. C70 (1996) 39-46.

[106] A. P. Bugorsky et al., "Measurement System of Muon Fluxes for Neutrino Experiment at the IHEP Accelerator," Nucl. Instr. Meth. 146 (1977) 367.

[107] P. S. Auchincloss, Measurement of the Total Cross Section for Neutrino - Nucleon Interactions. PhD thesis, 1987.

[108] S. Kopp and L. Loiacono, "Muon Monitor Data Sets for Study of Horn Current Scans." MINOS internal note 2404, 2006. 
[109] J. McDonald et al., "Ionization chambers for monitoring in high-intensity charged particle beams," Nucl. Instrum. Meth. $\mathbf{A 4 9 6}$ (2003) 293-304.

[110] R. M. Zwaska et al., "Beam tests of ionization chambers for the numi neutrino beam," IEEE Trans. Nucl. Sci. 50 (2003) 1129-1135, arXiv:hep-ex/0212011.

[111] I. Abt and B. Jongejans, "An Absolute Calibration of the Solid State Detectors in the Narrow Band Neutrino Beam at CERN," Nucl. Instr. Meth. A235 (1985) 85.

[112] E. Heijne, "Influence of muon induced secondary radiation on the muon flux measurement in the CERN neutrino beams." CERN-79-4, 1979.

[113] J. Koskinen, "Monte Carlo Description of the Geometry of the NuMI Beam for the Muon Monitors." MINOS internal note 2405, 2006.

[114] R. Keisler, "Neutron Induced Ionization in the NuMI Beam Monitors," 2005. Undergraduate Thesis. 


\section{Vita}

Žarko Pavlović was born on July 9, 1977 in Zagreb, Croatia to Milica and Željko Pavlović. He received an advanced graduate level degree in Physics (Diplomirani Inžinjer Fizike) from the University of Zagreb, Croatia in the spring 2003. He started working with Professor Sacha Kopp in the High Energy Physics Laboratory in the summer of 2003 and enrolled in the Graduate Program in Physics at the University of Texas at Austin in the fall of the same year. Over the course of his graduate studies, he earned the Miller Fellowship in 2007, the travel grant to attend the United States Particle Accelerator School in the winter 2004, and the Professional Development Award to attend the American Physical Society Meeting in Jacksonville, FL in the spring 2007.

Permanent Address: 3607 Greystone dr. 1233,

Austin, TX 78731

This dissertation was typeset with $\operatorname{AT}_{\mathrm{EX}} 2 \varepsilon^{1}$ by the author.

\footnotetext{
${ }^{1} \mathrm{AT}_{\mathrm{E} X} 2_{\varepsilon}$ is an extension of $\mathrm{ATT}_{\mathrm{E}} \mathrm{X}$. $\mathrm{LAT}_{\mathrm{E}} \mathrm{X}$ is a collection of macros for $\mathrm{T}_{\mathrm{E}} \mathrm{X}$. $\mathrm{T}_{\mathrm{E}} \mathrm{X}$ is a trademark of the American Mathematical Society. The macros used in formatting this dissertation were written by Dinesh Das, Department of Computer Sciences, The University of Texas at Austin, and extended by Bert Kay, James A. Bednar, and Ayman El-Khashab.
} 